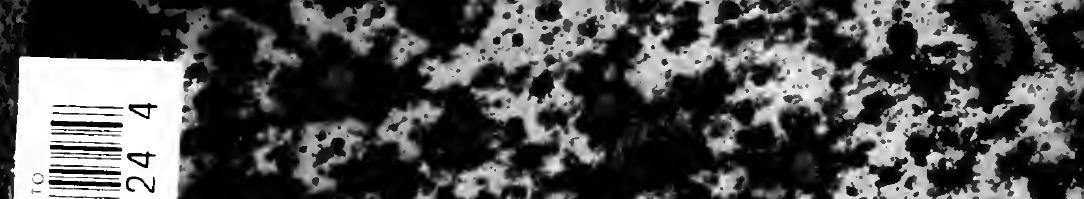

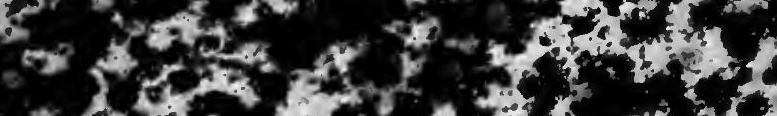

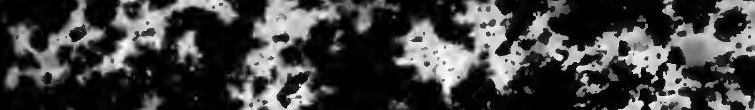

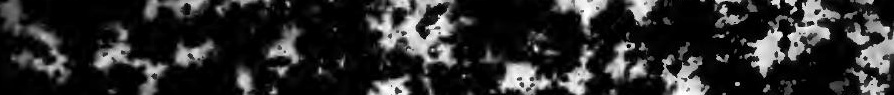
\& $2=0$ की

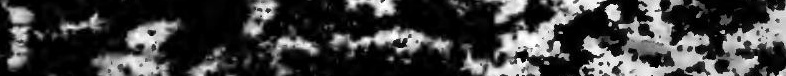

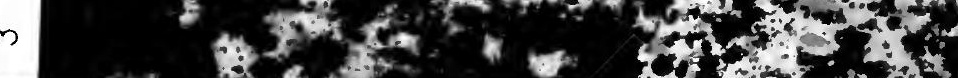

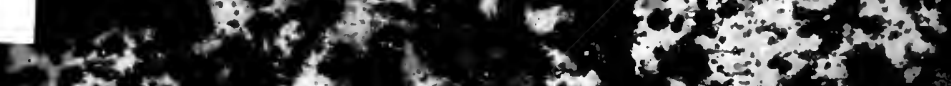

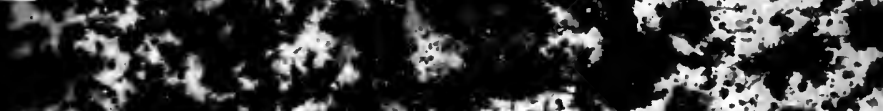

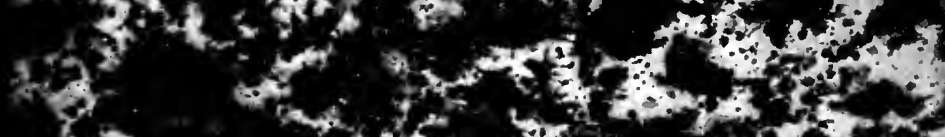
1.28

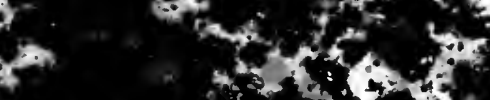

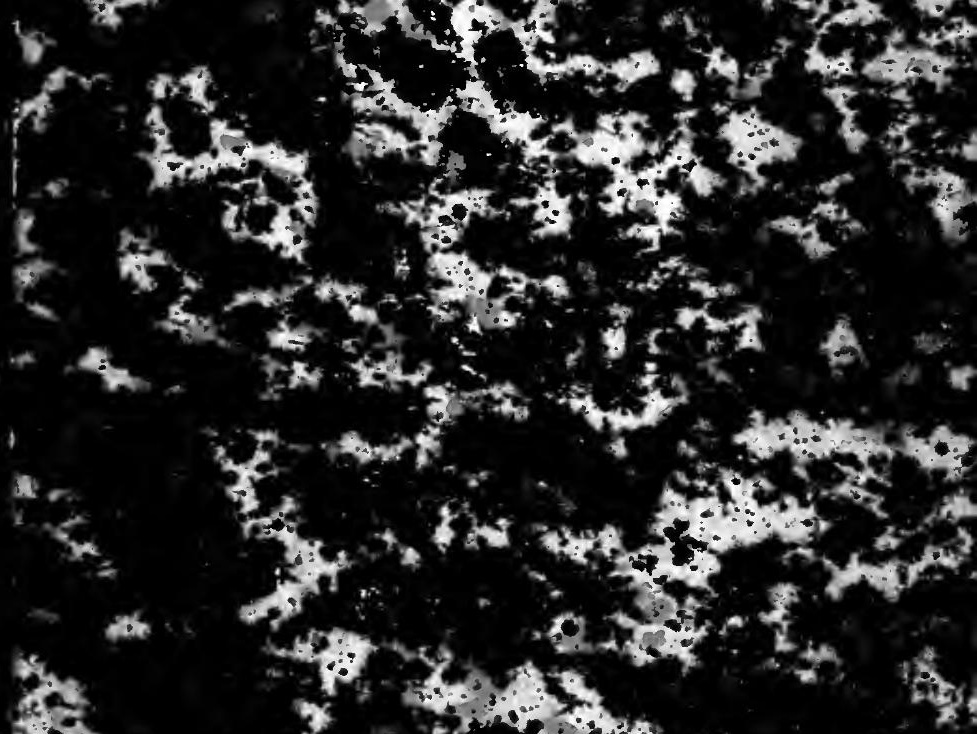

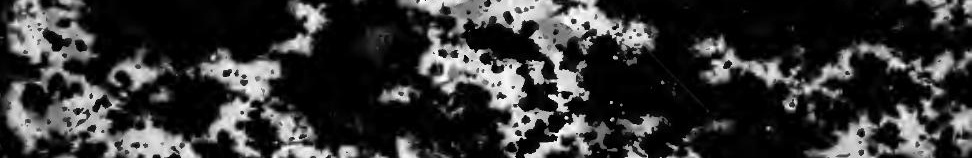

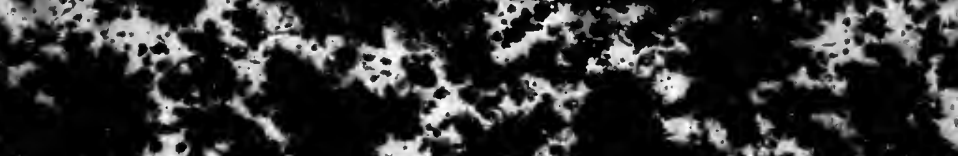

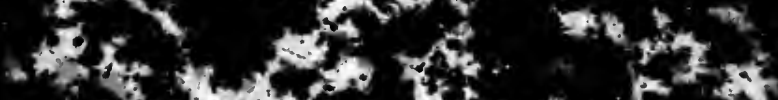

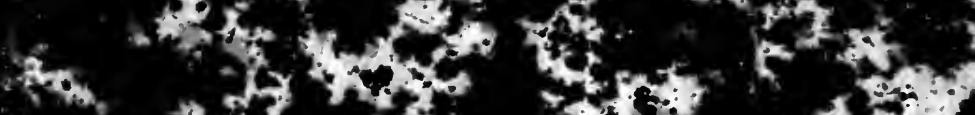

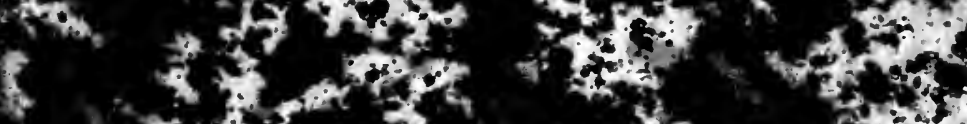
3.

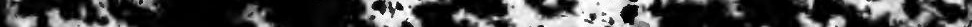




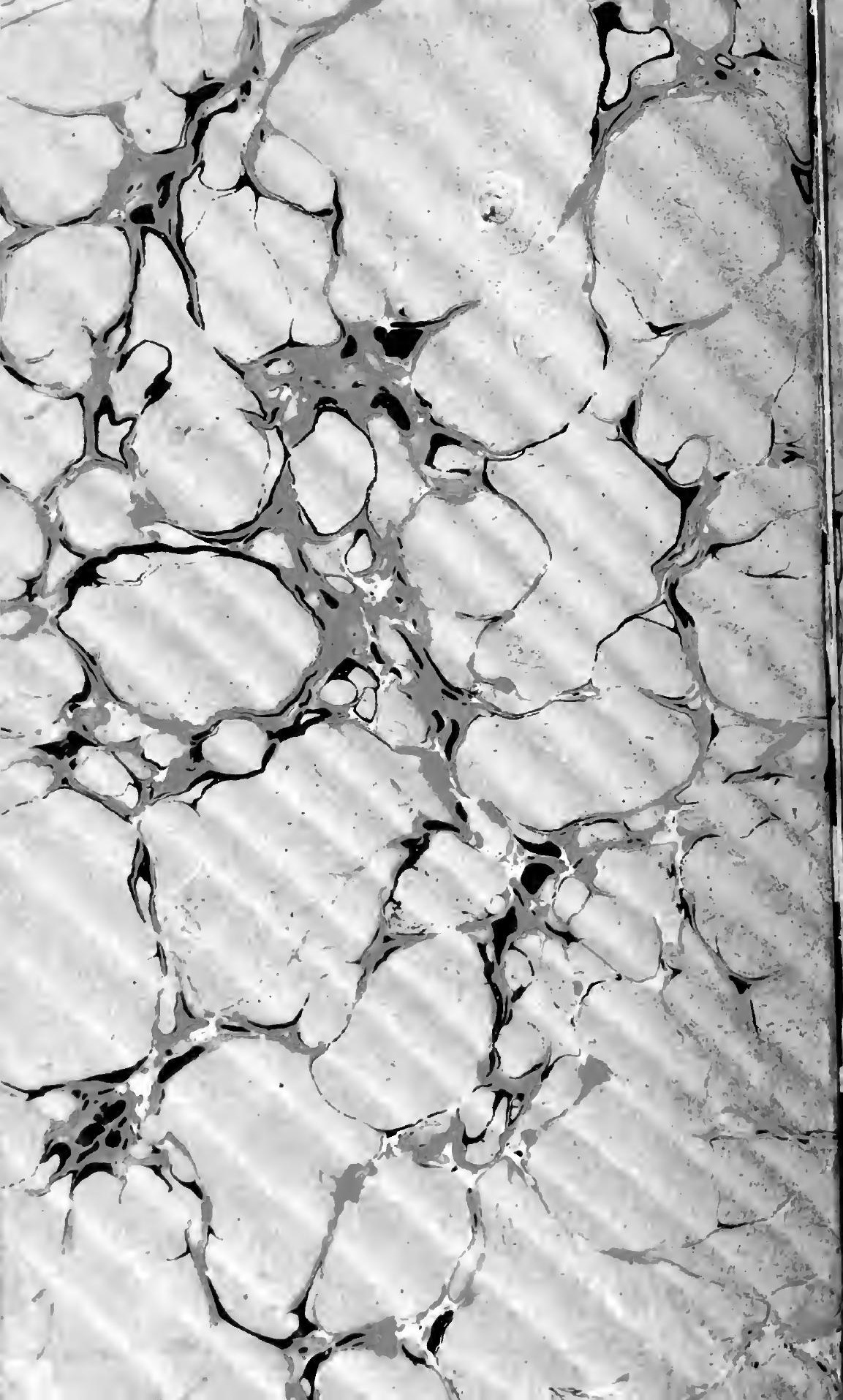





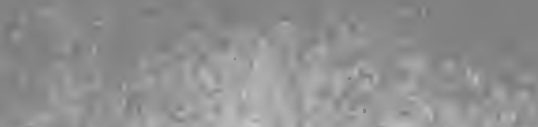

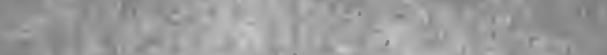

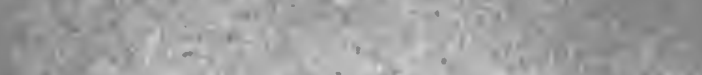

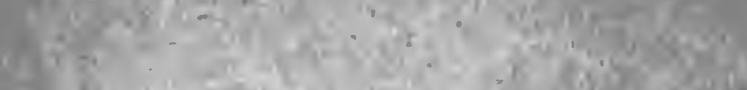

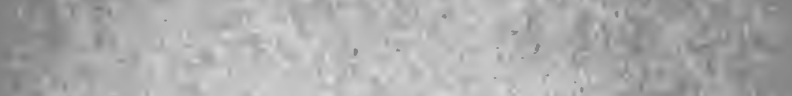

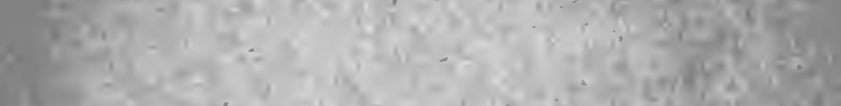

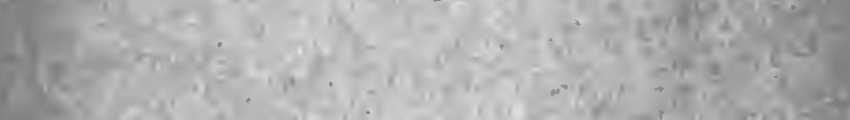

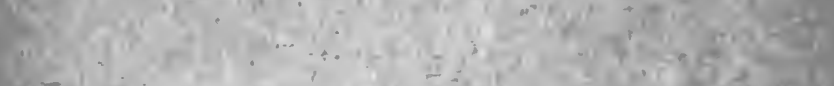

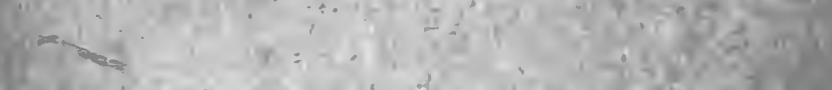

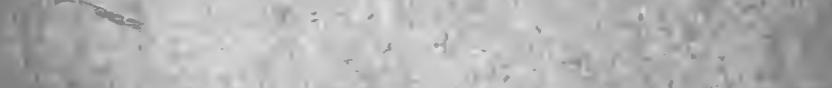

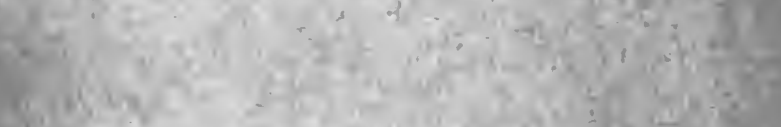

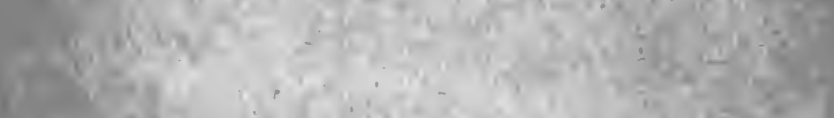

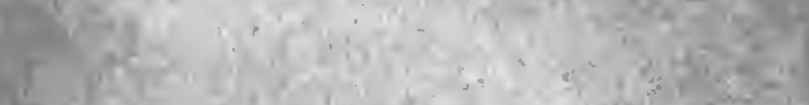

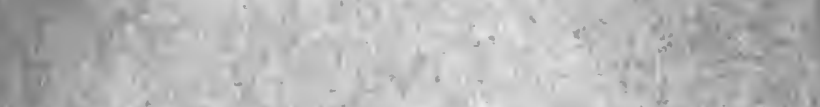
y

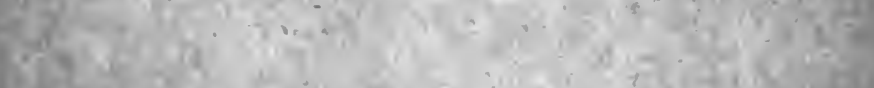
W.

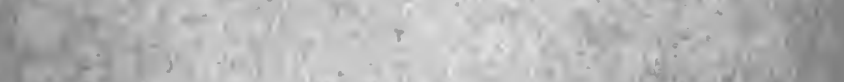

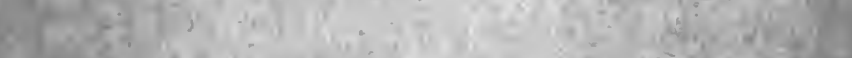

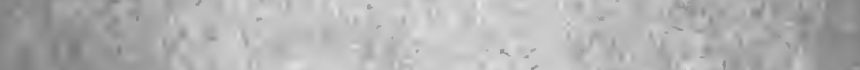

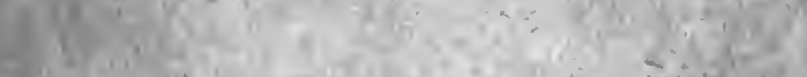

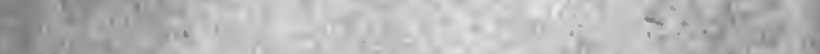

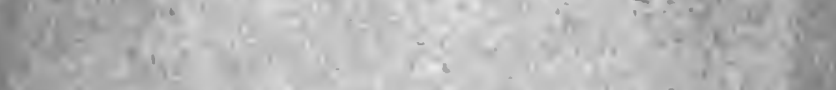

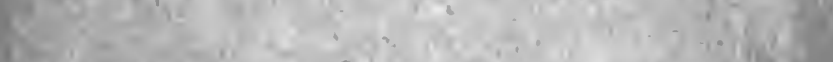

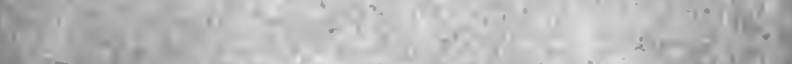

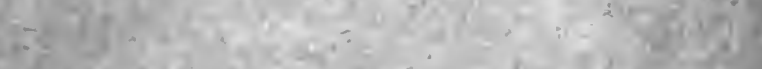

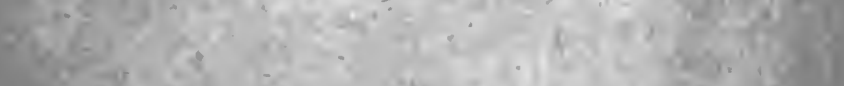

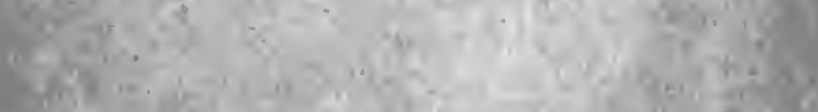

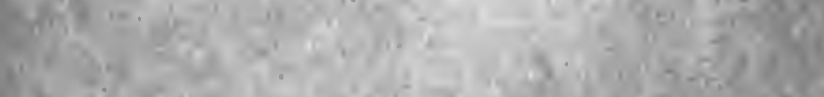
W.

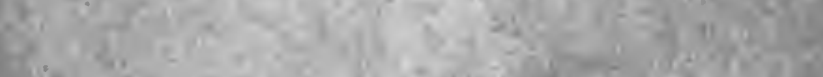

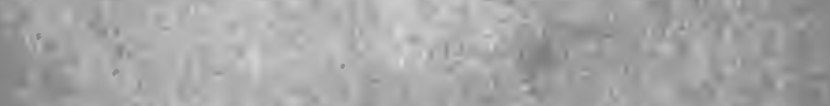

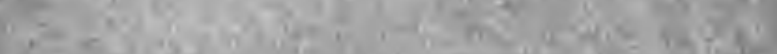

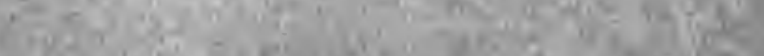

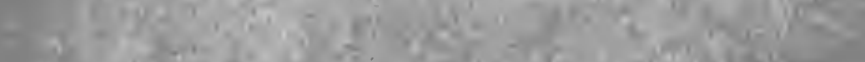

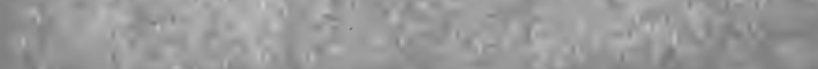

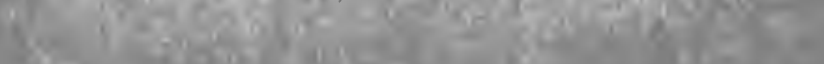
Pas

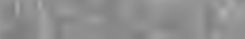



A Mrensins Beaussire

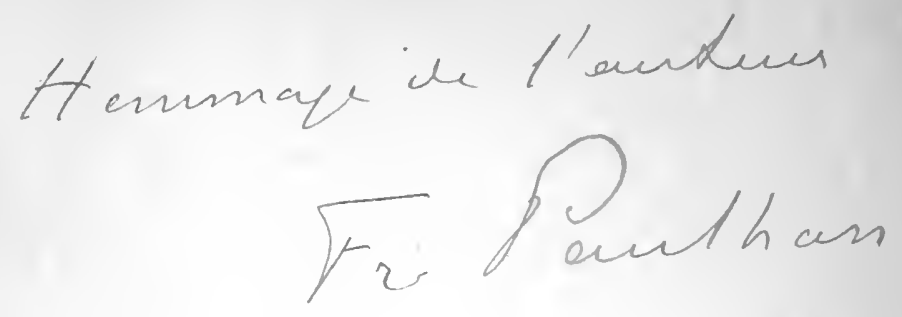

L'AC'IVITE NENTALE

ET LES ÉLÉMENTS DE L'ESPRIT 


\section{A LA MEME LIBRAIRIE}

\section{$-2000000$ \\ AUTRES OUVRAGES DE M. FR. PAULHAN}

Lu Physiologic de l'Esprit, I vol. in-32, de la BmLlotnique vTILE, arec figures dans le texte, cart. à l'anglaise.... 1 franc.

Les Phénomènes affectifs ct les lois de leur apparition essai de psychologie jénerale, 1 vol, in-18, de la Biblotmęove de Pmilosorme rostenporalng................. $2 \mathrm{fr}, 50$ 


\section{Psych. P326a}

\section{L'ACTIVITÉ MENTALE}

ET

\section{LES ELEEMENTS DE L'ESPRIT}

PAR

FR, PAULHAN

\section{PARIS}

ANCIFNNE LIBRAIRIE GERMER BAILLIËRE ET C $C^{\text {ic }}$ FÉLIX ALGAN, éditeur 108, Boulevard saint-Germain, 108 1889 


$$
\frac{232 k}{261411890}
$$




\section{L'ACTIVITÉ MENTALE}

\section{ET LES ÉLÉMENTS DE L'ESPRIT}

\section{N TRODUCTION}

A chaque instant notre esprit travaille. On peut dire, si l'on fait abstraction du temps du sommeil qui peut donner lieu à des discussions, que son activité est continuelle : sans cesse des phénomènes psychiques s'éveillent en nous, se déreloppent, disparaissent après en avoir suscité d'autres, sans cesse des tendances entrent en jeu, et, à notre comnaissance ou á notre insu, déterminent ou modifient le cours de nos actes, de nos pensées, de nos désirs, de nos perceptions mème, sans cesse des combinaisons nouvelles se forment et d'autres combinaisons se défont, simultanément et successivement un nombre indéfini de faits se p'ròduisent, s'associent, se repoussent, et cela constitue la vie de l'esprit, analogue à la vie de de-l'organisme, résultant comme elle de l'activité d'innombrables éléments, soumise commè clle à dés lois générales, expression de l'ordre abstrait des phénomènes.

Ces lois sont de généralitè variable; quelques-unes s'appliquent à tous les phénomènes sans exception, elles sont l'expression la plus abstraite de la vie psychique, elles ne l'épuisent pas, elles ne peuvent l'cxpliquer complètement, mais elles indiquent les relations essentielles et les plus générales des phénomènes et permettent de se faire une idée précise de la naluue de l'esprit. Les lois générales se retrouvent laus tous les phénomènes de l'activité psychique, les différentes classes de faits psychiques, la perception, l'idée, la volonté, les tendances 
nous en olfrent des formes diffirentes en mime temps qüils nons presentent a crie l'elles certaines atutros lois moins genébles fui ne se lalpoltent qu’i eux seuls.

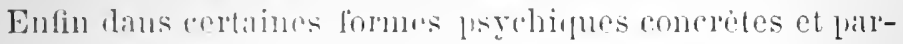
ticulieres. l'amome du mal, bar exemple. on la patrole

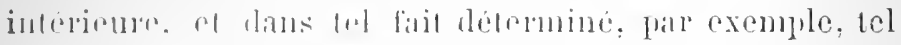

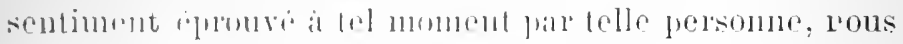
relrourous a rote des lois les plus générales et des lois moins gringriles, dautres lois phus particulières encore. In les luts de re tarail est lémde des lois les plus génerales do l’esprit ef le la maniè dont elles se manilestrent pour domner nassince anx prineipales classes de phénomenes psyrhiques: - la perception, le raisonnement: le juyement, la volonte. etc., nous apparaitront ainsi comme des formes paticulieres, dont nous aurons a determiner les caractères frécis et les éléments qui en sont en ruelipe sorte lit matiore, des lois ginérales de lauclivité mentale.

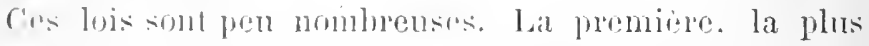

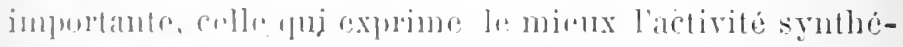

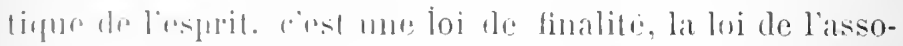

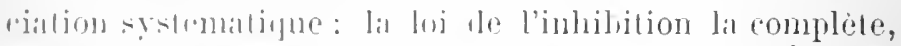

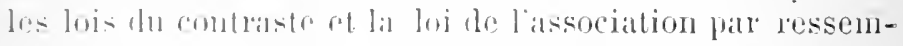
blance pondignite - yui ast loin d'avoir l'importance

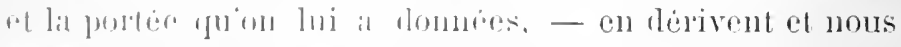
en montront los anuplitations. Mais ces lois ne penvent

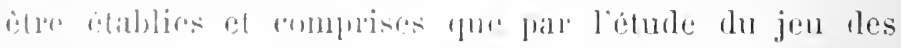

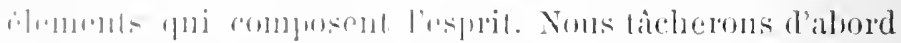

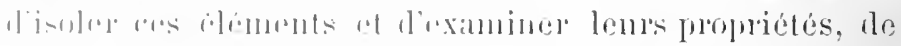

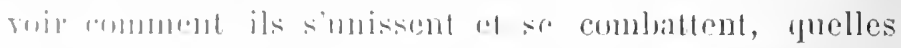

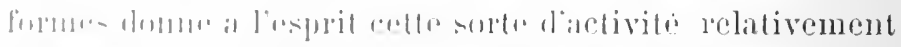

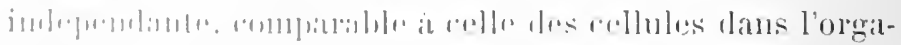

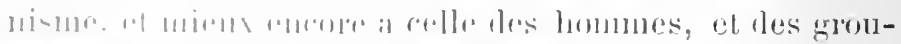

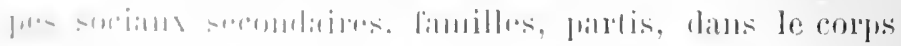

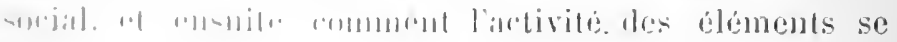


rattache à l'activité du tout et à. ses différentes formes, bien que celle-ci soit tout autre chose que la somme et la juxtaposition des activités qui la composent."

Et ceci nous conduit à une troisième étude : l'esprit est ou tend à être un tout unifié et coordonné. Nous aurons à l'envisager à ce point de vue synthétique. Etude de la vie des èléments psychiques, ètude des lois de l'activité mentale et des formes générales des phènomènes psychiques, étude de l'esprit, voila les trois parties dont se compose ce travail. 



\title{
PREMIÉRE PARTIE
}

\section{LA VIE DES ÉLÉMENTS PSYCHIQUES}

\author{
CHAPITRE PREMIER
}

\section{Les Éments psychiques.}

Tout fait psychique est un système, une synthèse d'élẻments plus ou moins bien coordonnés : que nous prenions une sensation de l'ouïe ou de la vue, une idée, un sentiment, une volition, nous trourons toujours que ce phénomène est complexe et que les éléments en sont coordonnés de quelque manière; c'est une loi de l'esprit qu'aucun phénomène psychique ne peut se produire avec des éléments totalement incoordonnés, - et c'est à la coordination mème que le phénomène doit son unité. Nous étudierons plus tard en détail cette loi de systématisation et ses diverses formes - mais nous ne l'envisageons ici qu'au point de vue de la manière dont un fait psychique est composé.

Les phénomènes psychiques diffèrent beancoup sous le rapport de la complication, de la complexité, et les éléments seront de nature diverse selon le composé qu'on examinera : dans l'audition d'un accord, par exemple, ces éléments sont des sensations auditives; dans la vision d'un tableau ces éléments sont des sensations visuelles, auxquelles s'associent dles images et des idées; dans la volition ces éléments sont des idées, des images motrices, 
des impulsions, ele. Yous examinerons phus loin en détail ces dillerentes dasses de fails jsychipues; pour le monent nous devons étulier la vie des élements psoreluiques el licher de freciser, non pits lant comment ces éléments se groupent, que lexistence des éléments psychiques et ce rüil faut cntendre par ce mol.

Il y a plusients sortes d'bléments pischiques - comme il y plusieurs sortes d'éléments sociologriques. Fst-ce lhomme, estece la fimmille qui represente l'unite dont la société est faite? C'est I'un el l'autre, el cela rarie selon l'éporgue el la societé, selon que la famille est plus ou moins forte, selon rue İindividu est plus ou moins indépendant, on peut aroir des déments sociaux dirers, les l'anilles peurent aussi s'agglomérer en tribus, les individus en associations diverses, en partis, - toules ces formes secondaires de lit vie soeiale ont une vie en quslyue sonto intépentante, ou peuvent l'avoir en eertains

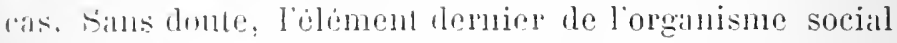
est lindividu - mais antre l'individu et le tout, il y a differentes prolites olatulsalions, differents systemes noins complexes pin jouent leur lóle daus lia vie du tout, 'pui sunt tre rériblules éliments socianx, les uns plus conplexes, les antres noins. ayint leurs tendances pro-

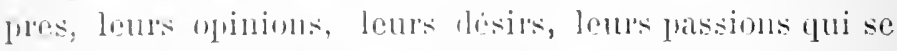
comblinent on se romblatlent al doment il l'ensemble qui

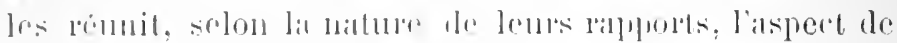

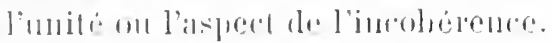

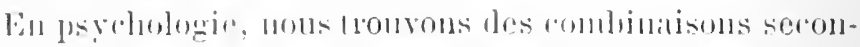

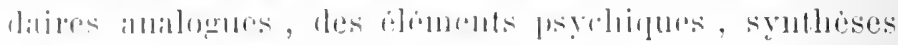

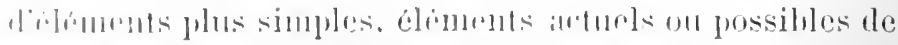

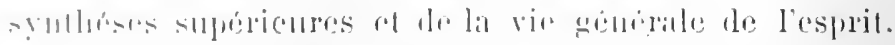

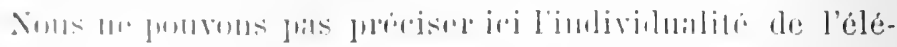

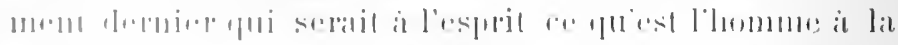

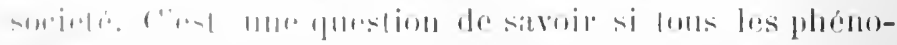

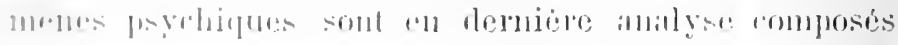


d'un élément unique, le "choc nerveux » de M. Spencer ", qui, quoique complexe à certains égards, car il correspond forcément à un état physiologique assez compliqué, serait simple, irréductible au point de vue mental (comme l'homme est simple au point de rue social, tout en étant. composé au point de vue psychologique), et formerait l'élément primordial de notre vie psychique. Celte question ne parait pas encore complètement résolue, et nous n'avons pas besoin de lui domner une solution. Les lois des combinaisons chimiques ne varient pas, si l'on suppose que tous les corps simples sont formés d'atomes semblables, diversement groupés, ou si l'on pense que les dernières particules dı plomb, par excmple, diffèrent de celles du chlore, ou si encore on pense que la question. est insoluble.

Mais si nous ne pouvons définir l'élément primordial de l'esprit, nous constatons la présence d'éléments de l'esprit très différents par leur complexité, comme les élé. ments sociaux dont nous avons parlé, et même quelque peu variables dans leur forme - et en cela ils ressemblent encore à ces mèmes éléments, - mais bien réels, et que l'on peut, en général, décomposer par l'analyse en éléments plus simples, et réunir par la synthèse en élénients plus complexes. Une idée, une perception, un raisonnement, une tendance sont ainsi des complexus d'éléments plus simples en mème temps qu'ils peuvent ètre des éléments de systemes plus complexes.

Une belle expérience de synthèse des éléments psychiques est celle par laquelle M. Helmholtz al montré la nature des sons des voyelles et du timbre des sons en général. M. Helmholtz, à l'aide d'un ingénieux appareil, fait vibrer it la fois divers diapasons accordés suivant un son fondamental et ses différentes harmoniques; selon les diversès

1. Voyez H. Spencer. Principes de psychologie, vot. I. Trad. franc. Ribot et Espinas. 
combinaisons que l'on produit, on fait apparaitre des voyelles diverses. Avec huit diapasons, Helmholtz a reproduit les sons ou, o, eu, avec quatre autres diapasons, il reproduit le son a, etc: La découverte de la nature du timbre des sons permet de considérer chaque instrument de musiquc, chaquc voix différente, comme donnant une synthèse d'impressions auditives, le sons divers réunis en un tout parfaitement unifié.

Mais à un autre point de vue chacun des sons produits par chaque diajason qui est un élément du son synthétique est aussi un complexus. Il résulte d'une quantité d'impressions produites par chaque vibration du diapason. Je n'insiste pas sul l'analyse des sensations auditives qui est bien connue '. Nous voyons ainsi une série d'impressions inconscientes se combiner pour former unc sensation et une certaine quantité de sensations se combiner pour former une sensation plus complexe et nous arous la uotion d'éléments plus ou moins complexes dont le jeu constituc les diverses apparences de l'activité mentale. Que d'ailleurs. les composés psychiques puissent, comme les composés chimirues, différer par leurs apparences de leturs composants, c'est un point dont il serait superlu de chercher longuement i montrer la réalité après les belles analy'ses de MM. Spencer, Taine, Bain ct Wundt et les remarfuables syntheses de M. Helmholtz; nous aurons d'ailleurs plus d'une fois l'occasion de le constater, et j'aurai í essayer d'en donner an moins une explication particlle.

Mais les plhénomènes de groupement et de systématisation rue nous remarfuons a propos le sensations et de perceptions, nous les retronyons dans tons les domaines le lat vie pischipuc - et elles sont plus faciles à remarquor dans les faits intellechucls ou aflectifs plus complexes. - Je suis chro noi me preparant a sortir; on me

1. Voyez Taiue. De l'intelligence, vol. I. 
dit : "Le temps est sombre, il va pleuvoir ; " ces mots éveillent en moi un système déterminé d'éléments psy* chiques, qui, selon qu'il s'accordera plus ou moins avec mon état mental présent, aboutira à ces mouvements coordonnés qui constituent l'acte de prendre mon parapluie ou à ces mouvements coordonnés qui constituent le fait de vérifier ce qu'on me dit ou de demander une confirmation. Si, étant allé quelques moments avant à la fenètre, j'avais vu le ciel clair et senti un air vif, je serais surpris et le systène éreillẻ en moi par les paroles qu'on m'a dites se combinerait arec cet état antėrieur pour me pousser á aller encore à la porte ou à la fenètre vérifier le temps; si j'avais vu le ciel chargé de nuages, le système nouveau aboutirait à l'acte sans trouble; de mème, si je suis préoccupé, je puis obéir machinalement ả la suggestion sans vérification ni doute.

Nous voyons dans des faits comme celui-là des éléments psychiques à l'œuvre, ils s'attirent, se repoussent, s'associent et se séparent, on peut suivre la série des phénomènes à partir d'un phénomène initial. Souvent les faits se compliquent encore darantage. Ouvrons un livre et prenons une phrase quelconqque, celle-ci, par exemple: "La science des étoiles a eu son berceau près du berceau mème du genre humain, dans les belles plaines de Sennaar "Ces mots, mis à la suite les uns des autres, éveillent en nous diverses idées qui s'associent et forment un tout; il y a dans le fait de lire une phrase en la comprenant une véritable synthèse psychique, des mots, des sons, des images, des idées s'associent dans un acte unique, convergent vers un mème résultat, et le sens total qui provient de l'association de ces différents éléments, est un système et non le résultat de la juxtaposition des mots, des images et des idées, mais l'ordre mẻme que prennent particulièrement ces éléments en ce cas. Et la phrase entière ainsi comprise peut à son tour entrer comme 
élément dans bien des combinaisous diverses. Nous pouvons, par exemple, la faire entrer dans une série de phrases résumant l'histoire de l'astronomie, mais nous pourrions aussi la faire enfrer dans une etude de la civilisation chaldéenne. De plus, chacun de ses éléments peut devenir le point de départ d'un nouveau complexıs, d'une nouvelle séric. L'esprit peut s'irrèter sur la phrase et travailler sur tel ou tel de ses éléments; nous jourons remarquer, par exemple, cette image par laquelle le licu où nait et commence à se développer une science s'appelle un berceau, ainsi que le lieu oì commence à se dévolopper un homme. Nous pourons remarquer, à un autre point de rue, qu'il est bien douteux que l'humanite ait eu pour berceau les plaines de Sennaar, nous pouvons examiner la contexture de la phrase, en faire l'analyse logique, nous pourons prendre claque mot et en faire le point de départ d'une longue série d'éléments psychiques constituant un commentaire plilologirue, nous pouvons passer par une série d'idées éveillées chacune par la précédente et arriver ainsi a des considírations qui paraissent n'avoir plus aucun rapport avec le point de depart. Mille autres combinaisons d'idées on d'inages sont possibles, qu'il serait trop long et d'ailleurs inutile de rechereher.

Dans tous ces cas nous entrevoyons le jen des éléments psychiques - c'est d'ailleurs à ce seul point de vue que nous devons envisager a present tous res phénomènes complexes. Il s'agit de hien voir fue laactivité de l'esprit est composéc de l'activité d'un certain nombre d'éléments varies, idées, tenlances, images, perceptions qui s'attirent et se repoussent, s'associent et se sepprent, se combinent et se dissolvent. Le mème élément pent passer l'un rompose a l'anlre, il peut so lecomposer en des eliments plus simples qui entremont dans de nouvelles syutheses d pourront rester séparés. Ces phénomenes sont tout it fint andogues a ceux que nous trouvons 
dans la vie sociale. On voit, par exemple, des groupes parlementaires se joindre pour voter sur telle ou telle question, tantôt à tel groupe, tantôt à tel autre, on les voit parfois se dissoudrc et les éléments qui les composaient entrer dans des groupes différents, et ne plus se retrouver ensemble. On voit des ouviers, des paysans se réunir, appelés par la loi, dans un régimeut, passer d'un régiment à un autre, obéir aux mêmes chefs, participer aux mèmes actes, combattre a comme un séul homme ". puis, le temps du service fini, rentrer chez eux, reprendre leur mettier, quitter leurs habitudes militaires, ne plus revoir leurs compagnons d'armes. Tout fait social est. cridemment la syuthèse des actes combinés d'un certain nombre d'hommes, actes tendant vers un mème but ou vers des buts harmoniques - de mème tout fait psychologique est la synthèse des activités combinèes d'un certain nombre d'éléments psychiques. Et les éléments psychiques, comme les éléments sociaux, peuvent aussi bien se combattre que s'associer, et se séparer que s'unir. Je ne veux pas dire qu'il y ait identité complète entre la vie psychique et la vie sociale, mais il existe entre elles des analogies profondes, la psychologie n'a pas moins besoin d'ètre rapp:ochée de la sociologie que de la physiologie. La tendance a expliquer la psrchologie par la physiologie domine en ce moment, mais si nị la sociologie, ni la physiologie ne peuvent précisément expliquer les phenomènes psychiques, ces deux sciences nous aident certainement à les comprendre, et si la physiologie leur donne une base, la sociologie leur donne un sens. Quant a la ressemblance particulière que nous venons d'indiquer, nous allons voir, par l'étude de la vie des éléments psychiques, qu'elle est parfaitement réelle.

- Nous avons vu que les elements sont, comme les groupes sociaux, de complexité différente, qu'ils peuvent se rattacher à des complexus rariables d'autres éléments, que 
les elements plus simples pulils comprenuent penrent eux-mèmes entrer lour à lonr, el quelyuefois simultanément, dans des combinaisons diverses. - Ces composés psychiques rarient évidemment splon les bements qui les forment et selon la forme propre du'tils affectent. Mls varient, par exemple. scelon qu'ils sont lormés d’idées, de sensations ou d'inages de telle on telle nalme, et d'inpulsions mulrices, ele, ils variont anssi selon rue les éléments qui les constituent se condoument d’me manière ou d'une autre, dans un sens on dans l'antre, selon telle ou lelle orientation - la forme de lat romlinaison doit dépendre au reste, à quelque degré, de la nature mème des composants.

On peut. pont étudier le composé psychique, l'élément de lesprit, se plater i deux points de rue corrélatifs qui se completent on se suppléent l'un l'autre. celui de lanalye et celui do la synthese naturelle on experimentale. Dans le fremier cas, on prenul nu romposé tout formé, ot l'on tiche l'en montrej les éléments; daus le

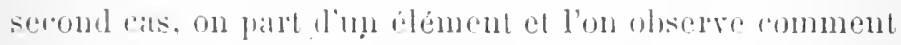
il se combine avec d'antres pom andiver an compose.

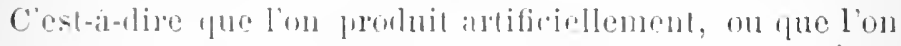

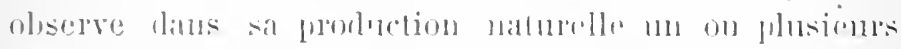

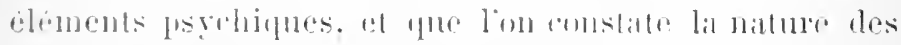

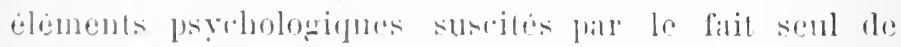

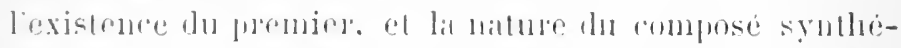

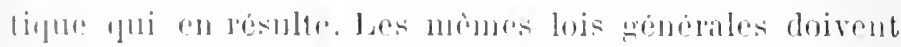

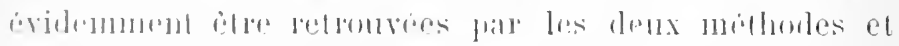

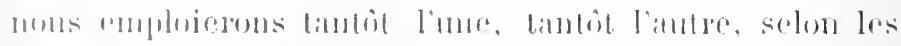

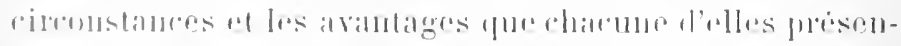

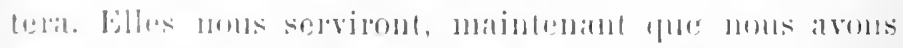

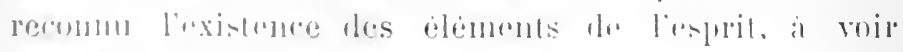
communt ils agissent. 


\section{CHAPITRE II.}

\section{L'activité indépendante les éléments psychiques.}

On a dit que l'activité de l'homme pouvait se résumer par ces deux formules : chereher son intérêt, éviter ce qui y est contraire. Cela n'est pas absolument exact, mais une formule de ce genre serait beaucoup plus juste appliquée aux éléments qui composent l'esprit qu'appliquée à l'esprit lui-même.

La loi d'association systématique, dont nous étudierons plus loin le fonctionnement, s'énonce ainsi : un fait psychique tend à s'associer et à susciter les éléments qui peurent s'unir avec lui pour une fin commune; la loi d'inhibition systématisée s'énonce ainsi : un fait psychique tend à empècher de se produire, à empêcher de se développer ou à faire disparaître les éléments qui ne sont pas susceptibles de sunir a lui pour une fin conmune. Ces deux lois paraissent résulter des propriétés mèmes des èléments de l'esprit; nous allons voir en effet comment ces éléments sont susceptibles d'agir d'une manière relativement indépendante, conment les opérations de l'esprit résultent de la mise en jeu de leur's affinités positives et négatives, de leurs désirs et de leurs répulsions, et comment l'esprit lui-mème, s’il existait à l'état parfait, à l'état de personnalité parfaitement coordonnée et unifiée, pourrait ètre considéré, en quelque sorte, comme le plus complexe des éléments, celui qui synthétiserait tous les autres.

La tendance à l'association systematique et à l'inhibition systématisée est une propriété des éléments psychiques : telle est la nouvelle loi par laquelle on peut compléter les deux autres, par rapport au but que nous poursuivons à présent, j'entends jar là que dès qu'un élément 
psychique existe, il tend par le seul fait de son existence, a en susciter certains autres, a en inhiber d'autres aussi. Ce n'est pas l'ensemble le l'esprit, s'il n'est pas lui-même bien coordonné; qui determine l'apparition on l'arrèt des phénomènes. ce sont les éluents. Cost-it-dire que cest ce qui est dejal systematisé dans l'espuit qui tend a aceperir une systématisation phus complète. Si c'est me sensation, elle temlat a ereiller des idees, a susciter des actes particuliers, préris, appropries, si c’estume tendance générale, une haljitude intellectuelle. une organisalion mentale préctalblie, elle tendra à faire interpréter de telle on telle manicre les sensations qui arrivent a l'esurit. Chaque élément, éest-it-dire chaque systeme agit pom soi, de lis provient un morle de fonctionnement de l'espuit qui peut allur en certains cas jusqu'au dedoublement de la personnalite, quand des systemes différents, non halmoniques coexistent en nous; ahacun travaille pour lui-mème el tent i s'assimiler de nouveaux èlements psychipues.

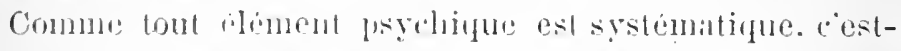
it-dire compose dólements qui ronvergent vers un même lósultat, romme lorsforr lat finalitó nexiste gas dans

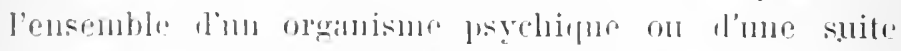
d'actes, on d'mu laisonnemont. on l'me passion fet en ce ras ces falits ur sont pas rebllement des cléments psychi-

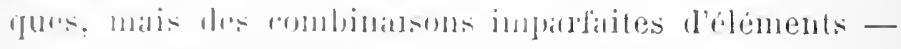

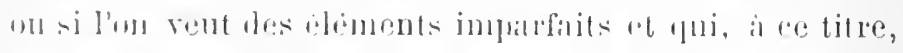

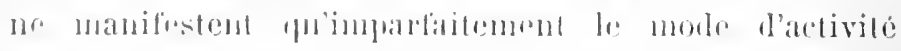

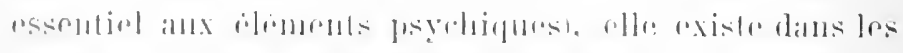

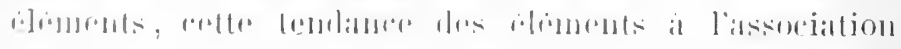

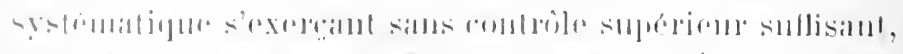

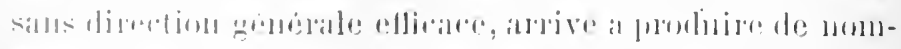

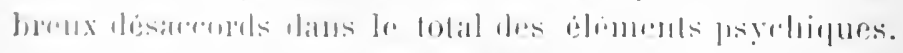

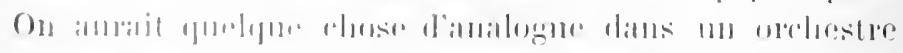

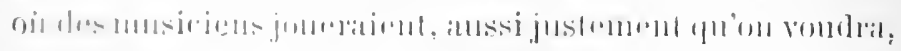

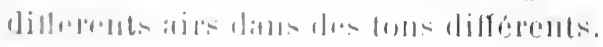


La vie organique nous offre un certain nombre de systèmes, d'actions coordonnées relativement indépendants, par exemple, les battements du ccur produits par les ganglions nerveux qui se trouvent dans les parois de cet organe. Le cuur arraché de la poitrine peut continuer à battre - un fait analogue a éte observé chez l'homme mème: une heure après la mort, on a vu le cœur d'un supplicie présenter encore des contractions rhythmiques. De mème pour la respiration. On sait que le centre principal de la respiration se trouve dans la moelle allongée, dans le bec du calamus scriptorius; ce centre ne parait pas fonctionner par voie réflexe, tout en subissant d'ailleurs par voie réflexe certaines influences, il agit automatiquement selon la nature du sang qui l'irrigue, selon que ce sang est plus ou moins riche en oxygène ou chargé d'acide carbonique ; or, " on peut, dit M. Richet, supprimer l'encéphale, couper la moelle au-dessus du bulbe, sectionner les deux pneumo-gastriques, autrement dit supprimer toutes les voies centripètes sans empècher le système incitateur d'avoir lieu."

Dans la vie psychologique proprement dite, nous trouvons des faits absolument analogues - on pourrait étudier successivement à ce point de rue toutes les formes de la rie mentale, depuis les sensations jusqu'au raisonnement, jusqu'i la volonté, toutefois, je me contenterai, pour le moment, de prendre quelques exemples qui me paraissent devoir donner une idée générale, nette et précise de la nature et du jeu des éléments psychiques, et d'étudier la question à un point de vue général, nous aurons ensuite a reprendre et it examiner séparément, it propos des lois de l'activité mentale, les diverses classes de phénomènes psychiques, et nous trouverons alors l'occasion de compléter par des détails nos idées générales sur l'activité indépendaute des éléments de l'esprit.

Lorsque dans une société, une association se dissout, 
c'est une loi, une forme de finalité qui se rompt, et les éléments, les hommes fui composaient l'association sont rendus a la vie individuelle, ils entrent alors dans de nouvelles formes d'activité sorialr. Si, par exenple, me filature se ferme, les ourriers ef les ouvrières qui $\mathrm{y}$ travaillaient et qui etaient réunis par me association sycstématique. se motlent a lravailler chacun de son côté, soit sfiparment, soit dans de nouvelles associations, ou quelques-uns, d'ailleurs, leuvent se rencontrer, et aroir cutre eux soit les mêmes rapports qu'auparavant, soit des rapports différeuts, - il en est de même des éléments psychiques, quand, pour une cause ou pour une antre, le lien qui les rattachait vient à se rompre: ils entrent dans de nouvelles associations, ou bien ils travillent chacun jour soi, au risque de ne prorluire que le l'incolérence. Cette activité isolée des éléments, nous la trouvons sous une forme fraplante daus les maladies mentales.

Le calembonl est une forme de ce désordle: en l'analysant on roit qüil ronsiste essentiellement en co quin son employe romme blement dius un romplexus parti-

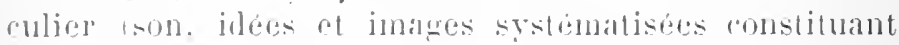

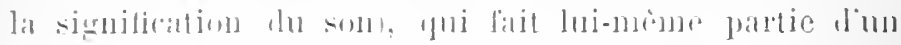

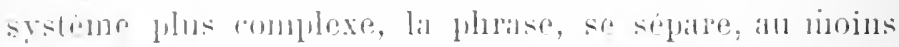

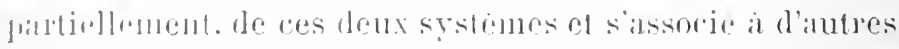

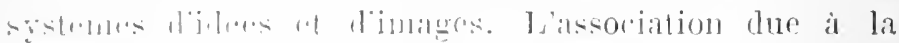

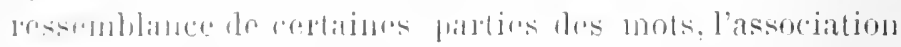

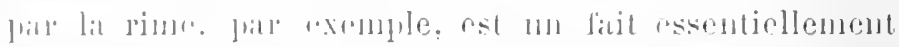

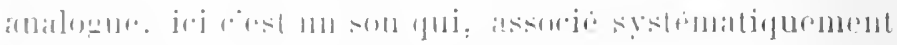

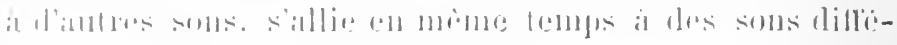

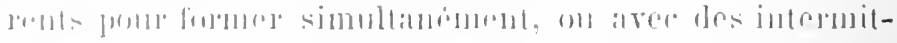

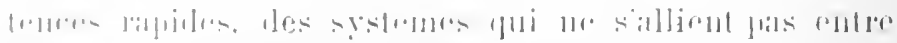

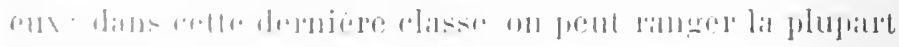

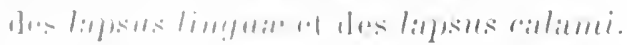

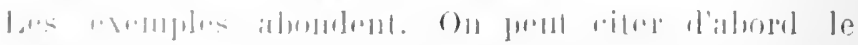

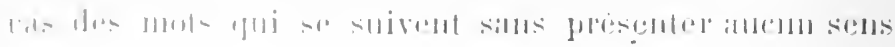


et qui sont appelés l'un à la suite de l'autre par la ressemblance, l'identité d'une partie de leur son. Un bel exemple de ce phènomène est fourni par la malade dont Trousseau a rapporté l'observation, et qui a écrit plús de cinq cents pages de mots qui sattiraient l'un l'autre par l'assonnance, quelquefois aussi par le sens, mais toujours avec une incohérence manifeste : " chat, chapeau, peau, manchon, main, manches, robes, jupon, pompon, rose, bouquet, bouquetièrc, cimetièrc, bière, elc...”.

A un degré plus élevé, nous trouvons le calembour volontaire ou à demi-volontaire. M. Regnard a cité plusieurs pièces de vers écrites par des fous, où se trouve à un lhaut degré ce mode d'association systématique élémentaire; quelquefois on $\mathrm{y}$ voit aussi un reste de coordination supérieure intellectuelle, certaines suites de mots ont un sens, comme dans la pièce suivante ou l'incohérence ne manque pas absolument :

J'aime le feu de la Fougère

Ne durant pas, mais pétillant;

La fumée est âcre de goût.

Mais des cendre de : là Fou j'erre

On peut tirer en s'amusant

Deux sous d'un sel qui lave tout,

De soude, un sel qui lave tout 2 .

D'autres fois, le sens disparaît à peu près complètement ainsi que dans ces vers, cités encore par M. Regnard, et composés par un délirant vaniteux, malade depuis vingtcinq ans :

Magnan I a mon soubait, mèdecin Magnanime

Adore de mon sort la force qui... t'anime.

Admirant son beau cràne..... autre renarl de Phedre,

Nargue Legrand du Saulle et sois un Grand du Cedre 3.

1. Cité par Luys. Actions réflexes du cerveau, page 170 .

2. Dr Regnard. Les maladies epidemiques de l'esprit, page 370.

3. Regnard. Ouv. cité, p. 390 . 
Il n'est pas indispensable d'ètre fou ou idiot pour faire des calculhours ot associer les mots d'après des ressemblances superficielles. Alols, an lien l'ètre une dissociation permanentedessylemes plus complexes, c'est une dissociation momenlanie qui domne lien an phénomène. Rien de plus naturel, quand on époure le besoin de se détendre l'esprit. que de rendre ì eux-mèmes les èléments psychiques retenus dins des systimes complexes dont la permanence nes pas essenticlle ä la vie, et de leur laisser une liberté dont ils alousent quelquefois. Pour continuer la comparaison faite plus haut et qui peut se suivre assez avant, les ouvriers de la filature ne travaillent pas toujours, ils ont leurs moments de repos, leurs récréations et vivent alor's specialement pour eux-mèmes, ils s'emploient, au moins en général, a des systèmes moins complexes.

Ce mème pllénomène qui résulte de la disparition d'un système supérieur, peut résulter anssi de ce que cessteme supérieur ne s'est pas encore constitué, en ce cals le ralembour est involontaire, il est simplement une ronfusion. I'n erfant entend lire un passage d'un livre oì il rst dit que. (hez certins animaux, par la conleur, les dimensons, la lennelle differe du mâle; l'enfant, pour Ini cer mots lemelle, différer' màle n'ont sans doute pas un sens hien net, et yni ne saisit pas l'ensemble de la

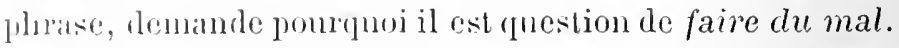
Chatur, en remontanl dans ses sourenirs d'enfance, trouvela sills doute des cas oì mue ressemblance de son lui a litil assored des dobes passablement incoherentes, el il nolsolvera farilement che\% les confints ou les illettes yu'il antar l'orcasion de voir.

We 111 somviens, fonr 11011 compte, que, étant tout

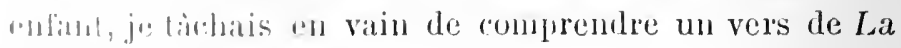
Cigiale "l la Finumi:

* Ie vous jaierai, lui dit-elle, A vint l'aout, foi l'animal,

lulirit el principal." 
Le principal et l'intérêt m'étaient des choses absolument inconnues ; principal me faisait l'effet d'un adjectif se rapportant a animal, et j'èbauchais une vague synthèse en interprétant intérêt d'une manière analogue, comme équivalant un peu à intéressant, mais je sentais bien que tout cela n'était pas satisfaisant et les divers systèmes, les diverses significations que je pouvais attribuer aux divers mots, dains mon ignorance de celle qu'ils devaient avoir dans ce passage, restaient en moi à peu près isolées.

De-même chez les peuples enfants, les éléments n'étant pas encore solidement associés les uns aux autres dans des systemes cohérents engendrés et fortifiès par un long exercice de l'intelligence et de ses formes èlevées, les éléments psychiques s'associent un peu ì tort et à travers. Le mythe verbal, dont M. Max Muller a tiré parti comme on sait, est un phénomène analogue à ceux que nous étudions ici et où se manifestent très bien l'activité indépendante et les affinités des élèments psychiques. M. P. Regnaud a fait récemment une étude des jeux de mots védiques et son étude parait psychologiquement très-juste.

Dans les langues modernes, dit-il, «le calembour est artificiel : c'est un effet voulu, personnel, sans attaches directes avec les lois qui président au développement du langage et qui, par cela même, reste isolé et infécond.

a Il n'en est pas de mème des jeux de mots qui remplissent le Rig-Veda, et qui contribuent à en rendre l'interprétation si difficile. Ils sont naturels, et sortent des entrailles mèmes du langage, sans que la volonté de celui qui les produit y ait généralement la moindre part. Loin d'ètre complices des erreurs qui en résultent, leur's auteurs en sont dupes et se sont pris aux illusions dont ils étaient les agents inconscients. C'est qu'ici il s'agit moins d'homonymie réelle que des différentes significations qu'un mème mot peut prendre en vertu de 
métaphores qui tiennent aux lois mèmes de la pensée à une certaine póriode de son développement. Eu rertu de ees lois, telle expression yui correspond primitivement it l'idee de briller' par exemple, passera souvent sans changer de forme, ou eu n’en changeant que légérement, soit a celle de briler, être ardenl (l'on ètre agilé an physique et passionne, desirer au moral), soit à celle de voir, connaitre, penser, soit i celle de brùler, souffrir. La confusion de ces différents sens n'ayant d'abord qu'un seul mot pour point de départ, linfluence des uns sur les autres, mème après la sejparation des formes verbales qui les ont tirés d'un sens primitif unique, l'adjonction à chacm d'eux d'épithètes qui ont passé par les mèmes plases significatives, sont autant de causes qui les ont entrerroisés, non pas de telle sorte qu'il y ait indétermination complète et que l'un se prenne pour l'autre, mais assez pourtant pour que l'un reflète l'autre et y fasse allusion.

"Ce n'est qu'un longr usage de la parole qui a détendu ces atlaches $\$$ I. Enfin on pent rajprocher de ces faits jusqu'a un certain point le phénomène de la composition poétique du moins chez cortains poètes. - L'idée et la rime s'appellent réciprofuenent el sourent cest la rime qui vient la premiere, les mots s'associent par leurs ressemblances, leur identite partielle de son et les idées viennent ensuite raccorder aussi bien que jossible le nouveau mot aux vers doja composés?. Les lapsus lingua et les lapsus calami

1. P. Regnaud. Le caractere et l'origine des jeux de mots védiques. liwue le l'histoire des re.jgions. Septembre-octobre 1887, p. 166-167.

2. Voyer Th. de Ban:ille. Petit traite de poésic francraise, pages 54, 6], 83. Il ne faulrait pas preulre trop an pied de la lettre la theorie de M. de lsauville, mais elle correspond hien, an moins partiellement, a la réalité. Eil nous atrons ici une occasion de remarquer comment un mème procédé général peut conduire selon la forme de ses applications particulieres à des incohrences on ì des beautés de fremier ordre. 
se rapprochent des phénomènes précédents en ce ru'ils sont des sortes de calembours involontaires, ils se produisent alors que l'association se reliche, lorsqu' les éléments psychiques ne sont pas assez strictement coordonnés, lorsqu'une partie le leur force associative peut se détourner du système principal et les coordonner avec d'autres éléments, de manière à faire apparaìtre quelquefois un nouveau système général, et à supprimer l'ancien. En mêne temps que la force de l'association, ces phénomènes nous montrent Ia force d'inhibition des èléments psychiques - celui qui l'emporte enrayant le développement, ou supprimant l'existence de celui auquel il se substitue.

Sous l'influence de diverses circonstances, ces éléments peuvent quitter un systẻme pour entrer dans un autre. Voici un lapsus assez compliqué où ce mode d'association est très visible. Je le cite parce qu'il est authentique et curieux, bien que la nature du lapsus paraisse le condamner à paraitre plutôt dans un journal amusant que dans une étude sérieuse.

Un jeune employé est chargé d'écrire une lettre à un percepteur pour l'inviter à faire « recouvrer les sommes dues d par certains contribuables. Autour de lui ses collègues tiennent des propos légers, si bien que, vaguement distrait par la conversation, et combinant les deux préoccupations, il se voit sur le point d'inviter le percepteur à faire « recouvrir les femmes nues, " il s'arrête heureusement après avoir écrit la moitié de la phrase. Qu'on remarque combien les deux phrases se ressemblent, elles ne diffèrent que par quatre lettres, une dans le premier mot, deux dans le troisiéme, une dans le quatrième; d'autre part le sens est aussi différent que possible. Il est bien évident que les éléments psychiques du second système, de celui qui était mis en activité par les propos de 
l'entourage, se sont combinés aree cenx qui constituaient la representation mentale les mots a écrire.

Les faits de ee gente jeltent me vive lumiere sur le mécanisme de lesprit. on freml sur le fait, si je le puis dire, laclivite rollulaire de l'ime; chaque impression, chaque complexus leul i s'associer les aulres éléments psychiques qui somt susceptibles de s'harmoniser avec lui, a il yarrient fuand les systèmes supérieurs sont momentanement dissous ou relaches. On voit combien l'interprétation senerale de ces pliénomènes s'aceorde aree les rues ingenienses de M. Binet, en particulier arec son interpletation de reptaines associations psychiques au moven de la loi de fusion ${ }^{1}$.

Dans los rèves nous royons souvent certains élements, certaines ilére, envahir pour aninsi dire l'esurit en s'assoriant les phenomenes presents, en délernumat leur

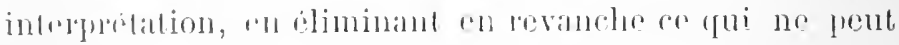

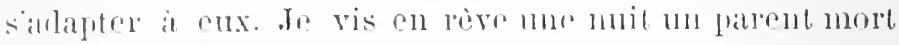

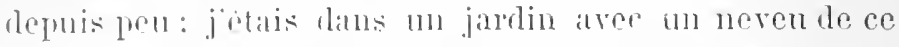

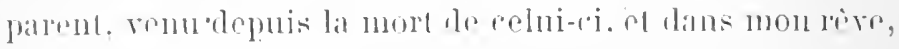

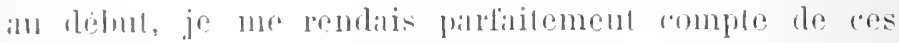

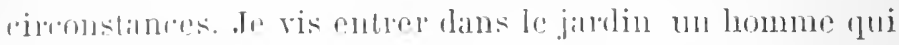
me pral'ut habillo conmue l'blat mon parent autant que je mons smbiens, fourtant, le costume gu’il portait u'avait

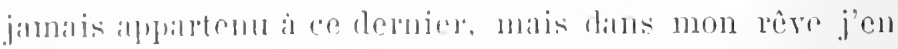

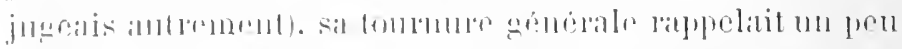

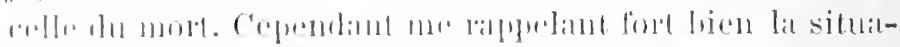

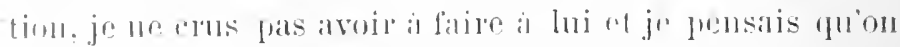

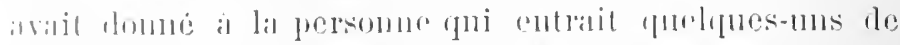

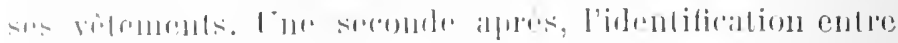

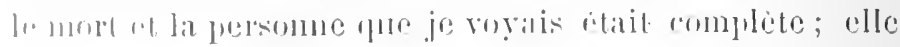

1. Binm. Psychologie du iasonnemene, J' 9i-11K. Voir aussi : Bro-

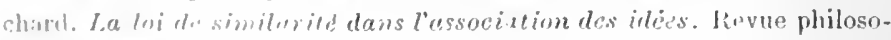
jhiquer, lnsil, t. 1. 
était devenue tout à fait mon parent, j'entendais sa voix que je reconnus très bien et je n'avais pas le moindre doute, l'idée mème de douter 'que mon parent fùt devant moi ne se présentait pas à mon esprit, rien ne me suggérait la pensée de sa mort et j'avais totalement oublié mes premières réflexions. Ces deux états psychiques paraissaient s'être succédé sans transition. Une partie du premier avait été totalement évincée alors qu'une grande partie, le lieu de li scène, la prèsence du neveu, enfin la présence de la personne dont l'identité avait été différemment interprétée dans les deux cas, subsistait encore.

La meilleure condition pour voir se développer cette propriété d'association systématique est peut-ètre l'état de somnambulisme. Ici, en effet, toutes les associations supérieures si elles ne sont point rompues, sont au moins relàchées et n'existent plus qu'à l'ètat latent, l'esprit est relativement vide; aussi une simple impression que l'on suscite éveille sans trouble et sans obstacle, au moins dans certains cas, et si l'on ne contrarie pas trop les associations anciennes et profondément enracinées, une sèrie systématisée de phénomènes.

Nous avons des exemples frappants de ce fait dans les suggestions faites pendant l'état catalepticque ètudié par l'école de la Salpêtrière. Par exemple, si, comme l'avait signalé Braid ', on dispose d'une certaine manière le bras d'une somnambule, les autres membres et la physionomie prennent une expression harmonique, si l'on approche les deux mains de la houche, comme dans l'acte d'envoyer un baiser, le sourire apparaît sur les lèvres. MM. Charcot et Richer ont obtenn des résultats tout à fait analogues sur la physionomie ${ }^{2}$. On suggère de mème des actes, par

1. Braid, Neurypnologie, trad. franc. de M. Simon, p. 170 et suiv.

2. Richer. Études cliniques sur la grande hysterie. 
exemple, « dans l'angle d'une pièce la malade est placée un pied sur les barieaux d'une chaise, et les deux mains saisissant les plis d'un rideau comme dans l'acte de grimper; à peine cette attitude est-elle communiquẹe que la malade, en un clin d'œil, a escaladé la chaise et qu'on a grand peine a la retenir et à lui faire laicher le rideau auquel elle se tient suspendue ».

Tous les pliénomènes de suggestion nous montrent une loi analogue, c'est toujours un fait qui, suscite dans un organisme oì le contrôle manque ì un degré r'cmarquable, détermine, soit des images, soit des actes appropriés. M. Richet a produit ainsi des personualités variées chez la même personne. Une fois le point de départ domné, le reste s'ensuit fatalement, par une systématisation spontanée; une honnête mère de famille entre en plein dans un personnage d'actrice et ne le prend pas par ses plus beaux cotés I. On sait que la systématisation ainsi produite s'étend jusqu'il cette partic de nos actes que la volonté consciente ne régle pas en général: l'écriture, par exemple, est modifièe 2. Toutefois la personnalité première n'est évidemment pas complétement anéantie ; - comment le serait-clle sans que l'organisme fìt anéanti ou complètement transformé - aussi la mème sugrestion peut-elle ne pas produire le mine efret sur deux personnes différentes. M. Beamis cite, en particulier, un cas où le vol dume cuiller, commis a la suite d'une suggestion, par deux persomes, s'accompagne de phénoménes qui, au point de vue pliysique et surtout atu point de vue moral, rarient semsiblement d'une

I. Richel. L'homme et l'intelligence, 1. 238.

2. Voir les expériences de MU. Ferrari, Hériconrt et. Richet. (Rerue Philosophique, t. xxı, p. 4l4.) - L'analyse de M. lloctis prouve, il est vrai. ju. relle transformation de l'écriture n'est pias complete et que le sujel iuterprète avec sa nature propre le persomnage suggéré ; mais cela n'a rien que de tres naturel et n'enlève jas sa portece a l'expérience. Voir in Revue I'hilosophique, T. xxil, p. 330 . 
personne à l'autre ${ }^{1}$. Évidemment l'organisation préétablie de l'esprit a une importance capitale dans n'importe quel fait psychique a l'ètat normal, el l'ètat de somnambule në détruit pas complètement ni n'interrompt même absolument cette organisation; bien surement, pour que les éléments psychiques puissent exercer leur pouvoir de systématisation, il leur faut un terrain propice. M. Beaunis a raison de voir dans l'expérience qu'il rapporte une épreuve qui montre d'une maniẻre tout à fait délicate le fond d'une inme, pour ainsi dire, ce qui reste quand les associations les plus superficielles ou du moins les plus instables sont détruites ou rendues lutentes, mais évidemment le fait que le nilieu où doit se développer le phénomène joule un rôle important dans ce développement mème, fait sur lequel nous aurons à revenir, ne diminue en rien le fail rue ce phénoméne aussi a un ròle considérable dans le déreloppement auquẹ il donne lieu. Dans certains cas curieux, la systématisation spontanée va plus loin encore. M. Richet endort un de ses amis et lui dit : * Viens avec moi, nous allons partir en " ballon.... Nous montons, nous sommes dans la lune!" Et, á mesure que je parlais, il royait les péripéties de ce fantastique royage. Tout d'un coup, il éclata de rive. «Vois done, me dit-il, cette grosse boule brillante qui est « là-bas? "C'était la terre que son imagination lui représentait 2 . On voit bien ici une spontanéité très marquée, l'esprit n'est pas passif, et à vrai dire un esprit ne saurait être complètement passif. Nous trouvons une spontanéité analogue dans certaines observations de M. Pierre Janet, Le sujet est placé dans une des phases intermédiaires de l'hypnotisme étudiẻes par M. Jauret, les hallucinations produites sont très intéressautes « elles sont très complètes, dit l'auteur, et existent immédiatement.dans tous

1. Beaunis. Études sur le somnambulisme provoque, p. 192 et suiv.

2. Ch. Richet. L'homme et l'intelligence, p. 180. 
les sens. Si je lui dis qu'il y a un mouton devant elle, elle le voit, mais aussitòt, sans que j'ajoute rien, elle l'entend bèler et imite son cri, puis elle le caresse et sent sa toison sous sa main ${ }^{I}$ ». Ici encore la systématisation spontanèc des éléments psychiques produit des effets que la volonté ne peut produire.

Il faut noter aussi dans cet ordre de faits les impulsions morbides. Marc raconte dans son ouvrage sur la folie, que passant un jour sur le Pont-au-Change et y voyant assis sur le parapet un garçon maçon fui se dandinait en prenant son dejeuner, il fut saisi du désir de lui faire perdre l'équilibre et de le précipiter dans la rivière: "Cette ideje, ajoute-t-il, ne fut qu'un iclair"; mais elle m’inspira une lorreur telle, que je traversai rapidement le paré pour m'élancer rapidement sur le trottoir opposé, et méloigner ainsi avec promptitude de l'objet qui avait fait naitre en moi cette horrible velléite. "Talma, à qui Marc rapporte le fait, lui dit avoir ćprouvé la même propension dans des circonstances à peu près semblables. Marc raptorte encore, d'après Pariset, qu'un littérateur conmu, s'etant trouvé un jour en face d'un des plus beaux tableaux de Gerard, fut pris de l'envie de crever la toile d'un coup de pied ; ce dẻsir s’évanouit bientòt, mais íl fut. assez vif pour lui faire tourner le dos an tablean 2 . Nous voyons ici naître un systeme très cohérent en lui-mème, mais tros mal cooldonné avec l'olganisation genèale de lat personne rhez qui il s'éveille, suscite par une perception dius des conditions psychiques qu'il est souvent difficile de préciser. Ce système ne peut toutefois se

1. P. Janet. Les phases intermediaires de l'hypnotisme. Revue scientifique, 1886, lor semestre, 1. 581.

2. Mare. De la folie consideré dans ses rapports avec les questions medico-judicirires, torne II, p. 479. Voy, encore Esquirol. Monomanie homicide au tome II le ses Malarlies mentales; Maurlsley, Le crime et lc folie: Kibot, Maladies de la volonte. 
compléter par un acte à cause des associations coordonnées qui se réveillent et luttent contre lui, mais dans d'autres cas, où les associations supérieures ont moins de force, le système nouveau est difficilement réprimé malgré les désiıs et la volonté générale du patient, le système nouveau se suffit, pour ainsi dire. Des malades sont obligés de se faire attacher, quelquefois, il est vrai, avec un Iien aussi fragile que possible; d'autres, enfin, finissent par céder à leur impulsion, qui alors envahit progressivement l'esprit et paralyse momentanément les tendances supérieures. On en trouvera des exemples dans les aliénistes qui ont traité la question, Marc, Esquirol, Maudsley; voici encore des faits qui montrent la même activité indépendante d'un systéme psychique. Marc a vu, dans une maison de santé de Paris, une demoiselle dont les discours et les actes étaient raisonnables, mais qui s'occupait à découper en petits morceaux ses vêtements et ses hardes. Interrogèe sur la cause de cette manière d'agir, elle répond: "Je ne puis m'en empècher, c'est plus fort que moi $\gg \mathbf{I}$. M. Ball dit avoir vu, dans le service de M. le $\mathrm{D}^{\mathrm{r}}$ Mesnet, un alcoolique hèréditaire chez qui les hallucinations étaient exclusivement auditives... il appréciait parfaitement le nature de ces fausses perceptions et ne croyait point à leur réalité ; « elles exerçaient cependant un empire irrésistible sur lui. Lorsque au milieu de la rue il s'entendait appeler par son nom, il se retournait presque toujours; lorsque les voix lui intimaient un commandement il obéissait presque invariablement. Un jour, passant sur les quais, il entend une voix qui lui commandait de jeter dans la seine les deux pièces de cinq francs qu'il avait dans sa poche ; il obéit machinalement, et, à peine l'avait-il fait, qu'il aurait voulu se jeter

1. Marc. De la folie, I, p. 88. Voir d'autres observations, même page ot pages suivantes. 
lui-mème a l'cau : car, disait-il, nous n'avions pas en ce moment vingt franes a la maison '. "Brierre de Boismont, dans un travail sur la monomanic, dit que, souvent, des personnes sont venues le consulter, en lui disant : Depuis un an, deux ans et plus, nous somnes tourmentés jar le souvenir de la mort de notre père qui s'est tué à cette époque. Nous arons d'abord résisté, mais à mesure que le moment fatal approche, notre résistance diminue, et nous craignons bien de noustuer quand il sera arrivé. " $\mathrm{Cc}$ déplorahle résultat, ajoute l'auteur, n'a été que trop souvent constaté ?. "On peut observer, chez les hysteriques, une sorte d'èmiettement, de dispersion de la volonté, une incohérence complète dans les actes et les paroles; chaque systeme suscité par une impression agit seul, et aboutit a l'acte sans contrôle de systèmes supérieurs. Une jeme fille profite de l'èloignement de ses parents, sort seule, rencontre un ouvrier. se laisse accoster et conduire dans un hòtel, y passe quelques jours, puis rentre dans sa famille, raconte en éclatant de rire ce qui lui est arrivé et ne demande pas même à épouser l'homme qui a abusé d'elle ${ }^{3}$. Il n'y a pas de cohérence dans les actes ${ }^{4}$. De mème il y a des systemes d'idées séparés dans la folie et des systèmes psychiques encore plus émiettés dans la démence, la paralýsie générale, etc.

Il ne fant pas croire, au reste, que l'activite relativement independante de systemes psychiques plus ou moins complexes se remarque seulement dans la folie et les etats analogues. Nous pouvons l'aperecvoir a chaque instant le notre vic mentale et de la vie mentale des personnes que nous rommaissons. I façon dont les idées nous arrivent,

1. Hall. Lerons sur les maladies mentales, H. 640.

2. Annaies l'hygiene publique et de médecine légule, 2e série, $t$. VIII, 1.. $4 \% 2$.

3. Legrand du saulle. Les hysteriques, p. 380 .

4. Voir lisbot. Les maladies de la volonte. Le regne des caprices. 
dont les souvenirs nous viennent, dont nos desirs se forment, dont nos décisions sont prises, implipuent dins une grande mesue l'ativite d'élements psychiques pui travaillent pour eux-mèmes, - pendant que nous sommes occupes dautre chose, - assez faiblement pour yue leur - mire passe longtemps inapergue, arec assez lle fonee pour que, à un moment donné. une nonvelle illóc, ilee de gènie, idée ordinaire, idee criminelle arrive a se juroduire et at se formuler sans être prévue, et s'impose alu moi qu'elle modifie, qui la modifie et dont elle fait désolmais partie integrante. Un grand nombre dides et de désirs naissent ainsi sans yuon s'y attende et bien souvent sans yuion puisse préciser leur origine et suive lem. développement.

Les mourements a l'etat normal et pathologique seraient un interessant oljet d'étude au point de rue de la loi dont nous nous occupons ici, mais il serait trop long de développer chaque détail. La pathologio du. langage nous olfre des exemples plus précis et peut-ètre plus concluants encore. On y roit lindépendance relative des systemes d'impressions qui sont les mots et des systèmes plus réduits encore qui sont les lettres. Souvent dans le désordre complet du langage quelques mots restent et penvent seuls ètre prononcés. "Un malade de la clinique de Weetplial, paralýsé depuis peu a la suite d'une attaque d'apoplexic, avait conserve lapparence diuc grande intelligence, mais il ne pouvait ni prononcer spontanément un mot, ni répéter cenx qu'on lui disait. Lorsqu'on prononçait devant lui une parole il ouvrait la bouche, faisait toutes sortes de yrimaces, et des efforts visibles pour parvenil à répéter ce fluon disait. mais il n'arrivait constamment qul at dise \& tschi-tschi» ou hien " "itoko ", il était égralement incapable d’imiter des sous isolés. Lorsqu'on l'invitait at faire une lecture, car il avait appris a lire, il produisait (quelles que fussent les lettres 
qui étaient dans le texte) comme en épelant, les sons a, $u$, œ, etc., qu'il était ensuite capable de répéter... A la dictée il écrivait convenablement de sa main gauche son nom et celui de sa femme ${ }^{1}$." Ce fait a l'avantage de montrer l'indépendance relative d'un grand nombre de systemes psychiques, mots écrits, mots prononces, sons. isoles: du reste, les exemples de pareils phémomènes abondent dans les ouvrages où sont étudiés les troubles de la parole. M. Bernard rapporte d'après M. Grasset un cas où l'on voit lien comment un système existe en luimème et forme une unité, non seulement par rapport aux autres sýstèmes, mais, en quelque sorte, par rapport a ses propres éléments. «Un officier, réduit à pardi et à $b$. , incapable de prononcer les mots enfant et patrie isolément, chante exactement le premier couplet de la Marseillaise, paroles et musique." MM. Escot, Onimus, Hallopeau, Brown-Séquard ont rapporté des faits semblables 2 . D'autres malades peuvent dire un air sur une seule syllabe el ne peuvent prononcer les paroles; on voit encore ici l’undépendance relative de deux systèmes qui sont unis en général el qu'on aurail pu croire indissolublement unis.

M. Beruard fait remarquer qu'il en est, en général, des airs et de leurs paroles comme du reste du langage des apheminues "fo sont des clichés dont les malades ne peuvent rien detacher, quils doment tout d'une pièce. » On le voit, le systime est un, les éléments en sont dans ces cas indissolublement agrolutines les uns anx autres, ils ne peurent se separer. Diantes exemples nous font voir an contraire d'autres modes d'atsociation et de dissoejation. M. Kussmaul cite, dapres: Graves, le lait d'un malade qui avail a ba suite d'une altatue perdu les noms projures of los smbstantifs, sauf les lettes alphabetiques intiales; pour le reste il u'avait fas perdu la

1. Kuesmaul L.s trubbles de la parolc, trad. franc., p. 607.

2. Bernard. De laphasie et de ses diverses formes, 1) 125. 
parole. Il se fit donc un dictionnaire des substantifs nécessaires pour la vie courante et le consultait aussi souvent quil était arrèté par un de ces mots. Voulait-il, par exemple, dire vache, il cherchait dans la lettre $V$. On voit comment des lettres peuvent exister isolément dans l'esprit ou plutòt s'associer seules avec un sens donné. Dans le begaiement on trouve encore de bons exemples de l'indépendance des systèmes psychiques qui composent les lettres, certaines lettres sont prononcées plus ou moins facilement ou quelquefois ne peuvent pas ètre prononcées selon les autres lettres qui les accompagnent ou qui les suivent. Enfin la surdité verbale, la cécité verbale, nous offrent encore d'autres cas d'associations systématiques indépendantes sur lesquels je n'insiste pas; ils sont très counus et il suffit ici de les rappeler ${ }^{1}$.

L'activité systématique des éléments nerveux se montrc peut-être encore plus visiblement, quand deux ou plusieurs systèmes agrissent à la fois dans l'esprit, quand deux éléments psychiques suscitent à la fois des systèmes d'idées et d'impressions qui ne sharmonisent pas entre eux. Cela se produit à l'état normal. J'ai examinć ailleurs la question en ce qui concerne les phénomènes affectifs ${ }^{2}$, et jai exposé dans la Revue scientifique ${ }^{3}$ quelques expériences qui montrent, à mon sens, comment chaque système, chaque èlément psychique suscité a, par lui-mème, une tendance à systématiser. Si, par exemple, j'essaye de réciter une pièce de vers, tout en ècrivant une pièce de vers différente, il se produit quelquefois certaines erreurs tenant à l'interpolation d'un mot ou d'une

1. Voir Kussmaul, Les troubles du langage; Bernard, De l'aphasie, G. Ballet, Le langage intérieur et l'aphasie; G. Bastian, Le cerveau comme organe de l'esprit, tome II ; Frré, Traité elémentaire d'anatomie médicale; Luys, Les actions reflexes du cerceau; Ribot, Maladies de la mémoire.

2. Les phenomenes affec:ifs, chap. III.

3. Numéro du 28 mai 1887. 
lettre qui passe du discours dans le texte écrit, mais en somme ces erreurs sont rares, à moins de rencontres fortuites, à moins que le mème élément ne se rencontre à la fois dans les deux systèmes. Les mots qui forment un vers et les vers qui forment une pièce tiennent bien les uns aux autres : en général, tout en récitant, je me représente d'un trait un ou deux des vers que je dois écrire, après cela; je n'y pense plus, la machine est montée, l'écriture se laiı. automatiquement, chaque élément étant coordonné avec les autres. De temps en temps je dois m'arrêter d'écrire, mais la récitation que je continue ne me gêne guère pour retrouver le fil de ce que j'écris. En récitant des vers, pendant que je faisais une multiplication très-simple, j’ai pu effectuer les deux opérations dạns le temps nécessaire pour en faire une seule, ce qui met hors de doute la simultanéité de l'activité des deux systèmes psychiques et le fait que chacun agit pour soi. C'est un peu - pour recourir encore aux comparaisons avec la sociologic qui pourraient eu psychologie ètre d'un grand usage - ce qui se passe, par exemple, dans une classe où des élèves lisent et calculent, tandis que d'autres font une page d'écriture.

M. Tannery a signalé a la société de psychologie physiologique, un fait assez curieux : lorsqu'il parle mentalement el que sa pensèe vient it ètre distraite, la parole intérieure se continue quelquelois d'ume liaçon incohélente, mais souvent avec plus de force I. Jai remarcqué aussi chez moi ce phénomìne : loisque je viens à penser un peu malgré moi et par distraction a une chose dont lides n'esl desagreable, il arrive souvent que le sysreme brychique dont l'activite est suspendue, tìche, si je puis dire, de maintenir sa prépondérance cu me faisant

1. Síance dn 25 octobre 1886. Voir dans la Revue philosophique, le janvier 1887, !. 103. 
parler tout haut; c'est du reste un fait analogue à celui de lire volontairement à haute voix pour éviter d'être distrait par le bruit ou par des idées; seulement dans co dernier cas le phẻnomène est voulu, dans le premier cas il est involontaire, e'est-a-dire que dans le premier cas il montre l'activité spontanée et indépendanle d'un systène psychique, dans le second il montre aussi la coordination régulière et l'influence d'un certian nombre de systèmes.

A l'etat semi-pathologique ou completement pathologique, l'intensité du dédoublement et laction séparée des éléments psychiques sont plus évidentes encore. Les faits de ce genre commencent à devenir très nombreux. L'hypnotisme en fournit un grand nombre. "On place sur une table un pot a eau, une curette et du savon ; aussitôt que son regard est attiré par ces objets, ou que sa main touche l'un d'eux, la malade, avec une spontanéité appalente, verse l'eau dans la cuvette, prend le savon et se lave les mains, elle le fait arec un soin minutieux. Pendant qu'elle tourne ainsi le savon entre ses mains, si lon vient a abaisser la paupière d'un seul ceil, de l'wil droit, par exemple, tout le còté droit du corps devient léthargique, la main droite s'arrète aussitôt, mais, chose singulière, la main gauche n'en continue pas moins le mouvement 1. "Les expériences de M. Dumontpallier ${ }^{2}$ sont encore plus extrardinaires. Je n'ai pas luesoin d'insister ici sur les phénomènes de dédoublement de la personnalite examinés par M. Pierre Janet; ils montrent admirablement cette independance relative

1. Richer, ouvrage cité, p. 693.

2. Comptes rendus des séances et mémoires de la Socièté de Biologie 1882, 1. 786. Voir aussi Magnan. Des hallucinations bilaterales de caractere différent selon le cóté affeclé (extrait des Archives de Neurologie). 


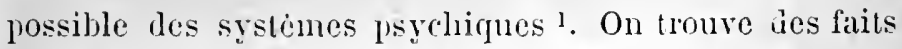
tres curienx a cet egard daus les dépositions les camisards, qui ont formé le Théatre sacré des Cévennes. Les camisalsts: quelques-mus an moins, prophétisaient, futedaient daus des langues yu ils connaiss:a ient a peine on qu'ils ne commissaicnt pas dans leur état normal. Il parait šcure produit chez eux de véritables dédoublements de la personnalice. a.... Ie les prie, au nom de Dieu, de se sourenir de ces choses terrihles qui ont été prononcées contre cenx qui, apres avoir été rendus participants du Saint-Esjrit, rejettent autant qu'il est en eux le don céleste qu'ils avaient goùté. Et je leur déclare solennellement et saus équivoque, par cet acte public et sur le sement que je fais devant Dieu, que je ne suis point l'auteur des agitations que je souffre dans mes extases, que ce n'est point moi qui m'agite moi-même, mais que je suis mù flar unc force qui est au-dessus de moi. Et pour les paroles qui sont prononcées par mes organes, je díclare: aroe les mènes protestations de vérite, qu'elles se forment sans dessein de ma part, et quelles découlent inopinement de nat bonche, sans que non esprit participe a cette operation nerveillense par ancune neditation précedente, ni par aucune volonté jrésente de parler surle-hamp. D Dnund Fage dit anssi : « Ma langue et mes lives furent subitoment forceses le prononcer avec véhémence ales paroles que je fus tout itomne d'entendre

1. J'jerre dinet. Les acies inconscimns ct le dedoublement de la inersonnalite pendant le somnambulisme provoque. Revue Philosophi¿que, liccomlne 1SS6, et L'ancsthés'e systematisde ct la dissociation des fhenemenes psychiques. Revue Philusophique. Mai 1887. Voir anssi Iibot, les Muladies de la personnalite; Richel, les Monerements incunscients; liall, le Inalisme cécbral, (dans le volume intitule le surphinumenic), al... 
n'ayant pensé à rien, et ne m'étant pas proposé de parler $D 1$.

Ce n'est pas seulement dans ce qu'on pourrait appeler les dédoublements simultanés de la personnalité, mais aussi dans les dédoublements alternatifs, que l'on peut voir se manifester l'indépendane relative des systemes psychiques. Ici encore nous voyons certains faits, certaines impressions devenir le point de départ d'une série systématique d'actes et d'idées en contradiction avec lia série d'actes et d'idées qui avait précédé et avec celle qui suivan. Un homme vient de parler ou d'agir dans un sens, en lui suggérant telle ou telle idèe, on le voit quelquefois parler et agir en sens inverse sans avoir conscience de ses contradictions. J'ai insisté ailleur's sur ces variations de la personnalité a l'état normal ${ }^{2}$. A l'ètat morbide, de pareils faits ne sont pas rares. Griesinger a cité un professeur qui se croyait empereur romain et qui contimuait a lire un cahier du cours d'histoire qu'il faisait dans son collège". Des faits de ce genre et de plus marqués se rencontrent à chaque pas dans les ouvrages de pathologie mentale; ils sont d'ailleurs bien connus et je n'ai pas besoin d'y insister t.

Des faits qui se ratlachent aux précédents el qui montrent comme eux l'indépendance possible du système psychique

1. P. 93 et III. J'emprunte ces faits à une réimpression du Thealre sacré des Cèvennes, rëimpression remaniée, mais dont l'éditeur affirme, dans sa préface, qu'il $n^{\circ}$ 'a fait que changer l'ordre des möceaux sans toucher au texte.

2. Revue Philosuphiqu: de 1882 . Voyez aussi, plus loin, le chapitre sur la personnalité.

3. Griesinger. Traité des muladies mentules. Trad. Doumic, p. 385.

4. Voir Griesinger, Traité des maladies mentales; Ball, Legons sur les maladies mentales; Azam, IIypnotisme, double conscience et alteration de la personnalité; Ribot, Les maladies de la personnalite; Richet, L'homme et l'intelligence; Berjon, La grande hystèrie che: l'homme; Bourru et Burot, I'ariations de la personnalité. 
et le rôle primordial de l'association systématique comparée aux principes de contiguäte ef de ressemblance, sont ceux qui nous montrent un systeme psychique, ou bien encolo des systemes pestés à l’étal latent, dont ni la lessemblance ni l'activita ne peuvent réveiller los élèments ot yni so reroillent d'emblée à un moment donné, selon lis loi de l'association systématique.

On sait une clıez les déments certaines facultés restent parfois intactes; ils penvent, par exemple, jouer aux cartes ou aux lames, bien que leur esprit soit généralement desorganisé. Che\% les idiots on trouve des faits analogues. Griesinger a vu, dans l'asile d'Earlswood, i Londres, un jeune homme qui avait fait tout seul un molèle remarguable d'un vaisseau de guerre ; cet individu avait mesprit très-borné, en particulier il n'avait aueune ilcie des nomlires. "Il est plus fréquent, ajoute l'auteur, de voir des individus plongés dans une idiote profonde exicuter d'assez hons travaux de dessin ou de peinture ; naturellencut ce n'est chez eux qu'un talent méeanique. M. Morel (Etudes cliniques: t. III, P. 49), parle d'un idiot qui ne lunounçait pas un mot et qui avait un talent remarqualsle sur le tambour ; son grand-père avait écé tambourmajol, et son piere avait tonjours eu le désir de se laire lni-mème tambour.... Ia mémoire des lieux, qui est quelquefois l res developpece chezcertains idiots, d'ailleurs i pen pros nuls, se ripproche un peu de ces talents suerianx 1. De mème dans la folie on voit persister certaines fitcultes dims un état tres florissant. Esquirol lappropte le as d'un general, attcint de manie, che\% qui "l: libline persiste tout l'eté, avee quelques intervalles A. l’unissun, pendant lesquellos le malate écrit des

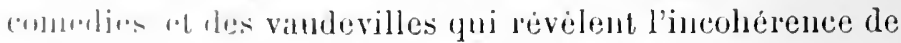
ser irlog... : malgró l'óganement le ses idées, le général

1 Griesinter, Traite des maladies mentules, trad. française, p. 432. 
congoit le perfectionnement d'une arme et en trace le dessin, il tèmoigrne le désir d'en faire exécuter un modèle.» Un jour il se rend clez le fondeur; ì sa rentrée il est repris par l'agitation et le délire. Huit jour's aprés, seconde visite au fondeur, a le modèle est exécuté et l'ordre d'en faire cinquante mille est donné. Cet ordre fut le seul acte du délire qui réréla au fondeur la maladie du général. Plus tard cette arme a été adoptée. " Ainsi, au milieu de l'incohérence généralè, un sýstème important est maintenu et a pu aboutir à sa fin. Il est à remarquer que le trait du délire qui révéla au fondeur la maladie de son client ne se révèle qu'après l'exécution du modèle, c'est-à-dire alors que le systeme, ayant abouti, derait tendre à disparaitre. La tinalité des éléments est ici évidente ${ }^{1}$. Quelquelois, dans des états de stupeur complète, d'anéantissement total, un seul système de mouvements coordonnés survit encore. Abercrombie raconte qu'un malade a dans un état d'apoplexie complète dout il ne gruérit jamais, ajustait fréquemment son bonnet de nuit avec le plus grand soin quand il prenait une mauraise position, l'abaissant d'abord sur ses yeux et en arrangeant le devant de la facon la plus exacte. Une autre personne, daus un état de parfaite insensibilité provenant d'une maladie grave du cerreau, fut souvent vue, le jour mème qui précéda sa mort, tirer d'un petit sac, à la tète de son lit, une montre á répétition, la mettre près de son oreille, lui faire sonner l'heure et la replacer ensuite daus le sac arec la plus grande précision ${ }^{2}$. o Nous pourous rapprocher de ces faits ceux oì un systène ou bien un ensenulule de systèmes s'éveille, interrompt les actes commencés qui reprennent quand le nouvel état disparaît à son tour. Il y aura

1. On trourera un grand nombre de faits très intêressants dans l'ouvrage de M. le Dr Parant : La raison dans la folie.

2. Ahercrombie, Inquiries on the intellectual powers, p. 111. 
a reprendre ces fails au point de rue de la loi d'arrêt, mais ils nous intéressent ici en ce qu'ils montrent bien l'action isolée d'un șstème d'ètats neuro-psychiques; ils nous montrent tris lien cette force de coordination inhèrente aux déments psychiques qui, la systématisation ue fourint s'effectuer immédiatement, attendent, pour alısi dile: que l'état qui les empêche d'alsoutir ait dispar pour reprendre la coordination interrompue, it peu pres comme me personne arrètée dans une course par le pissiage d'un régiment, par exemple, altend que lo régineul ait passé el reprend sa route des qu'elle le peut, iu point oủ elle en est restėe. Il est difficile de dire, toutefois, jusru'i quel point la comparaison est exacte. En elfet, nous pouvons hien roir un individu sur un lrottoir, mais nous ne savons pas comment se comportent des cellules corbubles yui sont andètes daus leur travail; la persistance d'une idee ou d'un souvenir a l'éat latent

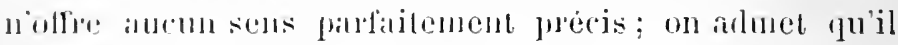
reste "lans les blements nervens une trace, un résidu, mais re nest gurie lá qu'un mot pour designer un phéuomenr inconnu : antant vilut galder le nuot d'association litunte. Yous reneontrons tres souvent ce phenomene, dialorid dins la vic normale : nous sommes a travailler, une personne nous dorange, nous ouldions notre travail four lui praler, et fubnd relle conrersation est finie, si

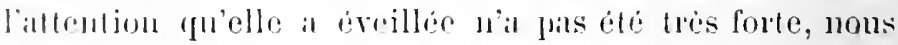

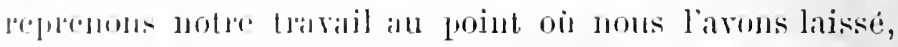

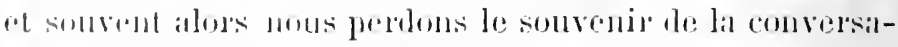

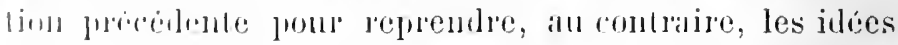

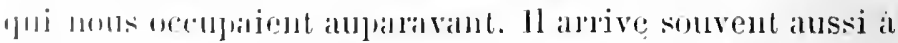

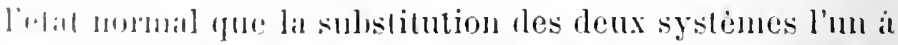

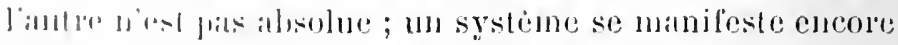
yluanl liantre est en jon far une sorte de tentance sentie,

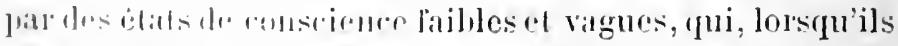
su fortifient un geu, cest-it-dire guand les temdances 
enrayees ont une forte organisation et une activité inpérieuse, donnent lieu i des sentiments d'impaticnce, d'irritation, auxquels on ne réfléchit pas toujours et dont on ne se rend pas toujours bien compte. Dans les cas semipathologiques ou pathologiques, ce phénomène s'exagère. Les rèves nous fournissent beancoup de faits de ce genre; les choses que nous avons vues en rève, les actes que nous arons commis nous ne nous les rappelons plus en bien des cas; quelquefois ils nous reviennent en mémoire pendant un rêve. Toutes les fois qu'il y a un changement, de ce qu'on pourrait appeler l'orientation générale de l'esprit, fait très important et sur lequel j'aurai a insister; il se produit une sorte de scission d'autant plus maryuéc. en général que le changement est plus fort. On oublie lacilement ce qu'on a fait pendant la nuit, si on s'est éveille et levé, jusqu'a ce que quelque ineident nous remette sur la voie. Lors du dernier tremblement de terre, je fus a deniréveillé par la secousse, assez faible d'ailleurs; je pensai inmédiatement à un tremblement de terre comme pouvant être la cause des oscillations qu'il me scml)lait éprouver, puis j'entendis du bruit, je m’imaginai qu'on cassait du charbon au rez-de-chausséc, que peut-ètre ccla m'avait fail croire à un ébranlement du sol; mes idées se confondirent, je me rendormis et quand je me réroillai, j’avais tout a fait oublié l'événement : ce ne fut que vers une heure de l'après-midi, lorsque j'entendis parler par d'autres personnes du tremblement de terre, que je me rappelai ce qui m'élait arrivé. Voilà done encore un exemple de système isolé. Il faut citer en ce genre l'oubli qui suit souvent les phénomènes hypuntifues : l'hypnotisé ne sait pas ce qu'il a fait précédenment, souvent mème le souvenir ne peut être réveillé par la vue des oljets qu'il a maniès pendant l'état sommambulique, et conme l'a montré M. Delbœuf dans des expériences que nous aurons encore a citer plus loin, l'association systématique peut 
seule rappeler le sourenir, si l'on réveille, par cxemple, le somnambule pendant qu'il est en train d'accomplir un acte. L'épilepsie est encore une névrose remarquable pour les faits qu'elle fournit dans cet ordre d’idées. Les malades accomplissent parfois, dans l'etat de petit mal, des actes dont ils n'ont aucun sourenir. Les faits de ce genre sont bien connus l. Tel est celui du magistrat qui, presidant une atudience, quitte son siège, s'avauce, parle d'une manière incohérente, retourne à sa place ef reprend ses fonctions saus aroir souvenir de ce yu’il vieut de faire. Les cas les plus intéressants pour nots sont ceux oil le systeme interrompu rentre en activité manifestement au point même de la coordination où il avilt été interrompu. Esquirol rapporte le cas d'une jeune dane, dont le père est épileptique, qui est prise de ses accos au milien d’un cercle, à la promenade, à cheval; " elle nest point renversee, les yeux sont convulsifs, le logard ust fixe: l'acres ne dure quime seconde, ef la malate reprend lat conversation, la phrase, oì elle les a laissegs sans se douter mullement de re fui vient de lui

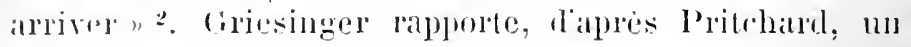

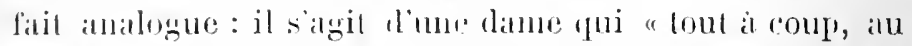
milien d'une conversation, śarêtait et se mettat a parler d'antre rluose; an bout de quelquesinstants, clle reprenat lat conversition à lat phatse, ol qui plus est, au mot même

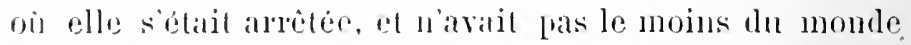
ronseience de l'interuption quelle arait faite ${ }^{3}$.

1. Vinil les observations de Tronsstiau, (Clinique médicale de l'HútelLicu, cilées par M. Luys, Actions reffexes du corrou, p. 129; voir alls:i I,ittr., la Double ronscience, in Fragments de philosophie rositier ot de sociologie contemporaine; voir aussi Iackson, les Tiunlors intellectuels momenlanes qui suivent les acces épileptiques, (Jierue seientifigue, 1876.)

2. Eequirol, Malatio's mentales, c. I, p. 277.

3. Pritclinol, Inmales wédiro-psychologiques, cité par Griesillger, Traite des maladies mentales, p. 129 
Nous pouvons rappeler encore ici ce fait que les tendances persistent ì l'état latent pendant des années entières sans se manifester, jusqu'i ce que les circonstances fivorables au développement du système dont elles font partie se présentent, soit que le milieu ait changé, soit que les tendances qui inhibaient celles-la aicnt disparu. Comme nous arons it revenir sur les faits de ce genre au point de vue de l'activité de l'esprit, je me borne à indiquer ici ce rui peut mettre particulièrement en relief l'activité automatique indépendante des éléments psychiques. Je rappellerai, par exemple, les retours de langues oublices depuis longtemps, de sentiments, ou peut-ètre plutòt de pratiques religieuses an moment de la mort, phénomènes très bien interprètés par M. Ribot ${ }^{1}$. Le retour d'une langue oublice ou inusitée depuis longtenlys, parait relativement assez frécquent. " Un homme, cité par M. Abernethy, ètait né en France, mais'il arail passe la plus gramrle partie do sil vie en Angleterre et, pendant plusieurs anmées, avait entièrement perdu l'habitude de parler français. Máis quand il fut soigné par M. Abernethy, a la suite des effets d'une blessure à la tète, il parla toujourss frauçais. Un cas semblable arrivi ì l'hôpital Saint-Thomas; il fut présenté par un homme tombé dans un état de stupeur ì la suite d'une blessure ì la tète. Quand il revint particllement à lui, il parla un langage rue per'sonne it l'hôpital ne comprit, mais qui fut bientòt reconnu pour le langage du pays de Galles. On découvrit alors qu'il était depuis trente ans alssent de ce pays et que, arant l'accident, il avail entièrement oublié son langage natal. Quand il fut complètement guéri, il oublia de nouveau ce langage et recouvra l'usage de la langue anglaise ${ }^{2}$.

\section{Ribot, Maladies de la mémoire.}

2. Abercrombie, Inquiries concerning the intellectual powers and the investigation of truth, p. 108. 
Des ras très curieux et fort interessants aussi et bien propres a montrer l'activité relativement indépendante des systèmes, sont ceux oì un systeme de mouverients ou d'irlées se transmet par hérédite d'me personne à une autre; le système parait d'aitleurs n'aroir aucune cohérence harmonique avec le reste de la personmalite de l'iudividu, cepeulant il doit évidemment se rattacher it d'autres phénomènes organiques qui ont arec lui un rapfort de canse à effet. Le fait n'en reste pas moins intéressant et frolnant dans la mesure oir l'activité isolic peut exister: car elle n'est jamais, comme nous l'avons dit, absolument isolée. Layeock rapporte le fait suivant cité par Darwin, dapres M. Francis Gillon. "Un personnage etait sujet a l'habitude que roici : lorsqu’il était étendu sur le dos daus son lit et profondement endormi, il élevait le blas doit lentement an-dessus de son visage jusqu'an nivean du front, puis l'allaissait par me secousse, en sorte quo le poignet tombait pesamment sur le dos de son iez. " Le geste ne se produisait pas chaque nuil et on ue lui conmaissait pas de cause apprécialule. Son fils et une fille de en fils heritiont du mene tice. Dajeres Lay-

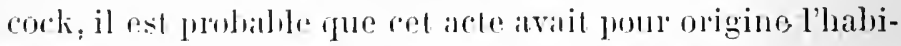
fude prise par le grandepie on wo do ses parents de se frapper la digure ou le menton puand il ne dormait pas ${ }^{1}$. M. Galton a trouve dans vingt-rleux limilles me tendance hópéditaire i la vision des anumber-forms »; l'hérédité se montre anssi a propos de celle curieuse disposition qui lail assorier mo conleur ave chafue royelle: Une lante berit a M. Galton rue ses sours voient comme elle les lolles rolorecs, mais les couleurs sont diflisentes. Y. Cialtun ajoute qu'il a pul lolever an moins trois antres castans lespuels l'hé rédite a joué aussi un rôle inportant ".

1. Iaycork, la Memnire ancrstrale (Revue scientifique, 1876, vol. II)

2. Galton, Inquiries into heman fuculty, jo. 140 et 151 . 
On trouve aussi dans l'ourrage de M. Ribot un certain nombre de faits analogues ${ }^{1}$.

On pourrait rapprocher de ces faits, ì d'autres points de vue, la mémoire qui se continue quelquefois d'un rève à l'aulre, et sourent d'un accès de somnambulisme naturel ou artificiel a l'autre, ainsi que la ressemblance des accès de folie ou d'épilepsie chez un mème sujet. Il vaut mieux pour le moment, poursuivre notre étude et en indiquer la portée et les conséquences. Toutefois, il n'est pas inutile de dire auparavant quelques mots d'une queslion traitée par les aliénistes mais qui intéresse notre sujet et dont il faut au moins interpréter les données.

On sait que la doctrine de la monomanie, fondée par Esquirel, admettait que l'esprit pouvait être troublé sur un point sculement; le malade était sain, sauf en ce qui concernait une idée particulière et ce qui s'y rattachait. Il n'est pas hesoin de faire remarquer combien cette idée, si elle étail juste, appuierail la théorie de l'indépendance fonctionnelle possible des systemes psychiques. Mais on l'a, depuis Esquirol, fortement attaquée; on a admis que, mème ruand le délire semblait localisé, l'esprit tout entier était en réalité malate. Inutile de dire que je nai pas a prendre parti dauns la discussion en laut qu'elle concerne la médecine mentale, mais il faut roir ce que nous pouvons en retenir pour la psychologie. En fait, il ne parait pas douteux actuellement que le désordre de l'esprit est plus général qu'il ne semble, un délire partiel suppose bicn, au moins, un trouble général qui se marquerail dans ce fait que les autres systemes psychiques, ceux qui sont ou semblent sains, ne jouent pas le rôle de réducteurs, pour employer une expression de M. Taine quils devraient jouer, ils nc peuvent arrêter, enrayer le systeme morbide. D'ailleurs; a plus on approfondit le problème, dit M. Ball, plus on demeure convaincu que les

1. Ribot, de l'Héridité. 
manifestations psychologiques de la folie constituent un ensemble dont on ne saurait logiquement démembrer les parties ». Mais le même auteur ajoute. "Il n'en est pas moins vrai que, dans certaines formes de délire, les aberrations mentales se groupent autour d'un centre commun et constituent une prédoninanre en faveur de certaines idées, de cerlaines tendances qui semblent absorber, pour ainsi dire, toul ce qui les entoure et imposent une sorte de conscription forcée à toutes les facultès de löindividu. Le délire ambilieux, le délire des persécutions dans l'ordre des conceptions délirantes, la folie homicide, la dipsomanie lans l'ordre des impulsions irrésistibles, nous offrent des exemples classiques de ce qu'on nomme aujourd'lui des délires partiels. o La théorie de l'indépendance relative des systemes psychiques et de la systématisation considérée comme proprièté des éléments psychiques u'al pas hesoin de lavantage. Elle ne saurait, en effet, altrihncr anx systènes partiels mne indépendance alsodue que les conditions organiques foreenent communes it lous rendent inpossibles. Elle trouve d'ailleurs un apui solicle dans de nombreuses observations des aliénistes, quelle que soit la manière dont on les interprête ${ }^{1}$.

1. Voir eneore, à cr sujet, du Diagnostic médico-légal de la pyromanic par l'rarainra indircet, par le Dr E. Marandon de Montiel, lans los Archire's de Neurologie, 18at, 1. II. 


\section{CIIAPITRE III.}

\section{ílléments poyehiques et facultés.}

On entend dire quelquefois quil y a lutte entre la mémoire et telle ou telle autre faculté. l'intelligence par exemple, ou la rolonté. C'est lit une façon de parler commode, mais purement metaphorirue: le conflit n'existe pas entre deux facultés, il existe entre deux ou plusieurs éléments psychiques; une idée, une tendance leuvent combattre un sentiment, une atue tendance, une croyance et nous avons vu déjà plusieurs cas de la force inhibitrice qui complète la force assoriative des éléments psychiques. Quant aux facultes, elles ne désignent guère que des manieres d’èlre des faits prschiques; nous les examinorons tout i lheure comme morles de systematisation. [s ychique, comme des formes diverses d'association systématique de différents èléments, nous derons dire ici quelques mots de leurs rapports avec la vie indépendante des cléments psychiques.

La mémoire, a-t-on dit, se résout en des mémoires ${ }^{1}$; la volonte se résout en des volitions. On pent generaliser cette manière de voir. Il semble bien que toutes les facultés de l'esprit sont aussi largmentees ou peuvent l'être. Nous aurons à examiner les conséquences de ce fait, mais il fant d'abord en hien determiner la signifieation. .

De tont re que nous renons de voir il parait résulter que les systèmes psychiques peuvent, dans des conditions

1. Ribot, les Maludies de la mémoire, p. 106 et suiv. 
appropriées, naitre et fonctionner dans une certaine mesure, independamment des autres systèmes qui composent l'esprit, c'est-i-dire sans se combiner avec eux. Chaque systeme parait aroir sa vie propre, comme chaque éèment biologifue a jusqu'is un rertain point la sienne, el dirllems un srsteme ne parait ètre que lactivité coordomnée de cellules ou de filmes nerveuses, cesta-dire yu'il existe pour chaque systeme une organisation particulière qui implique ou qui explique la mémoire, at moins au sens biologique, la sensibilité, l'intelligence (comme pouroir de coordination) et la rolonté (comme pouvoir de mourement ou d'arrèt). De plus ces qualites sont intrinseques pour ainsi dire it chaque système. Chez un individu donné, tel ou tel systène peut ètre très fortement doué au point de vue de la mémoire, de la senșibilité, de l'intelligence, ou de la rolonté, tandis que tel autre l'est trés peu. Pour la mémoire, je n'ai pas it insister leaucoul, les faits sont a frésent connus et interprètés 1. On sait que certinines ménoires très speciales peurent exister seules a un degre tres developpé. L'exemple des ralculatemrs prodiges est hien commu et tres frappant. Jai assiste a une seinne domnee par Inandi. On lui propose sept operations a la fois. me addition (quale nombles dr fualle chillres), me soustraction (huit ehilfres environ), une multipliation, une division, deux extractions rle racine cubique et mo extraction de racine carree. Il a fait toutes aes oremations sucessivement sans

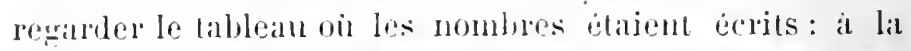
fin l. lit siance, il a pu esimmerer loutes ces operations

1. On le doit surtout aux travaux de MIM. '1rine, Griton, Ribot et Charcht. Voyez Rilsu!, Maladies de la mémoire, chal. III; Taine, de l'Inellygrece, vol. I; Galton, Inquiries into human facreley, Mental imagery, Numberform, colour association, etc.; G. Ballet, le La.lgugr interiener in liphasie. 1. 28. 
(données et rẻsultats), en commenģant par la dernicre, plus une multiplication de trois chiffres et nue soustraction de vingt-sept chiffres qu'il avait faites précédemment, sans hésitation et presque sans erreur. Il m'a dit qu'il pourrait se rappeler encore ces chill'es pendant quelifues jours, mais que, a part les chiffres, il n'avait pas une bonne mémoirc.

La sensibilité est spécialisée comme la mémoire, j'entends ici par sensibilité l'excitabilitẻ en général; je veux dire, par conséquent, que chez le mème individu certains systèmes sont plus facilement que d'autres mis en activité. Il n'est guẻre besoin, sans doute, d'appuyer sur cette considération; chacun a souvent remarqué combien cerlaines personnes discomrent volontiers sur certains sujets, combien elles sont sensibles a tout ce qui peut se rapporter í un point spécial, combien elles sont irritées, chagrines, ou au contraire joyeuses et épanouies à chaque allusion qui peut réveiller le système partịculièrement scnsible. Il est très important de remar'quer que ce systime ne tient pas bien souvent une place considérable dans la constitution de la personnalité. Le contraire se produit évidemment aussi, mais cela a moins d'importance au point de rue des sensibilités spéciales. C'est un fait bien connu qu'il y a " une manière de prendre les gens " c'est-à-dire d'appuyer sur tel ou tel ressort, de toucher “ l'endroit sensible », en flattaul une vanité particulière, on une haine ou une idée fixe. Tel enfant est sensible aux caresses, tel autre a la raillerie, tel autre aux coups; il y en a beaucoup, bien entendn, qui sont sensibles a plusieurs excitants à la fois. De même cerlains systèmes d’idées, comme certains systèmes d'actes sont éveillés plus facilement chez certaines personnes et l'effet varie d'une personne a l'autre. Tel récit laissera indifferente telle personne qui passe cependant pour sensible et frappera vivement une autre qui, d'une 
manière génèrale, eśt plus indifférente, si par des circonstances de constitulion ou par suite d'événements particuliers, il peut réveiller chez la derniere un plus grand nombre d'éléments jsychiques ou des éléments mieux exerecs, et plus aisénent excitables. Il parait probable d'aillen's que la sensibilite et la mémoire ont au moins quelpues conditions organiques communes : e'est ce qui est relativement le mieux organisé en nous qui se mel le plus facilement en activité. On sait combien les occupations professionnelles et les habitudes, comme la nature premiere, influent sur la manière de conceroir les choses; derant un accident un médecin pent ètre intéressé scientifiquement, une mère pensera a son fils. La gruerre n'est pas la mème chose pour un ufficier ambitieux el pour la fiancée liun soldat. Un psychologue est porté a analyser, a comparer l'esprit el le caractère des personnes qu'il connait; un négociant les envisage at un autre point de vuc: un ma thématicien sera enclin a remarpuer si les nomJres qui lufi passent devint les yeux offrent des caracteres de divisibilite par tel ou tel chifre, ete. Ce nest pas que les systemes psyluipues n'rxistent pats a l'etat latent ehe\% les personnes mirme rhez lesquelles ils ne se réveillent pas, nalis ils nont pis le degre de semsibilite voulu pour se met-

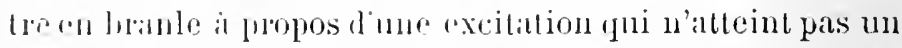
donge endnent de foree "t le qualite spécifique. Par exemple, le physiologiste fui exprimente sur mn chien ou un cobaye sait bien fur cel animal peut souffrir, mais sult organisation mentale ol ses babiutudes scientifiques lont fu'il ne songe pas a ce linit: on d flue, s'il y songe, re

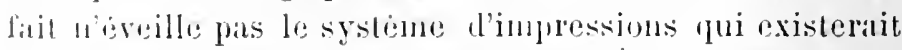
rles 111 spectatem on une sperlatrice antrement doués. Il llest pits sensilule en ce sens. Lin revaluche, lo spectatoul an la spectatlice qui söndignent sont moins sensibles a l'inporlance des recherehes seientifiques. Ce n'est pas düils lle silchent anssi que res recherches sout 
importantes, mais ce point de vue les frappe moins et ne détermine pas chez eux la mise en activité du système qui constitue le zèle scientifique et qui pourrait peut-être être réveillé par des moyens appropriés. On raconte que Fontenelle dinait de temps en temps avec un ami; ils mangeaient chaque fois, il la saison, me botte d'asperges dont ils étaient très friands, mais chacun les aimait i sa manière, Fontenelle i l'huile, son ami à la sauce. On apprêtait donc pour chacun sa part. Un jour, au moment de se mettre a table, et comme on allait commencer à préparer les asperges, l'ami est pris d'une attaque d'apoplexic et tombe mort, Fontenelle se précipite immédiatement dans l'escalier : a Toutes à l'huile », cria-t-il. Dira-t-on que Fontenelle n'était pas sensible ? Cela dépend. Il n'était peut-ètre pas très sensible à l'amitié, mais il serait injuste de lui dénier une vive sensibilité à l'endroit des asperges.

Ainsi la pluralité des sensibilités de l'homme se marque par ces deux faits : $l^{\circ}$ que chez un homme certains systèmes sont plus facilement excités que d'autres; $2^{\circ}$ que certains systèmes de sentiments qui sont communs à une grande pdrtie de l'humanité, le regret, le remords, la joie, et certains systèmes d'actes sont surtout déterminés chez chaque individu, par la mise en jeu d'un ou de plusieurs systèmes particuliers, à l'exclusion des autres.

La pluralité de l'intelligence n'est pas moins visible que celle des sentiments : il n'y a pas une intelligence, il y a des intelligences. L'intelligence ne doit pas ètre considérée comme une faculté ou un pouroir psychique ou nerveux existant a part des phénomènes et les réglant; elle fait partie des phénomènes, elle est unie à certains groupes de phénomènes, elle est la loi sclon laquelle s'arrangent certains groupes de faits; mais cette loi peut ne pas ètre semblable pour tous les groupes de faits qui existent dans un cerveau. Chaque système psychique a sa manière de se coordonner, il comprend d'ailleurs, un 
plus ou moins grand nombre d'éléments et selon que ces éléments seront plus ou moins nombreux et plus on moins complexes eux-mêmes, sclon qu'ils pourront s'abstraire plus ou moins facilement, se séparer les uns des autres, se décomposer en éléments plus simples, et se combiner facilement de différentes manières, selon qu'ils seront plus ou moins bien systématisés, nous verrons naître des intelligences particulières, variables d'un individu à l'autre pour le même système psychique, d'un système psychique à l'autre pour le même individu. Tout le monde, par exemple, n'est pas de la mèmc force au whist ou au jeu de dames et inversement une personne peut être très habile pour ces jeux et assez peu intelligente pour le reste. Les faits de ce genre sont d'une fréquence telle que chacun peut en observer ; certaines personnes sont des. commerçants ou des industriels distingués et n'ont, en dehors de là, aucune aptitude remarquable. Il y a des hommes de science qui sont complètement fermés aux lettres, et des hommes de lettres qui ne comprennent rien aux sciences; bien des personnes qui passent pour intelligentes et qui, en effet, le sont sur certains points, ont beaucoup de peine a suivre un raisonnement mathématique. L’inverse se présente aussi. Pour descendre des mathématiciens aux calculateurs, les petits calculateurs prodiges, Mangiamele, Mondeux, Inaudi, etc., ne paraissent pas avoir été remarquablement doués en dehors de leur intelligence spéciale, qui même dans ce domaine du calcul reste très spécialisée.

lintelligence que nous étudicrons plus loin avec plus de précision est, en somme, une manière particulière de coordination des éléments psychiques abstraits. Résoudre un probleme, c'est trouver des résultats qui se combinent de manieye at s'accorder avec certaines donnees; gagner une partic d'échecs, e'est combiner de telle manière les mouvements de ses pièces que l'échec et mat soit donné au roi de l'adversaire; guérir une maladie, c'est combiner 
diverses influences de médicaments, de diète, de repos, d'exercices, etc., de manière a rétablir ou à laisser se rétablir le bon fonctionnement de l'organisme. Il y a évidemment dans tout acte intelligent une coordination plus ou moins consciente d'images, de sensations et d'idées; cette coordination se distingue de celle qui produit les sentiments par un trouble psychique moins considérable, par une systématisation plus parfaite, de l'activité volontaire par le manque ou l'affaiblissement de certains éléments, impulsion et attention qui caractérisent les volitions, et de l'activité automatique par une systématisation moins parfaite et relativement plus compréhensive. Mais il n'est pas nécessaire d'entrer ici dans ces distinctions et d'autres qu'on pourrait trouver, et nous pouvons nous en tenir au sens un peu vague dans lequel on prend vulgairement ce mot : facultè de compréhension et de combinaison.

La pluralité des intelligences dans un sens comme dans l'autre parait indiscutable; tout le monde a vu avec quelle facilité chacun combine et comprend. Dans une certaine sphère, avec quelle facilité il se trompe et raisonne faux dès qu'il en sort. Il est en chacun des éléments intelligents et généralement il y en a un plus grand nombre qui ne le sont pas. On raisonne bien sur un point, on raisonne faux sur d'autres; cela se marque d'une manière frappante chez les personnes qui veulent juger des théories appartenant à des ordres de connaissances qu'elles possèdent mal : généralement elles sont incapables de faire le moindre raisonnement juste. Ce fait facile à constater est peut-être celui qui, si on l'examine un peu attentivement, montre le mieux combien l'intelligence n'est pas, bien souvent, une faculté générale de l'esprit ou du cerveau, mais une manière d'être particulière de systèmes psychiques qui peuvent rester isolés. Je prendrai comme exemple le fait suivant: J'ai assisté à des séances données par 
un liseur de pensées, Picliman, l'émule de Cumberland. Je ne parlerai pas ici de l'interprétation que I'on doit donner de ces pliénomènes; les expériences que j'ai vues ue mont pas paru suffisamment concluantes pour que je me sois litit une opinion bien arrètée; peut-être la suggestion par le tact et le sens musculaire ne peut-elle pas tout expliquer, mais je ne l'affirmerai pas. Je ne voudrais pas non plus nier qu'une partic des phénomènes ne fùt due au talent de prestidigitateur de Pickman. Quoi qu'il en soit, un certain nombre de spectateurs ne royaient en tout cela qu'une mystification et comme Pickman commettail parfois des erreurs, j’ai entendu dire : si c'était de la suggrostion, il ne se tromperait jamais. C'était là une raison en favenr. Cependant les personnes qui la présenl.ient, si elles vout parfois à la chasse, doivent savoir fuim manque parfois le gibier, si elles ont fail des problèmes elles ont pu voir qu'on arrivait quelquefois à des résultats erronés et qu'on peul se tromper même en faisant une multiplication, surement elles n'en concluent pas que le chasseur qui tue une piece est un prestidigitateur ou que la table le Pythagore est une mauvaise plaisanterie. Mais ici, elles s'avançaient sur un terrain qui ne leur était pas connu ct les úléments psychiques qui s'éveillaient en clles dans res conditions manfuaient completement de cohélence générale et s'unissaient en des systèmes qui ne s'hamonisaient pas avec l'expérieuce. Une intelligence abstrate n'eut pas manqué de remarquer que: $1^{\circ}$ une fores fuelconque peut atteindre un certain degré et ne pas êtro capalble d'aller au deli; 20 que le phénomène dont il stait question etait trop mal commu pour qu'on pùt dirremment il devait être all cas oì il serait réc ; $3^{\circ}$ que dos rorronstances particulieres pouvaient quelquefois obscurcin la firculte particulière de Pickman au cas où elle existerait, romme des circonstances particulieres amoindrissent toutes les facultes yossibles, etc. Ce n'est pas ì 
dire que ectte intelligence abstraite ne se puisse acquérir jusqu'à un certain point et nous verrons tout à l'heure, comment on doit l'interpréter. En somme, elle ne parait exister que par une bonne systématisation de tous les éléments psychiques, c'est-à-dire qu'elle fait bien encore partie intégrante de ces éléments, mais qu'elle est partie intégrante de tous ou de presque tous.

L'illogisme qui est si fréquent dans les raisonnements humains, comme chacun sait, n'est pas autre chose qu'une illustration du mème principe. Un homme illogique est celui chez qui coexistent ou se succèdent fréquemment des idées qui ne sont pas en harmonie, c'est-à-dire qui ne sont pas dans un rapport parfait cle finalité, mais ces idées ne sont que des systèmes d'images, de résidus moteurs, sensitifs, etc.; chacune existe à part, chacune a son intelligence propre, elle a son intelligence en soi, parce qu'elle est coordonnée et que l'intelligence n'est pas autre chose qu'une forme particulière de coordination de certains éléments; les systèmes pris à part sont intelligents, l'individu, leur somme, ne l'est pas.

Remarquons, en passant, que les idées les plus contradictoires en apparence peuvent très bien s'adapter l'une à l'autre quand on les conçoit d'une certaine manière, c'est-à-dire quand on leur retranche ou qu'on leur ajoute certains éléments sans les modifier sensiblement. Par exemple, on peut trouver que telle ou telle coutume est bonne et mauvaise à la fois, si on complète la pensée en entendant qu'elle est bonne dans certaines conditions, mauvaise dans d'autres. C'est ce fait qui a fait probablement la fortune de l'identité des contradictoires, absolument insoutenable quand on la présente conme on le fait parfois. Mais bien souvent les idées ne sont pas mises en harmonie quoiqu'elles puissent l'ètre sans trop de peine; d'autres fois encore elles ne peuvent réellement pas l'ètre. Les idées sur les oljets de la religion, Dieu, la vie future, 
etc., présentent souvent, non pas dans les catéchismes ou les cours d'instruction religieuse (où déjà elles sont passablement illogiques), mais chez les individus, une incohérence remarquable. La mème personne vous dira selon les moments : on n'a jamais ru revenir un mort, quand on est mort c'est pour longtemps, etc.; d'un autre côté, elle s'indignera contre " ces doctrines qui nous assimilent à des bêtes » et qui nient l'àme et l'immortalité. L'homme est manifestement considèé aprés sa mort, à la fois, comme pourissant dans la terre et comme vivant dans le ciel, mais il ne s'agit pas ici bien souvent d'une séparation radicale entre le corps et l'àme; on donne presque toujours un corps à l'àme - il faut avoir l'imagination abstraite et l'esprit cultivé pour la concevoir ou s'imaginer qu'on la conçoit autrement - et aussi une áme à ce corps. Le séjour sous la terre épouvante un peu, il semble que réellement quand on serit cu elle, on en sentira le poids. Il n'est pas difficile de voir dans ce fait la coexistence de deux systèmes d'idees, issus d'expériences différentes d'abord et ensuite d'expériences et d'enseignements rlivers, systemes (qui coexistent dans l'esprit sans s'unitier, et qui se composent chacun d'idées et aussi de raisonnements, car chaque.systène aboutil de temps en temps à des imaginations nouvelles, à des actes, à des exclamations. Ici encore chaque systeme est intelligent,

- pris en soi ; leur somme, l'individu considéré comme un tont. ne l'est pas au mène degré. Les religions et les thenries sur la vie future ancienues el nouvelles donnent. me bonne collection de faits de ce genre 1. Il faut les prendre surtoul évidemment non chez les philosophes

1. Vour les ouvrages sur les civilisations inférieures et les civilisations primitives, Lubbock, Origines de la civilisation; Tylor, la Civilisation primitive; Maspero, Histoire ancienne des peuples de l'Orient; Perrot. Histoinc le l'Art; Reville, Proligomenes de l'Histoire des Religions ct les Religions des peuples non rivilisés, otc. . 
ou les théologiens qui ne sont pas, cependant, exempts de contradictions, mais chez les gens incultes ou chez les peuples plus rapprochés des origines, chez qui les systèmes divers entrent moins en rapport et où la contradiction est plus nette et moins masquée.

Comme il y a des mémoires, des sensibilités et des intelligences, il y a aussi des rolontés. Chaque système se souvient, sent, pense et reut pour son compte, ce n'est que par leur harmonie et leurs combinaisons que l'individu pense et agit à son tour. On a sourent dit que toute idée était une tendance à un acte, cette loi qui peutètre gagnerait en exactitude et en précision à être quelque peu modifiée, est assez vraie pour que je fasse remarquer ici combien elle s'accorde avec tout ce que j'ai dit. Toute idée tend à un acte; il en est de même pour tout sentiment, c'est-à-dire en somme, que tout système psrchique tend à se compléter par des volitions et des phénomènes moteurs ${ }^{1}$, que chaque système a sa volonté. “ L'homme le plus vertueux, dit M. Renan ${ }^{2}$, ne peut empêcher que, dans les profondeurs de son organisation, des millions de créatures rudimentaires ne crient : "Nous voulons être. " Ces petits êtres ne sont pas moraux, ils n'ont pas lu Malthus, ils n'aspirent qu'à exister pleinement. "L'observation est juste et Schopenhauer l'arait faite en d'autres termes, il faut seulement la généraliser. Chaque organe représente une rolonté, un désir; le cœur veut battre, les poumons veulent respirer, les pieds veulent marcher, les intestins veulent digérer,la preuve en est que le cœur bat séparé de la poitrine, queles intestins d'un animal éventré, continuent leurs mouvements péristaltiques : cest que nos organes sont reliés à des systėmes d'éléments nerveux qui fonctionnent harmoniquement, les ganglions du cœur, le

1. Les Phénomenes affectifs et les lois de leur apparition, p. 21.

2. Reaan, Dialogues philosophiques. 
centre respiratoire de la moelle allongée, les organes coordinateurs des mourements dans la moelle allongèe, le mésocéphale, le cervelet, mais il existe des systèmes d'éléments nerveux et psychiques semblables qui ne se rattachent a aucun organe en particulier, mais qui n'existent pas moins jour eux-mêmes, sentant, jouissant, souffrant, voulant chacun pour soi. Nous assistons contimuellement à une action harmonique et aussi à des conflits de ces systèmes. Une certaine coordination d'éléments psychiques nous pousse à rechercher les honneurs, une autre a chercher le profit, une autre nous mène à la recherchc désintéressée de la vérité, d'autres nous poussent au jeu, à des distractions diverses, d'autres encore a telle ou telle occupation, et plusicurs de ces systèmes existent a la fois chez le même individu. Les faits de ce genre, les conflits de ce que nous appelons nos désirs, nos tendances et qui sont l'expression de l'activité séparée et souvent mal coordonnée des systèmes psychiques qui sont liẻs physiquement à un mème organisme, sont tellement fréquents que je n'ai pas besoin d'y insister. Ils sont connus de tous; on les trouve partout, dans les livres et dans la vic, il me semble bicn qu'il n'y a pas pour les interpréter, de théorie plus simple ou plus exacte que celle que j'expose.

Mais il y a encore d'autres moyens de constater la pluralité des volontés. C'est de roir combien le mode de vouloir change quelquefois, selon le système qui est en jen, comment le mème homme peut avoir sur différents points une volonté tantôt ferme, tantôt lıesitante, tantòt tenace, tantôt faible. C'est que le degré d'organisation des systèmes n'est pas le mẻme, ni leur degré de sensibilité. Tel homme restera inflexible sur certains points et vis-avis de cortaines personnes, qui cèlera facilement sur d'autres points ou vis-a-vis de certaines autres per'sonnes.

Dire que chaque système a son activité, c'est dire que 
chaque système a sa moralité, la moralité n'étant qu'une systématisation parfaite des actes. Je connaissais une personne qui était à la fois zélée pour la religion et pour les devoirs de son état, sa religion lui interdisait de travailler le dimanche, sa profession le lui commandait quelquefois, et généralement ce n'était pas la religion qui l'emportait. Je lui avais fait remarquer le fait sans avoir naturellement de réponse bien satisfaisante. On voit bien ici comment chaque système a sa moralité propre, et cela se voit encore en bien d'autres cas. Les conflits de devoirs sont bien connus, ils répondent précisément à ces conflits de systèmes psychiques qui ont chacun leur moralité propre et une moralité qui ne peut se réaliser sans nuire a la moralité du système voisin. Toute la casuistique, c'est-i-dire une bonne partie de la moralc, a pour matière ces conflits de systèmes. Un livre récent de M. Brouardel ${ }^{1}$, montre quelques cas fort intéressants, les cas où le devoir de l'homme et le deroir du médecin sont en conflit direct. Il n'est pas toujours aisé de dire lequel des deux doit l'emporter et où se trouve la vraie moralité. M. Brouardel même qui penche pour faire trionpher, partout et toujours, le devoir du médecin, ne peut s'empècher de trouver parfois des expédients ingénieux pour donner satisfaction au devoir de l'homme. Il $\mathrm{Y}$ aurait beaucoup à dire sur cette question du conflit des devoirs; je dois me borner ici à la rappeler et à la signaler en faisant remarquer qu'elle est une conséquence naturelle de notre conception générale de l'homme.

Il y a ainsi des mémoires, des intelligences, des raisonnements, des volontés, des moralités: c'est que chaque faculté tient aux autres. Un système psychique est un tout complet à certains égards et sclon que nous considérons son activité à un point de vue ou à un autre nous l'appellerons mémoire, intclligence ou volonté; en fait, ces

\section{Brouardel, le Secret médical.}


facultes sont des formes différentes de la tinalité psychique, et dans l'homme elles correspondent à des éléments diffélents différemment associés; mais en considérant un système psychique comme un tout, nous pouvons lui attribuer facilement la mémoire, la sensibilité, l'intelligence, la volonté, selon que uous considérons la permanence des éléments qui le composent, la facilité avec laquelle ils entrent en jeu, la manière dont ils se coordonnent et l'action plus ou moins énergique qu'ils exercent sur d'autres èléments psychiques.

Pour le moment nous devons répondre à l'oljection qui pourrait être faite aux théories que j'expose, de ne pas tenir compte de l'unité du moi : n'y a-t-il pas réellement, ell chacum de nous, une unité de sentiment, de volonté, de personnalité? Il y en a une, quelquefois, et à un certain point de vue, il y a surtout une unité organique, la moins imparfaite chez nous, mais ce fait n'cxclut pas l'autre si nous les interprétons bien tous les deux.

Il est des gens qui ont, d'une manière géuérale, une bonne mémoire, d'autres qui ont une bonne intelligence générale, d'autres qui ont uné volonté sans hésitation ni lutte et une per'sonnalité bien nette. Ce fait semble d'abord devoir être extrêmement rare ; si nême on Jousse les choses a l'extrême il semble impossible, mais supposons-le récl, il ne signifie pas autre chose que ceci : les systèmespsychiques rui ont une existence propre peuvent ue pats être en conflit et se rénnir dans un systeme supérieur dout ils sont à leur tour les élóments. Ils peuvent aussi, cho\% une uneme personne. avoir certaines ressemblances générales an point de vue de la vigueur, de la vivacité daus l'activité, ressemblances dépendant en certains cas des conditions organiques d'existence fui leur sont communes a tous. C'est lit règle dans le domaine biologique. Le systeme daction du ceur et le systeme d'activité du poumon sont bien capables, jusquu'à un certain 
point, et a un point de vue théorique, de subsister indépendamment l'un de l'autre, mais en fait, dans un organisme donné, ils sont étroitement liés ensemble; en modifiant volontairement le système de la respiration il est possible de modifier indirectement le système cardiaque, de plus un arrêt complet de la respiration finirait par amener un arrêt des battements du cœur, comme un ar rêt prolongé des battements du cœur entraînerait un arrèt de la respiration. L'indépendance visible des associations systématiques, le fait que la systématisation est une propriété des éléments psychiques, n'implique pas que les éléments psychiques soient toujours, ou même le plus souvent, ou même jamais, en désaccord les uns avec les autres, et qu'ils existent en aucun cas complètemənt isolés. Souvent ces éléments se réunissent en systèmes, ces systèmes en des systèmes supérieurs qui peuvent se comporter à leur tour comme un seul élément et ainsi de suite jusqu'à l'unification complète de la personne qui n'est jamais arrivée et jusqu'à la systématisation de l'univers qui n'arrivera probablement jamais. Nous verrons plus tard comment ces coordinations différentes, précédées de différentes décompositions et accompagnées d'inhibitions systématisées, constituent les différentes formes de la vie mentale. Examinons seulement ici comment ces synthèses supérieures s'expliquent avec notre conception de la vie des éléments psychiques.

A côté de l'association systématique des éléments il se produit un phénomène d'inhibition qui complète le premier, el concourt avec lui pour donner une forme définie à l'élément psychique. Les éléments secondaires qui entrent dans un élément supérieur abandonnent certaines de leuis associations, renoncent au moins momentanément à certaines de leurs activités, - comme des hommes qui, tandis qu'ils accomplissent une cuuve commune, négligent au moins certaines de leurs affaires individuelles, 
renoncentà la satisfaction de quelques-uns deleur's goùts.On comprend que ces deux propriétés générales combinées permettent aux éléments de former des combinaisons de plus en plus complexes et toujours unifiées. Ainsi lorsque plusicurs éléments seront fortement associès ensemble l'un d'eux ne pourda pas contracter une nouvelle association qui ne saurait s'accorder avec le sens général de l'association principale, - l'inhibition dure autant que l'association supérieure persiste sans se relàcher.

Ainsi lactivité des éléments est individuelle, mais, comme nous le verrons plus en détail, elle est détermince souvent pour une grande part, et on peut le dire, toujours en quelque chose, par la nature de l'ensemble dont ils font partie - comme l'activité des hommes est toujours une activité individuelle, bien que les formes de cette activité soient souvent déterminces en très grande partie et toujours à quelque degré par la forme même et la nature de la societe dont ils font partie.

Nous nous occuperons dans la seconde partic de ce travitil des lois générales d'association et d'inhibition, de leur application a tous les phénomènes, et des formes complexes qui les manifestent, pour le moment nous n'avons pas á nous placer a ce point de vue, bien qu'il ait été nécessaire de l'indiquer.

Il y a dans l'esprit des mémoires, des intelligences, des activités; que ces mémoires, que ces activités s'harmonisent ou se combattent, qu'elles se ressemblent ou qu'elles diffèrent, nous n'en avous pas moins une loi importante du fonctionnement de l'esprit, de même que dans une société les hommes en travaillant chacun pour soi peuvent arriver à l'harmonie ou à l'anarchie, de même les èléments psyehiques, en se souvenant, en comprenant, en reflechissant chacun pour soi, peuvent aboutir a la personnalité une, romprenant, sentant et voulant, ou a tous les désordres, à tous les caprices, a toutes les dépravations. 
La loi ne donne donc pas jusqu'ici l'explication de toutes les formes les plus élevées de l'espril en tant précisément qu'elles sont supérieures, cę sont ces formes supėrieures qui nous restent à étudicr, mais nous voyons qu'elles impliquent la mème propriété des éléments nerveux et psychiques, avec les circonstances particulières oủ ces tendances de chaque élément à l'harmonie et à la finalité pourraient être en somme le mieux satisfaites.

De tous les faits que nous avons cités, des synthèses partielles que nous en avons faites, il résulte donc ce fait général que sur une unité organique sans laquelle la vie serait impossible, peuvent coexister un grand nombre de systèmes psychiques qui atteignent un degré assez marqué d'incohérence et d'indẻpendance, chacun existant en soì et pour soi, et travaillant pour soi. Evidemment cette indépendance ne peut ètre complète, pour deux raisons capitales : la première est une cause efficiente, c'est l'unité de l'organisme qui est le support et, dans une certaine mesure, la cause des plıénomènes nervoso-psychiques, la seconde est une cause finale au sens positif du mot, c'est l'impossibilité pour un organisme de subsister' si l'indẻpendance et l'incohérence des actes devient trop grande. Mais il reste encore assez de marge pour que les faits se chargent de montrer à la fois que la systématisation est la loi la plus générale de l'esprit et que ce caractère de finalité appartient aux éléments psychiques qui peuvent d'ailleurs être plus ou moins compliqués et composés ; dans un cas idéal qui marquerait la perfection de la personne humaine, cette personne ne formerait qu'un seul élément, décomposable seulement d'une manière théorique, mais où l'existence isolée ou plutôt l'incohérence des élėments secondaires ne pourrait se montrer. 


\section{CIIAPITRE IV:}

\section{Les causes qui fusoriseut ou qui controrient lactivité des éléments psychirues.}

81.

Yous arons ainsi reconnu l'existence dans l'esprit d'éléments plus ou moins complexes, connosés d'éléments d'ordre inférieur, s'unissant pour former des éléments plus synthetiques et en tant qu'ils existent comme tout systématisé, agissant chacun pour soi, cherchant à susciter et a s'associer les faits psrchiques đui peuvent s'harmoniser areceux, se joindre a eux pour une fin unique, el au contraire à arrèter, à inhiber tout fait, toute activité qui les contrarie. Dèsquills se sont associés, un autre fait, une nouvelle sýnthèse en resulte, ainsi qu'une nouvelle formo de l'inhibition, et la nouvelle sinthèse est 'à son tour un elément psychique plus complexe que le précédent mais qui comme hi travaille pour soi jusqu'i ce qu'elle s'associe encore à d’antres pour former un élément d'orde superient, une sinthese plus complexe qui agira elle aussi de la mème maniere.

On comprend latilement qu’il puisse résuller fréquemment de eette activité relalivement indejpendante. - je dirais volontiers personuolle - des déments peschiques, dos luttes et des contlits; l'areord est somvent precede part drs antagonismes direcels ou indireets gui durent jusqü

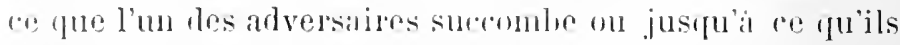
disparaissout tous denx, er qui arrive, ou jusqu'il ce que tous deux jarviennent a s'établir daus l'esprit, chaem sur soll teratu ot gardent desormais nue sorte de neutralite

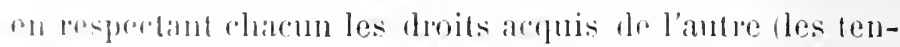
lintures scimblifipues et les systimes religienx rhez les salvants qui tienuent selon le not de Vogt « lenr esprit en 
partie double ) ou bien encore jusqu'à ce que l'un des deux systemes psychiques ou tous les deux à la fois abandonnent certains de leurs éléments et rendent possilile une synthèse systématique supérieure qui les réunisse. tous les deux, rui en fasse un seul tout (c'est l'opérátion mème que M. Fouillée recommande pour la synthèse et la conciliation des systèmes philosophiques ${ }^{1}$.) La lutte directe est produite par l'inhibition que chacun des systèmes psychiques tend à exercer sur l'autre, par l'association que tous les deux à la fois cherchent à contracter avec un autre élément que tous les deux veulent absorber et s'assimiler, comme lorsque, par exemple, nous hésitons entre deux théories et que les systèmes psychiques quiles représentent tendent chacun à interpréter à leur manière un fait qui vient à notre connaissance et à s'en faire un nouvel élément. La lutte indirecte se manifeste quand deux systèmes non harmoniquement reliés tàchent chacun d'attirer à soi les forces psychiques, l'activité de l'un tinit souvent, sans qu'il y ait el conflit direct, par éteindre ou amoindrir l'activité de l'autre, par exemple une occupation a laquelle nous nous habituons fait souvent disparaitre certaines habitudes prises sans que nous ayons eu jamais a choisir entre elles, sans que jamais une manière d'ètre de l'esprit se soit directement opposée à l'autre.

Le succès de la lutte des éléments psỹchiques dépend évidemment de conditions nombreuses et diverses, tel système est favorisé et triomphe soil à cause de sa force propre soit à cause des circonstances favorables qu'il a rencontrées. Nous devons ici rechercher les principales causes, les principales conditions qui peuvent favoriser un élément psychique ou au contraire lui nuire et rendre

1. Voyez les ourrages et les articles de M. Fouillée. La liberté et le determinisme. La science sociale contemporaine, etc. 
sa défaite probable. Il est bien entendu d'ailleurs que l'une de ces conditions peut ètre compensée et au delá par la réunion de plusicurs autres qui favorisent ou contrarient le. système opposé, mais chacune d'elles, prise en cllemême, et toutes choses égales d'ailleur's, est une chance de succès ou de dèfaite.

\section{§. 2.}

La nature même de leur organisation propre, et pour ainsi dire leur intelligence, leur sensibilité, leur activité particulière, ainsi que la nature de leurs rapports avec les autres systèmes qui composent l'esprit, voilà l'influence la plus importante qui puisse agir pour faciliter ou pour arrèter la naissance et le développement d'un élément psychique. Plus un composé est fortement organisé, plus les éléments qu'il comprend sont étroitement liés les uns aux autres, plus est considérable le nombre d'autres éléments auxquels ce composé est susceptible de 's'unir harmoniquement, plus aussi il a de chances de se développer ou tout au moins de se maintenir. Une croyance qui est associé a tous les sentiments de lhomme, qui a cté fortifiec par l'édncation, yui s'harmonise arcc toutes ses aspirations - élevées ou vulgaires - aura, qu'elle soit d'ailleurs vraie on fausse, beiluroup de chances pour résister aux tentatives faites pour l'ébranler, c'est-à-dire pour vaincre les autres croyances, les autres systemes psychiques ru'on essayera de faire naitre dans l'esprit, au contraire une opinion qui ne tient à rien, c'est-a-dire i laruelle nous ne tenons pas, sera sourent aussi facilement lenversée qu'elle a été facilement acceptée. On peut dire d'une manière généralc que l'organisation acquise est la condition de l'organisation future (effectuée dans le mème sens plus un systeme psychique, croyance, halibude, sentiment est devenu cohérent, plus il est solidement rattache aux laits fondamentaux de la vie psychique 
et organique, plus aussi il a de chances pour s'assimiler les nouveaux faits produits, pour déterminer leur interprétation ou pour arrêter le développement, pour empêcher l'existence même de ceux qui pourraient lutter contre lui. Au reste, l'influence de l'organisation rentre dans les lois de l'activité mentale, - l'organisation étant la forme essentielle de l'esprit, - bornons-nous à indiquer ici son rôle dans les conflits et le développement des éléments psychiques.

C'est à l'organisation qu'il faut rattacher certaines conditions qui jouent un rôle dans la sélection psychique: l'attention, par exemple, qui détermine si visiblement le rejet ou l'adoption de certaines impressions, de certaines idées. ou encore les goùts, les penchants que nous éprouvons et qui déterminent aussi soit le cours de nos idées et de nos rèveries, soit nos actes et par nos actes la série de nos perceptions, soit mème le triage de nos perceptions involontairement subies et l'ébranlement plus ou moins considérable qu'elles impriment à l'esprit. Il n'y a donc pas lieu de les étudier à part, nous les retrouverons comme formes de la loi générale de l'association systématique.

Mais d'autres causes viennent en aide aux éléments psychiques et facilitent plus ou moins leur formation et leur développement, en facilitant par les mèmes raisons leur organisation, ce qui est la mème chose. L'intensité, la répétition, la persistance des phénomènes tendent à rendre leur existence plus sủre et aussi il faciliter leur adoption. Ces causes ne sont pas d'ailleurs sans rapport avec les précédentes.

L'influence de l'intensité d'un phénomène est bien connue, un phénomène suscitera d'autant plus facilement les faits susceptibles de s'associer avec lui, et arrêtera d'autant mieux ceux qui pourraient s'opposer a lui qu'il aura lui-même plus d'intensité. Une image vive éveillera plus facilement des phénoménes affectifs qu'une image 
faible. Une douleur modérée est supportée sans cris ni mourements; une douleur plus forte arrache des plaintes ou détermine des actes; une douleur plus forte encore nous fait agir plus promptement ou plus vigoureusement, elle peut aussi déterminer un grand nombre de phénoménes incoordonnés, des rris, des trépignements, des gestes incohérents, ou bien des actes de volonté très énergiques pour les contenir. On sait combien une vive émotion laisse dans l'esprit des traces durables et profondes, comlieu le souvenir en persiste, et combien le caractère même et l'intelligence peuvent en ètre modifiés. Une dame effrayée par des voleurs prend tous les hommes qu'elle voit, mème son fils, pour des brigands qui veulent l'assassiner; une autre femme voit son enfant renversé par un cheval, les raisonnements, la vue même de son enfant qui se porte bien, ne peuvent la convaincre qu'il est vivant; une femme appelée voleuse dans une dispute, se persuade que tout le monde l'accuse d'ávoir volé (Esquirol): une autre frappée diun accés de manic a la vue des incendies allumés dans sa rue pendant la Commune, royait encore dans son délire les flammes quil'environnaient de tous còtés, six mois après l'explosion du début du mal I. On voit un effet instructif de l'intensité d'un phénomène quand on rencontre la persistance, sans aucune raison d'être au point de vue de la finalité générale de l'esprit, de quelques éléments psychiques associès jadis a un pliénomène d'une intensité considérable, comme, par exemple, dans ces faits cités par M. Luys : \& I)ux hommes daus la force de l'ige, dans la période de début de la paraýsie générale, avaient été exposés à des scènes terrifiantes. Lim avait èté surpris en flagrant délit d'adultère, l'antre avait lassé phusieurs jours à Paris an moment des derniers

(1) I.uys, Etudes de physiologie et de pathologie cerébrales. Actions reflexes du "ereau, p. 29. 
temps de la Commune, dans une anxiété poignante, sous le coup des réquisitions et des menaces de mort proférées contre lui, les deux malades, une fois sortis du milieu ou ils avaient èté frappés d'épouvante, ont néanmoins continué a conserver la même impression de terreur répandue sur leur personne. Leurs regards étaient furtifs, timides, leurs tètes inclinẻes, leurs roix dolentes, l’inquiètule peinte sur tous leurs traits; tout indiquait en eux une sidération profonde des forces nerveuses et un cachet spéeial imprimé à leur émotivité ; alors mème qu'ils pronongaient quelques phrases gaies, leur physionomie était toujours sombre.

"Pendant plus de six mois qu'ils ont été sous mes yeux, ces deux malades n'ont'pas changé d'aspect. Leur délire s'est organisé dans la série des idées contemporaines du début du mal, et insensiblement la démence les a surpris, figés en quelque sorte dans un état chronique de terreur.

" Un autre malade, M. D...., vivement impressionné lors des évènements de la guerre de 1870, étant capitaine de mobilisés, avait été vivement effrayé de scènes d'insubordination de ses hommes et de menaces de mort proférées contre lui. L'impression de terreur ressentie avait été si vive, que plusieurs mois après les évènements du déljut, ce malade présentait encore sur sa figure l'expression permanente d'un très vif effroi ; il croyait qu'on voulait le fusiller, et répétait sans cesse d'une façon automatique ces mots : " Je vous dis, Monsieur, qu'on veut m'assassiner ce soir. „ Peu à peu, par l'effet de l'cnvahissement de la démence, la physionomie perdit son expression de terreur primitive, mais néanmoins le malade, frappé profondément, répétait encore diune façon machinale, au bout d'un an, cette phrase précédente, sans participation de la sensibilité et sans aucune conviction $\gg 1$.

1. Luys, ouvr. cité. p. 72,73, voir aussi p. 74 . 
Un dément avait eu un duel au pistolet à Toulouse trente ans auparavant; «l'impression violente de la scène du duel, à la suite de laquelle il était tombé nalade, était restée gravée dans son esprit l'ume facon persistante, et tout en répondant avec justesse aux questions courantes de la vie ordinaire, il ne pouvait faire autrement que répéter ces mots sacramentels qui revenaient itérativement: «En attendant que le pistolet se fasse à Toulouse. n On lui disait : "Bonjour, Monsieur, comment allez-vous? - Pas mal et rous? En attendant que le pistolet se fasse à Toulouse. - Voulez-yous vous promener? - Oui, en attendant que le pistolet se fasse à Toulouse. » Une dame ayant cru être volée en 1870 eut, à la suite de cette émotion, un accès de manie et répétait sans cesse qu'on l'avait volée. Plus tard on lui rapporta ses valeurs, elle les toucha, les reconnut, et malgré cela, " tombée dans un état de manie chronique clle répéta itérativement pendant deux années consécutives avec une grande placidité de physionomie: " Je vous dis, Monsieur, que l'on m'a tout volé el que je suis dépouillée $* 1$.

Dilns ces fuits nous royous comment l'intensité, la violence d'une émotion peut faciliter l'organisation d'un système, rendre sa reproduction facile au point de déformer réllement l'organisation générale de l'esprit. Les éléments donl l’intensité a dé considérable ou ceux qu'ils ont logiquement suscités, acquièrent ume telle sensibilité que la moindre excitation les net en jeu. Chaque fois que de la force nerveuse se degage, elle prend la forme imposée far l'unotion violente qui a depuis longtemps disparu, nuais dont les traces subsistent, an grand détriment naludrellencul de la cohérence générale des phénomènes psicholoriques.

D'antes exemples sont fort propres anssi at nous montrer

1. Luys, ouv. cité, p. 146-147. 
le ròle de l'intensité ; ce sont ceux où nous voyons un état d'esprit d'abord faible devenir de plus en plus intense et son efficacité s'accroitre à mesure que son intensité augmente. Tel est, par exemple. le cas suivant que j'emprunte à M. Mandsley. Un homme de cinquante. ans, d'une grande vigueur physique, énergique, éloignẻ depuis plusieurs années de toute occupation active, éprouvait une impulsion au meurtre telle qu'il s'était vu forcé de se séparer des siens, de peur d'en devenir le meurtrier; l'impulșion « variait d'intensité mais elle ne disparaissait jamais entièrement. Quand elle était le moins forte ce n'était qu'une idée occupant constamment sa pensée, mais saus inclination positive à la mettre à exécution; une idée homicide plutòt qu'une impulsion homi.cide; de temps en temps elle acquèrait une énergie plus grande et atteignait au paroxysme. Cela durait peu, mais alors le sang lui montait á la tête, il éprourait une sensation de plénitude et de trouble dans cette partie, ainsi qu'un affreux sentiment de désespoir et un tremblement violent de tout le corps qui se couvrait d'une abondante sueur. La crise se terminait par un déluge de larmes, suivi d'un épuisement profond. Ces accès survenaient souvent durant la nuit et, alors, cet homme sautait hors de son lit dans un état de frayeur mortelle, frissonnait arec tant de violence que la chambre en tremblait; en même temps la sueur ruisselait sur son corps" 1 .

L'influence de l'intensité n'est donc pas douteuse. Mais qu'est-ce au juste que l'intensité? Nous n'arons pas absolument besoin de le préciser, car ce mot a, par lui-même, un sens suffisamment clair, toutefois il est intéressant de

1. Mandsley. Le crime et la folie, p. 137. Spencer et Bain ont étudié a lenr point de vue les effets de l'intensité. Voir aussi à ce sujet un article de M. Binet, L'intensite des images mentales dans la Revue philosophique de mai 1887. 
voir l'idée que nous pouvons nous en faire. Physiquement, l'intensité d'un son est proportionnelle à l'amplitude des vibrations. Psychologiquement, il est plus difficile de dire à quoi correspond l'intensité d'une sensation ou d'un sentiment. Y a-t-il quelque rapport entre cette qualité et le mode de vibration de molécules nerveuses? On est tenté de le penser, mais ces rapprochements si séduisants qu'ils puissent être ne sont guère que des déductions a priori trop hasardées et en somme ne nous offrent que des images qui plaisent à l'esprit sans lui rien apprendre et sans lui être fort utiles.

Un fait plus positif, c'est que l'on risque de confondre souvent la cause et ses effets, l'intensité et la plus grande complexité de l'organisation. Une image est un phénomène plus vif, plus intense qu'une idée abstraite, mais l'image renferme des éléments que l'idée abstraite ne renferme pas, la sensation est plus vive que l'image, mais l'image paraît biẹn être, en termes généraux, un abstrait de la sensation, de mème un sentiment fort contient des éléments que le même sentiment affaibli ne contient plus, si bien que cette question peut se poser : le fait devient-il plus complexe parce qu'il est plus intense, ou, au contraire, nous apparait-il plus intense parce qu'il est plus complexe? On voit que l'intensité se laisse, au moins en partie, ramener à l'organisation. Il semble bien, cependant, que l'expérience nous montre dans l'intensité du phénomène quelque chose qui diffère de la complexité de lor çanisation. Il semble bien que le même élément psychique peut être mis en jeu plus ou moins fortement. On peut admettre, avec M. Binet, qu'il se produit dans le cas de l'intensité plus grande, une désintégration d'une plus grande quantité de natière nerveuse el une production de chaleur plus considérable. Mais cela paraît impliquer encore une complexito plus grande de phénomènes chimiques at de phénomènes biologiques, et l’intensité 
nous paraitrait encore une forme supérieure de l'organisation. Le problème ne me paraissant pas susceptible d'ètre actuellement rẻsolu, et ne nous intéressant pas d'ailleurs d'une manière essentielle, je n'y insiste pas davantage.

\section{$\S 3$.}

La répétition a son influence aussi : d'une manière générale, et toutes choses égales d'ailleurs, un système psychique a d'autant plus de chances de s'imposer à l'esprit, de s'associer à ceux qui existent dejà, et, une fois établi, il sera suscité d'autant plus facilement qu'il aura été répété un plus grand nombre de fois. L'influence de la répétition n'est pas contestable. Un de mes amis, écrivant une lettre dont le destinataire habitait une ville renommée pour ses vins, remplaça sur l'enveloppe le nom du département par la date de l'année. L'habitude de faire suivre d'une date le nom de la ville, employé souvent pour désigner le vin qu'on récolte dans les environs, avait, grâce à l'inattention de mon ami, facilité un système qui ne s'harmonisait pas avec les autres systèmes en jeu. Les exemples de ce genre sont très fréquents, et à force de répéter un mot, une expression, on en vient à la placer dans des circonstances où elle n'est nullement en situation. On sait aussi combien il est utile de répéter certaines choses à certaines personnes pour arriver à les leur faire comprendre, et à les leur faire retenir. Un écolier apprend sa leçon en la répétant, en la relisant plusieurs fois de suite, si le sens d'une proposition, d'un théorème lui échappe, si un problème lui présente quelque difficulté, une nouvelle lecture lui facilitera le travail. Quand nous relisons un livre nous découvrons toujours de nouveaux faits, de nouvelles idées qui nous avaient peut-être frappés autrefois, mais que nous a vions oubliés et auxquels la répétition donne plus de chances pour résister aux causes 
d'oubli et se faire une place dans notre organisation mentale.

Nous arons vu précédemment l'intensité ètre une cause de persistance et le répétition, nous royons aussi la répétition être une cause l'intensite, et dans les deux cas le résultat est un aceroissement de lorganisation, non pas toujours de l'esprit en général, mais de certains éléments psychiques. Il arrive, par exemple, que jusqu'à un certain point auquel le pliénomène s’arrète, la répétition nous rend les plaisirs plus vifs, souvent on en vient a faire avec plaisir des choses qui ont été au début indifférentes, il est des ouvages qu'on a généralement besoin de relire pour les bien apprécier, un opéra, surtout si l'on n'en comnait pas la musique à l'arance, offre généralement plus d’intérèt à une seconde représentation qu'à la première, et ainsi de suite jusqu'i ce que l'on se hlase. Le pliénomène de l'augrmenlation du plaisir avec la répétition n'est nullement en contradiction avee le phénomène inverse qui est trés fréquent et qui succede sourent au premier; tant que de noureaux éléments psychiques sont engendrés par la répétition, taut qu’elle suggère de nouvelles idées, le nouveaux sentiments, tint que l'adaptation niest pas parfaite, le plaisir angmente, se maintient ou diminue selon le nombre et la qualité des nouveaux èléments formés, une fois que l'esprit a tiré l'un ourrage tout ce qu'il en pomvail tirer, une fois quil ne nait plus de nourelles idées, de nourramx sontiments (i) moins que les anciens n’aient été assez oubliés pour que leur réapparition at les elfets dume apparition), la satiété arrive, et l'indillèrence, on le dégoùt el l'enmui.

Nous royons se produire, sous l'intluence de la répétition, des effets analogues a ceux de lintensité. Une fois qu'un système a été suffisamment repété, il acquiert une sensililite tolle quil entre en jeu a la moindre excitition. M. Binet mulormait labituellement un de ses sujels 
en lui montrant une clef à laquelle il avait associé la suggestion du sommeil ; "cette suggestion, dit-il, avait si bien réussi, qu'il me suffisait, tout en causant avec elle, de jeter négligemment la clef sur la table pour qu'elle tombàt en catalepsie 1 . L'influence de la répétition sur les actes est depuis longtemps reconnue; c'est en répétant un acte qu'on le rend plus facile et jylus correct, le sysstème d'éléments que l'on associe ainsi acquiert une cohérence et une précision qu'il était bien loin d'avoir tout d'abord. Et l'influence de la répétition est bien manifestée par ce qui arrive lorsque les conditions de l'activité sont quelque peu modifiées et qu'il s'agit non plus tant de répéter rue de modifier et de systématiser à nouveau. Une plume qui ne ressemble pas à celle dont nous nous serrons, nous gêne pour écrire, le chasseur qui tire avec un fusil qui n'est pas le sien, et dont, par exemple, la crosse a une courbure différente de celle à laquelle il est habitué, risque de manquer fréquemment; les mots d'une langue étrangère qu'on n'a pas souvent répètés sont facilement mal prononcés; l'ouvrier qui se sert d'outils différents de ceux dont il se sert d'ordinaire, toutes choses égales'd'ailleurs, travaillera moins bien, etc.

Pourquoi, comment la répétition d'un acte, d'une pensée, rend-elle cet acte, cette penséc, plus précisc, plus forte. plus facile à répéter une autre fois? C'est évidemment a un accroissement de l'organisation, de la coordination que cet offot est dù et ce processus a une doulble face; d'un côté, le phénomène répété s'associe mieux, comme nous l'avons vu à ceux des autres phénomènes psychiques qui peuvent s'harmoniser avec lui, d'un autre côté, il devient lui-mème plus cohérent, plus logique, mieux systématisé. Il faut examiner ce dernier fait ou nous retrouvons l'activité des éléments psychiques,

1, Rexue philosophique, mai 1887, p. 475. 
Souvent, la première fois quiune idée est exposée, qu'un ouvrage est lu, qu'une sımphonie est écoutée cette idée, les théories exposées dans le livre: les images des mélodies èt des accords de la symphonie ne prennent pas dans l'esprit une forme nette, c'est-à-dire que les systèmes éveillés ont quelque chose de vague, d'incohérent, de mal défini; une idée, par exemple, éveille de petites idées accessoires qui n'ont rien de commun avec elle au point de rue logique; une mélodie se confond avec une autre, elle est défigurée par les éléments étrangers qui viennent se joindre a elle, etc. A mesure que le système psychique est répété, si l'idée est exposéc encore à la mème personne, si la mélodie est entendue de nouveau, les éléments qui composent le systeme s'unissent, ils travaillent à se coordonner, on peut dire qu'ils font sans que nous nous en doutions des efrorts pour se systématiser, comme le prouve ce fait qu'une mèlodie déjà cntendue, mais que nous avions vainement tâché de llous rappeler, vient un jour à chanter en nous sans l'intervention de notre volonté ni même de notre désir. Mais les éléments, jar cela seul qu'ils se coordonnent, écartent, tendent à supprimer les éléments parasites qui s'étaient introduits dans l'ensemble en s'attachant à l'un d'entre eux et le rendaient incohérent, et chaque fois que le système est remis en activité par les circonstances qui le favorisent est une nouvelle orcasion de lutte et de sélection. Mème chose arrive punr le système considéré dans son ensemble, l'intensite nouvelle que lui donne le fait d'être suscité de nouveau lui permet de s'associer de plus en plus étroitement aux autres èléments qui composent l'esprit, et de lutter avec plus d'avantage contre ceux qui s'opposeraient à lui. On comprenrl que la répétition, en favorisant l'organisation interne et externe d'un système psychique, est une condition tris favorable dans sa lutte pour l'existence. 
Il y a des exceptions au moins apparentes à l'influence de la répétition, quelqnefois la répétition d'un acte, d'une idée trouve plus d'obstacles que sa première apparition; cela se produit, par exemple, quand cet acte ou cette idée a provoqué une réaction de la part d'autres systèmes psychiques importants, lorsque, par exemple, lacte a eu des conséquences imprévues et fàcheuses. Mais il suffit, pour que la règle indiquée soit exacte, de tenir compte, comme nous l'avons fait, de la différence possible des conditions de production des pliénoménes ${ }^{1}$.

La persistance d'un phénomène psychique a des effets analogues ä ceux de la répétition; lit persistance, en effet, est une sorte de répétition a des intervalles de temps nuls on très petits. Quand les causes qui ont produit l'apparition d'un système psychique continuent a agir, le syslìme psychique, par le mécanisme déjà décrit, a d'autant plus de chance de s'imposer ì l'esprit, et, s'il disparait ensuite momentanément, de réapparaitre encore plus tard. Un son qu'on a longtemps entendu est rappelé trẻs facilement et souvent mème se représente à l'imagination sans qu'on le venille. "Lorsque dans la soirée je. me suis livré à un travail opiniàtre, dit M. Maury, les hallucinations (hypnagogiques). ne manquent jamais de se présenter. Il y a quelques années, ayant passé deux jours consécutifs a traduire un long passage grec assez difficile, je vis, a peine au lit, des images si multipliées, et qui se succédaient avec tant de promptitude que, en proie à une véritable frayeur, je me levai sur mon séant pour les dissiper. Au contraire, à la campagne, yuand j’ai

1. On confond souvent la répétition et l'habitude. L'habitude est plutôt une manière d'être de l'organisme, préparée par une première apparition d'un phénomène, développée par ses répétitions - nous n’avons donc pas à la considérer à part. V. Léon Dumont. De l'habitude, Revue philosophique, arril 1876. 
l'esprit calme, je n'éprouve que rarement ce phénomène » " «On sait, dit M. Baillarger, que les personnes qui se servent habituellement du microscope voient quelquefois reparaitre sjontanément, plusieurs heures après avoir cessé leur travail, un objet qu'elles ont examiné très longtemps; il en est de mème quelquefois quand on s'est appliqué a une longue freparation d'anatomie. J'ai moi-mème éprouvé une hallucination très remarquable de ce genre et qui s'est reproduite pendant plusieurs jours : arant de trouver pour mesurer les surfaces céréJrales un frocédé qui me permit de déplisser le cerveau sins tiraillement et de le mouler, j'avais essayé d'obtenir cette mesure en appliquant une gaze trè fine sur les circonvolutions; je faisais pénétrer cette gaze dans toutes les anfractuosites, et la maintenais de distance en distance avec des èjingles doubles. Ce procédé, outre qu'il élait moins exact fue le moulagre, exigreait une extrême patience; il ne me fallait pas moins de quatre ou cinq heures pour courrir un seul hémisphere. Te second jour de ce travil, je vis tout a coup la gaze courrir à chaque instant les objets qui ètaient devant moi ; c'est surtout dins une demi-olscurite et quand je cessais dappliquer mon esprit, que ce phénomène se produisait ${ }^{2}$. On retrouve ici les caractères de l'activité incohérente des éléments psychiques; la demi-olsscurité est évidemment uue condition favorable anx images qui ne sont pas en hammonie avec les excilations venues du dehors, puisqu'elle allaiblit la force, l'intensité de celles-ci, de plus, le relachement de lattention, en afraiblissant les liens qui remissent ef coordonnent le phénomene, permet à d'autres phénomènes de se produire plus librement. Dans ce cals-ci limage renaissante aequérait, gruace à la persistance frécédente de la sensation, assez de force pour

1. Maury, Le sommeil et les réves, p. 44.

2. Baillarger, Memotre sur les hallucinations, p. 460. 
vaincre les perceptions actuelles, puisque la gaze sem blait “ couvir les objets ». Ces exemples suffisent a montrer les effets de la persistance, on voit que la répétition $\mathrm{y}$ intervient aussi. Les analogies de ces deux conditions me dispensent au reste d'insister davantage.

\section{$\$ 4$.}

Nous ne saurions aroir l'idée d'énumérer toutes les circonstances, mème les circoustances générales qui favorisent on entravent l'activité personnclle poụr ainsi dire, relativement indépendante des éléments psychiques; à côté de l'organisation particulière des systèmes psychiques et des autres systèmes auxquels ils se rattachent, de l'attention, de l'intensité, de la répétition, de la persistance, il y a d'autres conditions générales qui agissent sur le systeme nerveux et tout en excitant d'une manière générale l'activité cérẻbrale, donnent la prédominance à tel ou tel système psychique par l'effet de conditions assez souvent inaccessibles, telles sont les circonstances qui activent modérément la circulation du cerveau, les substances qui, d'une manière on d'une autre, activent le fonctionnement de certains centres nerveux : le café, le vin, le haschich, etc.; ou qui irritent ou engourdissent,-et l'un de ces effets est souvent la suite de l'autre, - certaines parties de l'encéphale : la paralysic générale au début, certaines maladies nerveuses on mentales. Il faut joindre a cela les dispositions anatomiques et les circonstances phrsiologiques qui permettent à la force nerveuse excitée de se répandre plutôt dans tels pássages que dans d'autres (ceei n'est qu'une métaphore), et quelques associations physiologiques dont la raison est à peu près inconnue. On peut ranger dans cette catégorie de faits la plupart de ces phénomènes qu'on a appelés pendant longtemps des sympathies et que l'on a reconnus être dus à des actions réflexes, une partic des faits d'inhibition et de dynamogénie 
étudiés par M. Brown-Séquard et M. Féré, et aussi les phénomènes que dans son ouvrage sur l'expression des émotions, Darwin a étudiés comme déterminés par linthence directe dus steme nerreux. Cette classification est, du reste, tris-vague, et, comme on l'a fait remarquer, tous les phénomènes psychiques pourraient se ramener, a l'action dn systeme nerveux. Mais Darwin voulait dire sans doute qu’il était impossible de les zattacher à un principe determint. i une forme particulière de la mentalité. Peut-ètre cependant pourrait-on trouver un sens a certains de ces phènomènes en se plaçant au point de vue synthétique de la finalité générale de l'esprit et de l'organisme.

On a dit que le Darwinisme avait abusé des causes finales. Si l'on reut dire que Darwin a mis admirablement en hunière un grand nombre de sytématisations naturelles et qu'il a été conduit a rechercher à propos de tous les phénomènes leur utilité possible ciest-a-dire lenrs relations harmoniques avec d'autres phénomènes, on a parfaitement raison, sauf qu'il u'y a pas abus à les constater, mais il y a tinalité et finalité. Il ne faut pas confondre la finalité immanente consistant dans une coorlination d'éléments avec la finalité qui résulterait Ar lat realisation d'un plan divin antérienr an monde. Si cotte derniere finalite existait. elle serait un cas particnlier de la prénière.

Il n'est pas tonjours farcile d'ailleur's, de savoir si un lhenomene psycho-organigue est produit par me association systematique, ou hien s'il est dù a ces actions nervouses mal roordonnées qui sont encorr assez fréquentes dans lorganisme. Par exemple, le tremblement a été considere tour à tour comme devant être langé dans l'une r l'antre le ces categories. D'après Darwin a le tremblement, nest l'ancune utilité, souvent il est nuisible ${ }^{\prime}$ ».

1. Larwin, L'Expression des emotions, trad. Pozzi et Benoit. 
M. Mantegazza, aú contraire, a pensé que le tremblement, sous linfluence de la peur, offre des avantages en ce qu'il contribue a produire de la chaleur en réchauffant le sang que l'influence de la peur tend a trop refroidir. II. Mosso, qui eximine l'opinion de Darwin et de M. Mantegazza, se range du côté de Darwin, en se fondant sur les faits qui nous montrent le tremblement se produisant dans des eirconstances ou un réchauffement du sang ne pourrait qu'tre nuisible, et sur ce que le tremblement de la peur, empèchant l'animal de fuir ou de se défendre, ne peut lui ètre avantageux. On peut almettre, je crois, avec Darwin et M. Mosso, que le tremblement ne saurait rentrer dans la loi de l'association systématique; mais cette recherche ne rentre pas dans notre sujet actuel.

Les faits qui ne peuvent manifester aucune finalité grénérale, ne sauraient intéresser la psychologie que comme étant susceptibles de montrer lactivité relatirement indépendante des éléments psychiques, qui paraissent en certains cas exister en eux-mèmes et pour euxmèmes, sans relation de finalité, soit avec dautres sýstèmes psychiques, soit à l'ensemble de l'organisme ou de l'esprit. S'il en existe réellement ainsi d'ailleurs, ils ne sauraient nous arrèter longuement, car leur enchainement, leur production n'intéresse pas la psychologie, n'etant soumise à aucune loi psychologique. La psychologie ne commence, en effet, quavec les formes propres et régulières de l'esprit. Une sẻrie de plénomènes psschiques dont chacun serait déterminé successivement, par un processus purement mécanique, ou physiologique, n'appartient pas en tant que série, à la psychologie, les éléments sont des éléments psychiques, mais ils ne sont reliés en ce cas que par une circonstance qui n'a rien de psychologique ${ }^{1}$.

1. Masso, La Paura, p. 169, 170. 


\section{CONCLUSION}

Nous arons reconnu l'existence d'éléments psychiques plus ou moins complexes, toujour's composés, toujours, sauf celui qui réunirait tous les autres - prèts à entrer comme partie dans des ensembles plus vastes. Ces éléments ont une organisation propre et une activité relativement indépendante. Chacum, en tant qu'il est un système agit pour soi ; une fois réuni à d'autres éléments en un système supérieur, il n'agit plus, tant que l'association se maintient strictement, que pour ce système supérieur dont il fait partie; quand l'association se relàche ou disparait, il retourne à ses premières habitudes. On trouve ainsi des complexus d'éléments de plus en plus nombreux, de plus en plus étroitement liés. Dans cette lutte pour l'existence, la sélection est déterminée, les systèmes sont farorisés ou affaiblis par la nalure plus ou moins imparfaite de leur organisation propre et de leurs associations avec les autres éléments de l'esprit, par le degré do leur intensité, par leur répétition, par leur persistance et par d'antres circonstances moins importantes. Cos éléments psychiques ont une double tendance: susciter pour s'associer arec eux, pour les absorber, on pour être absorbés par eux, selon leur importance, les systemes psychiques qui peuvent s'harmoniser avec eux, s'unir avec eux en vue d'une fin commune, former a vec eux des composés unifiés, el au contraire inhiber, empêcher de naitre, de se développer; faire disparaitre ceux avec qui ils ne peurent entrer dans un mème système. De ces deux fitits généraux, par la différence des èléments et des groupenents, me pirarissent dériver, à certains égards, 
toutes les formes de la vie mentale et la nature même de l'esprit, mais il ne serait pas juste de dire en un sens que les ćléments de l'esprit expliquent l'esprit même, si l'on n'ajoutait,que l'esprit explique à son tour ses éléments, de mème que la société explique l'homme, comme l'homme explique la société. C'est ce que nous allons tàcher d'établir en étudiant d'ahord les lois de l'activité mentale, et ensuite la synthèse psychique la plus élevée, l'esprit, sous ses différents aspects. 


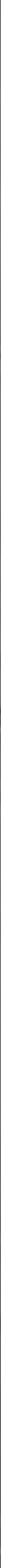




\title{
DEUXIĖME PARTIE
}

\section{LES LOIS DE L'ACTIVITÉ MENTALE}

\author{
LIVRE I.
}

La loi de l'association systématique.

\section{N T RODUCTION}

Nous avons reconnu en même temps que l'existence des éléments psychiques, leurs principales tendances: tendance à l'association et tendance inhibitrice. Ce sont ces tendances qui déterminent le mode de fonctionnément de l'esprit, qui caractérisent les lois de l'activité psychique. Reprenons donc par un autre côté l'étude de ces tendances, nous étudierons comment les phénomènes s'associent et comment ils s'inhibent, en nous attachant cette fois non plus à l'existence des éléments psychiques, mais aux composés de plus en plus élevés qu'ils forment et aux lois géuérales des phénomènes de l'esprit. Nous nous élèverons ainsi des formes inférieures de l'association sỹstématique à ses formes les plus élevées et nous tâcherons de préciser quelles associations et quels éléments constituent les différentes formes de la vie mentale et les différentes catégories de phénomènes psychiques: la sensation, la perception, l'idée, le jugement, le raisonnement, les sentiments, les tendances, la volonté et la personnalité. 
Nous arons formule ainsi la loi de l’association systematique : Toul fait psychique tend à s'associer et à faire naitre les faits psychiques qui peuvent sharmoniser avec lui, qui pellent conconrir atee lui vers une fin commune ou des fins harmoniques, qui, avec lui, peuvent former un système. Nous devons tàcher de retrouver cette loi et de préciser sa forme particulière dans tous les ordres différents de phénomènes psychiques, nous nous placerons pour cela à deux points de vue : dans tout composé psychique formé, une perception, une idée, une volition données, les éléments sont associés selon une loi de finalité. Dans tout composé en voie de formation, dans la genèse d'une perception, d'une idée, d'une volition, d'une faculté, ils se suscitent selon une loi de finalité. Il s'agit done d'établir, sous les réserves impliquées par ce qui précède que les faits psychiques sont des composés systématisćs, dont nous avons à déterminer les éléments; que les facultés, les traits dé caractère acquis, les formes fixécs de la perception, de l'intelligence, de la volition, de la sensibilité, sont des formes particulières de l'association systématique; que les faits ou les facultés abstraites, c'est-à-dire les habitudes qui vont se fixant hous présentent la mème loi. - C'est donc une revue complite le l'esprit ot de ses éléments qui simpose ici et que je ticherai de faire en passant le plus brièvement possible sur les points deja acquis a la science.

Il n'est peut-être pas très utile de dire que l'ordre que nous sommes obligés de suivre n'a rien de bien rigoureux; a mon point de vue l'homme est un appareil de systematisation qui recoit les impressions du monde exterinur, lus dicompose, fiat avec les éléments de seg imprescions de nouvelles syntheses et finalement réagit 1) manite it itugnenter la finalité en lui-mème, dans la sorictí et minte lans le monde extérieur len tenant compte de celto reserve que la finalité sociale n'est pas 


\section{L'ASSOCIATION SYSTÉmATIQUE - INTRODUCTION 89}

plus parfaite que-la finalité individuelle et qu'elle est aussi, en ce cas, une sorte de propriété des ćléments avant que d'ètre une propriété de l'ensemble.) Ces divelses actions et réactions composent par leurs groupements diverses tendances, et l'activité de ces tendances s'accompagne sclon le mode de cette activité, de phénomènes variés, perceptions, idées, sentiments. Au fond, le phénomène essentiel c'est la tendance; mais l'étude des autres phénomènes a son importance également si l'on songe surtout qu'cn les étudiant, c'est la tendance ellemème ou quelques-uns de ses éléments que nous étudions en somme par certains côtés et dans certaines conditions. 


\section{CHAPITRE PREMIER}

\section{Sensations et Percoptions.}

Dans les sensations et les perceptions, le róle de la finalité est assez évident. Si nous considérons ces phénomènes comme donnés, nous disons que la sensation est une synthèse systématique d'éléments inconscients, que la perception est une synthèse de sensations et d'images. Au fond, d'ailleurs, la sensation et la perception ne sont guère discernables. Mais dans certaines perceptions la part de l'intelligence est plus faible, dans d'autres elle est plus forte et cela suffit pour légitimer cette distinction.

Que la sensation soit une synthèse d'éléments inconscients, on ne peul guère, je crois, y contredire. L'expérience parait bien le démontrer et je ne pense pas qu'on ait rien répondu de péremp̣toire aux considérations présentées par Leibniz ${ }^{1}$, et aux analyses de M. Taine ${ }^{2}$. On invoque quelquefois, pour ne pas accepter les conclusions qu'on paraìt olligé de tirer des phénomènes, des raisons à priori : la conscience ne peut résulter de l'inconscience. On pourrait tout aussi bien dire 'fu'un corps liquide ne peut résulter de la combinaison de deux corps gazeux. Mais nous arons surtout it insister ici sur le caractère d'unité synthétique du phénomène résultant. La sensation n'est pas une somme, elle est une synthese, et, comme nous le verrons pour tous les autres phénomènes psychiques, son caractère d'unité lui vient dı fail de la systématisation de ses éléments, dès que cette harmonie

1. Nouveaux essais sur i'entendernent humain.

2. Taine, De.l'Intelligence. Vol. I. 
disparait, l'unité cesse et la pluralité la remplace. Tant que la roue de Savart, dont les dents frapuent tour it tomr une latte, tourne assez Ientement "les sensations étant discontinues sont distinctes, et chacune d'clles étant composéc est un bruit. Mais si la roue se met i tourner avec une vitesse suffisante, une sensation nouvelle s'élève, celle d'un son musical. Parmi les restes de bruit qui persistent encorc et continuent à ètre distincts, elle se dègage comme un élément d'espèce différente; cntre les diverses sensations élémentaires qui constiłuent chaque bruit, il en est une que l'opération a séparée; désormais celle-ci n'est plus distincte de la sensation élémentaire semblable qui la suit dans chacun des bruits suivants. Toutes ces semblables font mainlenant ensemble une longue sensation continue; leurs limites mutuelles se sont effacées, l'expérience, comme une analyse chimique, a retirć une sensation élémentaire du groupe complexe où elle était incluse pour la joindre à une sensation élémentaire absolument pareille et faire un composé nouveau, la sensation de son musical "1. Il est inutile de développer longuement le caractère synthétique de la sensation; d'une manière générale la fusion des sensations semblables, dont M. Binet a montré l'importance ", parait être due à ce que deux sensations semblables peuvent jouer un rôle identique dans un système psychique. Il en est de même des sensations harmoniques en général. Mais si la finalité, au point de vue de l'enscmble de l'organisme, n'est pas toujours évidente, l'harmonie, au point de vue de l'organe qui perçoit, ne peut ètre mise en doute - rappelons l'unité des sons qui forment un accord parfait et la synthèse des sensations visuelles différentes, données par un disque animé d'un mouvement

1. Taine, De l'Intelligence. Vol. 1, p. 178

2. Binet, Psychologie du raisonnement. 
de rotation, etc. La contre épreuve est faite par les battemeuts des sons inharmoniques, l'impossibilité de distinguer les objets éclairés par une lumicre trop vive; dés que la synthise systématique des éléments ne peut plus s'opérer', la sensation disparait ou se dédouble, l'unité perçue disparait avec l'unité de coordination.

Avec les perceptions plus intellectuelles: la finalité générale de lorganisme, ou tout au moins de systèmes psychiques plus importants, est plus visible. Si nous étudions d'abord' la perception formèe, nous trouvons quelle est un composé de sensations et d'images ${ }^{1}$. Les images servent à interpréter la sensation, à la classer, à lui assigner une place et un rôle distincts dans le jeu des tendances. Nous n'avons, pour en ètre convaincus, qu'à examiner la différence des perceptions éveillées par un objet qui nous est familier selon que nous le reconnaissons ou non. De loin, nous apercevons une personne sans la reconnaître, nous n'avons qu'une sorte de sensation yisuelle assez vague, mais qui se précise peu à peu. Au moment où la personne approche et où nous la reconıaissons, une assez grande ruantite d’images, de sentiments, d'idées, de tendances, s'éveille faiblement et constitue la perception et la reconnaissance de cette personne. Chaque perception - et toute sensation est perception a quelque degré - est ainsi constituée par l'association systématique des impressions éveillées par les excitations venues du dehor's arec des images, des idées, d'autres phénomenes psychiques plus ou moins nets. Cette synthese n'est pas toujours évidente ; cependant il fant bien reconnaitre que toute impression, jar cola mène qu'elle est percue, est classec ; elle prend place dans tel ou tel système, elle est reconnuc apte á faire partic de telle ou telle tendance, à déterminer, selon les circonstances, tel ou tel

1. V. Binet, ourr, cité. 
sentiment, telle ou telle volition. Reconnaitre un livre, c'est classer la perception qne nous en avons de telle sorte que nous saurons si nous en avons besoin, le prendre et l'ouvrir pour le lire. Ainsi non seulement la sensation consiste dans l'éveil systématique d'un ccrtain nombre d'éléments psychiques, mais en devenant perception, elle détermine l'éveil systématique aussi d'un nombre plus ou moins grand d'autres phénomènes aptes à former avec elle des combinaisons en vue d'une fin déterminée.

Enfin, les perceptions les plus complexes nous montrent encore mieux cette unification d'éléments différents. Nos perceptions de l'espace et du temps, par exemple, sont des sỹnthèses où l'on voit des éléments hétérogènes se combiner dc maniére à permettre la coordination de nos actes et se combinęr si étroitement que le phénomène de la perception parait simple et indécomposable à un esprit non exercè. On connait l'étude que Wundt a faite de $\propto$ la liaison particulière des sensations sensorielles périphériques et des sensations d'innervation centrale, qui engendre ici l'ordre des premières dans l'espace ". 1 . On peut considèrer aussi comme perceptions complexes les perceptions qui nous arrivent à la fois par différents sens et qui sont combinées par l'esprit, par exemple, lorsque nous voyons et entendons à la fois sonner une cloche, les deux perceptions sont unies, nous les considérons comme l'expression d'un mème fait extérieur. Mais le procédé synthétique est ici rendu bien évident par les cas où l'union des perceptions ne peut se faire, où, par exemple, nous cherchons autour de nous, pour en avoir la perception visuelle, l'objet que nous supposons produire le son qui nous parvient. On peut dirc que notre expérience, en tant quelle détermine un progrès de l'esprit, implique

1. Voyez Wundt, Elements de psychologie physiologique, trad. franc., de M. E. Rouvier. Tome II, chap. XI, p. 5. 
la formation de perceptions complexes de cette nature (ou de composés complexes de perceptions et d'images). Toutes les recherches que l'on fait sur les corps ont, en dernière analyse, pour principal but d'agréger une nouvelle perception (réelle ou possible) à des complexus déjà formés de perceptions, d'images et d'idejes, et la nature synthétique du composé définitif sc marque d'abord en lui-mème par l'aspect qu'il prend a nos yenx, en ce que nous considérons l'ensemble de ces pereeptions réelles ou possibles comme une seule chose et aussi par la colıérence, l'unité de direction qu'il permet à uos actes l'acquérir.

Nous arons déjà vu, outre le caractèrc systématique de la perception qui se produit, lc caracterc systematique des associations cu'elle forme avec d'autres élèments psychiques et arec l'esprit en général, et ce dernier fait est plus visible encore si l'on considère la genèse des perceptions, ou des autres images vives, celle du rêve, des illusions et des hallucinations. Il est d'observation vulgaire que nous entendons surtout ce que nous comprenons, on peut en juger facilement à la représentation d'un grand opéra, où l'on entend bien mieux les paroles que l'on sait a l'avance; une langue étrangère parlée à côté de nous, nous fait l'effet d'un murmure confus dont nous ne pourrions reproduire avec précision aucun son ; les personnes atteintes de surdite verbale, tout en conservant le sens de l'ouïe, ont des impressions analogues; de mème si nous avons vu de près une inscription, par exemple, nous la lisons plus facilement á une certaine distance ; les caractères d'une langue ignorée sont difficiles à discerner; l'écriture paraît confuse aux malades frappés de cécité verbale; d'autre part une écriture tracée normalement de la main gauche, à rebours, parait à un observateur non préveuu un gribouillage sans netteté, et l'on est tout surpris d'en voir la rrécision quand on a retourné le papier et qu'on lit l'écriture par transparence. 
Ces phénomènès ne se laissent guère interpréter que d'une manière: dans la perception normale les systèmes psychiques, les tendances qui existent en nous et qui subsistent continuellement soit à l'état latent, soit à l'état d'activité faible ou partielle et inconsciente, choisissent dans les données de la sensation tout ce qu'ils peuvent prendre et s'assimiler: les donnees des sens subissent ainsi une première décomposition et sont incorporées dans différents sýstèmes - quelques-uns de leurs éléments faisant partie de plusieurs à la fois; dans la sensation donnèe par la vue d'un chien, par exemple, certains éléments seront associés aux tendances du chasseur, d'autres aux tendances du dessinateur ou du peintre ou du naturaliste, ou du phýsiologiste, ou bien elles éveilleront des sensations d'admiration el d'affection même. Comme quelques-unes de ces tendances, sinon toutes, peuvent être réunies chez une mème personne, et beaucoup d'autres avec elles, on voit toute la complexité du fait de la perception. Une perception est une occasion de penser, de rêver, de sentir et d'agir de bien des manières différentes, en tant que chaculle de ces manières de penser, de rêver, de sentir et d'agir est déjà représentée en nous par un certain nombre d'éléments nerveux et un mode habituel de leur action combinée.

Dans toute perception cette association systématique déterminće par les habitudes préexistantes est très visible, des remarques bauales ne pernettent pas de la mettre en doute. Ce que nous percevons le mieux c'est, toutes choses égales d'ailleurs et sauf le cas d'un trouble extrême de l'esprit, ce qui nous intéresse le plus. D'une manière générale la reconnaissance est plus prompte que la connaissance qui implique une nouvelle organisation au moins partielle de l'esprit, un réarrangement des phénomènes psychiques. Ce que nous connaissons déjà, ce qui est familier est plus vite et plus facilement perçu que le 
nouveau, l'extraordinaire. On pourrait en douter en considérant les cas où la perception d'un objet mal connu ou inconnu excite une attention très vive, tandis que les objets connus passent inapercus, mais ces cas mêmes se laissent ramener a notre loi et ne s'expliquent bien que par elle. Si la perception nouvelle, en effet, attire l'attentention cest grràce à la peine mème qu'elle a à entrer dans l'esprit, il ne se troure pas de système psychique pour la receroir et se l'associer, comme d'ailleurs elle a pu déterminer l'excitation systématisée des éléments sensoriels elle tend a persister, et elle tàche alors d'attirer à elle certains éléments de manière à établir dans l'esprit de nouvelles associations harmoniques. Elle ne peut être reçue qu'à cette condition, une perception qui ne se rattacherait a rien, disparaitrait, et en fait toutes nos perceptions sont rattachées plus ou moins consciemment à des systèmes d'impressions ou d'actes. Seulement au lieu que dans beaucoup de cas c'est la force de l'habilude précédemment établie qui détermine la nature de la perception, ici c'est la force de la perception nouvelle qui tend a entrainer un changement dans les habitudes - la force de l'association systématique n'est pas moindre dans un cas que dans l'autre.

Il est un fait, dans la production des sensations, qui montre admirablement la part des habitudes acquises de l'organisme : c'est que chaque appareil sensoriel donne, quelle que soit l'excitation, des sensations toujours de mème ordre. Si l'on comprime le nerf optique on a une sensation lumineuse, il en est de même, si on le dilacère; l'excitation mécanique du nerf auditif ne donne que des sensations auditives, et quelquefois ce phénomène est encore plus marqué, on voit des complexus très différenciès ètre déterminés par une excitation mécanique générale.

- On peut, dit M. Ball, à l'aide d'un courant électrique, 
provoquer de véritables hallucinations de l'oüe chez les individus qui sont déjà pródisposés aux accidents de cette espèce. Sous l'influence de cette excitation, les malades entendent non seulement des hourdonnements, des siflements ou des bruits, nais des interjections, des mots, des phrises plus ou moins compliquées, quelquefois des prières ou des fragments de poésies, depuis longtemps oubliés $₫ 1$.

Un cas fort intėressant encore et qui montre bien l'interprétation particulière d'une excitation par un organe donné, est celui où l'excitation d'un sens produit des hallucinations d'un sens différent. " Un homme sujet à des hallucinations auditives demeure parfaitement tranquille dans l'obscurité ; dès qu'on apporte des lumières, des paroles grossières viennent frapper son oreille §2. M. Féré a traité, par les vibrations du diapason sur le crìne, une hystérique qui présentait une monoplégie du bras droit, avec anesthésie s'étendant à la face, où elle prédominait autour de l'œil. Liœil ne voyait que les couleurs supérieures et n'en distinguait que peu de nuances, " l'anesthésie visuelle, dit-il, ne cédait que lorsque les vibrations étaient appliquées sur l'émergence des nerfs sus et sous orbitaires, dont le domaine reprenait successivement la sensibilité $" 3$.

Le centre de lą perception, puisque l'hypothèse de l'énergie spécifique des nerfs ne paraît pas s'être confirmée, interprète à sa manière toutes les excitations qui lui arrivent. Nous retrouvons le même phénomène à propos des centres intellectuels et moteurs : quand nous avons une idée, une théorie favorite, tout nous a suggère; un

1. Ball. Leçon sur les maladies mentales, p. 109-110. M. Ball qui cile ce fait d'après Brenner pense que cette expérience aurait besoin d'être contrôlée, en raison de son importance.

2. Ch. Féré, Sensation et mouvement, p. 79-80, voir aussi p. 120 et suivantes.

3. Meme ouvrage, p. 117. 
mouvement habituel, un tic, il se produit it la moindre excitation. Il y a me halsitude de l'esprit, une association sýstématique de certains éléments toujours prète à fonctionner, et qui tend a s'associer tous les nouveaux phénomènes qui se produisent, en choisissant en eux ce qui peut s'adapter à elle. La perception spécifique est une habitude du centre sensoriel, une théorie, une croyance enracinéc, pourrait-on dire, si l'on ne craignait d'abuser de l'analogie. d'après laquelle le centre sensoriel interprète toutes les excitations qu'il reçoit, comprend tous les faits qui lui parviennent. Il a sa manière particulière de grouper les éléments psychiques derniers et cette manière est toujours la même. Et s'il est vrai que nos différentes sensations ne soient que que des arrangements différents d'un même élément dernier, le a choc nerveux » de M. Spencer, par exemple, on voit la part immense qui échoit ici à la systématisation acquise, et la finalité immanente des éléments. 'L'organisation est telle que le centre choisit l'excitation et en fait l'occasion d'une synthèse psychique déterminée l'excitation ne peut pénétrer daus l'organisme qu'en s'associant à d’autres éléments psychiques qu'elle met en activité de manière à former un tout unifié.

Cette interprctation de l'expitation par les centres nerveux est peut-ètre plus frappante quand elle nous apparaît en dehors des fiits où nous la trouvons d'habiude, par exemple, dans le cas bien commu de Gabriel Gallien. Gabriel Gallien était un berger du Languedoc qui, après aroil abusé de la misturbation, ne pouvant plus amener la jouissance vénérienne par ses moyens habituels, s'avisia pour y parrenir d'irriter l'urèthre avec une barguette de buis qu'il y introduisail. Au bout de plusieurs anners, ce nouveau moyen devint inutile it son tour, il finit alors par s'armer d'un couteau et par s'inciser le gland; " une pareille opération, loin de lui causer de la 
douleur, lui procura une sensation agréalule et produisił une abondante ejaculation spermatique" i. Nous assistons réellement ici, à la formation d'un sens, le sens génésique, à la naissance dune forme définitive de systématisation psychique. Il est probable que tous nos sens se sont ainsi formés peu à peu ct sont en réalité des habitudes acquises, des croyances devenues organiques.

Ces deux influences, celle des habitudes acquises, celle de l'impression présente, se retrouvent dans lcs phénomènes d'ordre morbide, rêves, illusions, hallucinations, que nous avons, au reste, déjà abordés, et la force de l'association systématique est d'autant plus visible, peutêtre, que la lutte des phénomẻnes est plus vive. Les figures que l'on voit facilement dans les nuages ou dans les veines d'une plaque de marbre, les illusions causées par des formes d'arbres ou de linges étendus, sont des exemples de ses degrés les moins élevés. Ces faits nous montrent combien l'esprit tend à systématiser les données des sens et à leur donner, à défaut d'ordre réel, un ordre imaginaire dépendant des habitudes quelquefois insignifiantes de l'organe qui les reçoit. Il faut signaler dans le mème ordre de faits, les illusions de l'ouïe ; jai eu autrefois et j'ai encore, mais à un degré moins èlevé, une grande facilité pour entendre des airs, des sons d'instruments, en me tenant près d'une chute d'eau, ou bien en me trouvant en vagon, enfin en me tenant près d'une cause de bruit continu, assez fort et indifférencié. M. Pavlofski, poursuivi comme nilıiliste et jeté en prison, éprouvait des hallucinations de l'ouie dues au bruit de ses pantoufles. "Pendant le jour, dit-il, je courais de côté et d'autre dans ma cellule, mes pantoufles criaient : ce

1. Dictionnaire des sciences médicales, vol. IV, p. 238. Le fait est emprunté par Fournier au Traité des maladies des voies urinaires de Chappart. 
bruit, par une bizarrerie inexplicable, me rappelait les refrains de chansons obscenes que j'avais entendu vociférer par des irrognes attardés dans la rue. Je m'efforce de penser à artre chose.... Ln vin je làche de faire cesser ce dégoùt que j'éprouve, je reux me persuader que ces chants ue sont que grotesques, naïfs, peut-être..... Mais tout à coup une voix de fausset aigüe et félée en même temps me les crie aux oreilles en accentuant avec ironie les passages les plus ignobles...., je jetais mes pantoufles avec fureur et me mettais a courir pieds nus sur les dalles froides du plancher. Ceci faisait passer les hallucinations de l'ouie $\boldsymbol{}^{2}$.

De mème que le raisonnement a pour but d'établir dans l'esprit un système d'idèes ou de tendances, de méme l'interprétation perceptive a pour but de nous donner un système de sensations, d'images et d'états plus faibles, aussi cohérent que possible. Cette interprétation perceptive est continuelle, elle est plus visible dans les états morbides ou semi-morbides parce qu'clle aboutit souvent à des résultats qui ne concordent pas avec la réalité et que l'on attribue le systeme produit à la force constructive de l'esprit, non à l'enseignement de l'expérience, comme si l'expérience pouvait servir à quelque chose et comme si elle pouvait seulement exister on dehors de la systématisation psychique. A propos de toute sensation il se fait ainsi dans l'esprit une sorte de raisonnement obscur et parfois de raisonuement inconscient. Dans les rêves, les sens sont généralement inactifs ou à peu près, l'association systématique, est très visible: dès qu'un des sens fait parvenir une impression a l'esprit, les images d'autres sens s'éveillent pour la compléter. Le Dr Gregory raconte que s'étant couché avec une houillotte remplie

2. Parlofski. Mémoires d'un nihiliste, publiés lans le volume de Tourguenef. Wueves posthumss. Souvenirs denfanoe. 
d'eau chaude ì ses pieds, il rêva qu'il marchait sur' l'Etna et qu'il sentait la chaleur sous lui. Une autre fois il rêva qu'il passait un hiver à la baie d'Hudson et qu'i souffrait beaucoup du froid. Il trouva qu'il avait, en dormant, rejeté ses couvertures. Peu de jours auparavant il a vait lu un récit de l'état des colonies pendant l'hiver $\$$. On voit ici comment des idées, récemment emmagasinées, étaient venues se joindre aux données d'un sens pour les compléter en se complétant elles-mêmes par l'adjonction d'images vives et de sensations réelles. On connaît également les faits relatés par M. Maury : Etant endormi, il se soumettait à diverses expériences, on lui chatouillait avec une plume les lèvres et l'extrémité du nez et il rêvait qu'on lui appliquait un masque de poix sur la figure et qu'en l'arrachant brusquement, on lui déchirait la peau des lèvres, du nez et du visage ; on faisait vihrer à quelque distance de son oreille une pincette sur laquelle on frottait des ciseaux dacier et il entendait le bruit des cloches, ce bruit devenait le tocsin et il se croyait aux journées de juin $1848^{2}$. Les faits de cette nature sont trop nombreux pour qu'il soit besoin d'insister. Certains cas font voir d'une façon toute particulière la force de l'association systématique en montrant une impression perçue seulement après que son adaptation a èté préparée par les phénomènes qu'elle a suscités elle-mème et qui sont perçus avant elle. C'est ce qui parait avoir lieu dans certains faits, c'est ce qui a lieu certainement dans quelques autres. Bonaparte dormait dans sa voiture lorsqu'elle faillit sauter par l'explosion de la machine infernale. Ce bruit épouvantable offrit à sa conception, dans

1. Abercrombie. Inquiries concerning the intelleotual powers, p. 201.

2. Maury, Le sommeil et les réves, p. 152. Voir aussi Delbouf, Lo sommeil et les réves. Mandsley, Pathologie de l'esprit, etc. 
la durée presque inappréciable du réveil en sursaut, le passage du Tagliamonte, la canonnade de l'ennemi, les Autrichiens, le prince Charles, les Français pressés autour de leur général, et en s'éveillant il s'écria: « Mes amis, nous sommes minés 1 . Le fait suivant est bien plus significatif: a J'étais un peu indisposé, raconte M. Maury, et me trouvais couché dans ma chambre, ayant ma mère a mon cheret. Je rêve de la Terreur; j'assiste à des scènes de massacre, je comparais devant le tribunal révolutionnaire, je vois Robespierre, Marat, Fouquier-Tinville, toutes les plus vilaines figures de cettc époque terrible; je discute avec eux; enfin après bien des événements que je ne me rappelle qu'imparfaitement, je suis jugé, condamné à mort, conduit en charrette, au milieu d'un concours immense, sur la place de la Révolution; je monte sur l'échafaud, l'exécuteur me lic sur la planche fatale, il la fait basculer, le couperet tombe; je sens ma tête se séparer du tronc; je m'éveille en proic à la plus vive angoisse, et je me sens sur le cou la flèche de mon lit qui s'était subitement détachée, et était tombée sur mes vertebres cervicales, à la facon d'une guillotine. Cela avait eu lieu à l'instant, ainsi que ma mère me le confirma, et cependant cétait cette sensation externe que j'arais prise, comme daus le cas cité plus haut, pour point de lépart d'un rève oủ lant de faits s'étaient succédé " 2. On a noté que des phénomènes analogues se produisaient ¿ l'état normal: par exemple, le médecin qui fait une saignee peut voir le sang couler avant de voir la lancette jénétrer dans le bras du patient. De tels cas éclairent vivoment le mécanisme de la perception; on comprend que les faits ne peuvent être perçus qu'en prenant place

1. A. Garnier, Traile aes facultes de $l^{1}$ ame, I, 470 .

2. Maury, Le sommeil et les réres, 139-140. 
dans des systèmes d'éléments psychiques. Si un phénomène est attendu, e'est-à-dire si un système psychique auquel il s'adaptera est déjà en activité et n'attend que lui pour se compléter, la synthèse sera très rapide, sinon il sera obligé d'éveiller un șstème approprié, la perception sera retardèe d'une manière sensible, - bien qu'il ne s'agisse que de fractions de seconde, - et les phénomènes suscités pourront ètre perçus avant le phènomène qui les produit si, logiquement, celui-ci ne doit venir qu'après eux, si le système est ainsi plus cohérent.

L'influence de l'activité de l'esprit, de la foree de l'association systématique dans la genèse des représentations vives, est bien marquée encore dans le somnambulisme. On y voit un sourenir, une idée préconçue déformer la réalité pour la rendre suseeptible de s'harmoniser avec elle. Une malade, écrivent MM. Binet et Féré, dans une viṣion imaginaire, arait lutté corps à corps contre l'hallucination de l'un de nous et lui avait appliqué un violent coup de poing en pleine figure. Le lendemain matin, comme son prétendu adversaire entrait dans la salle, elle s'aperçut qu'il portait une ecchymose à la joue " 1 . Mème observation à faire à propos des hallucinations de la folie. a Les hallucinations, dit Esquirol, sont ordinairement relatives aux occupations de corps et d'esprit auxquelles se livrait l'hallueiné, ou bien elles se lient à la nature de la cause qui a produit l'ébranlement du cerveau. Une femme a lu des histoires de sorcière, elle est préoceupée du sabbat où elle doit assister, elle s'y voit transportée, elle voit toutes les pratiques dont elle a faseiné son esprit "2. On voit mieux dans ce fait l'influence d'un système prẻpondérant, fortement ancré dans l'esprit, c'està-dire associé solidement à des éléments organico-ps ỹchiquies qui sont facilement mis en activité.

1. Binet et Féré, le Magnétisme animal, p. 134.

2. Esquirol, Maladies mentales, p. 197. 
On peut constater aussi l'effet inverse, une perception imposée à l'organisnıe par le monde extérieur et déterminant de nombreuses associations systématiques. C'est ce qui arrive à chaque instant à l'état normal, chaque perception tend à déterminer en nous des idées et des actes appropriés, c'est ce qui arrive aussi dans le rêve et la folie. Mais l'examen de ces phénomènes rentre plutôt dans l'étude des phénomènes intellectuels et des faits de volition.

En résumé, dans le domaine de la sensation et de la perception, l'association systématique se manifeste d'une double nanière. Nous voyons d'abord qu'elle est la loi qui réunit les éléments de toute sensation, de toute perception acquise. Une sensation est essentiellement la synthèse systématique de phénomènes inconscients, une perception est une synthèse de sensations et d'images. Mais, le plus souvent, si ce n'est toujours, des images et des idées s'ajoutent à la sensation et à la perception, de manière à les modifier quelque peu, de sorte que la distinction entre les phénomènes est quelque peu illusoire. Si du point de vue statique nous passons au point de vue dynamique, nous voyous la force de l'association sysiématique se montrer par ces leux faits essentiels : d'un côté les habitudes de l'esprit et de l'organisme, les systemes deja formés tendent à imprimer à la perception et a la sensation des caractires tels que cette sensation et cette perception puissent s'harmoniser avec eux, concourir avec eux à une fin unique; d'un autre côté, la sensation et la perception qui sont déji, elles aussi, des systemes psychiques, tendent à modifier déjà lessystèmes existants de manière à les rendre susceptibles de s'adapter à elles ou tendent à mettre en activité ceux qui, existant déjà, Jeuvent former avec elles des systemes coherents. Nous commencons déjà à cntrevoir, même à propos de phénomenes elomentaires, lat force de la finalité générale de 


\section{L'association Systématique - PERceptions 105}

l'esprit, considéré comme le systène général des phénomènes psychiques ou au moins de certains éléments psychiques plus ou moins complexes, mais très complexes déjà, habitudes mentales, tendances, sentiments, qui interviennent pour faciliter la formation de telle ou telle perception, de telle ou telle hallucination capable de s'accorder avec eux. 


\section{H A P I T R E II.}

\section{Lintelligence. \\ Images, idées, jugements, ruisonnements.}

La nature des images mentales est bien connue, gràce aux travaux récents d'un grand nombre de psychologues '; nous n'en parlerons ici que pour montrer l'application de la loi d'association systématique. Une image étant, à certains égards, la reproduction affaiblie d'une perception, doit présenter ce caractère essentiel d'être comme la perception une synthèse d'éléments, il n'est pas besoin d'insister longuement sur ce point. Si maintenant nous considérons la formation et la renaissance des images, il ne sera pas difficile non plus d'apercevoir dans ces faits l'application de notre loi. Considérons les images qui viennent se grouper autour d'une perception, nous les rerrons s'unir a la sensation pour donner un tout un et concret; par exemple, les images qui viemuent se joindre à une perception visuelle, sont souvent les images tactiles ou sonores représentant les perceptions que peut nous donner le corps doni nous avons encore une perception visuelle, c'est-à-dire que ce sont celles qui, jointes à la perception actuelle, peuvent servir de guide à nos raisonnements; a nos seutiments, à nos actes ; l'unité de fin est

1 Voir surtout Maurg, le Sommeil et les réves. Taine, De l'Intelligence. Galton, Inquiry into human faculty. V. Egger, La parole - intérieure. Stricker, Etudes sur le langage et la musique, et les ourra.. ges de M. Charcot et de ses disciples : G. Ballet, Le langage inierieur et les formes de l'A phasie; Bernard, De l'A phasie; Binet, Psychologiedu raisonnement, etc. 
manifeste. Elle ne l'est pas moins dans les synthèses d'images qui constituent, par exemple, un souvenir et qui sont composées d'éléments trés divers : tactiles, musculaires, visuels, auditifs, olfactifs, etc., combinés en un seul composé apte à entrer comme unité dans une coordination supéricure : raisonnement, sentiment, rolontè, activité raisonnée.

Les idées se rattachent aux images par différents intermédiaires. Je ne puis dire ici que quelques mots de leur nature prop̣re que j'ai étudiéc longuement dans un autre travail 1. Les images peuvent être plus ou moins vives, plus ou moins nettes, elles peuvent aller jusqu'à un effacement très marqué, comme divers observateurs l'ont signalé, comme j'ai pu le vérifier par ma propre exץérience to comme, je crois, un grand nombre de personnes peuvent le constater en s'observant elles-mêmes. Si nous prenons un complexus d'images, par exemple, la représentation d'un cheval, nous pouvons supposer que certaines de ces images, visuelles, motrices, auditives, s'affaiblissent de plus en plus, il reste alor's un complexus d'éléments assez faibles, reliés à des tendances motrices et susceptibles de déterminer, par leur association avec d'autres éléments, soit des sensations, soit des sentiments, soit des actes. C'est là ce qui constitue une idée. L'idée a toujours quelque chose d'abstrait et de général, elle est un commencement d'habitude de l'esprit. Elle réunit en les synthétisant divers éléments, divers résidus sensitifs et moteurs, mais de telle sorte que ces éléments, ces résidus ne peuvent bien souvent ètre facilement reconnus par l'observation directe et que leur vraie nature ne se montre avec évidence que quand elle s'exagère, quand,

1. L'Abstraction et les idés abstraites, Revue philosophique, 1889. La première partie seulement a paru dans les numéros de janvier et de férrier. 
par son association avec d'autres éléments, elle donne naissance à des inages concrètes.

Avoir l'idée d'une chose, c'est connaître cette chose, c'est pouvoir soit la décrire, soit la reconnaitre, soit s'en servir; mais la décrire, c'est s'en servir encore, par exemple, s'il faut répondre à une question d'examen, c'est la faire entrer comme élément dans un système; la reconnaître, e'est également lui assigner une place dans notre vie mentale ou dans le monde extérieur, c'est la savoir apte a jouer tel ou tel rôle, à entrer dans telle ou telle combinaison; s'en servir, c'est la faire entrer dans un système de mouvements. Partout et toujours nous retrouvons dans l'idée une synthèse formée, car elle est composée d'éléments unis et susceptibles d'être employés ensemble en vue d'une même fin. Elle est aussi une occasion de nouvelles synthèses systématiques, si nous considérons ses emplois possibles. L'unité des idées se manifeste par ce fait que tous les éléments qui la composent, sensitifs ou moteurs, sont coordonnés et tendent vers un même but: rendre possible notre action sur l'objet représenté, ou, d'une manière plus générale, l'adaptation à l'objet comme a une des conditions possibles de notre existence. Le caractère de finalitè de l'idèe est rendu plus évident encore par ce fait que les idées d'une mème chose varient avec la nature, la profession, les tendances habituclles des personnes en qui se forme cette idée. L'idée d'un appareil de physique n'est pas cxactement le même chez le fabricant qui le vend ou chez le savant qui l'emploie. Mais ici, comme dans les cas de la genèse des pereeptions que nous avons vue plus haut, c'est le système général de l'esprit qui intervient pour diriger, soit directement, soit indirectement (en suscitant des expériences particulieres), la formation d'un système particulier d'élénents.

Le jugement parait ètre une forme spéciale de l'idée, si l'on dit avee $M$. Taine que tout jugement est analytique 
L'Association SYSTÉMATIQUE - MAGES ET IOÉES 109 et dégage un abstrait d'un concret, l'idée nous apparaît comme un complexus d'éléments qui, par leur association avec d'autres phénoménes pourront constituer autant de jugements. Chaque jugement associe un de ces éléments avec un autre système de phénomènes - en particulier avec les mots qui servent a l'exprimer. Si je dis, par exemple, que la terre est à peu près ronde, je sépare un des attributs de la terre de ses autres attributs, et je l'associe à un système particulier, je le rends apte à entrer dans des combinaisons variées et à y prendre, par rapport aux autres parties de mon idece totale, une place prépondérante. Ma phrase, en effet, s'associera très bien à une série de phrases où les conséquences ou les preuves de cette forme seront examinées - les autres qualités de la terre étant oubliées ou sous-entendues.

Le jugement est donc l'acte par lequel un élément abstrait d'une idée complexe est rattaché à un nouveau sỹstème d'éléments. Mais le mot idée s'appliquera aussi bien à un jugement qu'à l'ensemble des éléments d'où un des derniers jugements a été extrait, ce mot équivalant à celui de représentation faible. L'essentiel est de voir qu'il existe en nous des composés très complexes d'éléments psychiques abstraits, et que l'un de ces éléments peut être spécialement associé à un nouveau système qui l'extrait pour ainsi dire du premier, non pas complètement, mais en le mettant du moins spécialement en activité, en le faisant servir de trait d'union entre les deux systèmes. L'acte par lequel l'élément d'un système abstrait est ainsi associẻ à un autre système, ou du moins mis particulièrement en activité de manière à pouroir contracter cette nouvelle association, cet acte est un jugement. L'élément ainsi mis en jeu peut s'associer à tout autre chose qu'au système de mots constituant l'expression du jugement.

Examinons à notre point de vue cette opération mentale. Un homme pressé court à travers champ, il se trouve devant 
un fossé, il va le franchir lorsqu'il se dit : ce fossé est trop large, et il s'arrête. Il a p̧orté un jugement sur le fossé qu'a-t-il fait au point de vue psychologique? Il a choisi un des caractères du fossé qui est également plus ou moins profond, plus ou moins rempli d'eau, plus ou moins garni de plantes et de fleurs, plus ou moins droit ou sinueux, etc., et il a choisi ce caractère parce que c'était le caractère qu'il avait besoin de considérer en ce moment. Toutes les associations systématiques qui composent une idée sont, en général, à l'état latent, prêtes à s'éveiller au moment opportun, l'acte par lequel l'esprit éroque une de ces associations, celle dont il a besoin, ei lui donne momentanément une existence indépendante, est un jugement. C'est ce qui arrive quand nous donnons un renseignement sur une personne ou une chose, par exemple, quand nous nous déterminons à accomplir ou à ne pas accomplir tel acte à cause de telle réflexion qui nous vient et qui n'est que le souvenir évoqué à propos ou la constatation même d'une qualité d'un objet, d'une manière d'être d'une personne.

Il n'est pas nécessaire, d'ailleurs, pour porter un jugement, que la considération de l'élément isolé d'une idée nous soit immédiatement utile à quelque chose - ceci arrive dans la plupart des cas : si nous pensons qu'un livre est instructif, c'est en général au moment oì nous arons à le consulter, ou bien au moment où il nous faut donner notre opinion sur lui. Mais lorsque l'esprit est porté ả la rêverie il arrive que des jugements, sans utilité apparente, sans finalité immédiate, viennent s'offrir à lui. La loi de systématisation se manifeste encore en ce cas d'abord dans le jugement considéré en lui-même comme une synthèse, et aussi par ce fait que le jugement exprimé, s'il n'eutre pas immédiatement dans un systeme psychique plus complexe, est susceptible d'y entrer une autre fois et qu'il sera alors plus facilement suscité. 
On a tiché de distinguer le jugement de l'association des idées. "La penséc lumaine, a dit M. Pierre Janct, lorsqu'elle est élevée et complexe, renferme plusieurs sortes d'éléments, des matériaux d'abord, si l'on peut ainsi dire, les sensations et les images juxtaposées, et en second lieu, lintelligence de leur disposition, l'idée du rapport qui les unit. Dans un jugement, par exemple, il y a évidemment les termes, homme, mortel, chien, mammifère, mais il y a plus que cela ; car, si ces termes se succèdent simplement dans la pensée sans que je puisse voir entre eux aucun rapport, il n'y a pas de jugement. Entendons-nous : des termes successifs sont toujours rattachés par quelque chose, dira M. Binet, une association par contiguïté. une habitude, une vibration commune qui les fait naitre l'une à la suite de l'autre. Sans ancun doute il y a une raison qui fait naître dans un esprit B à la suite de $A$, mais tant que cette raison n'est pas connue de moi, tant qu'elle ne se présente pas à moi comme la conscience d'un rapport entre $\mathrm{A}$ et $\mathrm{B}$, il $\mathrm{y}$ a dans mon esprit simple juxtaposition des termes par l'association, il n'y a pas jugement. Ce n'est pas du tout la même chose, et M. Binet l'a parfaitement compris, que l'association par ressemblance et le jugement de la ressemblance. Il y a donc quelque chose de nouveau dans mon esprit quand je conçois ce rapport de ressemblance, et l'association mécanique des termes n'en était que la préparation.

“ Pour beaucoup de psychologues, cette différence ne paraît pas très importante, car ils considérent la conscience comme un épiphénomène dont la présence ou l'absence ne modifie pas la nature des phẻnoménes. J'aroue ne pas bien comprendre comment une école positive, c'est-à-dire préoccupée avant tout des faits et de leurs plus petits détails, peut négliger ainsi un fait capital comme la présence ou l'absence de la conscience. Quand on fait de la psychologie, quand on analyse les idees de 
l'homme, on ne doit pas trouver indifférent que l'homme pense ou ne pense pas, comprenne ou ne comprenne pas. Il n'y a donc jugement que lorsque je comprends le rapport de ressemblance entre Pierre et Paul, et expliquer simplement la juxtaposition de Pierre et de Paul dans mon esprit ce n'est pas du tout expliquer le jugement " I.

J'ai donné tout au long le raisonnement de M. Pierre Janet, car il me semble y voir en même temps qu'ume bonne critique de la théorie associationniste anglaise que j'avais moi-même adoptée autrefois, une occasion d'éclaircir la nature du jugement. En fait, le jugement n'est pas une simple juxtaposition ou association d'idées; supposons que l'idée d'une cruche soit indissolublement unie dans l'esprit d'un homme à l'idée d'un perroquet, le tout ne pourra, à aucun égard, passer pour un jugement. Mais la théorie associationniste prend un autre aspect si l'on remplace les principes de ressemblance et de contiguïté par le principe de la finalité immanente. Un livre qui est devant moi a une couverture jaune-orangé. Le jugement que je porte sur sa couleur n'est pas seulement une juxtaposition de la couleur jaune-orangé et des autres qualités qui, pour moi, constituent ce livre : c'est une synthèse systématique; et j'entends par là que certains rapports sont établis entre cette couleur et les autres qualités rapports qui, étant perçus par moi, servent à faire naître en moi certaines autres idées ou d̀ diriger certains actes : je sais, par exemple, que si j'ai le besoin ou le désir de percevoir la couleur jaune-orangé, je n'ai qu'a susciter les sensations tactiles et musculaires approprićes pour prendre et regarder ce livie, je sais aussi que cette couleur peut me faire reconnaître ce livre parmi un certain nombre d'autres. Ce caractère que j'ai isolé et considéré à part, peut me servir à relier ce système d'impressions,

1. Revue philosophique. Vol. 22, pages 191-192. 
L'association SYSTÉmatique - IMAGES ET idées 113

d'images et d'êtats faibles qui constitue mon idée de ce livre avec un grand nombre d'autres faits, tendances, ou désirs. Le jugement consiste done bien dans l'isolement relatif d'un des caractères d'un objet, d'un être, dans le fait de considérer à part ce caractère qui deviendra le point de départ d'une nouvelle série coordonnée de phénomènes. Toutes les fois que nous isolons ainsi, que nous considèrons à part, dans un complexus systématisé d'attributs, un des attributs et que nous continuons cependant à rattacher cet élément à ce complexus, comme une partie à son tout, tout en le disposant à entrer dans de nouvelles associations systématiques, nous portons un jugement. Il est bien sùr qu'un des éléments essenticls du phénomène c'est le iien logique entre les deux états dout la synthèse constitue le jugement, c'est-à-dire l'aptitude pour ces deux états à se coordonner en vue d'une fin commune. Dire : tel homme est un fripon, c'est associcr, synthétiser des états de conscience dans un sens défini, de manière à les rendre capables d'exercer sur la conduite une action précise, de manière à les faire converger vers une fin commune. Le mot et l'idée : fripon indiquent dans quel sens on doit comprendre l'individu dont il est question et de quelle manière il conviendra d'agir avec lui dans telle ou telle circonstance. Par le jugement, on le voit, nous isolons un des caractères d'un être et nous faisons de ce caractère l'élément principal, au moins en certains cas, celui qui, dans des circonstances que l'esprit saura apprécier, devra déterminer le sens de l'orientation de la pensée et de la conduite.

Mais je ne puis admettre que la conscience qui accompagne le jeu des éléments psychiques et des tendances, soit un élément essentiel du phénomène; ce qui importe ce n'est pas que ce jeu particulier des phénomènes quil constitue un jugement se révéle à nous par des faits subjectifs, e'est qu'il se produise. La perception interne 
nous fait connaître un phénomène, elle ne le constitue pas, elle est un nouveau plénomène qui vient se greffer sur le premier, unc nouvelle synthèse qui embrasse la première, mais ne lui donne pas l'existence ${ }^{1}$. Les exemples de jugements inconscients ne sont pas difficiles à trouver, la plupart de ceux que nous portons sont mème de cette nature. Toutes les fois que nous nous servons d'un objet, il arrive que certaines de ces qualités sont spécialement celles qui nous sont utiles, les autres sont momentanément négligées, celles-là, au contraire, sont en rapport étroit avec des séries coordonnées d'actes et de pensées, ou de sentiments. Il y a bien ici, par conséquent, les conditions du jugement, mais il n'y a pas de jugement formulé en mots, ni perçu par l'esprit comme jugement. Les phénomènes sont bien coordonnés comme ils le sont dans le jugement, mais cette coordination elle-même n'est pas considérée à part. Sans doute, comme le dit M. Janet, « expliquer la juxtaposition de Pierre et de Paul dans mon esprit v ce ne serait pas expliquer un jugement de ressemblance entre les deux, mais montrer les éléments communs de nos idées de Pierre et de Paul, montrer pourquoi et comment, par conséquent, l'idée de l'un peut ètre, dans certain cas et à certains égards, substituable à l'idéc de l'autre, et comment ces idées peuvent se remplacer dans l'esprit dans de certaines conditions, ce serait hien expliquer le jugement de ressemblance, et alors même que ce jugement n'aurait pas été expressément formulé par l'esprit, alors mème qu'il n'aurait pas été l'oljet direct d'un acte de perception intérieure re jugement n'en existerait jas moins. Si je jette une pier're avec l'intention d'atteindre un but, cet acte suppose que japprécie le poids de la pierre, la distance à laquelle

1. Voir a ce sujet mon travail: La perception interne et la conscience. Revue scientitique, 1888. T. I. 
L'ASSOCIATION SISTÉMATIQUE - IMAGES ET IDÉES 115 il faut l'envoyer, etc.; il y a une série de jugements par lesquels je fais abstraction d'une partie des qualités de la picre, par exemple, la couleur, la nature minéralogique, etc., pour ne considérer guérc que le poids et aussi la forme, - et d'une partie des qualités des objets qui m'environnent, de presque toutes, à vrai dire, pour ne considérer guère que leur's dimensions représentant l'espace qui me sépare du but visé; de plus, ces données sont associées à des ébauches de mouvements, à des sensations tactiles et musculaires. Il y a là une série de jugements bien nets et cependant ils peuvent s'accomplir tous, ou presque tous, sans conscience précise. Ce n'est donc pas la connaissance d'un rapport qui constitue le jugement, c'est ce rapport mème, mais ce rapport est un rapport de finalité non une simple juxtaposition d'éléments.

A côté de ces jugements analytiques on peut, à un certain point de vue, reconnaître des jugements synthétiques. Ce sont ceux qui se forment lorsque de nouvelles expériences viennent compléter les idées que nous nous faisons des choses ou des gens. Lorsque, par excmple, on a découvert les propriétés antiseptiques de l'acide phénique, lorsqu'on a découvert que l'absinthe pouvait occasionner des accès épileptiques, de nouveaux jugements se sont formés, nos idẻes de ces objets se sont complétées. Au fond, ces jugements ne diffèrent pas essentiellement des autres. Ils sont encore des extraits d'une idée complexe, dans lesquels l'un des attributs est principalement mis en évidence. Seulement ils se produisent au moment oì l'idée se complète, et les jugements doivent régulièrement commencer par lá, car, en général, au moment où un caractère nouveau est attribué à un objet, c'est ce caractére qui est mis en évidence, les autres restant presque inaperçus et désignés collectivement par le nom de l'objet.

L'hypothèse, la croyance, la certitude, peuvent au 
point de vue psychologique, se ramener à des formes varièes de la finalité. Dans la certitude, la finalité est complète, l'état de conscience ou la tendance qu'il représente sont en complète harmonie avec tous les phénomènes de l'organisme, des rapports très étroits les associent systématiquement avec eux. Dire que nous sommes certains que deux et deux font quatre, c'est affirmer que cette idée s'accorde avec toutes nos idées, toutes nos perceptions, que tous nos actes s'accomplissent logiquement d'accord avec elle. On comprend que la certitude complète, rigoureuse, soit rare au point de vue psychologique, on comprend aussi qu'elle s'ètablisse d'autant plus facilement que l'esprit est moins riche en idées, en tendances, car alors il y a moins de chances d'en trouver qui, par leur impuissance à former un accord systématique, fassent naître le doute. D'une manière générale il est facile de comprendre que c'est dans les parties les moins complexes, mais les mieux organisées de l'esprit, dans les instincts, que l'on trouve la plus grande certitude, j'entends la plus grande certitude subjective et relative, la seule qui nous intéresse dans une étude psychologique. Ici le doute ne se produit pas, la réflexion n'intervient pas, il n'y a pas de lutte, pas d'hésitation. L'acte réflexe pur montre à certains égards le plus haut degré de certitude, en supprimant même la connaissance que nous avons de cetle certitude, connaissance qui est encore un signe que celte certitude n'est pas complète, car évaluer les qualités et la force de ses opinions, c'est peul-être encore témoigner de quelque doute á leur égard.

$\Lambda$ un degré inférieur vient la croyance, la probabilité, la quasi-certitude; ici l'association systématique générale existe encore, mais elle est plus faible. Elle existe, puisque nous arissons en général conformément à nos croyances, et que ces croyantes sont en harmonie arec les données de l'expérience, c'est-a-dire avec nos perecptions, 
mais certains éléments n'entrent pas dans l'association systématique, cette association est moins complète, et les éléments qui y entrent sont moins étroitement liés, l'association est plus faible. Il y a, au reste, comme on sait, tous les degrés possibles dans la croyance, depuis la certitude jusqu'au doute absolu. Je crois, je suppose, que le temps sera beau dans la journée et je me dispose à partir pour une course à la campagne. Si certains indices se montrent, si le temps paraît quelque peu couvert au Nord, si le vent souffle du Midi, ma croyance se mêlera de quelques inquiétudes, et bien que mon opinion soit, en somme, que le temps sera beau, elle n'est pas assez systématiquement associée à tous les éléments de mes perceptions pour que la certitude soit complète, c'est-àdire pour que le système formé par la perception et l'opinion que j'en tire puisse se compléter facilement par des mouvements dépendant logiquement de la croyance, par l'acte de me préparer et de partir. Si le système entier finit par s'établir ce n'est que péniblement, lentement, avec des oscillations successives, et son existence est toujours menacée par un système opposé, composé d'idées contraires, de prévision d'un temps défavorable, systématiquement associées avec certains éléments de la perception, les nuages et le vent soufflant d'un certain côté. Si nous descendons un degré de plus, dans le doute, la division de l'esprit devient encore plus grande, et les systèmes opposés ne peuvent, ni l'un ni l'autre, determiner l'orientation de l'esprit.

Mais nous pouvons encore descendre un degré, nous trouvons alors des systèmes d'images et d'idées qui n'ont pour ainsi dire pas d'associations systématiques avec les tendances importantes qui constituent essentiellement notre personnalité. Nous avons affaire à des rêves, à des idées flottantes; ces phénomènes manifestent encore la loi de finalité en ce sens qu'ils se composent eux-mêmes 
d'éléments coordonnés, et que d'ailleurs ils sont en harmonie avec certaines des tendances de l'organisme, mais ils restent isolés dans l'esprit; le plus souvent, il n'y a même pas de discussion à leur égard, ils ne sont ni repoussés, ni adoptés par la plupart des systèmes psychiques qui ne s'éveilleraient que si les idées de la rêverie tendaient a déterminer des actes, ce qui n'arrive pas ordinairement. On le voit, ce qui distingue l'un de l'autre tous les phénomènes intellectuels que nous venons de passer en revue, c'est la force de l'association systématique et la diversité des éléments qui entrent dans cette association.

\section{$\S 4$.}

Les images, les idées, les jugements paraissent bien être les principaux éléments de l'intelligence. Ils se combinent de plusieurs manières, mais toujours nous les retrouvons, et les formes plus complexes des faits intellectuels ne sont guère différentes, au point de vue de la psychologie abstraite, des formes les plus simples. Nous avons examiné la croyance dans ses rapports avec le doute et la certitude. Ce qu'on entend généralement par une croyance est un système de jugements que l'on tient pour vrais, c'est-à-dire en somme un complexus de croyances. C'est dire qu'il s'agit encore ici d'une association systématique. Des théories récentes ont fait intervenir la volonté dans la formation de nos croyances. On a voulu légitimer ainsi l'acceptation de théories non scientifiquement démontrées. Sans doute, le fait de la croyance ne s'explique absolument ni par la force des idées, comme l'a cru Hume ${ }^{1}$, ni par aucune des suppositions

1. Voyez Hume, Traité de la Nature humaine, trad. de MM. Renouvier et Pillon. La question de la nature de la croyance est discutée, et les opinions de Hume bien critiquées dans la préface de M. Pillon. 
de l'école empirique, mais, si l'on veut ne pas s'en tenir simplement a une explication verbale, ou faire de la croyance une sorte de catégorie, il faut y voir un mode particulier de l'association systématique, celui que nous venons d'indiquer pour le jugement, sauf en ceci que le jugement parait indiquer plutôt un acte propre de l'esprit, - la croyance, une acceptation d'un jugement offert par autrui ou encore d'un jugement qui est à quelque degré incertain et que des considérations d'ordre hétérogène nous poussent à admettre comme vrai. Mais ces nuances n'ont rien qui nous intéresse pour le moment.

Le raisonnement peut être considéré comme une série de jugements, dépendant logiquement les uns des autres, une synthèse de jugements. Tout ce qui vient d'être dit du jugement, au point de vue de la loi que nous étudions, est donc applicable au raisonnement avec ceci en plus que le raisonnement présente encore une synthèse supérieure celle des synthẻses qui forment les jugements. Le raisonnement est l'établissement d'un rapport logique entre les éléments de diverses idées ou perceptions et, par suite, une formation de nouvelles idées au moyen de nouvelles perceptions et d'anciennes idées ou d'anciennes idées seulement; ainsi naissent et s'établissent de nouvelles tendances, de nouvelles raisons ou conditions de nos actes. L'inférence, l'induction, la déduction présentent toutes ce même caractère.

Dans tous les cas, il y a une nouvelle synthèse formée au moins en grande partie avec des éléments empruntés à d'autres phénomènes psychiques qui peuvent, d'ailleurs, subsister à l'état latent, car un même élément peut faire partie, selon le moment, de différents systèmes. Le raisonnement, comme tous les phénomènes intellectuels, comme tous les phénoménes conscients, appartient à la période d'organisation des tendances. Dans ses formes rudimentaires il est à peine conscient et la plupart du temps n'est pas 
connu comme raisonnement, c'est que ses formes rudimentaires sont les moins complexes, et souvent, par suite, les mieux organisées, et, à cet égard, les plus voisines de la perfection. Mais il $\mathrm{y}$ a une analogie essentielle entre toutes ces formes d'inférence et de raisonnement. Dans l'inférence du particulier au particulier, les caractères A, B, C, D.... E, F, G, H, s'étant présentés une première fois avec un caractère $\mathrm{X}$, si les caractères $\mathrm{A}, \mathrm{B}, \mathrm{C}, \mathrm{D} \ldots .$. I, J, K, L, viennent à se présenter, l'esprit s'attendra instinctivement à retrouver le caractère $\mathrm{X}$; dans l'inférence du particulier au général, l'esprit fait abstraction des caractères secondaires E, F, G, H - I, J, K, L, et considère à part l'association de $\mathrm{A}, \mathrm{B}, \mathrm{C}, \mathrm{D}$ et de $\mathrm{X}$ en considérant la présence de $\mathrm{X}$ comme une conséquence nécessaire de la présence de A, B, C, D. Enfin, dans la déduction, dans le passage du général au particulier, l'association de $\mathrm{A}$ B C D et de $\mathrm{X}$ est déjà acceptẻe par l'esprit, elle y est représentée par une proposition-verbale, par une idée, par une tendance plus ou moins consciente ${ }^{1}$, et le caractère $\mathrm{X}$ sera attribué immédiatement, implicitement ou explicitement à tout objet, à tout acte, à toute personne présentant les caractères A B C D. Toutes ces formes de raisonnement ne sont que des degrés divers de l'abstraction de cette vérité générale : A B C D est suivi ou accompagné de $\mathrm{X}$. Dans le premier cas, la vérité générale n'est pas encore dégagée des circonstances particulières au milieu desquelles elle se manifeste; dans le second cas, elle se dégage; dans le troisième, elle existe a part sous des formes variables. Il s'agit donc d'une synthese abstraite, d'une systématisation d'élements empruntés par l'esprit ì difrérents cas particuliers,

1. Comme l'a profondément vu M. Pannier dans son article sur Le Syllogisme et la connaissance. Revue philosophique, septembre 1882. 
destinés à fournir, par de nouvelles associations postérieures, une direction particuliere a l'intelligence et a la volonté.

Pour éclaircir la nature du raisonnement et de ses diverses formes, prenons des exemples et analysons-les. Un chien que l'on a frappé pour avoir dérobé quelque chose ne se laissera plus (s'il est facilement éducable), tenter par le mème objet ou des objets plus ou moins analagues, dans des circonstances semblables, si, par exemple, ils sont placès sur une table, ou dans un buffet, et non par terre. Evidemment la vue de l'objet et la tendance à le prendre éveillent le souvenir des coups, ou des reproches qui ont suivi le vol; il $\mathrm{y}$ a, comme le dit M. Binet, " une association entre deux ètats de conscience au moyen d'un état intermédiaire qui ressemble au premier état, qui est associé au second, et qui en fusionnant avec le premier, l'associe au second "1. Remarquons toutefois que le caractère particulier de l'association qui constitue proprement un raisonnement est la finalité de ses éléments qui forment un tout cohérent, si ce tout n'est pas unifié au point de vue de la fin, on n'a qu'une divagation. Quand les expériences ont été fréquemment répétées dans des conditions différentes, les caractères essentiels présents dans toutes les expériences s'unissent plus fortement ensemble, ils finissent par constituer un système qui a son existence particulière, tout en restant associè aux éléments particuliers qui l'ont complété en diverses occasions, et en restant prèt à s'associer à de nouveaux éléments. Nous sommes ici dans la période de l'induction. Enfin, on voit par un nouveau progrès, le système d'éléments généraux s'organiser de plus en plus et ces éléments finissent par former une tendance plus ou moins consciente, et accompagnée ou non d'une proposition verbale,

1. Binet, Psychologie du raisonnement, p. 141. 
une véritable habitude de l'esprit à laquelle doivent s'adapter les noureaux phénonènes intellectuels que les circonstances suscitent. Par exemple, nous apprécions à peu près justement, d'après nos impressions visuelles, la distance d'un arbre, d'un homme, d'une maison, sans nous rappeler les nombreuses expériences qui nous ont permis de passer d'une impression visuelle à une évaluation de la distance, à une possibilité de sensations musculaires et tactiles, déterminées et comptées, nous ne faisons même pas usage d'une proposition générale. Cependant nous pouvons quelquefois, si nous le voulons, rappeler $\dot{a}$ notre esprit un certain nombre d'expériences passées, et l'on pourrait aussi formuler en propositions verbales les idées générales qui règlent notre jugement; mais dans certains cas, cela n'est pas possible et tel homme qui apprécie correctement une distance serait bien cn peine de dire les principes généraux sur lesquels il se fonde ct même ne les comprendrait pas si on les lui énonçait. C'est que ces principes généraux n'existent en lui que sous forme de tendance fixée, dont les éléments ne peuvent être isolés pour être rattachés à des mots ou à des idées autres que celles qui doivent concourir à sa mise en activité. C'est un fait analogue - avec la différence de l'ètat normal à l'état morbide - à celui du malade qui ne pouvant prononcer des mots isolément, prononce la phrase qui les contient, les mots ne peuvent être rappelés ici que par l'ensemble de la plırase, comme certains élénents qui entrent dans une habitude mentale ne peuvent être rappelés que par les élèments qui sont ètroitement associés, systématisés avec cux et par aucune autre considération, aucun autre fait psychologique. La tendance inconsciente est, en somme, le plus haut degré et le plus abstrait de l'idée générale, au moins dans certaines conditions et à certains égards - c'est le cas oì la systématisation psychique se presente sous sa lorme la plus parfiate. Ce sont 
naturellement les états psychologiques moins complexes qui ont pu acquérir jusqu'ici cette manière d'ètre.

Comine on le roit, le raisonmement et le jugement ont des rapports très étroits. On peut dire, avec plusieurs auteurs que le raisonnement est une complication du jugement, mais le jugement lui-mème est compliqué, et il est impossible de trouver à cet égard matière à une distinction suffisante. On peut dire, à un autre point de vue, que le raisonnement désigne plutôt l'acte, le procédé parlequel l'esprit arrive à formuler un jugement, Le jugement apparaitra ainsi comme le point d'arrivée d'un raisonnement, et mème comme le résultat d'une série de raisommements, et comme pouvant servir de point de départ à des raisonnements nouveaux. Au fond et pour la question traitée ici, ces questions de terminologie n'ont pas une grande importance. En fait, un jugement est la conclusion d'un raisonnement qui l'a précédé, il sert lui-même de point de départ, ou de terme intermédiaire à des raisonnements nouveaux qui aboutiront à de nouveaux jugements. Il serait absolument oiseux de rechercher lequel des deux, du jugement ou du raisonnement, a précédé l'autre. Ce sont deux formes différencièes d'une opération primitive qui n'était ni l'un ni l'autre.

J'ai essayé de montrer comment les faits intellectuels impliquaient tous l'existence d'une loi de finalité. Pour le jugement et le raisonnement il n'était peut-être pas besoin d'une longue démonstration, mais j'ai tenu d poser la question sous sa forme la plus générale et à la résoudre de manière à ce que la solution put s'appliquer à tous les faits sans exception. Invoquer comme preuve de finalité le raisonnement de Dupuytren faisant l'opération du trépan d'après de simples conjectures que. l'évènement justifie, de Pascal vérifiant la pesanteur de l'air par la mesure de la colonne de mercure du tube de Torricelli au pied et au sommet du Puy-de-Dôme, de 
Newton découvrant les lois du monde en voyant tomber une pomme, eùt été plus facile mais moins probant, je crois, et en tout cas ce n'est pas seulement le fait de la finalité qu'il fallait établir, mais la nature de son mécanisme.

On pourrait maintenant passer à des faits généraux d'un ordre moins abstrait, c'est toujours la même loi génèrale qu'on retrouverait dans tous les phénomènes. Il est impossible ici de passer, même rapidement, en revue tous les produits de l'intelligence humaine, mais il est bien reconnu que les sciences sont des systématisations de faits réels, c'est-à-dire de perceptions, d'images et d'idées, que la philosophie est une tentative de synthèse générale des relations abstraites des phénomènes, c'est-à-dire d'idées suggérées elles-mêmes par des coordinations d'images, d'idées et de perceptions; la religion est, au point de vue intellectuel, une synthèse du monde comme la philosophie; l'art est une synthèse d'images, d'idées et de perceptions dépendant de l'organisation créatrice de l'artiste. Si nous examinons, par exemple, une science quelconque, nous voyons qu'elle est un ensemble de lois d'autant mieux coordonnées que la science est plus avancée, condensant chacune un nombre incalculable d'expériences et d'observations, (c'est-à-dire de perceptions et d'images) établissant par suite entre ces perceptions et ces images un lien de finalité, en les fäisant converger vers un but commun en les employant toutes a fournir les éléments d'une loi abstraite dont la connaissance déterminera notre conduite et nous permet. tra soit de nous modifier de manière a nous adapter au monde, soit de modifier en quelque chose le monde pour l'adapter à nous.

$\S 5$.

Nous avons étudié jusqu'ici le raisonnement, le jugement, les phénomènes intellectuels en général, comme 
des faits donnés, en étudiant à présent les conditions, les circonstances de leur formation, nous $\mathrm{y}$ verrons de nouvelles applications de la finalité psychique. En effet, tous les jugements possibles ne se forment pas en nous, et surtout toutes les idées ne deviennent pas des croyances, toutes les croyances ne deviennent pas des certitudes. Nous aurons à étudier plus tard, à propos de l'arrêt, de l'inhibition, la partic négative de cette loi, beaucoup plus importante qu'on ne parait généralement le croire. Nous avons à examiner, pour le moment, sa partie positive, a voir comment les idées naissent en nous, comment les jugements se forment, comment les croyances s'organisent.

Pour introduire un ordre, un peu factice mais réel, en somme, dans le sujet, nous étudierons d'abord les synthèses intellectuelles déterminées surtout par les associations provoquées par des perceptions, ensuite les synthèses intellectuelles dans la formation desquelles la plus grande part échoit aux habitudes internes, dispositions acquises, idées préconçues, tendances déjà organisées, manifestées par des phénomènes intellectuels ou affectifs. Les deux cas nous seront également des preuves de l'activité synthétique essentielle de l'esprit.

D'abord nous trouvons que chaque perception un peu importante joue un rôle dans notre vie intellectuelle. C'est un fait bien général que nos idées nous viennent de l'expérience, c'est-à-dire que nos perceptions déterminent dans le cerveau un travail systématique. Cette systématisation, - avec l'abstraction et la dissociation des éléments qui nécessairement l'accompagnent, - aboutit à des idées, à des raisonnements, à des conceptions plus ou moins vastes, plus ou moins profondes qui se traduisent ensuite par des actes plus ou moins compliqués et importants. Depuis les pauvres idées des animaux dont les instincts ne sont pas assez fixés pour que l'expérience ne 
puisse les modifier en quelque point, jusqu'aux obser'rations et aux expériences les plus élevées du savant, nous pouvons constater des formes très varièes de ce mène phénomene dont la generalité apparait faeilement. Mais ici, comme partout, les erreurs sont spécialement ins-. truetives; une synthese imparfaite laisse mieux voir les matérianx et lactivité intellectuelle sera plus visible peutêtre quand clle aboutira à une erreur, bien qu'elle soit plus imparfaite. Les exemples ne manquent pas, de systèmes intellectuels erronès, suscités par des perceptions qu'il s’agit d'interpréter - nous n'avons à examiner d'abord ici que les cas oi la perception elle-nème n'est. pas sensihlement morlifièc.

Un cas assez curieux est celui des onomatopées. Ici la perception n'est pas précisénent alterée et pourtant l'esprit la croit autre qu'elle n'est, il se trompe sur elle et porte un jugement faux sur son compte. "Si nous croyons parfois entendre, dans certains sons de nos idiomes, une imitation des bruits de la nature, nous devrions nous rappeler que ces mêmes bruits, dans d'autres langues, sont représentés par de tous autres sons dans lesquels les peuples étranger's croient également sentir des onomatopées; de telle sorte qu'il serait vrai de dire que nous entendons les bruits de la nature a travers les mots auxquels notre oreille est habituée depuis l'enfance "1. Ajoutons que les imitations que l'on fait du tonnerre, dès cris des animatux, du vent, etc., dénotent une illusion de même ordre. Il s'établit une certaine convention d'après larguelle tel bruit produit par les organes vocaux de l'homme, inite tel bruit naturel, et des croyances crronées particulières se produisent ainsi.

Voici un fait cité par M. Max Simon oì nous retrouvons bicun cette faculté de l'esprit d'encadrer une perception

1. M. Breal, Mélanges de Mythologie et de Linguistique, p. 401. 
dans un système de faits qui peut ètre un roman. L'esprit veut comprendre et il interprète toujours, en se trompant s'il n'est pas dans des conditions favorables à une activité régulière. "Une personne ayant de l'asthme depuis plusieurs années et qui s'était endormie dans un ètat d'anxiété respiratoire, se voit en rêve dans une rue montueuse, que gravit une lourde voiture : la chaleur est étouffante, les chevaux sont essoufflès; ils ont beaucoup de peine à marcher et bientôt l'un d'eux s'abat. La respiration du pauvre animal est haletante; il est couvert de sueur. Le conducteur fait tous ses efforts pour relever le cheval abattu, et le dormeur vient lui prêter assistance. La personne qui fait ce rêve se réveille: elle est ellemême en pleine transpiration et souffre d'une extrême oppression" 1. Dans le mème ordre de faits nous trouvons, citée par le même auteur, une malade du pensionnat Saint-Lazare qui ne pouvait voir couper, entendre déchirer une étoffe sans se plaindre qu'on la coupât, qu'on la.déchiràt, et sans se mettre dans une violente colère. M. Simon pense qu'il s'agit là d'une illusion provoquée par un phénomène d'action réflexe ; " on sdit en effet, que certains bruits : la main humide passée rapidement sur le bois vernis ou ciré, le grincement d'un couteau sur certains corps, etc., produisent chez beaucoup de personnes une sorte de frisson extrêmement désagrẻable; eh bien! notre malade, au bruit de l'étoffe déchirée, éprouvait vraisemblablement ce frisson qu'elle interprétait dans le sens de son délire "2. Quoi qu'il en soit, l'activité de systématisation intellectuelle déterminèe par. la perception ne peut ètre mise en doute.

Aux associations déterminées par des perceptions, il faut rattacher celles qui sont déterminées par le manque

1.. Mar Simon, Le Monde des réves, pages 39, 40, Cf. p. 41.

2. Max Sïnon, Ouvr. cite, p. 271. 
d'une perception attendue ou habituelle. Les anesthésies partielles ou générales, produisent chez les malades certaines idées, certains jugements particuliers. \&e malade se croit mort, il croit que ses jambes sont de bois "1. Quelquefois il se produit une sorte de dédoublement de la personnalité, les aliénés hémiplégiques "s'imaginent être couchés avec un autre individu, parce que le contact de la moitié paralyséc et anesthésique de leur corps produit chez eux la sensation de la présence d'une personne ètrangère $\triangleright 2$.

Enfin, un cas particulier est celui où la sensation détermine l'apparition de phénomènes intellectuels qui n'ont aucun rapport avec elle. Il se passe ici quelque chose de tout à fait analogue à ce que nous avons vu à propos de l'interprétation par les appareils sensoriels. Une excitation venue du dehors détermine une séric de phénomènes psychiques, mais ces phénomènes résultent simplement de la mise en jeu d'une habitude psychique qui ne correspond nullement á la nature du phénomène extérieur. L'individu est, en ce cas, soit d'une manière permanente, soit d'une manière temporaire, tellement absorbé par une préoccupation dominante ou une habitude prise, que la force psychique dégagée par l'excitation venue du dehors s'emploie tout entière au profit du système dominant. Comme un nerf olfactif dilacéré donne des impressions lumineuses, une sensation auditive, musicale, arrivant $\dot{a}$ un cerveau préoccupé, déterminera une suractivité des tendances principales. C'est ainsi, je pense, qu'il faut interpréter un fait raconté par M. Legouvé, dans ses mémoires. M. Legouvé faisait une pièce en collaboration avec Goubaux, il travaillait pendant la matinée. "Après

\section{Griosingor, Maladies mentales.}

2. Ball, Lecons sur les Maladies mentales, p. 97. Voir d'autres faits analogues, mème page et pages 161, 162, 163, 172, etc. 
le dejeuner, une heure de musique qui nous servait de récompeuse et d'auxiliaire. Il y a un lien mystérieux entre les arts. Une mélodie vous dicte souvent un bon vers, et plus d'une fois, pendant le travail, c'est Beethoven, c'cst Weber, e'est Schubert qui m'ont aidé à me tirer d'affaire dans une scène difficile \1. Darwin éprouvait des impressions analogues, il fut obligé de renoncer à écouter de la musique parce que, au lieu de le distraire, elle le faissait penser trop vivement à l'objet de ses travaux 2 . Enfin la même chose est arrivée à Stendlıal : «H. Bcyle, ce profond observateur, raconte qu'un jour (il aimait alors je ne sais quelle personne), la musique le rendit plus énamouré que jamais ; il crut d'abord que cet art avait sur l'amour une influence particulière. Mais il se rappela que l'année précédente où il songeait au moyen d'armer les Grecs, la mème musique avait éveillé son ardeur avec la même intensité, mais en la tournant du côté de ses recherches d'alors " ${ }^{3}$. La musique, par le caractère quelque peu vague et diffus de l'excitation qu'elle nous donne, est l'art qui se prête le mieux à ces transformations, et son charme lui vient en grande partie de ces tendances, quelquefois inconnues de nous, qu'elle va réveiller au fond de notre être assez pour nous émouvoir, trop peu pour que l'émotion cesse d'être esthétique.

Dans les phẹnomènes intellectuels eux-mêmes, nous remarquons aussi cette double force coordinatrice de l'élément nouveau et des habitudes anciennes; toute idée implantée dans le cerveau cherche à se subordonner d'autres éléments, ou au moins à les coordonner aver elle, les anciennes tendances cherchent aussi à se subordonner les idées nouvelles. Il y a quelquefois lutte, mais parfois

1. Legouré, Soixante ans de souvenirs, t. III, p. 77.

2. La vie et la correspondance de Charles Darwin. 3. Guyau, les Problemes de l'esthetique contemporaine, p. 30. 
l'une ou l'autre des idées réussit à se développer', parfois l'une et lautre persistent et l'on a soit une coordination supérieure, soit un manque d'harmonic dans l'ensemble. Nous sommes toujours portés à croire ce que l'on nous dit, à accepter les idées qui nous sont suggérées, si aucune tendance ne s'y oppose. Le fait se reproduit à chaque instant. Si quelqu'un vient me dire qu'il a trouvé une pièce de dix centimes en passant dans la rue, si je ne le sais pas particulièrement menteur, si je ne connais pas une raison spéciale pour qu'il ne dise pas vrai sur ce point, il est sùr que je le croirai, et cette idée restera dans mon esprit, de manière à déterminer, si l'occasion s'en présente, une direction particulière de mes idées et de mes actes. La force associative de l'idée se montre très bien quand on supprime la résistance des tendances organisées, par exemple, dans le somnambulisme. On sait qu'il suffit, en général, et à-moins que l'idée suggérée ne heurte des tendances bien profondes, de suggérer une idée à une personne hypnotisée, pour qu'elle la croie vraie et que ses autres idées et ses actes soient logiquement en rapport avec clle. J'ai déjà cité le cas de M. Richet annoncant à un ami hypnotisć qu'il est transporté dans la lune, le sujet accepte la réaliti de ce voyage et son esprit construit un systeme dimages et de raisonnements en harmonic avec l'idée suggérée. Il voit des bètes fantastiques, et comme M. Richet lui annonce vouloir les ramener avec lui : "Je te recomais bien lit, disait-il, tu ne sais seulement pas comment nous ferons pour redescendre et tu veux te charger de ces gros animaux-lá... Il disait cela tris scrieusement et se fächait tout de hon. "Les tendances concrètes représentant les associations produites par l'exprerience de la vie de tous les jours ne paraissent s'êtro manifestées que par un mot. \& Quel beau récit de voyare it faire, mais par malheur on ne nous croira 
pas " '. Ainsi lincrédulité, la résistance que remrontrait chez luil lidée envahissante il la recomnaissait. malis il la plagait chez d'autres persomnes, comme les milides yui ont une moitié du corps anesthésié, attribuent cette moitié à une autre personne. Autour de l'obstacle qui se présente, l'ilée envahissante construit un nouveau système d'iunages et d'idées. De tels exemples me paraissent mettre complètement en lumière le pouvoir de systématisation des phénomènes intellectuels qui éveillent à la fois des idées, des hallucinations, des sentiments et des tendances motrices.

La force de systematisation des habitudes de l'esprit n'est pas moins évidente. On sait que nous accueillons en général très favorablement les idées qui s'accordent avec les nôtres; au besoin nous défigurons aisément les idées des autres pour les accorder aux nôtres, ou bien encore, et peut-être plus volontiers, cle manière à les rendre telles que leur absurdité soit frappante et que les nôtres en triomphent aisément, le tout, bien entendu, sans mauvais vouloir conscient. Quiconque a discuté, si peu que ce soit, sur un sujet quelconque, a pu s'apercevoir que bien souvent ses arguments ne sont pas compris et que les réponses qu'on oppose à ses raisonnements n'ont pas de rapport logique avec ce qu'il a dit, - et les deux interlocuteurs pourraient souvent faire, chacun pour son compte, la même remarque. L'adversaire, gràce à des associations d'idées, gràce à une organisation psycho-physique différente, n'a pas saisi les raisons qu'on lui donne. Le milieu

1. Ch. Richet. L'Homme et l'Intelligence. Du somnambulisme provoque. On trouvera d'autres exemples. « Ln jour, dit M. Richet, je dis a $\mathrm{M}^{\mathrm{me}} \mathrm{X} \ldots$ qu'elle était changée en perruche, puis, je ne sais pourquoị je n'y pensais plus, lorsque, tout d'un coup, elle me demanda très sérieusement si elle pouvait manger du chénevis qu'on avait mis dans sa cage. "Voir aussi Richer, l'Hystéro-épilepsie. Bernheim, de la Suggestion. Binet et Féré, le Magnétisme animal. Beaunis, Etudes sur le Somnambulisme provoque. Delbeuf, Revue philosophique, passim. 
n'était pas favorable aux nouvelles idées qu'on voulait y introduire, et elles n'ont pu s'y établir. De plus, la lutte a été si courte et si peu vive, qu'elle n'a pas excité dans le cerveau assez de trouble pour éveiller le sens intime. Cela se produit quelquefois bien qu'on ait étudié la question et examiné avec conscience les arguments; les mots parlés ou écrits, n'éveillent pas les idées qu'ils devraient éveiller, ou bien ne les éveillent que très faiblement, et elles sont bientôt étouffées. La parabole du grain qui tombe sur le roc ou dans les broussailles, s'applique ici merveilleusement. Ce qui arrive aussi e'est que les éléments d'idées éveillées par le mot, sont arrangés par l'esprit qui les reçoit de façon à déformer plus ou moins la doctrine, l'idée qu'il s'agit d'examiner. En fait, on n'est presque jamais parfaitement compris. Les erreurs sont d'une fréquence extrême et les hommes éminents n'en sont nullement exempts. Un cas assez curieux est la critique que fit Flourens de l'origine des espèces de Darwin. "Selon M. Flourens, la grande erreur de Darwin est d'avoir personnifié la nature....." Quoi ! M. Flourens n'a-t-il pas compris, s'écrie M. Huxley, la nécessité logique de ces arguments simples qui servent de base à tous les raisonnements de Darwin? A-t-il pu confondre une déduction irréfragable, tirée des rapports reconnus qui subsistent entre les organismes et les conditions qui les entourent, avec une forme substantielle métaphysique, une personnification chimérique des forces de la nature? Ce serait à n'y pas eroire, si d'autres passages du livre ne venaient faire cesser toute hésitation à cet égard.

a On imagine une élection naturelle, que pour plus de ménagement on me dit être inconsciente, sans s'apercevoir que le contre-sens littéral est précisément là : élection inconsciente. J'ai dejà dit ce qu'il faut penser de l'election naturelle. Ou l'élection naturelle n'est rien, ou c'est la nature. Mais la nature douée d'élection, mais la 
nature personnifie !.... Derniere erreur du dernier siecle!..... Le dix-neuvième ne fait plus de personnifiration " 1 . Au reste la théorie de la sélection naturelle qui nous parait ajourd'hui si claire, eut beaucoup de peine, je ne dis pas a se faire admettre comme vraie, mais a se faire comprendre. On s'en rendra compte en lisant le second volume des lettres de Darwin et le récit de M. Iuxley sur la réception faite it l'origine des espèces ${ }^{2}$.

La manière dont certaius peuples acceptent, ou plutôt paraissent accepter les nouvelles religions qu'on leur prẻche, est une nouvelle preuve de l'activité systématique des habitules psýchiques, des idées acquises. Ces peuples, dans la religion 'qu'on leur offre, choisissent inconsciemment ce qui s'accorde avec leurs habitudes d'esprit, rejettent le reste, et, par la systématisation des éléments acceptés avec les croyances, les théories, les ilées déjà existantes, défigurent complètement la doctrine quon leur a transmise. Les nègres du Congo; dit M. Girard de Rialle, tout en paraissant convertis au christianisme, n'en sont pas moins fétichistes, ils considirent les prètres comme les sorciers, les ouagangas des blancs. Dans les localités où l'appui du gourernement a permis ì ces ecclésiastiques d'extirper les anciennes croyances et les pratiques terribles ou repoussantes de l'ancienne religion fétichique, les nègres n'ont pas acquis pour cela des notions thẻologiques plus élevées; ils considèrent JésusChrist comme le grand fétiche des blancs. Les prédications sur la vie future, l'autre monde, les récompenses et les peines après la mort, n'ont pas été comprises, et toutes ces idées se sont confondues dans la cerrelle des noirs

1. Huxley, Les sciences naturelles et les problemes quielles font surgir, p. 424.

2. Ch. Darwin, La vie et la correspondance de Ch. Daruin. T. II. Liourrage a été traduit en français par M. II. de Varigny. 
avec l'expatriation forcée de leur's compatriotes, embarqués comme esclaves sur des bateaux négriers, transportés en Amérique et que l'on ne revoyait jamais ${ }^{1}$. De mème, dans un ordre de faits analogues, M. Ferri a remarqué que "l'hommme doux et honnête adore un Dieu d'amour et de pardon et que l'homme pervers et immoral se forme un Dieu cruel et haineux " 2 .

L'influence des idées dominantes est très marquée aussi dans l'état pathologique. M. Foville raconte l'histoire intéressante d'un capitaine de la garde impériale atteint du délire des persècutions arec hallucinations multiples, qui entra à Charenton peu de temps avant la guerre de 1870. Le malade conservait une certaine lucidité en dehors de l'objet propre de sa folie, mais toujours il interpréta les faits dans le șens de son délire. "M. Y.... n'a pas cessé de se montrer rebelle aux vérités les plus évidentes, insensible aux plus douloureuses réalités. La succession des événements malheureux, l'investissement de Paris, la capitulation de Metz, par suite de laquelle son régiment et ses camarades devenaient prisonniers, les combats sous Paris dont il voyait les divers épisodes de ses propre yeux, le bombardenent des forts qu'il entendait sans interruption, l'insurrection de Paris et ses suites déplorables, le second siège et la réorganisation d'une armée française, tout est resté pour lui comme non avenu. Charpue événement lui était raconté par plusieurs persomnes différentes, et jamais il n'a voulu en croire un seul mot. Il u'a jamais cessé de soutenir que la France était en paix, l'empereur aux Tuileries; que les communications étaient libres et que c'était pour faire cause commune aree ses perséculeurs que l'on refusait d'envoyerses lettres a ses parents el de lui faire parvenir

1. Girarl de Rialle, Mythologie comparé, I, 223.

2. Ferri, cité par Garofalo, La Criminalogie, 1. 141. 
leur réponse; que tout ce bruit fail autour de la maison par la canonnade était l'cuvre de quelques officiers de son régiment, ses ennemis déclarés, acharnés à le tourmenter et dont il citait les noms... Je lui donne des journaux qui racontaient en détail les grands événements qui se succédaient d'une manière si lamentable pour la France et pour son arméc. Il les lisait devant moi sans la moindre emotion, et me les rendait en disant avec un sourire ironique, que c'ètait une feuille imprimée par ses ennemis, uniquement pour le tromper.... Il m'est arrivé de lui remettre le mème jour cinq ou six journaux différents, portant la même date, relatant les mêmes faits; il les a lus avec la mème incrédulité, assurant qu ils étaient tous de faux journaux, imprimés à sa seule intention par des persécuteurs tellement acharnés qu'ils ne reculaient devant aucun sacrifice d'argent " ${ }^{1}$. On trouvera des faits analogues en grand nombre chez tous les auteurs qui ont traité de la folie ${ }^{2}$.

On le voit, tous les phénomènes intellectuels, considérés en eux-mèmes, nous ont apparu comme des formes particulières d'association systćmatique de certains èléments fournis par les perceptions externes et internes: images vires, images faibles, idées, éléments moteurs. Selon la forme de l'association, selon le but qu'elle doit remplir, nous avons des images, des idées, des jugements, des raisonnements, des croyances. Quant ì la formation, ¿ l'apparition de ces phénomènes intellectuels, elle nous a paru amenée, selon une loi de finalité, par la nature de

1. Cité par Despine. De la folie au point d: vue philosophique ou spécialement psychologique, p. 325 et suivantes.

2. Voyez en particulier Esquirol. Des Maladies mentales, II, 13, 14, 15. Les Annales d'Hygiène publique et de Médecine légale, aux articles concernant les aliénés criminels. Voil, par exemple, l'A ffaire Severac, far Chambeyron, médecin de l'hospice des alienès de Rennes, au tome XVIII, P. 374, etc, 
la cause qui la produit, des éléments psychiques momentanément prépondérants, par l'influence coordinatrice des perceptions et par la nature des croyances, des idées, des tendances antérieurement acquises et dont l'activité tend a se systematiser arec les nouvelles idées qui se produisent. On pourrait compléter ceci par une étude sur la mémoire et l'habitude. L'habitude, en tant qu'impliquant une association plus étroite et plus parfaite de phénomenes intellectuels, rentre évidemment dans notre loi. La mémoire désigne le réveil de certains phénomènes intellectuels dans certaines circonstances. Nous l'avons donc implicitement étudièe en indiquant les conditions de réveil des idées à l'état normal, et en remarquant que c'est l'idée dont nous avons besoin qui se présente gẻnéralement à l'esprit. Si nous étudions une question de psychologie et que le mot âme se présente à nous, nous nous souviendrons de son sens psychologique, non du sens qu'il a dans l'expression l'ime d'un canon, etc, Du reste, cette question sera plus longuement examinée à propos de la critique des lois de contiguïté et de ressemblance.

Il resterait à se demander maintenant quel est le rôle général des phénomènes intellectuels, sensations, perceptions, raisonnements, dans la vie de l'esprit, et comment ce rôle est réductible à la loi de finalité. Nais il est a noter d'abord que nous avons pris dans un sens très large les mots phénomenes intellectuels et il est une distinction qu'il importe de faire, celle du phénomène jsychique qui est essentiellement un jeu des tendances et de leurs éléments, et des faits de conscience qui l'accompagnent. Si l'on entend par phénomène intellectuel la ronscirnce rue nous avons dins certains cas des associations et des dissociations interues des éléments de nos tendances, Ies phénomènes intellectuels correspondent à des

1. Voir pour le developpement Les phenomenes affectifs et les lors de leur apparition. 
actions, à les groupements harmoniques de ces éléments, alors que ces actions, ces groupements s'effectuent d'une manière assez systématisée pour ne pas donner lieu ì des plénomines affectifs, avec assez de trouble pourtant pour ne pas ètre automatiques. Mais on a montré plusicurs fois et nous arons cu occasion d'y revenir, que, dans le jeu des tendances, la conscience n'etait pas un élément essentiel. En quoi donc consiste essentiellement le jeu de tendances qui se manifeste en certains cas par ces phénomènes subjectils qui sont, pour le sens intime, les phenomènes intellectuels? Il consiste, d'une manière générale, en une association, précédée quelquefois ou accompagnée d'une dissociation d'éléments psỹchiques telle que le système ainsi formé soit un en lui-mème et soit apte à entrer comme dilement dans me tendance plus complexe, laquelle se manifeste, en génèral, plutôt par des phénomènes affectifs, et en tout cas correspond à une part plus grande de la personnalite. Toutes nos connaissances, toutes nos idées, tous nos raisonnements (en frenant ces mots dans un sens qui puisse s'appliquer aussi a des actes inconscients de l'esprit) ont pour röle, dune manière générale, de déterminer l'application des tendances motrices, quand cellesci entreront en jeu. Savoir: par exemple, que l'eau apaise la soif: c'est onvrir une direction a la tendance organique qui se manifestera quand notre organisme nous donnera l'impression de la soif. Savoir la physiologie, c'est avoir des connaissances qui peuvent diriger un grand nombre d'actes de diverse nature, depuis les soins à prendre de son corps jusqu'aux cours ou aux lirres que l'on peut faire sur cette science.

D'une manière génẻrale, l'intelligence a surtout pour élements des phénoménes alsstraits, susceptibles d'entrer dans un gratud nomlsre de tendances, de systèmes psychiques divers. Les phenomenes affectifs sont plus souvent produits far le jeu de tendances concrètes, 
intéressant davantage l'ensemble de la personnalité. Par excmple, savoir que deux et deux font quatre est une connaissance abstraite, elle est représentéc en nous par l'association de divers éléments psychiques, par une certaine manière d'ètre qui pourra se manifester en bien des circonstances et s'associcr à des tendances très variẻes. Au contraire, le plaisir que produit, par ex́emple, une bonne digestion est lié à une fonction vitale déterminée, concrète et ne peut s'associer à plusicurs tendances organiques. A la distinction indiquée: au point de vue de leur manifestation au scns intime, entre les phénomènes intellectuels et les phénomėnes affectifs, nous pourons donc ajouter, $\dot{a}$ un point de vue moins abstrait et plus synthétique, cette différence que les idées et les perceptions, les éléments de l'intelligence, sont des éléments possibles pour un grand nombre de tendances l'idéc ou la perception d'un fruit, par exemple, étant susceptibles de s'associer à des actes très variés et à des tendances très diverses : faim et actes destinés à la satisfaire, amour de l'horticulture et soins donnés aux arbres, esprit scientifique et recherches de botanique, etc. - et que les operations intellectuelles, jugement, raisonnement, etc., sont toutes des synthèses, précédées ou non d'une décomposition, d'éléments abstraits extraits des tendances ou des perceptions, ou bien encore, comme les mots et les autres signes, représentant les perceptions ou les tendances. L'intelligence est donc destinée d'une manière générale à analýser et à synthétiser les données de l'expérience sous toutes ses formes, do manière a former des éléments abstraits systématiquement associés, idécs, haloitudes d'esprit, jugements, croyances, qui, par leur association arec les tendances motriees, permettent a ces tendances de.s'atsocier liarmonirfuenent entre elles, et d'arriver à se satisfaire, c'est-itdire a se traduire par ales actes coordonnos qui lont servip 
L'ASSOCiation Systématique - L'INTElligexce 139

le monde extérieur au développement de la finalité humaine et qui par cela mème, augmentent sa propre finalité. L'intẹlligence, à ce point de vue, a donc pour effet général, de préparer et de développer par l'association systématique de certains éléments abstraits, la sýstématisation des tendances de l'homme, et par suite de son milieu. C'est son rôle daus la vie de l'esprit, mais il ne faut pas oublier que l'on ne peut séparer absolument l'intelligence de la sensibilité et de la volonté, et que ce qui nous parait i un foint de vue être un phénomène intellectuel, apparait i un autre point de vue comme un élèmeni d une volition. 


\section{CHAPITRE III.}

\section{Phénomènes affectifs et tendanees.}

$\S 1$.

La loi de finalité est plus évidente peut-être encore dans les phénomènes affectifs et dans les tendances en général que dans les opérations intellectuelles. Que la faim, par exemple, ait pour effet de donner à l'organisme les aliments dontil a besoin, que le manque d'oxygène dans le sang détermine un état d'essoufflement qui a pour effet de remédier à ce manque d'oxygène par la rapidité de l'acte respiratoire, cela n'est pas douteux, mais il y a peut-être licu d'examiner brièvement le problème à un point de vue très général, et d'étudier quel est le mode particulier de finalité des phénomenes affectifs et des tendances. Comme dans les chapitres précédents nous examinerons d'abord les phénomènes une fois donnès et ronstitues, nous examinerons ensuite les circonstances. les conditions de leur production.

Si je réunis ici les phénomènes afrectifs et les tendances en génèral, ce n'est pas que les deux ordres de faits me paraissent se confondre absolument. D'un côté, en effet, nous arons nombre de tendances rui ne se manifestent pas par des phénoménes affectifs au moins ordinairement, et telles sont, par cxemple, la plupart des fonctions organiques continues ou at rythme très rapide : la circulation, la respiration, la digestion, par exemple; l'autre part, il est des phenoménes affectifs qui accomjagnent dos oporations intellectuelles; c'est-i-dire un jeu 
d'éléments abstraits comme, par exemple, le plaisir que donne un beau raisonnement. Mais d'une manière générale, comme je l'ai indiqué, les phénomènes affectifs sont la manifestation à la conscience de l'activité de tendances importantes dans certaines conditions que j'ai précisées ailleurs ${ }^{1}$. Nous pouvons donc, sous les réserves qui viennent d'ètre indiquées, et en en tenant comple dans le choix des faits, parler simultanement des sentiments et des tendances qu'ils accompagnent d'autant plus légitimement que parler des unes après avoir parlé des autres serait s'exposer à des répétitions forcées et sans utilité.

La finalité dans les tendances organiques est tellement évidente qu'il serait oiseux de s'y arrêter. La respiration, la digestion, la circulation ont toutes pour effet lc maintien de la vic. Quand le physiologiste cherche les fonctions d'un organe donné, il ne fait pas autre chose que chercher la finalite particulière de cet organe et comment il s'associe systématiquement avec les autres organes du corps. On trouve même déja dans l'organisme des tendances, des fonctions qui ont un autre but que la vie de l'individu : la fonction génératrice qui a pour but la vie de l'espèce. ll serait hors de propos de s'arrêter ici avec admiration devant tous les détails de ce système où l'on a voulu voir la marque d'une intelligence créatrice, d'un fait de conscience divin, comme si la finalité des faits de conscience n'était pas sous la dépendance de la finalité organique, bien loin de l'avoir précédée. Pour en venir au point de vue qui concerne plus spécialement la psychologic, les instincts offrent des applications non moins frappantes de la loi de finalité. Il n'est pas bien

1. Les Phénomenes affectifs. En rèsumè, la tendance en activitè s'accompagne d'un phènomène affectif, émotion, sentiment de désir, plaisir, peine, etc., quand elle n'aboutit pas sans quelque difficulté, et que son activité persiste cependant. 
utile de décrire an long les instincts des animaux, des abeilles en particulier ou de ces insectes qui ont pu sembler un moment une pierre d'achoppement pour la théorie évolutionniste bien qu'elle ne paraisse en somme devoir en éprouver aucun dommage. Dans l'homme nềme les actes réflexes plus ou moins simples, plus ou moins conposés, qu’ils se passent dans la moelle épinière, dans la protubérance anmulaire, dans les tubercules quadrijumeaux, sont de merveilleux exemples de finalité organique, de systematisation d'impressions et de mouvements.

Si nous passons de ces phénomènes aux tendances qui s'accompagnent plus ordinairement d'émotions, de sentiments, de désirs, nous trourons les mêmes caractères généraux avec cetle différence que si ce système d'éléments est plus vaste, plus complexe, la coordination est moins parfaite que dans la sphère de l'automatisme.

Il ne samrait itre question de faire ici une étude complote du caractère, mais au point où nous en sommes il convient d'en dire quelques mots. Ce que l'on appelle caractère n'est que la forme particulière que prend chez un individu la finalité générale de lorganisme, les qualitès et les défauts indiquent la forme particulière d'association des élements qui composent une tendance. Nous ne jourons ici que nous en tenir aux lignes générales et indiquer ce gui concerne spécialement la grande loi quie nous étudions en ce moment. A ce point de vue on peut diviser les tendances cn trois catégories, celles qui ont pour résultat ou pour lont, (si l'on veut entendre par but le résultat vers lequel convergent tous les éléments diun systeme lsycho-organique,) la vio ou le bien-être de l'individı, celles qui ont pour hut la vie et le bien-ètre de la socicté on de l'espèce, celles qui ont pour but la systématisation idéale ou réclle de l'univer's en général. Dans chaque catégoric, le caractère de finalité est bien marqué, el assec evident par lıi-même. 
Parmi les tendances qui ont pour but la vie et le bienètre de l'individu, il faut signaler, outre li plupart des tendances organiques, tous les sentiments qui s'y rapportent et qui ont pour objet de les faroriser, de leur permettre de s'exercer dans les meilleures conditions possibles - ainsi, à certains égards, l'amour du gain, l'amour du luxe, ou au moins du confort, la gourmandise, le besoin de remuer, de marcher, d'exerecr ses muscles, etc. Dans la seconde classe nous rangerons l'instinct sexuel qui est sourent ramené à la première par les calculs de l'individu, et toutes ses dépendances: l'amour en général, les affections de la famille; l'amitié, la sympathie en général, l'ambition quand elle a un but élevé, etc.; dans la truisieme l'instinct moral, é qu'on a appelé le sens du divin, l'amour de Dieu compris comme un législateur de l'univers, ou de tout ce qui peut remplacer le Dieu personnel des déistes, la formule suprême d'où tout se déduit, l'harmonie universelle, la substance des panthéistes, etc., l'amour du beau, l'instinct esthétique, à de certains ègards et dans de certaines conditions.

Je n'ai pas la prétention d'énumérer ici toutes les tendances possibles, il me suffit de faire remarquer que chacune d'elles consiste en une association très vaste; comprenant comme éléments un grand nombre de phénomènes intellectuels et de tendances secondaires, coordonnés vers une fin unique ou vers des fins harmoniques. La gourmandise, pour prendre une des moins élevées, implique comme principal élément un goùt fin et exercé qui est pour ainsi dire le centre du systeme. Pour arriver a produire les impressions gustatives; qui ne devraient être qu'un moyen de contrôler la qualité nutritive des aliments mais qui ont donné lieu à des développements qui ont souvent fait perdre de vue leur vraic fonction, et qui sont le centre vers qui tout converge, il faut travailler pour gagner l'argent qui permettra d'acheter des virres 
choisis avec art, dresser avec soin la personne chargée de les apprêter, lui donner des instructions, la surveiller au besoin, et pour cela avoir fait un certain apprentissage, faire de l'exercice ou prendre des précautions pour entretenir l'appétit, se metre dans les conditions les plus farorables pour apprécier un bon morceau, au point de vue de l'éclairage, de la température, de la compagnie, etc.; on peut roir dans Brillat-Savarin les conditions multiples requises pour être un vrai gourmand, conditions qui tendent toutes vers un même but : la sensation du goùt ou la séric de sensations de goùt saramment harmonisées qui se produisent à un moment donné, - et je néglige toutes les petites tendances qui se combinent et se répetent pour l'accomplisscment d'un grand nombre d'actes tels que déplier sa serviette, prendre sa fourchette, découper, soulever son verre, ete. Sans doute on peut être gourmand à noins de peine, et le système d'idées, d'images, d'actes, de perceptions sera alors moins vaste, mais il yen aura toujours un et il aura essentiellement le mème caractìre, l'association des éléments vers un but, c'est-it-dire la finalité au sens positif du mot. Et si nous prenions la plus petite des tendances rui s'associent pour former une tendance psychique, par exemple, l'acte de remuer le doigt, si nous considérions l'idèe la plus simple de toutes relles qui se produisent à l'occasion de la sensation gustative, nous trouverions encore, comme nous. l'avons vu, cette tendance et cette idée composées ellesmêmes d'éléments coordonnès, capalıles sans doute de se subdiviser à leur tour. Que serait-ce si nous jrenions une des tendances superieures de l'homme, l'anour de la perfection, par exemple, tel qu'il peut se manifester dans un esprit four qui la perfection n'est pas 111 mot respectable et vidr, on l'amour de la science; qui pourrait dirce ce qu'un tel sontiment suppose d'actes, de sentiments secondaires, d'idees et l'experiences tendant tous vers ce mème but: 
la recherche et la connaissance de l'ordre naturel des pliénomènes?

Si au lieu d'étudier les tendances à un point de vue plutòt objectif, nous étudions, par le sens intine, leur's manifestations affectives, nous arrivons à des conclusions analogues. Nous voyons, par exemple, que le plaisir correspond à un accroissement de la systématisation de l'organisme ou d'un des éléments qui le composent, la douleur à une diminution de cette systématisation, quand cet accroissement ou cette diminution atteignent un degré suffisant d'intensité et de rapidité, le désir correspond à une tendance sentie qui ne peut ètre satisfaite et qui tend a nous faire accomplir les mouvements nécessaires pour lui procurer les éléments qui lui manquent, l'espérance accompagne une tendance satisfaite faiblement par des images, elle diffère du désir en ce que dans le désir la tendance est plus impérieuse, néanmoins le désir s'accompagne souvent d'espérance et l'espérance de désir. Mais il est inutile de s'arrêter plus longtemps à ces détails qui dépendent des faits généraux que nous avons examinés.

On pourrait objecter que je n'ai guère considéré que les tendances qui ont pour but un accroissement de systématisation et que la finalité de celles-là n'a rien de douteux, mais qu'il en est d'autres, la haine, la crainte, etc., qui paraissent présenter plutôt un caractère opposé. L'objection s'évanouil, je crois, si l'on y regarde de près. A considérer la tendance en elle-mème, son but fut-il l'anéantissement de l'univers, elle se présente toujours elle-même comme un composé d'éléments qui tendent vers une fin unique ou vers des fins harmoniques, en ce sens la loi de la finalité s'applique à toutes les tendances, de plus cette tendance est généralement en harmonie avec d'autres tendances de la même personne, et ceci est encore une application de notre loi. Si, par exemple, on veut renverser un édifice, c'est qu'il gêne la circulation, 
c'est qu'il empèche l'air et la lumière de se répandre abondamment, si l'on veut détruire un ordre social quelconque, c'est qu'on le tronve maurais, c'est qu'il froisse des aspirations, qu'il comprime des énergies qu'il faudrait, pense-t-on, laisser libres de se développer, - àmoins que ce ne soit pour des raisons inverses, - mais dans tous ces cas, il est bien évident que le but quon poursuit est en harmonie avec certaincs ides et certains sentiments. Il n'est pas jusqu'an suicide qui ne témoigne de la finalité psychique, la suppression dn mal étant équiralente, i certains égards, à l'acquisition d'un bien.

\section{2.}

Si, au lieu de prendre les tendanees toutes formées, nous examinons la facon dont elles naissent, dont elles s'organisent et se déreloppent, et l'influence qu'elles cxerceut sur les autres phénomènes psychiques, nous sommes de plus en plus amenés à reconnaître l'importance de la loi d'association systématique. Il est manifeste que nos tendances cherchent à s'organiser, à former des systèmes de plus en plus complexes et ensuite d'autres systèmes généraux qui englobent, relient et harmonisent les premiers. Quand une nourelle tendance se forme, quand un nouveau sentiment prend naissance, il se combine au moins avec quelques tendances secondaires et aussi, en général, aree quelques tendances supérierres. Peu à peu, les diverses circonstances de la vic mettent cette nouvelle tendance en ripport arec celles qui existaient arant elle, et arec celles qui ont pris naissance el se sont déreloppées aprés elle. Des associations et aussi des arrêts se produisent peu à peu et la tendance se tronve comprendre un plus grand nomlse d'éléments et tote astocice a un plus grand nombre de faits organiques ou pircho-organiques. Elle entre dans des systemes de plus en plus complexes, it moins, Jien entendu, que la 
lutte ne lui soit défarorable et qu'elle ne soit éliminée. Si l'organisme arrivait à la perfection, si la personnalite devenait une, la tendance serait completement harmonisée avec toutes les autres. Mais ce fait ne se produit pas, lc travail incessant des phénomènes psychiques qui s'arrangent en systèmes et des systèmes qui se coordonnent en systèmes plus rastes, est toujours entravé par les circonstances complexes de la vie, avant d'être détruit par la mort, ou changé en travail inverse de dissolution, par quẻlque crise ou quelque maladie. L'organisation du caractère n'est jamais complète.

Nous pourons encore ici étudier d'abord l'action du sentiment qui s'établit, ensuite la part des tendances déjà organisées dans la synthèse.psychique. Nous arons chaque jour des prcuves nombreuses du caractère envahissant des tendances qui commencent à entrer en activité, surtout si la cause qui les a produites continue à se faire sentir. Il est vrai qu'il s'agit ici des tendances organisécs et subsistant toujours à l'état latent, c'est-à-dire probablement sous forme de modifications imperceptibles des centres nerveux. Mais la manière dont une tendance passe de l'état latent à l'état d'activité, est assimilable, pour ce qui nous intéresse à présent, à la manière dont une tendance s'établit pour la première fois dans l'esprit. Quand un besoin organique, la faim, la soif, le besoin de respirer commence à se faire sentir, il se manifeste d'abord par une impulsion faible, à peine remarquée, si le besoin n'est pas satisfait l'impulsion persiste, elle détermine des séries de sentiments et d'idées, nous commencons à sentir la faim, la soif, la suffocation; certains jugements, certaines idées s'éveillent en nous, certaines phrases se formulent : il faut partir, il est l'heure de diner, on étouffe ici, etc., en mème temps les autres préoccupations s'effacent et ne reparaissent plus que par intermittence. Et toujours le besoin persiste, - je laisse 
iéi de côté, bien entendu, le cas où c'est la tendance naissante yui est enrayée - il devient de plus en plus imperieux, on passe de la gêne respiratoire à la suflocation, de lappetit a la faim, du désir de boire à la soif, les idées se pressent et naisseut en abondance, toutes orientées dans le mème sens, dirigées ver's une seule fin, l'intelligence n'est plus occupée que de trouver les moyens de satisfaire la tendance éreillée, et, avec des intermittences, le systeme formé devient de plus en plus complexe, de plus en plus envahissant, jusqu'a ce qu'enfin il puisse se compléter par les actes appropriés et que la tendance soit satisfaite.

Prencz une passion quelconque, l'amour, la liaine, l'ambition, vous trouverez toujours que son itablissement est marqué par une orientation nouvelle de l'esprit; dès qu'un nouveau but s'impose à notre actirité, les objets nous apparaissent sous un jour nouveau, nous les voyons autrement, des choses qui nou's amraient laissés tout a fait indifférents ou que nous n'aurions même pas aperçues nous frappent dès que nous voyons la possibilité d'en tirel parti pour la nouvelle tendance, dès qu'elles peuvent s'associer harmoniquement avec la nouvelle coordination psychique qui s'est établie. Nos anciens sentiments euxmèmes se modifient plus ou moins pour s'adapter à nos nouveaux désirs. Nous aimons ce que nous n'aimions pas, et ce qui nous plaisait nous déplaît; l'amour, l'ambition peuvent donner du courage a qui n'en avait pas; le goùt du travail peut venir au paresseux le plus determiné s'il a une fois trouvé le genre d'activité qui convient à sa nature, et les études qui lui déplaisaient autrefois, peuvent lui devenir agréables une fois qu'il entrevoit leurs rapports arec ce qui l'intéresse, etc. Les faits abondent et chacun peut les observer.

11 en est un sur lequel il faut insister, car il montre parfaitement l'effet de trouble et d'harmonie aussi qui 
r'sulte de la formation d'une nouvelle tendance; ce sont les changements psychiques qui s'accomplissent à la puberté. La puberté est essenticllement constituée par ce fait que les organes de la génération deviennent aptes à fonctionuer normalement. Ln nouveau fait s'impose a l'organisme, une nouvelle tendance se constitue. Aussitôt l'ètre tout entier se modifte d'une maniere harmonique, logique, si l'on peut le dire. An point de vue organique, les organes sexuels secondaires se caractérisent; che\% l'honme la voix mue, le corps grandit, se forme, prend un aspect plus màle; chez la femme les seins se développent, to bassin s'élargit, etc. Au point de vue physique tous les sentiments qui peuvent servir a satisfaire dans les meilleures conditions la nouvelle tendance naissent ou se fortifieıt: le courage, le désir de plaire, la corquetterie, tous ces sentiments normaux ou déviés, dont le but commun, quoique quelquefois invisible, est de préparer l'acte qui doit donner naissance à un nouvel ètre. Que l'on essaye d'analyser-les idées, les sentiments divers, les séries secondaires d'actes qui se produisent quand l'homme est sous l'empire de la passion amoureuse, que l'on remarque comment presque tout dans l'esprit, mème les choses autrefois les plus indifférentes, prend un aspect particulier et se trouve rapporté par quelque point à la tendance dominante, on sera émerveillé de voir comment une tendance organique a pu, pour un moment, s'associer systématiquement l'organisme toul entier et donner une unité de fin à un grand nombre d'éléments autrefois divisés $\mathbf{1}$

1. Toyez en particulier l'admirable Essai sur l'amou', de Schopen. hauer. Nul n'a vu aussi profondément dans celte question, même ou surtout Stendhal, qui arec toute sa finesse est resté assez superticiel. On trouvera un bon choix de faits intéressants dans $L / 4$ Physiologie des passions, de M. Letouroeau, au chapitre des passions affectires. Au reste, je reprendrai tout a l'heure plus en dètail cet exemple de tendance. (Voir partie III, livre 1.) 
A propos de l'influence exercée par les tendances habituelles nous avous it constater des fatits absolument analogues à ceux que nous avons remarqués en traitant des sensations, des perceptions et des idées. La perception est le résultat d'une habitude des centres sensoriels, le sens comnun est le résultat d'une habitule des centres intellectucls, les habitudes des centres moteurs produisent des effets exactement semblables. De même que toute excitation qui porte sur le nerf optique produit une sensation lumincuse, de même toute excitation qui parviendra à un complexus fortement organisé d'éléments motenrs organico-psychiqnes, produira un acte toujours le même. Pour peu qu'une tendance souvent exercée puisse s'assimiler quelques éléments d'une perception, ces éléments suffisent à la mettre en jeu. Nous pouvons remarquer ce fait pour tous les modes de l'activite, depuis les plus simples jusqu'aux plus compliqués.

Cette propriété qu'ont les tendances de s'associer les phénomènes qui naissent, est très visible à l'état normal, elle se manifeste par la manière différente dont chacun réagit en préscnce d'un même évènement. Nous avons eu ¿ signaler déja les différences qui séparent les idées que diverses personues se font d'une mème chose. Il suffit de rappeler ici que ces idées accompagnent génẻralement des tendances motrices et des sentiments variés. La vue d'un moulin n'éveillera pas les mèmes tendances chez un meunier, chez un propriétaire qui a de la farine à moudre et chez un peintre. Ce ne sont pas des idées mais aussi des tendances actives qui seront mises en activité, au moins dans certaines circonstances, et si nous supposons Ine rhacun d'eux cherche un emploi de son activité spéciale ou la satisfaction de ses désirs personnels. Une miladio rare éveillera des sentiments trés variés dans l'erjurit du jatient, dans celui de ses parents, de ses amis, de ses ennemis, d'un medecin qui voit avant tout dans sa 
profession l'occasion de soulager les malades, d'un médecin plutôt passionné pour la science que pour son art, on d'un ambitieux heureux de trouver l'occasion de se faire connaitre, chacun réagira et agira selon sa nature propre, et les systèmes de mouvements et d'actes qui trouveront ¿ se compléter d'une manière différente à propos des perceptions données par un mème fait, peuvent être très variés et très nombreux.

La force associatrice de la tendance organisée est peutêtre encore plus visible, elle aussi, quand elle s'exerce a contre-temps. L'observation ordinaire et la pathologie nous permettent souvent de le constater. Par exemple, chacun de nous a seslocutions favorites, il les place souvent a propos, souvent aussi hors de propos, la moindre ressemhlance entre les circonstances qui doivent les amener et des circonstances quelconques, suffit pour mettre en activité les centres moteurs, et logiquement les organes de la parole, de la manière habituelle. Les enfants, pour qui manger est l'affaire la plus importante de la vie, sont très disposés à porter à leur bouche tous les objets qu'on leur donne; les éléments qui dans la perception de ces objets ressemblent à ceux qui se trouvent dans les perceptions éveillées par les aliments qu'on leur a donnés: suffisent pour exciter la tendance dominante qui s'associe systématiquement les éléments qui peuvent s'adapter à elle et néglige le reste. On voit aussi d'autres habitudes se former et déterminer des actes de la mème manière. Un enfant de huit mois a parmi ses jouets favoris une boite en fer blanc qu'il aime surtout à cause de son ouverture dans laquelle il met tout ce qui peut $y$ entrer. Cette habitude de mettre un objet dans un autre lui tient au cœur. Dès qu'on lui donne un objet qu'il ne connait pas, il y cherche quelque apparence douverture. "Une fois, on lui donna un bouchon de carafe que, surtout à cause de sa transparence vitrée, il s'obstina à croire 
ouvert par le bout eỵlindrique. Il chercha à faire entrer par là les jambes d'un petit pantiu, ensuite un berceau de poupée, et enfin, d'un air dépité, le bout de son index »1. A l'état pathologique on retrouve des faits analogues. a J'ai eu dans mon service, dit M. Luys, une malade, jeune encore, qui jendant longtemps arait été attachée a la Salpètrière comme fille de lingerie, pour plier lẹs linges et rouler les bandes. Dans les dernières années de sa vie, cette femme étant devenue complètement aveugle et paralytique, présentait les phénomènes suivants : étant dans le decubitus dorsal, venait-on à mettre entre ses doigts une bande non roulée, un bout de corde même, immédiatement ce contact éveillait en elle d'anciens souvenirs : clle se mettait a opérer des mouvements de roulement avec ses mains, automatiquement, sans savoir ce qu'elle faisait, comme si c'eùt été un appareil d'engrenage mécanique "2. On connait aussi l'histoire du militaire qui, dans certaines crises somnambuliques survenues à la suite d'une blessure à la tête, se mettait en position comme s'il allait assister à une scène de combat quand on lui plaçait une canne dans les mains. Evidemment les habitudes subsistent à l'état latent, et, dès qu'une perception se présente, elle agit par ses analogies avec les autres perceptions, elle se syntlétise avec les traces laissées par l'habitude; ce qui dans une canne ressemble à un fusil, ce.qui dans une corde ressemble a une bande de linge, suffit your compléter le systène psychirque et mettre en activité la tendance. C'est une application de la loi de fusion de M. Binet, combincée avec la loi d'association systématique.

Il y a une véritable interprélation motrice et sensitive des donnces de la perception, comme il y a une

1. I3. Pérez, Les trois premières annees de l'enfance, (1're édition), 1. 172-173.

2. Luys, Le cerveau el ses fonctions. 
interprétition intellectuelle el une interprétation sensible. Les systemes d'habitudes qui constituent nos organismes sont pareils à une corde tendue et prête à vibuer au moindre clioe en donnant sa note propre, ils attendent toujours un ébranlement venu soit du dehors, soit des organes, soit d'une autre partie du cerveau; ils se completent dès qu'ils trouvent des éléments capables de s'harmoniser avec eux, ils trient ces éléments, décomposant les perceptions, les idées qui naissent dans l'esprit, les sentiments mème, pour s'assimiler tout ce qui pourra s'unir avec eux. Le fait, dejà indiqué, que la musique éveille des systemes d'idées et de sentiments très différents selon les différentes organisations, est à rappeler ici, car ces systẻmes d'idées correspondent à des tenlances puissantes et aboutissant à des actes. Les idécs de M. Legouvé aboutissaicnt à la production de pièces de thétre, celles de Darwin à la production d'ouvrages scientifiques. Mais les effets sont de toute autre nature quand la tendance dominante est d'un ordre inférieur. M. Moreau de Tours cite, d'après Louyer-Villermay; une nymphomane chez qui une statue, un tableau, la vue d'un homme, le contact le plus simple, un mot, suffisait pour exciter des désirs violents ${ }^{1}$. Marc raconte qu'une paysanne, adonnée a la masturbation, fut apportée à l'hôpital SaintLouis, où « dans le dẻlire le plus effréné, elle offrait le scandale perpétuel d'une sorte de mouvement automatique qu'elle n'étail pas maitresse de réprimer, malgré les violents reproches qu'on lui adressait..., ce qui causa surtout notre surprise, c'est (pue les forces sensitives s'étaient éveillées, et en quelque sorte concentrées dans l'intérieur de l'organe utérin, au point que la vue seule d'un homme suffisait pour déterminer en elle un spasme voluptueux des parties de lit gènération : loutes les impressions

1. Moreau de Tours, Les aberrations du sens genésique, p. 221. 
qu'elle éprourait, renaient retentir dans ses organes : la main de toute personne yui n'était pas de son sexe, poséc dans la sienne, elle en avait la sensation dans le ragin. Plus lard, la vue les élèves qui l'entomraient, même la seule exploration de son pouls, suffisait pour produire le spasme voluptueux "1. Au point de vue psychologique, ce cas est un des plus beaux exemples qu'il y ait de l'interprétation des données de la pereeption par une tendance organisée.

En somme, tous ces cas d'interprétation se raménent ¿ lit mème forme : un système rlominant choisit, dans les cxcitations venues du dehors ou des organes, les éléments 'jui peuvent s'adapter à clle, la force nerveuse dégagée par l'excitation s'emploie surtout à susciter un système coordonné, déterminé, pour une part considérable, par la nature de l'organisation mentale à qui parvient l'excilation. L'état nerveux ainsi produit, et l'état de conscience gui l'accompagne - que ce soit un phénomène intellectuel, un phénomène affectif on une volition sont des synthèses systématiques d'états antérieurs de l'esprit et des éléments nouveaux qui lui arrivent.

En résumé, les phénomènes affectifs et les tendances comme les phénomènes précédemment examinés, nous ont paru manifester à un double point de vue la loi rle systimatisation. Considéres conme rles phénomènes douné, ils consistent en un systime d'eléments condonnes

1. Mare. De la folie, ete. Vol. II, p. 210. Marc cite le fait d'aprés Bayard qui l'emprunte a Alibert. On trouvera, chez les aliénistes, d'autres faits intéressants de même nature. Voir en particulier, le mémoire du I) IIenri Bayarl : Examen médical de cclle question : la nymphomanie peret-clle etre une cause d'interdiction, ore les faits qui tendraient "rrablir sunt-ils non pertirents? dans les Annales d'Hygiene publique et de medecine légale. Tome XV1II, 1. 416 et suiv., et surtout inux [rages 422, 433 . Voir anssi Moreau de Tours, ouv. cilé, I.egrand du Siulll", Les Irystriquques (appendice sur les Nymphomunes), le médio: cre traité do Biéville: le Nymphomanic, Butlon, La l'uberté. etc. 
vers une fin unique - considérés au point de vue de leur genèse et de leur activité, ils nous montrent i la fois leur activité systématique propre et l'activité systématique des autres éléments psychiques et de l'esprit en général, qui acceptent, interprètent, repoussent et décomposent les nouveaux éléments, tandis que ceux-ci exercent sur les habiturles aequises des actions analogues de manière i former de nouvelles synthèses.

Les sentiments mème qui semblent résulter non d'un accord, mais d'une discordance ne sont pas en dehors de la loi, et nous en avons eu des exemples. Ce sont les sentiments pénibles qui, d'une manière générale, paraissent offrir le plus de diffieulté à s'y laisser ramener; ils y rentrent cependant. D'après la loi d'association systèmatique tout phénomène psychique est un composé coordonné d'éléments plus simples, et tout fait psychique tend i susciter les phénomènes susceptibles de s'unir avec lui en un système plus complexe. Or il est évident, d'un côté, qu'une douleur est le symptôme de plusieurs faits psychiques concourant au même résultat, lorsque ces faits sont en désaccord avec des tendances importantes; d'autre part que la douleur entre bien comme élément daus les systèmes psychiques qui tendent à nous en préserver; la douleur que cause le feu détermine le retrait du doigt qui s'en est trop approché. Si le second fait n'est pas absolument général et montre surtout la fiualité grénèrale de l'esprit, il n'en est pas de mème du premier. Mais pour bien comprendre les phénomènes de la douleur et la façon dont ils lentrent dans notre loi, il faut tenir compte de la pluralité réelle de certains états de conscience qui paraissent, a première rue, ne former qu'un tout, - on peut prondre pour exemple les impressions diverses et opposées que nous donne parfois la mème personne ou le mème événement. Dans ce cas l'association systematique est moindre, clle cxiste eependant 
a quelque degré, car les plínomines, si peu coordonnés qu'ils soient, offrent encore quelque unité en tant que leur ensemble agit de manière à imprimer à notre conduite une certaine unité de direction, mais cette unité est due surtout a la partie de l'esprit qui les reçoit el les interprète. En eux-mêmes la pluralité et la division existent à quelque degré, et toujours le caractìre d'unité des phénomènes est en raison de la coordination de leurs éléments ${ }^{1}$.

Quand a la forme particulière d'association que reprèsentent les tendances et les phénomènes affectifs; nous la présenterons ainsi : les tendances sont des syntheses d'eléments psycho-organiques de nature diverse, parfois inconscients, parfois accompagnés de conscience, qui, lorsqu'elles se complètent par les éléments voulus, aboutissent à des actes coordonnés. La fin essentielle de ces actes, le but qui détermine le développement de la série des phénomènes coordonnés, qui lui donne une forme et une signification est, soit une satisfaction organique, soit une satisfaction psychique, soit une satisfaction sociale, soit une satisfaction religieuse, esthétique ou morale, c'est-it-dire l'accroissement de la systématisation de l'or'granisme, de l'esprit ou du monde représenté dans l'esprit par des idees et des sentiments alsstraits. Quant aux phénomènes affectifs perçus par le sens intime : emotions, sentiments, impressions diverses, etc. ; ils sont simplement des signes de l'activité des tendances daus le cas où leur organisation, leur satisfaction est encore imparfaite.

1. Se rappeler, à cet égard, ce qui a été dit de la funalité comme propriété des éléments psychiques. J'aj traité plus longuement la question dans la troisième partie de mes Phénomenes affectifs. Voir, pour un cas particulier, mon article sur l'Amou' du Mal, Revue philosophique, 1887, Tume I. 
CHAPITRE IV.

\section{Le pouvoir personnel. Conscience, Attention, Volonté.}

Il convient peut-itre d'examiner a part un mode particulier des phénoménes, celui yui a èté longtemps considéré conme formant a lui seul presque toute la psychologie je veux parler de l'attention et de la volonté. auxquelles on peut joindre la conscience rélléchie. On a souvent reproche aux psychologues qui, de diverses manieres, se rattachent à l'ecole expérimentale, d'aroir nègligé toute une partie de l'esprit, — et la principale - d'aroir ru l'esprit " du delorss " comme on l'a dit avec une métaphore qui

- manque peut-ètre de clarté ; de naroir tenu compte que des opérations inférieures: de l'activité pour ainsi dire inconsciente, et je ne roudrais pas dire que l'on ait toujours eu tort. Il n'est pas a nier que les lormes psychiques supérieures n’aient été longtemps négligées par les psychologues qui tichaient de séparer la psychologie de la neetaphysique. Lorsipu'on s'en occupait, c'était pour les étudier dans leurs formes inférieures ou norbides, ou bien encore en tant qu'on jouvait y retrouver les faits mieux étudiés appartenant aux degrés moins élevés de la mentalité. Et il n'y avait rien lá que de légitime puisque les formes inférieures étant les moins complexes pouvaient logiquement être étudiées arant les autres qui dépendent d'elles en un sens et ru'elles aident it comprendre. Mais bien que depuis quelque temps l'etule des fonctions supérieures ait quelque peu repris faveur je crois que la question de ce Iue lid philosophie spiritualiste appelait 
"le pouvoir personnel " n'a guère été abordée qu'incidenment, sauf a propos de l'attention volontaire, et plutòt pour montrer que la question n'avait pas l'importance qui lui avait eté attribuée que pour l'examiner à fond en elle-mème. Comme cette question se pose forcement ici, nous devons l'étudier - assez briévement - et tout d'abord, essayer de la poser d'une façon précise. Si le pouvoir personnel n'est pas le fait essentiel en psychologie, si mème il n'est pas d'une importance telle qu'une bonne partic de la psychologie et toute la psychologie abstraite peut-être ne puisse ètre constituéc sans y avoir égard, cependant comme il nous offre une forme assez particulière des phénoménes, comme cette forme marque un moment de la formation de l'esprit ainsi que la manière dont cette formation s'accomplit, il nous intéresse a ce titre, et il n'est pas inutile de rechercher si et comment il peut se ramener à notre loi générale.

Jouffroy a défini avec netteté et en l'exagérant le pouvoir personnel. "Ce qui distingue une chose d'une autre, a-t-il dit, e'est qu'elle a des propriètés on des capacités naturelles différentes : l'homme ayant des capacités spéciales, est, à ce titre, comme toutes les choses possibles, un être diune espèce particulière, et qui mérite un nom particulier. Mais, indépendamment de cette spécialité de nature qui lui est commune avec toutes les choses du monde, car toutes les choses du monde ont leur nature speciale, il jouit d'un privilege tout particulier, et qui le sort de la foule, cest celui de pouroir disposer de ses capacités naturelles. Il a non seulement des capacités spéciales comme chaque chose en a, et par exemple, celles de penser, de se sourenir, de se mouroir, mais, de plus, il goureme ses capacités, rest-i-dire qu’il les tient daus sa nain et sien sert comme il veut. Ainsi, il se meut comme il reut, il dirige sa mémoire, il applique sa pensée où il ve1t : Pll um mot, il est maitre de lui et des capacités qui 
sont en lui. Or il n'en est pis ainsi dans les choses; elles ont aussi des capacités naturelles, mais il n'y a point en elles de pouvoir autonome (jui s'approprie ces capacités et qui les gouverne. Ainsi l'arbre a beaucoup de capacités naturelles, mais elles se développent en lui sans sa participation; ce n'est pas lui qui les dirige, c'est la nature; elles existent en lui, elles opèrent en lui, mais elles ne lui appartiennent pas, et ce qu'elles produisent ne saurait lui être attribué.

a Le pouroir que l'homme a de s'emparer de ses capacités naturelles et de les diriger, fait de lui une personne; et c'est parce que les choses n'exercent pas ce pouvoir en elles-mèmes: qu'elles ne sont que des choses.....”

- De l'existence du pouvoir personnel dans l'homme et de son absence dans les choses, résulte une différence entre les capacités naturelles de l'homme et celles des choses. En effet, nous régnons sur nos capacités naturelles, et nous nous en servons, tandis que les choses ne disposent pas des leur's et ne s'en servent pas.... La capacité de marcher ne serait en nous qu'une simple propriété conme celle de sécréter la bile, si nous n'avions le pouvoir de marcher ou de ne pas marcher, de marcher vite ou lentement, a gauche ou a droite selon notre volonté. Mais comme nous gouvernons cette capacité naturelle, clle devient en nous une faculté. Telle est la véritable force de ce mot. Si done, pour le dire en passant, nous u'étions, comme le prétendent quelques physiologistes et mème quelques philosophes, qu'une espèce d'alambic, où les idées, les images, les souvenirs, les déterminations et les actes se distillent sous l'influence d'une excitation extérieure, il faudrait commencer par réformer la langue qui consacrelait de vaines distinctions entre des choses identiques....."

Il y aurait beaucoup a critiquer ou à interpréter dans la description de Joufloy, on ne peut nier qu'elle 
se rapporte it un fait récl, et qu'elle en domne une idéc exacte. Il est vai qu'il y a une différence entre voir et legarder, entre réflèchir et rèver, entre l'automatisme et la volonté : quelle est la nature de celte différence?

Prenons l'exemple mème indiqué par Joufroy, et analysons-le : je puis matther plus on moins vite. Il arrive que je marelie plus ou moins vite sans m'en rendre compte. sans que la volonté intervienne, parce que l'air est plus vif, parce que le temps est lourd, mais aussi je fuis prendre me allure déterminee apress me délibératton raisonnée. Je me dispose a prentre le train, je suis a deux lilomitres de la gare, je dois aller a pier, et jai ringt minutes devant moi, je puis hesiter pour savoir si je dois me retourner ou continuer ma route, et si je prends ce dernier parti, je sais qu'il me faut aller au pas de six lilometres à l'heure environ, je connais a peu pres les impressions musculaires el autres que fail naitre cette allure on une allure plus rapide, et je les suscile volontairement. Nous arons ici un exemple de lactivité du pouvoir personnel qui peut servir de type. En quoi consiste alu juste celle aclivité?

Elle consiste en ceci que le noureau fait psychique yui tend a s'établir a été mis successivement en relation avec un grand nombre de tendances et que ces tendances. soit séparément, soit réunies, ont essayé de se l'assimiler, l'ont eprouve pour voir s'il pouvait entrer' comme élément dans un systeme coordonné dont elles feraient partic. Lallure une je devais jurendre a pu ètre refresentée conme devant amener une certane faligue, l'mn autre côté l'intérèt que je fuis avoir a ne pas retalrder datvantage non voyage, et par snite mon arrivere a lii se présenter at l'esprit el servir a applécier lá valen te la considération frécédente, mes dispositions fertonnelles a ce moment-lia, l'état de fatigue on de repos de nes nutscles, la temperature, la qualite de la route, 
tout cela a pu être évalué; d'un autre còté, lidée des personnes qui m'attendent a lì se présenter aussi. Une grande quantité d'éléments psychiques sont ainsi mis en activité, ils doivent s'harmoniser, se coordonner d'une certaine facon de manière à produire soil l'arrêt ou le retour, soit la marche en avant. Il y a une sorte d'essai le divers systèmes psychiques, chacun tendant a s'imposer, jusqu'à ce que tous se soient completement, ou à peu près, réunis pour déterminer une manière d'ètre définitive. Le pouvoir personnel consiste ici dans cet essai fait par un certain nombre de tendances, d'un nouveau phénomène qui tend à s'établir dans l'esprit. Il s'oppose à l'automatisme en ce que celui-ci se produit quand il n'y a pas éveil de sentiments et d'idées complexes, quand, par exemple, une personne craint d'ètre en retard et se met à courir sans que l'idée des inconvénients possibles de la course, - si elle a une santé générale délicate, oı une maladie organique particulière - se présente à elle, sans qu'il s'établisse une sorte d'épreuve de la tendance mise en activité par les autres tendances de l'organisme et la forme même de leur association.

Réfléchir - qu'il s'agisse d'un acte à accomplir, ou d'une croyance á adopter, - c'est éprouver la résistance du nouveau phénomène suscité dans l'esprit en le mettant en rapport successivement avec un certain nombre de tendances, de systèmes psychiques établis dẻjả dans l'esprit, ou bien encore, prévoir les rapports de ce phénomène arec les systèmes psychiques, perceptions et idées que l'expérience déterminera un jour. Il n'y a pas lá de difficulté sérieuse. On dit, je suppose, que des ossements humains ont été trouvés dans le terrain jurassique, un homme n'a besoin que de posséder des notions élémentaires de géologie pour rejeter immédiatement la réalité de ce fait, qui ne peut se systématiser nullement avec ses idées acquises, en tant au moins qu'il tendrait ì 
signifier que les ossements lnunans sont antérienrs a la formation des couches jurassiques par des dépôts marins. Mais si l'on annonce, par exemple, que des silex ayant une certaine forme ont été trouves dans des terrains plincènes, heaucoup de réflexions pourront ètre suscitées et le pouroir personnel des archéologues et des géologues tronvera i s'exercer. Quelques-uns d'abord pourront mettre les interprétatious possibles de ce fait à l'épreuve de leurs croyances scientifiques ou religienses. S'ils considèrent que l'existence de l'homme à l'époque miocène soit inconpatible avec elles, el si ces croyances sont trop profondément enracinées, trop fortement organisées pour être détruites ou modifiées, la nouvelle tendance ne pourra s'adapter, la réflexion lui sera funeste, il faudri. rechercher une autre interprétation plus acceptable, c'està-dire chercher un aulre système d'idées qui puisse se syslématiser avec les perceptions ou les idées nouvelles (silex ayant une forme particulière et trouvés dans telles circonstances données), et trouver en même temps des idées acquises, des syslèmes psychiques déjà formés el consolidés qui puissent s'assimiler le nouvel élément. Par exemple, on dira que la forme des silex est due à des causes mécaniques, au frottenent, à ce qu'ils ont été frappés de la foudre, ou exposés ì la thaleur du soleil, etc. En même temps les savants consciencieux éprouveront ces diverses interprétations possibles, non seulement it l'aide des idées deja acquises, mais a l'aide de l'expérience, cest-a-dire des systinatisations psyeliques produites par les perceptions. Ils comparent les silex nouvellement trouvés à des silex que le travail hmmain a, d'une manière ou d'une autre, inclubitablement façonnes, ils rechercheront, en examinant le lieu de la découverte, si les silex sont bien contemporains de la conche géologique dans laquelle on les retrouve, etc., etc. 'Toutes ces manilestations du pouvoir jersonnel, et l'on pourrait 
en multiplier les exemples, sont essentiellement des épreuves faites d'un état nouveau par les divers systèmes qui constituent l'organisation primitive et dues aux obstacles que lui opposent certaines tendances dejjà formées. C'est par là que les actes réfléchis et voulus, les croyances sérieuses et raisonnées se distinguent des actes réflexes et des opinions adoplées à la légère - ces dernier's phénomènes, en effet, sont caractérisés par ce fait que leur présence n'éveille nullement un grand nombre de tendances, de systemes psychiques, ils s'associent sans lutte à un système bien préparé pour les recevoir. Un homme trop convaincu de la vérité d'une théorie sera exposé à accepter sans vérification suffisante, sans épreuve décisive, sans réflexion, les faits qui la confirment, à rejeter ceux qui ne peuvent s'accorder avec elle. Il n'y a plus ici une sẻrie de systèmes s'éveillant successivement et éprouvant l'associabilité d'un nouvel élément, une seule tendance s’éveille et s'assimile le fait qui vient de se produire. De même si une mouche se pose sur mon visage, je ne me mettrais pas à réfléchir, a peser le pour et le contre, pour savoir ce que je dois faire, je la chasserai machinalement d'un geste de la main ; sans éveiller d'autres tendances, la sensation produite a déterminé la production des mouvements appropriés.

Nous n'avons pas toutefois expliquẻ suffisamment encore le pouvoir personnel, n'ayant pas montré en quoi il mérite particuliẻrement ce nom de personnel. Mais ceci non plus ne parait pas devoir être une difficulté insurmontable.

Bicn que nous ayons à cxaminer dans le prochain chapitre les rapports de la loi d'association systématique avec la constitution de la personnalité, nous pouvons dire ici déjà que la personnalité consiste essentiellement dans l'ensemble des tendances réunies et associées d'après 
quelques principes généraux, c'est un système plus ou moins bien coordonne de tendances, c'est-à-dire de phénomènes psychiques de tonte sorte. Or, il est assez naturel que l'on appelle " piersonnel " un acte, une croyance on la personne entière est intéressée, oì la personne entière a pris part - c'est-ì-dire un acte ou une croyance qui a été éprouvée par toutes les tendances qui entrent dans la personnalité ou au moins par le plus grand nomJre et les plus importantes d'entre elles. Il est quelquefois des actes, des théories qui semblent d'abord s'imposer a nous: qui s'adaptent merveilleusement a certaines parties de notre personnalité et les éveillent rapidement, risquant, si d'autres tendances n'interviennent pas, de faire adopter des idées fausses, de faire accomplir des actes nuisibles ou coupables. En ce sens le premier mouvement est souvent le mauvais - en effet, une idée tend facilement à n'éveiller dans l'esprit que ce qui est favorable à son admission définitive - mais si l'esprit est-bien organisé, si l'assimilation de la nouvelle impression ne se fait pas trop vite, d'autres tendances latentes, indirectement ou directement froissées par le nouveau phénomène, entreront en activité et jugeront á leur tour la nouvelle idée. Nous n'avons à nous occuper ici que de celles qui s'éveillent selon la loi d'association systématique, mais un certain nombre prenuent la forme du contraste que nous examinerons plus loin. Dans un esprit bien équilibré, dont la persommalité est fortement constituée, c'est-à-dire che\% lequel les diverses tendances sont suffisamment solidaires les unes des autres et assez étroilement reliées entre elles selon une loi d'association systématique, une tonlance ne peut entrer en activité sans que les autres tendances ne s'éveillent failılement, mais assez pour pouvoir entrer complètement en activité si le besoin s'en fait. sentir, cest-it-dire encore selon la loi d'association systématique. Le pouvoir personnel se réduit done à un mode 
parliculier de la finalité de l'esprit - celui où toutes les tendances de l'organisme; ou au moins les principales, sont en connexion assez étroite pour que l'activité de l'une éveille faiblement d'abord, et surtout éveille complètement s'il le faut l'activité des autres, afin que nulle nouvelle tendance, nulle croyance, nulle théorie nouvelle ne puisse s'établir dans l'esprit qui ne soit susceptible de s'acconmoder au moins aux plus importantes des tendances acquises et à l'ensemble qu'elles forment, c'esta-dire il la personnalité.

Ce qui précède nous permet aussi de comprendre comment le moi semble s'isoler de ses états, se tenir à part comme un ètre qui persiste tandis que ses ètats paraissent et disparaissent tour à tour, qui juge, qui choisit, qui décide. Il en est réellement ainsi, il y a bien un moi qui persiste et qui agit, mais ce n'est pas dans une substance métaphysique qu'il faut chercher sa réalité. Cette collection systématisée de tendances, qui s'éveille faiblement ou fortement chaque fois qu'un phénomène important se produit, qui reste sensiblement la même pendant un tenips très long, car les tendances ne varient pas très vite, ni surtout l'ordre dans lequel elles sont coordonnées, nous finissons par les reconnaitre, par les considérer à part, par les regarder comme un tout naturel et indivisible - et en effet nous les voyons agir généralement dans le même sens, et elles sont bien réellement, à certains égards, abstraites des phénomènes particuliers rui peuvent en certains cas les compléter. Si j'ai à juger une théoric psychologique, je n'ai pas en général besoin de faire revirre en moi tout ce que je sais de psychologie; les tendances qui correspondent à des faits souvent répétés, souvent organisés, s'éveillent d'unc manière abstraite, je sens qu'elles s'accordent ou ne s'accordent pas avec la nouvelle théorie qui m'est proposée. Si, par exemple, on me dit que tout fait psychique 
est comme tel parfaitement connu par le sens intime, je sens immédiatement que cette irlée ne s'accoude nullement avec mes opinions - et sans doute je puis, si je veux, me développer tous les motifs que j'ai de croire que ce désaccord existe récllement et aussi toutes les raisons qui me font trouver mon opinion bonne, mais si mes opinions sont suffisamment formées, je n'en ai pas besoin, - il se peut que je le fasse par scrupule de conscience, mais immédiatement je sais que je ne pense pas comme mon interlocuteur et que j'ai des raisons de ne pas penser ainsi, et même je le sais sans me le dire, sans le formuler comme je viens de le faire. Mon esprit rejette la nouvelle pensée, et l'état de conscience qui se manifeste alor's est un etat abstrait, contenant implicitement, mais non explicitement, tout ce que je viens de dire que j'en puis tirer. Toutes les tendances qui sont en nous suffisamment organisées sont susceptibles de se manifester ainsi et de manifester leur coordination par des ctats abstraits, par l'éveil seulement de quelques-uns de leurs éléments, sans que les faits qui les ont constituées, les images, les perceptions, les idées nombreuses qui relèvent d'elles, à quelque degré, aient à entrer sur la scène de l'esprit-mais elles se manifestent par un état de conscience que nous reconnaissons bien - ce qui équivaut à dire que cet état s'associe à certaincs idées. Il en rósulte que l'ensemble de ces tendances souvent ćveillées d'une manière abstraite nous est connu comme se manifestant a la conscience d'une certaine façon; l'état de conscience ainsi suscité pendant la réflexion, la volonté et en général pendant lous les actes du a pouvoir personnel, o c'est a jeu près ce fui correspond an sens du moi. Ce sens du moi est ainsi en action dans les actes volontaires et réfléchis, ee qui justifie encore le $110 \mathrm{~m}$ du pouvoir personnel, mais jusqu'a un certain point seulement, car ce sens du moi n'est qu'un phénoméne accompagnant le jeu des tendances 
dans les conditions que je viens d'indiquer, et d'autre part il est loin de constituer la personualité dont, comme nous le voyons, il n'est qu'une sorte de représentation abstraite, correspondant à l'éveil coordonné de quelquesuns des éléments des tendances dont la réunion constitue le moi.

Toutefois, s'il ne constitue pas la personnalité, il la représente, et il la représente d'une manière efficace, car ce simple éveil d'éléments abstraits et coordonnés suffit pour déterminer l'adoption ou le rejet de certaines croyances, comme s'adaptant ou he s'adaptant pas à notre personnalité, à l'ensemble de nos tendances considérées oul plutôt senties, non pas les unes après les autres, mais dans leur coordination, dans leurs rapports réciproques. Il est important de bien constater ce fait qui justifie suffisamment le nom de pouvoir personnel et qui permet d'en expliquer la nature par les lois de la psychologie expérimentale ${ }^{1}$.

Nous voyons au reste, que le pouvoir personnel varie beaucoup, et que le sens du moi, s'il est indispensable poul rendre possible un fait de volontè ou de réflexion ne suffit pas ả le constituer. Le fait que je viens de citer en effet, celui oǹ le moi organisé repousse une opinion qui ne s'adapte pas à ses habitudes est à peine un fait de volonté, il n'est pas un fait de réflexion, mais il nous niontre comment le moi, en.tant que groupe d'éléments abstraits et forme de coordination, peut s'éveiller facilement et se séparer des autres phénomènes. Il nous montre aussi la transition entre la volonté et l'automatisme marquée par la coordination croissante des phénomènes et l'éveil d'un moins grand nombre d'entre eux.

1. Il est a peine besoin de faire remarquer ici que les tendances organiques, celles qui constituent proprement la vie des organes, sont largement représentées dans le sens du moi. 
Souvent il arrive, si la lutte est vive, si la réflexion est intense, que des phénomènes particuliers, idées, images, etc., s'èveillent, mais ils sont facilement considérés à part des tendances; c'est que ces phénomènes sont variables, tantôt l'un, tantôt l'aulre, selon le cas, se présentera à l'esprit, c'est tantòt telle tendance, tantôt telle autre qui, pour maintenir son organisation, aura besoin de se compléter par tel ou tel phénomène particulier. Au contraire, les tendances elles-mêmes et leurs coordinations sont relativement stables. Il semble donc que, dans ce jugement: je vois ceci, je pense a cela, ił n'y ait pas seulement, comme l'a dit M. Taine, le rapport d'un composé à un de ses éléments ${ }^{1}$, il y a aussi le rapport de tendances abstraites à des phénomènes concrets qui viennent en certains cas compléter une ou plusieurs d'entre elles. 11 me semble que cette conception du pouvoir personnel permet de rendre compte et d'expliquer la partie vraie des doctrines spiritualistes sur la couscience, la volonté, la responsabilité, stc.; tout en restant absolument indépendante de toute hy pothese sur la substance, elle n'implique en effet ni le libre arbitre, ni la séparation de l'âme et du corps, ni rien de semblable. Il ne s'agit jamais que de certaines circonstances particuličres du jeu des tendances et d'une forme, que j'ai tâché de déterminer, de la loi d'association systématique.

Le pouvoir personnel et le sens du moi peuvent être à leur tour des objets de réflexion, e'est un cas de ce qu'on appelle le repliement de l'esprit sur lui-même; malheureusement cette métaphore qui peut très bien s'employer en certains cas est beancoup trop vague et deviendrait absolument inexacte si l'on roulait s'en servir pour rendre comple réellement du phénomène. En fait, le sens du Inoi pui est l'objet de la réflexion est la réviviscence d'un

1. Taine, Les philosophes classiques au $\mathbf{S} I X^{\circ}$ siecle. 
état passé qui entre à son tour en contact avec certaines idées. Ici j’ai tâché de l'analỵser, c'est-à-dire que les eléments du sens du moi se sont associés en moi arec des ilées particulières et des mots par lesquels j'ai essayé d'exprimer sa nature, l'opération s'étant faite selon $n$ un mode particulier d'issociation systématique, celui qui préside a toutes les manifestations graphiques de la pensée. Le phénomène n'aurail pas èté différent au fond, sij'arais exprimé mes idées par la parole, mais la forme et les èléments du systeme auraient changé. Le phénomène aurait encore été essentiellement le mème au point de vue de la psychologie générale, tout en différant par des points importants si j'avais eu à considérer le moi à un autre point de rue, à rechercher, par exemple, son importance au point de vue de la formation de tel ou tel sentiment.

$\S 2$.

Ce qui précide nous permet de voir les rapports de la volonté et de l'automatisme à un point de vue plus général. Nous voyons que le progrès, en psychologie, comme en économic politique, par exemple, s'accomplit parfois selon une sorte de spirale qui nous ramène à un point voisin du point d'où nous ètions partis, quoique supérieur. L'automatisme et l'indépendance des éléments psychiques se retrouvent au commencement et it la fin d'une évolution mentale, mais avec des différences importantes. Au début l'enfant a de la peine à coordonner pour écrire - les mouvements de ses doigts, ces mouvements se produisent à tort et à travers, automatiquement; la volonté n'a pas sur eux l'empire qu'il faudrait, ces èléments psychiques ont une existence trop indejpendante et ne sont pas soumis au pouvoir personnel. A cette période d'anarchie succède une période d'organisation, peu à peu le pouvoir de la volonté devient efficace, l'intervention contiuue du moi, c'est-i-dire, des tendances fondamentales, 
des désirs permanents, régularise l'activité, coordonne les éléments, puis, à mesurc que l'organisation devient plus parfaite, les éléments psychiques ont une tendance à reprendre une certaine indépendance, à revenir, sinon à l'anarchic, du moins à la liberté. On écrit alors sans qu'un acte de volonté intervienne, sans que des tendances complexes soient éveillées, les éléments psychiques, intellectuels et motcurs, qui déterminent l'acte de l'écriture sont suffisamment coordonnés, sont associés assez fortement entrc eux pour n'avoir plus besoin de la contrainte, de l'influence exercée sur eux par la personnalité générale. De même des travailleurs unis pour la première fois dans une curre commune tâtonnent, se trompent, ne savent pas se reconnaître, mais une fois qu'un supérieur les aura placés, installés, aura montré à chacun la tìche qu'il avait a faire, ils pourront apprendre à se passer de lui et finiront peut-être par exécuter leur travail sans ses instructions d'abord, sans son intervention directe, et plus tard sans sa surveillance. Ici, le supérieur est un homme comme les autres, mais il représente la coordination déjà ètablie dans la société. Dans l'esprit, ce qui dirige, ce sont des faits psychologiques qui cux, ont acquis déjà une coordination suffisante et qui tendent à l'imposer aux éléments restès encore en dehor's de la synthèse psychique qui est le moi. Anarchic, despotisme et liberté daus un fonctionnement harmonique, paraissent être les trois termes de l'érolution normale d'un fait psychologique et social. Il importe de remarquer les différences essenticlles du premier et du dernier élal, le premier étant l'incohérence mêne, le dernier la liberté du bien.

\section{$\S 3$.}

L'attention volontairc, la délibération, la volonté, la conscience reflechie, sont des formes diverses du « pouvoir jersonnel ", il faut indiquer brièvement en quoi elles 
consistent et comment elles peurent entrer dans l'analyse générale et diıns la définition que nous venons de donner. Dans la délibération et la volonté c'est spécialement l'idée l'un acte fui entre en rapport avec les diverses tendances ou avec les phénomènes qui les représentent; dans la conscience réfléchie c'est un phénomène psychique, quel qu'il soit; dans l'attention, c'est un phénomène quelconque. A ce point de vue, tous les actes du pouvoir volontaile seraient des formes de l'attention, mais on pourrait dire aussi bien que l'attention volontaire et la conscience léfléchie sont des eas particuliers de la volonté, d'autant que l'attention et la réflexion psychiques impliquent a divers égards des éléments moteurs. Ceci n'est guère qu'une question de définition, ecpendant le premier ordre indiqué a l'arantage de se prêter á une classification plus large en permettant ensuite de rapprocher l'attention volontaire de l'attention spontanée ${ }^{1}$.

Nous n'arons guère jusqu'ici considéré dans le pouroir personnel que la réflexion, mais il ỹ a quelque chose de plus à examiner, c'est la décision, c'est le moment où la croyance se fixe, où la volonté se décide. Pendant quelque temps les diverses tendances dont l'activité s'est.manifestée ont essayé le nouvel état qui tend à s'imposer: mais l'activité est restée assez faible, au moment de la décision,

1. L'attention spontanée se distingue de l'attention rolontaire en ee qu'elle n'implique pas que la coordination de la personnalité ait pris part au phénomène de l'attention. Por exemple, nous pouvons être captirés quelques minutes par un spectacle qui, si nous refféchissions, ne nous arrêterait pas. Réciproquement il faut quelquefois l'intervention, l'éveil systématique d'un nombre important de tendances ponr nous rendre attentifs a un fait quı autrement passerait inaperçu ou nous rebuterait. L'attention rolontaire estaussi une application indirecte de l'attention spontanée : la tendance capable de s'associer systématiquement le phénomène, objet de l'attention, ou sa représentation étant éveillée secondairement. Toyez à ce sujet Ribot, Psychologie de l'Attention. 
brusquement, une orientation nouvelle s'établit dans l'esprit, une coordination particulière apparait, les tendances dont l'intervention n'est plus utile ou qui ne pourraient s'accorder avec le nouvel état, retournent à l'état latent. Je suis quelque temps indécis entre sortir ou bien rester a travailler': j'hésite, les diver's avantages de l'un ou de l'autre parti se présentent à mon esprit, des tendances diversess'éveillent en moi et essayent de s'harmoniser avec la représentation de l'un et de l'autrésystème d'actes à accomplir. Il s'établit une sorte de lutte plus ou moins vive, puis tout d'un coup, et bien souvent sans que la conscience du fait soit très nette, on se trouve avoir fait son choix, un des termes de l'alternative s'est imposé à l'esprit ou a èté accepté par lui, une des tendances ou un systèmé de tendances a vaincu, aussitôt les autres tendances disparaissent ou retourment au second plan, et les éléments psychiques en activité se trouvent tous associés selon le mode voulu pour l'exécution de l'acte décidé. A l'essai, à l'éveil partiel de tout un groupe de tendanees, a succédé la coordination selon un certain mode, de tous les phénomènes actuels, ou de presque tous. Si j'ai décidé de sortir, tous mes actes sc dirigent vers cet acte, je me lève, je prends mon chapeau, etc. : si je reste, - a part peut-être quelques regrets, protestation des tendances évincées, toutes mes pensées viennent converger vers le travail que j'ai a faire, je prends ma plume, je consulte des livres, j’écris. Dans les deux cas, après un tàtounement, aprés que les diverses tendances yui constituent le moi se sont manifestèes assez jour permettre un chuix qui convienne a la persomnaliti, l'orientation se forme d'une manière hrusfue, en général, et les phonomenes psychiques se trouvent associes dans un systeme déterminé.

Nons retrouvons ici le jeu des élements psyehiques, mais nons frouvons aussi le lonetionnement general de la persominlite. Chaque tentance, chaque desir, chaque 
croyance, a lutté, a combattu pour elle-mẻme, seulement le moi, l'ensemble des tendances déjà organisées, est intervenu, et un équilibre s'est établi, la force de l'un des éléments quiluttaient, s'est mieux accordée que la force de l'autre avee notre organisation mentale et l'orientation de l'esprit s'est établie par la mise en actirité de tendances, d'idées, de sentiments, convergeant tous vers le mème but. Mais le phénomène ne prend son aspect propre que par la subordination caractéristique des tendances opposées, par leur inhibition que nous étudierons plus loin. Remarquons seulement que la décision dans la rolonté est marquée par l'association de certains sentiments, de certaines idées, de certains désirs arec les éléments moteurs qui doivent servir à leur réalisation, le reste de l'esprit étant, dans son ensemble, farorable à cette association.

\section{$\S 5$.}

Ici, comme dans les chapitres précédents, les phénomènes où le pouroir personnel a disparu sur un point ou sur quelques points ou sur tous les points, et ceux où il s'est amoindri, nous montreront peut-être mieux sa nature propre par la comparaison qu'ils imposent avec les faits de l'état normal. Je citerai d'abord, comme transition entre l'état normal et l'état morlside, bien qu'appartenant encore a l'état normal, les faits où nous royons un mot, un geste, un acte, échapper, pour ainsi dire, à une personne, par inattention. On dit quelquefois sans mauvaise intention un mot capable de ficher son interlocuteur, c'est qu'on n'a pas suffisamment mis en rapport l'idẻe de ce mot avec les tendances actuelles du moi avant de le prononcer.

L'habitude nous montre en beaucoup de cas l'affaiblissement du pouvoir personnel. On sait que quand nous arons accompli souvent le mème acte, surtout dans des 
circonstances diverses, la tendance qui lui correspond tend à s'organiser et à se mettre en activité en échappant plus ou moins au contrôle des autres tendances. Les exemples de ces faits sont nombreux. Un ivrogne d'habitude a beaucoup de peine à s'empêcher de boire, un joucur à s'empècher de jouer. Ils ont beau se faire des serments sincères, une fois que la tendance dominaute est éveillée par la vue de la bouteille, par la vue des cartes, de l'or et du tapis vert, la coordination, dans le sens habituel, s'accomplit presque fatalement. La délibération sc produit bien encore, mais faiblement, le sens dı moi s'èveille, diverses tendances entrent en jeu, mais l'arrèt ne se produit pas ou ne se produit que faiblement, le moi assiste impuissant au triomphe d'une des tendances qui le constituent, sans que les autres tendances puissent cnrayer le mouvement: les roues de l'engrenage ne mordent plus. La liberté est rederenue de la licence et le contrôle du moi s'est trop affail,li pour y remédier.

A l'élat morbide on trouve fréquemment cette impuissance du pouvoir personnel. M. Lnys cite quelques fragments d'une lettre d'un jeune homme qui, occupé pendant plusieurs jours de suite à faire des calculs d'intérèt composé, en garda une grande tension d'esprit et dut continuer malgré lui son travail intellectuel. « Ayant besoin, du plus grand calme et du repos auquel je ne pouvais atteindre, je me mis, sans la moindre volonte de ma part, ¿t compter, à refaire exactement les mènes problèmes qu'au bureau " '. On trouve des phénoménes completement analogues dans la folie impulsive. Le sens du moi se manifeste, les principales tendances de la persounalité sont bien éveillées, mais elles restent impuissantes, une idée qui ne peut s'accorder avec elles tend a déterminer et détermine parfois des acles entièrement

1. Luys. Le cerveau et ses fonctions, 1. 145. 
opposés au caractère général de la personne qui les accomplit malgré elle, et qui essaye de les repousser jusqu'au moment où l'orientation nouvelle s'établit brusquement, où les tendances organiques qui forment la base de la personnalité ne peuvent plus résister à la nouvelle systématisation que l'idée morbide tend à introduire, et où pour un temps, les tendances habituelles sont réduites a l'impuissance, reléguées dans l'arrière fonds mental qui persiste toujours, mais seulement à I'état latent. Un dipsomane " M..... est calme, poli ; ses manières sont agréables, sa conversation est intéressantè, il s'occupe de littérature. Lui fait-on des observations sur les suites de son excès, il rougit, paraît honteux, s'excuse, se repent et promet de résister à son funeste penchant. Il déclare, avec l'acrent du désespoir, qu'il est entraîné malgré lui dès qu'il voit la possibilité de satisfaire son goùt pour les liqueurs. Si je cause avec M. G......, après lui avoir représenté tout ce que sa conduite a d'humiliant et de hideux pour un homme qui a reçu une bonne éducation, qui a une femme et des enfants, après l'avoir fait conveuir que sa santé s'altère, que son intelligence s'affaiblit, M. G.... apprécie la justesse de mes observations, me remercie de mon bon vouloir pour lui, pleure de honte et de chagrin, forme les plus sévères résolutions et consent à ne plus sortir de la maison s'il retombe à l'avenir dans les mêmes excès.

«Malgré ses beaux sentiments, malgré ses belles protestations, malgré ses promesses, chaque fois qu'on permet à M..... de sortir de l'établissement, il se contient pendant trois ou quatre jours, après lesquels il boit et rentre dans un état d'ivresse complète........ Tous nos efforts depuis dix-huit nois pour aider ce malade a triompher de son funeste pencliant ont été inutiles jusqu'à présent $\gg 1$.

1. Esquirol, Maladies mentales. Tome II, p. 79-80. 
Il y a là un manque d'équilibre; un élément psychique domine, il n'est plus enraye, il travaille pour lui seul, au grand dommage de l'eusemble de la personnalité avee lequel ses tendances personnelles ne concordent plus ; le moi n'agit plus, il reste impuissant et bientòt il se laisse gagner en partie. C'est, en effet, ce qui se produit lorsque le mode d'orientation de l'esprit change brusquement. Ici encore l'état morbide nous permet de nieux comprendre l'état normal. Tous ces états qui proviennent de la sensibilité interne, tous ces phènomènes cérébraux qui reprèsentent dans l'esprit les tendances organiques, - celles qui constituent la vie du corps, - et aussi les représentations motrices, tous ces phènomènes qui accompagnent chacun de nos actes passent soudainement d'un système psychique a l'autre. La puissance de l'idée morbide est telle, en certains cas: gu'elle finit par se les associer, par les détacher des tendances supérieures auxquelles elles étaient auparavant liees. Les mouvements du cœur, la respiration, toutes les fonctions organiques en un mot, qui jusque là s'unissaient pour donner aux tendances supérieures et habituelles les conditions de leur existence, sont maintenant au service de l'idee morbide qui a envahi le moi et accapare momentanément toutes les forces de l'organisme qu'elle systematise a son profit, selon une forme nouvelle. Mais arant cela il se produit souvent un de ces phénomènes qui marquent si bien la vie individuelle des systemes psychiques; les tendances habituclles se sentant menacées, ot préroyant leur défaite. usent de l'empire qui leur reste encore pour mettre, an moyen de secours ctrangers, l'idee morbide dans l'impossibilite de profiter de son triomplie momentaué, et de se compléter par des artes. "lne dame, prise parfois d'impulsions homirides, demantlat a être maintenue à l'aide d'une camisole rle force, et annongait ensuite le monent ou tout danger stait funset oin olle pouvait reprendre la liberte de ses 
mouvements. Un chimiste tourmenté de mème par rles desirs homicides, se faisait attacher les deux pouces arec un ruban et trouvait dans ce simple obstacle le moxen de résister à la tentation. Une domestique, d'une conduite irréprochable, supplie sa maitresse de la laisser partir, parce que, en voyant un enfant qu'elle soigne, elle est dévorée du désir de l'èventrer. Lne autre femme, d'une grande culture intellectuelle et pleine d'affection pour ses parents, « se met à les frapper malgré elle, ct demande qu'on vienne $\dot{a}$ son aide en la fixant dans un fauteuil $\gg$. Un mélancolique, tourmentè d'idées de suicide, se leva la nuit, alla frapper à la porte de son frère et lui cria : * Venez vite, le suicide me poursuit, bientòt je ne résisterai plus 1.

Dans l'exercice de l'attention les mêmes faits se produisent. Chacun sait qu'à de certains moments nous ne pouvons, malgré notre désir, prèter notre attention à une lecture. "Il m'arriva une fois, dit sainte Thérèse, que voulant lire la vie diun saint pour voir si je pouvais trouver de la consolation dans ce qu'il avait souffert, j'en lus quatre ou cinq fois de suite quatre ou cinq lignes sans pouvoir jamais y rien comprendre, quoi qu'elles fussent en langue vulgaire, ce qui me fit jeter le livre; et la même chose m'est arrivée diverses fois, mais je ne me souviens que de celle-là, 2 . Inutile d'insister et de multiplier ici les faits, on en trouvera d'intéressants dans les ouvrages de M. Ribot sur les maladies de la volonté et sur l'attention. Ils n'offrent, à notre point de vue, aucune difficulté d'interprétation. L'affaiblissement ou la disparition du pouvoir personnel est toujours marqué par l'impossibilité d'une coordination totale, d'une systématisation qui fasse

1. Ribot. Les maladies de la volonté, p. 76-77. Voir tout le chapitre.

2. Sainte Thèrese. Autobiographie. Traduction Arnauld d'Andilly, p. 184. 
concourir vers un même lut l'état noureau et une partie suffisante des tendances qui constituent la personnalité.

$$
\S 6 .
$$

En résumé le pouvoir personnel nous parait, Itui aussi, être essenticllement un eas de l'association systémalique: celui oì les ètats nonveaux qui tendent à s'implanter dans l'organisme sont tités, éprourés, par la plus grande partie de nos tendances diverses et par l'association de ces tendances, parces systèmes de phénomènes dont la coordination supérieure constitue la personnalité et par la personnalité considérée comme un tout relativement unifié. Dans ce cas le sens du moi est produit par l'éveil faible de ces tendances coordonnées. L'épreuve et la décision, la délibération et la volonté, la réflexion et l'adoption d'une croyance constituent les deux modes du pouvoir personnel. Le premier consiste dans l'épreuve faite par les divers systèmes, dans les tentatives de coordination générale de l'esprit, englobant comme un de ses éléments le systène nouveau, le second consiste dans le fait de l'établissement de cette coordination générale, qui se produit si souvent, $\dot{a}$ un moment donné et parfois après une longue hésitation, done manière brusque. L'esprit comme une solution saturée qui cristallise immédiatement lorsqu'on y introduit un petit cristal du sel dissous, finit par prendre une orientation déterninéc par la nature du nouveau phénomène qui en fait partie intégrante el aussi par la nature des habitudes antérieurement aequises, ou bien par la nature des tendances qui ont déterminé le rejet du nouveau système. Toute nouvelle adaptation amène un certain changement, peut-c̀tre inperceptible, dans le moi, dont la majeure partie toutefois persiste longtemps. Il n'est pas inutile de remarquer que dans les complexus qui constituent la personnalité, un grand nombre, les facultés intellectuclles, par exemple, et les fonctions organiques 
peuvent s'associer également à des sỵstèmes tout a fait différents. Si un homme, par exemple, aprés avoir été honnète, devient voleur et assassin, il n'y a pas de raison générale (bien que cela arrive parfois) pour que les fonctions organiques ne contribuent aussi bien a entretenir et ¿ dérelopper le nouveau caractère qu'elles servaient à entretenir et à déroiopper l'ancien, et les opérations intellectuelles s'opèrent de la mème manière. Je les comparerais volontier's à la masse d'indifrérents politiques et de travailleurs qui, dans un pays, entretiennent la vie organique et payent l'impòt quelle que soit la forme du gouvernement, qui ne font pas d'opposition, que tous les partis sont certains d'avoir avec eux, s'ils sont au pouvoir, à condition de ne pas détruire complètement la vie socials, et que chacun tâche de s'associer. De mème qu'on fait intervenir la volonté du peuple dans des questions dont la plus grande partic de la nation ne s'est jamais occupée, de mème que tout se fait "au nom du peuple», tout se fait au nom du moi, et c'est lui qui toujours est censé agir. En réalité le conflit ne s'élève souvent qu'entre quelques systèmes psychiques et le moi s'associe à celui qui a su le prendre, ou c'est du moins ce que fait une grande partie de la personnalité dans les cas ordinaires et principalement la personnalité organique. Les véritables cas de l'exercice du pouvoir personnel, ceux où un moi fortement constitué éproure vigoureusement et rejette ou admet les états nouveaux que les circonstances suscitent sont plus raresqu'on ne pense - il était pourtant indispensable de les étudier. 


\section{CHAPITRE V}

\section{L a leraon naité.}

\section{$\$ 1$.}

Comme les sensations et les perceptions sont des systèmes d'élémentsplus simples, comme les idécs sont des systèmes d'éléments empruntés à de nombreuses perceptions, les tendances sont des associations coordonnées d'idées, de perceptions réelles ou possibles d'images motrices, d'éléments récls et s'associent à leur tour à des systèmes de plus en plus vastes. Chaque trait de caractère résulte de la coordination selon un certain mode d'un certain nomJre de tendances. L'avarice, par exemple, est une systématisation dans un sens très déterminé de ces tendances qui font travailler pour gagner de l'argent, qui cmpêchent d'acheter certains oljjets à cause de la dépense qui en résulte, quí détournent de la charité, etc. Il y a là un nombre immense d'idées, de perceptions, de monvements, 'd'images, de sentiments, de tendances qui convergent tous vers' un même but : l'acquisition continuelle et l'accumulation des richesses. Mais ces tendances générales, ces traits de caractère, s'associent á leur tour jour former des groupes psychiques plus importants dont la réunion plus ou moins unifiée au point de vue de la fin et associée aux tendances de la vie organique qui ont un rôle des plus importants, est la personnalité même.

Tous les phénomènes sont ainsi des éléments de la personnalite, nous les avons étudiés dejá, venons maintenant aux formes les plus hautes de leurs syntheses. Nous avons d'abord à considérer les traits de caractère. Ce qu'on 
appelle chez une personne qualités ou défauts, ce sont quelques formes principales que prennent chez elles la coordination des tendances secondaires, ainsi que je l'ai indiqué. En tout cas, je crois que c'est dans la psychologie mème, non dans l'étude des tempéraments, que les éléments de la science des caractères doivent ètre cherchés, bien que l'étude des tempéraments puisse donner des renseignements très utiles. Analysez tous ces mots qui servent à indiquer une qualité ou un défaut: bon, honnête, pervers, rusé, fin, grossier, méchant, délicat, prodigue, avare, généreux, vaniteux, humble, etc, vous verrez, si je ne me trompe, que dans chaque cas, ce not indique une forme particulière de l'activité mentale, une fin commune atteinte ou du moins visée par de nombreux éléments psychiques coordonnès, á moins qu'il n'indique simplement l'absence d'une fin commune particulière. C'est ici que l'on peut trouver exacte la théorie du fait dominateur de M. Taine, qui, d'une manière générale, s'applique à une humanité abstraite, idéale, plutôt qu'à l'homme illogique et mal fini que la réalité nous montre, mais aussi peutètre à une humanité un peu trop simplifiée. Chez l'homme vaniteux, les idées, les actes, les paroles convergent vers ce but : donner à soi-même et aux autres une haute idée de l'importance de sa personne à propos de choses parfaitement insignifiantes ; chez l'homme fin, les sens et l'intelligence, les facultés en général, sont toujours prêts à apercevoir les moindres nuances, les plus petites particularités des choses, à les combiner, à s'ell servir soit pour émettre des idées ingénieuses ou des remarques piquantes, soit pour en tirer parti au point de vuc pratique, pour agir d'une manière déterminéc sur une personne, etc. L'écueil de ce genre est que les nuances empêchent parfois de voir les couleurs. Il n'est pas besoin de faire remarquer que l'intelligence, la combinaison des idées a ses types, comme le caractère, qui s'applique plus 
particulièrement i la combinaison des sentiments et des actes. Dire qu'un esprit est vif, lent, profond, superficiel cela indique une manière d'ètre générale des combinaisons intellectuclles qu'il produira et le désigne comme apte à prendre telle ou telle place dans le consensus social. A côté des qualités qui sont simplement unc forme de la combinaison, une manière d'ètre d'un esprit donnant toujours des produits plus ou moins semblables, il y a des qualités qui tiennent aux éléments même qui sont coordonnés, ainsi un esprit peut se plaire à combiner des images visuelles, et c'est un des dons du peintre, ou des images sonores, et c'est une qualité du musicien, ou des idées abstraites. Chacun a ses images particulières et la distinction des types moteur, auditif, visuel, indifférent, est bien établie, de même chacun a ses idées, plus ou moins mêlées d'images sensibles ou d'éléments moteur's, et pour chacun de nous, certaines idées sont plus facilement que d'autres combinées par l'esprit : l'un a plus de goût pour les mathématiques, un autre pour les sciences biologiques, un autre pour la philosophie, ete. De même encore pour le caractère: il y a des personnes portées surtout ver's les plaisirs des sens, d'autres vers les plaisirs de l'intelligence, d'autres ver's l'exercice de la volonté, sous différentes formes: activité motrice: voyageurs, activité sociale : hommes d'état, ambiticux, cte. Enfin, la fonetion sociale.que tout le monde exerce et que l'on choisit quelquefois d'après ses aptitudes propres, est encore souvent une forme du caractère, on elle le devient avee l'àge. Je n'hésite pas à croire qu'il peut y avoir beaucoup de vrai dans une théorie des types professionnels, d'une manière générale chaeun est plus apte à certains emplois qu'à d'autres, et certaines vocations, celle de militaire, de prêtre, sont souvent très marquées au point que l'activité déterminée ver's laquelle un individu se sent porté est quelquefois la seule dans layuclle il puisse tenir convenablement sa place. Mais 
l'activité sociale d'une personne n'est pas seulement l'exercice de son métier, presque tout ce que nous faisons a une portée sociale, et c'est ici que se montre le rôle de l'association systématique pour les cas cités précédemment et oủ elle n’était pas suffisamment évidente. Ėtre gai, ètre entreprenant, etc., c'est plutôt un rẻsultat qu'une fin au point de vue de la psychologie, mais c'est un élément de coordination sociale. En raison de ses qualités et de ses défauts, l'individu est propre ì rendre tel ou tel service ì la société. Il est des circonstances où trop de finesse nuit, il y a des fonctions qui ne peuvent ètre remplies que par des personnes douées de peu de délicatesse physique et malheureusement pour notre socièté certains de ses èléments utiles ne peuvent accomplir leur fonction qu'à la faveur d'un manque de délicatesse morale, portant au moins sur quelques points. Les traits de caractère qui paraitraient sans signification au point de vue de la psychologie, en prennent une si l'on considère la coordination des éléments sociaux.

On sait la force de systématisation que possèdent les traits de caractère et comment ils tendent à se subordonner le plus grand nombre possible d'éléments psychiques. Cliacun connaît des vocations qui se sont fait jour au travers d'obstacles très graves, et il arrive parfois que le système de tendances qui domine ainsi l'organisme et tend a plier toutes les circonstances de manière à ce qu'elles puissent s'harmoniser avec la tendance maîtresse, ne correspond pas à une faculté de premier ordre. Des facultés moyennes, au point de vue scientifique ou artistique, peurent arriver a se subordonner l'organisme entier malgré des obstacles qui auraient pu paraître insurmontables. Le peintre Ducornet, qui ne paraît pas avoir eu précisément du génie, arriva à être un artiste distingué, étant " privé des bras par vice de conformation et n'ayant pour soutenir son corps que des jambes exiguës, 
sans autre mouvement articulaire apparent qu'au bassin et au pied oì l'on ne compte que quatre orteils. "Quelques détails sur sa manière de travailler donneront une idée frappante de la force d'association systématique que peut avoir un trait de caractère, et prourent combien les organes peuvent, sous l'influence d'une organisation cérébrale particulière, s'adapter aux fonctions auxquelles ils semblaient répugner le plus. "Ducornet marche-t-il sur ses pieds ou sur ses mains? peut-on se demander après l'avoir vu travailler, assis sur un tabouret, tenant sa palette d'un pied et dirigeant de l'autre une brosse habile. Devant lui s'élève la toile à couvrir ; elle se meut au gré du peintre immobilisé par l'emploi de ses extrémités uniques, destinées à lui servir, néanmoins, aux doubles fonctions qui, chez l'homme normal, sont réparties entre les membres supérieurs et inférienrs. Le doute augmente encore quand Ducornet veut tracer ou peindre de larges parties, alors il saisit avec la bouche son crayon ou son pinceau, et promène hardimentl'un ou l'autre dans une grande surface. Ce mode plus expéditif lui permet ainsi de s'éloigner ou de se rapprocher immédiatement de son travail, a laide de ce qui remplissait auparavant dans sa personne loffice de bras et de mains. n Mais la force du systeme psychique ne s'exerce pas uniquement sur le cerveau et sur le reste de l'organisme, elle modifie le milieu qui s'adapte à son tour. Ducornet avait évidemment des appareils particuliers pour peindre, puisqu'on nous dit que sa toile s'éloignait el se rapprochait a volonté, on nous apprend de plus que son père le transportait sur ses épaules pour qu'il ne se fatiguàt pas les pieds; a c'est lui qui monte Ducornet sur son échafaudage, l'en descend, l'habille, en un not le complète " $\mathbf{1}$.

1. Dictionnaire de la conversation. Article Ducornet, par J -B. Delestre 
Chacun de nous a d'ailleurs, comme Ducornet, bien que généralement à d'autres égards, besoin d'être complété, et il n'y a pas de donte que chaque système dominant , chez un individu ne modifie non seulement la personnalité de cet individu, mais aussi celle des autres personnes qui ont quelques rapports avec lui, et aussi indirectement, et dans une mesure variable, le milieu matériel. C'est ainsi que les coordinations familiales et sociales se forment et s'établissent, que les arts et les métier's se créent et se développent et c'est aussi une des raisons qui font varier la géographie.

$$
\S 2 .
$$

La part que prend à la vie de l'esprit l'organisme proprement dit, l'etat des organes autres que le cerveau considéré dans sa fonction psychique, est un point intéressant mais assez obscur. On ne peut douter que son influence ne soit considérable sur la formation de la personnalité. M. Ribot l'a mise en lumière récemment ${ }^{1}$, et ses conclusions sur ce point ne paraissent pas aroir èté sérieusement combattues. J'ai dù en dire quelques mots dans le chapitre précédent, à propos du pouvoir personnel, je dois en parler encore ici au point de vue de l'association systématique en général.

Conme le cerreau, l'esprit, s'adaptent à l'organisme, l'organisme s'adapte au cerveau. Cette adaptation est générale, le cerveau et l'organisme, le cœur, les poumons, etc., sont parties d'un mène sýstème. Si l'on admet que le sens du corps et l'état des organes fasse la base de la personnalité, - el pall sens du corps il faut entendre toutes les impressions conscientes ou non qui viennent des organes et influent sur l'orientation de l'esprit, - on admeitra aussi que, ì chaque moment, l'état des organes

1. Ribot. Les maladies de la personnalité. 
doit exercer une certaine influence surl'état de l'esprit. Cela arrive continuellement : les effets de la digestion lente et pénible ou facile, de la circulation ralentic, exagérée, puissante ou faible, de l'état du sang, de la respiration libre ou gênée sont indéniables. Il faut admettre en même temps que l'état de l'organisme s'adaptera, s'harmonisera en quelque manière avec cet ètat de l'esprit, c'est-à-dire qu'il sela influencé par lui '. Cela revient a constater que. le corps et l'esprit constituent d'une manière générale un même système. La surcharge stomacale détermine des nausées qui débarrassent l'estomac, le manque d'eau clans l'organisme produit la soif qui doit y remédier, de même l'attention parait attirer un afflux de sang et une diminution du volume de sang qui circule dans les membres 2. Il paraît se produire ainsi une association systématique très difficile à expliquer et à analyser, dont on ne peut déterminer même rigoureusement les conditions, entre certains élats du corps et certains élats cẻrébraux et psychiques, de sorte que lorsque les conditions physiologiques viennent a changer', l'orientation de l'esprit change également, les souvenirs même s'effacent et disparaissent en dépit de la contiguïté et de la similarité. Lor'sque l'organisme revient au contraire it son état premier, le souvenir reparait et l'orientation générale de l'esprit redevient ce qu'elle était avant. On comprend que cette brusque interruption de sourenir ne se produise pas toujours, car les systemes psychiques peuvent ctre rappelés a l'activité selon la loi de finalite par d'autles systemes psychiques, avec accompaguement peut-être d'un ćat des organes

1. Voyez Hack Tuke. Le corps et lesprit. Traduction franc, de M. Parant Maudsley, Pathologie de l'esprit, trad. franc. He M. Germont, ป.. 31 "t suiv. (Influence des organes sur le rêve). Cabanis. Rapport d" physique et du moral, etc.

2. Musso. La Paura. 
quelque peu différent, mais le fait se produit assez souvent pour qu'on puisse l'envisager comme répondant réellement à une loi naturelle dont les manifestations, comme celles de beaucoup de lois, peuvent varier de formes et ètre quelquefois difficiles à reconnaître. Il est essentiel de remarquer, d'ailleurs, qu'il ne s'agit ici que de cas exceptionnels, ou d'adaptations cachées et très complexes; d'une nanière générale la systématisation de l'esprit et de l'organisme est si évidente, si forte et si complexe, que éest une banalite de le constater. Mais elle se produit souvent et probablement toujours, alors même qu'on ne saurait à première vue la reconnaître.

C'est sans doute dans cette classe de coordinations organico-psychiques que rentrent, à quelque degré, certains phénomènes de l'hypnotisme. On voit que bien souvent les sujets hypnotisés ont ouhlié au réveil tout ce qui s'est passé f,endant l'état somnambulique, et ne peuvent rien se rappeler, à moins que les phénomènes de cet état ne soient rappelés selon l'association systématique qui rattache d'un autre côté les phénomènes de somnambulisme aux phénomènes normaux ${ }^{1}$. Dans un nouvel accès, au contraire, ils se souviennent parfaitement de ce qu'ils ont fait pendant l'état somnambulique précédent. On peut rapprocher de ces faits le cas de Félida $X$., la malade bien connue du docteur Azam. Le fait que Félida; à l'état de condition scconde, se rappelle ce qui a eu lieu dans l'intervalle de ses accès, peut recevoir un commencement d'explication, bien imparfait encore, si l'on admet avec $\mathbf{M}$. Azam que cet état s'accompagne d'une suractivité circulatoire des centres nerveux et qüil est en somme supérieur à l'état normal. D'ailleurs les somnambules ordinaires se rappellent, à l'état hypnotique, les phénomènes de la vie

1. Voir en particulier Delbœuf. Art. cité, Revue philosophique, janvier 1886 . 
normale, peut-être simplement parce que ces faits sont en association systématique arec les impressions que l'on excite en elles; peut-être encore chez elles l'idée provoquée est-elle toute puissante, ou du moins plus puissante qu'i l'état normal, et éveille-l-elle facilement tous les faits psychiques qui peuvent s'accorder arec elle, probablement à cause d'un affailulissement général des tendances non éreillées qui n’exercent pas leur force inhibitoire ordinaire. Mais il résulterait de la que les somnambules doivent oublier tout ce qui se rapporte a elles tant qu'on ne le leur rappelle pas ou qu'on ne le leur suggère pas, et c'est bien lả en rẻalitè le phénomène qui semble constituer l'automatisme et la passivité observés pendant l'état de somnambulisme. Ainsi il semblerait que les phénomènes ordinaires à l'état normal sont éveillés pendant le somnambulisme exactement comme les phénomènes du somnambulisme sont rappelés pendant l'état normal, sauf que ceux-ci aryant été associés à un ètat organirue tout particulier et ayant été heaucoup moins mêlés que les autres à la vie psychique complète, c'est-a-dire s'etant associés à un nombre beaucoup moins considérable de tendances, sont réreillés bcaucoup plus difficilement, à moins que cet éveil ne soit facilité parle retour du même ètat organique qui a accompagne leur production.

D'autres exemples intéressants de la part du sens du corys, de l'etal des organes dans le jeu de l'association systématifue, sont fournis par les rêves ordinaires. Nous avous eu a sigrmaler la facilité avec laquelle les rèves sont oublics. D'autre part un même état dess organes produit les rèves analogues. On en tronvera de nombreux exemples dans l'ourrage de M. Maudsley que j'ii déjả cité, et si l'on considrere que la répélition d'un mème état est le fondencut nône du souvenir on sera porte a rapprocher ces lints de reux que je vieus de citer. "Un repras lourd et indigr:se ] nis fuclque temps avant de se mettre au lit, 
est une cause bien connue d'une forme de cauchemar dans lequel ou rêve qu'on a sur la poitrine une montagne ou un monstrc qui l'écrasent par leur poids. Il n'est pas facilc de dire si le rêve est l'effet direct de l'action sur le cerveau de l'estomac distendu ou de la gène des fonctions des poumons ou du cœur; mais quel quc soit le mécanisme intime, il est intéressant de noter que l'interprétation mentale de l'oppression est en rapport avec sa cause »1. Un fait d'un autre genre, mais qui rentre bien dans notre loi, c'est le rève continué d'une nuit à l'autre. M. Yung en a cité un qui s'est continué trois fois de suite 2 . De même on sait que l'ivresse donne, selon le degré où elle est menée, un certain ton à l'esprit, unc orientation particulière qui pour un même vin, et pour une même dose, est, dans des circonstances équivalentes, sensiblement la mème chez une mème personne. Souvent le caractère est notablcment modifié, les facultés intellectuelles se transforment aussi, il y a un nouvel arrangement, une coordination différente des éléments psychiques, lc changement des ètats organiques modifie jusqu'à un certain point la personnalité. Un fait produit par une certainc personnalité organique ne peut êtrc rappelé facilement que par la même personnalité organique. M. Ball en cite, d'après Beard, un cas intéressant: Un employé de commerce, venu a New-York pour s'occuper des affaires de son patron, se grisa complètement; pendant trois ou quatre jours il parcourut la ville, s'occupant des commissions qui lui avaient été confiées, ct lorsque l'ivresse fut dissipée il n'avait aucun souvenir de ce qu'il avait fait, et croyait avoir à recommencer sa tournée ${ }^{3}$. On sait que

I. Maudsley, Pathologie de l'esprit. Trad. française de M. le $\mathrm{D}^{\mathbf{r}}$ Germont, p. 36 .

2. Yung. Article sur le sommeil. Rerue internationale des sciences, 1882, p. 494.

3. Ball. Leçons sur les maladies mentales, 
certaines affections organiques comme la phtisie, paraissent donner à l'esprit une orientation particulière.

Les maladies du cerveau et du systeme nerveux offrent des faits très intéressants à notre point de vue, par cxemple la ressemblance des accès d'une maladie chez une même personne. "Il est des malades, d'aprés Dagonet, qui commettent, chaque fois qu'ils reprennent leurs habitudes de boisson, les mêmes actes, les mêmes tentatives de suicide "1. M. Ball cite des faits analogues a propos des dipsomanes 2. M. Ritti, à propos des folies intermittentes, note qu'un de leur's caractères les plus importants est que « l'accès ultèrieur, ainsi que l'a dit Falret, ressemble en tous points, par les symptòmes psychiques, intellectuels et moraux et même par les idées délirantes exprimées, ainsi que par sa marche, aux accès précédents. Le malade passe habituellement par toutes les phases de son état antérieur. Ce caractère s'observe aussi dans la folie à double forme, tous les accès sc ressemblent à tel point que sil'on en a observe un avec soin, on retrouve dans ceux qui suivent les mêmes symptômes, les mêmes conceptions d'éléments, les mêmes actes, la même marche, etc. La durée de chaque accès ne présente que peu de variations. Aussi toutes ces conditions permettent-clles, si les accès antérieurs ont été bien observés, d'indiquer, pour ainsi dire d'arance, dès le début de l'accès, les différentes manifestations qui vont se produire, et même d'assigner' l'époque à laquelle le malade passera d'une période à l'autre ${ }^{3}$ D. On sait la part que M. Maudsley attribue au tempérament épileptique dans la formation de certaines irlées religieuses. "Le tempérament épileptique par luimeme, abstraction faite des accidents convulsifs habituels,

1. Ditgonet. De l'alcoolisme, p. 50 .

2. Ball. Orev. cité, p. 672.

3. Kitti. Traite clinique de la folke a double forme, p. 4. Voir aussi p. 178 . 
semble particulièrenent favorable a cet enthousiasme absorbant, dans lequel l'esprit élevé au dessus de luimème, par une sorte d'extase ou d'exaltation de toutes ses énergies, fait naître en lui l'opinion l'un divin, d'une. possession divine $n 1$. Des faits très curieux sont ceux auxquels l'hérédité prend part. M. Ritti a rapporté I'histoire d'une malade atteinte de folic a double forme, dont la tante avait, elle aussi, passé son existence dans les alternatives d'excitation et le tristesse; " on a même remarqué, ajoute-t-il, que $\mathrm{M}^{\mathrm{me}} \mathrm{X}$..., dans les diverses phases de son état circulairc répète les mèmes choses, fait les mèmes actes que sa tante ». Dans la maladic désignée par M. Krishaber sous le nom de Névropathie céribro-cardiaque, on trouve encore de bons cxemples de l'influence de l'ètat de l'organisme sur le ton génèral de l'esprit et du changement d'orientation mentale qui suit un trouble psychique, bien que les faits ne s'expliquent pas entièrement par les modifications de la cénesthésic. Les malades gardent leur intelligence intacte, mais ils trouvent leur personnalité changèe, les objets ne font plus la même impression sur eux, quelquefois " ces sensations sont si profondément perverties, si différentes de celles de la vie normale, que le malade conçoit des doutes sur la réalité des choses qui l'entourent, voire mème sur l'identité de sa propre personne, mais sa mémoire et son jugement étant rèstés debout, il se rappelle ses sensations exactes, les compare et comprend qu'il est en proie à des illusions multiples et incessantes " 2. Il arrive que le malade commence à avoir des doutes sur sa personnalité, et que plus tard il sentira sa personnalité changée. Il dit

1. Maudsley. Natural causes and supernatural seemings, p. 407. Vuir aussi Le crime et la folie.

2. Kirishaber. Le la neuropathic cérebro-eardiaque, I, 222. Voir aussi Taine, De l'intelligence, appendice au premier volume à partir de la $3^{\text {e }}$ édition. 
d'abord a je ue suis plus moi», et ensuite "je suis un autre ». Le premier stade, comme l'a vu M. Taine, correspond au moment ò̀ "les sensations nouvelles étaient trop nourelles, elles n'araient pas été répétées un assez grand nombre de fois pour faire dans la mémoire un groupe distinct, une série cohérente, telle serait la chenille dont nous arons parlé dans le premier quart d'heure qui suit sa métamorphose en papillon" ; dans le second stade, un autre moi est formé. « Il faut du temps pour que la clienille s'habitue à ètre papillon; et si la chenille garde, comme c'était le cas, tous ses souvenirs de chenille, il $\mathrm{y}$ a désormais un conflit perpétuel et horriblement pénible entre les deux groupes d'actions ou impressions contradictoires, entre l'ancien moi qui est celui de la chenille, et le nouveau moi qui est celui du papillon ». Nous résumerons le fait, à notre point de vue, en disant, qu'une ancienne forme d'association systématique est en train de se défaire dans le premier stade, que dans le second on voit surtout l'organisation d'une forme nouvelle.

Tous ees faits et bien d'autres qu'il est impossible même de rappeler ici nous montrent une relation étroite entre l'état organique et l'état mental. Nous voyons que l'association psychologique, outre les phénomènes de conscience et les processus cérébraux qui leur correspondent comprend aussi un grand nombre d'élements organiques. Il semble récllenent que à chaque penséc, à chaque émotion, à chaque fait jsychique correspond ou tend a colrespondre un état spécial non-sculement du cerveau et descentres nerveux, mais de l'organisme entier el réciproquement. Voici pour finir deux cas d'adaptation du corps à l'idée ou d'adaptation réciproque de l'esprit et du corps qui m'ont par'u particulièrement intéressants el ıui peuvent par leur forme bizare mettre mieux en lumière la loi que nous étudions. .'enıpunte le prenier à M. Hack'T'nke: Un enfant fut mortu it la jambe par un petit terrier. La blessure 
était lègère et guérit sans difficulté, le chien ne parut présenter aucun signe de rage, mais deux mois apres environ, l'enfant se plaignit d'une forte douleur à l'endroit mordu. Cette douleur prit ensuite la forme d'une aura épileptique. La semaine suivante l'enfant dit que, au moment où l'aura gagnait l'abdomen, il avait eru y sentir le chien qui l'arait mordu et qui le déchirait avec force. - Pendant cet acces, il aboya et son visage prit une expression de doute, de douleur et d'égarement. La salivation marqua la fin de l'accès. Le lendemain, il voulut mordre et égratigner tous ceux qui lapprochèrent, et imita quelques-uns des actes et des mourements d'un chien. Ainsi il prit son oreiller avec les dents et se mit à grogner comme un chien qui tient un rat. Il se mit à refuser la nourriture à moins qu'on ne lui permit de la laper. D'autres fois, on le menaçait de coups de fouet s'il ne cessait daboyer, il tournait alors en rond sur lui-mème et criait comme un chien battu.... Cet enfant finit par guérir ${ }^{1}$.

L'interprétation de ce fait peut paraître discutable. On peut attribuer une grande partie de la systématisation singulière qui s'établit entre l'esprit et le corps à une sorte d'auto-suggestion. Cette interprétation laisse subsister d'ailleurs une grande partie de l'intérèt du fait, mème à notre point de vue actuel, car l'influence de l'esprit sur l'organisme ou mème de l'organisme sur l'esprit peut toujours ètre considérée si l'on veut comme une auto-suggestion et ce qu'il s'agit de rendre vraisemblable, c'est précisément qu'il $y$ a toujours auto-suggestion à quelque degré, que toujours le corps et l'esprit, ou si l'on préfère, le cerveau et le reste de l'organisme s'adaptent continuellement el que tout état de l'un modifie plus ou moins l'état de l'autre.

1. Hack Tuke. Le corps et l'esprit. Traduction française de M. V. Parant, p. 145. 
Un cas plus complet encore et non plus accidentel et passager, mais permanent, est celui que rapporte Pinel d'une idiote de la Salpètrière qui " par la forme de sa tête, sa gaité, sa manière de vivre, scmblait se rapprocher de l'instinct d'une brebis. Pendant deux mois et demi qu'elle a resté a l'hospice de la Salpètrière, elle marquait une répugnance particulière pour la viande et mangeait avec avidité les substances végétales, comme poires, pommes, salades, pain....; ; elle ne buvait que de l'eau et témoignait une reconnaissance vive pour tous les soins que la fille de service lui prodiguait; ces démonstrations de sensibilité se bornaient à prononcer ces deux mots: bé, ma tante, car elle ne pouvait prononcer d'autres paroles, et paraissait entièrement muette par le seul défant d'idées, puisque d'ailleurs sa langue semblait conserver toute sa mobilité ; elle avait aussi coutume d'exercer des mourements alternatifs d'extension et de flexion de la tête, en appuyant, à la manière des brebis, cette partie contre le ventre de la même fille de service, en témoignant sa gratitude. Elle prenait la mème attitude dans ses petites querelles avec d'autres enfants de son àge, qu'elle cherchait à frapper avec le sommet de sa tête inclinée. Livrée à un instinct aveugle qui le rapprochait de celui des animaux, elle ne pouvait mettre un frein à ses mourements de colère.... On n'a jamais pu parvenir a la faire asseoir sur une chaise pour prendre du repos ou faire ses repas, et elle dormait le corps roulé et étendu sur la terre à la manière des brebis. Tout son dos, les lombes et les épaules étaient couverts d'une sorte de poil flexible et noiratre, long d'un pouce et demi ou de. deux pouces et qui se rapprochait de la laine par sa finesse.... 1 .

1. Pinel. Traité médico-philosophique sur l'alienation mentale, seconde edition, pages 182-184. 
On peut rapprocher de ces phénomènes les histoires de mystiques conme Louise Lateau. Mais le cas de Pincl est plus probant encorc, bien que, dans lignorance oi l'on est des causes qui ont produit les anomalies en question, il soit difficile d'en tirer tout le parti qu'on voudrait. Mais il me semble prendre une certaine signification si on le rapproche de tout co que nous savons des corrélations des organes chez les animaux. Une partie de l'organisation animale se comprend fort bien au point de vue de la systématisation organique, par exemple le rapport entre les dents et l'appareil digestif et la forme ou la dimension des membres; d'autres corrélations paraissent donner à l'animal une unité particulière dont le sens nous échappe. Le fait cependant qu'un phénomène ne peut, dans un être organisé, se produire sans amener la production d'une autre série de phénomènes toujours les mêmes qui concourent arec lui à former la nature d'un animal, nous conduit logiquement à admettre que ces phénomènes sont unis par un lien; qu'ils ont soit une cause unique, comme, par exemple, le manque de pigment qui fait que les lapins blancs ont les yeux rouges, soit une mème fin, comme lorsque la variation d'un organe entraîne corrélativement la variation d'autres parties du corps, de manière à donner à la systématisation générale de l'animal une forme différente. Il faut citer aussi, comme des exemples remarquables de systématisation organique ces faits qu'on a réunis sous la dénomination bien imparfaite d'influence directe du milieu. Ces cas se ramènent à une systématisation organique déterminée par l'ensemble des perceptions; des tendances latentes s'éveillent, et ce sont celles qui peurent former avec le milieu un système plus complexe. Il arrive ainsi que la nature de l'ètre semble changer complètement, c'est-à-dire que les éléments varient et que la forme de leur coordination varie aussi très fortement. Dans un lac des environs 
de Mexico certains animaux, les axolotls, vivaient, se reproduisaient et mouraient sous forme de têtards à branchies extérieures. On en rapporta un certain nombre à la ménagerie du muséum d'histoire naturelle de Paris, et, la , on s'aperçut que plusieurs d'entre eux se transformaient completement; leur's branchies s'atrophiaient et ils devenaient un animal amphibie respirant par des poumons. De mème, mais inversement: "Les tritons vivant dans l'eau pendant la première période de leur vie, respirent par des branchies, plus tard ils se tiennent habituellement sur le bord des mers; les branchies disparaissent, les poumons les remplacent. Cependant si lon force ces animaux à rester dans l'eau, la métamorphose ne s'accomplit pas if 1. Dans l'Origine des espèces, Darwin cite une grande quantité de ces corrélations qui modifient plus ou moins, dans certaines circonstances, la persomnalité organique 2. Les métamorphoses des insectes sont également intéressantes à notre point de vue, le phénomène est ici extrêmement complexe, une personnalité se dissout, une autre persomnalité prend sa place - ce qui nous importe pour le moment, et ce qui est si évident qu'on ne songe pas à le remarquer, c'est que la personnalité psychique suit exactement la personnalité physique, les instinicts de la larve et de linsecte parfait correspondent exactement à leur état physique. Si les hypothéses de sir John Lubbock sur l'origine des insectes peuvaient être vérifiées, l'importance de leurs métamorphoses deviendrait encore plus considérable, eu égard à la loi que nous étudions ici ${ }^{3}$. C'est que l'àme est essentiellement la systématistion des actes du corps. Cette vérité nous est masquée dans l'homme où le rôle immense joué

1. Charles Martins. Lamarck, sa vie et ses cuvres.

2. Darwin. Origine des especes.

3. Sir John Lubbock. Métamorphose et origine de's insectes. 
par la forme des séries d'actes par rapport ì la forme physique des organes qui les accomplissent, nous empèche de voir les relations des systèmes psỹchiques et de la constitution organique. L'homme est un être social et sa psychologie ne peut être comprise si on fait complètement abstraction du milieu dans lequel il vit et dont il est un élément. L'animal, au contraire, est plus conıplet considéré en lui-même, et la plus grande partie de sa mentalité — surtout si l'on fait abstraction des relalions sexuelles et de la protection instinctive ou réfléchie donnée aux petits - est simplement la systématisation des fonctions organiques, et n'a d'autre but que l'accomplissement de ces fonctions. La coordination en vue de la société nous empêche de voir suffisanment chez l'homme la coordination psycho-organique. Les faits que jai cités tout à l'heure, sont de nature, je crois, à nous en faire soupçonner l'importance et la force - mais il me semble que nous la comprenons mieux en la constatant chez les animaux.

Nous pouvons même descendre plus bas sur l'échelle de la mentalité, les plantes nous offrent aussi d'excellents exemples pouvant rendre compte de la personnalité organique et de ses variations. Les rapports de position de la tige et de la racine, par exemple, sont toujours les mêmes. Il y a là une sorte de coordination organique qui est comme une forme très basse de la mentalité. Si on retourne une plante qui pousse de manière à mettre la racine en l'air, la racine et la tige se recourbent et reprennent la direction voulue. On sait qu'un même élément, la feuille peut manifester des tendances bien différentes, se transformer selon le cas, en sépale, en pétale, en pistil, en étamines, etc. La plante entière subit des modifications si on change ses conditions d'existence, et ces changements s'accomplissent de manière à conserver toujours une coordination bien marquée de ses 
diverses parties. A mon avis, nous ne sortons pas même ici de la psychologie, - la systématisation générale des acłes et des tendances d'un ètre est son domaine essentiel.

Si j’ai tant insisté sur la personnalité organique, c'est uu'elle est une part considérable de la systématisation totale de l'individu, e'est ensuite que je tenais à montrer comment la personnalité physique était liée à la personnalité psychique, comment la moindre de nos pensées, de nos idées, le sentiment le plus fugitif peut aussi ètre systématiquement associé, par un lien que nous ne soupçonnerions pas à première vue, avec l'état de nos organes et de leurs fonctions, du cœur et de la circulation, de l'estomac et de la digestion, des poumons et de la respiration, etc. Sans doute cette association systématique n'est pas toujours bien étroite, elle n'est pas indissoluble, et elle peut être rompue au profit d'associations psychiques mieux coordonnées encore mais elle existe surtout comme tendance, et il en résulte que la persomulité psychique et le sens du corps jouent un rôle darrs la mémoirc, dans la reproduction des idées et des sentiments, et peuvent servir à expliquer l'apparition, daus certaines circonstances, de certains phénomènes psychiques que nous ne pourrions comprendre si nous ne faisions attention qu'aux phénomènes de la conscience, ou aux tendances surtout psychologiques, c'est-à-dire, pour presque tous les cas, surtout sociales.

En somme, la personnalité physique est, comme l'a dit II. Rihot: la base de la personnalité psychologique. Elle coustitue i elle seule une notable portion de l'individu. La colrélation des organes détermine pour une part ntable la corrélation des instincts, et mème des phénomenes fsychiques: idees, sentiments, passions, volitions, tendances, etc. Cela est vrai surtout pour tout ce qui dans l'esprit se rapporte à la vie de l'individu. Cette corréhation entro lo pychique et le physique peut aller jusqu'a 
la coordination de certaines idées avec certains états organiques de manière a pouvoir expliquer des cas obscurs de l'association des idées et de la mémoire des faits psychiques, par la considération des étais organiques qui ont accompagné ces phénomènes psychiques et ont été associés avec eux.

\section{$\S 3$.}

La personnalité organique, tout en restant toujours à peu près identique à elle-même pendant une période donnée, varie de mille manières, avec l'àge, les maladies, etc. Mais pendant même qu'elle reste la même ou du moins qu'elle ne varie que d'une manière peu sensible, diverses personnalités psychicques peuvent s'appuyer sur elle, et surtout disposer d'elle, c'est-à-dire la faire entrer dans telle ou telle forme de coordination générale. Je veux dire que, dans des circonstances ordinaires, un homme normal, pourra sans que l'état de ses organes varie d'une manière appréciable, être magistrat ligoureux ou père de famille débonnaire. Il est bien probable, d'après ce que nous venons de dire, que des modifications organiques correspondent à ces manifestations psychologiques, mais, pour le moment, nous les négligerons pour ne nous occuper que des systématisations psychologiques elles-mêmes, c'est-à-dire des coordinations diverses dans lesquelles l'organisme peut être employé.

Chacun de nous a un certain nombre de grands systèmes de tendances dont la réunion forme sa personnalité complète et chacun de ces systèmes est constitué essentiellement par quelques éléments psychiques dominants auxquels sont associés un grand nombre de tendances et de phẻnomènes divers. Ces systèmes généraux se supplantent l'un l'autre selon les circonstances, et leur 
collection, lorsqu'elle n'est pas relièe par une loi de coordination générale, rappelle à beaucoup d'égards les faits bien connus de dédoublement de la persomnalité. Les amnésies périodiques elles-mèmes présentent plusieurs degrés et les cas éloignés sont très différents au point de vue de la netteté de la séparation, et de la dualité ou de la multiplicité de l'esprit. Depuis le cas cité par Macnish, dans lequel on voit que même " cette mémoire organisée qui permet de parler, de lire, d'écrire, n'est pas un fonds commun aux deux états, „ jusqu'au cas du somnambule qui se rappelle dans un accès ce qu il a fait pendant l'accès précédent et jusqu'a l'ivrogne, qui, ayant perdu un paquet pendant qu'il élait ivre, ne peul le retrouver qu'en s'enivant de nouveau, en passant par le cas de Félida X., ou de la malade du docteur Dufay, on voit la scission du moi diminuer de plus en plus d'importance, et les deux vies différentes se pénètrer de plus en plus et se confondre presque. Chez l'homme à l'état normal (remarquons que l'ivrogne étalslit déjà une transition entre le malade et l'homme sain) on trouve de même une sorte de division du moi. L'homme se compose pour ainsi dire de plusieurs moi qui ont un fonds commun et se confondent jusqu'à un certain point, mais non pas complètement. On peut couper artificiellement une personnalité en plusieurs morceaux et montrer que cette division correspond à quelque chose de réel. On peut de plus, ce qui ne peut se faire pour l'état morbide, voir les causes de cette pluralité de la personne.

Cette division du moi est moins marquée dans l'état normal que dans l'ètat pathologique ; elle se caractérise d'ailleurs de la même maniere par la formation de plusieurs groupes distincts de phénomènes et de tendances et elle est peut-être plus profonde qu'on ne serait porté à le croire après un eximen superficiel. Elle porte, en effet, nonseulement sur les faits de conseience, c'est-c̀-dire sur la 
partie de l'esprit la moins organisée et la plus mobile, mais aussi sur quelques-unes de ces habitudes qui commencent à devenir à peu près inconscientes. Elle n'a pas, d'ailleurs, passé inaperçue et l'on pourrait relever dans la littérature beaucoup de passages qui l'impliquent ou la signalent, mais on a sans doute vu surtout dans les expressions employées une simple métaphore.

On comnait suffisamment les vers de Racine, le cantique qu'il tira des paroles de saint Paul ; la dualité du moi y es' énoncéc avec une clarté qui ne laisse rien à désirer :

\footnotetext{
Mon Dieu quelle guerre cruelle

Je trouve deux hommes en moi.

L'un veut, que plein d'amour pour toi,

Mon coeur te soit toujours fidèle;

Liautre, à tes volontés rebelle,

Me rérolte contre ta loi, etc.
}

Ces deux hommes que Louis XIV reconnut, d'autres personnes les connaissent aussi, et le combat qui s'élève dans l'esprit a divers propos n'est plus une chose rare ${ }^{1}$. Pensera-t-on, peut-être, que la question de la personnalité ne doit pas intervenir ici et qu'il est facile d'alléguer que c'est la même personne, la même àme qui est sollicitée par deux penchants? Examinons les faits, je trouve d'un côté l'homme pieux, celui qui croit fermement aux dogmes d'une église, à la norale enseignée par Dieu, chez qui une quantité considérable d'idées, de sentiments, d'actes, viennent s'associer harmoniquement avec cette

1. On retrouve de nombreuses traces, dans la littérature, de ce combat entre divers systèmes de tendances qui prennent généralement la forme d'une dualité. Le debat du corps et de l'cime remonte au moyen-åge, (G. Paris. La litterature française au moyen-age, p. 21\%). Cf, Le débat du corps et du cueur de Villon et dans la littérature contemporaine Tartarin-Quichotte et Tartarin-Sancho dans le Tartarin de Tarascon de M. Daudet. 
croyance. Cet homme communie, il va à la messe ou au prêche, il lit la Bible ou les Pères de l'Église, il contribue de son argent à entretenir en prospérité la communauté religicuse, il fait la charité, il prie, de temps en temps il se préoceupe du salut de son ime, il se repent de ses fautes, prend de bommes résolutions, il s'indigne contre les incrédules, etc. Il y a là tout un ensemble fort complexe de tendances, une très vaste synthèse de faits coordonnés en une même orientation qui pourrait devenir au besoin, et qui est devenue quelquefois, la caractéristique principale d'une personnalite, la tendance maîtresse ver's laquelle convergent tous ou presque tous les phénomènes de l'esprit et de l'organisme, si bien que l'extéricur même, l'habitude du corps, le regard, le geste, finissent par s'harmoniser et se subordonner à la manière d'être de l'esprit. Mais quelquefois, à côté de cette tendance, il peut y en avoir d'autres qui ne s'accordent pas avec elle et qui tiennent dans la personnalité totale une place égale à la sienne, le croyant peut ètre ambitieux, avide du pouvoir politique comme Richclieu, il peut être porté á l'anour comme Louis XIV, et nous trouvons alor's deux groupes de tendances très diverses et parfois opposées qui subsistent et se développent sur le même organisme. Chacunc de ses personnalités psychiques se suffit à ellemème, elle a ses lois propres et ses modes d'association secondaire particuliers. Ce qui dans l'une s'associera ádes sentiments de honte ou d'indignation, s'associera daus l'autre a des sentiments de desir violent, d'orgueil et de plaisil.

Ces personnalités psychiques rliférentes qui ne disposent que d'un organisme, ne peurent s'entendre sur la manière de le faire agir, aussi les conflits sont fréquents et la "guerre cruelle ne déclare. Mais la lutte des groujes de tendances n'est pas le seul fait qui témoigue de retle division de la personne humaine. Elle so montre 
également par les successions périodiques de deux systèmes diffẻrents de tendances et de phénomènes qui tour a tour dominent et font agir le corps. Il est assez fréquent de dire ou d'entendre dire qu'il $y$ a deux personnes chez un individu désigné : l'homme et le magistrat, par exemple, ou le professeur, l'homme privé et l'homme public. Ici encorc c'est la même personne, si l'on veut, qui est à la fois homme privé et homme public. Mais ces deux moi, que l'on soude plus ou moins artificiellement en une individualité vivante et concrète, n'en sont pas moins des groupes de faits et de tendances différents, sẻparément associés entre eux dans chaque groupe et non associés d'un groupe à l'autre. Ce manque d'association se traduit par ce phénomène que l'un de ces groupes ne sait généralement pas ce que fait l'autre, il l'oublie ou l'ignore; une personne en qui domine momentanément une de ces sous-personnalités est généralement surprise et souvent froissée si on lui rappelle ce qu'elle a dit ou fait alors qu'elle était dominée par un système de tendances différent, alors qu'elle etait, pour ainsi dire, un autre moi. C'est que la condition même du réveil des phénomènes psychiques, la finalité, manque très souvent d'une manière évidente dans les rapports des personnalités soudées sur un même corps. Pour la mème raison la connaissance d'une des personnalités d'un individu ne nous apprend rien sur les autres, nous pourrions savoir que César ètait ambitieux et ignorer qu'il fut débauché. Chaque sous-personnalité est formée d'un nexus de tendances rqui se lient les unes aux autres et peuvent se déduire jusqu'á un certain point les unes des autres, mais qui bien souvent ne se lient pas aux tendances d'un autre groupe et ne se déduisent pas d'elles. La théorie de la faculté maitresse et du fait dominateur peut etre vraie pour chacune des sous-personnalités sans s'appliquer aussi bien à leur ensemble. 
Bien entendu, ce que l'on troure chez l'homme ce n'est pas seulement deux groupes de faits, deux centres, deux complexus de tendances qui se combattent ou se succèdent et se remplacent par moments. Nous sommes plus compliqués que cela. A vrai dire, on ne peut savoir quel est le nombre de moi que contient un individu. Lhomme religieux et l'impie, l'homme public et l'homme privé, ne constituent pas une personnalité complète ; on troure chez un homme le moi du monde et le moi de l'intérieur, le moi de la ville et celui de la campagne, le moi des réunions d'amis, etc. Ces différentes personnalités se croisent, s'associent parfois les unes avec les autres, et les unes contre les autres. Lhomme public peut être religieux, tandis que l'homme privé est indifférent, mais souvent il ne se ramène pas à une unité supérieure, ct l'on ne peut attribuer à la personne complète ou considérée comme telle presque aucune qualité. Nous arons vu dans le premier chapitre de ce travail jusqu'où peut aller le morcellement de l'esprit, nous indiquerons plus loin jusqu'où peut aller son unité. Mais l'unité parfaite, au point de rue psychique, n'est guère qu'une conception idéale, qui n'existe que d'une manière bien fugitive, si l'on peut admettre qu'elle se produit parfois.

Nous constatons ainsi que c'est la loi d'association systématique qui est l'expression du rapport général des phénomènes psychiques et des tendances dańs les souspersonnalités que nous venons d'examiner en ellesmèmes et toutes formées. Si nous étudions la manière dont elles se forment et aussi les circonstances de leur apparitiou dans le cours de la rie, nous retrouverons encore la même loi comme fait essentiel. Il n'est pas difficile de voir yue dans la vie normale, ordinaire, certaines causes bien determineses doivent agir presque toujours de manirire à froduire différents systèmes de groupes psychipues, par un whangement que, nous pouvons obscrvel 
dans les conditions d'existence de l'individu et par l'effet des associations harmoniques diverses produites par des ensembles de perceptions, des conseils, des renseignements, des exemples, et de manière à amener la naissance et le développement des groupes psychiques et psychophysiques qui se développent parallèlement, quoi qu'ils paraissent ètre parfois en contradiction les uns avec les autres, qui s'ignorent parfois et n'entrent pas toujours en conflit. Ces influences plus ou moins constantes, plus ou moins longues, plus ou moins fortes, plus ou moins rẻgulières, ce sont l'éducation morale, l'éducation religieuse, le milicu moral, intellectuel ou physique, la profession, etc. Chaque perception, chaque idée, chaque sentiment nouveau qui nait dans le cerveau, suggéré ou communiqué par les diverses influences extérieures, tend à produire une systématisation de l'esprit dans un certain sens, et des impressions systématisées tendent à susciter des systèmes d'idées et de tendances. Chaque influence crée ou développe directement ou indirectement en nous diverses coordinations psychiques, ces coordinations en entrainent d'autres à leur tour et l'on voit ainsi se développer chez un même individu plusieurs petits moi psychiques reposant sur un fonds organique commun, mais relativement très séparés les uns des autres. Certains sarants se vantent, de laisser l'homme religieux à la porte de leur laboratoire. Voilà un exemple de scission opérée dans une individualité par l'influence de conditions d'existence différentes, peut-être avec l'aide de l'hérédité. Diverses influences tendent à donner à un homme certaines tendances religieuses : c'est peut-être l'hérédité, c'est sùrement pour un grand nombre l'éducation, le désir d'expliquer l'inconnu, les relations sociales, les besoins du cocur, l'imitation, etc. D'autres influences tendent á développer l'esprit ḋexamen et le scepticisme à l'ègard de ce qui n'est pas scientifiquement démontré ètre 
vrai ou probable : la réflexion, l'hahitude des recherches scientifiques, la considération des erreurs commises par nos devanciers et par nous-mèmes sur un grand nombre de points pour aroir jugé trop rite, etc. Voila donc deux tendances opposées, l'esprit critique et la foi qui naissent et se développent séparément, chacune ayant ses habitudes propres, chacune ayant son organisation et respectant celle de l'autre. Elles sont nées par adaptation d'une partie de l'esprit à des conditions d'existence, c'est-it-dire par la force de systématisation de groupes de perceptions rariées et leur coordination arec les tendances acquises, et elles vont se développant, se fortifiant et s'enrichissant chaque jour, l'une par la continuation des habitudes de piété, pal tout ce qui peut contribuer à rendre la foi solide et agréable à l'esprit, par les épreuves qui en font une consolation précieuse, par les controverses bénignes qui la rendent plús cohérente et plus capable de résister, l'autre corrélativement par de nouvelles découvertes, par des perfectionnements de la méthode, par l'exercice fréquent de l'intelligence qui acquiert plus de précision et de vigueur. L'unité complète du moi exigerait que de ces deux tendances, par exemple, l'une fùt subordonnce à l'autre (philosophia ancilla theologie) ou au moins dùt se mettre daccord avec elle, ou bien encore que l'une des deux disparùt. Mais en général ce n'est pas ce qui arrive. Tel homme de science se vante d'avoir la foi du charbonnier en matière de religion et d'être en revanche très difficile à contentel' en fait de preuve scientifique. Royer-Collard a prétendu a tort qu'on ne faisait pas au scepticisme sil part. On la lui fait presque toujours, et chez nous tous, tant que nous sommes, mème chez les plus sceptiques, il est bien rare qu'il n'y ait pas un point faible, un coin de croyances et d'illusions que nous abandonnons ì la foi et où nous ue laisserons pas pénétrer le doute, soit que nous ne.le 
voulions pas, soit que nous ne pensions pas a faire la critique nécessaire.

Mais ce qui nous importe ici, c'est la loi d'association systématique que nous voyons à l'œuvre dans le développement de chaque personnalité. La même loi se retrouve encore dans la succession des sous-personualités dans le cours de la vie normale. Généralement la personnalité qui se manifeste est celle qui a intérêt à se Inanifester, celle qui peut profiter des circonstances présentes, nous royons le caractère d'une personne changer d'un moment à l'autre si elle change de milieu. Le plus simple des hommes peut devenir solennel aussitôt qu'il a mis un vètement de forme particulière. Un père est facilement austère et vertueux devant ses enfants, mème s'il ne l'est pas partout ailleurs. Nous avons tous quelque chose de l'axolotl, il y a en nous des moi que nous ignorons et que les circonstances peuvent réveiller. Un incroyant peut ètre fortement saisi par une cérémonie religieuse et sentir s'éveiller ou se former en lui un moi qu'il a perdu ou qu'il n'a connu jamais, l'incrédulitė mème peut ètre une circonstance favorable. Les cérémonies catholiques impressionnent quelquefois plus un incroyant peu habitué à elles et par conséquent plus frappé par la grandeur de l'ensemble, que des fidèles habituels choqués plus ou moins par des détails vulgaires; un moi sympathique, embrassant un grand nombre de tendances, respect du mystère, admiration, sentiment de l'immensité du monde, réflexions sur la destinèe, combinées avec des impressions physiques, obscurité relative de l'église, lueur des cierges, lumière affaiblie des vitraux, odeur de l'encens - s'éveillera peut-être chez lui, quoique d'une manière passagère, plus facilement que chez celui pour qui le fait d'assister à la mème cérémonie est une habitude machinale.

Aussi arrive-t-il que les influences exercees sur la 
nature primitive de l'individu par des milieux diffèrents, par des situations variées, déterminent la manifestation de qualités absolument opposées. On peut être timide comme homme dans le monde et fort décidé comme chirurgien sil faut faire une opération sur une personne qu'on n'aborderait pas sans embarras dans un salon. Il est des gens qui sont insupportalyles dans leur famille et charmants hors de chez eux. Ils passeront pour hypocrites et feront parler de dissimulation volontaire, mais plutôt il y a deux personnalités soudées lune a l'autre, ei s'il y a eu un moment dissimulation voulue, il est très possible que cette dissimulation cesse bientòt. L'habitude est une seconde nature.

Aussi, a moins de connaître la vie entiere d'une personne, à moins de pouvoir préciser les influences ancestrales qui ont agi pour former ses dispositions innées et celles rui ont agi sur elle depuis sa conception, ne saura-t-on jamais tout a fait ce qu'elle est. On ne peut deviner tous les groupes psychiques qui existent virtuellement en elle, tous les modes d'association que peuvent prendre ses états psychiques, et il est parfois impossible de savoir comment tel individu agira dans telle ou telle circonstance oủ il ne s'est pas encore trouvé, car la systématisation amenée par ces circonstances variera évidemmment selon la nature de causes internes qui nous echappent. Qu'on remarque que chacun de nous a en lui, à diverses profondeurs, sept ou huit sous-personnalités qui, à un instant donné, peuvent être momentanément équivalentes à ume personnalité définie; outre celles dont nous avons parlé jusqu'ici, il faut tenir compte de celles qui nous ont été transmises par nos ancêtres; il est an fond de chaque homme civilisé un sauvage et probablement une brute qui se réveillent à l'occasion.

A voir les choses comme elles sont généralenent, l'homme n'est pas un, il est plusieurs, et il est plusieurs 
parce qu'il a été façonné par des séries diverses, par des ensembles tout à fait différents de circonstances extérieureś qui, par l'intermédiaire des perceptions, ont déterminé la formation de systèmes non convergents ou opposés et que la force de son organisation a été insuffisante pour unifier et coordonner tous ces contrastes. On voit que les variations de la personnalité à l'état normal sont, à beaucoup d'égards, un phénomène sociologique. Bien des gens doivent se contenter - ils ne s'en plaignent pas toujours - de cette coordination morcelée que nous avons étudiée. Il existe chez eux un certain nombre d'orientations générales de l'esprit, de sous-personnalités qui entrent parfois en conflit, mais qui souvent ne s'aident ni ne se nuisent directement, qui présentent seulement un désaccord logique. Mais si nous considérons en ellesmêmes ces sous-personnalités nous royons qu'elles consistent essentiellement en une coordination d'un nombre toujours très considérable d'éléments psychiques, unis étroitement par des associations systématiques, et que c'est notre loi qui préside soit à leur développement dans l'individu, soit à leurs manifestations successives.

\section{$\S 4$.}

Il ne nous reste plus maintenant pour terminer cette revue des faits psychiques qu'à examiner brièvement la personnalité considérée dans ses formes les plus hautes. Ici nous n'avons plus, - ou nous ne devrions plus avoir, si l'idéal était de ce monde, - à parler de luttes, de conflits, d'illogisme ; ici l'homme entier est ou devrait être un seul élément psychique extrèmement complexe et pourtant indécomposable, j'entends ne pouvant ètre décomposé sans perdre absolument ce qui le fait être lui-même, comme une machine dont tous les rouages sont essentiels et qui ne peut ètre comprise dans son fonctionnement 
synthétique que par la considération des réactions de toutes ses parties les unes sur les autres et de l'unité finale qui en résulte. De même que la sensation, la perception: l'idée, le jugement, la tendance, le groupe de tendances sont des systèmes complexes d'éléments inférieurs qu'on ne peut dissocier sans faire perdre au phénomène supérieur son caractère synthétique, comme l'eau cesse d'être de l'eau pour n'ètre plus que de l'oxygène et de l'hydrogène si on la décompose, la per'sonnalité une embrasserait tous les éléments de l'organisme, elle serait la forme qui les dirige vers une fin unique ou vers des fins convergentes. Nous n'avons qu'à appliquer ici à la psychologie la théorie de Claude Bernard en physiologie générale. La personnalité, l'àme, le moi, l'esprit, est essentiellement une idée directrice (en prenant le mot idée en un sens un peu particulier), une forme de l'arrangement des phénomènes organiques et psychiques.

Je n'insisterai pas sur ce fait que la personnalité est une coordination, la thèse me parait dès maintenant suffisamment établie, on ne peut guère que convenir avec M. Ribot que "l'unité du moi n'est donc pas celle de l'entité une des spiritualistes qui s'éparpille en phénomènes multiples, mais la coordination d'un certain nombre d'états sans cesse renaissants, ayant pour seul point d'appui le sentiment vague de notre corps. "

"Cette unité parfaite existe-t-elle? se demande $\mathbf{M}$. Rifot. Au sens rigoureux, mathématique, évidemment non. Au sens relatif, elle se rencontre rarement el en passant. Chez un bon tireur (jui vise, ou un habile chirurgien qui opère, tout converge physiquement et mentalement. Mais notons le résultat: dans ces conditions, le sentiment de la personnalité réelle disparait, l'individu conscient étant réduit à une idée ; en sorte que la parfaite unité do conscience et le sentiment de la personnalité s'excluent. Nous revenons par une autre voie à la mème 
conclusion : le moi est unc coordination. Il oscille entre ces deux points extrêmes où il cesse d'être; l'unité pure, l'incoordination absolue. Tous les degrés intermédiaires se rencontrent en fait sans démarcation entre le sain et le morlbide ; l'un empiète sur l'autre •.

Outre cette parfaite coordination dans l'espace, la personnalité impliquerait une parfaite coordination dans le temps. Les gens à persomnalité puissante et bien formée sont ceux qui, non-seulement sont tout entiers à à la chose qu'ils exécutent, mais aussi savent coordonner vers un mème but tous leurs actes successifs préparant de loin ce qu'ils feront un jour, ne disant pas un mot, ne faisant pas un geste qui n'ait un but, qui ne se rapporte $\dot{a}$ l'idée dominante, toujours prête à déterminer les actes qui l'expriment ou la réalisent, à interpréter les données de l'expérience de manière à se fortifier, à s'étendre, à se développer. On a pu donner cette définition d'une vie dignement remplie " une grande pensée de lajeunesse réalisée dans l'àge mùr ", et en effet, plus le rôle joué par une personnalité est considérable, c'est-à-dire, plus le nombre des tendances, desidées, des sentiments des perceptions systématiquement associés est considérable, plus l'influence coordinatrice sur le monde intérieur est grande, cette influence se manifeste de mille manières, depuis celle du général qui commande à des milliers d'hommes, et dont les victoires décident du sort de peuples entiers, jusqu'à celle de l'ingénieur qui réunit dans une œuvre commune des foules de travailleurs, et par des issues nouvelles, change les conditions du commerce et de la vie organique des nations, jusqu'a celle du penseur qui communique ses théories, modifie l'orientation des esprits, et détermine ou facilite un changement dans la direction de l'humanité, plus aussi la personnalité est vraiment grande et vraiment forte, plus l'homme a le droit de se dire réellement quelqu'un. 
Inversement moins est grande l'influence coordinatrice de l’individu sur le monde extérieur, moins est considérable la somme des éléments psychiques organisés en lui, et plus la personnalité s'amoindrit. Et si la coordination cesse, la personnalité se divise, c'est-it-dire que en tant que personnalité une, elle n'existe plus. Les phénomènes pathologiques le montrent de manière à ne laisser aucun doute. Il est inutile d'insister ici sur des faits bien connus, rappelons seulement le cas de Félida X... ', de Louis V.., ${ }^{2}$, de la malade du docteur Dufay ${ }^{3}$, des dédoulblements de la personnalité dans le rêve et de ceux que l'on opère artificiellement pendant l'état d'hypnotisme ${ }^{4}$. Partout dès que la coordination cesse, la personnalité totale disparaît. L'unité est impliquée par la personnalité, et ce qui fait la personnalité, comme ce qui fait une perception, une idée, un raisonnement, une tendance, c'est essentiellement une synthèse systématique, une association coordonnée d'éléments psychiques.

Une autre question se pose maintenant à propos de la personnalité, c'est celle de la systématisation sociale. Nous avons reconnu que l'homme, pris dans son ensemble, était une synthèse, mais le domaine de l'association systématique est plus étendu que le champ que nous avons parcouru et nous demandons maintenant à comprendre l'homme comme nous avons compris ses éléments. Chacun de ses éléments, groupe de phénomènes coordonnés, est à son tour associé, comme un tout avec

1. Voyez Azam. Hypnotisme, double conscience et altérations de la personnalité.

2. Voyez Bourrut et Burot. Variations de la personnalite.

3. V. Revue scientifique, 1876.

4. Voyez Richet. L'Homme et l'intelligence. Delbøuf Le sommeil et les réves, Revue j̧hilosophique. Pour l'étude complète des phénomènes pathologiques et leur interprétation, voir l'ouvrage déja cité de M. Ritsot. 
d'autres éléments. Il tire sa signification et sa valeur, non seulement de l'association qu'il constitue, mais aussi des associations plus complexes dans lesquelles il peut entrer. En est-il de même pour l'homme? S'il en est ainsi, nous devons nous arrêter, très peu d'ailleurs, parce que nous cmpiéterions sur une autre science, à examiner le fait.

Il serait possible, à priori, que l'homme n'eùt pas d'autre fin que sa propre existence, ou, pour exprimer en termes positifs cette proposition philosophique, le jeu de tous les éléments anatomiques qui composent un homme pourrait converger vers un seul effet : la conservation de la vie. Cette convergence est bien réelle, il est sûr que ses diver's organes servent à entretenir et à conserver son existence, et un philosophe italien a pu voir avec raison dans les phénomènes psychologiques la manifestation d'une fonction de protection 1. Mais la vie même de l'homme implique d'autres fonctions que celles qui l'ont spécialement pour fin; sans la société l'homme ne saurait vivre, sans l'amour il ne saurait vivre non plus, puisqu'il ne pourrait naître. Le chimiste n'aurait plus rien à faire s'il avait déterminé tous les éléments des corps, trouvé leurs diverses combinaisons. Le psychologue eut-il décomposé tous les faits psychiques et montré leur rôle dans le fonctionnement de l'esprit, n'aurait pas achevé sa science. Certains faits psychiques, en effet, ne trouvent pas leur signification en eux-mêmes, ni dans la vie individuelle, mais on ne peut les comprendre que par l'examen de la vie sociale et de la permanence relative de l'espèce. Il semble que plus les sciences s'élèvent, c'est-à-dire plus les phénomènes qui en font l'objet se compliquent, plus aussi les limites qui les séparent sont mal délimitées. Les mathématiques se distinguent plus nettement de la physique, que la physique de la chimie, la physique est plus

\section{Voyez G. Sergi. I fenomeni psichici.}


facilement séparable de la chimie que celle-ci ne l'est de la biologie, la biologie est plus difficile cncore à différencier la psychologie, et la psychologie et la sociologie sont réellement, à certains égards, confondues. La sympathie pour nos semblables, l'amour sexuel, les sentiments religieux se rattachent à la science socialc d'un côté, comme ils se rattachent de l'autre à la biologie. La psychologie ne peut les étudier sans tenir compte des conditions de l'existence humaine, c'est-à-dire de la société, et le psychologue qui étudie l'homme pris en lui-même ne peut terminer sa science sans entrer quelque peu dans la science supérieure, tant il y a de faits dans la vie individuelle qui ne se comprennent réellement que par la considération de la vie sociale.

A ce point de vue de nouveaux travaux s'imposent à la psychologie et la loi de finalité reçoit des applications plus précises encore. Un homme, en étant à certains égards un tout, est à d'autres égards un élément, il est un élément de la société dont il fait partie et dans laquelle il a un emploi particulier qui résulte de ses aptitudes, de ses goùts, de ses tendances. Aucun élément social ne ressemble a un autre, et le rôle de chacun dépend, au moins en partie, de sa nature. De lá nécessité, pour comprendre la société et pour comprendre l'homme, d'étudier les divers types intellectuels et moraux, les intelligences et les caractères, de rechercher si l'existence de types sociaux, comme ceux'dont M. Tarde a défendu la réalité, peut être établic et ces divers types déterminés arec précision. Inutilc de dire que ce n'est pas ici que ces études peuvent être faites, ce que je tiens à faire remarquer c'est que lenr but est d'établir des types multiples de systématisation déterminée. Tel enscmble de tendances, d'idées, d'images, de sentiments, apparaitra comme pouvant ou devant constituer un soldat, un prédicateur, un juge, un philosophe, etc. Autrement dit la loi générale de finalité 


\section{L'ASSOciation SYSTÉmatique - PERSONNalité 215}

sera appliquée d'une maniére différente dans chacun de ces types, et chacun nous présentera une nouvelle forme de coordination de divers éléments psychiques, chacune de ces formes se caractérisant non pas seulement par sa valeur et ses qualités, ou ses défauts, en tant qu'il s'agit d'assurer la vie de l'individu, mais aussi par la nature propre individuelle qu'elle constitue, en tant que cette nature doit influer sur la vie de l'ensemble.

Peut-on aller plus loin encore ? La socièté humaine peutelle ètre à son tour une partie d'un système plus vaste? Cette question sort de la psychologie, et il n'y a pas lieu de chercher ici à la résoudre. 


\section{('II A PITRE IV.}

\section{Conclusion.}

L'ètude de l'esprit et de ses éléments nous a donc conduits à cette conclusion que la loi d'association systématique est la loi essentielle de la psychologie. Tout phénomène psychique qui se produit est le résultat d'une association systématique d'éléments plus simples et il tend à susciter l'apparition d'autres éléments qui puissent s'associer systématiquement avec lui et concourir à une fin commune. A tous les degrés de la hiérarchie psychologique, depuis la sensation jusqu'à la personnalité, nous retrouvons ces deux lois, et nous avons tàché d'en préciser les formes diverses. Remarquons que la première énonce un fait donné, la seconde une tendance, c'est-à-dire un fait encore, mais un fait qui n'est qu'une condition essentielle, la condition principale, du fait considéré. J'entends que un fait psychique est toujours unc synthese systématique d'éléments plus simples, mais que, en certains cas, sa force coordinatrice peut être cnlayée et ne se manifestera pas. Par excmple, la sensation de faim tend a déterminer une série d'actes qui aboutiront à l'introduction de la nourriture dans l'organisme, mais cette tendance peut ìtre arrêtée, inhibée pard'autres et ne pas aboutir; le fait de la faim ll'est pas moins une condition essentielle de l'éveil systematique d'un certain nombre de faits psychiques. Lit tendance existe alors mème qu'elle est enrayée, et la loi usychologique est la mème que l'éveil des phénomènes se frorluise ou non, comme la loi physique est la mêne pour le ballon qui s'élève et pour la pierre qui lomlute. 
Ce que nous en avons dit, en parlant de la vie des éléments psychiques suffit à montrer le mécanisme de l'association systėmatique et nous dispense d'une longue analyse. Dans les associations suscitées par un fait psychique quelconque, dans les séries de faits qui s'appellent et s'enchainent nous trouvons toujours un même procédé. L'état primitif renferme des éléments qui à leur tour en appellen: d'autres auxquels ils sont étroitement associés, de sorte que l'état primitif et l'état qui lui succède sont ainsi réunis parles éléments communs qu'ils possèdent et qui sont systématiquement associés d'un côté avec les autres éléments du premier état, de l'autre avec les autres èlẻments du second. Ce qu'il y a de remarquable dans les formes élevées de l'association systématique, e'est que le premier et le second état forment un tout coordonné, et non plus, comme dans certains cas d'activité indépendante des élénıents, un mélange hẻtérogène. C'est que, ici, les éléments n'agissent que dans le sens du système dont ils font partie, ils sont étroitement reliés aux autres éléments de ce système, et seules celles de leurs affinités qui peuvent s'harmoniser avec la tendance dominante en activité se manifestent en suscitant de nouveaux éléments. L'association systématique est donc complétée iei par des phènonėnes d'inhibition que nous allons étudier en détail.

Quant au mode général d'association par un intermédiaire qui tient a la fois aux deux états successifs I, nous le retrouverons partout, il se manifeste dans toutes les formes d'association, dans l'association par contraste, dans l'association par ressemblance et contiguïté, comme dans l'association systématique et dans un cas comme

1. C'est le mode d'association qui a èté étudjè, surtout pour quelques cas particuliers, par M. Brochard. La loi de similarité. Revue philosophique, et M. Binet. Psychologie du ruisonnement. 
dans l'autre c'est toujours les affinités des éléments et leur pouvoir de systématisation que nous trouvons comme condition essentielle du phénomène.

De plus, pour bien comprendre la loi d'association systématique telle que nous la constatons dans l'humanité, nous devons tenir compte de la nature imparfaite de l'homme, c'est-à-dire de la non-existence en général de lunite de la personne. Nous avons vu ainsi que si la finalité pouvait être considérée comme une loi absolue de l'esprit humain, c'était à la condition de la considérer dans les èléments, non dans l'ensemble et quelquefois dans les éléments des éléments ${ }^{1}$. La personnalité est un fait comme les autres, qui lorsqu'il se produit implique une association sytématique parfaite de ses éléments, mais l'unité de l'homme est bien incomplète, et cette loi d'unité supéricure qui serait la personnalité n'existe pas toujours, les éléments de la personnalité existent sans la loi générale qui les réunit, et chacun se fait centre et travaille pour lui. Mais les groupes de tendances, les tendances elles-mêmes, et les éléments des tendances peuvent aussi disparaître momentanément en tant que formes particulières de la loi d'association, et alors les éléments sont livrés pour ainsi dire à eux-mêmes et manifestent des énergies qui ne sont plus en harmonie avec celles des autres éléments. Si le lien qui maintient des ouvriers travaillant à une même entreprise vient à se rompre, si la société fait faillite, si l'entreprise est reconnue impraticable, si comme le tunel sous-marin qui devait réunir la France a l'Angleterre elle ne peut être autorisée, les ouvriers se remettent $\dot{a}$ chercher de l'ouvrage ailleurs, quelques-uns travaillent individuellement, d'autres cherchent du travail dans d'autres grandes entreprises. De

1. Encore est-il possible que, si bas que l'on descende, la systématisation reste toujours quelque peu imparfaite. 
mème quand une coordination psychique disparait, les éléments qui la composaient cherchent fortune ailleurs, chacun selon ses aptitudes et ses goùts. Une idėe comme celle des corrélations organiques qui pouvait être autrefois une des causes de la croyance à l'existence des créations spéciales, peut, si cette ancienne croyance se dissout, devenir gràce a de nouvelles associations (par exemple de nouvelles explications, de nouveaux rapprochements avec des faits découverts depuis ou autrement considèrés) un élément de croyance à la théorie de l'évolution. Un système psychique s'est détruit, ses éléments sont allés ailleurs, se sont réunis à d'autres systèmes. Et cette décomposition psychique peut aller très loin, nous avons eu occasion de voir que des tendances très peu complexes, que des éléments psychiques relativement assez simples pouvaient en certains cas reconquérir une indẻpendance relative, mais partielle et précaire, car l'indépendance trop grande des éléments de l'esprit finit par amener la disparition de l'organisme et par suite des éléments psychiques eux-mêmes.

Quand l'esprit s'organise au contraire, les synthèses deviennent de plus en plus vastes et la systématisation plus parfaite, depuis les actes à demi-incohérents du petit enfant qui tàtonne pour prendre le sein, chez qui l'esprit est encore confus, mal coordonné et ne présente pas même des éléments bien précis, jusqu'aux hautes spéculations scientifiques de Newton, jusqu'au génie militaire de Napoléon, jusqu'à ces hautes mánifestations qui marquent le degré le plus élevé de coordination du plus grand nombre d'éléments qu'ait pu embrasser jusqu'ici l'esprit de l'homme, la gradation se fait insensiblement, marquée toujours par la détermination plus précise des éléments, c'est-à-dire par le caractère de moins en moins imparfait de leur unité, c'est-à-dire encore par la systématisation de plus en plus grande des éléments d'ordre 
inférieur qu'ils renferment et par la systématisation de plus en plus grande aussi des systèmes supérieurs qu'ils forment et des synthèses de ces systèmes supérieurs. Le développement des organes et du cerveau, l'expérience de la vie, les circonstances, sont les facteurs essentiels qui fournissent la matière de ces processus érolutifs.

Mais si la systématisation croissante en est la forme essentielle, si l'association systématique est ce qu'il y a de plus important dans l'esprit, ce sans quoi l'esprit ne serait pas l'esprit, il existe en psychologie des lois moins importantes, mais très générales aussi qui nous aident a comprendre les phénomènes. Nous avons vu que les phénomènes réunis pour constituer un fait supćrieur (des idées par exemple qui prennent part à une croyance) si ce fait supérieur vient à disparaître, reprennent pour ainsi dire possession d'eux-mêmes et tendent à provoquer d'autres associations. Un mot, je suppose le mot arbre éveille certaines idées dans un traité de botanique, il en éveille d'autres dans l'expression arbre généalogique. Il y a donc une partie de sa force associative qui passe parfois à l'état latent; quand un élément est employé dans un système, il ne manifeste pas normalement les affinités qui pourraient le faire entrer dans un autre systeme. Ces affinités persistent-clles? Elles sont donc arrètées, inhibées, il y a là un fait général, une loi que nous devons cxaminer. $\Lambda$ côté de la loi d'éveil des phénomènes, il y a la loi d'arrêt des phénomènes. Chaquc fois que nous suscitons des faits psychiques nous en empêchons d'autres de se produirc, chaque fois que nous développons unc tendance, nous nuisons a l'existence on an développement d'un certain nombre d'autres et à la manifestation d'un nombre encore plus grand. Il convient it prosent de formuler et d'étudier ce nouveau fait général qui constitue une partie importante du fonctionnement le l'esprit, cette nouvelle loi qui complète la première. 


\section{LIVRE II.}

\section{La loi d'inhibition systématique.}

\section{INTRODUCTION}

La loi d'inhibition ou d'arrêt peut se formuler ainsi : Tout phénomène psychique tend à empêcher de se produire, à empêcher de se développer ou à faire disparấtre les phénomènes psychiques qui ne peurent s'unir à lui selon la loi de l'association systématique, c'est-à-dire qui ne peuvent s'unir avec lui pour une fin commune. Cette loi est, comme on voit, le complément de la précédente; jointe à elle, elle exprime le résultat de cette lutte pour l'existence incessante et acharnée dont l'esprit est le théàtre, dont les systèmes psychiques sont les acteurs.

. Soixante-dix fois par seconde environ, le cœur envoie au cerveau du sang oxygéné : le sang amené par les artères dans les milliers de capillaires de ses différentes parties, et en particulier dans les petits vaisseaux si abondants de la substance grise corticale, assure le fonctionnement vital des centres nerveux, continuellement les matériaux se brùlent, la substance s'use et se renouvelle. En même temps, les organes des sens, la peau, l'œil, l'oreille, les organes internes envoient au cerveau des excitations incessantes, continuellement un nombre incalculable se produit de ces phénomènes cérébraux qui constituent la vie physique ou qui la représentent, et continuellement aussi, chaque phénomène suscité tend 
a déterminer une longue série de phénomènes. Comme des graines dans un champ, comme des œufs de poisson dans la mer, mais en plus grande quantité et avec une fréquence incomparable, des germes de phénomènes psychiques, des embryons de tendances apparaissent et se développent ou meurent. Nous avons vu selon quelle loi ils se développaient, étudions à présent l'autre face du fait de la sélection, l'arrêt et la persistance latente ou la mort des tendances.

Les aliments introduits dans le système digestif subissent une série d'opérations qui les rendent assimilables, ils deviennent notre chair et notre sang et se transforment de diverses manières en s'incorporant au milieu qui les a reçus, - de mème, un homme qui entre dans une socièté, dans un corps social organisé, quel qu'il soit, doit diriger ses actes de telle sorte qu'ils concourent avec ceux de ses nouveaux collègues, de ses nouveaux concitoyens, vers une fin commune. Rien ne peut être reçu par un corps organisé, d'ordre biologique ou d'ordre sociologique, rien ne peut faire réellement partie d'un organisme qu'en prenant place dans le système déja établi, sauf à modifier plus ou moins ce sýstème. De même, aucun phénomène psychologique ne peut se produire s'il ne forme une combinaison coordonnée avec quelques-unes au moins des tendances existant avec lui dans l'esprit, aucun état de lâme ne peut durer, aucun processus psychique se développer si cette combinaison coordonnée n'embrasse un assez grand nombre d'éléments et ne peut s'accommoder à la vie de l'organisme.

Le fait est général, chaque nouveau fait qui se produit est modifié, rectifié, déformé, il est décomposé et souvent rejeté en partie, il n'en reste que ce duli peut s'harmoniser it l'état de l'esprit, le reste est rejeté définitivement ou conservé à l'etat latent comme nous le verrons avec détails. Le grain jeté par le semeur ne germe pas toujours, 
et ne produit pas toujours sa fleur et son fruit; il faut qu'il soit de bonne qualité, qu'il tombe sur un sol favorable, qu'il soit suffisamment arrosé, sinon il se pourrit, il avorte, il produit une plante rabougrie et qui végète misérablement. En psychologie, le résultat dépend aussi de la nature du grain, mais le sol n'est pas inerte, il est organisé déjà et aussi bien préparé à étouffer tel germe qu'à fournir à tel autre les éléments d'un développement magnifique. La lutte et la sèlection sont incomparablement plus actives et plus compliquées.

Les physiologiste, M. Brown-Séquard en particulier, ontètudié dans ces dernières années, des faits qui se se rapprochent de ceux qui nous intéressent en ce moment. M. Brown-Séquard a donné le nom d'inhibition à cette action qu'exerce un phénomène nerveux pour empècher la production d'un autre phénomène nerveux dont le siège peut ètre assez éloigné ${ }^{1}$. Nous observerons en psychologie des faits analogues, sauf qu'ils paraissent avoir une signification différente que nous chercherons à dégager, saufaussi qu'ils se présentent avec quelques autres caractères. Nous allons les passer en revue en les vérifiant successivement, comme nous avons fait à propos de notre précédente loi, pour chaque catégorie de faits psychiques, et je m'attacherai à mettre en lumière, d'abord l'inhibition des phénomènes, ses différents degrès et ses différents modes, l'arrèt des tendances, ensuite la persistance des forces inhibées.

1. Voyez Brown-Séquard. De l'inhibition et de la dynamogénie. Voyez aussi les travaux du même auteur dans les Comptes-Rendus de $l$ Academie des sciences passim. 


\section{CHAPITRE PREMIER}

\section{Sensations et Perceptions.}

On ne remarque pas en général quel nombre immense d'excitations arrivent a nos organes, venant du monde extérieur, qui pourraient dans des circonstances favorables déterminer des sensations, des perceptions, et à la suite toute une sèrie de phénomènes enchaînés, des tendances et des orientations de l'esprit, et meurent stérilement faute d'être arrivées au bon moment et pour avoir été étouffées par des excitations plus fortes. Chacun sait pourtant que le bruit de l'orchestre couvre souvent la voix des chanteurs, et que le soleil nous empêche de voir les étoiles. Continuellement les astres nous envoient leurs rayons, et ces rayons nous parviennent, mais l'ébranlement quils causent aux organes passe totalement inaperçu, il ne peut arriver à déterminer ces synthèses dans lesquelles nul fait psychique n'apparaît, et pendant le jour, dans les conditions ordinaires, les étoiles n'existent pas pour nous. Et si l'on était porté à trouver le fait insignifiant, je ferais remarquer qu'il ne faudrait que le généraliser pour suppriner une partic de la vie mentale de l'humanité. Si pendant que le soleil éclaire un hemisphere, sa lumiere était réfléchie sur l'autre par uue lune qui serait toujours au-dessus de l'horizon en l'absence du soleil, ce qui est astronomiquement possible, et qui serait en mème temps un réflecteur assez puissant four nous empècher de voir la clarté des étoiles, tous les mythes, toutes les croyances, toutes les pensées, tous les sentiments qui se rapportent aux étoiles eussent été à 
jamais empêchés de se produire, une science entière, l'astronomie, serait restée remarquablement incomplète, et notre connaissance de l'univers aurait été notahleunent réduite; nos idées sur la vie, la vieillesse et la mort des astres, presque toute la philosophie générale qui implique la connaissance des résultats de l'analyse spectrale et des mouvements des étoiles ne se seraient peut-être jamais formée. La connaissance du système solaire mème serait impossible, les planètes restant inconnues; nos idées sur l'ordre du monde seraient probablement vagues, saus base solide et sans portée. C'est qu'une perception, et surtout tout un ordre de perceptions supprimées, tout une partie de la personnalité disparaît aussi.

Certains phẻnomènes psychologiques vulgaires impliquent une sélection et un arrêt du mème ordre. Le phénomène de l'irradiation est un fait de ce genre : un objet peu éclairé, placé sur un fond lumineux nous paraît moins grand que s'il est placé sur un fond sombre. On a admis pour expliquer ce fait, mais on conteste beaucoup aujourd'hui cette explication, que les parties très lumineuses "ébranlent non seulement les points de la rétine où elles viennent se peindre, mais encore les points les plus voisins, de façon à empiéter sur les parties des images moins éclairées " ${ }^{1}$. De même, deux carrés de dimensions égales, l'un blanc sur fond noir, l'autre noir sur fond blanc, paraitront inégaux, et le noir paraitra le moins grand. L'excitation produite par l'objet qui réfléchit le plus de lumière, étant plus forte, tendra à l'emporter sur l'autre et à la faire disparaître, mais l'explication est hypothétique.

Un exemple nous montrera micux cette lutte des impressions, et l'arrêt produit sur quelques-unes par les circonstances qu'elles rencontrent, en nous montrant comment

1. Küs et Duval. Physiologie, p. 544. 
la modification de ces circonstances modifie aussi le rėsultat. Les daltoniens, comme on sait, ne peuvent distinguer nettement certaines couleur's, et confondent, par exemple, le rert et le rouge. M. Delbœuf a présenté de ce fait une explication particulière. En suppposant que la rétine comparéc à une membrane montée naturellement a un certain ton, mais susceptible de setendre ou de se détendre sous l'action des forces extérieures, soit spécialement adaptée à la lumière verte, la sensation du vert sera provoquée par la production du mouvement vibratoire qui caractérise la composition de la membrane sensible. Les rayons bleus et violets tendront à lui imprimer un mouvement plus rapide, et les rayons jaunes et rouges un mouvement plus lent. La rétine tend à se maintenir à l'état 0 , et elle offrira une certaine résistance aux autres couleurs que le vert, résistance accrue par la présence des rayons verts dans les couleurs complexes. Les rayons rouges ne peuvent être reçus, ¿ moins que des circonstances spéciales ne leur viennent en aide, et c'est ce que fera l'interposition d'une solution de fuchsine qui arrête les rayons verts, ou une projection de lumière pourpre qui renforce les rayons rouges. On voit la lutte et la sélection, et comment les circonstances extérieures peuvent faire triompher telle ou telle excitation, et au contraire empêcher telle autre d'aboutir à la sensation qu'elle tend à produire. Quelle que soit d'ailleurs la théorie adoptée pour rendre compte du daltonisme, le fait subsiste et l'interprétation au point de vue de la loi d'arrêt, paraît bien devoir être la même, c'est ce qui ressort, semble-t-il, des ingénicuses expériences de MM. Delbœuf et Spring. On peut produire des daltoniens artificiels en leur faisant regarder les objets a travers une solution de chlorure de nickel, une plaque taillée dans une tourmaline verte, ou un morceau de verre colore an moyen du cuivre. La fuchsine rélablit la vue normale du 
daltonien artificiel, et inversement le daltonien dont la vue est corrigeje par la fuchsine redevient daltonien quind il recourt en outre au chlorure de nickel ${ }^{1}$,

Le phẻnomène de la vision des couleurs complémentaires, quelle que soit d'ailleurs la théorie qu'on croit devoir l'expliquer, qu'on l'attribue à une fatigue de la rétine ou a une fatigue des centres nerveux, reconnait une cause analogue. Il s'agit toujours d'un processus entravé ; à cause de la fatigue d'un organe, les causes qui tendent à produire une sensation ne peuvent aboutir après s'ètre manifestées cependant par certains phénomènes systématisés. Des faisceaux lumineux, réfléchis vers l'œil par une surface grise sont décomposés par les organes si ceux-ci sont fatigués par la vision antérieure trop prolongée du rouge, seuls les rayons autres que les rouges pourront déterminer une sensation.

Si nous considérons maintenant les perceptions; nous trouvons des faits analogues. Ce qui précède suffit pour faire comprendre combien le fait seul de l'apparition d'une perception implique de processus qui n'aboutissent pas. De toutes les excitations qui frappent à la fois nos sens, un faible nombre seulement arrive à déterminer la mise en activité d'une partie notable de l'esprit. Rappelons pour la vue, par exemple, que seuls les objets qui viennent se peindre sur la tache jaune, à cet endroit où la rétine s'amincit, et de tous les éléments qui la composent ne conserve guère que les cônes, sont nettement perçus et capables à un haut degré par conséquent de s'associer harmoniquement des images et des idées. Le reste passe inaperçu à ce point que la psychologie de cette vision secondaire n'est pas encore suffisamment explorée. Remarquons également que cette vision imparfaite est une excitation qui tend à déterminer la vision parfaite. Quand

1. Deltœuf et Spring. Le Daltonisme. Revue scientifique du 23 mars 1878. 
nous nous trouvons dans un milieu qui nous est inconnu, nous arons une tendance a tourner les yeux dans tous les sens, it regarder partout. Mais on ne peut regarder partout a la fois, ici encore de nombreuses excitations sont enrayées et ne peuvent déterminer le système psychique qu'clles appellent logiquement.

On sait aussi, et nous avons eu déjà à parler de ce fait, combien les sensations qui ne se rapportent pas aux objets de nos préoccupations passent facilement inaperçues si ces préoccupations sont vives. L'esprit fait un choix entre les sersations, et cette sélection implique l'avortement d'un grand nombre de germes. "La plupart des chercheurs scientifiques, dit M. Paul Bert, dans une notice sur Claude Bernard, sont des espèces de somnambuiles quĩ ne voient que ce qu'ils cherchent, que ce qui est sur la trace de leur'sidées ; leur ceil est fixé sur un point, et non seulement ils ne percoivent pas ce qui passe à côté de ce point, mais même ce qui s'y présente sans avoir été prévu. Claude Bernard semblait, suivant l'expression d'un de ses élèves, avoir des yeux tout autour de la tête, et c'était avec stupéfaction qu'on le voyait, au cours d'une expérience, signaler des phénomènes évidents, mais que personne hormis lui n'avait aperçus." Le cas de Claude Beruard, même alsstraction faite de l'effet de contraste un peu cherché peut-être, est ainsi présenté commc une rareté, et en somme il ne fait pas exception a la loi générale, seulement le choix s'exerçait che\% lui d'une maniere plus large. Le cas d'Archimede, et les cas semblables où l'attention trop forte empêche l'esprit de s'adapter it certaines circonstances, de percevoir certaines impressions, ot de laisser se dírouler les systemes de phénomènes qu'cllos tendent à éveiller, sont bien connus et faciles a intriprites. Remarquons seulement qu'ils ne sont que la forme cxtreme d'un fait continuel, car jamais l'esprit n'est alsolument inactif, et, toujours par consequent, la 
perception des objets extérieurs est facilitée ou entravée dans une certaine mesure. Il est assez visible que c'est là ce qui se passe à chaque instant.

Des cas intéressants et instructifs et qui montrent bien le mécanisme de la sélection. sont ceux où le choix de l'esprit aboutit à une perception erronée, illusion ou hallucination, à cause du rejet de certaines excitations et de leur impuissance à éveiller le système d'images qui se coordonnerait avec elles. Je demande la permission de reproduire ici une observation que j'ai déjà citée dans un autre volume.

J'ai chez moi un petit harmonium. Unjour, je passais près de lui sans le regarder et en marchant assez vite. Aussitôt après l'avoir dépassé, j'eus une sorte d'impression visuelle assez vive, me représentant distinctement les touches blanches et noires de l'instrument. Etonné de voir l'harmonium ouvert à ce moment, je me retournai et m'aperçus qu'il était en fait complètement fermé. Une carte roulée, en fort papier blanc, que j'avais posée sur l'instrument était la cause de ma méprise.

Ce fait ne me paraît pouvoir être expliqué que de la manière suivante: la perception nouvelle qui tendait à s'établir était celle d'une surface blanche continue sur l'harmonium. Mais des perceptions visuelles précédentes et nombreuses m'avaient représenté l'harmonium offrant, quand il était ouvert, une surface blanche (les touches blanches), interrompues seulement par les petits intervalles existant entre les touches, et, en certains endroits déterminés, par les touches noires. La tendance à la nouvelle perception était en partie d'accord avec les traces laissées par elles, mais il $\mathrm{y}$ avait desharmonie complète sur quelques points. Ce nouvel état s'est présenté dans de mauvaises conditions, puisque je marchais vite et sans regarder l'harmonium. Ayant à lutter pour s'établir contre des habitudes prises par les organes qui servent à la 
perception visuelle, il a été rejeté en grande partie. Seulement, ce qui dans celte impression complexe pouvait exciter systématiquement les inages habituelles a pu être admis par l'esprit, et a réveillé par association, grâce aussi peut-être à l'ébranlement provoqué par la partie rejetée de l'excitation, la représentation visuelle des touches noires, des petites fentes entre les touches, et des circonstances accessoires. Tout cela s'est effectué sans que j'en eusse conscience.

Il y a comme on voit deux parties à distinguer dans cette opération mentale : l'erreur est causée par le rejet d'une partie de l'excitation, et aussi par le réveil d'anciennes impressions. Nous nous sommes occupés dans le livre précédent de cetle dernière partie du phénomène, c'est sur la première que nous devons à présent porter notre attention, et nous manifesterons ainsi par notre état mental la loi même que nous étudions.

On retrouve encore cette inhibition bien marquée dans un lapsus visuel raconté par, M. Egger. Ici encore le système sensible éveillé par certains éléments de l'excitation, de lasensation, rend impossible l'adoption d'autres éléments avec lesquels il ne peut s'associer harnoniquement et arrête le processus. "Je me trouvais dit M. Egger sur une place de Bordeaux, quittant quelques amis, je fais une volte-face rapide durant laquelle mes yeux rencontrent une série d'affiches de toutes couleurs qui couvrent un mur. Le temps et l'attention m'ont également manqué pour en remarquer spécialement aucune; mais, aussitôtle mouvement terminé, mon imagination me représente cette inscription en gros caractères: VELPEAU. C'est un état faible comme les visa intérieurs du souvenir." L'une des alfiches portait l'inscription : Ville de Pau. C'était la canse du lapsus. La préférence accordée au nom de Velpeau provenant de ce que "aucun mot, dit M. Egger, n'est plus proche du suscitant V.., L.... PAU, que le 
suscité. Il y a eu lutte entre le système.d'éléments éveillés par la perception complète et ceux des systèmes habitucls d'idées, d'images qui ont été suscités par certaines parties de cette impression, le résultat a été l'arrêt des premiers processus et l'obstacle mis à leur force d'asso-

- ciation. Remarquons d'ailleurs que dans les deux cas qui précédent, les éléments de perception rejetés d'abord ont été reconnus par des expériences postérieures, et que, se représentant dans de nouvelles conditions, ils ont pu réussir où ils avaient échoué d'abord; à leur tour les perceptions et les idées qu'ils ont éveillées ont empêché les illusions premières de déterminer dans l'esprit la coordination particulière qu'elles tendaient à y introduire, en définitive, elles n'ont pu me faire croire qu'un harmonium était ouvert, ni à M. Egger que le nom de Velpeau se trouvait sur l'affiche, et l'arrêt de ces processus déterminés par l'illusion a été amené par un mécanisme analogue à celui qui avait amené l'arrêt des premiers éléments fournis par les perceptions. Si les habitudes de l'imagination perceptive pouvaient se systématiser plus aisément avec les éléments de l'impression qui d'abord sont seuls parvenus à la conscience, les habitudes générales de l'intelligence, le raisonnement, ont eu de la peine à coordonner cette donnée imaginative avec les notions acquises déjà, et par une association bien simple, l'ensemble des idées qui ne pouvaient s'accorder avec la nouvelle impression arrivant à l'esprit, a déterminé les mouvements voulus pour opérer une vérification.

Les illusions ne peuvent guère s'établir dans l'esprit que par suite de l'arrêt qu'elles font subir aux processus perceptifs. Toute illusion implique des perceptions arrètées - la sensation se produit probablement au moins dans bien des cas, mais elle n'éveille pas les images qui la changeraient en perception, elle passe inaperçue dans l'organisme et disparaît sans avoir pu exercer son 
influence. En arrêtant volontairement certaines impressions, en donnant i l'esprit une orientation roulue, on peut se créer des illusions. En regardant couler sous un pont une rivière assez rapide, je pouvais me figurer à volonté que c'était le pont qui se déplaçait, et sentir l'impression du mouvement. Il y a bien certainement ici arrêt de quelques perceptions externes et internes. Les personnes qui dans un salon, peu après la mort du maréchal Ney, l'entendirent annoncer, alors que c'était simplement M. Maréchal Aîné qui arrivait, éridemment ne perçurent pas certaines parties des paroles prononcées.

De même pour les hallucinations, - une hallucination tient la place d'une perception, elle l'arrête, et l'empêche de se produire. Il n'y a peut-être pas de différence essentielle entre les hallucinations et les illusions. C'est du moins ce que les recherches de M. Binet paraissent établir 1 ; cependant, à notre point de vue, l'hallucination se distingue de l'illusion en ce que l'arrêt des perceptions qu'elle produit est plus considérable. L'halluciné qui croit voir une personne imaginaire assise sur un fauteuil ne voit généralement pas le fauteuil derrière la personne. M. Marillier $z$, qui a exposé avec netteté et précision des hallucinations observées sur lui-même, a constaté le fait. «Is... s'écartait alors un peu de moi, elle se plaçait devant un fauteuil de ma chambre qu'elle me cachait, et sa tête me cachait aussi une partie d'une gravure pendue au-dessus du fauteuil : mon hallucination faisait donc écran comme un corps opaque. Je voyais à la fois le mur de ma chamlire et la personne qui était placée devant, et il m'était impossible de saisir aucune différence de netteté ou d'intensité entre ces deux images ". Brierre de Bois-

1. Voir Binet. L'Hallucination. Revue philosophique, 1880.

2. Voyez Marillier. Etude de quelques cas d'hallucination observes sur moi-meme. Revue Philosophique. Tome 21. 
mont, après avoir cité le fait, que nous retrouverous tout à l'heure, d'une hallucination recouverte par une perception visuclle, ajoute : « il est plus ordinaire de voir les images fantastiques recouvrir les objets extėrieurs et en dérober la vue au malade". Le physiologiste allemand, Gruthuisen, affirme avoir parfaitement reconnu que les images flottantes couvraient les meubles de l'appartement dans lequel il se trouvait 1. M. Baillarger signale le même phénomène ${ }^{2}$. Un cas particulièrement intéressant est celui dans lequel l'inhibition produite par une hallucination au lieu d'arrêter simplement les perceptions actuelles, arrête par association systématique des perceptions qui tendent à se produire un certain temps après. C'est ce que l'on remarque dans les phénomènes de l'état hypnotique que M. Bernheim a appelés hallucinations négatives, et MM. Binet et Féré anesthésie systématisée.

Inversement, les perceptions exercent une action inhibitrice sur les images et les empèchent de se transformer en hallucinations. L'image faible tend naturellement à se compléter systématiquement par les éléments qui lui manquent pour ètre un ètat fort. Si les perceptions n'intervenaient pas, elles se changeraient facilement en hallucinations, - non pas toujours, car d'autres phénomènes aussi exercent sur elles une action d'arrèt, mais beaucoup plus souvent. Que des hallucinations soient ainsi inhibées par les perceptions, on en a la preuve par les cas où les perceptions venant à disparaitre, l'hallucination se produit. Ayant un jour bu une assez grande quantité de café, je sentais une certaine excitation nerveuse, et je ne sais trop pourquoi je vius a penser aux processions de la FêteDieu que j'avais vues peu auparavant et aux deux rangées de jeunes filles habillées de blanc qui figuraient á la cérémonie. Fermant tout a coup les yeux, j'eus la vision assez

1. Brierre de Boismont. Des Hallucinations, p. 594.

2. Baillarger. Des Hallucinations, p. 334 . 
confuse et très courte de ces deux files qui se sont fondues en deux lignes blanches, lesquelles se sont elles-mêmes rapidement évanouies. Tous les auteurs ont indiqué le rôle joué dans la production des hallucinations par l'obscurité, le silence et l'occlusion des paupières, toutes circonstances généralement favorables à l'hallucination, comme une demi-obscurité ou un bruit confus paraît favorable à la production des illusions. M. Taine a consacré un beau chapitre dans son traité de l'Intelligence à étudier la tendance hallucinatoire et le rôle de réducteurs des perceptions ${ }^{1}$, et la preuve de ce rôle peut être considérée comme faite.

Nous tirerons de ces faits une autre conséquence, c'est que bien souvent au moins, la tendance enrayée persiste par quelques-uns de ses éléments; l'inhibition en supprime les effets, mais ne supprime pas leur cause. Les atomes d'oxygène qui sont combinés à des atomes d'hydrogène dans une goutte d'eau ne manifestent pas toutes leurs affinités, mais ces affinités per'sistent, c'està-dire qu'il en reste une des conditions essenticlles de ce que nous considérons conme le résultat de l'affinité; dès que les circonstances favorables se produiront, l'affinité se manifestera, en termes moins abstraits, de nouvelles combinaisons auront lieu. De même, une image faible garde sa force associative, c'est-à-dire que dès que les conditions le permettront, elle produira une perception, une hallucination, ou bien un mouvement. Et cela n'est pas vrai seulement pour les images qui correspondent à des phénomènes cérébraux plusieurs fois produits, mais ces sensations avortées que nous avons vues paraissent persister plus qu'on ne croirait lorsqu'elles sont arrètées dans leur développement. Il arrive assez souvent que, plus tard, l'inhibition venant à cesser, pour une

1. Taine, De l'Intelligence, rol. I, livre II, chap. ler. 
cause ou pour une autre, le processus interrompu recommence et se poursuit, ou du moins, le phénomène, si fugitif qu'il ait été, a laissé une trace, une image plus faible, et cette image à l'occasion agit selon les lois ordinaires, à peu près comme la perception aurait fait. Ainsi, il arrive que nous n'entendions un mot qu'après l'avoir pour ainsi dire deviné. Nous restons devant notre interlocuteur à chercher ce qu'il a dit, et nous arrivons à susciter non pas tout à fait la perception qui n'a pu se produire, mais son image assez vive et assez nette. Nous comprenons d'une manière quelconque, par un geste, un sourire de la personne qui a parlé, un souvenir qui nous arrive, ce que notre interlocuteur a voulu dire, et il se produit alors une sorte de souvenir, de localisation dans le passé d'uue perception qui n'a pas eu lieu, mais qui aurait pu se produire. L'état des organes qui, pour une cause ou une autre, avait empêché la perception s'est modifié quelque peu par l'idée, le souvenir, la nouvelle perception qui arrive ensuite, et les éléments qui avaient pu être admis et qui étaient restés inaperçus, peuvent déterminer alors une image de la perception qu'ils tendaient à produire. Nous remarquons cette série de phénomènes dans les deux lapsus visuels que j'ai cités plus haut. En effet, l'illusion en ce cas n'a pas été immédiate, il n'y a pas eu d'abord perception, mais après un temps assez court, ceux des éléments de l'impulsion produite qui peuvent se développer et qui paraissent avoir été quelque peu arrètés aussi déterminent la vision erronée.

Nous trouvons une curieuse démonstration de la persistance des impressions et de leur force associative dans les cas de somnambulisme et de dédoublement de la personnalité étudiés par M. Pierre Janet. Il faut que quelque chose dans le somnambule reconnaisse le fait qu'il ne doit pas percevoir. Ceci nous indique déjà quelque peu comment l'arrêt peut s'effectuer, il se produit au moins en 
certains cas sur un processus déjà commencé, et ce n'est guère en effet que lorsque une impression a commencé à faire sentir son influence que l'esprit peut juger cette influence et la favoriser ou la repousser. C'est ainsi que l'Académie ne peut refuser de recevoir qu'une personne qui a fait acte de candidat, et elle peut arrêter la satisfaction du désir sans le supprimer jusqu'à la prochaine vacance. Les recherches de M. Pierre Janet ont eu pour résultat de nous renseigner sur quelques phénomènes obscurs et de nous permettre de nous faire quelques idėes encore trop vagues sur ce que peut être la persistance d'une partie d'impression et la tendance associative qu'elle conserve. Le cas qu'il a étudié est évidemment une forte exagération de l'état normal, nous y trouvons un dédoublement très complet et très remarquable de la personnalité et ce fait curieux que les sensations qui passent inaperçues pour l'un des moi sont parfaitement perçues par l'autre, c'est-à-dire que les sensations non perçues par le moi sont associées à un autre système important de tendances, d'images et d'idées. Or, dans l'état normal, les associations des éléments de la sensation qui passent inapercues dans la perception, tout en ayant plus d'importance qu'on ne serait parfois porté à le croire, sont certainement moins complexes et moins étendues. "Pendant le sommeil hypnotique complet, écrit M. Janet, je mets sur les genoux de la somnambule cinq cartes blanches dont deux sont marquées d'une petite croix. "Quand vous serez réveillée, lui dis-je vous ne verrez pas les papiers marqués d'une croix. "Je la réveille le plus complètement possible une dizaine de minutes plus tard, et elle n'a conservé aucun souvenir de mon commandement, ni de ce qu'elle a pufaire pendant le sommeil. Comme elle s'étonne de voir des papiers sur ses genoux, je la prie de les compter et de me les remettre un à un, L... prend l'un après lautre trois papiers, ceux qui ne sont pas marqués, et me 
les remet. J'insiste et demande les autres, elle soutient ne plus pouvoir m'en remettre car il n'y en a plus. La physionomie ne semble pas altérée, elle peut causer librement et se souvient de tout ce qu'elle a fait, même de m'avoir répondu qu'il n'y avait que trois papiers sur ses genoux. Je prends tous les papiers, et je les étale sur ses genoux à l'envers, de manière à dissimuler les croix, elle en compte cinq et me les remet tous. Je les replace en laissant les croix visibles, elle ne peut prendre que les trois non marqués et laisse les deux autres. C'est là l'expérience de MM. Binet et Féré et il semble naturel d'en conclure comme eux que les croix sont vues et reconnues d'une manière quelconque. Ce n'est pas L... qui peut nous expliquer comment, car elle n'a jamais vu de croix sur ces papiers, elle en est bien sùre. A ce moment, je m'écarte d'elle et profitant d'un moment de distraction suffisant j'interpelle Adrienne, (Adrienne est la seconde personnalité qui a un nom particulier, la seconde coordination générale de phénomènes psychiques qui remplace la première à l'appel de ce nom) et je lui demande deme dire ce qu'elle a sur les genoux. La main droite prend un crayon placé à sa portée et écrit: « Il y a deux papiers marqués d'une petite croix. " 1 En revanche, les objets perçus par L... restaient invisibles à Adrienne, les deux personnalités étaient à cet égard au moins complémentaires.

De tout ce qui précède on peut tirer en résumé les conclusions suivantes: L'inhibition peut s'exercer sur les sensations, les perceptions, les illusions, les hallucinations, en un mot sur toutes les représentations vives. Les faits qui tendent à produire une perception ( phénomènes physiologiques de mise en activité des organes

1. Pierre Janet. L'Anesthésie systèmatisée et la dissociation des phénomenes psychologiques, Revue philosophique. Tome xvir p. 457-458. M. Binet a signalé des phénomènes de même nature chez les hystériques. Voir Ravue philosophique. Février 1889, et Revue scientifique 1889. Tome 1. 
de la sensation et de la perception à la suite d'impressions extérieures), ceux qui tendent à produire une hallucination (images), sont arrêtés, leur's tendancès à l'association ne peuvent se manifester, le processus qu'ils tendent à produire est enrayé. Cet arrêt peut ètre causé par des phénomènes psychiques variés : tendances, habitudes organiques, expériences antérieures, hallucinations, perceptions, images, etc. Mais il se produit toujours dans les mêmes conditions, c'est toujours par l'influence d'un système psychique qui ne peut s'accorder avec l'état qui tend a se produire que cet état ne se produit pas, que le processus avorte, que l'inhibition se produit. Nous voyons en mème temps que d'une manière générale la tendance enrayée persiste, c'est-à-dire que quelques-uns des éléments psychiques qui la composaient restent prêts à reprendre leur développement dès que les circonstances ne s'y opposeront plus, dès que l'inhibition cessera. 


\section{CHA P I TRE II.}

\section{dintelligence. \\ Images, Idées, Jugements, Raisonnements.}

Les images et les idées impliquent à plusieurs égards de nombreuses actions d'arrèt. Le fait seul de l'existence d'une image suppose l'arrêt de la force qui tend soit à la transformer en hallucination, soit à déterminer des mouvements coordonnés en rapport avec sa nature propre. On peut dire la mème chose à propos de l'idée. La force motrice de l'idée est bien connue à présent des psychologues - Setchénoff a développé dans ses Etudes psychologiques le rôle de l'action d'arrèt dans la formation de la pensée : « Je vais, dit-il, montrer au lecteur la première et la principale conséquence qu'a pour l'homme l'art d'arrêter le terme final de l'action réflexe. Le résultat se résume dans la possibilité de penser, de réfléchir, de raisonner. Qu'est-ce, en effet, que la réflexion? C'est une suite de représentations, d'idées liées entre elles, suite qui existe à un moment donné dans la conscience et ne s'exprime par aucun fait extérieur dépendant de ces actes psychiques. Mais, comme le lecteur le sait déjà, il ne peut se produire aucun phénomène psychique dans la conscience sans une excitation sensitive venue du dehors. La pensée est donc soumise à cette loi, elle renferme donc le commencement et la continuation d'une action réflexe, la fin seule, le mouvement semble faire défaut.

« La pensée est une action réflexe psychique réduite à ses deux premiers tiers " 1.

1. Setchénoff. Etudes psychologiques, traduites du russe par Victor Derély, p. 135. 
Il y aurait bien des détails à relever, et à examiner de plus près, à discuter mème dans ce passage, mais nous pouvons le considérer au point de vue général auquel nous nous plaçons ici comme très suffisamment exact. Bain a dit de même : a Penser, c'est se retenir de parler et d'agir $1 \%$. Nous pouvons donc considérer toute image et toute idée comme étant par cela seul qu'elles existent, l'indice d'un arrêt, arrêt soit des tendances motrices, soit des tendances hallucinatoires. Nous avons indiqué ce dernier cas dans le chapitre précédent, il n'y a donc pas à y revenir ; pour le premier, il n'offre pas de difficulté particulière - la systématisation que l'idée tend à déterminer est empêchée par l'influence inhibitrice d'autres systèmes dominants. Je suis assis à ma table, et je travaille dans une pièce qui n'est pas chauffée, j'ai des impressions de froid, et je me représente des moyens de me réchauffer, mais les systemes dominants, ceux qui font courir ma plume sur le papier, empêchent l'idée d'aboutir, et lui empruntent les óléments qui peuvent s'accorder avec eux en devenant un fait propre à illustrer la loi que j'étudie.

Si nous recherchons comment une perception devient une idée ou donne naissance à une pensée, nous aurons encore de bons exemples d'actions d'arrêt. - Je regarde ma montre pour savoir l'heure, je vois la position des aiguilles et je me dis : il est dix heures et quart, puis mes regards se dirigent vers d'autres objets - la première perception visuclle disparaît, l'idée à laquelle elle a donné naissance reste sous forme d'état faible, d'élément entrant dans l'orientation générale de l'esprit, et aussi, sous forne de phrases, de mots coordounés ou de substituts de ces mots, car il se peut que la phrase ne soit pas tout it fait présente à mon esprit et que je sente cependant

\section{Bain. Les sene et l'intelligence.}


L'inhibition systématique - images et idées 241 que je pourrai la rappeler dès que j'en aurai besoin. Voyons ce qui s'est passé : les sensations et les perceptions causées par la montre, ont éveillé, gràce aux traces laissées par des expériences antérieures, des coordinations d'éléments psychiques, je sais que lorsque les aiguilles de la montre sont dans une position déterminée, il me reste un nombre déterminé de fractions de temps, heures, quart d'heures, arant le déjeuner. Il y a donc jusqu'ici association systématique, les éléments ainsi éveillés donnent une certaine forme à l'allure générale de l'esprit, ils peuvent, par exemple, me faire hâter mon travail, si je trouve que j'ai un peu perdu mon temps jusqu'ici, ou faire ralentir ma pensée, si je me suis fixé une tâche et si je juge que j'ai du temps de reste pour l'accomplir, ou bién laisser les choses comme elles étaient avant. Mais je détourne les yeux de la montre : immédiatement toute une partie du système précédent disparaît, ce sont les perceptions visuelles, de plus comme je n'ai pas besoin à présent de me représenter le cadran de ma montre et la place des aiguilles, elle n'est pas même remplacée par une image moins vive qui lui ressemblerait. Cette image, il me serait possible de la rappeler si j'en avais besoin, mais la force associative qui aurait ce résultat est inhibée pour le moment par l'orientation générale de l'esprit qui donne une autre forme à la coordination psychique. L'idée seule reste, et d'une manière qui sera très vague, très faible, jusqu'au moment où le besoin de savoir l'heure se fera de nouveau sentir.

Pour les objets dont nous concevons une idée, le pro. cessus est toujours le même, il y a association systématique d'abord, sélection ensuite, et rejet d'une grande partie de l'état primitif, nos images ne contiennent pas tous les éléments de nos perceptions si vives qu'elles soient, à moins qu'elles n'arrivent aux degrés les plus élevés de l'hallucination qui parait psychologiquement équivaloir 
à la perception même; les images faibles ne contiemnent pas tous les éléments des images vires, il y a eu un nouveau choix, un nouvel arrêt, on n'a rqu'ì comparer pour en être sù les diverses formes de l'imagination visuelle ou de l'imagination auditive. M. Galton' dit que chez certaines personnes la vision mentale atteint presque à l'hallucination : les objets gardent leur forme, leur couleur, leur's positions réciproques, dans les images moins vives, nous voyons certains éléments disparaitre, le champ de la vision mentale se rétrécit, les formes sont moins nettes, les couleur's moins vives, l'image diffère plus de la sensation, dans les formes inférieures, la couleur disparaît parfois, la forme reste vague, c’est-à-dire que certains éléments disparaissent et manquent. Au-dessous, la vision mentale disparait, l'idée restant. Les personnes interrogées qui présentent cette forme d'esprit, et qui sont généralement dun certain àge et plus ou moins habituées aux études alsstraites déclarent que les mots : «voir par l'esprit » ne sont pour elles qu'une pure métaphore.

Doutera-t-on peut-être que les différences de l'hallucination et de l'image faible ne soient celles que j'indique, je peuse que l'expérience peut offrir des preuves convaincantes, et que liuterpretation de différents documents ne saurait ètre douteuse. Voyons en quoi se marque le vague de la couleur, par exemple, ou le vague de la forme. Si je ne puis préciser la couleur d'une image mentale, c'est probablement au moins dans la plupart des cas que les élements de limage vive on de la perception qui auraient pu ètre associés diuns mon esprit avec les mots exprimant telle ou telle couleur ont dispraru ou n'ont fras ete éreilles. De mêne pour la forme, ce fui la rend moins nette, e'est le manque de certains éléments. Il n'y

1. Galton. Inquiry into human faculty and its development. Mental imagery. 
a pas de doute que l'on n'en trouve une preuve dans la description que fait M. Taine de sa propre imagination: "Je revois sans difficulté à plusieurs années de distance cinq ou six fragments d'un objet, mais non son contour précis et complet; je puis retrouver un peu mieux la blancheur d'un sentier de sable dans la forêt de Fontainebleau.... mais je ne puis tracer intérieurement l'ondulation du chemin, ni les saillies de la roche; si j'aperçois en moi-même l'enflure d'un muscle végétal, ma demi-vision s'arrête là, au-dessus, au-dessous, à côté, tout est vague, même dans les résurrections involontaires qui sont les plus vives, je ne suis qu'à demi-lucide; le fragment le plus visible et le plus coloré surgit en moi sans éblouissement ni explosion; comparé à la sensation, c'est un chuchotement où plusieurs paroles manquent à côté d'une voix articulée et vibrante " 1 .

Si au lieu des images visuelles, nous considérons les images auditives, le même fait se présente. On peut comparer les images sonores, vives, qui pour certaines personnes constituent une partie importante de la vie intérieure avec les images faibles qu'un certain nombre d'observateurs peuvent distinguer en eux-mêmes. "La parole intérieure, dit M. Egger ${ }^{2}$, a l'apparence d'un son, et ce son est celui que nous nommerons parole ou langage.... » et M. Egger retrouve dans la parole intérieure les principaux caractères de la parole extérieure, rythme, la hauteur, l'intensité des sons, et même le timbre. Au contraire chez moi, par exemple, les images auditives sont généralement faibles, le timbre n'est plus perceptible ou l'est à peine. Voilà donc au moins un élément très important qui ne se manifeste plus dans l'image alors qu'il existe certainement dans les perceptions qui ont rendu

1. Taine. De l'Intelligence, Volume I.

2. Egger, La parole intérieure, p. 67 et suivantes. 
l'image possible. Pour les images motrices, les résultats sont généralement équivalents, "une articulation supprimée, dit M. Bain, est la matière du souvenir, la manifestation intellectuelle, l'idée de la phrase " 1 . Et il n'est pas douteux que les images motrices comme les autres ne puissent être plus ou moins vives 2 .

De même les idées, comme nous l'avons vu, paraissent différer des perceptions et des images à la fois par leur association plus fréquente avec un certain nombre de nouveaux éléments : systèmes d'idées, raisonnements, croyances, etc., et aussi par la suppression de certains autres éléments. Plus complexes d'un côté, elles sont moins complexes de l'autre, il y a une sorte de compensation, mais une partie seulement de ce phénomène nous intéresse ici. Nous avons entrevu d'abord les faits d'arrêt dont il s'agit, il faut maintenant les considerer en euxmêmes, et avec plus de développements.

Quand nous nous occupons d'une chose ou d'une personne, l'idee que nous nous en faisons est toujours une idee abstraite. Du complexus d'images ou de tendances que peut être pour nous cette personne ou cette chose, nous extrayons ce qui peut se coordonner avec nos préoccupations, avec les tendances qui dominent actuellement notre esprit. Le reste ne s'éveille pas, et cependant ce reste tendrait à ètre suscité par les éléments que nous appelons à l'activité, et cela est rendu bien évident par les cas où l'attention gémérale venant à se rclàcher, les éléments sont livrés à eux-mèmes et éveillent des séries de phénomènes parasites qui sont coordonnés en

1. Bain. Les sens et l'intelligence.

2. Je ne puis m'occuper ici que de l'interprétation générale des phénomènes. Pour leur étude détaillée, je me permettrai de renvoyer a me articles sur l'Abstraction et les Ides abstraites. Revue phil., 1889. et Le Langage interieur et la Pensée. Revue philosophique. Janvier 1886. 
L'INHIBITION SYSTÉMATIQUE - IMAGES ET IDÉES 245 eux-mêmes, mais ne peuvent entrer dans le système général qui se manifeste actuellement. Par exemple, si un propriétaire qui doit s'occuper de ce que peut rapporter par an un hectare de vigne, compte les bénéfices: vente des raisins ou du vin, vente des sarments, et les dépenses: journées de travail des ouvriers, frais occasionnés par les bêtes de somme, fumures, remèdes divers, soufre contre l'oüdium, ammoniaque et sulfate de cuivre ou bouillie bordelaise contre le mildew ou le black-rot, etc., toutes ces idées de raisins, de sarments, d'ouvriers, de maladies et de remèdes, sont réduites ici à ce qu'elles ont d'essentiel à certains égards, aux élèments qui leur permettent d'entrer dans certaines coordinations psychiques, le raisin n'est pas ici un fruit recouvert d'une pellicule bleu-noir ou jaunâtre, l'ammoniaque n'apparaît pas comme un composé d'hỹdrogène et d'azote, agissant vivement sur les nerfs de la sensibilité générale des muqueuses du nez, il n'est pas besoin que le propriétaire se représente le mildew sous forme de petites tàches brunes, avec efflorescences blanchàtres, apparaissant sur les feuilles vertes, - le raisin est simplement une chose dont le quintal se vend un certain prix à peu près déterminé, le mildew est simplement l'occasion d'une dépense pour le prévenir, ou d'une perte en raisins ou en vin. On voit l'arrèt subi par les forces qui tendraient à présenter à la conscience des éléments associés aux éléments qui y sont présents, mais qui ne sauraient être utiles dans la circonstance. Il en est de même chaque fois que nous pensons à une chose et que nous la considérons sous un certain aspect, notre idée est toujours le résultat de l'arrèt d'un grand nombre de tendances associatives.

Mais les idées que nous avons considérées sont engagées dans un raisonnement, en est-il toujours ainsi, ou ce que nous avons dit s'applique-t-il ì toutes les idées - ou bien 
encore nous est-il possible d'avoir des idées complètes représentant à l'esprit, sans arrêt, toutes les propriétés d'une chose, toutes les qualités d'une personne? Il est hors de doute que nos idées peuvent ètre plus ou moins complexes, qu'elles peuvent réunir dans un même état de conscicuce, ou synthétiser inconsciemment, un plus ou moins grand nombre d'éléments empruntés à nos perceptions, à nos sensations et à nos actes. Mais alors mème que notre ilée serait aussi complexe que possible, elle n'en serait pas moins un cas de la loi d'arrêt. Que serait en effet cette idée complexe sinon un état de l'esprit qui permettrait d'éveiller à volonté, d'une manière suffisamment complète telle ou telle représentation plus particulière? Il est manifestement impossible, que l'esprit se représente à la fois d'une manière complète tous les caractères d'une personne ou d'une chose, l'idée complexe que nous supposons ne serait done que l'éveil d'un élément capable de s'associer systématiquement avec tous ces caractères, capable par suite d'en éveiller au besoin la représentation. Cette idée complexe serait donc un état très abstrait, l'éveil de certains sentiments abstraits fortement coordonnés entre eux et avec une foule d'autres états restant pour le moment a l'état latent. C'est-à-dire que cette idée complexe serait la manifestation la plus nette de l'action d'arrêt, ici, toutes les associations particulières possibles seraient enrayées. Avoir l'idée complète d'une chose ce serait n'avoir aucune idée actuelle des diverses particularités qui composent cette chose, mais avoir présents à l'esprit les éléments qui peuvent à volonté selon le besoin ru'on en a, éveiller l'idée ou l'image de n'imforte: lefucl de ces earactères particuliers. Cette idée complexe existe réellement d'une maniere plus ou moins inlarfinte, plus ou moins conforme au lype ideal que j'inclifur: elle ne se confond pas avee le mot, mais le mot s'assorib souvent a elle et la remplace dans une certaine 
mesure, et il est peut-être plus facile de vérifier sur le motce que je viens de dire de l'idée. Le mot cheval, est susceptible de s'associer avec n'importe quelle couleur, avec n'importe quelle forme appartenant à un cheval, il peut éveiller l'idée d'une quelconque des qualités de cet animal, il les représente toutes, sans en représenter particuliẻrement aucune; sans représenter non plus en particulier aucun des ètres concrets qui forment l'espèce désignée, il les représente tous, et nous pourons l'aroir dans l'esprit et l'employer même rapidement sans évoquer une seule image individuelle et concrète. Il y donc là une action inhibitoire très marquée et qui est mise en évidence par̀ ce fait que si les circonstances qui la produisent viennent a disparaitre, ses effets disparaîtront, et que les associations qu'elle a empêchées se produiront tour à tour selon qu'elles seront favorisées par les circonstances nouvelles. On ne saurait appeler évidemment une idée complète l'acte qui consisterait à envisager tour à tour ses différents caractères. C'est bien pourtant cet acte qui nous permet d'arriver à avoir cette idée mais il est seulement un moyen de réveiller certains éléments psychiques que nous abstrairons ensuite d'une des représentations partielles. L'idée complexe peut ètre le résultat de cette opération. Et d'ailleurs, cette opération mème suppose un arrêt successif des différentes tendances à l'association : quand nous pensons à un caractère en particulier, nous ne pouvons penser également à tous les autres ou nous ne le pouvons au plus que très faiblement, c'est-à-dire avec un arrêt très marquè.

Ainsi de la perception à l'idée abstraite, nous trouvons entre autres caractères, un arrèt graduel de l'activité de certains èléments. La suppression de certaines parties de la perception donne une image vive, une inhibition portant sur quelques-uns des éléments de l'image vive est impliquéc par la production d'une image faible, enfin 
l'idée abstraite suppose un nouvel arrêt, et la suppression de quelques-uns des éléments de l'image.

Le rôle de l'inhifjition dans la forma'ion et la consolidation des croyances, des jugements, des raisonnements a du ètre indiqué par ce qui précède. Nous devons y revenir cependant pour le rendre plus évident, et en apprécier plus exactement la généralité. Nous avons déjà entrevu ce fait qu'un jugement en empêche un grand nombre d'autres. Au moment où nous disons par exemple que la terre est ronde, nous ne pensons pas, ou nous ne pensons que très peu à ses autres propriétés, un des caractères du jugement est précisément de choisir une des propriétés de l'objet pour la mettre spécialement en lumière, et cela implique forcément que les autres restent dans l'ombre, il ne peut y aroir de jugement sans sélection, c'est-à-dire saus ar'êt portant sur les tendances qui ne peuvent actuellement se développer.

Mais un jugement ou une croyance implique aussi en tant que l'esprit fonctionne bien, la non-existence de jugements contraires portés sur le mème objet considéré sous un même rapjort. Le principe de contradiction, en psychologie, est un cas de la loi d'inhibition systématique. Croire que la terre tourne empeche de croire qu'elle est immobile. Et comme bien souvent nous avons des raisous de porter des jugements différents sur une même personne ou sur un même objet, il se produit alors une inhibition qui en certains cas s'exagère jusqu'à l'erreur. Par exemple les jugements que porte un homme sur ses semblables sont assez variables. En général ils oscillent brusquement d'un extrème à l'autre, l'homme est toujours forte à abonder daus son propre sens, et c'est une appli"attion de la loi d'association systématique. S'il vient de lire diu anteur quelque chose qui lui ait plu, il sera assez lorte a lui trouver un tres grand talent, s'il vient le lire du nosme antenr quelque chose qui lui a déplu, il ne 
sera pas éloigné de croire que c'est un idiot. Il y a dans chaque cas exclusion manifeste de l'esprit d'un groupe de considérations qui devraient intervenir pour aider à former un jugement complet. Ici encore une qualité d'une personne est bien considéréc it part, mais l'esprit la grossit considérablemeut et le jugement porté est trop général. Il n'y aurait pas grand mal si l'esprit ne le prenait pas souvent pour un jugement complet et impartial. Généralement un acte d'une personne, s'il vient d'ètre commis, s'il a attiré notre attention, éveille seulement l'idée des autres actes, des qualités de cette personne qui peuvent nous aider à porter sur elle un jugement semblable à celui que le dernier acte tend à faire naître en nous. Au contraire les actes qui pourraient éveiller un jugement contraire passent inaperçus, la représentation des qualités de la personne qui ne paraissent pas en. harmonic avec l'acte sous l'impression duquel nous restons et qui peuvent être cependant fort réelles et même assez développées - car.l'homme a souvent des tendances illogiquement réunies en lui, - cette représentation qui tendrait à ètre éveillée par l'idée de la personne qui les possède, par son nom, par les représentations visuelles ou autres que nous pouvons en avoir, ne se produisent pas, elles sont arrètées par I'établisscment dans l'esprit d'un système qui ne s'accorderait pas avec elles, - car l'idée que l'on se fait it un moment donné d'une personne est généralement beaucoup moins complexe, mais aussi beaucoup plus cohérente et logique que la personne même. Il en résulte que les jugements sont généralement exagérés, la systématisation a ćté trop ctroite, l'inhibition a été trop forte, il en est résulté une erreur.

Il est intéressant à cet égard de remarquer la peine qu'on a pour attribuer à la même personne des qualitès opposées et des actes qui manifestent ces qualités. Si ces actes sont attestés seulement par des témoins dont on ait 
quelque raison de suspecter le témoignage, on les rejette comme faux. Si ces actes sont certifiés par des témoins dignes de foi, on n'en tient pas compte, on ne les nie pas expressément, on les rejette simplement, on n'y pense plus. Dans les deux cas un jugrement a tenté de se former, il n'a pas pu y parvenir, ou plutôt dans les deux cas des perceptions ont éveillé des idées, ces idées comme toutes les idées ont tenté de s'associer un certain nombre d'éléments psychiques, mais ces éléments étaient engagés dans d'autres systèmes très stables, et ils ne pouvaient appartenir à la fois aux deux systèmes dont les fins étaient opposées, de là est résulté l'arrêt de l'activité nouvelle. Si l'acte se passc sous nos yeux mêmes, notre attention est plus fortement excitée par lui, il arrive encore pourtant que tout en voyant un acte on ne le comprend réellement pas, on le voit, on l'admet for'cement comme vrai, mais on n'en tire aucune conséquence; si l'on est pressé par une autre personne qui cherche à vous ouvrir leș yeux, on cherche à échapper à la discussion, ou bien on tâche de trouver des raisons, de combiner des idées qui puissent expliquer l'acte sans cependant détruirc l'opinion ancienne, autrement dit, on cherche à éveiller un système psychique qui puisse satisfaire à quelque degré la tendance à la coor:dination nouvelle, qu'on nc peut enrayer complètement, sans pourtant nuire à la coordination dominante, et cela se fait généralement en négligcant plus ou moins volontairement quelque circonstance du fait. Remarquons au reste que le changement d'opinion n'impliquerait pas moins un arrêt, seulement cet arrêt porterait sur ccux des éléments de l'ancienne tendancè qui ne pourraient plus entrer de la mème manière dans la nouvelle association. L'acle le plus simple et le plus facile pour un esprit peu raffiné est de changer complètement l'orientation de son esprit, et comme cela arrive souvent, de passer brusquement d'une 
opinion à une opinion opposée. Tenir compte de tout, décomposer chaque fait, en isoler les éléments et en déduire les tendances qui ont pu l'inspirer, tìcher de comprendre la coexistence ou la succession dans l'auteur de l'acte de tendances opposées ou qui paraissent telles est évidemment beaucoup plus compliqué, - mais du reste manifeste également notre loi puisque les associations systématiques que tend à déterminer l'idèe de tel acte, ou la croyance a l'existence de telle tendance sont toujours arrètées à quelque degré.

Au reste, quand une idée est fortement enracinée dans l'esprit ct surtout si elle est harmoniquement associée à un état permanent de l'organisme, comme cela se trouve dans certaines formes de folie et aussi dans certaines formes de l'état normal (confiance dans l'avenir', croyances optimistes des gens chez qui les fonctions de l'organisme s'accomplissent aisément et avec activité, les démonstrations les plus rigoureuses, les discussions les mieux conduites restent souvent complètement impuissantes à changer l'habitude de l'esprit, à déterminer un système d'idées autrement orienté que celui que l'on combat. Cet état de l'esprit est très bien exprimé par le mot d'une application fréquente ; « quand bien même vous me conraincriez, vous ne me persuaderiez pas ». Lorsqu'une idée tient aux systémes fondamentaux qui constituent la personualité mème, c'est eu rain qu'on essaye de l'ébranler. On ne saurait guère, par exemple, convertir par des raisonnements abstraits ou par des discussions philologiques et historiques, une personne qui a fondé sur la religion tous ses actes et toutes ses croyances, qui a associé à la religion ses affections les plus chères. Il est des cas où l'évidence même ne convaincrait pas. Toutes les passions fortes, l'amour, par exemple, ont frequemment ces effets: a Si tu m'aimais, tu m'en croirais plutôt que tes yeux », 
disait une amante prise cn flagrant délit d'infidélité, et psychologiquement elle n'avait pas tout à fait tort.

Souvent dans la folie une idée fixe due on ne sait trop à quelles causes, arrète ainsi toute idée saine que l'on tente de suggérer. Et parfois l'idée juste est momentanément admise, mais bientôt, - que l'aliéné en ait ou non conscience, - elle est arrêtece, les efforts qu'on fait pour la maintenir restent vains et souvent les éléments psychiques conservés satisfont autrement leur' besoin de systematisation, ils sont incorporés dans d'autres complexus qui peuvent s'accorder avec l'idée dominante. Un malade se plaignait de ce que ses ennemis, afin de le faire passer pour un voleur, venaient mettre des objets de prix dans son oreiller et sa paillasse. On fait découdre son oreiller; après avoir attentivement regardé, il reste tranquille toute la journée. Le lendemain, șes idées ètaient les mèmes qu'avant l'expérience ct, lorsqu'on voulut faire une nouvelle tentative, il dit que ses ennemis avaient soin d'enlever d'avance les diamants 1. Ainsi l'inhibition n'avait pas été complete du premicr coup, mais bientôt l'idée nouvelle avait élé écartèe, ct le fát réel qui fut encore perçu ne doma plus lieu qu'í des systémes d'idées qui pouvaient s'accorder très bien avec l'idéc principale; le malade, voyant que les oljets n'y sont plus, conclut, non pas qu'on ne les a pas apportés: ce qui gonerait sacroyance, nais hien quon les a enleves. Il n'en est pas foujours aiusi, ot puchufuefois il se produit uue sorte de scission de l'arlivilé intellectuelle, les deux systèmes d'idées coexistent quelque temps, puis l'un des deux finit encore par évincer l'autre, et l'iuhibition systématique se produit. Un négociant épronve quelques pertes légeres et se croit ruiné; on lui pressente en vain l'état de ses affaires, qui est trés brillant, il semble convaincu de son illusion, et n'en conclut pas moins qu'il est

1. Brierre de Boismont. Des IIallucinations, p. 138. 
ruiné. M. Maudsley essaya de convaincre de son erreur un aliéné, fort intelligent, et d'une haute culture intellectuelle, persuadé qu'il y avait un complot tramé contre lui, et que partout où il allait, il était suivi et surveillé. L'aliéné reconnu la justesse du raisonnement et avoua s'ètre dit souvent à lui-mème qu'il fallait ètre fou pour croire ce qu'il croyait, après quoi il garda entic̀rement ses convictions 1 . De même une femme voit son enfant renversé par un cheval; les raisonnements, la vue mème de son enfant qui se porte bien ne peuvent la convaincre qu'il est vivant. Dans un autre cas, que je trouve comme le précédent dans l'ouvrage d'Esquirol, qui est emprunté par lui à Pritcliard, on voit un jeune homme continuellement dominé par l'idée de son insuffisance à faire ce que l'on attend de lui. Regardé comme un bon écolier a il avait unc telle défiance de lui-mème qu'il redoutait de réciter ses leçons, craignant de ne pas les savoir. Son éducation terminée, il entre dans le commerce chez son père, qui lui donna des appointements considérables. Sa vie paraissait heureuse lorsqu'il annonce à son père qu'il ne veut pas le tromper plus longtemps, qu'il sent n'avoir pas la capacité nécessaire pour remplir sa táche, qu'il fait tort à son commerce. Le contraire était la vérité : aussi les parents de ce jeune homme s'efforcèrent de le rassurer mais il répétait: "Mon frère a plus de talent que moi, je ne remplirai jamais bien mon emploi. n Le père, homme d'esprit, proposa à son fils de changer de résidence et d'oceupation, l'envoya à Manchester faire de grandes acquisitions. Celui-ci remplit cette nission avec le plus grand succès. Mais bientôt M... retomba dans sa défiance ordinaire, se plaignit de nouveau de son incapacité tout en reprenant ses premières occupations. Les mêmes craintes de nuire aux intérêts de 
son père se réveillèrent, il renonga aux avantages que lui offrait la maison paternelle, partit secretement pour Liverpool dans l'intention de s'embarrjuer pour l'Amérique. Ne pourant s'embarquer, il fut contraint de retourner' dans sa famille. Il recommença bientòt sa tentative d'évasion. Reinstallé chez son père, il s'empoisonna. Confié aux soins du docteur IItch, ce médecin recomnut dans son malade un homme fait pour les affitires, intelligent, actif, économe, connaissant les marchandises, calculant très bien et ayant un très bon jugement.

"Trois mois suffirent pour remettre ce jeune homme en itat de rentrer dans sa famille, où pendant un an il s'occupa des affaires d'une manière très satisfaisante. $\Lambda$ celte époque il perdit encore toute confiance dans sa capacité, quitta ses parents, depuis il fit de fréquentes absences. On le laissa contenter ses caprices de déplacement; bientôt ses sentiments s'altérèrent, il évitait son père, lui parlait brusquement, n'en causait qu'avec colère ; il était soupgonncux envers les memlores de sa famille. "I J'ai cilé cet exemple tout au long, comme montrant parfaitement une série de systèmes psychiques, d’influences coordinatrices qui luttent contre l'idée fixe, et dont lactivité est toujours arrêtéc au bout d'un temps plus ou moins long.

Si l'on considere nos idées, nos croyances les plus larges, celles qui, par l'orientation qu'elles donnent frèquemment it de nombreux éléments psychiques sont pour ainsi dire la forme même d'une intelligrence, le fait est bien plus évident encore. Les exemples que j’ai cités plus haut de doctrines mal comprises impliquent également á un haut degré des actions d'arrèt, ils ne peuvent s'expliquer qu'en admetlant yue l'esprit rejette une portion uotablo de re qui lui est oflert. Bien des systemes psychiques qui nitissent sont emperches de serbeveprer d'abord

1. Esquirol. Des maladies mentales. T'. I, p. 61-62. 
sont rejetės ensuite et disparaissent presque completement sinon tout à fait; ils ne déterminent dans l'esprit aucune coordination de quelque importance. Par exemple, il est bien surr que les critiques que nous arons citées de la sélection naturelle de Darwin impliquent ce fait que les arguments de Darwin n'ont pas èté compris, que les mots imprimés ont à peine éveillé une perception visuelle, que cette perecption visuelle n’a pu déterminer la coordination d'éléments intellectuels qu'elle tendait à réveiller ou que, en tout casces élémentsintellectuels nont puavoir sur l'activité générale de l'esprit l'influence qu'ils auraient dù aroir. Ils tendaient à arrèter les autres sýstèmes, ceux qui se sont formés conformément aux habitudes de l'esprit, et ils ont èté arrètés par eux.

Si l'on se rappelle l'analyse du raisonnement telle qu'elle a étẻ faite dans la partie précédente de cette étude, on reconnaitra facilement que l'inhibition en est une condition nécessaire. Les idées et les jugcments qui s'enchainent, se coordonnent pour aboutir à un nouveau jugement exprimé ou qui pourrait l'ètre, impliquent chacun individuellement une certaine quantitè d'actions d'arrêt, mais la série entière, considérée en elle-même, en implique également. L'esprit qui est occupé à raisonner ne pense généralement pas à autre chose qu'à l'objet de son raisonnement; toutes ses autres tendances intellectuelles sont à quelque degré enra ๖eées, l'attention spontanéc qui seule nous intéresse pour le moment est une cause très forte d'arrêt pour un grand nombru de phénomènes et de tendances. Un raisonnement absorbe les forces psychiques, et, comme le jugement, il tend a cnrayer tout raisonnement contraire qui tendrait à se produire.

$\Lambda$ propos des phẻnomenes intellectuels, commie à propos des perceptions, nous pouvons remarquer la persistance des tendances associatives arrètées dès que l'association supérieure qui unit des elements dans un ş̌stėme en 
inhihant certaines de leurs tendances associatives vient à se rompre, les forces arrètées se manifestent et des associations incoherentes se produisent. Lorsque nous changeons d'opinion sur un sujet, nous royons un nouveau classenent des phénomènes s'opérer d'après leurs affinités arrètées jusqu'ici, et qui entrent en jeu. Pour prendre un exemple que j’ai déjà donné, on est quelquefois embarrassé d'expliquer quelques actes de certaines personnes d'après l'idèe qu'on se fait de leur caractère, et alors, on néglige ces actes, on les oublie, ou bien encore on n'en tire aucune conséquence, mais les idèes de ces actes, telles qu'clles se forment en nous n'en ont pas noins leur force propre, et cette force se manifestera si le système inhijsiteur vient ì disparaitre; on voit immediatement alors ces ilées déterminer une systématisation d'éléments ; les actes auparavant oubliés ou négligés, sont reconmus, classés, interprétés. De même quand une nouvelle théoric finit par se faire jour et s'établir, on s'aperçoit qu'elle explique une quantité de faits qui ne pouvaient s'expliquer par la theorie qui régnait jusqu'alors, et qu'on négrligeait on fu'on incorporait dans des systemes pen complexes. C'est ainsi que le darwinisme a donné une signification a certains faits, comme les organes rudimentaires, le développement de l'eml,ryon, dont la représentation dans l'esprit ne pouvait guère auparavant déterminer des systématisitions psychipues importantes, et se raccordait lant licen fue mal a cerlaines conceptions métaphysiques onl religieuses.

Le langage dont les modalités diverses, les combinaisons, les variations nous donnent des exemples très nets di: tontes les formes de l'artiviti intellecluclle peul nous servir a clablir plus clairement encore, l'importance des lois psychologriques que nons ctudions. Le changement du sens des mots est min exemple de sélection systematirue silns resse continnio. On peut ronsiderer d'abord 
les variations individuelles, car il arrive que les mots dont nous nous servons prennent jusqu'ì un certain point la marque de notre personnalité. En mème temps qu'ils représentent des idées qu'ils ne représentent pas dans le langage courant, officiel, ils cessent d'en représenter qui leur sont généralement attachées. Souvent ce changement de sens se fait peu à peu dans l'esprit, quand nous nous sommes servis plusieurs fois d'un même mot, nous prenons l'habitude de l'employer de préférence dans certaines conditions et dans certaines circonstances, il contracte de nouvelles associations, et inver'sement d'autres associations se rompent, et c'est ainsi par une série d'associations systématiques et d'inhibitions systématisées qu'il arrive à son sens le plus précis et le plus net, mais pas toujours le plus facile à comprendre pour les personnes dont l'esprit n'a pas fonctionnè dans le même sens. Nous remarquons des changements de cette nature dans la vie intellectuelle de chacun de nous. Mais ils sont dûs le plus souvent, à des causes sociales, et il ne faut pas oublier; en les citant, qu'ils ne valent en psychologie que par les opérations intellectuelles, individuelles qu'ils représentent, dans le détail desquelles il est impossible d'entrer, et qui d'ailleurs ne présentent aucune difficulté pour se rattacher à notre loi d'inhibition. C'est ainsi que vezzozo vicieux, devient un synonyme de charmaut, cadran qui indique à l'origine quelque chose de carré s'est étroitement associé à l'idée d'un cercle et a naturellement rompu ses anciennes attaches ${ }^{1}$. De même, vertueux se prend facilement en mauvaise part aujourd'hui, et des expressions qui furent à la mode au dernier siècle chez nous sont devenus difficiles à employer. Qui oserait célébrer comme le faisait Diderot le plaisir que doit éprouver une

1. Voyez Darmesteter. La vie des mots étudié dans leur signification. 
femme à serrer dans ses bras un homme vertueux et sensible 1? M. Brunetière a indiqué quelque part la transformation curicuse du sens de seduire (seducere) et des idées et des sentiments qu’il éveille. Un point qui nous interesse ici au point le vue du mécanisme de la sélection et de l'arrèt, c'est que la similitude des sens ne parait crécr aucun désavantage pour les mots. Cela indique la perfection avec laquelle l'arrêt systématique s'accomplit. Si la ressemblance des sons amenait la confusion des sens, il n'y a pas de doute que l'activité de l'esprit, ne tendìt généralement à éliminer des éléments nuisibles a la coordination, mais un son dans la phrase où il est cuclavé n'éveille que les idées qu'il doit logiquement éveiller, les autres associations qu'il pourrait amener ne se produisent pas ${ }^{2}$, il y a arrêt d'une partic de son activité virtuelle. M. Gaston Paris indique cette loi du langage en termes qui impliquent bien l'application a ce cas particulier de la loi que nous étudions. "Les mots verre, vers, ver, vert, sont parfaitement homophones : si l'un d'eux périt, ce ne sera pas probablement à cause de l'existence-des autres, l'homophonie ne peut, me semble-l-il, faire renoncer it un mot que quand elle frappe deux mots quelque peu voisins de sens, et peut amener de l'équivoque ${ }^{3}$; ainsi il est possible que l'ancien français ver de verrem ait cessé d'exister à cause de la confusion quelquefois possible avec ver de vermen."

En résumé, tous les éléments de l'activité intellectuelle examinés les uns après les autres nous ont paru fournir des cas d'arrêt extrèmement nombreux sans lesquels ils ne pourraient ni exister ni se développer; ni même se maintenir dans l'esprit. L'activité intellectuelle peut être

\section{Viderut. Lettres a $M^{110}$ Voland.}

2. Vuyez M. Bréal. Comment les mots s'associenl dans notre esprit.

3. Ces mots ne sont pas souligués dans le texte. 
considérée d'une manière générale comme une coorlina. tion d'éléments abstraits, ainsi que j'ai tiché de l'établir dans le livre précédent. Cette coordination ne peut se faire que par l'arrèt d'une certaine partic des tendances associatives des éléments qui en font partic. Nous avons vu que chaque élément psychique a pour ainsi dire sa personnalité propre, il tend à vivre et à se développer. S'il entre dans des combinaisons supérieures, il est ob)ligé de s'orienter d'une certaine manière, il n'agit plus que dans un sens, seules les qualités qui le rendent apte à faire partie de cet ensemble continuent à se manifester. Un mot a plusieurs sens intercalé dans une phrase n'éveille que les idées quii peuvent s'accorder avec le sens général de la phrase - c'est-a-dire qu'il y a arrêt d'une grande partie des processus possibles, lont une condition essentielle subsiste toujours. Et cette condition nous en voyons la permanence dans ce fait que, si les autres circonstances changent, si la coordination cesse, les éléments psychiques, reprenant à de certains égards la liberté de leur jeu, manifestent par de nouvelles associations ces affinités qui n'ont pas cessé d'exister, et qu'il ne faut considérer d'ailleurs, si l'on ne veut pas ressusciter les qualités occultes, que comme des conditions essentielles de certains groupes de phénomènes. 
CHAPITRE III.

\section{Plhénomènes affectifs et tendances.}

\section{$\S 1$.}

J'ai essayé d'établir aillcurs que les phénomènes affectifs conscients, dont la condition primordiale est une impulsion, une tendance, impliquaient également un certain arrèt de cette tendance, il faut qu'une impulsion rencontre quelque opposition pour que son fonctionnement donne lieu à des phénomènes affectifs : émotions, plaisirs, douleurs, etc. Je ne reviens pas sur cette démonstration; nous avons d'ailleurs à nous placer ici à un point de vue quelque peu différent, et nous étudierons simultanément les phénomènes ạffectifs et les tendances pour les raisons que j'ai indiquées dans un chapitre précédent. Le jeu des tendances est l'oljjet principal de notre étude, et spécialement l'action inhibitrice qu'elles exercent les unes sur les autres, ou qu'elles exercent avec réciprocité sur les autres phénomènes psychiques en général, les phénomènes suljectifs n'étant à considérer que comme signes des tendances qui les produisent.

Tout fait psychique, avons-nous dit, tend a empêcher de se produire, à empềcher de se développer, on à faire disparaitre les autres phénomènes psychirques qui ne peu. vent s'harmoniser avec lui pour une fin commune. Etudions sous ces trois aspects le jeu des tendances.

Qu une nouvelle tendance ne puisse réussir it s'organiscr dans l'esprit si elle contrarie les habitudes antécédentes, cest un fail d'expérience commune. Une préoceupation tres forte sera souvent suffisante pour préscrver 
une personne de prendre certaines habitudes malgré les occasions et les exemples. Un amour profond est généralement considéré comme d'une heureuse influence sur les mueurs d'un jeune homme, et les parents le favorisent quelquefois; a notre point de vue, ils tàchent de déterminer une orientation fixe des éléments psychiques qui arrète les associations que ces éléments pourraient contracter - et qu'ils désirent arrêter. S'ils ne réussissent pas toujour's complètement, ils n'en ont pas moins créé une influence qui exerce une action d'arrèt réelle quoique insuffisante. Quelquefois nous tàchons de nous créer à nous-mèmes des habitudes d'esprit ou de corps qui nous empêchent de prendre tel ou tel goùt. On cesse de fréquenter telle ou telle personne, telle ou telle société pour ne pas se créer de tendances à la paresse, à la dissipation, au jeu, a la débauche. L'idée des tendances qu'on veut éviter suffit à déterminer l'arrèt des tendances existant déjà et que l'on considère comme une cause possible des premières. Il faut ranger dans cette catėgorie tous les cas oì des hommes, à cause de leurs dispositions innées ou acquises, manifestent toujours une aversion pour les plaisirs que l'exemple ou les conseils tendent à leur faire partager. Ces faits sout fréquents. Quelques cas oì l'action inhibitrice trop forte aboutit à des erreurs mettent davantage son influence en lumière. "Que de fois, dit M. Luys, ne nous arrive-t-il pas à nous autres médecins d'entendre chaque fois que nous abordons un de nos clients fiévreux, alité, et que nous lui adressons les mots d'usage : "Comment allez-vous? o nous répondre invariablement " très bien " tout d'abord, pour se reprendre immédiatement, et commencer le récit de ses souffrances $\gg{ }^{1}$. L'organisation accuise a arrèté ici la tendance

1. Luys. Etudes de physiologie et de palhologie cérébrales. Des actions reflexes du cerveau, p. 134. 
les idées dominantes à s'associer arec les images auditives ou motrices et les eléments moteurs du langage alticulé. C'est un réllexe jsychique, pour employer l'expression de M. Fichet déterminé par l'excitation du nerf acoustique et metlant en activité probablement les centres de l'audition, peut-ètre le centre dont la lésion entraine la surdité verbale (première circonvolution temporale gauche) et le centre moteur (pied de la troisieme frontale gauche) pour aboutir a l'excitation des muscles de l'appareil rocal - qui a rrête la tendance de certains éléments psychiques a déterminer l'éveil d'autres phénomènes psychiques dans quelques-unes des mèmes régions. Ce n'est qu'après que la tendance liabituelle a été satisfaite que l'autre peut s'établir. L'influence inhibitrice de la tendance habituelle se manifeste souvent chez les enfants qui ont toujours à acquérir quelque nouvelle tendance, comme elle se manifeste aussi dans le fonctionnement des éléments sociaux. Ainsi un enfant qui s'est habitué à se traîner alor's que ses jambes n'étaient pas assez fortes pour le porter, est fort enclin à conlinuer a se trainer au lieu de marcher alors que ses jamles se sont fortifices, bien que ses parents essayent de lui faire comprendre que la marche du bipede est seule digne d'un homme. En sociologie des faits sembla-. bles sont bien connus. Un progrès accompli empêche d'en réaliser un autre plus considérable. Ainsi tous les systèmes perfectionnes de pavage, d'éclairage, de locomotion, etc., se heurtent a des habitudes prises, i des organisations anciennes qui ne se peuvent défaile facilement. Si l'on n'avait pas autrefois fait un eertain progres il serait a present plus facile de faire un progrès plus grand. II. Spencer a multiplié les exemples de ces inhibitions suciales 1. Si je les indique ici, c'est fue: outre que la

1. Sirencer. Introduction à l'étrede de la science sociale. 
sociologie ne se distingue point très nettement de la psychologic et qu'il y a en plusieurs occasions un grand intérèt a rapprocher les deux ordres de phénomènes, les faits socianx dont il est question se manifestent dans l'esprit des individus par des phénomènes psychiques intéressants à notre point de vue. Les fonctionnaires qui connaissent l'état de choses actuel et qui savent les longs efforts qu'il faudrait faire pour y porter remède, et les dépenses et les inconvénients qui en résulteraient, se montrent généralement très conservateurs, par intérêt social, intérêt collectif d'une compagnic, on intérêt personnel, au point qu'on a pu quelquefois sans injustice les accuser de l'être beaucoup trop. C'est-à-dire que chez eux les nourelles tendances sont fortement arrêtées par les coordinations déjà formées. C'est là un point absolument général et qui se manifeste toujours de quelque manière alors mème que les anciennes habitudes finissent par être désorganisées et que la nouvelle systématisation s'établit.

De même tout fait psychique tend à empêcher de se développer une nouvelle tendance qui ne peut s'harmoniser avec lui. Les cas de lutte de divers sentiments sont d'ordre normal. Ils n'est pas de jour où nous ne puissions en remarquer des cxemples. Elle se produit soit à propos de sentiments très peu importants, soit à propos des fondements même de notre caractère. Dans cette catégorie rentre, par exemple, l'effort que fait en nous l'instinct de l'économic, ou tout autre considération, pour empêcher le goùt d'une chose qui nous tente de se développer au point de s'associer avec les mouvements des jambes qui nous conduisent chez le marchand, comme aussi la quan. tité de sentiments variés qui, chez un vieillard, viennent tenter d'arrêter le développement d'un amour naissant pour une jeune fille. Les impulsions pathologiques nous montrent ce phénomène daus toute sa netteté. Ici des circonstances morbides que l'esprit ne peut vaincre, portent 
en lui limpulsion et ne la laissent pas disparaitre, mais les tendances antérienres font au moins tout ce qu'elles peuvent pour l'empècher de ce développer. Un homme de bonne famille, raconte Spurzheim, sentit des son jeune àge un penchant au vol; il entri dans la carrière militaire, espérant ètre retenu par la discipline, et fut sur le point d'être condamne à mort. Il étudia la théologie et se fit capucin, son penchant le suivit dans le cloître. Là il ne dérobe plus que de menus objets et se livre a son naturel sans s'en inquiéter, après quoi il avoue ce qu'il a fait et invite le propriétaire à venir reprendre son bien ${ }^{\mathrm{l}}$. Ici on le voit la lutte finit par un accord, le développement de la tendance n'a pu être complètement arrêté, mais il a été au moins enrayé et la nouvelle habitude a vécu ainsi en paix avec les autres. La tendance nouvelle, dans la folie, se manifeste souvent sous la forme d'une voix qui donne des ordres aux malades, et ceux-ci font souvent des efforts pour résister sans pouvoir toujours y arriver, l'inhibition ne peut se produire, ou plutôt elle se produit en sens inverse, ce sont les tendances habituclles qui sont arrêtées par l'influence de la nouvelle venue. Une jeune fille fort jolic, tres douce, bien élevee, ayant des principes religieux, quitte sa maison et se réfugie dans une forèt pour obeir a une voix. Un homme riche, également poussé par. une voix, vend ses biens et jette dans un puits tout l'argent qu'il retire de cette rente. Un dame que des roix empêchent de toucher aux mets qu'on lui présente, sait bien rue ces voix proviennent d'une affection nerveuse; elle s'apercoit souvent que les roix lui font faire des choses déraisonuables, malgré tout, elle est forcée d'obéir. Ponssée a sc noyer, elle éprouve une resistance intélieure qui lempèche de céder, mais elle craint de ne jouvoir toujours resister.

1. Sifurzheim. Observations sur la pherénologie', p. 17:3. 
Enfu, des faits interessants d'inhibition nous sont fournis par les cas de monomanie lomicide que j’ai déjá cu l'occasion de citer et dans lesquels nous royons les malades tàcher, par divers moyens, d'arrèter le développement de la tendance qui les assiège. La moindre influence peut quelquefois empècher celle-ci d'aboutir à des actes. Un malade se fait attacher les pouces arec un ruban I, un autre prévient la personne qu'il est tenté de frapper et s'enfuit ${ }^{2}$, un troisième se fait des remontrauces, crie a ses anis de se saurer et les remercie de la résistance qu'ils lui opposent ${ }^{3}$, etc.

De mème que linhibition empèche les tendances de naitre et de sc développer, de mème elle peut en interrompre les manifestations et peut-ètre à la fin les faire disparaitre. Au milieu d'un diner, si quelqu'un raconte une histoire fort intéressànte, le mourement des fourchettes se ralentit. Si péndant quun ,romeneur se lirre à ses méditations le vent lui emporte son chapeau, aussitòt ses méditations cessent; quand deux personnes causent en marchant, si la conversation devient très animée, il est rare qu'il n'y ait pas inhibition de l'activité locomotrice, etc. Cette influence inhibitrice des systémes psychiques est employẻc dans l'éducation; si un enfant manifeste de mauraises dispositions passagères, s’il s'entète à faire un acte qu'on lui interdit et s'il n'obeiit pas aux couseils ou aux ordres, ou peut, si par cxemple sa mauraise santé fait craindre de mauvais effets de l'obéissance imposée, agir sur lui et inhiber ses tendances actuelles en dètournant son attention, en suscitant en lui des perceptions, des idées, qui l’intèressent et qui l'empèchent de continuer à désohéir. On agit de mème sur les

1. Esquirol. Mémoires sur la Monomanie homicide.

2. Pinel. Traité médico philosophique de l'alienation men!ale, p. 160.

3. Spurzheim. Obserca:ions sur la phrénologie, p. 121. 
grandes personnes en éveillant chez elles des idées étrangères à leurs désirs actuels au lieu de heurter de front ces désirs. Les inhibitions de ce genre se produisent à toute heure, par la raison bien simple que dès qu'une de nos tendances entre en activité, elle empèche plus ou moins celles qui se manifestaient arant elles de continuer d'agir, mais certains faits les mettent particulièrement en lumière. Théophilacte, patriarche de Constantinople au $x^{e}$ siècle, avait, dit-on, plus de deux mille chevaux dans ses écuries: "Ln jour do Jeudi Saint, comme il célébrait la messe, son maître stabulaire vint lui dire à l'oreille que sa jument favorite mettait bas en ce moment. Aussitôt il laisse là l'office, court à ses écuries, et aprè̀s avoir assisté à l'heureuse délivrance de la bète, il vient reprendre la messe où il l'avait laissée " I. Esquirol racontc le fait suivant d'une idiote, Quéneau, très sensible à la musique: "M. Desprès, élève interne de l'hospice, chante un air compliqué, Quéneau redouble d'attention, fixe ses yeux sur l'élève, contracte ses traits et parvient à se mettre à l'unisson avec le chanteur. Des fruits qu'elle aime beaucoup sont mis à sa portéc, elle manifeste, par ses regards et par ses gestes, le désir de les prendre, mais au moment où elle est prète à s'en emparer, M. Desprès bat la mesure et chante; aussitôt . Quéneau bat la mesure, abandonne les fruits qu'elle saisit avec avidité dès que le chant a cessé n?

L'inhibition systemalique est souvent causće par le changement de milieu, les pereeptions nouvelles et les systemes jesychiques qu'elles organisent, mettent fortement en jeu certaines tendanees et interrompent celles qui ne pouvaient s'harmoniscr avec elles. Nous avous dèja étudié l'association systématique de l'étal de l'es-

1. Raissac. Le Diable, p. 362.

2. Esquirol. Des maladies mentales, T. II, p. 306. 
prit et mème de l'état des organes avec les impressions faites par le milieu, elle implique une inlibition systématique des tendances organiques et psycho-organiques qui étaient en harmonie avecun milieu différent. Les métamorploses chez les plantes ou les animaux, que mous avons precedemment citées et commentées ne se comprennent hien que si l'on fait interrenir l'arrêt en mème temps que l'association. Qui a vu des amis dans un café et qui les retrouve dans un salon voit que leur manière d'ètre n'est pas la mème. D'une manière générale la présence de personnes qu'on ne connait pas, mais qui ont une situation sociale importante it un point de vue quelconque (talent, position, fortume, influence, etc.), change jusqu'i un certain point la manière de penser et de sentir, la manière de s'exprimer et modifie mème jusqu'à un certain point le son de voix des assistants. Le caractère d'une personne est ainsi variable selon les milieux, et certains défauts, certaines idées ne se manifestent très souvent que dans un milieu donné, partout ailleurs ils sont fortement arrêtés. Une nymphomane chez qui " une statue, un tableau, la rue d'un homme, le contact le plus simple, un mot suffisaient pour exciter les désirs violents », était aussi sujètte à des rèves lascifs " qui agissaient sur ses sens avec une force surprenante. Au reste dans la société cette dame s'imposait une telle réserve, que rien ne transpirait de ces dispositions qui la désolaient amèrement " 1 .

De mème que l'influence inhibitrice des sentiments est souvent employée dans l'ćducation et aussi dans les relations sociales, on a tenté, quelquefois avec succès, de l'appliquer au traitement de la folie. «Les heureux effets

1. Annales d'hygiène publique et de médecine légale, t. iv, p. 422. Art. de M. H. Bayard : Examen medico-legal de cette question : la Nymphomanie peut-elle ctre une cause d'interdiction? 
qu'on se propose, écrit Esquirol, ne s'ohtiennent que par des sccousses, des commotions, des évènements imprévus, des conversations vives el animées et courtes, car ce n'est point par de longs arguments qu'on peut espérer d'être utile aux aliénés. Il faut toujours prarler avec vérité, sincérité à ses malades, n'employer que le langage de la raison et de la hienveillance, mais vouloir guérir les aliénés par des syllogismes et des raisonnements, c'est mal connaitre l'histoire clinique de l'aliénation mentale.

"Il importe surtout de substituer a une passion imaginaire, une passion réelle..... Les passions excitantes, l'amour, l'ambition, ont été appelées au secours des aliénés. Un mélancolique se désespère : on lui suppose un procès; le désir de défendre ses intérêts lui rend son énergie habituelle. Un militaire devient maniaque : après quelques mois on lui dit que la campagne va commencer ; il demande la permission de rejoindre son génèral, il se rend à l'armée et y arive très bien portant. "

"Une dame confièe à mes soins, dit-il ailleurs, rui croyait ètre damnée et aroir le diable dans le corps, fut guérie par la crainte des bains froids, qu'elle redoutait infiuiment, dont on lui faisatit la menace chaque fois qu'elle s'abandonnait à ses idées et à ses craintes chimériques. " Eufin « j’ai vu, ajoute le même auteur, la substitution d'une passion à une autre guérir la lypémanic en s'adressant a l'amour-propre, en l'irritant, en l'exaltant. J'ai fait taire des terreurs imaginaires ; j'ai vu l'amour prendre la place du délire mélancolique chez deux ou trois jeunes lypémaniaques de la Salpétrière. "Les moyens physiques qu'indiquait Esquirol, de guérir on le soigner la folie, peuvent se rattacher au mème principe. "Les sites bien éclaires, gais, pittoresques, conviendront ans lypémaniatgurs. Ceux qui sont tombés malades rans les pays chauds auront beaucoup de chances pour recouvrer leur 
L'INHibition systématique - tendances

raison dans les climats froids, et réciproquement: les nostalgiques ne se rétablissent qu'en revoyant leur pays.»

$$
\S 2 .
$$

Il se produit souvent dans l'ordre des sentiments et des tendances des phénomènes tout à fait comparables à l'anesthésie systématisée des somnambules. C'est une sorte de suppression systématisée de tout un groupe de tendances. L'inhibition se rattache toujours à la systématisation psychique, en ce sens que c'est une influence organisatrice qui la prołnit, mais dans les cas particuliers qui se présentent à nous maintenant, l'inhibition est complexe et, portant directement sur une tendance, elle agit indirectement sur d'autres tendances qui sont systématiquement associées à la première. De mème que la mise en activité d'une tendance amène la mise en activité des tendances qui peuvent s'unir avec clles en vue d'une fin commune, de même la mise en non activité d'une de ces tendances amène l'arrèt des autres, alors mème que quelques-unes de ces autres pourraient, en d'autres circonstances, s'accommoder du nouveau système dominant. On en a des exemples dans des faits d'adaptation organique, par exemple, les modifications qui se produisent chez les eunuques corrélativement à l'ablation des organes sexuels, et dont quelques-unes au moins paraissent illogiques. Il est à remarquer du reste que l'opération faite après la puberté n'a pas les mêmes influences et que les modifications qu'elle produit sont moins nombreuses et moins profondes. C'est que les nouvelles qualités se sont développées et se sont associées aux conditions organiques qui persistent après l'opération. De même si, je suppose, un jeune homme, sous l'influence d'une passion vive, amour ou ambition, a rompu avec certaines habitudes de flànerie, s'il s'est plié à une régularité de vie qüil n'avait pas auparavant, il se peut très 
bien que la disparition de l'amour ou de l'ambition fasse reparaitre les anciennes tendances et disparaître les nouvelles qui, bien que s'accordint avec la passion dominante passagère, auraicut pu ètre utiles, malgré la disparition de celle-ci. En ce cas l'assoriation entre la personnalité cutière, l'ensemble des tendances et les nouvelles habituturess n'était pas suffisamment effectuće. L'influence des halbitudes premières avait probablement arrèté jusqu'à un certain point la nouvelle systématisation, et bien que cotte systématisation s'étendit jusqu'í la conduite, elle n'avait pas embrassé des éléments psychiques persistants dont l'influence l'a fait rejeter alors que quelques-uns de ses principaux éléments ont disparu. Si cependant les nouvelles habitudes avaient duré assez longtemps pour qu'une longue pratique les eùt harmoniquement associées à une partic considérable de la personnalité, elles eusșent pu sulsister après la disparition de la cause qui les avait produites, et en fait, il arrive bien qu'une qualité, une habitude acquise sous l'influcnce d'un sentiment vif quelconque persiste après que ce sentiment s'est éteint. Ir. de Quatrefages a signalé un fait d'importance sociale mais de nature psychologique qui montre bien la forme particulière d'inhibition dont nous nous occupons à présent. C'est peut-être, dit-il, dans les arts, les industries "que se manifeste le mieux l'influence exercée par une race supéricure venant s'imposer aux races inférieures. En ruelques années, celles-ci oublient ct perdent leurs industries, celles mêmes qui leur assuraient une sorte de supériorité sur quelques points. Les Maoris ont substitué nos cotonnades aux fines nattes qu'ils tressaient pour leurs femmes-chefs, les Polynésiens ont remplacé par nos plus grossicrs canots les doubles pirogues qu'ont admirées Cook ct Bougainville et dont la plate-forme portail tantôt une morleste cabane fouvant servir d'abri a une famille, tantòt un groupe de guerriers qui, réunis aux rameurs, 
formaient un équipage de cent quarante ì cent quatrevingts hommes $\gg$.

Nous avons ici une occasion de préciser à certains égards la nature de l'association systématique et bien que nol.re sujet actuel soit plus spécialement l'inhibition, il n'est pas inutile de voir comment une forme particulière de l'arrèt peut nous faire mieux conıp'endre l'association. Il ne suffit pas que dans l'organisme deux tendances ou deux phénomènes soient cn harmonie, et puissent servir à une même fin, pour qu'ils soient récllement associés entre eux, il faut. aussi que l'un puisse susciter l'autre. Il n'y a pas, en un sens, harmonic préétablic entre les tendances, il y a conditionuement des unes par les autres. Seulement la nature de l'esprit est tclle que le conditionnemênt se fait généralement selon une loi de finalité et que réciproquement la finalité est une cause du conditionnement. Si deux tendances sont susceptibles d'être harmoniquement associées, elles finissent généralement par le devenir. C'est ainsi que nous conservons plusieurs survivances se rapportant à des croyances disparues, que nous agissons conformément à des théories acceptées autrefois. Ces théories, ces croyances, ne sont plus, mais ce qui, en elles, s'adaptait à la nature générale de l'homme et s'unissait systématiquement à ses tendances permanentes, a fini par s'associer réellement avec elles, et par persister avec elles quand les croyances-causes ont disparu. Cettc association paraît se produire à la longue par le fait même de la vie qui suppose que les divers systèmes psychiques sont à quelque degré mis en rapport entre eux, éprouvés les uns par les autres, et placés dans des conditions qui les forcent à s'unir plus étroitement ou à disparaitr'c. Nous en avons indiqué plus haut des exemples concrets. La tendance associative naît 
et se fortifie peu à peu, nous arons des cas où elle est très faible et où elle cesse bientòt de se manifester, nous en avons d'autres oì elle est déjà très visible, où les nouvelles habitudes acquises persistent plus ou moins longtemps aprés la suppression te la cause qui les a produites. Fnfin dans certains autres cas, les associations quielles ont contractées arec des systemes psychiques permanents suffisent à leur assurer une durèe indéfinie.

$\$ 3$.

La persistance des tendances arrètées se manileste de la même manière que la persistance des autres phénomènes, par leur retour à l'activité dès que l'inhibition cesse. Nous en avous déjà vu des exemples avec les cas du patriarche de Constantinople et de l'idiote de la Salpètrière, il serait possible de les multiplier, on pourrait citer, par exemple, les faits nombreux de vertige épileptique arrêtant pour un moment une activite psychique quelconque qui recommence ensuite au point même oì elle s'est interrompue, et les faits analogues présentés par les aliénés ou les hypnotisés. Dans la vie normale le mème phénomène se présente: je suis à travailler, on me fait une question, il y a un arrèt immédiat du système en activité et lorsque j'ai répondu, ce système recommence à se développer à partir du point où il s'est interrompu. En somme, nos tendances, - sauf les cas de disparition complete qui arrivent pent-être, mais qui sont plus rares qu'on ne pense, - persistent généralement en nous it quelques degrés, représentées par certains de leurs éléments qui, des que les conditions seront favolables, en eveilleront d'autres et recommenceront à donner a l'esprit une orientation particulière. On sait combien Iessouvenirs, les habitudes de l'enfance, inhibées pendant longtemps par' les coordinations qu'amènent les diverses circonstances de la vie et la nécessité de modifier incessamment les habitudes de l'esprit au moins pendant la jeunesse et 
de conserver ensuite longtemps ces modifications acquises, ont une persistance longue el opinicitre et sont susceptibles de revenir lorsque la vicillesse ou la malatie ont detruit les coordinations supérieures qui, en retenant daus de certaines formes mentales les éléments psychiques, les empèchaient de manifester leurs tendances associatives premières. Par exempie, un individu émigre, il habite un pays qui n'est pas le sien, il en apprend la langue; les objets usuels, les sentiments mème, enfin tout ce qu'il a à nommer s'associe aux nouvelles formes de langage qu'il est obligé de prendre pour se faire comprendre et sans lequel ses idécs: ses croyances, ses actes ne pourraient ètre coordonnés avec les idées et les actes de ses noureaux concitoyens, c'est à-dire avec les nouvelles conditions d'existence qưil s'est faites. Les nécessités de la vie lui font toujours employer cette langue, il finit par oublier mème sa langue maternelle, c'est-à-dire que les premières associations sont si bien arrètées que la volonté mème, ou une tendance quelconque ne peut les reproduire, mais si la nouvelle coordination vient à cesser, si une maladie vient détruire les systèmes les plus récents, la force inhibitrice cesse et les affinités inoccupées depuis tant d'années se manifestent encore, et à mesure que la dissociation des systèmes récents se continue on voit en quelques cas se manifester successivement deș associations de plus en plus anciennes. Tel est le cas de l'homme qui ayant appris successivement trois langues différentes et ayant perdu par maladie l'usage de celle qu'il employait en dernier lieu, revint d'abord à la précédente, et enfin a la première de toutes, ì celle qu'il avait parlé étant enfant ${ }^{1}$.

Après la persistance des habitudes de l'enfance, il faut

1. Abercrombie. Inquiries concerning the intellectual powers, ete. Voir plusieurs cas intélessants, Rilıot, Maladies de la mémoire. 
noter celles des habitudes de nos ancêtres et spécialement les faits d'atarisme. Que l'on adopte la théorie de la pangenèse de Darwin on la théorio de la perigenèse des plastidules de Hackel on fuelyne autre ${ }^{1}$ on bien eneore quion nien aecepte aucume et fu'on trouve la question insoluble ou non résolue, le fait est indéniable, nous portons en nous virtuellement des tendanees qui furent celles de nos ancêtres humains et peut-être celles de nos ancêtres animaux. Les éléments de notre organisme et de notre esprit ont des désirs, des besoins qui leur sont transmis par une longue suite d'influenees dont l'origine remonte à des milliers d'années. Les matériaux de notre corps se renouvellent, il n'est sans doute pas d'atome dans les cellules de notre corps qui ne soit destiné a ètre remplacé, à disparaître dans le tourbillon vital, emporté par les opérations chimiques dont la vie est la synthèse, mais la matière passe et la forme reste, au moins en partic, elle reste et agit, influençant les nouvelles combinaisons qui se produisent. Ainsi nos cellules et les organes qui les réunissent et l'urganisme qui est la synthèse de ees organes gardent des habitudes permanentes que l'expérience modifie et perfectionne, mais en apprenant i agir de nouvelles manières, en entrant dans de nouvelles combiuaisons, elles n'ont pas entièremeut désappris leurs premières manières d'ètre. La mer' dit-on, est encore agitéc par le sillarge des vaisseaux de Pompée, et notre être vibre encore, jourraiton ajouter, des résirs qui ont agrité nos ancêtres de l'îge de pierre et peut-être aussi des tendances qui ont synthétisé les aetes vitaux rudimentaires du protoplasma qui a récu dans les mers lamenticunes ou cambriennes. Depuis lors la complication des systemes pliysiologirues et psychiques s'est

1. Voir un exposé des récentes théories sur la génération et l'hérédité, d'apres le cours de M. Balliani dans la Revue philosophique, 1889, t. I. 
indéfiniment accrue, mais c'est encore la même tendance, lo mème désir de vie et d'harmonie qui se manifeste en nous et ce sont eneore les memes formes dissociation qui se montrent lorsque les circonstances permettent anx phus anciennes habitudes de se manifester. Et nous en avons de merveilleux exemples oì la psychologie ne les cherche pas assez souvent. La cellule fécondée, le germe d'un nouvel ètre va reproduire successivement des ébauches des divers états par où l'homme a passé, s'il est vrai, comme la seule hypothèse acceptable nous l'indique, qu'il ait son origine dans les êtres inférieurs. N'est-ce pas là, à notre point de vue, la remise en activité de ces tendances inapergues pour la conscience, oubliées depuis des millions d'années peut-ètre, mais qui ont continué d'ètre prèsentes dans lïntimité des tissus, arrètées, entravées par toutes les circonstances de la vie actuelle, mais persistant toujours? J'ai essayé d'établir et de rappeler dans un chapitre précédent la systématisation singulièrement étroite de l'esprit et de l'organisme, il semble que chacun de nos actes, que chacune de nos pensées, ait son retentissement sur la vie organique, et il n'est pas douteux que cette coordination générale de l'organisme et de l'esprit ne se renouvelle à chaque instant et qu'une quantité innombrable d'impercejtibles modifications ne se produise à chaque instant dans les éléments qui nous composent, il n'est pas douteux non plus qu'elles laissent une trace. Les influences les plus faibles exercent une action persistante sur la matière, une expérience connue est celle-ci : Posez un pain à cacheter sur un rasoir, soufllez, une légère couche humide se dépose sur l'acier, par la condensation de la rapeur d'eau de l'haleine, oxcepté sur la partie préservée; qu'on laisse l'instrument sans le toucher pendant un temps mème très long, vous verrez, en soufllant de nouveau, reparaitre la forme ronde du pain à cacheter. La conservation 
des modifications organiques est bien connue aussi. Cliez les syphilitiques, mème complètenrent guéris en apparence, les éléments organiques gardent la trace du mal, et i la moindre occasion, une blessure, par cxemple, des acridents se produisent qui limoignent de la modification subie par lo corps. La divination de la pensèc par le sens musculaire et le sens dutact, comme l'ont nontrée les expériences de M. Cumberland, fait pressentir jusqu'où va la modification que l'esprit impose d l'organisme. Mais si nos pensées et nos actes se réfléchissent dans les organes et les modifient plus ou moins, chaque partie de nous a pour ainsi dire jusqu'd un certain point la marque de l'organisme auquel elle appartient. J'ai cité plus haut des faits qui montrent juscu'à quel point la reproduction d'un état organique très particulier peut entrainer la reproduction d'un état psychique avec lequel il s'est associé.

Ainsi l'ovule et le spermatozoüde ont reçu certainement des modifications inappréciables des actes, des pensées, des desirs des individus auxquels ils ont appartenu. Ils ont contracté ainsi des dispositions particulières, une tendance systématisatrice spéciale qui déterminera la forme que prendront, selon les circonstances, les nouveaux phénomènes que la croissance va produire. Ce n'est pas sur un terrain vierge rue tombent les premières excitations, ce n'est pas un terrain vierge que fécondent les premiers aliments. Il a en hui des tendances qui lui viennent de loin et que les circonstinces vont manifester et comme il a la forme des etres inferieurs, des "organismessans organes", c'est leur manière d'ètre qu'il reproduit tout d'alord, et à mesnre qu'il se développe nous voyons ses virlualites latentes, que l'organisme alulte n'avait pu favoliser, mais qu'il gatdait comme un heritage de ses ancotres, se manifester daus les limites du possible organidjument et psychiquement, et être de nouveau inlidées 
par un développement nouveau qui permet lapparition d'une nouvelle couche, plus récente, de ces souvenirs anciens. Lal génération, l'hérédité tout entière, le développement de l'ètre nous parait ainsi comme la manifestation de tendances persistant malgré l'inhibition dont elles n'ont cessé d'ètre frappées, sauf à de rares intervalles, dans la série des organismes qui les ont contenues, et ces tendances ne sont que des propriétes de la matière organisée en général, ou des propriétés particulières de la matière organisée de telle ou telle manière, d'éléments systématisés selon une loi donnée. Le léveloppement qui s'effectue après la naissance prête à des considérations analogues, la manifestation des habitudes psychiques qui a caractérisé tel ou tel état de l'évolution humaine, s'explique par les mêmes considérations, et de même l'apparition des tendances qui ont caractérisé telle ou telle race dans un membre de cette race, telle ou telle famille dans un membre de cette famille, telle ou telle personne chez son enfant ou son descendant direct.

Le développement de l'embryon est évidemment une condition exceptionnellement favorable pour la réapparition des tendances inhibées pendant l'àge adulte - l'ètat des organes ne permettant pas alors que les formes supérieures de l'organisme et de l'esprit puissent se montrer - mais dans la vie adulte, si ces formes supérieures sont détruites, ou provisoirement arrêtées, à leur tour, les formes inférieures reparaissent. Laycock dit aroir souvent observé que dans les affections cérébrales qui atteignent l'homme d'une haute culture intellectuelle, il y a une réversion à l'état mental de l'enfance des ancètres et mème de l'homme sauvage. Nous avons dejii vu 1 un cas emprunté a Galtou oì une habitude ancestrale parait déterminer certains plénomènes. Un fait de mème

1. Page 4r. 
nature est que la paille qui a servi pour la litière des lions et des tigres ne peut ensuite servil aux chevaux parce que l'odeur de cette paille les épouvante quand on l'introduit dans leur étable. "Bien des générations de chevaux domestiques, ajoute Laycock, ont dù se succéder depuis que le cheval sauvage, que nous devons supposer l'ancêtre de l'animal domestique, a été exposé aux attaques de ces représentants de la race féline ". Il est a croire qu'il y a ici une réminiscence ancestrale, mais il est possible aussi que l'expérience des premières générations, des ancêtres sauvages, et l'imitation des générations successives aient une part dans la manifestation de cet instinct. Laycock admet encore que chez des idiots et des fous, il peut $\mathrm{y}$ avoir un retour de tendances animales. "Cette variété de dégradation est caractérisée par des mouvements insensés ou par une tendance à abandonner la société pour rechercher la fréquentation des classes les plus basses de l'humanité, à errer dans les bois et les lieux inhabités (mélancolie vagabonde), à vivre comme les solitaires dans les souterrains, sans se laver, ni se peigner, à suivre les goùts du sauvage, comme le cannibalisme et les manies sanguinaires, á obéir aux instincts féroces, comme la lycanthropie, le vampirisme, et les mours pratiquées par la secte des Agharis, chez les Hindous, que l'on dit aller nus, se nourrir d'ordures, et manger a petits morceaux la chair qu'ils tirent d'un crâne humain " 1 . Cette interprétation est certainement intéressante, mais à mon avis les données que nous avons sur la psychologie primitive de l'humanité ne nous permettent pas de l'accepter sans réserve. On sait que M. I Lombroso aussi a vu dans le type criminel un retour à des

1. Laycock. La mémoire ancestrale. Revue scientifique du 19 aouic 1870. 
tendances ancestrales 1 . Son opinion a été vivement combattue ${ }^{2}$ et il ne semble pas qu'elle puisse être une theorie génèrale du type criminel. Mais il est très possible qu'elle s'applique fort bien a un certain nombre de faits, et il est une considération dont on n'a peut-être pas assez tenu compte. Nous pouvons, en cffet, parfaitement supposer que le type criminel sans ètre constitué par un retour au type primitif soit causé par un retour partiel à ce type, rendant l'indiridu incapable de prendre une place régulière dans une socièté civilisée. Combinez certaines tendances qui devaient se trouver chez l'homme primitif, la difficulté de s'astreindre à une tâche réguliére, le goùt du farniente, la brutalité des mcurs, le peu de raffinement des sentiments moraux, le courage et le mépris de la mort, peut-être une tendance à faire moins de cas de la vie humaine que nous n'en faisons à présent, tous ces traits qui pouvaient constituer alors un homme parfaitement normal, feront à présent un irrégulier, un déclassé, et si ses passions sont vives, un criminel que l'on pourra fort bien appeler un criminel-né parce qu'il apportait en naissant les tendances qui devaient fatalement, étant donné notre organisation sociale actuelle, le rendre criminel.

L'arrêt de développement, on le sait, amènè des phénomènes qui reproduisent souvent une forme ancestrale, nous prenons ici sur le fait et l'inhibition et la persistance des tendances. Le nom seul d'arrêt de développement indique suffisamment qu'il s'agit de l'inhibition des tendances supérieures et la forme des phénomènes qui en résulte montre bien comment cette inhibition s'accompagne d'une réviviscence des tendances anciennes. Darwin

1. Lombroso. L'homme criminel.

2. Voir particulièrement Tarde. La criminalité comparée, ch. I. 
expose; d'après Vogt, l'al'êt dont est frappé le développement du cerreau chez les idiots microciphales. a Le cuine de ces idiots est plus petit et les circonvolutions du cerveau sont moins compliquées que chez l'homme it l'état normal. Le sinus frontal, largement développé, formant une projection sur les sourcils, et le prognathisme effrayant des machoires donnent á ces idiots quelque ressemblance avec les types inférieurs de l'humanité. Leur intelligence et la plupart de leurs facultés mentales sont d'une extrême faiblesse. Ils ne peuvent articuler aucun langage, sont incapables de toute attention prolongée, mais sont enclins à l'imitation. Ils sont forts et remaquablement actifs, gambadent, sautent sans cesse et font des grimaces. Ils montent souvent les escalier's à quatre pattes et sont singulièrement portés à grimper sur les meubles ou sur les arbres. Ils nous rappellent aussi le plaisir que manifestent presque tous les jeunes garçons à grimper aux arbres, et ce fait que les agneaux et cabris, primitivement animaux alpins, aiment à folìtrer sur les moindres élévations de terrain qu'ils rencontrent. Les idiots ressemblent aussi aux animaux inférieurs sous quelques autres rapports, ainsi on en a connu plusieurs qui flairaient avec beaucoup de soin chaque bouchee arant de la manger. On cite un idiot qui. pour attraper les poux, se servait indifféremment de sa bouche on de ses mains. Les idiots ont d'ordinaire des habitudes dégoùtantes; ils n'ont aucune idée de la decence; on a remarqué que certains avaient le corps couvert de poils o 3. Enfin un fait général qui montre it

1. Ch. Darwin. La descendance de l'homme. Trad. E. Barbier . $1.34,35$. Je dois dire ici que $\mathrm{N}$. de Quatrefages a contesté l'interprétation le Vogt et s'est refusé à voir dans les anomalies présentẻes par les idiots microcéphales des caractères ataviques. D'ailleurs M. de Quatrefağes ne uie nullement l'atavisme d'une façon générale, il en cite luimème dey exemples et c'est sur des phénomènes ataviques qu'il s’appuie 
merveille la persistance des systèmes inlubes, c'est le retour au type que manifestent très fréquemment les espèces domestiquées ou modifiées par l'homme dès que les conditions d'existence changent, des que la contrainte imposée ne se fait plus sentir, c'est-a-dire dès que les systèmes inhibiteurs qui n'ayant pas encore acquis assez de cohésion dans l'organisme ont besoin d'ètre maintenus par l'influence de circonstances extérieures particulières viennent à disparaitre et que les éléments de l'organisme peuvent reprendre leurs anciennes associations : "Que les plantes cultivées et les animaux domestiques redeviennent sauvages; dit Heckel, qu'ils soient soustraits au milieu de la rie domestique, alors apparaissent des modifications, qui ne sont pas seulement le résultat d'une adaptation à de nouvelles conditions d'existence, mais qu'il faut aussi considèrer comme un retour atavique partiel a la forme ancestrale primitive, d'où provient le type domestique. C'est ainsi que l'on peut, en cessant de cultiver les variétés de choux si extraordinairement diverses, les ramener peu à peu à la forme ancestrale originelle. De mème les chiens, chevaux, bœufs, etc., redevenus sauvages retournent souvent plus ou moins aux types éteints. Une immense série de générations peut s'écouler avant que la puissance de cette hérédité latente s'amortisse complètement " 1 . On remarquera qu'il s'agit

pour èmettre sa théorie de l'bomme primitif au teint se rapprochant de celui des races jaunes et à chevelure tirant sur le roux. (Voyez L'espece humaine, p. 181), conception qui ne parait pas d'ailleurs beaucoup moins hypothètique que celle de Darwin. Il ne m'appartient pas de porter un jugement déc'sif sur les points controversés, et d'ailleurs le fait génèral de l'atavisme qui n'est pas mis en doute suffit à ce que nous avons à dire ici de l'inhibition et de la permanence des tendances arrétées et de la remise en activitè des tendances.

1. Hæckel. Histoire de la création des étres organtsés d'uprès les lois naturelles, p. $18 \%$. 
dans une grande partic des faits qui précèdent, de l'inhibition et de la permanence des tendances purement organiques aussi bien que des tendances psychiques. Les formes du corps et les formes de l'esprit, les coordinations organiques et les coordinations psychiques sont soumises aux mêmes lois. La systématisation psychique et la sýstématisation organique ne peuvent être complètement séparées l'une de l'autre et l'on peut seulement se demander comment les distinguer, et si la coordination psychique doit être considérée comme un cas particulier de la coordination organique, ou si au contraire toute coordination organique ne peut pas être considérée comme étant essentiellement de naturc psychique. Les deux affirmations peuvent, au reste, se concilier dans une même thèorie.

Ainsi partout dans la vie des sentiments, des passions, des tendances, nous trouvons l'inhibition, c'est bien une forme essentielle du fonctionnement des phènomènes psychiques que nous avons étudiés dans ce chapitre. Les sentiments, les passions, les tendances qui arrêtent souvent la systematisation vers laquelle tendent d'autres éléments psychiques, perceptions, images, ou idées sont arrêtés à leur tour soit par ces phénomènes, soit par d'autres tendances. A chaque moment des arrêts de ce genre se produisent et l'activité d'une tendance ne peut se produire qu'en déterminant l'arrếl de plusieurs autres. Des raisons générales et des exemples particuliers ne laissent aucun doute à cet égard. Mais les tendances arrètées ne sont pas détruites, elles persistent à l'état latent, c'est-it-dire qu'il en reste dans l'esprit des éléments, les coordinations particlles qui, plus tard, quand les circonstances seront redevenues favoraliles, reproduiront des phènomènes semblables à ccux du passé. Nous arous vu jue ces tendances inlibées peuvent pisser en 
germe d'un individu à ses enfants et persister ainsi pendant de longues séries de génèrations. Finissent-elles par disparaitre complètement? Il semble bien au moins qu'il en reste toujours quelque trace et que, ì cet égard, nous soyons toujours à quelque degré ce que nous arons èté jadis ou ce que nos ancêtres ont été arant nous; c'est ce que paraissent prouver les cas de retour de sourenirs perdus, de tendances disparues et qu'on aurait pu croire mortes it jamais, mais éridemment le rappel des actes anciens peut devenir de plus en plus improbable, peutètre nème en certains cas tout à fait impossible. La question n'est pas d'ailleurs des plus importantes et la permanence générale des tendances nous suffit, et nous aide singulièrement à comprendre le phẻnomène de l'inhibition. 


\section{CHAPITRE IV.}

\section{Le pouvofr personnel.}

\section{$\S 1$.}

Ce que j'ai dit dans un précédent chapitre de la nature du pouvoir personnel suffit à montrer que l'inhibition en est un caractère essentiel, nous arons trouvé, en effet, que l'exercice du pouvoir personnel consistait cn ce que une croyance, une idée, une tendance était mise en lapport avec les principaux groupes de tendances gui constituent la personnalité, celles dont l'éveil faible et coordonnè tel qu'il est généralement déterminé par celte sorte d'essii, d'examen, s'accompagne de la conscience du moi. Il est évident que eet essai d'une tendance par d'autres tendances qui cherchent à se systémaliser avec elle est, à des degrés divers: un arrèt de la tendance. Pour les cas oi l'intervention du noi aboutit i un rejet de la croyance, a un non accomplissement de l'acte, il ily a pas de difficulté, la tendance a l'acte, l'action associative qui anuat amené l'établissement d'une nouvelle croyance sont bien arrètées. On propose ì quelqu'un d'aller se promener, les pereeptions ainsi produites éveillent des systèmes d'idées el de sentiments qui sont mis en rapport avec les antres tendances existant deja, elles tendent pal alles-mèmes ì déterminer une orientation de l'esprit telle qu'elle éveille par association systimatique des images motrices déterminées et quelle suscite des mourements appropries. si le moi n'intervenait pas, l'acte serait décidé, conme dans l'état d'hypuotisme, par le seul firit de la présence des systèmes eveilles directement par les paroles prononces, mais le fait que la nouvelle tembnte entre en ridprort avec d'autres du'elle suscite secondairement peut 
amener des arrèts; la systématisation pour ètre lrop vaste et embrasser un grand nombre d'éléments peut ne pas aboutir si ces éléments ne peuvent y entrer', et l'activité systématique des nouvelles tendances éveillées, inhibe l'activité de la tendance qui les a appelées à l'activité. Ici, par exemple, l'idée de la promenade en voulant se coordonner avec tous les éléments du moi, suscite des idées de travail à faire, de visites à attendre, etc., qui ne peuvent s'accorder avec elle et qui peuvent arrèter le processus qu'elle aurait déterminé. Que l'inhibition ait ici un caractère sýstématique, cela ne fait aucun doute, mais ce qu'il im!orte aussi de remarquer c'est qu'elle est amence par une tendance à l'association systénatique. C'est par'ce que l'impulsion nouvelle tend à déterminer dans l'esprit une coordination trés complexe qu'elle est arrètée, aussi voyons-nous les gens qui ne réfléchissent pas, les gens qui agissent très vite, agir le plus souvent d'une manière incohérente, tantôt dans un sens, tantôt dans l'autre, parce que. chez cux, l'idée des actes n'a éveillé qu'une faible portion de l'esprit, et si elle est parvenue a faire naitre cette coordination particulière qui se termine par des actes en harmonie avec elle, elle n'est pas entrée en rapport avec les autres tendances qui, chacune à leur tour, peuvent déterminer en d'autres circonstances des actes qui ne seront coordonnés qu'arec elles-mèmes. Les petites coordinations ont nui a la grande, comme la coordination générale nuisait dans le cas précédent a la coordination particulière d'une idée et d'un acte. Si cette disposition devient habituelle on a les caractères légers, les incohérents, les menteurs inconscients, l'état loystérique de l'imagination et de la volonté. Les éléments psychiques sont livrés à eux-mèmes et chacun agit pour soi.

Ici comme partout les faits pathologiques sont très instructils, en nous montrant le pouroir d'inhibition 
supprimé, ils nous font comprendre ee pouvoir. Il y a des cas, en effet, où le pouvoir personuel paraît anéanti, l'arrêt ne se prodluit plus, le moi est éveillé, les tendances ordinaires entrent en activité, mais soit a cause de leur faiblesse, soit it cause de l'intensite du phénomène morbide, larrêt ne se prorluit plus, ciest comme un engrenage dont les dents se cassent. Dans une discussion sur la responsalibité des actes commis par les épileptiques, M. Billod rapporte le fait qu'il a cilé ailleurs d'une dame irrésistiblement poussée à exécuter certains mourements et à proférer certains mots qu'elle ne peut pas retenir. L'ennission de ces paroles s'accompagne d'me lutte interieurc. Dans la mème discussion, M. Legrand du Saulle parlant des épileptiques, dit que les troubles intellectuels précèdent parfois la crise : "Les malades ne savent plus alors réunir et fixer leurs idées; ils sentent tristement qu'ils ne sont plus les mêmes, et sont poussés à des actes étranges, al la violence, par une force à laquelle ils ne peuvent jas résister" 1 . On trouvera d'ailleurs dans les Maladies de la volonté de M. Ribot, une étude approfondie du pouroir d'arrêt et de ses troubles ${ }^{2}$.

Lor'sque la nouvelle tendance n'est pas rejetée mais acceptée par le moi, lorsque la réflexion aboutit à une adlésion, lorsque la déliljération aboutit a une volition active, à unc impulsion consciente, il peut paraître au premier abord que le rôle de l'inhibition n'est plus le même. Toutefois la refiexion et l'examen des faits amenent, je crois, a conclure autrement. Il y a d'abord un léger arrêt de la tendance qui finit par triompher, et il y a ensuite arrêt d'un certain nombre d'autres tendances, ce dernier lait ne présente d'ailleur's aucune difficultè.

1. Annales d'hygiène publique et de médecine légale, $1875,2^{\circ}$ série, tome XLIV. Discussion sur la responsabilité des actes commis par les Apileptiques.

2. Les maladies de la colonté. Introduction et passim. 
La grande différence qu'il y a au point de vue de l'inhibition entre le cas où la nouvelle tendance est rejetée, et le cas oủ elle ne l'est pas, c'est que l'arrêt cst définitif (pour cette fois au moins) dans le premier cas, et qu'il est provisoire dans le second. Dans le premier comme dans le second la tendance est arrêtée, mais après examen elle continue à être arrètée, elle est remise en activité dans le second. Je comparerais volontiers le sort de ces deux tendances à celui de deux individus, arrêtés par la police, dont l'un est reconnu innocent et relàché, dont l'autre est présumé coupable et définitivement retenu. Pour que le moi puisse examiner une tendance, c'est-à-dire pour que cette tendance puisse essaỹer de s'associer à des éléments importants des autres tendances dont la synthèse constitue la personnalité, il faụt qu'elle n'aboutisse pas immédiatement. D'une manière générale et toutes choses égales d'ailleurs, la longueur de l'arrêt est proportionnel au degré d'importance de l'intervention du moi. Un homme qui se préoccuperait constamment d'agir logiquement et conformément à sa personnalité vraie, qui aurait toujours le désir de ne commettre aucun acte que rigoureusement examine, et reconnu comme ne devant occasionner ni regret ni remords, qui voudrait en un mot raisonner absolument et complètement tous ses actes s'exposerait a n'agir qu'avec une lenteur cxtrême. Ce u'est pas le défaú de la plupart des honmes, ct il n'y a pas à leur faire un reproche d'arrêter trop longtemps les impulsions et les idees qui arrivent pour vérificr leur nature et leur portée ; généralement elles sont acceptées ou rejetées sans examen séricux, le moi ne s'émeut pas facilement, et dans beaucoup de cas quelques éléments psychiques a peine sont mis en activité.

Le phénomène de l'arrèt dans le cas où l'impulsion finit par être acceptèe est bien mis en lumière par les expériences sur la durée des phénomènes psychiques. Si 
le sujet de l'expérience doit réagir d'une certaine maniére lorsqu'il percevä un signal lumineux, la réaction se fait au bout d'un temps determiné, variable selon les individus, après la perception du signal. Mais si la perception lumineuse peut varier, si par exemple le signal donné peut aroir différentes couleurs, et si la réaction ne doit se produire qu'après l'apparition d'une couleur désignée, le temps de réaction est angmenté. Analysons le phénoméne: l'esprit est préparé à l'expérience; c'est-a-dire qu'il est orienté vers la réaction, les muscles qui doivent agir dans un cas domné sont déjà faiblement excités, il § a une tendance évidente à l'acte, et elle est déjà arrêtée pendant l'attente. D'un autre côté, lorsque le signal lumineux se produit, la sensation qu'il excite tend à se coordonner avec les images motrices éveillées, avec les phénomènes qui constituent cet état particulier d'excitation faible. Cette tendance est telle que le sujet est porté a réagir quelle que soit l'excitation produite. Si un signal quelconque doit déterniner la réaction, la synthèse des phénomènes n'est pas ou presque pas arrêtée (elle l'est en réalité à quelque degré toutes les fois que la conscience apparait), mais si l'esprit doit distinguer, reconnaître cette impression, il se produit un arrèt plus marqué, et le temps de réaction augmente 3 . Cette augmentation du temps de réaction marque la durée de la discrimination, c'est-à-dire du temps nécessaire pour qu'une impression puisse ètre examinée, être appréciée par certaines habitudes mentales. Remarguons qu'il ne s'agit ici que d'un acte de réflexion très élémentaire, et que le pouvoir personuel intervient à peine. Il intervient cependant et les enfants se laisseraient, au moins au début, entrainer a répondre chaque fois à l'excitation quelle qu'elle fùt. En jeu gui pent faire la récreation des personnes á goùts

1. Voyez Ribot Ia psychologie allemande contemporaine, et Buccola, La legge del temponei fenomeni psichici. 
très simples et qui amuse les enfants, pigeon vole. nous montre a l'uurre le pouroir personnel et l'arrèt que lacte de volonté et de réflexion le plus simple doit occasionner. On sait en quoi consiste ce jeu, un des joueurs énonce une proposition juste ou fausse en levant le doigt appuye auparavant, comme celui de ses compagnons, sur un oljet quelconque. Si la proposition est juste : pigeon vole, cheral court, oiseau chante, ete, les autres personnes doivent lever le doigt; si la proposition est fausse : cheval vole, àne chante, etc.,.ils doivent le laisser immobile. Celui qui se trompe ou hésite trop donne un gage. Or, le fait seul cru'un des joueurs lève le doigt. dispose instantanément les autres, d'une manière génèrale, à en faire autant, de plus l'excitation de l'ouïe par les paroles prononcées doit être aussi un excitant (Cf.les expériences de M. Féré sur la dỹnamogénie et les faits cités plus haut), il existe donc chez le joueur une certaine tendance arrêtée par la nécessité de vérifier l'assertion lancée. Si le pouroir personnel est insuffisant, et cela arrive souvent chez les enfants, l'impulsion n'éreille aucune idée, elle se produit sans arrèt marqué, par action réflexe; dans le cas contraire il se produit un arrêt plus ou moins appréciable selon la perfection de l'organisation acquise, et l'un des écueils à éviter est précisément le trop de longueur de cet arrêt. Remarquons encorè ici qu'il s'agit de réflexions très simples.

A mesure que les actes de volonté, de réflexion se com. pliquent, l'inhibition provisoire devient plus marquée. D'un côté il faut évidemment un certain temps pour que de nombreuses tendances puissent entrer en rapport avec un processus nouveau, de l'autre il est rare que quelqu'une de ces tendances n'ait pas quelique difficulté a s'associer avec ce processus, et par conséquent ne l'arrète dans une certaine mesure. Au fond, c'est là l'une manière générale la cause de l'arrêt. Ici l'arrêt est double, les deux 
tendances en désaccord s'arrêtent réciproquement, avec des alternatives visibles de suecès relatif et de défaite relative pour l'me et pour l'autre, jusqu'à ce que l'arrêt de l'une d'entre elles se produise définitivement et qu'une orientation de l'esprit s'établisse par l'arrêt de tous les phénomènes, de toutes les tendances qui ne peuvent pas s'accorder avec celle que l'esprit a definitivement choisie.

Le développement de la volonté esi le développement de ce pouvoir d'arrêt momentané ou définitif. "Ce qui distingue la volonté dit très bien M. Bernard Pérez, du reflexe, de l'instinct, de l'habitude, c'est avant tout le degré de conscience qui est en général assez net dans l'acte dit volontaire. Mais la conscience ne suffit pas pour constituer la pleine volonté, il faut un intervalle, si court soit-il, entre l'idée, le désir et l'acte. Cet intervalle est rempli par le jeu des motifs et des mobiles préparant le choix. C'est ici l'œuvre de la délibération" 1. Et la façon dont il résume ses observations sur les jeunes enfants est en parfait accord avec la théorie. "A mesure, dit-il, que l'expérience de l'enfant se forme, un plus grand nombre de sensations, de sentiments et d'idées, c'est-à-dire de mobiles et de motifs déterminants, s'interjosent entre l'action et la détermination autrefois simple et instantanée» 2 . On ferait une remarque analogue en examinant des degrés diver's du développement du pouvoir personnel, depuis les hystériques, cliez qui, dans certains cas, le pouvoir personnel est presque nul, et les gens légers qui agrissent pcrpétuellement d'une manière your ainsi dire réflexe, à la merci des idées en l'air et des sentiments peu solides que les perceptions éveillent

1. Bernard Pérez. L'enfant de trois at sept ans, p. 291.

2. Bernard Pérez. La psychologie de lenfant (les trois promieres (nnies) p. 125. 
continuellement en eux selon divers modes, jusqu'aux personnes dont la réflexion est le carietère dominant et qui se mettent tout entières dans chaque parole qu'elles prononcent, dans chaque aete qu'elles accomplissent. Et il ne s'agit ici, bien culendu, que des volitions positives, non des tendances déftnitivement arrètées.

Nous avons déjà cité des faits dans lesquels le pouvoir d'inhibition est suspendu ou détruit. Nous devons considérer à un autre point de vue maintenant ces faits ou des faits analogues, en les reprenant au point de vue de la volonté active. C'est un fait adnis que l'habitude, en facilitant les actes, finit par les soustraire à l'influence de la volonté. Généralement, en effet, le moi n'intervient plus pour vouloir un acte peu important ou qui nous est familier. Après avoir péniblement appris à écrire, nous écrivons automatiquement, et plus vite; quand le moi n'intervient plus, l'inhibition ne se produit plus, ou presque plus, la tendance active n'éveille plus les autres tendances. Notons en passant qu'il y a là un écueil pour la systématisation générale de l'organisme. Il n'est pas bon de laisser disparaître le contrôle de la volonté sur des actes ou des séries d'actes, car plus tard peut-être la tendance automalique ne pourra plus être arrêtée, et plus voisine de la perfection en elle-même, parce que sa coordination propre est plus complète, elle pourra nuire à la coordination générale de l'organisme. C'est ainsi que des actes accomplis d'abord avec l'intervention de la volonté, peuvent cnsuite être accomplis automatiquement et finir par l'être, malgré la volonté même.

\section{\$2.}

Le fait de la volition renferme d'autres inhibitions que celle de la tendance principalc qui, avec le consentement de l'esprit, c'est-à-dire harmoniquement avec les tendances éveillées, détermine un acte, ou qui est rejetée par l'organisation psychique. Nous avons vu, en effet, que parmi les 
tendances éveillées à propos de la résolution qu'il s'agit de prenule, un certain nombre sont généralement hostiles au nouveau processus qui tend is s'établir. Si je délibère pour faire un voyage ou un achat, les raisons que j'ai de ne pas le faire viendront se présenier à la conscience, suscitées quelques-unes au moins, par les tendances qui constituent le moi. Tant que la délibération dıre, des tendances opposées naissent, se développent, disparaissent presque sans qu'une coordination définitive se dégage, puis brusquement cette coordination définitive se fait, l'orientation de l'esprit change, - les plénomènes se sont classés, et ce fait qui est caractérisé d'uu côté par une association systématique que nous avons déjà examinéc, est caractérisé aussi par une inhibition. Les phénomènes qui ne peuvent trouver leur place dans la nouvelle forme de l'esprit disparaissent, le moi change en quelque sorte, en ce sens que les tendances en activité qui le composent ne sont plus tout à fait les mêmes. Si, par exemple, je me décide à faire un voyage, il $\mathrm{y} a$, au moment oì je prends mon parti, inhibition plus ou moins complète des tendances qui m'auraient poussé à rester chez moi. En général on oublie momentanément les raisons qui ont fait hésiter, les sentiments qui ont longtemps contesté la. décision enfin prise. Sans doute, ces sentiments, cesidées peuvent se manifester encore, mais elles sont au second plan, elles déterminent des regrets sourds, des impressions pénibles de divers genres, mais elles ne luttent plus, e'est-it-dire que certaines de leurs associations sont rompues. Quand on a décidé de faire une rhose, on peut épronver quelquefois des regrets, on pense pu'on anrait pu agir autrement, mais on ne se represente plus l'autre terme de l'alternative comme étant actuellement possible, ou on ne se lo represente que sous forme de proposition verbale, el à moins que la délibération ne recommence, ce qui arrive partois, les ideset les sentiments qui peurent persister 
encore et qui sont opposés à la décision semblent sur un uiu autre plan, ils forment, pour ainsi dire, partie d'un autre moi, on sent que quelque chose les empèche d'arriver á la pleine conscience et de jouer un rôle actif dans I'esprit. On a, si j'en crois mon expérience propre, un sentiment très net de ce genre d'arrêt quand il vient à cesser ou bien quand il s'est produit et que l'on compare les leux élats. Je me représente une chose que je sais ne pas devoir arriver, par exemple, que demain je partirai pour l'Italie, je puis penser aux préparatifs à faire, je puis rêver aux agréments du voyage, etc., je puis désirer, je puis me représenter même assez vivement certaines choses, mais quoi que je fasse, je sens que tout cela n'est pas sérieux, que cette activité est arrêtée, qu'elle ne peut pas avoir de suite, et je me suis d'autant mieux rendu compte de cet arrêt qu'il m'est arrivé, comme à tout le monde je pense, de commencer par rèver á des choses qui me paraissaient impossibles, que je me représentais pour. tant comme réelles et qui ensuite devenaient possibles et étaient l'objet d'une volition ou d'une série de volitions. La différence entre les deux états est très marquée. De même quand j'ai pris une décision qui contrarie certains de mes goùts, je puis penser que je ne l'ai pas prise, je puis me représenter moi-même n'ayant pas pris cette résolution et n'ayant pas à faire ce qui me contrarie, mais je n'en sens pas moins que la décision est prise, qu'elle s'accomplira, que mon esprit est orienté d'une certaine manière, accordé sur un certain ton et que sans que je m'en mêle davantage, l'acte suivra, et, désormais, presque automatiquement; les tendances contrariées sont inhibées en tant qu'elles tendraient à détruire celte organisation, à déterminer des séries d'actes autres que ceux que mon esprit a décidément choisis et qui ont été l'objet d'un acte de volonté. En un mot elles ont perdu le pouvoir de diriger, au moins à certains égards, l'organisme, 
elles sont, pour un temps, impuissantes a s'associer les phẻnomènes d'activité musculaire qui s'harmoniseraient avec elles.

Si l'on me permet encore une comparaison, avec les faits sociaux, et je crois qu ici la comparaison est très apte ì nous faire comprendre le phénomène en question, je comparerai ce qui se passe dans l'esprit après une volition, aree ce qui a lieu dans une assemblée parlementaire quand un vote important vient d'avoir lieu après avoir soulevé des discussions nombreuses et suscité une vive opposition. Les membres de l'assemblée dont l'opinion n'a pas prévalu, existent encore, ils gardent leur manière de voir: ils peuvent l'émettre en dehors et ne pas désespérer de la voir triompher un jour, ils regrettent ce qui s'est passé, mais tous ces regrets, tous ces chagrins qui se produisent dans l'assemblée ne peuvent ricn sur' la réalité, l'opinion et les sentiments de la minorité sont à moins d'une révolution, radicalement impuissants, ils ne peuvent avoir aucune action sur les actes qui résulteront du rote de la loi, ces actes s'accomplissent, si vifs que soient les regrets, si ardente qu'ait été la lutte; l'orientation politique est faite, une nouvelle coordination sociale s'est établie, et pour le moment au moins, les sentiments de ceux qui n'ont pu l'empècher ne peuvent rien contre elle.

Il n'est guère besoin d'insister longuement sur ce fait que l'activité volontaire suppose l'inhibition d'une grande quantité de tendances qui ne sont pas directement intéresséesàce qui se passe à ce moment même dans l'esprit. Quand nous délibérons fortement, lesperceptions ne déterminent guire en nous lus systemes psychiques qu'en d'aulros temps elles pouraient éveiller; l'activité mentale frulant à se coordonncr selon un mode déterminé, les systinnes psychiques qui sont reliés selon un mode difféInnt í telon tel blément actuellement en activité ne sont 
pas, en général, suscités par lui, mais cette inhibition ne diffère pas de celle que nous avons déjá étudièe a propos des phénomènes intellectuels et des tendances. Il me suffit donc de faire remarquer qu'elle se produit pendant la délibération comme pendant l'éveil de tonte activité hautement coordonnée, et de rappeler ce qui s'est dit aux chapitres précédents, on pourrait ici multiplier les faits sans aucune utilité.

De mème je n'ai pas besoin d'insister sur la persistance des tendances inhibées, j'en ai indiqué certains cas dans ce qui précède et j'ai traité longuement la question au chapitre précédent; j’ai examiné implicitement la persistance des tendances arrètées par l'intervention de la volonté, en étudiant la persistance des tendances arrêtẻes en général. Il y a d'ailleurs une infinité de degrés entre les actes volontaires et les actes involontaires et au point de vue spécial qui s'offre maintenant, ce qui s'est dit des derniers peut se dire tout aussi bien des autres.

$\S 3$.

Il est bien entendu que tout ce qui est vrai de la volonté s'applique égalements à l'attention volontaire, et a la réflexion, en tant que ces phénomènes peuvent passer pour des cas particuliers de la volonté; l'attention et la réflexion impliquent des arrèts multiples, si nous portons notre attention sur un fait, nous arrètons une foule d'idées qui pourraient naitre en nous à propos de diverses excitations, mais nous arrètons aussi la représentation mème du fait, nous l'empêchons d'aboutir trop vite à des conclusions ou a des actes, nous la mettons en rapport avec des groupes d'idées nombieux et variés. On dit ì un ccolier qui se trompe dans une version : faites attention, pour que la phrase qu'il a mal interprétée ne soit pas traduite trop vite, pour que les mots qui la composent soient arrètés un moment dans l'esprit et menés pour ainsi dire 
d'une idée à l'autre jusqu'à ce qu'ils trouvent celles qui convient, auquel cas l'attention resse. L'atention volontaire portée sur un objet d'art indique que l'on ne l'a pas fjen goilé au premier abord car ce serait alors in cas d'attention spontanec et non d'attention volontaire, elle implique aussi que nous voulons mettre les perceptions que nous donne l'objet d'art en rapport avec des systèmes de sentiments, de goùts ou d'idées pour mieux l'apprécier et le comprendre, que nous retenons les sensations devant l'esprit et que nous arrêtons les jugements qu'elles tendent à déterminer en nous et dont nous nous méfions. Partout et toujours quand le pouvoir de contrôle intervient, nous nous trouvons en présence des mêmes phénomènes et je résumerai ainsi la part qu'y prend l'inlibition systématique : tout acte de pouvoir personnel (délibération et volition, attention volontaire, réflexion, etc.), implique un arèt de la tendance nouvelle qui s'offre à l'esprit, arrêt relativement définitif, si la volition esl négative, arrêt provisoire, si la volition est positive, (j'entends positive ou négative par rapport à la nouvelle tendance, car une volition en soi est toujours positive), un arrêt des tendances eveillées par la délibération et qui ne sont pas en accord avec l'état définitif (cet arrêt portant surlout sur les relations possibles de ces tendances avec la direction réelle de l'organisme) et un arrêt des tendances qui, sans contrarier directement la tendance dominante, lui nuiraient, si elles entraient en activité en détournant a elles une portion notable des forces nerveuses et psychiques. 


\section{CHAPITRE V.}

\section{La person naité.}

L'assuciation et l'arrèt sont les deux faces de la systématisation psychique. Nous les arons retrouvées dans tous les faits psychologiques que nous avons successivement passés en revue, et toujours l'inhibition était déterminée par la forme mème de l'association. Les choses ne changent pas quand nous passons des perceptions aux idees et aux raisomements, et des opérations intellectuelles aux tendances. Il en est de mème si nous passons des tendances aux actes rolontaires qui sont un cas particulier du jeu des tendances, aux groupes de tendances qui constituent pour ainsi dire des fragments de personnalité, et à la personnalité elle-même. L'arrèt prend une forme particulière selon la nature de l'association qui le produit.

La manifestation successive de ces sous-personnalités que nous avons étudiées dans un chapitre précédent implique que, pour chaque groupe de tendances qui entre en activité et vient a son tour prendre la direction de l'organisme, il se produit un arrèt des tendances qui se manifestaient aupararant. Ce phènomène est absolument anologue à celui que nous avons analysé à propos de la rolonté. L'orientation de l'esprit change, les anciens sentiments, les anciennes idées sont momentanément écartées et si elles persistent encore en certains cas, si elles se maintiennent dans l'esprit, elles n'ont plus aucune influence, elles ne determinent plus les actes, elles sont mises en dehors des conditions de l'activiié. Ce qui caractérise surtout l'inhibition dans la sous-personnalité, c’est ru'elle est composér l'une longue série d'inhilions systematisées. En effet, un des groupes principaux 
des tendances qui sont en nous, s'il vient à dominer pour un temps se manifeste par une longue série coordonnée de desirs, de sentiments divers, d'idées, de rolitions et d'actes, série interrompue par les alternances de domination d'antres groupes. Les systemes de tendances par exemple qui, à côté de l'homme privé, du père de famille, constituent le magistrat, le professerr, le militaire, se manifestent en général à de certains intervalles et offrent alors une série coordonnée plus ou moins longue d'idées, de sentiments et d'actes qui ont tous ce caractère de converger vers des fins associées - les diverses idées, les divers sentiments qui passent par l'esprit d'un magistrat pendant la durée d'une audience (en négligeantles distractions possibles, qui constituent sculement une intermittence plus fréquente des sous-personnalités). Chacune de ces idées, chacun de ces sentiments détermine des inhibitions particulières, et ici ces inhibitions sont amenẻes non seulement par l’influence du phénomène mental actucl, mais encore par la nature même de la série entière qui donne a l'esprit une certaine orientation plus ou moins percue par le sens interne. Un chasseur blesse une pièce de gibier, il la ramasse - en un autre temps, si le groupe de tendances en jeu était différent, si, par exemple, il avait trouvé un oiseau blessé de la mème manière en se promenant dans la campagne, il aurait eu pitie de lui et aurait soil essayé de le guérir, soit tenté an moins de le metlre dans de bonnes conditions pour qu'il se guérit lui-nême. A la chasse sa pilié peut s'éveiller anssi, car le plaisir de lat rhasse nest pas absolument incompatible, nème an point te vue de la logique, avec le desir de ne pas voir sonffrir, frien qu'il tende an moins à l'alténuer - mais sa pitié se manifestera autrement, sans doute il clooisira la maniere la moins douloureuse et la plus prompte pour arheres victime. Un sentiment analogue, dans des cirronslaluess qui diffèrent, rest-it-dire assorié à des groupes 
divers d'idées et de tendanees, détermine des actes différents en harmonie avec l'un ou l'autre groupe. L'acte n'est pas en rapport systématique seulement avec le sentiment qui nous parait ètre sa cause immédiate, mais aussi arec tout le système auquel se rattache ce sentiment ; toutes les autres manifestations actives qui pourraient èlre la conséquence de ce sentiment sont plus ou moins empèchées et elles le sont d'autant plus que le groupe de tendances est mieux organisé, que la sous-personnalitė est plus cohérente: l'indignation de Brutus contre le complot de ses fils pouvait se traduire de bien des manières diverses, mais les devoirs du magistrat dictèrent la série d'idées et dactes particuliers qui pouvaient se coordonner en même temps avec la personnalité en jeu, et Brutus fit périr ses enfants. De mème, dans une composition musicale, un accord pourrait ètre suivi d'un certain nombre d'accords différents, mais la nature de l'accord futur est déterminé en quelque sorte par la série des accords qui ont précédé le dernier. Il se produit donc à chaque moment un groupe d'inhibitions en rapport à la fois avec la nature de l'état, idée, sentiment, qui domine au moment mème oủ l'arrèt se produit et avec la nature des autres états qui sont associés avec lui. Nous avons donc, pendant tout le temps qu'un groupe de tendances associées règne dans l'esprit et détermine des séries et des simultanéités de sentiments, d'idées et d'images, une série concordante d'arrèts systématisés en rapport a la fois avec chaque terme et avec l'ensemble de la série d'états psychiques. Chaque fois que le groupe de tendances cède à un autre la direction de l'organisme, la forme des arrèts se transforme corrélativement et une nouvelle série se produit, chaque fois que la premiere forme d'association reparait la première forme d'inhibition reparait aussi. C'est ainsi que nous voyons les desirs et les répulsions de telle 
ou telle personne pour telle ou telle chose se manifester et disparaître corrélativement.

La persistance latente des tendances momentanément enrayces ne peut être l'objet d'aucun doute - nous les voyons reparaître quand la fatigue ou une raison quelconque tend à amener un changement de l'orientation de l'esprit. L'unité du moi n'est qu'apparente: toutes les tendances qui se manifestent clairement à la conscience tendent bien vers le même but, mais les tendances opposées n'ont pas tout à fait disparu et ne sont que momentanément paralysées il en subsiste au moins certains éléments.

On pent en multiplier les exemples: un soldat, est au fort d'une bataille, - je suppose que c'est un bon soldat - tout le groupe d'instincts qui peut le rendre propre à sa destination actuelle se réveillera à ce moment: patriotisme, instinct de destruction, haine de l'étranger, colère irréfléchic, amour de la gloire, instinct de l'obéissance aux chefs, imitation, adaptation inconsciente a une situation donnéc, peut-être aussi un certain lectour à des tendances ancestrales - les autres sentiments, les autres idées disparaissent. Mais à d'autres moments, à cause de la fatigue des instincts trop longtemps mis en activité, ou bicn par suite d'un changement de circonstances; c'est en vain qu'on fera appel au dévouement et au courage qu'il a montrés précédemment et sur lesquels on aura cru pouvoir compter; c'est le côté égoïste de l'individu qui se manifestera, la peur de la mort, ou les sentiments de famille, ou la haine d'un chef et avec eux tont le cortège re sentiments et d'idées qui peut leur venir en aide, la tendresse, la prudence, la crainte, le regret, la mélancolie, des idées sur l'égalité, sur la justice ou l'injustice appliquées d'une manière particulière, une peur irréfléchie, re. On a mille occasions de voir que les tendances arretées n'ont pas disparu, souvent mème l'arèt ne leur nuit en 
rien, et quelquefois il parait leur permettre de se développer.

Si nous examinons à son tour la personnalité aussi parfaite que nous pouvons nous la représenter, nous trouvons qu'elle nous offre avec la forme la plus complète de l'association, la forme la plus complète aussi de l'inhibition systématique. De même que, par hypothèse, tous les états d'esprit, toutes les tendances convergent vers un mème but ou ver's un système de fins, de mème, et par une conséquence nécessaire, rien ne se produit qui puisse tendre à des fins opposèes. Cet état paraît d'ailleurs purement théorique, mais plus l'association systématique, telle que les faits nous la montrent, tend à s'en rapprocher, plus l'inhibition systématique tend à s'en rapprocher aussi. Lorsqu'on nous parle des faits exceptionnels qui constituent une personnalité éminente, lorsqu'on nous montre par exemple les heures employées au travail, et des années successives occupées par un labeur excessif vers un but important, des recherches ininterrompues, des méditations prolongẻes - nous entrevoyons tout ce que cela indique aussi d'habitudes rompues, de tendances arrètées, de renonciations à des plaisir's sains ou malsains, innocents ou coupables, quelquefois au bonheur de la vie, quelquefois à la vie elle-mème. Généralement, dans nos conditions d'existence actuelle, la personnalité ne se forme pas sans se déformer - et l'inhibition si elle est moins systématique au point de vue de la coordination générale des fonctions organiques n'en est aussi que plus visible. La forme mentale qui tend à absorber pour son usage toutes les forces psychiques, ne peut que rarement s'associer à toutes les tendances utiles a la vie et au bon fonctionnement de l'esprit, - il en résulte que bien souvent le développement excessif d'une manière d'ètre qui constitue l'unité relative de la personnalité, telle que nous pourons la constater dans 
la vie reelle, ne s'obtient que par l'arrèt trop prolongé de tendances dont le jeu serail nécessaire à l'harmonie générale de l'esprit et du corps et cette unité forcée qui provient de la domination d'une seule passion plutòt que d'un concours de toutes les tendances, n'aboutit souvent qu'à un état plus ou moins morbide ou monstrueux au point de vue psychologirpe comme au point de vue social. Chez ceux mème qui réussissent le mieux, toujours quelque partie souffre. Littré s'est fait une remarquable personnalité d'érudit à force de travail et d'application, il a laissé au moins une grande œuvro, et il a été un des hommes qui ont contribué pour une part notable à diriger le courant intellectucl de leur époque, mais il s'est ruiné la santé, et d'un organisme exceptionnellement vigoureux, il a fait, avant le temps, un corps usé, souffrant et maladif. Chez d'autres, ce sont les sentiments de famille qui pàtissent et qui pourtant se seraient développés volontiers, ou quelquefois une qualité de l'esprit qui s’atrophie, des gouts littéraires ou artistiques qui disparaissent, comme chez Darwin. Pour une personnalité à peu près harmonique comme Gœthe, combien avons-nous eu de génies déformés?

Si nous réfléchissons à l'évolution de lindividu à la formation mème de cette unité psychologique qui est la personnalité même, nous sommes frappés de la somme incalculable d'arrêts qu'elle implique, de destructions qu'elle occasionne. Qu'on pense seulement au continuel changement d'idées, de penchants, de goùts, de tendances qui se produit depuis la naissance de l'enfant jusqu'au complet développement du moi on se rendra compte que, si la vie physiologique est caractérisée par la mort continuelle des éléments de l'organisme, la vie psychologique n'est pas moins caractérisée par l'arrèt, la désorganisation lente des phénomènes de l'esprit - nos manières de voir, de sentir, de jouir, de souffrir, de penser, de concevoir, de 
raisonner et d'agir changent continuellement et chaque. changement est marqué aussi bien par la rupture d'anciennes associations que par l'établissement d'associations nourelles. Toutes nos premières manières d'ètre sont successivement suspendues, les éléments psychiques, c'està-dire les formes élémentaires qui les constituaient et qui sont le mode d'arrangement des atomes de l'organisme persistant arec des modifications pendant le renouvellement de ces atomes, sont séparés les uns des autres, décomposés, arrangés de nouveau pour être séparés de nouveau, voir s'interrompre leurs nouvelles combinaisons et renoncer à la satisfaction de leurs affinités passagères - c'est là un travail continuel dans. l'esprit qui se forme, (comme dans celui qui se dissout ${ }_{j}^{\prime}$ et toute évolution suppose une dissolution préalable ou simultanée une nouvelle association imposée à des éléments psychiques suppose la rupture et l'arrèt des associations précédemment établies, les gouts de l'enfant disparaissent, se renouvellent, disparaissent encore, depuis la naissance jusqu'à la puberté de nouvelles tendauces se montrent, les idées se forment peu à peu, continuellement de nouvelles connaissances rectifient les anciennes, et le travail se poursuit ainsi, et cette mort partielle de l'esprit se renouvelle perpétuellement jusqu'a ce qu'un équilibre toujours bien instable paraisse s'établir.

Et ici, nous sommes amenés à retrouver la persistance des anciennes tendances. On pourrait croire que les nouvelles associations les ont fait disparaitre, mais il n'en est rien, et toujours les anciennes affinités subsistent, menacant l'œuvre nouvelle. A la première occasion, un choc, une maladie, une émotion vive, un phénomène ou un autre, peut, en détruisant l'association supérieure, en faisant disparaitre l'orientation de l'esprit, en dénouant le lien qui relie ensemble les éléments psychiques, rendre ces éléments à leurs affinites premières et faire 
reparaitre les premières associations. Les malades atteints par exemple de paralysie générale, alors même qu'ils ont été de bons peintres, reviennent a la manière de dessiner de l'enfance et, comme les enfants ne tiennent compte ni de la perspective, ni des rapports de dimensions des diverses parties du dessin, notre personnalité est ainsi à la merci des forces brutales qui peuvent la faire revenir en arrière ou la faire disparaitre d'une minute à l'autre - et la faiblesse relative de la coordination suprême nous est expliquée en grande partie par la force relative que conservent encore les systèmes arrêtés; en même temps qu'elle nous est une occasion de la constater et de l'étudier. Il n'est pas douteux d'ailleurs, que cette force ne soit variable selon les individus. Chez les uns les tendances arrètées se manifestent toujours à la conscience, et il faut un très petit changement pour qu'elles repremnent la direction de l'organisme; chez d'autres, au contraire, elles sunt refoulées et décomposées au point qu'elles paraissent avoir complètement disparu, et pourtant une désorganisation mentale peut les reproduire d'un moment à l'autre si elle se fait assez lentement pour laisser place à des manifestations psychiques.

Les faits anormaux et pathologiques nous montrent aussi la réalité et l'importance du phénomène de l'inhibition en nous faisant voir ce qui se produit quand cette inhibition cesse ou en nous montrant comment on peut aider à la rétablir et comment le jeu régulier et coordonné des tendances peut reformer la personnalité en inhibant l'activité désordonnée des éléments livrés à euxmèmes et contractant des associations incohèrentes. Je ne crois pas devoir revenir ici longuement sur cette question qui a été implicitement traitée à propos des tendances. Indiquons seulement le nouveau point de vue et examiuons le fait en général. Les cas de dédoublement, de dissolution le la personnalité, en mème temps qu'ils nous 
marquent un trouble de l'association systématique. marquent également un trouble de l'action d'arrèt qui s'exerce trop ou ne s'exerce pas suffisamment et en tout cas s'exerce mal. Tel est, par cxemple, le cas de l'homme, qui d'après Wigan, " avait le pouvoir de poser devant lui son double. Il riait très fort à ce double qui riait aussi. Ce fut longtemps pour lui un sujet d'amusement, mais dont le résultat final fut lamentable. Il se convainquit graduellement qu'il était hanté par lui-mème (haunted by himself). Cet autre moi le chicanait, taquinait et mortifiait sans relâche. Pour mettre fin à cette triste existence, il régla ses affaires et ne voulant pas commencer une autre année, le 31 décembre à minuit, il se tira un coup de pistolet dans la bouche "1. On voit se produire dans les cas pareils des séries de phénomènes cohérents en eux-mêmes, mais incohérents par rapport au moi qui marquent évidemment un trouble du pouvoir inhibiteur amenant un commencement de décoordination. Il parait $\mathrm{y}$ aroir, au contraire, surtout un arrèt excessif dans des cas comme celui de Félida $X . .$. et de Louis V..., où le malade, en passant d'un état à l'autre, oublie ce qui s'est pạssé pendant une longue période de son existence et présente plusieurs orientations différentes de l'esprit qui ne peuvent suffisamment s'associer entre elles, la présence de l'une étant une cause d'inhibition pour les phénomènes et les tendances qui caractérisent l'autre.

Il est intéressant de contrôler les inductions précédentes par ce que dit Griesinger au sujet du moi, de ses troubles et de la manière dont on peut l'aider à se reconstituer. Griesinger, un des aliénistes qui ont vu le plus clair en psychologie, a traité plusieurs fois la question de la personnalité, il a signalé la pluralité des personnalités à l'état normal ${ }^{2}$, il indique les troubles de la personnalité

1. Cité par M. Ribot. Maladies de la personnalité, p. 111.

2. Griesinger. Ouvr. cité, p. 55. Trad. franc de M. Doumic. 
qui se produisent fréquemment dans des cas de folie et où nous n'avons pas de peinc à reconnaitre un défaut $d u$ pouvoir iuhibiteur, dù soit à l'affaiblissement du moi, soit à la force des éléments psychiques engendrés par la maladie; dans la folie, dit-il, "avec le début de la maladie cérébrale, il se développe ordinairement, également de l'intérieur vers l'extérieur, des masses de sensations, de penchants et d'idées tout a fait nouveaux et qui, jusque là, avaient été complètement inconnus sous cette forme i l'individu: (par exemple, sensations de grande anxiété auxquelles se lient l'idée d'un crime commis, l'idée de persécutions, etc.). Au début ces idèes nouvelles se présentent devant le moi ancien comme un toi étranger qui souvent excite l'étonnement et la frayeur. Souvent les individus ressentent cette pénétration dans le cercle des idées anciennes, comme une prise de l'ancien moi.par une puissance obscure et irrésistible, et lc fait de cette prise de possession est exprimé par des images fantastiques. Mais cette duplicité, cette lutte de l'ancien moi contre les nouveaux complexus d'idées non adéquates, s'accompagne toujours d'une sensation pénible de combat, d'état émotionnel et d'agitations violentes ». Ainsi nous voyons que la coordination du moi ne peut plus inliber, rejeter ces éléments qui la troublent; si la maladie fait des progrès le désordre s'accentıe, "le malade n'est plus, sous aucun rapport, le même qu'il était jadis, il est tout autre, son moi est devenu un moi nouveau et faux. D'autres fois il semble qu'il se forme plusieurs masses peu cohérentes entres elles d'idées dont chacune veut représenter le moi, et par cela même l'unité de la personne peut disparaître complètement ${ }^{1}$.

La manièe de recomposer le moi par le traitement moral est autant que possible d'exciter une activité

1. Même ouvrage, p. 56. 
systématique qui inhibe le jeu désordonné des éléments psychiques. C'est ainsi que nous interpréterons les conseils de Griesinger pour le traitement moral de la folie, conseils qui concorlent complètement avec ceux d'Esquirol que j’ai déjà cités dans un autre chapitre.

"Au lieu de discuter logiquement les idees fixes, an lieu de les fortifier en les adoptant, il vaut mieux, quand les circonstances exigent une action directe sur le malade, contredire simplement ses idées sans les combattre, le renvoyer à une époque à venir, où il s'étonnera d'avoir pu croire à de semblables erreurs, lui rappeler le passé où il n'aurait jamais pensé que de pareilles choses fussent possibles, etc. Mais le meilleur système, et celui auquel on a le plus généralement recours, c'est de toucher le moins possible au délire des aliénés, et de le laisser s'affaiblir en ne lui fournissant aucun aliment, en appelant son esprit sur d'autres idées qui ne concordent pas avec son délire. Au premier rang parmi ces moyens se trouve le travail, puis viennent les distractions, les conversations qui doivent toujours avoir pour objet des choses raisonnables et qui sont du goût des malades, dans lequel on évitera tout ce qui a trait à son délire, et où l'on s'efforcera au contraire de placer des idées capables de ramener son esprit dans la bonne direction " $\mathbf{1}$.

Ainsi l'arrêt doit être produit indirectement, la tentative de l'inhibition brusque et systématique opposée par une discussion est, comme nous le verrons à propos de la loi du contraste, fort propre à fortifier les idées et les sentiments que l'on veut combattre, l'inhibition indirecte amène moins de troubles - mais voici qui concerne spécialement la personnalité : "C'est alors que l'on doit fortifier le moi ancien qui commence à se reconstituer, pour éviter qu'il ne retombe et ne se détruise. On y arrive

1. Même ouvrage, p. $551-552$. 
en réveillant et en cntretenant de nouvelles idées et de nouvelles sensations en harmonie avec l'individualité psychique antéricure du malade; d'où la règle de ne guider le convalescent que dans le sens des choses qui l'intéressaient jadis, et l'on comprend qu'ici la mème méthode ne peut pas s'appliquer a tous les cas, et qu'il faut savoir prendre chaque malade par son côté favori." Il s'agit de venir en aide à ce qui reste de l'ancien moi, pour rétablir les anciennes associations et par conséquent inhiber les nouvelles. "C'est ici qu'intervient dans toute son utilité la connaissance que le médecin peut avoir des hommes, alors qu'il doit saisir les différences provenant du caractère, des penchants, des habitudes; du degré d'éducation, et qu'il doit rechercher par quel côté le malade est sensible, " c'est-à-dire, les associations systématiques inhibitrices qu'il est le plus facile de susciter : a Ainsi, il y a sous ce rapport certaines différences entre les deux sexes. 11 est des individus chez qui l'intégrité de la pensée et de la volonté est liée de la façon la plus intime avec les occupations extérieures de leur vic; certains ouvriers ne retrouvent l'unité complète de leur individualité antérieure qu'en reprenant leurs travaux, a certains musiciens il faut le son de leur instrument, etc... Tout ce qui a une certaine analogie avec la vic antérieure du malade, tout ec qui lui rappelle ses liens de famille ou ses anciennes occupations, concourt à fortifier son moi». De même que le moi disparaît quand l'inhibition systématique cesse, il se reforme et l'on peut le ramener en reproduisant la coordination systématique ancienne des éléments psychiques - et nous avous ici une nouvelle preuve de la persistance de tendances arrètées, c'est qu'on peut bien fortifier l'ancien moi et le faire revenir a son etat de force relative, mais il serait a peu pres impossible d'en susciter un nouveau. "On manque toujours son but, dit Griesinger, quand on veut développer clez le 
malade des sentiments qui n'ont aucun rapport avec le fond du caractère qu'il avait avant de tomber malade: Par exemple, on perdrait son temps à tenir des propos religieux à un homme léger et frivole, tout aussi bien qu'a vouloir forcer un individu qui déteste la musique, à joner d'un instrument. Il n'y a qu'un seul cas oì l'on doive se départir de ee précepte d'entretenir soigneusement et de fortifier la personnalité antérieure du malade, e'est lorsque quelque défaut de caractère qui a longtemps dominé le moi a contribué éventuellement au développement de la maladie. Dans ces cas, qui se présentent de temps en temps, où la folie semble la conséquence finale d'une existence rouée au mal, on ne pourrait guère espërer la guérison que s'il se produisait un changement total dans l'existence norale de l'individu; mais chacun sait de quels faibles moyens nous disposons pour arriver à un tel résultat, combien il est difficile d'opérer une modification aussi radicale, et surtout, combien il est rare, quand on est arriré au succès, de ne pas voir bientòt la rechute se produire. „ C'est que l'ancien moi, tout démembré qu'il est, persiste encore en partie, il en reste des éléments qui ont conservé leurs anciennes affinités, des fragments, des systémes incomplets qui tendent à se complèter et qui peuvent $\mathrm{y}$ arriver si les circonstances sont favorables, s’ils y sont aidés par des perceptions, des idées, des sentiments suscités d'une manière appropriée.

Ainsi l'inhibition systématique nous parait une des conditions essentielles du maintien de la personnalité; quand la personnalité se forme, elle se produi ; quand clle disparaît, la personnalité se dissout. Elle contribue également à la production et au maintien des groupes de tendances qui constituent des sous-personnalités, et à la production et au maintien de la synthèse psychirfue aussi complète que possible qui constitue la per'somnalité la moins imparfaite que nous connaissions, et fui n'est, 
comme l'ont fait remarquer M. Taine et M. Ribot, qu'un état très instable. Et cette instahilité est due pour une bonne part à la persistance des tendances inhibées qui menacent la coordination totale ct sont toujours prêtes à profiter du relaichement de cette coordination pour reproduire des systèmes psychiques coordonnés en eux-mêmes et par rapjort à leurs éléments, mais incoordonnés entre eux. Une forme plus élevée de la personnalité serait celle ou se montrerait parfaitement dans sa plus haute manifestation l'inhibition systématisée comme corrélatif de l'association systématique, mais c'est un cas fui ne se présente pas.

Pent-être une forme plus élevée encore se produiraitelle si les tendances qui menacent la coordination générale venaient ì disparaitre, à s'effacer completement, si les éléments psychiques se morlifiaient de manière. à ne plus pouvoir entrer dans d'autres systemes que ceux qui donnent à la personne son unité, si les éléments de ces élements se modifiaient dans le même sens, et ainsi de suite : l'inhibition alors pourrait disparaitre - mais un tel cats est tout au plus admissible au point de vue le plus abstrait, au point de vue d'êtres psychiques sans passé qui ait laissé en eux une trace, ou completement dégagés des souvenirs, des dispositions qui leur seraient restés de ce passé. Ni l'une ni l'autre de ces conditions ne parait pouvoir se réaliser pour l'homme et nous avons le droit le négliger l'hypothise en question - qui, on pourrait le discuter, si cela en valait la peine, n'est peut-être pas recevalıle au point de vue même le plus abstrait possible. 


\section{RÉSUMÉ ET CONCLUSION}

Dans cette revue de toutes les classes de phénomènes psychiques, l'inhibition systématisée nous est apparue partout comme une condition essentielle de la vie psychologique, partout nous l'avons trouvée en corrélation avec l'association systèmatique. En même temps qu'une coordination psychique se forme, nous voyons que d'autres coordinations se défont, que les tendances qui n'entrent pas dans le systėme nouveau sont, à des degrés divers, inhibées, empèchées d'aboutir, arrètées dans leur développement, la forme de l'inhibition étant toujours déterminée par la forme de la coordination nouvelle qui la détermine. En somme, l'association systématique et l'inhibition systématisée sont les deux faces du processus de coordination de l'esprit, l'une n'existe pas sans l'autre, la perception, l'image, l'idée, le jugement, le raisonnement, les sentiments, les tendances, les volitions, la personnalité soumises à des analyses particulières nous ont montré la généralité de ce fait, sa raison d'ètre et son importance, nous n'avons ici qu'à indiquer à un point de vue général et en résumant et synthétisant ce qui vient d'ètre dit, les formes essentielles de l'inhibition psychologique.

Elles sont au nombre de deux qui se complètent l'une l'autre et se produisent simultanément quand l'esprit fonctionne bien. Elles peuvent s'indiquer ainsi : 1o un élément psychique ne peut entrer a la fois dans deux systèmes non coordonnés entre eux; $2^{\circ}$ l'activité d'un élément psychique tend a arrêter celle des autres éléments qui ne peuvent se coordonner avec lui.

La première forme consiste en ceci que lor'squ'une des 
affinitẻs des éléments psychiques est satisfaite, les autres sont momentanément inhibées; si, par exemple, l'idée du triangle est employée pour un moment dans le jugement : la somme des angles d'un triangle est égale à deux angles droits, les autres jugements: où cette idée pourrait entrer aussi comme élément, ne sont pas suscités en nous, et nous ne pensons pas explicitement que dans tout triangle un côté est plus petit que la somme des deux autres et plus grand que leur différence. Le jugement lui-même a d'ailleurs ceci de caractéristique qu'il suppose la mise à part d'un des éléments d'une idée complexe, c'est-à-dire larrêt d'une grande partie des affinités d'un mot et d'une partie de l'idée complexe ; cet arrêt est déterminè par la forme systématique de la coordination, c'est-à-dire de l'ensemble dont l'élément considéré, mot, idéc, ou tout autre que l'on voudra, fait partie. Ce qui le prouve bien, et ce qui prouve en même temps la persistance des affinités inhibées, c'est que dès que cette coordination se relàche, dès que la systématisation supérieure se dissout, l'arrêt ne se produit plus de la même manière et que le même mot, la même partie d’idée peut éveiller à la fois plusieurs séries discordantes; plusieurs affinités se satisfont alors a la fois, eomme dans la lettre de ce fou qui corivait vin mille francs, et où le son vin était associé ¿ la fois à l'idée du nombre vingt mille et à celle du jus de raisin.

Isa seconde forme consiste en ce que l'action d'arrêt occasionnée par une activité systématique s'exerce non seulement sur les éléments du systeme dont il inhibe certaines affinités, mais aussi sur les éléments qui ne louvent fas entrer dans le système et dont il empêche l'activití. Peut-être aussi celte seconde forme est-elle un cas de la première, et peut-être pourrait-on soutenir que tons les élóments concourent à la fois à un même fait d'une maniere plus ou moins marquée, et que la première loi 
suffit pour établir que d'autres phénomènes psychiques non coordonnés avec le fait dominant ne peuvent se produire. Nous arons eu l'occasion de voir, à propos de la personnalité physique que le retentissement dun phénomène psychique dans l'organisme entier. et l'association systématique qu'il y déterminait était beaucoup plus considérable qu'on ne serait porté tout d'abord à l'admettre. Cependant il me paraît bien difficile d'établir qu'il $n^{\prime} y^{\prime}$ ait pas mème dans l'activité systématique la moins imparfaite, un certain nombre d'éléments qui res. tent indifférents, puisque aussi bien nous sarons qu'il y en a dans tous les cas qui restent hostiles et chez lesquels le contre-coup de l'association systematique dominante se montre seulement par l'inhibition de leurs tendances propres. C'est là du moins le fait le plus ordinaire à ce qu'il semble et linterprétation la plus simple et la plus satisfaisante qu'on en puisse donner. Au reste, il n'est pas besoin d'insister davantage sur le fait lui-même de l'arrêt des phénomènes et des tendances qui ne peurent s'harmoniser avec l'état dominant, il en a été donné assez d'exemples dans les pages qui précèdent. Bien entendu encore ici il ne se peut agir que de l'inhibition exercée par une activité coordonnée sur un esprit bien organisé. Ce que j’ai dit dans la première partie de ce travail sur la vie indépendante des éléments psychiques suffit à indiquer dans quelles limites l'inhibition se produit, et c'est sous les réserves imposées par l'imperfection de l'homme et l'incohérence plus ou moins grande mais toujours réelle de sa nature que nous derons formuler la loi de l'inhibition systématisée.

"Tout phénomène psychique tend à arrêter à sa nais. sance et dans son développement ou à faire disparaître tout autre phénomène psychique qui ne peut entrer avec lui dans une association systématique."

Je crois que les deux grandes lois qui ont été étudiées 
ici sont celles qui expriment essenticllement la nature même de l'esprit, mais le moment de chercher à déterminer arec plus de précision ce que c'est qu'un esprit et de tâcher de retrouver dans une synthèse psychique concrète le jeu de nos deux lois, et de ramener ces lois a cette synthèse n'est pas encore venu. Il y a, en effet, une autre loi psychique, moins importante que les deux précédentes, et dérivée d'elles, mais à laquelle on n'a pas fait encore, à ma connaissance, la part qu'elle mérite dans la constitution de l'esprit : c'est la loi du contraste. Elle est, comme nous le verrons, une manifestation particulière des deux grandes lois dont il a été question jusqu'ici mais elle les réunit toutes les deux dans une forme très spéciale et suffisamment caractérisée pour mériter d'être étudiée à part. D'un côté, ellè les complète, de l'autre elle parait s'opposer à elles, et l'analysc seule de la loi et des phénomènes dont elle est l'expression abstraite pourra nous montrer, sous la discordance apparente, les - rapports de causalité et aussi de finalité immanente des formes de l'esprit. 


\title{
L I V RE III.
}

\section{L'association par contraste.}

\author{
I N T R O D L C T ION
}

L'association par contraste a été jusqu'ici plutôt entrevue qu'étudièe par les psychologues. Bain lui a consacré seulement quelques pagres de son livre : Les sens et l'intelligence, Griesinger, MM. Kussmaul, Binet et Féré, Bernard Pérez l'ont indiquée en des passages fort intéressants que j'aurai a citer tout à l'heure; mais on ne parait pas en avoir jusqu'ici suffisamment généralisé la portée et approfondi la nature. Elle est, je crois, beaucoup plus importante que les associations par contiguïté et ressemblance qui l'ont pourtant éclipsée et auxquelles Bain essaye de la ramener au moins partiellement. Je ticherai d'établir tout à l'heure que la contiguïté et la ressemblance ne sont pas en réalité des principes généraux dassociation psychique, mais des formes secondaires, dérivées, ne pouvant guère oftrir au psychologue que des . manières de classer les faits, utiles en pratique, mais sans grande valeur scientifique, je roudrais maintenant essayer de montrer l'importance de l'association par contraste, et de faire voir en quoi elle est réellement une loi générale de l'esprit, quoiqu'elle ne soit pas une forme fondamentale de la mentalité au mème titre que l'association et l'inhibition systematiques.

La loi de contraste peut se fornuler ainsi : \& Un état psychique tend à être accompagné (contraste simultané), ou suivi (contraste successif) d'un état qui lui est opposé, 
qui est au moins à certains égards son contraire. "On a des exemples bien connus des deux formes de cette loi dans le fait decouleur's complémentaires que je rappelle ici pour fixer les idées et préciscr le sens de la loi, mais ses applications sont très générales et nous les retrouvons aussi dans le jeu des idées, des sentiments et des tendances. Il s'agit donc de montrer la réalité et la généralité de la tendance au contraste et de faire voir comment elle est parfois empêchée d'aboutir, tout en se manifestant par des effets sensibles, comment elle peut, dans certains cas, aboutir pleinement, et quels sont ses rapports avec les autres lois générales de l'esprit. Nous étudierons successivement ses deux formes, le contraste simultané et le contraste successif, en en cherchant les manifestations dans tous les modes de la vie de l'esprit, disons tout d'abord que ces deux formes de la loi se ramènent à une même cause principale, le mécanisme essentiel en est le mème, tout en différant quelque peu par sa nature et un peu plus par ses effets d'une forme a l'autre. 


\section{CHAPITRE I.}

\section{Le contraste simultané.}

\section{$\$ 1$.}

D'après l'énoncé de la loi du contraste, il faut admettre que toute tendance, mise en activité, s'accompagne immédiatement de l'activité d'une tendance opposée à elle. Ceci semble ètre a priori en contradiction avec l'expérience directe et aussi en contradiction avec les lois de l'esprit que nous arons déjà examinées, en particulier avec la loi d'inhibition. Ne renons-nous pas de voir, par exemple, que tout phénomène $\mathrm{s}$ sychique empèchait la production et le développement des autres phénomènes qui ne pourraient s'harmoniser avec lui? Et ne semble-t-il pas évident qu'une tendance ne peut qu'ètre gènée par l'intervention d'une tendance opposée? D'un autre côté, l'observation directe ne pourrait-elle pas prouver avec évidence que si je suis bien disposé pour quelqu'un, par exemple, je ne suis pas, au mème moment, et sous un même rapport, mal disposé pour lui? L'examen et l'analyse des faits permettront, j'espère, de lever ces contradictions apparentes. Etablissons d'abord leur réalité.

Pour ce qui concerne les perceptions et les images vives, nous connaissons le contraste simultané des couleurs - si, par exemple, nous regardons fixement un petit carré de papier rouge, nous ne tardons pas à le voir entouré d'une hordure verte, si le carré est jaune, la bordure sera bleue, violette, etc. Cette tendance à voir la couleur complémentaire est très générale, bien qu'elle n'aboutisse pas toujour's à des phénomènes très nets.

Pour les images, nous arons aussi ce fait connu que la représentation mentale très vive dune couleur, peut 
amener la représentation consécutive d'une couleur complémentaire. Je ne pense pas que l'on ait établi la possibilité du contraste simultané, provenant de l'influence exercéc par une image, et je n'ai pas l'inagrination visuelle assez vive pour réaliser moi-même l'expérience; mais voici une expérience plus accessible a tout le monde et qui montre bien le pouvoir de l'image au point de vue de lit production du contraste : regardez fixement un petit carré de papier rouge, et portez ensuite le regard sur une surface grise, vous aurez, par le contraste successif, la vision subjective d'un carrè vert et, par un effet de contraste simultané, cette image d'un carré vert, se lordera d'une bande rouge. Il ne s'agit pas ici d'images pures, mais d'illusions, puisque la vision de la couleur complémentaire est exçitée par des rayons lumineux partant du monde extérieur, cependant, le ròle que joue l'image est assez marque pour qu'on puisse supposer que ce qui s'applique aux sensations, par rapport à la production du contraste simultané, s'applique également aux images. Il semble bien que, dans ce dernier cas, la part des rayons venus de l'extérieur, soit surtout de renforcer, de mettre en évidence les tendinees psychologiques au contraste existant dans. les organes de la perception. Et on pourrait anssi bien tirer la mème conclusion, mais avec noins d'évidence peut-ètre, du contraste produit par la sensation.

Le fait du contraste n'existe pas seulement pour les représentations visuelles, il correspond à une tendance générale de l'esprit et nous pouvons la faire ressortir dans tous les genres de sensations. Elle est moins apparente pour l'onïe ou pour le gont que pour la vue, mais nous la mettrons en évidence d'une manière indirecte.

Le contraste des couleurs ne se manifeste pas uniquement par la production de couleur's complémentaires simples, il se mar'fue aussi, et c'est un cas particulier du mène phénomene, parla modification que subissent deux couleurs 
rapprochées. Des teintes opposées, le rouge et le vertbleu, placées l'une près de l'autre se font naturellement ressortir, le rouge paraît plus rouge et le vert paraîtra plus vert « d'une manière générale: lorsque deux surfaces colorées sont juxtaposées, chacume d'elles se trouve modifiée comme si elle avait èté mélangée d'une certaine quantitè de la couleur complémentaire de l'autre" ${ }^{1}$. On sait que l'art a tiré parti de ces influences, et des effets produits par l'association des couleur's 2. "C'est pour aroir connu ces lois, dit Charles Blanc, pour les aroirs étudiées à fond, après les avoir par intuition devinées, qu'Eugène Delacroix a été un des plus grands coloristes des temps modernes, et l'on peut même dire le plus grand, car il a surpassé tous les autres non seulement par le langage esthétique de son coloris, mais par la rariété prodigieuse de ses motifs et par l'orchestration de ses couleurs ${ }^{3}$.

Mais si ce phénomène: deux sensations opposèes se font ressortir l'une l'autre, est un effet du contraste des sensations, et un cas de ce fait général : l'existence d'une sensation rend l'esprit apte á éprouver plus facilement une sensation opposée, il ne paraît pas douteux que le contraste ne se produise dans tous les ordres de perceptions. Si le rouge tend à nous faire paraitre plus vert le vert qui est à côté de lui, de mème un son strident nous paraitra plus strident s'il éclate pendant une mélodie douce, que si nous l'entendons au milieu d'un tumulte discordant. Malheureusement pour la plupart des sons, des goùts, des odeurs, la tendance au contraste qui ne peut pas être très vive par la raison bien simple qu'il n'existe pas d'opposition aussi précise dans le domaine des sons, du goùt ou des odeurs que dans le domaine des couleurs et que, par exemple, ni le timbre de la flùte ne s'oppose à celui du

1. Rood. Théories des couleurs, p. 120.

2. Voir en particulier : Helmoltz. L'optique et la peinture.

3. Charles Blanc. Grammaire des arts du dessin, p. 564. 
trombone, ni l'odeur de l'héliotrope blanc à celle de la viande gàtée, ni le goùt du sucre á celui de la craie, comme le vert soppose au rouge, cette tendance au contraste, dis-je, est encore masquée par des causes diverses. Il n'y a pas de champ des sons, des odeurs, des goùts, ni même du tact tout ì fait analogue au champ visuel et où des sensations différentes puissent se produire, les sensations restent moins séparées, elles tendent soit ì se confondre, soil a produire une impression dominante de malaise qui rend difficile l'analysse délicate dont nous aurions besoin. Mais il existe un moyen de tourner celte difficulté, c'est d'avoir recours au contraste successif, c'est ce que nous ferons tout a l'heure et nous aurons l'occasion de voir que ces deux formes du contraste sont l'expression de tendances analogues et que, par suite le fait du contraste peut être reconnu dans le domaine entier de l'activité perceptive.

Ce n'est pas seulement au point de vue de la qualité, pour ainsi dire, de la sensation que le contraste se manifeste. L'intensité, l'ëclat donnent aussi lieu it des effets de contraste. La couleur rouge légèrenent orangée du géranium qui paraît très vive quant on la met à côté de la plupart des fleurs de couleur analogue, pàlit remarquablement si on l'approche de la fleur de la verreine. De même la voix d'un chanteur nous paraitra d'autant plus forte que les voix de ses compagnons scront plus faibles, je n'insiste pas sur ces faits connus que j'aurai d'ailleurs l'occasion de retrouver encore it propos du contraste successif. Peut-être pensera-t-on que les faits de contraste de cette nature rentrent plutôt dans le domaine de l'intelligence que dans celui de la perception, mais liutensité est bien un des éléments de la pereeption, il est sùr d'ailleurs que la perception renferme des phénomènes intellectuels et aussi que dans les cas précédents, la percertion considérée dins son ensemble est modifiée. 
$\S \ddot{2}$

Si nous passons aux faits intellectuels proprement dits, des phénomènes fréquents, et d'observation journalière nous montrent la tendance au contraste. Lorsqu'on éveille chez une personne des idées qui s'accordent mal avec ses croyances ou son expérience, il s'ensuit généralement une sorte de conflit, dans lequel l'issociation par contraste se marque par la force supérieure qu'acquièrent momentanément les idées préexistantes et par l'énergie avec laquelle clles se manifestent, c'est-àdire par le nombre et la force des nouvelles associations qui se produisent en opposition avec l'idée suggérée. Si, par exemple, vous affirmez à une personne qu'un objet est jaune alors qu'elle le voit rouge, son premier mouvement sera de nier ce que vous dites, de vous répondre: " Mais non, il est rouge ! Elle ne peut accepter à la fois les deux perceptions, ou même la perception et l'idée contradictoires que l'on tend à faire naître en elle, et l'effet du contraste est de donner plus de force à l'idée déjà existante, de la faire associer avec les mots qui expriment la négation ou avec les idées qui peuvent expliquer notre affirmation tout en maintenant la vérité de croyances antérieures. Il se peut, en effet, que la personne ne réponde rien, surtout si elle soupçonne qu'elle est le sujet d'une expérience, mais alor's encore, on voit la suractivité imprimée à l'opinion déjà établie chez elle se manifester par la formation de nouvelles idées. Cette réaction, par l'effet du contraste, se manifeste même dans l'état d'hypnotisme, les personnes mises en état de somnambulisme artificiel se refusent parfois plus ou moins à admettre l'hallucination qu'on tend a leur imposer ${ }^{1}$.

1. Un des caractères psychiques de l'état des personnes hypnotisées parait bien être en général la suppression des associations par contraste ou du moins l'affaiblissement extrème d'une grande partie des idées suggérées par ce mode d'association. 
Ce qui précède peut en somme se résumer ainsi : Toute idée suggérée en opposition avec une croyance, avec une idée antérieurement établie dans l'esprit tend à donner de la force à cette idée et à cette croyance. Mais comme elle a fortifié ses antagonistes elle peut à son lour et par un mécanisme analogue être fortifiéc par elle - c'est une application de la même loi du contraste. En effet, si l'idée suggérée au lieu d'être immédiatement rejetée, parvient à se maintenir quelque peu dans l'esprit, il est à remarquer bien souvent que les idées éveillées en opposition avec elles, selon le mode déjà décrit, ont pour effet de lui donner une certaine force. C'est le cas, par exemple, des soupçons que l'on tend à rejeter avec indignation mais qui, s'ils ne sont pas bien évidemment absurdes et ridicules, peuvent finir par s'ancrer d'autant plus profondément qu'ils auront été plus vivement combattus d'abord, au lieu que la distraction ou l'inattention les auraient beaucoup plus facilement empêchés de se développer. De mème une objection adressée à l'une de nos croyances acquiert souvent si nous nous l'approprions bien, une force d'autant plus considérable que nous nous efforcons davantage d'y répondre. On évite cet inconvénient en ne tenant pas compte des oljections.

Et c'est bien ainsi que les choses se passent dans la plupart des discussions; les arguments d'un des interlocuteurs ont juste l'efficacité voulue pour surexciter les opinions de l'autre. Ils ne pénètrent pas assez avant pour les ébranler, mais ils font sentir qu'ils les menacent, et les opinions menacées se défendent, elles éveillent tout un cortège de phénomènes : idées, images; raisonnements aptes at se systématiser avec elles, c'est-i-dire à les fortifier. De nouvelles associations se forment, de nouvelles cohesions s'établissent, - et si quelque élément imparfaitement coordonne existait dans l'esprit, il y a des chances que l'activité éveillée par contraste le fasse 
disparaitre. - C'est ainsi que la discussion a du moins ce hon effet, quand les adversaires sont intelligents, que ehacun en sort souvent bien plus convaincu et arec quelque raison, car surement ses opinions sont mieux cooldonnées, ses idées sont plus nomlireuses et plus précises et ses arguments plus varies et plus complexes.

Aussi est-ce un excellent moyen en bien des cas pour réveiller chez quelqu'un des opinions anciennes encore persistantes, mais un peu engourdies, de les attaquer brusquement, et selon a qui on a affaire, maladroitement ou adroitement. On peut obtenir un effet analogue en suscitaut chez son interlocuteur, non pas un état de conscience qui contrarie une opinion dominante, mais un état qui contrarie une manière de penser systématiquement associè a l'opinion dominante ou dérivant d'elle logiquement. Cela peut ètre une occasion de vérifier le degré de coordination des opinions d'une personne. La propagation desidées anti-religieuses ou la persécution, - surtout si elle n'est pas très rigoureuse, - ravirent non seulement le zèle, mais les croyances religieuses, de mème les persécutions politiques, de mème simplement le fait d'un changement de gouvernement; on est souvent d'autant plus convaincu de l'excellence d'une forme politique, et pour des raisous diverses dont je ne retiens que ce qui se rapporte à mon sujet, que l'on ne la possède pas actuellement. Chacun des actes du gourernement que l'on subit est une occasion de le comparer á celui qu'on désirerait, et la comparaison est genéralement il l'avantage du dernier jusqu'à ce qu'il s'établisse à son tour. Mais les faits de ce genre se compliquent de phénomènes affectifs et nous aurons l'occasion d'y revenir.

Il faut rappeler ici ce que disent les aliénistes au sujet des précautions à prendre en parlant aux aliénés, ils recommandent de ne pas contrarier leurs idées et de ne pas discuter avec eux - on ne fait ainsi que fournir des 
aliments à leur délire; j'ai déjá cité à propos d'un autre sujet un passage d'Esquirol, voici ce que dit Griesinger : "Quant à la première indication, qui est d'affaiblir les sentiments et les idées morlbides des aliénés, l'expérience a démontré qu'on arrive rarement à un résultat favorable en les combattant directement..... Il est également mauvais de chercher à combattre directement, par un raisonnement logique, les idées délirantes des malades.... Toute discussion directe, surtout passionnée, augmente ordinairement le délire en ce qu'elle excite le malade à justifier ce qu'il dit; elle l'irrite et l'aigrit d'autant plus que la dialectique qu'on lui oppose est plus forte et plus serrée, et que ces raisonnements lui sont présentés avec un air de moquerie et de persiflage. Que, devant un malade qui se croit complètement ruiné on étale son or et ses billets de banque, que devant un autre on démolisse le mur dans lequel il croit que ses ennemis se cachent, on ne fera que les irriter l'un et l'autre, qu'attirer leur attention sur leurs idées fausses " 1 . L'action du contraste est ici bien visible et exactement analogue d celle que nous avons reconnue déjà- la suggestion d'une idée est une cause de renforcement pour l'idée opposće.

Ainsi nous apereevons la fréquence de l'association par le contraste, nuais nous ne pouvons déterminer sa généralité qu'après en avoir examiné le mécanisme. Nous avons cncore d'ailleurs, a l'examiner au point de vue intellectuel par d'autres côtés, et nous pouvons rappeler à cet ègard ce qu'en ont dit dejà quelques auteurs : Bain, qui a lien vu la fréquence du contraste, l'a surtout reconnu dans les opérations intellectuelles; il ne parle guere de son rôle dans le jeu des sentiments et des tendances, et ce qu'il dit de l'émotion produite par le contraste ne peut ètre considéré comme suffisamment approfondi et

1. Griesinger. Ouvrage cité, p. 549-550. 
ramené aux principes généraux les plus importants, mais il indique bien le rôle de ce mode d'association dans le fonetiommement de l'intelligence. "Rien, dit-il, ne peut être plus naturel quand nous considérons une propriété que la disposition à retourner à l'autre propriété qui constitue le contraste de la première, son opposé, la chose qu'on nie quand on affirme la prenière. "Grand " n'au rait aucun sens pour nous, n'aurait jamais été exprimé par un mot, et n'aurait jamais signifié une qualité, si nous n'avions eu devant nous des choses inégales par la grandeur dont les différences ou contrastes affectaient notre esprit. Le " grand " n'est grand que parce qu'il y a quẻlque chose qui est «non grand o ou « petit " mème quand nous nous imaginons ne considérer que la seule propriétė de la grandeur, nous avons dans l'esprit implicitement la propriété de la petitesse, c'est-à-dire l'alternative ; et c'est seulement quand noùs passons à la considération explicite de l'alternative, que l'autre, la grandeur, devient la propriétẻ implicite. Voilà ce que nous faisons quand nous passons d'un terme d'un contraste à l'autre; les deux termes doivent être présents, bien que l'un d'eux seulement soit, pour le moment, un objet de considération explicite. Ce qui prouve que l'autre objet ne cesse d'être présent à l'esprit, en quelque sorte, c'est que,- lorsque nous sommes restés longtemps sans nous occuper expressément de l'alternative, nous oublions la valeur du principal $₫ !$

Ce qui précède nous montre une généralisation de l'association par contraste - nous l'avons vue d'abord déterminée par des jugements et des raisonnements, elle nous paraît ici, d'après les olsservations fort justes de

1. Bain. Les sens et l'intelligence. A rapprocher de l'ingénieuse théorie sur l'identité de genre des contraires, de M. de Roberty, clans son volume sur l'Inconnaissable. 
Bain comme déterminée par n’importé quelle idée, et éveillec, faiblement il est vrai, mais d'une manière que l'allention peut rendre perceptible dans une multitude de cas. Nous aurons plus tard, en expliquant l'association par contraste, a en préciser le degrè de généralité que la connaissance de ses conditions psychiques nécessaires et suffisantes peut seule nous pernettre de faire. Mais nous derons auparavant insister sur ce point que, si l'association par contraste nous parait déja avoir une part importante dans le fonctionnement de l'esprit formé, elle en a une également dans la formation de l'esprit. C'est à l'arde des idées éveillées par le contraste que nous apprenons a distinguer les objets el les personnes, - cette série de rectifications qui de la conception primitive vague, uniforme, ni concrete ni abstraite tinit par aboutir à des idées alsstraites synthétiques et analytiques, et à des conceptions concrètes très riches est en partic déterminée par des associations de contraste. Le fait qu'une idée nous sugere parfois son contraire el par suite nous force i la vérifier ost trës freptuent et lrès important dans l'óvoIution de lesprit. Mais cet effet du contraste que nous avons leneontre dans les sensations et par lequel des oljets rontrastants nous paratissent l'un ef l'antre ètre, four ainsi dire. plus que jamais ce fu'ils sont, est d'une extreme inportance dans le développement de l'esprit.

Lst-il bien légitime de roir dans ce phénomène si connu ol si frépuent unc forme de l'association jar contraste? Cela nest pas évident à premiere vue, pourtant rela peut s'etablir, je crois, pour les idées comme pour les schsations. Un liat psychique, dit la formule de la loi, tend it determiner un lait psychique opposé a lui ou it l" fortifier. Nous en arons eu déja des exemples, une crovance mal attapuée est géneralement fortifiec par l'allingue mente? Mais qu'est-ce que fortifier une illé 1) whe croyance : c'est essentiellement l'associer à un 
plus grand nombre d'éléments psychiques stables et permanents; par exemple, si notre opinion sur telle ou telle doctrine vient à s'associer systématiquement avec un fait évident, patent, c'est-à-dire avec une perception virtuellement permanente, ou avec une de nos tendances les mieux organisées elle en sera notablement fortifiée; par consérquent éveiller et fortifier une tendance ne sont pas des opérations très distinctes, une opinion est deja quelque peu fortifiée par le seul fait qu'elle a été appelée de nouveau à l'activité, et la force qu'elle acquiert, elle ne l'acquiert qu'en devenant de plus en plus ce qu'elle est : un lien logique entre des éléments psychiques plus ou moins nombreux; plus ces éléments sont nombreux et complexes, plus la croyance existe et se fortifie, plus l'idée devient riche et stable a la fois. Lidée d'une locomotive est à la fois beaucoup plus riche et beaucoup plus stable, elle existe beaucoup plus chez l'ingénieur qui en dirige la construction que chez le gamin qui la voit passer. Or, ce que nous appelons effet du contraste, c'est que chaque fait, chaque idée perçue en contraste avec une autre acquiert plus de précision et plus de force, c'est-a-dire qu'il y a en général plus d'éléments psychiques éveillés et que ces éléments sont plus étroitement et plus fortement coordonnés. Il y a lì un phénomène tout-à-fait analogue à celui du renforcement de deux couleurs voisines, dont chacune nous parait plus vive par le contraste. Un homme grand et un homme petit placés l'un près de l'autre nous paraitront l'un plus grand, l'autre plus petit, de mème si nous pensons simultanément ou à peu près à un trait de générosité et à un trait de bassesse, l'un paraìtra plus généreux, l'autre plus bas. "Le contraste entre deux objets différents, dit Bain, est une ressource toujours prète et qui abrège le travail en excluant tout de suite les idees susceptibles d'ètre confondues avec celle que nous avons en vue. Pour graver dans l'esprit l'idée d'un 
cercle, nous le mettons à côté de l'ellipse. Avec un groupe d'objets destiné il faire comprendre le nombre abstrait qualre, nous mettons un groupe composé de trois et un autre de rint oljets. Nous mettons le blanc et le noir à côté l'un de l'autre. Pour mieux expliquer en quoi consiste le luxe, nous citons des exemples de mours simples et frugales. Tout maitre doit savoir trouver des contrastes et des oppositions aussi bien que des exemples et des faits particulicrs. Et encore: "Les vérités présentées sous la forme d'antithèses bien marquées s'apprenneut facilement avant d'avoir été comprises" 1 , ce qui semble indiquer que le contraste vague existant entre les sens généraux de deux mots suffit a les rattacher l'un et l'autre à des états psychiques assez importants et à les fixer dans l'esprit. Ici, dans les cas cités par Bain, l'un des principaux effets du contraste est de bien préciser chaque idée en lui enlevant certains éléments qu'elle pouvait s'associer vaguement et aussi en lui annexant certains autres éléments par les nouvelles coordinations qu'elle fait naître.

Dans la formation d'une idée précise par l'influence du contriste, nous voyons qu'il s'établit aussi un certain nombre de coordinations nouvelles se rattachant à l'idée principale que le contraste détermine. Par exemple si pour éveiller l’idée du luxe on eite des exemples de morms simples el frugales, l'idée du luxe ne peut se former fre frare que les faits cités font songer immédiatenent à des faits de nature entièrement opposé. De nême pour nos autres idées. La vue d'une enfint qui buvait lans le creux de sa main fit trouver à Diogène, dil-on, yur son ecuelle etait un luxe. Il est hien sùr que le conllatslo a fait son effet, et que son idée du luxe a été completer ainsi par une atsociation par contraste. Toutes les

1. Bain. Science do l'éducation, cité par M. Bernard Pérez, Lienfant de trois it sept ans, 11. 35, en note. 
fois que nous apprenons une nouvelle manisre d'etre courageux, ou bienveillant, ou modeste, nous apprenons inversement une nouvelle manière d'ètre likche, ou malveillant ou orgueilleux, et nos deux conceptions opposées s'enrichissent et se déreloppent simultanément. II. Bernard Pèrez a signalé limportance du fait de contraste daus le developpement de l'esprit. Nous ne dirons pas avec M. James Sully: écrit-il, que le contraste joue un rôle très limité dans l'aequisition; son principal usage est, d'après l'illustre psychologue " d'éveiller l'attention - et par la de graver plus profondément dans l'esprit ce "qui est inusité, exceptionnel, en contraste avec le cour's " ordinaire de l'expérience, comme la vue d'un gréant ou d'un nain, le rugissement du Niagara, ete." Le contraste a, selon moi, une part aussi considerable dans l'aequisition que dans la reproduction des idées, ce mot entendu dans le sens le plus large. Il a pour effet de mettre en relief non seulement l'extraordinaire et le démesuré, mais aussi l'ordinaire et le banal dans notre expèrience quotidienne $>1$.

A mesure que nous examinons plus de faits, il senble que la part du contraste dans la vie intellectuelle s'élargit, - les perceptions, les jugements, les raisonnements, les idées nous paraissent présenter des exemples nombreux de la mème loi - mais nous ne comprendrons bien sia généralité et son rôle qu'en la rattachant à des faits trés généraux, en démontrant qu'elle est impliquée par le fonctionnement normal de l'esprit et aussi en examinant les cas pathologiques dans lesquels elle s'afraiblit et ceux dans lesiuels, au contraire, elle montre une exageration de sa force habituclle.

Comment se fait-il que l'activite d'une idée fasse naitre une idéc opposèe? Prenons le cas d'une idée

1. B. Pérez. L'enfant de trois a sept ans, p. 34. 
éveilléc par une perception qui doit tendre systématiquement a la supprimer. On apprend à quelqu'un qu'une personne de sa connaissance, je suppose, a commis un crime. Son premier mouvement, si clle a confiance entière dans le caractère de cet ami, sera de se récrier et de dire que c'est impossible, - c'est-à-dire que l'idèe suggérée éveillera sans retard l'idée immédiatement opposẻe. Que s'est-il done passé? Simplement ceci : l'idée suggéréc implique une association entre des éléments psychiques existant déjả et qui n'étaient pas associés ensemble ; liidée de crime doit s'unir arec les phénomènes psychiques qui constituent la représentation mentale d'une personne que l'on s'était habituéc à considérer comme très honnête. Mais pour que cette association se forme il faut que cette dernière représentation se produise, et elle ne peut se produire sans réreiller les éléments psychiques qu'une longue habitude et des expériences nombreuses ont coordonnés avec elle, ont mis avec elle en rapport de finalité. Et ces idées s'éveilleront d'autant plus facilement qu'elles sont mieux organisces et aussi que leur existence est plus menacéc. Si l'accusation est absurde elle est rejelcee trìs vite et l'activité des idées de contraste peut ne pas se. prolonger longtemps, mais si l'accusation se présente avec force, avec logique, Ie systeme menacé s'éveillera plus vivement ef tendra a s'associer un plus grand nombre l'éléments psychiques ; de nouvelles irlées, de nouvelles considerations, de nouveaux jugements naitront, tous orientés dans le même sens, e'est-à-dire en harmonie avec la croyance menacéc, en contraste arec l'idéc suggéde. Il laut se rappeler ici ce que nous avons dit de la vie individuelle des systemes psychifues - un systeme psychique existe pour soi et lon pent le comparer it une personnalité, la personnalité n'étant que le flus conlJllox dos systemes, - des qu'un systeme se sent menace il rageit ot se defend, táche d'arrèter son adversaire et 
d'appeler à lui ses alliés - ce sont lí les deux formes générales (association et inhibition) de l'actirité psychique. Toute croyance menacée éreille les arguments, les idées qui peurent lui renir en aide, ct cela d'autant plus virement que l'attaque est plus forte. Si nos croyances sont attaquées par des ignorants ou des imbéciles, nous nous contentons de sentir sommairement leur force et de hausser les épaules, mais si elles sont attaquées par des personnes dont nous estimons l'intelligence et l'instruction, l'attaque ètant plus dangereuse, la lutte est plus vive et la réaction plus forte, nos croyances menacees saffirment davantage - de plus a la complexité de l'attaque répond la complexité de la défense, à chaque argument mis en avant, s'oppose tant bien que mal, une considération nourelle, - une fois les deux systèmes opposés mis en présence, l'un et l'autre se déreloppent parallelement. Il y a donc une double action - d'abord un système psychique est éveillé par le fait que quelques uns de ses éléments sont sur le point de s'unir à d'autres pour créer un système de tendances opposé au premier, de fin contraire, ensuite les şrstèmes qui ne peurent saccorder tendent á s'imposer à l'esprit et pour cela se développent chacun de son còté de telle manière que chaque progrès que fait l'un est, en lant que la chose est possible, accompagné d'un progrès analogue et correspondant de l'autre. Et nous voyons par lia que l'association par contraste est une combinaison particulière de l'association systématique et de l'inhibition systématisée, ces deux grands faits qui semblaient devoirl'exclure et qui la produisent au contraire, gräce it la vie relativement indépendante des srstimes psychiques.

Ceci nous pernet de nous rendre compte des limites entre lesquelles doit s'exercer l'association par contraste. Nous voyons par les faits précédents et parl'analyse des raisons de ces faits que les idlees, les opinions, les croyances 
contrastantes doivent se produire chaque fois qu'il s'établit une certaine lutte dans l'esprit, chaque fois que des éléments psychiques tendent à ètre séparés d'un système auquel ils sont joints habituellement, pour être incorporés à un autre système. Or, cela arrive d'autant plus aisément que l'activité est derenue moins automatique - c'est-à-dire que la systématisation générale de l'esprit est moins parfaite. Dans le cas où une idée, une conviction rencontre certains obstacles, elle s'établit difficilement et peut même finir par être rejetée - dans ces cas-là, il y a production d'idées de contraste - et ces idées peuvent être très fortes et rester même supérieures à l'idée qui tend à déterminer l'impression nouvelle. Quand la nouvelle idée produite peut s'établir plus facilement dans l'esprit, quand elle s'accorde mieux avec les habitudes de l'intelligence, l'association par contraste est moins visible: - si l'on énonce une pensèe qui n'est pas absolument en contradiction avec nos idées mais qui est telle que la pensẻe opposée paraîtrait s'associer mieux avec nos croyances antérieures, cette pensée opposce nous viendra probablement a l'esprit, mais clle arrivera sans violence, - elle ne tendra pas; comme dans le cas précédent et au mème degrré, à déterminer un grand nombre d'associations nonvelles qui lui viennenten aide : la différence de fin n'étant pas très marquée, la lutte sera moins vive et les idées contrastantes suggeérées cn moins grand nombre. Si nous passons maintenant à cette sorte d'activité mentale antomatique qui fait la plus graule partie de notre vie intellectuelle, nous voyons que l'association par contraste, si clle ne disparait pas, ne se montre pas tonjours d'une naniere qui la lasse reconnatre aisement. Aimsi le papier blanc sur lequel jeeris ne me sugrère pas sensiblement l'ibée du noir, de même si je penso machinalement a un hommete homme, je ne m'apercerai pas que

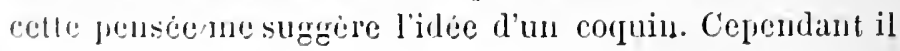


y a des raisons de croile que dans ce cas l'activité par contraste ne disparait pas - il y a toujours une tendance à la naissance d'idées de contraste, mais ces idées sont ì peine excitées et n'apparaissent pas nettement à la conscience. Nous n'avons pour les mettre en évidence qu'à appuyer un peu sur les idées que nous avons ; si nous réfléchissons à l'honnèteté d'une personne nous en arriverons facilement à des réflexions comme celle-ci : ce n'est pas lui qui ferait ce qu'a fait tel ou tel autre - et l'idée de l'honnête homme appellera dès que nous $\mathrm{y}$ insisterons l'idée d'hommes qui ne sont pas homnêtes. Quand une idée est faiblement éveilléc, l'idée opposée est, pour ainsi dire, dans la pénombre de la conscience, toute prête ì paraitre au grand jour si la première idée se dévcloppe. Si l'on pense, au reste, que la conscience est toujours l'indice d'une certaine lutte entre les tendances ou les éléments intellectuels, si l'on songe que toute conscience est le signe d'un certain arrèt des tendances, d'une certaine difficulté des opérations intellectuelles, on scra très porté à admettre a priori que chaque idée, chaque croyance, s'accompagne, au moment où elle sc produit, d'une tendance plus ou moins forte à la production de l'idéc contrastante. L'expérience montrant, conme je viens de l'indiquer, que dès que l'idée se précise, une idée contrastante veut apparaitre, le raisonnement et l'observation paraissent bien ètre d'accord pour nous conduire à la mème conclusion : la généralité de l'association par contraste dans le domaine des fails et des opérations intellectuelles.

La forme de l'association par contraste n'est pas toujours aussi nette que daus le prenier exemple choisi pour étudier son mécanisme. Peut-être mène sera-t-on porté à ne pas retrouver le même mécanisme dans les autres faits. Une analyse minuticuse nous montre cependant, me semble-t-il, que tous les cas sont bien semblables, 
évidemment quand nous pensons par exemple à l'honnèteté d'une personne, an sujet de laruelle nous n'avons aucune raison davoir un doute, nos tendances ne sont pas vivement froissées, et ne réagissent fas comme si par exemple on vient nous dire que nous nous sommes completement trompés sur son compte, mais cependant, certaines de nos habitudes sont froissées. Si nous n'avions jamais vu que des cygnes blancs, et si nous n'avions janıais entendu parler de cyones noirs, il est clair que l'idée de la blancheur du cygne éveillerait moins facilement l'image d'un cygue noir, mais elle éveillerait probablement quelque idéc d'une couleur foncée associée phus ou moins indirectement au cygne, de mème que la vue de la neige immaculée qui recouvre le sol, fait facilement songer à la boue noiràtre qui en sera la conséquence - de mème lorsque nous pensons à un honnête homme, nous arons fatalement et par suite d'associations indissolubles, une tendance a nous dire que tous les hommes ne sont pas honnètes - nous savons qu'il en est ainsi et une association momentanément contrariée tend a réapparaitre. Il se pent yue la conscience ne s'en apergoive pas, mais le fait est réel, et la prenière occasion, persistance de lidee, changement brusque, va le mettre en lumiere. C'est ainsi que naît, comme nous le verrons, le contraste successif qui bien souvent ne fait que rendre évilente lia tendance au contraste simultane, ce que nous aurons at développer bientôt, mais qu'il fallait pourtant indiquer ici.

Ainsi depuis l'acte du pouvoir personnel, de la réflexion oil l'esprit hésite entre les deux termes d'une alternative posée sous la forme du contraste : croire telle chose ou ne pas la croire - car alors, meme que trois on quatre opinions se presentent, la lutte s'engage en grénéral à chaque moment, entre chacune d'elles et sa nogation, - et oil les systemes opposes se développent longuement, jus'qu'a l'activite ordinaire, fuasi antomatique, nous voyons 
la persistance de la tendance au contraste, mais en mème temps son affaiblissement correspondant. d'ailleurs it l'aflaiblissement de l'idée principale. Dans l'activité antomatique, la teudance au contraste est í peu près enrayée en mème temps que l'inlibition cesse, et nous arons vu en effet que le contraste supposait l'arrèt.

Ce qui précède nous permet d'entrevoir le ròle joué par l'association par contraste dans l'organisation de la vie intellectuelle - l'association par contraste a pour effet de régulariser l'activité, d'empècher une idée, une croyance de se développer plus qu'il ne faudrait pour la coordination générale des idées et des croyances. C'est du moins ce qui se produit dans les cas les plus favorables, quand l'esprit est pondéré, bien doué au point de vue intellectuel. L'association par contraste a donc sa raison d'ètre au point de vue de l'organisation de l'esprit, elle a sa place dans le fonctiomement systematique de l'intelligence, et si nous avons vu tout a l'heure qu'elle dépendait de l'inhibition, nous royons maintenant qu'elle peut ètre une forme de l'association systématique, au reste les cas morbides oủ cette forme d'association est exagérée ou supprimẻe nous aideront à comprendre sa fonction normale.

Mais auparavant nous devons nous demander si ce que nous venous de dire des idées et des opérations intellectuelles peut s'appliquer aussi aux perceptions. Les analogies générales des idees. des images, des perceptions nous portent à croire qu'il en est ljien ainsi, toutefois il est difficile de donner une explication preice, satisfaisante du contraste simultané dans les perceptions. Si nous nous souvenons pourtant de la part importante que les images et les idées prenuent it la production des perceptions, si d'ailleurs le contraste suecessil est comme nous le verrons plus longuement tout it l'heure explicable dans les deux cas de la mème manière, on admettra volontiers comme hypothese probable qu'il en est de mème pour le contraste 
simultané. Peut-ètre pourrait-on préciser encore plus l'hypothèse, mais sans espérer arriver à quelque chose qui ressemble suffisamment ì une certitude. S'il ćtait prouvé par exemple que lirradiation est due, mème particllement à une diffusion de l'excitation nerveuse on pourrait admettre que dans cerlains cas, lorsque la force d'irradiation serait moindre, ce qui arriverait quand l'excitation au lien de partir d'un objet brillant ou blanc viendrail d'un oljet simplement plus èclairé que le fond sur lequel il se détaclie, les éléments voisins de ceux que l'impression atteint directement seraient sollicites at vibrer à l'misson de ceux-ci, et réagissant contre cette sorte de choc en retour produiraient en vibrant la couleur complémentaire. Encore l'explication ne suffirait-clle pas pour tous les cas, et la conception indiquée de l'iriadiation est trop contestée pour qu'on ait le droit d'en faire le fondement d'une théorie.

$\$ 3$.

Dans diver's états plus ou moins morljides, nous voyons l'association par contraste modifiee. Notons d'aboril que l'on peut obscrver a cet égard des differences sensibles d'une personne à l'aulre, de mème que nous constaterions des différences dans l'étendue et la ténacité des associations systématipues, dans la force des actions d'arrèt. Un hỹonotisé accepte, en général, l'hallucination qu'on lui sugreire; dans la vie normale il est un certain nombre de personnes qui se rapprochent de son état, en ce que, sur certains points au moins, clles sont portées à suljir d'une manière excessive l'influence de telle personne, ou bien it accepter sans rearir, sans virilier, les opinions toutes faites, telles fu'elles leur sont transmises. C'est l'état de credulite. Par contre nous avons tous ju olsserver des incredules par tempèrament, des gens it intelligence contrariante a qui il suffit de dire une chose jour qu'immédiatement l'opinion contraire domine chez eux. Entre ces 
deux extrèmes, nous avons tous les intermédiaires, les états moyens étant représentés par les gens raisonnalbles et ealmes qui réfléchissent et pésent mùrement le pour et le contre. Le mode d'association varie évidemment d'une personne a l'autre; chez les unes, une idèe aussitôt suggérée détermine une orientation systématique de tout l'esprit, au moins de toute la partic consciente, et aussi de toute cette partic qui est actuellement associée avec les centres de la parole et les mouvements volontaires en général. Il arrive souvent que l'idée contraire, suggérée quelques instants après, s'établit aussi facilement en chassant l'autre. Chez d'autres personnes les idées éveillées par contraste s'organiseront à part et les deux systèmes opposés se développeront parallèlement; chez d'autres enfin les idées éreillées par contraste s'organiseront immédiatement arec une force suffisante pour faire rejeter sans lutte l'idée suggérée. Ces différents modes d'association peuvent très bien, du reste, se manifester à différents moments et relativement à des sujets différents chez le mème individu. L'analogie nous permet, je pense, d'armettre que, même dans ce eas où les tendances ou les idées éveillées par contraste ne sont pas perceptibles, elles existent cependant, a un très faible degré - comme l'idée qui en éveille d'autres par contraste existe aussi a quelque degré, bien qu'elle soit quelquefois si vivement rejetée qu'elle ne puisse ètre perçue par la conscience. De mème il arrive quo nous apercevons une couleur complémentaile sans avoir conseience de la vision de la couleur réelle qui a déterminé le phénomène subjectif.

Dans le rève, dans le somnambulisme, nous trouvons fréquemment un amoindrissement de l'association par contraste. Linhibition ne se produit plus ou se produit it peine, de mème les idées contrastantes ne se sont pas éveillées on le sont si faiblement qu'elles ne sont pas perçues et mème qu'elles ne servent ì rien. Nous acceptous 
dans le rêve Ies idées qui sont le plus en désaccord arec notre expérience, arec nos croyances, et bien qu'il en résulte parfois un certain malaise, bien souvent l'idée contrastante n'apparait pas. Nous royons des bateaux qui naviguent dans l'air et une certaine surprise vague, une certaine curiosité sont la seule marque de la réaction des tendances. Nous nous royons transportés dans des pays nouveaux, notre personnalité mème est plus ou moins changee, nous retrouvons des personnes mortes et soulvent nous ne pensons ni qu'elles sont mortes ni qu'elles sont vivantes, l'image est suivic des phénomènes coordonnés qu'elle suggère, et nous avons occasion de remarquer ici combien les idées contrastantes sont associées, car si nous ne pensons pas que la personne qui nous apparait soit morte, nous ne pensons pas non plus explicitement (qu'elle soit vivante, nous nous contentons d'enchaînerquelques images ou quelques idèes qui impliquent plus ou moins, sans l'affirmer nellement, la croyance it son existence. A l'état de veille un processus semblable, si faible qu'il soit, suffirait pour amener, en choquant l'expérience acquise, la production de l'idée opposée. Mais l'état de rêve se caractérise par la lenteur et la faiblesse le ecrtaines associations et le contraste ne se produit pas visiblement. Il n'en faudrait pas conclure qu'il ne se produit absolument pas, dans quelcues cas la tendance au contraste se montre indirectement, et des faits pareils nous font admettre que cette tendance peut se manifester réellement soit it l'état de veille, soit a l'état de rêve, sans que nous nous en apercevions, alors que nous arons des ratisong générales tirées de la nature mìne dn fonctionnement de l'esprit de croire à sal réalité. Voici par' exemple un rêve oỉ le fait est évident, et qui nn it été communiqué par lat personne mềne qui l'a fait. Cette personne rèva une nuit qu'elle reroyitit une anie morte depuis guelque temps. Elle so promenc ct catuse assez longuement avec cette amic, 
sans penser i sa mort qu'elle connaissait parfaitement d'ailleurs mais qu'elle oubliait. Seulement la voix de lia morte s'affaiblissait peu ì peu et son amie en éprourait une impression pénible. A lia fin du rére, la morte - nou recomnue pour telle, - lui dit que parler la fatiguait beaucoup, qu'elle ne parlerait plus, qu'on n'entendrait plus sa voix. Et cela mème n'éveilla pas l'idée de sa mort - ne l'éveilla pas du moins explicitement, mais il est bien visible quc les illusions du rève tendaient à quelque degré à faire naitre selon le mode de l'association par contraste l'idée de la mort de la personne apparue, toutefois cette idée se manifestait trop faiblement peut-être et le peu qui en survivait ne pouvait que donner une certaine forme appropriée au phénomène du rêve. La loi de contraste se manifeste ici un peu comme la loi de la pesanteur dans le cas du ballon qui s'enleve. Elle ne perd pour cela rien de sa réalité.

En tous cas nous royons d'une manière générale, dans les rèves, le défaut de réaction des habitudes et des tendances, le manque d'association par contraste aboutir à des croyances incohérentes, ¿̇ des idées sans solidité, et lon comprend facilement que l'organisation de l'esprit ne saurait être maintenue ou formée si les idées peuvent sans éveiller la possibilité de leur négation, c'est-a-dire l’idée opposée, déterminer facilement l'orientation de l'esprit.

Dans l'état de sommambulisme artificiel nous trouvons des faits complètement analogues, tous les obscrvateurs ont signalé la passivité de l'esprit de l'hỵpnotisé, cette passivité résulte du manque d’inhibition et du défaut d'association par contraste. L'hypnotisé roit tout ce qu'on lui dit, il accepte tout ce qu'on lui suggère - ou presque tout ${ }^{1}$. Si on lui fournit un point de départ il systématise

1. Voir les ouvrages déja cités de MM. Richet, Richer, Bernheim, Binet et Féré, Beaunis, Delbauf et ceux de MM. Liègeois, Chambard Yung, Cullerre sur l'hynoptisme. 
autour de l'idée suggérée - quelquefois l'association par' contraste se manifeste chez lui, mais dans le domaine de l'activité psychique que la suggestion lui ouvre. Il verra très bien par exemple les difficultés d'une entreprise particulière, au milieu d'un ensemble de conditions impossibles imposées par la suggestion et dont il ne pourra comprendre l'absurdité. L'ami que M. Richet fait voyager dans la lune n'accepte pas facilement lidée de rapporter les animaux étranges qu'il rencontre, mais le fait même du voyagre ne soulève en lui aucune opposition. L'idée de rapporter les animaux parait bien faire naitre en lui celle de ne pas les rapporter, mais l'idée de la réalité du voyage qui lui est suggérée autrement ne fait pas naître en lui l'idéc que le voyage est impossible. La suppression de l'inhibition, de la réaction des tendances habituelles, et par suite de l'association par contraste paraìt être de règle dans l'hypnotisme. Elle aboutit comme dans le rêve à des croyances incohérentes, à des idées absurdes.

Même olsservation á faire pour la folie. Ici encore la formation d'idées fausses et de convictions incohérentes est caractérisce par l'affaiblissement de l'association par contraste : Griesinger fait remarquer 1 que pour la délibération " il faut une détermindtion réciproque des pensées, points d'ar'êt et suspension, léunion et examen, il faut que les idées de contraste puissent se produire; il faut enfin que le moi jouisse du calme nécessaire. Tout cela n'a pas lieu chez les aliénés. La maladie cérébrale fait naître en eux des dispositions et des penchants qui devienuent le point de départ d'émotions; quand celles-ci donnent à leur tour naissance à de faux jugements (idées fixes, lo malade ne peut pas les rectifier, il ne peut pas s'apercevoir de son erreur : au début paree que l'émotion persistante ne laisse pas aux idees de contraste le calme 
nécessaire pour qu'elles se développent d'une façon convenable, et que plus elle sẻ prolonge, plus les conséquences qui en résultent (faux jugements) se fortifient et se consolident, et plus tard parce que ces faux jugements sont devenus partie intégrante du moi. „ Et M. Baillarger ajoute, dans une des notes ajoutées à la traduction française du traité de Griesinger a ....... cette explication peut ètre excellente, mais qu'on l'adopte ou non, il est certain que les idées de contraste sont absentes chez les aliénés et que de ce fait découle l'inutilité du raisonnement pour combattre leur erreur. Ces idées de contraste que Pariset appelait idées intermédiaires sont celles qui séparent la conception de la croyance et l'impulsion de l'acte. On peut bien comprendre ce qui se passe alors dans la folie par ce qu'on observe dans les rêves. "Le rôle de l'association par contraste est indiqué dans ces dernières lignes d'une manière trop vague et à quelques égards inexacte, mais il est intéressant pour nous de retenir ce fait que dans certaines formes de la folie l'association par contraste ne se produisant plus, la réaction de certaines tendances ètant supprimées, il en résulte un fonctionnement désordonné de l'esprit - les idées délirantes n'ont plus de contre-poids pour arrêter leur développement.

En revanche dans quelques autres formes de troubles mentaux, nous pouvons reconnaìtre un fait entièrement opposé l'association par contraste prend une importance et une fréquence excessive. Tandis que dans los cas précédents - et surtout dans le rève et le somnambulisme les croyances suivent naturellement les illusions, les images suggérées, dans certains cas de folie, spécialement dans ce que Ion a appelé la folie du doute, nous voyons que la perception, le raisonnement n'entrainent pas la croyance. A propos de n'importe quelle idéc, à propos de n'importe quel acte qui vient d'être accompli 
par le malade, des idées de contraste s'èlèvent, et l'esprit du sujet oscille entre l'affirmation et la négation, sans pouvoir se fixer sur l'une ou sur l'autre. L'idée de l'acte commis ou de la perception éprouvée évoque sans cesse l'irle contraire que cet acte n'a pas été commis, que cette perception n'a pas été éprouvée, ou bien encore à propos de tout le malade recherche anxieusement les causes et se demande pourquoi les choses sont ainsi et non pas différemment, c'est une complication du phénomène précédent, le malade ne doute pas de la réalité de ce qu'il constate, mais l'idée de la non-réalité lui vient et il se demande pourquoi ce n'est pas cette non-réalité qui est vraie. On comprend qu'un pareil délire puisse prendre facilement des allures philosophiques et métaphysiques - les recherches sur la nature intime des choses comportant fréquemment soit un doute sur cette nature mêne soit une recherche des causes qui ont fait que cette nature est ce qu'elle est et non autre chose.

Certaines formes psychologiques des divers délires que l'on a classés sous le titre général de folie du donte rentrent seulement dans le cadre des faits que nous recherchons - nous ne considérons en efret ici la folie qu’au point de vue d'un mécanisme particulier qu'elte est eapable de nous réyéler et on comprend que des états l'abreration mentale que leurs causes ou certains caractères géneraux peuvent faire rapprocher au point de vue clinique et therapeutique dans un mème groupe ofrent des formes assez variées de groupement pschologique.

Esquirol a raconté un cas remarqualle. Il faut, pour le complendre, au point de vue de l'association par contraste, bien remar'llè l'orientation générale de l'esprit de lat malade. "Mlle F..., dit Esquirol, ....est d'un carlactere gai et d'une humeur douce. Elevée dans le commeres des la premiere jeunesse, Mlle $\mathrm{F}$....., craignait de faire tort aux autres; plus tard, lorsqu'elle faisait un 
compte, elle appréhendait de se tromper au préjudice de coux poul qui ètait ce comptc. „ Ce sont là certes des tendances louahles, sa conduite entière paraît s'y être conformée, mais il arriva que, sous l'influcnce de causes incommes, une association continue vint déterminer des idées, des croyances entièrement en contradiction avec lc caraclère de ces tendances; sans cesse et à propos de tout la malade craint d'avoir fait tort à quelqu'un, d'avoir volé un objet de valeur - c'est-à-dire que la tendance dominante se trouve froisséc à propos de tout et réagit continucllement à propos d'idées qui se heurtent avec elle ce qui est comme nous avons vu le mécanisme général du contraste intellectuel ${ }^{1}$. Le cas est très complet et très intéressant, mais peut-ètre d'autres cas cités plus récemment offrent-ils une interprétation plus évidente. On voit quelquefois la négation s'attacher pour ainsi dire à chaque pas à l'idée, à la croyance, à la perception qui se forme, le malade en vient à douter de tout, il se produit à la fois un défaut d'association systématique, - l'idće ne pouvant associer assez fortement les éléments qui constituent la croyance à sa réalité, - et un excès d'association par contraste, suggèrant à propos de chaque fait, de chaque opinion l'idée que ce fait n'existe pas, que cette opipion en est fausse. "Mon travail se fait bicn jusqu'à aujourd'hui, dit un malade, et sans aucune erreur, et cependint j'ai beau me dire continuellement: « Je suis au travail, je fais eeci, je fais ccla, je ne puis pas me rendre compte que cela est vrai."

"Je crois pouvoir me résumer en disant: personnalité completement disparue; il me scmble que je suis mort il y a deux ans et que la chose qui existe ne me rappelle rien qui ait rapport avec l'ancien moi-mème ${ }^{2}$. La façon

1. Esquirol. Maladies mentules, T. II, p. 63 et suiv.

2. Cf. les chapitres sur la personnalité comme synthèe systèmatique. 
dont je rois les choses ne me rend pas compte de ce qu'elles sont ou qu'elles existent, d'où le doute » $\mathbf{1}$.

Dès qu'un acte intéresse une tendance dominante chez le sujet, cette tendance parait réagir d'une manière exagéréc et l'idée contrastante surgit. Le malade craint de n'avoir pas fait ce que en réalité il vient de faire; on voit la présence d'une perception, ou le souvenir d'un acte qui suit immédiatement l'acte lui-même évoquer ainsi l'idée que cette perception est fausse, que cet acte n'a pas été accompli ${ }^{2}$. Un médecin atteint de la folie du doute, après aroir soigneusement examiné les malades qui venaient le consulter, leur remettait des ordonnances rédigées avec le plus grand soin; mais à peine le client était-il sorti de son cabinet qu'il se précipitait pour lui arracher le papier des mains dans la crainte d'avoir commis quelque faute, d'avoir prescrit une dose toxique de quelque médicament, ou d'avoir contrevenu sous d'autres rapports aux indications du traitement. " D'autres fois le doute se présente, comme je l'ai dit, sous forme dune interrogation que l'esprit se fait à lui-mème. Un prince russe se demandait pourquoi les hommes n'étaient pas aussi grands que des maisons. "Un antre malade se demande pourquoi le poële qui réchauffe sa chambre est appuyé contre le mur, an lieu d'ètre an milieu de la pièce; un troisième; Jourquoi il n'existe qu'une lune au lieu de denx ${ }^{3}$. Ici l'idee du contraste, le doute au sujet de la réalité percue se présente seulement comme une tendance de l'esIrit it imagriner la possibilité de la ron existence d'un fait, et cette nouvelle idée entraine des associations d'éléments coordonnes.

Les exemples de l'affaiblissement et de l'exagération de

1. Ball. Lecons sur les maladies mentales, p. 490.

2. Ball. (Juvrage cité, p. 493.

3. Ball. ouvrage cité, P. 495 . 
l'association par contraste me paraissent fort instructifs au point de vue de l'étude de ce qui se passe dans le fonctionnement normal de l'intelligence. Il semble bien que les coordinations intellectuelles ne se puissent faire que par des rectifications successires; des habitudes correspondant à des réalités sourent percues, à l'expérience antéricure sont plus ou moins dèrangées par les nouvelles idėes, les nourelles croyances qui tendent a s'établir en nous et réagissent en déterminant des idécs de contraste qui enrayent au moins partiellement le déreloppement de l'état nouveau. Quand l'esprit fonctionne bien, a la suite de plusieurs associations par contraste a peine senties et qui peurent mème passer fréquemment inaperçues. la nouvelle idéc, la nouvelle croyance se dépouille de tont ce qui peut choquer les notions acquises, les idées fixées déjà, soit qu'elle perde quelques-uns des éléments qui la constituent, soit qu'elle modifie à son tour les croyances antérieures par un mécanisme exactement analogue à celui par lequel elle est modifiée elle-même par celle-ci - et une série d'associations par contraste a fini par aboutir à un état de ș̣stématisation. Mais nous derons supposer que ces associations se font alors mème que nous ne les apercevons pas et que la tendance au contraste subsiste toujours, mème quand elle ne se manifeste pas par des phénomènes susceptibles de la faire immediatement reconnaitre. Nous arons vu en effet des cas oir l'idée contrastante se réreille suffisamment pour donner une allure particulière à la série des phènonomènes psychiques. Mais les cas où l'association par contraste est suppriméc ou exagérée nous permettent de généraliser le fait. Nous pourons en effet roir ce qui arrive dans le rève, l'hỵpnotisme, la folie et nous remarquons dans un cas que les conceptions délirantes ou simplement lesidées fausses, les erreurs sont déterminées par un affaiblissement des associations par contraste qui 
auraient été capable de les enrayer, et dans l'autre cas que la trop grande force, ou la fréquence trop grande de ces associations détermine des idées erronées, des suppositions ridicules, des doutes insensćs que l'affaiblissement de la réaction ferait disparaître. Nous en concluons que si cette tendance au contraste présente des excès et des faiblesses qui donnent lieu à un fonctionnement pathologique de l'intelligence, c'est qu'elle existe à quelque degré dans son fonctionmement nolmal et qu'clle sert à faire le contrôle des idées qui se préscntent en suggérant l'idée opposée, ou plutôt en la tenant prête à apparaître s'il le faut pour assurer un conflit, une lutte pour l'existence et une sélection. Ainsi la réalité de l'association par contraste nous est montrée fréquemment par l'expérience directe et normale dans le domaine des idées et des opérations intellectualles, les formes pathologiques qui nous font voir ses affaiblissements et ses exagérations et les résultats qui s'ensuivent nous prouvent la nécessité logique ct l'existence permanente de la tendance mentale qui produit cette association. Au reste, je crois que la nécessité et la fréquence de l'association par contraste deviendront de plus en plus évidentes à mesure que nous avancerons dans cette étude.

$$
\$ 4 \text {. }
$$

Dans le romaine des tendances, de l'activité motrice, nous retrouvons l'association par contraste. Ici encore elle nous apparat comme universelle et nécessaire. La vie purement physiologique nous montre déja des phénomenes tres frappants et tout-it-fait analogues a ceux que nous venons de voir it propos de l'intelligence et du jeu des idees; c'est, patr exemple; la mise en action simultane des muscles antagonistes. « Les muscles d'un mene membre agissent presgue toujours tous a la fois 
dans l'exécution d'un mouvement, et pourtant dans chaque membre, une moitié des muscles a généralement une action diamétralement opposće à celle de l'autre moitié. Dans l'avant-bras, les muscles de la face antéricure ont pour action de fléchir les doigts et de fermer le poing : ce sont les fléchisseurs. Ceux de la face postérieure sont extenseurs et tendent à ourrir les mains. On dit pour cette raison que les uns sont les antagonistes des autres.

"Dans l'exécution d'un mouvement, jamais un muscle n'agit sans que son antagonistc entre en contraction pour lui faire subir une sorte de pondération et de contrôle. Cette opération est nécessaire pour modérer et rectifier le mouvement...... Deux muscles antagonistes sont les régulateurs l'un de l'autre; quand ils se font opposition dans une juste mesure, les mourements sont précis et bien coordonnés » 1 .

Cette conception de l'action des muscles antagonistes u'a pas été acceptée par tous les physiologistes, - émise par Winslow et ensuite par Duchenne de Boulogne, elle a été confirmée par les expériences de M. Beaunis. “Les muscles antagonistes ne sont donc pas comme on l'admet génèralement, les uns actifs, les autres passifs dans un mouvement donné; mais, au contraire, ils interviennent tous directement dans ce mouvement, et le mouvement total n'est que la résultante des actions qui se passentau même moment dans les muscles antagonistes " 2 .

Il y a, je pense, une analogie frappante entre cette mise en jeu des muscles antagonistes, aboutissant, quand elle se fait harmoniquement, a des mouvements précis et bien pondérés, et le jeu de l'intelligence, comme nous l'avons décrit tout à l'heure, la mise en activité d'idées, de tendances antagonistes aboutissant à des conceptions précises

1. Lagrange. Physiologie des exercices du corps.

2. Beaunis. Eléments de physiologie, t. II, p. 253. 
et hien coondonnés ausi. Lactirite nerreuse, dans un cas comme dans lautre. parai prendre essentiellement la meme forme. et lactirite nerreuse qui détermine lactirite musculaire est un expmple frarrant de lassociation par contraste conduisant à lanalite génerale.

Dans le domaine partioulier de la rspohologie. le fait parait bien etre le mome, mais il nahoutit pas toujours a lharmonie rinale. et dailleurs ces derniers cas ne sont pas les moins instructifs. Dans la rie des tendances les choses se passent exactement comme dans la rie intelectudle. lassociation far contraste est au fond toujours identique. Il sagit toujours de léreil dune tendancer menaco et des asonciations spstematiques qui en diritent. On rous sugsere lide dun acte: par elle-meme toute ido tend a se reliser. mais lenchainement de qhoromenes qui se rrojuit et qui tend a se completer tar des mourements. réreille secondairement des idres. dos tondances opposes a la premiere. des sentiments contratants. et selon les cas il arrive ou bien que lune des deur tendanese éreilless. que lun des stromes gspohiques lemporte presque sans lutte : ou bien que la lute continute et gue ni lune ni lautre des dever tendanes nabutit a lacte ou bien encore que lante sacomplit lentement. arec hesitation, mais

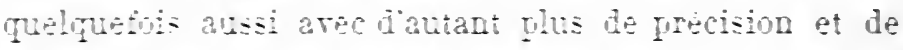
rectitude due das tendances oproses ont éte mises en jeu et que crte asocistion far contraste a permis. par

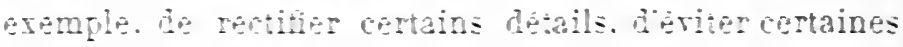
tautes. Ale tenir rompto de flus de cironsiances.

Cette forme de contraste est tres frequente. loaucoup Flus rion ne serait porte a le croire. mais il nest en tout rav fav douteux Tur quand nous naccomplissons pas immediatement un acte pui nous est suggore do noimpork plelle maniore. Jar quelque autre persone: ou par wos-mine a rrotos de nomporte quelle circonstance. 
l'idée de cet acte éveille l'idée des inconvénients qu'il peut entraîner, des difficultés que son accomplissement rencontrera, et que par suite la tendance a un acte déterminé éveille la tendance immédiatement opposée, c'est-àdire, soit la tendance à ne pas faire cet acte, soit en d'autres cas la tendance a faire un acte de fin contraire. Il se passe souvent ici un fait que nous avons déjà signalé à propos des phénomènes intellectuels : les deux tendances, les deux systémes de sentiments, d'idées, d'images motrices qui tendent à s'imposer à l'esprit se développent, s'organisent parallèlement, chaque désir se complique et se compense par un désir contraire. On dirait que plus une des tendances se complique et se fortifie, plus elle embrasse d'éléments psychiques divers et plus ces éléments psychiques sont étroitements coordonnés, plus aussi le système opposé se complique et se fortifie luimème. C'est souvent lorsque nous sommes tentés le plus fortement de faire une chose que nous sentons le mieux aussi le plaisir qu'il y aurait à ne pas la faire. C'est souvent au moment où l'émigrant se dispose à s'expatrier que l'amour de la patrie se fait sentir le plus vivement à lui, que la douceur de rester aux lieux où ont vécu ses ancêtres s'insinue dans son esprit avec plus de force et de pénétration. C'est an moment de se marier que le jeune homme apprécie quelquefois avec le plus de conscience le plaisir de la vic de garçon, et certaines habitudes, un repas d'amis, par exemple, sont une preuye de ce sentiment. De mème e'est souvent au moment de renoncer à une croyance, si elle était assez enracinée en nous, que nous y tenons le plus. On s'attache souvent à ce qu'on va perdre. L'idée de l'absence ou de la mort d'une personne aimée, alors même que rien ne la représente comme imminente, c'est-à-dire un commencement très faible de dissociation de certaines associations psychiques qui nous sont chères, nous les rend plus chères encore. Dans tous 
ces cas nous retrourons le caractère de l'association par contraste, une tendance menacée réagit et provoque l'apparition d'idées, de sentiments, d'images et de dispositions entièrement opposés à ecux que suggérait soit la personne qui nous parle, soil le systeme psychique éveillé par les ciconstances. Il arrive aussi, si la tendance suggérée est de nature à aboutir à des actes et cherche à se compléter par des volitions ou des images motrices déterminant des mouvements, que l'on aperçoit beaucoup micux les raisons d'agir el de s'abstenir, que l'on éprouve plus vivement les sentiments qui nous joussent a l'action et ceux qui nous retiennent, sans que l'une ou l'aute des tendances opposées puisse l'emporter définitivement - et si enfin le décision vient à se produire, elle paraît due parfois bien moins à la raison, ou ì la force des sentiments, qu'il un caprice, à un état mental amené par une circonstance fortuite qui vient brusquement déterminer une orientation relativement stable de l'esprit.

D'une personne a l'autre la force de l'association par contrate varie notablenent. Il se passe pour l'activité ce yui se prise polu l'intelligence : chez certaines personnes les tenlances contrastantes ne sont pas sensiblement éveillecs et elles agissent selon les conscils et les ordres (niron leur donne, d'autres au contraire semblent réagir instinctivement de maniere is faire le contraire de ce qu'on leur suggere ou an moins it refuser d'accomplir l'acte conseillé ou commandé. Il ne faudrait pas eu conclure que dans le premier cas, l'association par contraste ne se produit plus, - elle pent aroir lien sans ètre frereptible a la conscience, mais elle n'a lieu que dans les limites exigées par la coordination dominamte et en tant ru'clle pent aider cette coordination et donner plus de precision i l'acte. Mais il arrive tres souvent que l'association par contriste se produit d'une maniere un peu différente - et de manicre à constituer un phénomène 
d'ordre social autant que d'ordre psychologique. Il arrive souvent, par exemple, que, si quelqu'un abonde trop dans le sens de nos propres opinions nous serons portés à réagir et à parler contre ces opinions pour rétablir l'équilibre. Une personne égoïste peut quand elle a quelques bons sentiments, se corriger si elle s'aperçoit que sou entourage tend a lui faciliter beaucoup trop l'exercice de ce défaut. De mème on nous disposera mal pour une personne à force de faire son éloge et, inversement on desarmera notre haine si on attaque trop vivement, à notre sens, celui qui en est l'objet. Dans tous ces cas le phénomène essentiel est le même : les idées, les croyances, les perceptions qui s'accordent avec une de nos tendances ont pour effet de réveiller la tendance absolument opposée. Si la première tendance se développe trop, et si notre équilibre psychique est. rompu, il arrive que la tendance éveillée par contraste agit à son tour assez vigoureusement pour devenir beaucoup plus forte qu'elle n'était avant : la conversation avec des gens qui outrent nos propres idées est un moyen de nous en faire abandonner quelques-unes. Il est à remarquer, à propos de ces faits, qu'ils ont souvent pour fin un équilibre psychique réalisé par un nombre de personnes plus ou moins grand. Si, dans une sociêté, la majorité nous parait pencher trop dans un sens que nous approuverions volontiers en gẻnéral, nous réagirons trop et nous pencherons trop en sens inverse. Notre équilibre psychique est détruit en une certaine mesure au profit de l'équilibre général, de façon a ce que l'ensemble des opinions nous paraisse donner une moyenne ou plutôt une résultante juste. Le fait peut se produire dans une société entière, - ou dans un corps qui la représente - mais une forme plus affaiblie de ce genre d'association est celle que présente le spectateur modéré qui sans ètre très modéré ni très avancé, n'est pas fàché qu'il y ait des intransigeants et des conservateurs, 
jugeant que les uns et les autres se font contre-poids, de mème que le chat et la souris forment un heureux asscmblarge, car sans chat nous scrions bien incommodés par les souris, et saus souris quelle serait la raison d'ètre des chats?

L'association par contraste s'explique pour les sentimeuts et les tendances de la même nanière que pour les idées et les jugements - c'est toujours une tendance opprimée qui réagit, une association qu'on veut rompre et qui résiste Si un amateur de philosophie indécis lit l'œuvre d'un matérialiste convaincu, bien que sur plusieurs points les opinions de l'auteurse rapprochent des siennes, il est surtout frappé par les arguments (ju'on peut opposer à son système, il devient beaucoup moins porté à abonder dans son sẹs et assez porté, au contraire, à exagérer ses propres doutes età s'en créer de nouveaux. C'est que les idées suggérées par la lecture tendaient a rompre certaines associations déjà formées en lui pour en consolider d'autres. Les èléments des associations attaquées réagissent vigoureusement et tendent à fisterminer une certaine quantité de phénomènes psyrhiques qui les appuient, qui puissent s'unir à eux jour une fin commune. Si l'attaque a éte naladroite, il. arrive ainsi que les systemes attaqués sont consolides comme fuand une persecution reveille la foi religieuse - clle tend par elle-même à supprimer un certain nombre de croyaneses, de sentimrnts rui. d'après les lois que nous avons éturliées, réagissent et se complètent de sorte queleur lores peut, somme toute, s'être développée. Les contrastes de cette nature sont trés l'réfuents. On en a un cas bieu commu dans le developpement de la pudeur au moment de la pubertó, l'amour platonique, dont nous mons a parler plus loin, est frar certaines de ses formes un exenule de contrastr: entre la tendance qui tend a s'imposer et les sentiments éveilles. Nous voyous ici, comme lorsqu'il s'agissait de l'intelligence, que l'explication du phénomène 
tend à nous en faire admettre la généralité. En effet - sauf dans l'automatisme pur, dans l'acte réflexe oì l'impulsion ne rencontre sensiblement aucun obstacle et oì la tendance au contraste passe complètement ou à peu près à l'état latent, - si facile, si aisé que soit l'accomplissement d'un acte, cet acte est tout au moins quelque peu arrêté, enrayé si peu que ce soit, c'est-à-dire que pour lui l'alternative être ou n'être pas se pose à quelque degré, c'est-à-dire encore que l'association paì contraste se produit, si faiblement que ce soit et d'une manière si imperceptible que l'on veuille. Et c'est cet éveil d'idées et de tendances contrastantes qui donne à l'acte non encore complètement organisé la coordination qui lui permet de s'associer à un grand nombre d'états psychiques. Nous trouvons au reste tous les degrés de l'association par contraste depuis le cas où nous réfléchissons longuement à une proposition importante qui nous est faite et qui doit engager une partie considérable de notre existence, où l'alternative faire ou ne pas faire est longuement examinée, où l'idée de ne pas faire ce qu'on nous propose est pleinement éveillée et s'associe à un nombre très grand de tendances, de scutiments et d'idées, jusqu'au cas où l'idée de l'acte suggère á peine l'idée que nous pourrions ne pas agir et où même cette idée ne nous arrive parfois qu'après l'acté accompli.

\section{$\S 5$.}

Les phénomènes pathologiques ou semi-pathologiqnes nous aident encore ici à mieux comprendre le rôle et l'importance de l'association par contraste en nous montrant ce que l'esprit devient quand elle disparait ou quand elle s'exagère.

Elle s'affaiblit considérablement dans l'hypnotisme nous voyons alors le sujet dans la dépendance à peu près complète de l'hypnotiseur qui le fait agir comme il 
lui plaît. Il faut sans doute voir aussi un affaiblissement de l'association par contraste dans certains phénoménes spéciaux - par exemple dans la pureté d'expression des attitudes suggerées. Le manque d'association par contraste provient ici du manque d'action d'arrèt et le cas ne présente pas de difficulté d'interprétation, remarquons toutefois que l'inhibition et l'association par contraste cessent de se produire seulement pour ce qui concerne l'orientation suggérèe à l'hypnotisé.

Les cas d'exagération du contraste simultané des sentiments et des tendances sont très fréquents dans les états anormaux. Au reste ils ne diffèrent pas essentiellement des cas analogues que l'état normal peut offrir. Voici un fait raconté par Tolstoï dans ses Mémoires et où la tendance contrastante est singulièrement amence : "Dmitri causait avec sa mère. Il soutenait que la vue ne peut être belle quand l'horizon est limité. Varegnka ne disait rien. Je me tournai de son côté, elle était debout, accoudée sur la rampe du petit pont et regardant devant elle. Quelque chose l'occupait sans doute beaucoup, l'impressionnait même, car elle était visiblement absorbée et ne s'aperecrait pas qu'on la l'egardait..... étant amoureux de Sonietchlia, je ne pouvais considérer Varegnlia que comme la sceur de mon ami. Toutefois, elle me plaisait tant, en ce monnent, que je sentis le besoin irrésistible de faire ou de dire quelque chose qui lui fut désagréable... Vous naimez sans doute pas la nature? me dit Varegnka sans tommer la tête. - Je trouve que c'est une oceupation fatstidieuse, répondis-je, tres content de lui avoir dit quelque chose de désagréable en môme temps qu'origrinal $n$ '.

Daus les cas pathologifuos, le phénomene est plus marepus, la tendane plus puissante. "J'ai connu, dit M. Maury, une dame atteinte d'hystérie et qui dans ses

1. Tolstö. Mémoires. Trad. Halpérine, p. 336. 
accès, faisait et disait ce qu'elle voulait précisèment ue pas dire et ne pas faire. Sous l'empire de la crainte qu'aucun mot inconvenant ne sortit de sa bouche, elle pronongait, malgré elle et sans bien savoir ce qu'elle disait, des mots obscènes. Traversant, le soir, une longue galerie solitaire de son chàteau, un bougeoir à la main, elle était prise d'une peur extrême de se trouver là dans l'obscurité; et à peine cette pensée lui était-elle venue qu'elle soufflait sa boúgie. On observe encore dans le phénomène du vertige des faits de même ordre " ${ }^{1}$.

Kussmaul cite un certain nombre de faits qui peuvent se rapprocher du précédent au point de vue de l'association par contraste - ce sont ces phénomènes morbides qu'il a appelés dysphasies par trouble du pouvoir d'arrèt et qui consistent en ce que " le moi n'a pas le pouvoir de réfréner le cours de certaines séries d'idées qui sont en mouvement. " Voici comment est décrit le fait qui nous intéresse : "Un autre phénomène reposant sur un affaiblissement du pouvoir d'arrêt de l'âme est celui qui survient dans des états de fatigue et de faiblesse physique ; par hasard, une pensée ou un évènement qui coïncide avec ellc éveille une idée de contraste qui ne se laisse pas étouffer et qui cherche à sc manifester avec une grande puissance. Un fait de ce genre se produit quand une femme nerveuse, ébranlée dans tout son ètro par des nouvclles tristes, se met à rire d'unc façon spasmodique. Tout l'auditoire peut, au milicu d'un long sermon très fatigant, être pris d'un accès de gaité invincible par suite d'une circonstance insignifiante, un ronflement, par exemple. A côté de ces faits nous pouvons placer le suivant : " $\mathbf{M}^{\mathrm{me}} \mathbf{B}$..,, belle-mère d'un médecin très l'ecom" mandable, sans jamais avoir éprouvé d'accidents para"lytiques, arriva assez rapidement á des troubles d'inteln ligence fort singuliers. Un visiteur entre chez elle; elle

1. Maury. Le Sommeil et les Rêves, $4^{\circ}$ èdition, p. 156. 
n se lève pour le recevoir avec un air de bienveillance, " et en lui montrant un fauteuil : "Cochon, animal, fichue D bète! " - " Madame vous invite a vous asseoir, " dit le " gendre, qui interprète la volonté de la malade, si étran"grement exprimée, comment peut-on expliquer ce fait " autrement qu'en disant que la dame sentait et pensait "tout le contraire de ce qu'elle disait " 1.

Les faits de ce genre sont assez nombreux dans la littérature médicale, nous voyons toujours par un mécanisme plus ou moins accessible à nos recherches deux tendances opposées se développer en même temps, naître l'une de l'autre. M. Despine raconte qu'une dipsomane, citée par Trélat, quand elle sentait venir son accès, "mettait dans le vin qu'elle buvait les substances les plus dégoùtantes et même des excréments. C'était en vain. En même temps, elle se disail des injures: " Bois done, misérable! bois "done, irrogne! bois, vilaine femme qui oublie tes pre" miers devoirs et déshonore ta famille! "La passion et la maladie étaient toujours plus fortes que les reproches et le dégoùt qu'elle cherchait à s'inspirer. Sa mère et son oncle étaient également dipsomanes.

" Le D ${ }^{r}$ Verga cite un ingénieur de Milán qui présentaịt cette particularité que, quand les aliments arrivaient au pylore, ils y rencontraient un obstacle; alors araient licu des èructations fatigantes pendant lesquelles le malade était foreé de prononcer des blasphèmes, de dire : Dieu maudị! quand il aurait voulu dire : Dicu béni! Et au lieu de dire: Vierge sainte, il pronongait des paroles entièrement opposces. Félix Plater cite un homme reli. greux qui éprouvait un trouble intérieur qui le forçait is lenser, contre sa volonté, des choses impies. Lorsqu'il

1. Kusmaul. Les troubles de la parole, trad. française de Ruff, p. 281. La dernière observation est empruntée à Trousseau: Clinique chirurgıcale de l'Hótel-Dieu, T. II, p. 674. Paris 1877 . 
voulait prier Dieu, il était assailli d'idées blasphématoires. Ces idẻes diminuèrent avec l'àge, mais elles se présentèrent encore dans sa vieillesse. "Nous avons connu, dit le $\mathrm{D}^{r}$ Hildebrand, un sayant qui, lorsqu'il assistait à la messe, sentait des blasphèmes lui venir parfois a l'esprit, dans les moments où les cérémonies demandaient le plus de recueillement. Ces petits accès d'alienation blasphématoire, affectant la troisième forme de monomanie, pcuvent n'ètre dùs qu'à une névrose passagère du ccrveau, de mème que la mème folie blasphématoire que manifestaient les démonomaniaques du moyen-àge " 1 .

Une forme assez curieuse et demonstrative de l'association par contraste se produit quand les actes ou les discours voulus d'une personne sont en contradiction flagrante - parfois à son insu - avec ses pensées intimes. On peut voir clairement en certains cas l'action et la réaction de deux systemes opposés l'un sur l'autre. Chaque système se développe et tend à se systėmatiser davantage, mais en respectant la part de l'autre au moins dans certains cas. Quelquefois il s'établit ainsi une sorte de compensation. Un mari infidèle pourra se sentir comme obligé à plus d'égards envers sa femme. Un honme ivre se préoccupe de marcher droit. Une tendance habituclle se manifeste souvent ainsi par des discours, des propos sur la tendance opposée, ou sur cette tendance mème, le contraste peut se marquer dans les deux cas soit par l'approbation donnée à une tendance opposée à celle que l'on a soi-mème, soit par le blàme infligé à ses propres sentiments quand on les rencontre chez d'autres sans les reconnaitre, soit par l'attention mème que nous portons aux actes qui chez les autres dénotent des habitudes opposées aux nòtres. Un avare se préoccupera de constater et de juger chez les autres l'avarice on la générosité; un homme pieux, la piété ou l'irréligion; un homme

1. Despine. De la folie, p. 3iI. 
instruit, lignorance ou la science; un homme intelligent, l’intelligence el la bètise. Ún phénoméne semblable se produit quelquelois dans les maladies mentales. L'excitation sensuelle qui se froduit chez les femmes cn étal d'excitation maniaque se traduit souvent au dehors, dit M. Ball; " par d'interminables discours sur la chasteté, sur le bonheur de la vie religieuse » et il ajoute : a toutes les fois qüune malade en état d'excitation maniaque rous tiendra de pareils discours, soyez certain que, sous ce masque, se cache un fond d'excitation lubrique 1.

C'est sans doute encore un effet de contraste exagéré, qu'il faut reconnaitre dans certains phénomènes impulsifs. Esquirol note que les impulsions homicides ont souvent pour objet des personnes chéries du monomaniaque. Après avoir cité un certain nombre de faits, il ajoute : "Tous ou presque tous les individus dont nous venons de rapporter les observations, étaient d'une constitution nerveuse d'une grande susceptibilité ; plusieurs avaient quelque chose de singulier dans le caractère, de Jizarre dans l'esprit. Tous, avant la manifestation du désir de tuer, étaient incapables de nuire; ils étaient doux, lons, honnêtes gens et même religieux. "Et plus loin : Le monomaniafue, dit-il, immole des ètres qui lui sont indiflérents ou qui ont le malheur de se rencontrer sous ses pas au noment oi il est saisi par l'idec du meurtre; mais, plus souvent, il choisit ses victimes parmi les objets qui lui sont les plus chers. Une mere tue son enfant et non l'enfant de l'étrangère; un mari veut tuer sa femme avec laquelle il a vecu dans la plus donce harmonie depuis viugt ans; une fille veut tuer sa mère qu'elle adore" 2.

On voit que l'association par contraste parait caractériser cortaines formes de maladies mentales ou nerveuses.

1. Liall. Lregon sur les maladies mentales, p. 298.

2. Isquiro!. Oitir. cité, t. II. Mémoire sur la monomanie homicide. 
Griesinger en donne d'autres exemples et, comme Kussnlaul, il a, au moins partiellement, généralisé ses observations. Il explique, au moyen de l'association par contraste, eertaines formes morloides: " Lal melancolic avee idées de jossession du démon, dit-il, jarait ne survenir que ehez les femmes (presque toujonrs chez des hysteriques) et chez les enfauts. L'explication la plus ficcile de ce phénomène psyehologique se trouve dans tous les cas, qui ne sont pas rares, où les sciries d'ilées, à mesure qu'elles arrivent, s'accompagnent d'unc contradiction intéricure quii s'attache involontairement a clles, et qui a pour r'csultat d'amener une division, une séparation fitale de la personnalité. Dans les cas très développés où ce cercle d'idées, qui accompagnent constamment la pensée actuelle en lui faisant opposition, arrive a avoir une existence tout-à-fait indépendante, il met en mouvement de lui-mème le mécanisme de la parole, il prend un corps et se traduit par des discours qui n'appartiennent pas au moi (ordinaire) de l'individu..... Par exemple, chez une paysanne, entre trente et quarante ans, il se développa une maladie revenant par accès et qui bicntôt devint de plus en plus fixe. La malade " est obligèe de garder le lit, tombe dans une apathie complète, elle ne peut plus rassembler ses idėes, et l'anomalic mentale se présente chez clle sous forme de contradiction intérieure contre ses propres pensées et ses déterminations, une opposition. immédiate, constante contre tout ce qu'clle vient de penser ou de faire. Une " voix intéricure, " mais qu'clle n'entend pas dans son oreille, se révolte contre tout ce qu'elle veut, par cxemple déjà contre le simple sejour au lit auquel son état la condanne; en particulier contre toute élévation de sentiments, la prière, ete., la voix veut toujours le mal quand la malade veut le bien, et lui crie parfois tout à coup, mais sans qu'on puisse l'entendre extérieurement : "Prends un couteau et tue-toi. " La 
malade, qui est une femme raisonnable, dit qu'elle a de la peine à croire que ce soit un être étranger, un démon qui est dans son corps, bien qu'elle ait la certitude que ce n'est pas elle-mème qui fait tout cela " 1.

$\S 6$.

Ainsi pour les sentiments et les tendances comme pour les phénomènes intellectuels, - et les uns etles autres sont bien souvent mêlés ensemble, comme on a pu le voir, entre autres cas, par celui qui précède - les phénomènes pathologiques nous permettent de mieux comprendre le fonctionnement normal et en nous montrant l'eflet produit par l'affaiblissement ou l'exagération d'un mode d'association, nous montrent l'effet produit par son mécanisme habituel. Nous voyons que pour les impulsions, les tendances, les actes, etc., l'association par contraste est une condition de précision et de complexité. Lue tendance qui réveille des tendances opposées a des chances de n'arriver que si elle s'accorde avec un nombre très considérable de phénomènes jsychiques, et ì moins qu'elle ne soit très violente la lutte des impulsions oplposées est une raison pour que l'orientation définitive de l'esprit ne s'établisse pas à la légère.

Et nous royons encore ici que la tendance au contraste est une loi générale de l'esprit. A moins que les tendances ne soient parfaitement organisées, que l'esprit n'en soit arrivé à un véritable automatisme, une tendance est toujours quelque peu entravée dans son fonctionnement et mème elle l'est probablement à quelque degré dans l'antomatisme le plus parfait que nous connaissions. Mais cet arrêt suppose l'influence d'une tentance différente ot il scra principalement exercé par la tendance opposée a celle qui clicrche a se manifester, tendance qui sera

1. Giesinger, Ouvr. cite, p. 285 et 280. 
plus directement qu'une autre attaquée par celle-ci. Supposons que l'on suggère à un homme liidée de vendle une maison a laquelle il tient beaucoup - cette détermination ne pent s'imposer à lui sans que l'idée de la maison soit éreillece et lidée de la maison entraine forcément l'apparition des sentiments qui lui sont étroitement associès, c'est-à-dire que la tendance suggérée, dès qu'elle doit s’associer certains éléments psychiques; éveille les sentiments systématiquement associés à ces éléments psychiques, et, parmi eux, ceux surtout qui peurent s'opposer à elle, par suite de cette vie des systèmes psychiques qui par cux-mêmes tendent naturellement à subsister ct à se dévclopper comme j'en ai donné de nombreux exemples. On voit que la tendance suscitée et la tendance absolument opposée tendent à s'éveiller réciproquement à cause des influences diverses de leurs éléments et en mème temps cherchent à cxercer l'une sur l'autre une action d'arrèt, à cause de l'influence qu'elles ont comme tout coordonné. C'est de ces deux actions différentes que naît l'association par contrastc, elle résulte à la fois d'un arrèt et d'une association systématique - l'association sýstématique étant déterminẻe par les éléments de la tendance suggeérée, l'action d'arrèt par l'influence de l'enscmble des deux tendanccs. Il y a là deux forces opposées qui se neutralisent jusqu'à un ccrtain point. Les affinités des éléments sont opposées en quelque chose aux affinités de l'ensemble, certains des éléments attirent ce que repousse l'ensemble dont ils font partic. Et le systeme éveillé au moyen de l'association par contraste se trouve dans la situation, il tend à arèter une activité à laquelle certains de ses élèments sont plus ou moins associés.

Nous comprendrons peut-ètre mieux la nature de l'association par contraste sinultane cn examinant les faits analogues qui se passent dans la société, où l'association par contraste jouc un trés grand rôle. Lor'squ'une activité 
empiète sur unc autre, l'autre est réveilléc, et commence it s'exercer et à réagir. Si un passant s'aperçoit qu'un voleur lui dérobe sa monire, il manifeste aussitôt une activité immédiatement opposéc à celle du voleur et si ce dernier ne craint pas que la lutte soit trop inégale, il se produit une lutte de ces deux activités opposées, parfaitement semblable à celles de deux tendances qui cherchent toutes deux a s'associer certains éléments de l'esprit. De mème unc compression gouvernementale trop forte, en enlevant à l'activité indivirluelle, ou sociale, des modes de s'exerecr auxquels elle tenait soulère des protestations et peut déterminer un courant d'opinion, une veritable tendance sociale qui lui scra absolument opposéc. Que l'on tàche de restreindre, par cxemple, certains usages de l'eau que les marchands de vins font dégénérer en abus, immédiatement les éléments sociaux dont on change le mode liabituel d'activite éveillent les anciennes tendances et deviennent le point de depart d'un mouvement d'opinion qui se traduit par diverses manifestatious tendant vers une mème fin : protestations isolées, articles de journaux, protestations collectives, ele. Nous dirons ici ce que nous disions a propos de la psychologie individuelle: comme il n'est guere.d'acte social et surtout gouvernemental qui ne froisse certains intérêts particuliers ou certains intérêts de groupe, comme les intérêts froissés réagissent el déterminent alu roins des plaintes, des conversations, des lettres, des articles de journaux, ete., l'association par contrastre est, all point de vue sociologirfue, comme alu point de vue psychologique, un lait géméral.

On s'en apercoit lien en remaryunt les courants d'opinion yui naissent presrfue simultanément et se dirigent en seus inverse. Toute action qui tend à modifier l'éfuilibre le lat soreictio ou d'un groupe social, it changer son orientatalion, salcompalgue generalement d'une action en sens 
inverse, qui parfois empêche la première d'aboutir; parfois ne peut s'opposer efficacement i elle, et parfois aussi, quand le fonctionnement social est bon, permet de faire le changement d'orientation sociale en tenant compte des intérèts, des aspirations, des besoins d'un très grand nombre d'èlẻments sociaux.

Les conditions sociales ont une influence considérable sur les sentiments individuels, et les déterminent souvent selon le mode de l'association par contraste. Un sentiment très puissant se précise, se développe, s'épure sous l'influence de l'opposition qu'il rencontre dans le milieu. Au milieu de la liberté de mœurs, de la licence même du xvir $^{\mathbf{e}}$ siecle, on voit apparaitre quelques-uns des plus beaux et des plus touchants exemples de pure tendresse et d'amour conjugal 1 . Mais ces faits ne nous présentent rien de nouveau après ce que nous avons vu du contraste en psychologie.

Comme l'association par contraste est un mode général de l'activité sociale: elle est aussi un mode général de l'activité physiologique. « Dans l'hypothèse de Wundt, à laquelle je crois devoir me ranger, dit M. Beaunis ${ }^{2}$; toute excitation nerveuse déterminerait dans le nerf deux modifications de sens inverse : une modification positive, pouvant agir à son tour comme excitant sur la substance

1. E. et J. de Goncourt, La Femme au XVIIIO siecle.

2. Beaunis. Elements de physiologie, $3^{\mathrm{e}}$ èdit., tome Ier, p. $684 . \mathrm{M}$. Beaunis indique très nettement la généralité de ce que j"appelle ici l'association par contraste dans le domaine de la psychologie. * Le fait essentiel, primordial, qui domine toute la question, dit-il, c'est cette dualité qui se trouve au fond de tout acte psychique; c'est cette double tendance à l'activité d'une part, à l'arrêt de cette activité d'autre part, qui fait que l'acte psychique n'est que la résultante de ces deux tendances contraires. » Toutefois ce mode de contraste simultané differe visiblement de celui que j'ai ètudié, et la ,théorie invoquée ne me semble pas pouvoir rendre compte des phénoménes psychiques, elle ne me parait pas au moins suffire à en donner une explication générale et compléte. 
nerveuse voisine et ainsi de proche en proche jusqu'au muscle; et une modification négative, qui tend à détruire ou a annuler la premiere; et, suivant que l'une ou l'autre de ces modifications prédomine, on aura ou bien un mouvement, ou bien un affaillissement (ou un arrêt de ce mourement). "- Il me semble que notre théorie sur l'association par contraste en psychologie est fortifiée par la considération du contraste physiologique et du contraste social qui, sans être identiques, expliquent partiellement ou impliquent le contraste psychologique. 


\section{Le contraste successif.}

$\$ 1$.

Les raisons qui expliquent la nature et la nécessité du contraste simultane, nous donnent aussi en partic l'explication du contraste successif. A côté de ces causes qui scnt communes aux deux formes de contraste, il en est d'autres qui nous rendent particulièrement raison du contraste successif, et qui, bien qu'clles se ramènent au même principe général que les premières, en diffèrent cependant par plusieurs points. Nous aurons à les cxaminer tout à l'heure. Commençons comme nous l'avons dejà fait pour le contraste simultané par constater les faits.

Pour les perception visuelles, le fait di contraste est bien connu : regardez fixement une croix rouge pendant quelques secondes, puis dirigez le regard sur une surfacc grise, vous apercerrez une croix verte, et d'une manière générale, une couleur complémentaire de la première couleur perçue. On peut faire mais arec beaucoup moins de précision, des remarques analogues sur le goìt. Apres un mets sueré, des confitures, un fruit naturellement doux páraitra acide, aigre, ou du moins sa douceur ne sera pas perçuc; après un fruit un peu doux, la moindre acidité, la moindre âpreté du vin rouge est fortement sentie. «Lorsque nous arons goùté une substance amère ou saléc, l'eau ([ue nous goûtons immédiatement après nous parait douce, et lorsque nous arons consommé une quantité de matière sucrée, nous éprouvons une certaine acidité dans la bouche. Ces faits nous rappellent presque involontairement les cculeurs contrastantes, mais nous manquons de toute hase 
exacte pour pouroir poursuivre cette comparaison " '. Au ! oint de rue du tact, mème remarque à faire: un objet ncus paraîtra lisse si nous renons de toucher un objet plas lugueux. Ce mème objet nous paraitra lugueux si celui que nous avons touche auparavant était hien poli. Plongez les deux mains, une dans l'eau froide, l'autre dans l'eau chaude, retirez-les ensuite et mettez-les a la fois dans de l'eau tièrle, et.la main qui était auparavant dans l'eau froide vous dounera une sensation de chaleur, tandis que l'autre rous fera éprourer une sensation de froid. Il y a bien lí une association non douteuse par contraste simultané. Mais pour la sensation visuelle aussi, nous pouvons renrarquer des contrastes de plus d'une nature, à côté du contraste des couleurs, nous avons aussi des contrastes d'intensité lumineuse ; un lieu légèrement sombre, paraît absolumeut obscur quand on vient du grand jour, et l'on ne saurait y voir immérliatement - et réciproquement le plein air est éblouissant quand on sort d'une cave. Même remarrue à faire pour les sensations de l'oüe : le silence est plus frappant apres un bruit intense, et un bruit d'autant plus fort en général (car il y a des exceptions, par exenple dans la paracousie de Willis) qu'il est percu au milieu d'un plus profond silence. Tous ces faits sont connus et je n'ai pas besoin d'y insister plus longuement - ils montrent bien comment une impression particulire de doucenr ou d'ipreti, d'éclat lumineux, de grandeur, de petitesse, de chaleur, etc., tend a se produre apres une impression qui frésente des caractéres absolument opposés - car bien sonrent cette dernièe injuression est la condition mème de son apprarition, $e^{t}$ sins elle, son existence serait impossible; la sensation, conserutive peut même ètre, dans les mèmes conditions exterieures, absolument diférente, puisigu'un papier

1. Jjernstein. Les sens, I1, 255. 
gris, par exemple, paraitra rouge, vert, violet ou jaune, selon que la couleur précédemment perçue était le jaune, le violet, le vert ou le rouge, puisque la mème eau paraitra chaude ou froide a la main, sclon que cette main aura été plongée auparavant dans de l'cau froide ou de l'eau chaude.

Les faits dejà cités s'appliquent à l'intelligence aussi bien qu'i la perception, et nous savons qu'il n'y a pas de différence essentielle à établir entre les deux ordres de faits. Ceux que nous allons voir nous moutreront réunies l'activité intellectuelle et l'activité affective et motrice il serait ici sans utilité d'essayer de séparer les divers cas de la loi de contraste, puisque aussi bien la loi est partout la mème; nous étudierons donc surtout les tendances et, simultanément, les phẻnomènes intellectuels ou affectifs dont elles s'accompagnent.

Dans la vie physiologique, nous trouvons certains faits qui paraissent se rapprocher beaucoup du contraste successif que la psychologie nous montre. L'alternance de la veille et du sommeil parait bien avoir quelque ressemblance réelle avec les contrastes psychiques. Ici aussi on retrouve la fatigue comme raison principale de l'alternance; nous examinerons tout à l'heure son rôle dans les associations psychiques par contraste - et de plus, on peut voir ou entrevoir les causes directes de l'alternance des deux états. On connait la théorie de Preyer, sur l'aceumulation des substances ponogènes dans l'organisme. Dernièrement, M. Léo Errera essayait d'ètablir que " la fatigue est, au sens propre du mot, un empoisonnement dont le sommeil est l'antidote normal" ', et il ajoutait des réflexions que je cite ici, parce qu'elles peurent donner lieu à des rapprochements curicux, et à des

1. Lèo Errera. Pourquoi dormons-nous? (Revue scientifique, 23 juillet 1887 ) p. 114 . 
hypothèses physiologiques qui seront peut-ètre un jour vérifiables : "Qui sait si beaucoup d'actes physiologiques (respiration, mourements du cceur, mietion, défécation) ne sont pas comme le sommeil, sous la dépendance de corps spécifiques agissant, soit sur les centres nerveux, soit sur les organes périphériques? Le rythme organique serait dì alors á ce qu'un acte physiologique donné, engenule des substances qui tendent a f,rovoquer l'acte contrairø, comme la descente du pendule fournit l'énergie uécessaire pour le faire remonter. Un travail tout récent de Zuntz et Geppert (Biol. Centralbl. 25 mars 188.5) ne conclut-il pas qu'en dehors de la teneur du sang en oxygène et en acide carbonique, il y a encore une substance particulière et inconnue produite surtout par l'activité musculaire qui intervient normalement pour régler les mouvements respiratoires."

Quoi qu'il en soit, et pour rester sur le terrain de la psychologie, l'existence au moins frécfuente de l'association par contraste, ne parait pas niable, et lo contraste successif parait plus communement admis que le contraste simultane. Certaines formes de cette loi, sont d'observation rulgaire (triste comme un lendenain de fète tel qui rit vendredi, dimanche plemera - quand le diable. devient vieux il se fait ermite, etc.) Au point de vue scientifirue, sauf en re oui regarde les couleurs complémentaires, le contraste successif a été peu étudié ; cependant MM. Binet et Féré ont indiqué avec beaucoup de précision me de ses formes, a propos des faits ru’ils ont desigues par le nom de polarisation psychique : il existe c'est lit conséquence qu'ils tirent de lemrs expériences sur les hystériques de la Salpêtrière) rles émotions complémentaires, comme il existe des couleurs complémentaires. L'émotion de la gaité, par exemple, a pour complémentaire l'émotion de la tristesse, de méme que la sensation du vert a pour complémentaire la sensation 
du rouge. Des deux parts le rapport est le mème. C'est l'aimant qui nous permet d'établir ce parallèle, mais la simple observation des faits normaux le fait dejai pressentir. Tout le monde sait que la sensation prolongée d'une couleur amène normalement à un degré quelconque l'apparition d'une couleur complementaire. C'est ce qu'on appelle la sensation consècutive (négative). 11 est également incontestable, quoique le fait soit d'une observation plus difficile, que, lor'squ'une émotion a duré un temps assez long, elle fait place pour un moment à une émotion de genre contraire, qu'on pourrait appeler avec assez de justesse, l'émotion consécutive $" 1$. Et les deux auteurs ajoutent cette remarque intéressante que: "Si la faiblesse ou la fatigue des yeux favorisent le développement des images consécutives, l'afficiblissement ou l'épuisement, produit par l'àge et les maladies, farorise ègalement le déreloppement des émotions consécutircs. C'est ainsi que peuvent s'expliquer les changements d'humeur si fréquents des nérropathes et en particulier des hystériques qui subissent presque immédiatement l'inversion des émotions et des passious. "On peut rapprocher ces faits généraux de la loi du rythme de M. Spencer ${ }^{2}$, mais le philosophe anglais n'a peut-être pas donnè à sa loi une précision suffisante.

Nous trourons daus la vie ordinaire ce fait que nous éprouvons souvent un état d'esprit absolument opposé à celui qui l'a précédé. Chez les personnes en qui la domination d'une passion a été très longue et très exclusive, le contraste peut aptaraitre arec une intensité surprenante. La tentation de saint Antoine parait ètre le résultat d'une association par contraste successif. Sainte Thérèse nous raconte que parfois elle était, malgré elle,

1. Binet et Féré, la Polarisation psychique (Revue philosophique, premier semestre 1885, p. 401).

2. Voyez Spencər. Premiers principes. 
dans des dispositions l'esprit entièrement opposées à celles qui dominaient généralement en elle - il paraìt $\mathrm{y}$ avoir ici at la fois un effet de contraste simultane et de contraste successif et nous pourons remarquer aussi que lassociation par contraste ne se manifeste pas moins dans le domaine des idées que dans celui des sentiments. "Cela marriva, dit la sainte ${ }^{1}$, une fois la veille de la feste du Saint-Sacrement pour laquelle jay beaucoup de dérotion, quoyque non pas si grande que je le devrais, et ne dura qu'un jour. D'austres fois il m'a duré lunit jours, quinze jours, trois semaines et mème davantage, et particulièrement dans les dernières semaines de carème qui est le temps où je m'appliquais avec plus de ferveur à l'oraison. Le démon remplissait mon esprit de choses si frivoles que je m'en serois mocquée en un autre temps. Il paraît être alor's maistre de l'àme pour l'occuper ainsi qu'il lui plaist de mille folies, sans qu'elle puisse penser a rien de bon. Il ne lui représente que des choses impertinentes, ridieules, inutiles à tout, et qui ne servent qu'a l'embarrasser et comme à l'étouffer de telle sorte qu'elle ne se reconnait plus elle-mème. Ainsi il me semblait que les démons se jouaient de moi comme on se jonerait d'une velote, el (u'il m'estoit impossible de m'échapper de leur's mains."

Un grand nombre de nos sentiments, on ne l'a pas assez remarqué peut-être, impliquent la production d'une associalion far contraste successif, le fait est bien évident pour le remords si on en accepte l'explication que Darwin en a donnce 2. Le remords se produirat, d'apres cette theorie, lorsfue les penchants socianx, un moment vaincus par une tendance egoïste reprennent, une fois l'acte conpalule commis et la tendance égoüste satisfaite, la foree

1. Sainte Thérèse. Autobiugraphic. Trad. Aınaul d'Andilly, p. 184.

2. Voyez. Larwin. La descondance de l'homme et la sélection sexuelle. 
quils ont normalement. Le remords serait donc une sorte d'élat srunthétique impliquant la succession de deux groupes de tendances opposés, orientés d'une manière très différente et comprenant comme éléments des sentiments actuels tris forts et le souvenir d'un acte accompli en opposition avec ces sentiments. Une explication complète du remords exigerait une analyse plus longue et la détermination des conditions du phénomène synthétique, mais ceci peut nous suffire pour le moment comme montrant un cas intéressant d'association par contraste successif, dans lequel un mode d'activité de l'esprit est suivi par un mode d'activité aỹant une orientation tout-à-fait opposée à la précédente. Mais si nous examinons nos sentiments conscients, nous verrons que la plupart sinon tous impliquent une association par contraste - tantôt contraste simultané, tantôt contraste successif. Nous avons vu déjá la généralité du contraste simultané: étudions à présent les sentiments au point de vue du contraste successif.

Le regret est analogue au remords en bien des cas il se produit évidemment lorsqu'il $\mathrm{y}$ a contradiction entre la tendance dominante et un phénomène passé, mais il ne peut nous intéresser ici que lor'sque l'activité d'une tendance détermine elle-même l'activité postérieure d'une tendance inverse. Il n'est pas rare de constater que lorsque nous arons hesité entre deux partis et que nous arons fini par nous décider, les regrets arrivent Jientôt - la satisfaction d'une tendance ayant été logiquement suivie par l'activité de la tendance opposée. Dans le désir nous vorons souvent un fait parfaitement analogue; ainsi lorsque nous sommes restés longtemps assis, nous éprouvons le désir de nous lever et réciproquement. Les tendances qui ont été trop longtemps comprimées; et ce sont naturellement celles qui sont le plus directement opposées à la tendance dominante, finissent par surgir et sc 
manifestent par des désirs plus ou moins sourds, plus ou moins inconscients d'aljoril et qui se développent peu it peu. Liespérance se prête sourent it une explication analogue, elle ne se distingue du désir qu'en ce qu'elle implique à un bien moindre degré l'intervention des éléments actifs, des tendances motrices qui y sont plus fortement enrayées. La crainte se ramene jarfois à des conditions semblables, mais cela est, je crois, plus rare.

\section{$\S 2$.}

A l'élat morbide et dans les états semi-pathologiques, intermédiaires entre l'état morljide et l'état normal, le phènomène du contraste est souvent trè visible. On a signale sourent et depuis longtemps le fait du contraste entre les rèves et la réalité et aussi entre l'état mental de certains aliénés et leur caractìre antérieur. Les personnes qui souffrent de la faim rêvent souvent de bons repas, et du reste a cet égard le phénomène du rêve n'est qu'une forme plus vive de ce qui se passe à l'etat normal, où c'est bien souvent ce qui nous manque qui nous affecte et nous fait soulhir ou même en ljien des cas nous procure du plaisir-e'est a ce qui nous manque que nous pensons surtout, que nous rêrons quand nous sommes éveillés. A l'état norljide, Pinel avait olsservé que l'on voyait dans tous les asiles consacres aux aliénés de l'un et de l'antre sexe des malades "recommandables par une ric sobre et laborieuse, les moeurs les plus irrejprochables et une extrome delicalresse de sentinents, tomber par queline cause physique ou morale dans un egarenent conplet de la raison, el contracter les vices qui forment un contraste frapuant avec leur caractere grinitif durant le cours de leur alienation, el revenir enfin, lors de leur guérison, anx dunces impressions de leur heureux, naturel. Que dhommes tres solmes, dans les intervalles d'une manie 
périodique, se livrent avec un penchant insatiable à l'ivrognerie au retour de leurs accès. Combien d'autres, daus les mêmes circonstances, ne peuvent s'empêcher de voler et de faire des tours de filouterie, tandis que dans leurs moments lucides on les cite comme des modèles d'une probité austère. Ne voit-on pas de même des carac ères doux et bienveillants se changer par les suites de l'aliénation en esprits turbulents, querelleurs et quelquefois entièrement insociables? J'ai cité ailleurs des exemples de jeunes personnes élevées dans les principes les plus sévère et dignes d'ailleurs de l'estime publique, tomber dans l'aliénation et alarmer la pudeur par la saleté de leurs propos et l'indécence de leurs gestes. Lors de leur rétablissement, tout rentrait dans l'ordre, et c'était alors autant de modèles de mœurs les plus pures et de toute la candeur de l'innocence $\triangleright 1$. Shakespeare faisait chanter une chanson libre par Ophélie devenue folle.

Dans certaines formes de la folie, le contraste successif est particulièrement visible. J'emprunterai quelques faits à l'étude de M. A. Ritti sur la folie à double forme. L'allure mème de cette maladie, qui consiste dans l'alternance d'une période d'excitation et d'une période d'affaissement, appelle facilement l'ilée d'une association par contraste. Mais la psychologie et la physiologie morbides ne paraissent pas encore suffisamment arancées pour nous expliquer le mécanisme de cette succession d'états opposés, et le fait tel qu'il se présente à l'observation ne peut pas prouver beaucoup - tout au plus pouvons nous y voir une application hypothétique de l'associa-. tion par contraste. Il n'en reste pas moins que certains phénomènes particuliers, dans cctte forme de folie, parais. sent explicables par les lois générales de l'esprit, en particulier par la loi du contrastc, et rentrent à cet égard

\section{Pinel. Traité médico-philosophique sur l'aliénation mentale.}


dans notre sujet. Ainsi M. Baillarger qui a le premier fait remarguer la surexcitation génesique pesdant la periode maniaque de la folie a donble forme, l'a considérée comme " une sorte de réaction après une longue période d'engourdissement des organes génitaux " 1 . Une femme, " après etre restéc tout l'hiver muette, immobile et accroupie dans un coin, renaissait au printemps et pendant l'été. Une intarissable loquacité et de violents signes de nymphomanie annongaient la fin de son immobilité et de son mutismen. Un cas intéressant est celui on le malade manifeste, dans une période de la folie, ses regrets ou ses remords de ce qu'il a fait pendant la période d'apparence opposée, - et les faits de ce genre, que l'on remarque souvent, semblent venir à l'appui de l'opinion qui verrait dans la forme essentielle de la folie i double forme une manifestation exagérée de la loi du contraste successif. M. Ritti signale comme très fréquent le fait que, pendant la période de dépression, la malade se repent de ec qu'elle a faitpendant la période d'excitation. En voici un exemple: Une fenme atteinte de la folie a domble forme, fut entraince au vol par une tendance irrésistijse et fut arrètée. "Un examen attentif de lả situation fit reconnaitre son état pathologique. Revenue à son bon sens, elle regreliait vivement ses actes. Quand la période d'excilation survenait, sa volonté était impuissimte pour dominer sa tendinee an vol. Elle dérobait tout ce qu'elle royait sans même y metlre du mystère; elle prenait des objets qui ne pouvaient lui etre d'aucune utilite et recherchait, dans ces latrins insigniliants, d joner de maturitis tours anx autres. Elle ent des hallucinations, fut effrayee par l'apparition du diable, et son argitaton ctait souvent extrême. Puis elle retombait clans un profond abattentent, lel gu’il a eté décrit plus haut.

1. Ritti. Traile clinique de la folie à double forme, 1. 138. 
Ce délire à forme alternante dura plusieurs années. C'était ordinairement toutes les quatre semaines que ces changements avaient lieu. Dans la seconde période, cette femme se reprochait vivement ce qu'elle avait fait pendant la première, et elle tombait dans le plus profond accablement, ètrangère à tout ce qui se passait autour d'elle "1. On trouve parfois aussi chez ces malades des contrastes de sentiments assez curieux : Une malade prenait en aversion, pendant la période maniaque, son mari et sa fille qu'elle aimait beaucoup durant son état normal.

Ainsi, pour l'association par contraste successif, comme pour l'association par contraste simultané, l'état morbide nous montre des exagérations de ce qui se passe a l'état normal, et l'un nous aide à comprendre l'autre. Mais nous ne nous ferons une idée juste et complète de ce mode d'association, comme du premier, qu'en comprenant ses raisons d'être générales et permanentes.

\section{$\S 3$.}

Les associations par contraste successif peuvent se diviser en dẹx groupes, distincts à quelques égards, sans l'être absolument ; le premier se laisse ramener aux causes qui produisent le contraste simultané, dont le contraste successif est, en ce cas, une sorte de prolongation; le second est dù à des causes différentes mais qui consistent essentiêllement au fond dans le mème fait général, la réaction d'une tendance. Dans le contraste simultané et dans la première forme du contraste successif, il s'agit de la réaction d'une tendance qu'un nouvel état tend à dissocier, dans la seconde forme du contraste successif

1. Ritti. Ouvr. cite, p. 129-130. L'observation est empruntèe à un mémoire de M. Begmann. 
de la réaction d'une tendance qui n’a pas pu s'exercer pendant un temps trop long - et cette secoude forme à son tour peut domner lieu à des effets de contraste simultané.

Pour conceroir que les causes qui produisent le contraste simultané puissent produire aussi le contraste successif, il suffit de supposer qu'une idée, une tendance étant amenée à l'activité par l'action d'une tendance opposće, suivant le mode que nous avons analysé plus haut, la premiere tendance vienne à disparaitre momentanément par une cause quelconque (quelquefois l'activité inhibitoire de la tendance qu'elle a suscitée), alors Ia seconde reste seule et l'ensemble des phénomènes qui avait d'abord l'aspect d'un contraste simultané prend celui d'un contraste successif.

Ce cas n'est pas purement théorique. Si, par exemple, on fait une objection à une théorie que nous croyous fermement viaie, il peut arriver que la théric et les idées coordonnées rui s'y rattachent, éveillées en nous selon la loi du contraste simultané, finissent par évincer absolument l'idée de l'objection à la suite de la lutte que j'ai indiruée, le fait, comme chacun a pu l'observer, se produit quelquefois tres rapidement. Il arrive alors que l'état definitif de l'esprit est absolument opposé à celui qui a un monent, sous l'influence d'une lecture, d'mue conversation, ou sous toute autre influence, tenté de se produire, et qu'il a élé récllement amené par cet acte auquel il s'oppose. Il y a done bien lì un effet de contraste successif se produisant à la suite d'un contraste simultané fitu la persistance de l'état psychique eveillé selon ce dernirer mode d'association.

Quand des sentiments fui dormaient en nous sont réveilles par une attaque brutale, quand des idées auxquelles nous ue songions pas sont remises an premier plan par unr: contuadiction brusque, ces sentincuts, ces idées surlivent atux sentimonts et aux idés contraires qui, imposés 
momentanément à l'esprit, les ont amenés à l'activité par le contraste, mais qui ne tenant à rien dans notre organisation mentale, ne peuvent pas se maintenir. En ce cas les tendances éveillées se développent et déterminent, pendant un temps plus ou moins long, l'orientation de l'esprit. Les exenuples ell sont très communs - on détermine presque toujours une réaction immédiate, et qui se prolonge, quand on froisse chez quelqu'un des sentiments profonds et des idées enracinées. Cette persistance des tendances éveillées s'explique très simplement par les propriétés des éléments psychiques Remarquons, d'ailleurs, qu'il n'est pas facile de dire où cesse le contraste simultané, où commence le contraste successif ; l'idée ou la tendance qui ne peut s'associer suffisamment à des éléments psychiques stables pour se maintenir dans l'esprit, ne disparait pas sans laisser des traces qui persistent sensiblement quelque temps, et qui se manifestent par le caractère mal unifié des composés synthétiques qui les réunissent avec la tendance opposée - c'est ce que nous tronrons dans les fai's de remords, de regrets, etc., qui impliquent à quelque degré une persistance, si faible soit elle, un souvenir de la tendance vaincue.

\section{$\S 4$.}

Dans le second groupe il s'agit de la réaction d'une tendance longtemps arrètée et qui, lorsqu'elle peut enfin s'imposer à son tour à l'esprit et prendre la direction de l'organisme, donne à notre activité générale un caractère absolument oprosé a celui qu'elle avait auparavant, - et la réaction est d'autant plus vive, toutes choses égales d'ailleurs, que la tendance est plus importante et que l'arrèt avait été plus long et plus complet. Mais entre ces deux groupes de faits, il existe des intermédiaires nombreux que l'on peut rattacher snit à l'un soit at l'autre et qui la relient par une série de grarlations insensibles. 
Il arrive par exemple que le contraste simultané ne se produit pas tout de suite d'une manière apparente. Si l'on nous dit une chose qui blesse en nous quelques sentiments assez peu actifs, il se peut que nous n'y prenions pas garde, il faudra que l'on revienne à la charge, que l'attarue se prolonge et se multiplie, que l'opposition se déreloppe pour que les tendances froissées finissent par réagir sensiblement et donner lieu à des représentations, à des idées, à des émotions qui sont en contraste marqué avec l'activité suggérée par les paroles de notre interlocuteur. Ici le mode de contraste est intermédiaire entre le contraste simultané et le contraste successif ct aussi cntre les deux modes de contraste successif, et ce caractère sera encore plus marqué, si nous supposons que la tendance éveilléc fasse disparaitre assez vite les idées et les sentiments opposés qui ont fini par déterminer son activité ce qui se produit assez fréquemment. Il nous suffit au reste d'indiquer ces phénomènes et de rappeler que les groupes que nous avons établis, tout en correspondant a des modes divers de production des phénomènes ne s'opposent pas complètement l'un à l'autre et que ces différents modes jeurent se combiner de diverses manières.

Revenons au second mode. Le nombre des associations systemaliques latentes, des habitudes, des organisations psyclıo-organifues qui existent en nous est incalculable. Ces associations, ces systemes psychiques ne sont pas toujours en autivite, mais elles ne cessent pas d'exister virtuellement, c'est-it-dire qu'il en reste toujours une trace, une disposition particuliere de l'esprit que nous nous fignurons volontiers sous la forme d'une modification injuerecplible de la substance cérébrale. Quoiqu'il en soit, lis prosistance des tendauces, des causes des pliénoniènes psychipges ne peut etre douteuse. De plus, certains systemos perelingues comprennent comme éléments les inplessions norveuses qui proviennent at tout instant 
d'organes physiques permanents, et dont l'existence concrète ne saurait s'interrompre. Toutes les parties de notre corps sont ainsi, en un sens, partics de systèmes organico-psychiques. Les poumons sont en rapport avec les éléments nerveux et psychiques qui peuvent arrêter, continuer, ralentir ou activer la respiration, l'état de l'estomac - ou peut-être du sang, — est un élément de ce système psychique qui se manifeste à nous par le sentiment de la faim, de sorte que lorsque ces organes physiques sont dans un état détcrminé, on peut constater l'éreil selon la loi d'association systématique, de tel système psychique déterminé, de tel sentiment, de telle émotion pénible ou agréable, de telle tendance à des mouvements coordonnés. Mais un grand nombre de ces excitations qui déterminent dans l'esprit l'éveil d'un système psychique se produisent à des intervalles réguliers. Les fonctions organiques sont manifestement rythmiques. Il y a ainsi continuellement une opposition, un contraste entre deux états successifs. Ce changement d'orientation d'un système psychique plus ou moins important se marque très visiblement en ccrtains cas par la nature des phénomènes qu'il déterminc. Ainsi l'odeur d'un ragoùt, qui est très agréable quand on a faim, peut ètre répugnante après le repas. Le contraste se marque ainsi à quelque degré dans l'exercice normal des fonctions organiques. Il se marque encore d'une autre manière: si les besoins organiques ne sont pas satisfaits, la tendance qu'ils déterminent devient de plus en plus forte, de plus en plus vive et lorsque les circonstances cessent de s'opposer à ce qu'elle aboutisse et se manifeste par des actes, ces actes présentent un caractére d'intensité d'autant plus considérable, toutes choses ègales d'ailleurs, que la tendance a été plus longtemps arrètée. Fréquemment la privation trop longue aboutit a des exces. Inversement une longue inspiration raleutit pendant quelques secondes le 
rythme normal de la respiration. - A mesure, en effet, qu'un état organique se produit, se prolonge et s'accentue, les sentiments qu'il détermine sont (en faisant abstraction des circonstances particulières qui masquent souvent l'enchaînement régulier des phénomènes en le compliquant sans le détruire) de plus en plus vifs, il en résulte naturellement un contraste très fort entre l'état antérieur: celui de la privation, de l'arrèt de la tendance et l'état qui lui succède : celui de la satisfaction. L'impulsion est telle en ce cas, que même lorsque les circonstinces viennent a la favoriser, clle persiste un certain temps, la tendance ne peut être immédiatement satisfaite et c'est ce retard dans la satisfaction qui produit le sentiment de plaisir éprouvé si vivement lorsqu'une tendance longtemps comprimée peut enfin reprendre son activité. Ce contraste qui est ainsi manifesté dans certaines formes de sentiments alors qu'il s'est produit, est annoncé à l'avance par certaines autres émotions synthétiques, comme le désir, l'espérance qui impliquent un contraste entre l'état actuel et celui que tendent à déterminer des tendances encore inlibées.

Le contraste prend ici, comme on le voit, une forme par- . ticulière; il se manifeste non pas précisément par l'apparition d'un état positif opposé à un ètat précédent, mais par la satisfaction d'une tendance opposée à sa non-satisfaction. C'est la forme la plus fréquente du contraste en ce qui concerne les fonctions organiques et la raison en est que l'on ne peut dire que, parmi les tendiuces physiologiques, les unes s'opposent absolument aux autres - la marclie, la respiration, la circulation, la digestion ne s'opposent pis les unes anx autres comme le rouge s'oppose an vert-bleu ou la sympathie it J'antipathie et le mépris at l'estince Les conditions de lansociation par contraste sont remplies cependant, call noms rovons bien se succeder ici deux elats opposes 
dont le premier est la cause de l'autre - l'arrêt d'une tendance et sa domination d'autant plus étendue et plus forte que l'arrèt avait été plus long et plus rigoureux.

Mais pour peu qu'on y réfléchisse on voit vite que presque toutes nos fonctions, au sens le plus génèral du mot, c'est-i-dire l'activité de tous les systèmes organico-psychiques qui existent en nous sont, ì quelque degré , périodiques et soumises à un rythme plus ou moins marqué, à des intermittences d'action et de repos l'orientation de l'esprit change partiellement il chaque instant et très sourent d'une manière notable. Nous ne sommes pas toujours occupés à lire, à écrire, á jouer à n'importe quel jeu, à faire notre méticr, à ètre fonctionnaire, administrateur, médecin, avocat; les sentiments, les tendances, mème les plus ancrées en nous, celles qui correspondent aux habitudes les plus invétèrées, aux affections de famille; ont des moments de repos relatif. Elles persistent toujours à quelque degré il reste toujours quelque chose de la disposition systematique qu'elles ont donné à l'organisme mais elles ne se manifestent pas d'une manière active, elles n'existent plus qu'en puissance, c'est-à-dire par quelques-unes de leurs conditions organico-psychiques. Un arocat reste avocat même quand il fait une promenade, cependant les systèmes psychiques qui le rendent apte à exercer sa profession ne sont pas en activité. Il n'en reste que des éléments, mais ces éléments subsistent avec leurs affinités et à la moindre occasion les habitudes d'esprit reparaissent. Un mathématicien sur le point d'expirer avait perdu l'usage de la parole, il ne reconnaissait plus, ne voyait plus; un de ses amis se penche vers lui et lui demande le carré de douze : cent quarinte-quatre, répond immédiatement le moribond. Il y a ainsi en chacun de nous des systèmes de pensées, de sentiments, d'images et d'actes qui sont momentanément dissociés et qui restent 
ainsi tant que ces éléments réunis à d'autres combinaisons ne peuvent se coordonner entre eux - mais plus la coordination a été habituclle, plus elle embrasse d'éléments fréquemment mis en aclivité, plus il y a de chances pour que telle ou telle circonstance lui donne l'occasion de se manifester de nouveau.

Des circonstances particulières, externes ou internes, peuvent empêcher pendant longtemps ces systèmes de se reformer. Un chasseur forcené est bien obligé, à moins qu'il ne passe braconnicr ou n'ait une propriété cnclose, de s'abstenir de chasser plusieur's mois de l'année; un médecin, un avocat, peurent être empèchés pendant un temps assez long de soigner les malades ou de plaider un père peut être séparé de ses enfants. C'est là une cause continuelle d'associations par contraste analogues à celles que nous avons déjà examinées et qui peuvent prendre plusieurs formes. D'abord si les sentiments comprimés ont atteint un certain degré de force, ils seront réveillés par des circonstances insignifiantes; la tendance arrètée entre en jeu, sans pouvoir se compléter, a la moindre occasion favorable, - un père qui est séparé de ses cnfants, s’il reneontre un enfant étranger, pensera au sien, beaucoup plus facilement et plus vivement que s'il n'en était pas séparé. L'association par contraste est ici compliquée d'une association systématique; ce qui frappe dans la réalilé extérieure, c'est ce qui peut s'harmoniser avec les tendances comprimées, c'est-it-dire ce qui contraste avec l'état d'esprit imposé par les circonstances, c'est chez un homme qui n'a rien à manger que la vue de l'étalage d'un marchand de comestibles, déterninera le plus vivenent des sentiments et des ilóes opposées à l'état organique el psychique que sa silnation lui inpose, et c'est parce que cet étal lui est imposé que l'idée d'un état opposé el les sentiments qui ronviendraient a celui-ci se produisent en lui ; il ya 
bien récllement une association par contraste. Ce n'est que lorsqu'une tendance est suffisamment enrayée dans son processus qu'elle peut donner lieu à ces phènomènes de rèverie, de désirs conscients que nous avons déjil examinés, le remords est un cas analogue de la même loi, mais il faut évidemment que la tendance enrayée conserve une certaine activité, il faut que l'arrêt prenne la tendance à un moment de son développement, il fau ${ }^{+}$ qu'il l'empèche d'aboutis', mais qu'elle ne l'empèche pas de naitre et de déterminer quelques associations psychiques. L'association par contraste, nous développerons ce point tout à l'heure, est une combinaison de l'association systématique et de l'inhibition.

\section{$\S 5$.}

Dans les cas que nous venons d'examiner, l'opposition se produit entre deux états d'une mème tendance, ce n'est pas la seule forme de l'association par contraste comme le prouvent les faits que nous avons citès tout à l'heure, il arrive souvent qu'une activité est remplacée par une activité opposée. Toutefois les deux cas ne diffèrent pas autant qu'on pourrait le supposer. Une orientation positivement opposéc de l'esprit peut résulter simplement de la mise en non activité ou en activité d'une tendance déterminée. La tristesse, par exemple, peut succèder à la gaìté, simplement parce qu'une certaine quantité de tendances qui étaient en activité auparavant viennent à entrer en repos. L'ennui résulte souvent d'une cause semblable, et succéde à l'animation et au plaisir. Ici l'état contrastant est déterminé par le changement de l'activité d'une tendance en non activité, ou réciproquement, mais il y a un nouvel ètat synthétique de l'esprit qui résulte des nouvelles conditions de son activité, et qui est le sentiment, opposé dans chaque cas, qui suppose une synthese psychologique des tendances éveillées et une appréciation de leur activité par rapport au moi. 
Ce fait est fréquent dans le mode de contraste que nous venons d'examiner. D'autres fois de nouvelles tendances opposées aux précédentes sont réellement mises en activité - on voit ainsi parfois l'amour surceder a la haine etl'estime au mépris, et cela implique les jugenents, et les tendances motrices se combinent jour des fins en opposition complète avec celles qui étaient impliquées par le précédent fonctionnement de l'esprit - c'est encore le mode de contraste qui se produit quand la sensation subjective d'une couleur sucède à la perception de la couleur complémentaire.

Ces cas ne paraissent pas offrir de difficulté particulière. La cause qui les produit est celle qui produit les formes préédentes; nons avons vu que lorsqu'une tendance est restee inactive pendant un temps relativement long, qui peut d'ailleurs être seulement de quelques secondes - comme cela a lieu pour la respiration, - par exemple, cette tendance si elle a une certaine importance dans l'organisme, est prête à se remettre en activité à la moindre occasion, d'un autre côté, les tendauces qui sont en activité à un moment donné ne jeuvent pas rester continucllement actives, il y a là une double cause de phé-. nomenes contrastants.

Il est très difficile, conme l'on sait, de laisser le bras étendu horizontalement pendant quelıues minutes seulement. La fatigue survient bientôt. Mais tont état prolongé du corps et de l'esprit finit en général par anener aussi une fatiruc plus ou moins consciente, un état tel que nos tendances organiques risquent d'en être troulslèes. C'est nue raison - non pas toujours, mais d'me manièe generate, - joul que eet état cesse, car at l'etat normal la systematisation psyclro-organique est asse\% malrquée pour determiner l'aret des phenomenes gui ne seraient pas en areorlatec les teudances dominates, et dont le dévelopjument mirat a ces tendances. Cette inhibition geut 
étre déterminée soit par une volition, soit par une associalion systématique ne comprenant pas l'intervention du pouvoir personnel. Si nous regarlons quelque temps un objet trop brillant nous finirons par détourner volontairement les vetix, de mème si nous éprouvons quelque part des émotions trop persistantes et si nous n'arons aucune raison de rester, nous nous en irons, mais aussi une préoccupation qui nous obsède ne nous obsède pas continuellement, et sans l'intervention de la rolonté, il se produit, comme l'a fait observer M. Spencer, de fréquentes rémissions; de mème si l'on a pris une position gènante on remue, sans y prendre garde, au bout d'un moment, pour se mettre mieux a l'aise.

Il y a bien des chances, comme l'indique ce fait genéral de la périodicité et du rythme des fonctions organiques et psychiques, pour que le complexus de phénomènes qui succède à un complexus différent soit un de ceux qui ne pouvaient se manifester librement en même temps que celui-là. En effet, nos systemes psycho-physiologiques tendent à être presque constamment mis en jeu par les excitations venues du dehors, ou par voie réflexe, par les idées et les autres phénomènes psychiques. Or, si l'excitation persistant le système psycho-physiologique ne peut être ércillé, la tendance acquiert souvent plus de force, de telle sorte que, dès que la possibilité d'aboutir existera, soit par suite d'un changement dans les circonstances extèrieures, soit parce que son développenent lui permet de changer l'orientation de l'esprit et d'inhiber a son tour les tendances qui s'opposaient à elles, la tendance se manifestera par l'éveil d'un complexus de plıénomènes. D'un autre côté il est bien sùr que ce qu'une tendance en activité tend le plus a exclure selon la loi de l'inhibition sy̧stématisée, c’est la tendance qui lui est directement opposée - ainsi la bienveillance envers me personne exclut la malveillance, etc., il est donc naturel que 
lorsque deux tendances opposées coexistent en nous et que l'une s'est manifestèe pendant un temps assez long, ce soit l'activité de l'autré qui la remplace. Il arrive, par exemple, que la même personne nous plaît par certains côtés et nous déplaìt par d'autres. Si pendant un certain temps c'est la première impression qui a dominé en nous, la seconde paraitra ensuite avec plus de force et son apparition sera certainement causée en grande partie par la domination trop longue de la première. Il arrive aussi que l'on s'illusionne, presque volontairement sur la nature d'une personne, on ne remarque dans sa conduite que ce qui peut en donner une idée favorable, le reste passe presque inaperçu - il ne faut pas croire pourtant que ces expériences soient entièrement perdues, ìl se passe souvent alors un fait qui est la reproduction avec les différences que comporte l'état normal du dédoublement de la personnalité observé et eréé par M. Pierre Janet et que j'ai eu déjà l'occasion de citer. Il se forme en nous non pas tout-à-fait deux personnalités, mais deux groupes de tendances opposés, dont l'un garde la direction de l'organisme et détermine l'orientation consciente de l'esprit, dont l'autre passe presque inapereu et s'organise insensiblement - et ces deux groupes sont complementaires, ce que l'un ne peut s'adapter est saisi par l'autre, chacun ne connait que lui-mème. T'outes les petites particularités, les paroles, les actes, que nous ne voulons pas voir sont enregistrées et classées dans l'inconscient, et un jour, lorsque l'orientation consciente do l'esprit change, lorsqu'un trait plus visible que l'autre nous a forcés d'ouvir les yeux, où lorsque la tendance inconsciente développéc peu a peu finit par déterminer l'iuhilution de la tendance opposée nous sommes surpris - si nous n'avons pas des notions suffisantes de psychologiv - de tronver en nous des sentiments qui nous paraissent tont naturels et trés développéset qui sont entièrement 
opposés à ceux que nous croyions avoir seulement jusque-la. Et nous nous rappelons alors une foule de détails, de circonstances qui nous avaient à peine frappésou qui nous avaient frappés à notre insu - et nous nous souvenons aussi de certaines impressions désagréables qui nous avaient semblé tout-ä-fait passagères et insignifiantes, qui ne se reliant a aucune des tendances dominantes ne nous étaient pas revenues en mémoirc, et qui maintenant se trouvant en harmonie avec le nouveau système dominateur clont elles étaient l'cxpression fugitive, reviennent en nous, se coordonnent et se développent. Bien souvent les phénomènes n'apparaissent pas sous une forme aussi caractéristique, mais il n'est pas rare de voir une même personne manifester successivement deux manières de voir alssolument opposées, deux sentiments contraires à l'égard d'une personne ou d'une chose ; c'est une forme affaiblie du cas précédent. 'Et cela peut se généraliser ; cette pluralité, cette diversité desidées et des sentiments est très fréquente. Si tous ces éléments psychiques ne se coordonnent pas en sc rectifiant réciproquement de manière à former des idées, des tendances générales ou chacun d'eux joue son rôle, ce qui arrive souvent, puisque la vie indépendante des éléments psychiques composés est loin d'ètre une chose rare, il se forme ainsi plusieur's coordinations opposées, chacun des élements s'associant avec ceux qui s'harmonisent le micux avec lui et l'activité contraire de ces deux groupes qui se succèdent dans la direction de l'organisme à des intervalles plus ou moins réguliers est évidemment une cause fréquente de la forme de contraste que prend l'association des faits psychiques.

Les faits généraux qui expliquent le second groupe d'associations par contraste successif peuvent aussi produire des associations par contraste simultané - c'est l'inverse de ce que nous avons constaté tout à l'heure. 
Supposons, en effet, qu'une tendance ait été en activité assez longtemps pour fatiguer l'organisme, la tendance opposée pourra commencer à se manifester avant que la première ait complètement cessé d'agir, et l'on aura un effet de contraste simultané produit par les causes qui déterminent généralement le contraste successif.

D'ailleurs, si l'on y regarde de plus près, on roit qu'il faut non pas confondre, mais rapprocher les deux groupes de causes, les deux mécanismes différents que nous arons distingués. Dans un cas comme dans l'autre. l'association par contraste est due à la réaction de l'organisme opprimé par la persistance de l'activité d'une tendance. Seulement il arrive quelquefois que cette activité soit d'abord coordonnée, utile ou agréalble, et qu'elle ne commence à être nuisible qu'au bout d'un temps plus ou moins long, et c'est alors d'une manière générale, le contraste successif qui se produit - dans d'autres cas son existence seule suffit pour géner considérablement les habitudes psychiques et organiques et l'éveil des tendances opposées se fait immédiatement. Il se produit alors, en général, une association par contraste simultane. Nous arous vu que cette association pouvait, par la combinaison de tendances opposées, aider ¿̀ la précision et augmenter la complexité du fonctionnement de l'esprit en préparant une tendance synthétique plus large, le contraste successif aussi a son utilité évidente en nous aidant à conserver un équilibre organirjue et mental relatif.

$$
\$ 6 .
$$

Il me semble que nous avons ainsi rendu compte de tous les faits de contraste successif énumérés tout à l'heure, ils se ramènent tous, en somme, a la réaction des tendances comprimées. Depuis les phénonènes de la vision les coulenrs complémentaires, jusfu'aux alternatives de tristesse et de joie, de bienveillance et de méchanceté 
nous trouvons partout et toujours le même phénomène: un désir contrarié, une tendance non satisfaite, qui réigissent et se manilestent d'autant plus qu'ils ont été plus ou moins empèchés de le faire, et que les tendances opposées sont fatiguées par une activité correspondant à leur repos.

La loi d'association par contraste successif nous est apparue comme un résultat nécessaire du fonctionnement de l'esprit selon les lois de l'association systématique et de l'inhibition. Elle se présente done avec une portée très génẻrale; bien qu'elle ne donne pas toujours lieu à des phénomènes trés nets, elle existe toujours comme tendance. Un phénomène n'est pas toujours remplacé par un phénomène absolument opposè, si je regarde vaguement et très peu de temps un papier rose, et que je regarde ensuite un papier gris, il n'est pas sùr que j'aurai la perception subjective de la couleur complémentaire, il faudrait que la vision ait été assez prolongée pour qu'il $\mathrm{y}$ ait une fatigue réelle des organes - de mème après une douce gaité on n'est pas forcément triste, mais on est peu porté à la gaîté après avoir trop ri.

D'une manière générale, la réaction n'est extrème, le contraste n'est très marqué que dans le cas où la capacité de l'esprit pour le premier phénomène a été, ou peu s'en faut, complètement épuisée et où le second, le phénomène produit par contraste successif, s'accorde d'autant mieux avec les nouvelles conditions d'existence qu'il diffère plus de l'autre. On croit que, ici encore, comme pour le contraste simultané, nous arons une série de cas plus ou moins marqués et que si les phénomènes peuvent ètre plus ou moins nets, cependant la tendance à déterminer un fait le contraste existe toujours, et résulte du fonctionnement normal de l'esprit. Elle se manifeste plus ou moins par l'aspect que prennent les phénomèues suivants. La tendance au contraste se traduit ainsi de mille et 
mille manières qui souvent passent inaperçues - par l'accueil plus cordial d'un ami gu'on n'a pas vu depuis longtemps, ou d'un indifferent qui est resté quelque temps malade chez lui sans voir personne, ou d'un compatriote inconnu qu'on a quelquefois plaisir à rencontrer en pays etranger. L'association par contraste successif que l'observation nous fait voir souvent dans la vie normale, dont les cas pathologiques nous montrent la déformation, parait donc bien, si l'on analyse suffisamment son mécanisme, être le résultat d'un mode général du fonctionnement de l'esprit. 


\section{RÉSUMÉ ET CONCLUSION.}

La loi du contraste est en somme un résultat nécessaire des deux grandes lois de l'association systématique et de l'inhibition systématisée dans les conditions du moins où elles s'exercent chez l'homme. Un système psychique quelconque qui s'établit, une nouvelle croyance i laquelle nous adhérons, un nouveau sentiment qui s'impose à nous, fendent par le seul fait de leur cxistence, à exclure de l'esprit, au moment actuel, tout ce qui ne peut pas s'accorder avec eux - c'est le fait général dont la loi d'inhibition est l'expression abstraite, - mais pour s'établir, l'état nouveau est obligé d'employer certains éléments des états qui lui sont complètement opposés si nous nous sentons portés à admettre la vérité de telle ou telle théorie scientifique ou philosophique, le darwinisme, l'évolutionnisme, l'existence de l'homme tertiaire, la réalité des atomes chimiques, il se produit en nous une systématisation particulière d'un certain nombre de données de l'expérience, d'idées acquises d'une manière ou de l'autre; mais d'un autre còté il tend ì se produire une désagrégation de certaines idées antérieures qui ne peuvent s'adapter aux nouvelles conditions d'existence qui leur sont faites, et auxquelles les nouvelles idées empruntent un grand nombre d'éléments pour les classer d'une manière différente. C'est par l'intermédiaire de ces éléments communs que les systèmes opposés paraissent s'éveiller - car les anciennes affinités de ces éléments ne peuvent guére disparaitre immédiatement, et les anciennes habitudes persistent encore quelque peu. Une fois ces éléments mis en jeu, la loi d'associa- 
tion systematique et les lois de la vie indépendante des systèmes psychiques nous expliquent le reste: l'éveil de l'ancien etat d'esprit et les cooldinations secondaires qui en résultent. L'association par contraste simultané est done essentiellement une association systématique déterminée par une activité sur laquelle s'exerce une inhibition imparfaite. - Comme dans les coordinations actuelles de notre vie, tout est généralement imparfait, la tendance au contraste simultané peut ctre legardé comme un fait général.

Le contraste successif est, comme nous l'avons dit, essentieltement de même nature. Ici encore nous retrouvons une association systématique précédée d'une inhibition imparfaite; la tendance comprimée réagit avec d'autant plus de vigueur qu'elle a été plus longtemps comprimée, et aussi qu'elle a pu préparer sa réaction, c'est-àddire qu'elle a été enrayée en quelque manière imparfaitement, - l'arrêt tout en étant très long peut-être et tres rigonreux quand la tendance cherche à se développer au-delà de certaines limites, - lui a permis cependant de commencer a s'organiser et de se développer jusqu'a un certain point. C'est au moins ce qui se produit souvent. Mais le contraste successif est marqué encore par le fait de l'arrêt exercé en retour par les tendances contrastantes. Qnoi qu'il en soit, le contraste successif ne se produit que lorsque l'inhibition exercée sur la tendance contrastante vient à être insuffisante pour en arrêter le développenent. La mème formule s'applique donc rigoul'eusement aux deux formes principales de comtraste que nous avons étudiées à part, mais qui, dans la réalité se confondent julus on moins et s'encherêtrent. C'est ainsi fut par le necanisme que nous avons étudié, l'associatimn par contraste, gràcs à la multiplicilé et à l'indépendance relative des systemes psychiques, mait du fonctionroment des deux grandes lois qui tout d'abord semblaient 
L'ASSOCIATION PAR CONTRASTE SUCCESSIF

devoir l'exclure : la loi de finalité et la loi d'inhibition. Nous avons eu, de plus, l'occasion de constater qu'elle prenait sa part, en bien des cas, it la systématisation géuérale de l'esprit. 


\section{LIVRE III.}

Les associations par contiguïté et ressemblance.

\section{$\S 1$.}

Il y a dans tout composé quelque chose qui ne peut se retrouver dans ses éléments, à savoir l'ordre même de ces éléments, leurs relations réciproques et les résultats généraux qui en dérivent. C'est la connaissance dé ces faits et de ces lois, résultant de la combinaison des éléments qui constitue véritablement la connaissance d'un composé. Elle n'achère pas cette connaissance mais elle lui est indispensable, et celle-ci n'existe pas sans elle. Je n'hésite pas a croire que l'homme qui aurait en un tas, sous les yeux, tous les matériaux d'une pendule, mais qui ne pourrait les reconstituer soit réellement, soit idéalement, connaitrait bien moins la pendule que celui qui en verrait simplement l'extérieur et qui saurait que sa fonction est de marquer les heures. La comnaissance des éléments, utile, et même nécessaire pour comprendre pleinement la nature du composé, pour en modifier rationnellement lemécanisme, jour en diriger d'une manière éclairéc le fonctionnement, doit toujours s'associer a la connaissance des relations de ces éléments, et de la loi particulière qui exprime la nature propre et specifique d'un objet en énonçant le résultat général des relations de ces éléments, la fin vers laquelle ils convergent tous.

beux écueils sont a eviter dans la construction d'une sciruce, l'empirisme ot la métaphysique : la métaphysiquo bon un sens donne, celui que lui attribuait Auguste 
Comte, car si l'on entend par métaphysique la recherche des principes généraux de la philosophie, la portée du mot change tout it fait), consiste essentiellement dans une explication vicieuse de l'harmonie des éléments, l'empirisme consistera essentiellement dans la suppression de cette harmonie. En biologie, par exemple, la métaphysique consistera à expliquer l'unité du corps humain et les relations des organes par une entité quelconque, àme, archée, principe vital, etc.; l'empirisme consisterait à ne tenir aucun compte de la loi d'ordre et de finalité à laquelle se conforment les phénomènes vitaux, à ramener les processus vitaux à des phénomènes physico-chimiques, et ceux-ci à des phénomènes mécaniques, et ì croire que l'on a ainsi trouvé tout ce qu'il y arait à ehereher. Sans doute, la réduction des phénomènes vitaux aux phénomènes physico-chimiques et de ceux-ci aux phénomènes mécaniques est désirable si elle est possible; mais alors même que l'on aurait réduit à une formule mathématique tous les phénomènes matériels dont la synthèse constitue la vie, il resterait encore à considérer l'ordre même de ces phénomènes, leurs relations réciproques et le résultat général de ces relations, et e'est lia proprement l'objet de la science biologique. - C'est en ce sens qu'il faut interprêter l'idée directrice de Claude Bernard. L'illustre physiologiste ne voyait pas d'autres éléments dans la vie que des phénomènes physico-chimiques, mais le groupement de ces phénomènes lui paraissait appartenir à un autre domaine que celui de la chimie, et malgré les critiques qu'une analyse minutieuse pourrait faire naitre à l'egard de quelques-unes de ses formules il a bien distingué la loi, l'idée directrice à la fois des phénomènes dont elle exprime l'arrangement et de la cause occulte dont l'utilité est nulle.

Si nous considérons l'autre science voisine de la psychologie, la science sociale, nous trourons des occasions 
de remarques semblables. A côté d'idées théologiques: la Providence, le droit divin des rois, etc., on trouve des idées métaphysiques : la société, considérée comme une sorte d'entité mystéricuse; le progrès, qui est devenu pour certains une manière de dogme métaphysique ou religieux; l'àme de la patrie, qui cst pricipalement une conception poétique, et qui, susceptible d'ailleurs d'une interprétation positive très acceptable, scmble bien être parfois conçue comme une sorte d'cntité vague et mal définic, et des idées empiriques, qui portent à méconnaitre les conditions générales de la vie des sociétés, à les considérer comme une sorte de juxtaposition d'éléments semblables par les qualités individuelles desquels l'ensemble paraît entièrement constitué.

Le premier écucil, la métaphysique, est peut-ềtre aujourd'hui plus facilement évité quand il se présente sous ses formes traditionnclles. Le second est peut-être actucllement plus dangereux. L'école spiritualiste u'a pas su toujours se garder du premier, et l'école expérimentale a fréquemment échoué sur le second. Le premier c'est en psychologie l'explication de la synthèse des phénomènes par l'àme et les facultés considécées comme des ètres métaphysiques; le second c'est la suppression de la synthese psychique et le fait de ne tenir aucun compte, dans l'étude de l'esprit, de ce qui est l'esprit mème. Une des causes des erreur's commises est, sans aucun doute, la prédominance trop grande de l'esprit d'analyse. La grande école de psychologie qui va le Inatley et IIume à Bain ct Stuart Mill, a montré avec le talent ou le génie de lanalyse un manque excessif de dispositions à la synthèse. M. Spenrer, qui continue à certains égards celte ecole, en a rajeuni les doctrines et les a en partic corrigées parce qu'il possédait à un degré éminent ce qui manquait à ses devanciers, le don de la synthese - encore est-il resté quelquefois lui-mème arrèté daus l'empirisme. 
Au reste, il ne faut pas voir dans la méthode analytique, la seule cause et surtout la cause logique de l'erreur commise. L'analyse, en effet, pourrait s'appliquer aussi bien it l'ordre des éléments d'un fait composé qu'à ces éléments eux-mêmes; mais en fait, et cela au reste n'est pas très malaise a comprendre, les philosophes, les psychologrues qui s'appliquent surtout à l'analyse, portent leur attention sur les éléments qu'ils dissocient plutôt que sur les relations synthétiques supérieures des phénomènes, - de plus ils prennent souvent un élément qui se présente à eux sans chercher à reconstituer le groupe dont il faisait partie. Les lois abstraites qu'ils dégagent proviennent plutôt de l'abstraction d'un caractère particulier de certains phénomènes considèrés isolément, de la comparaison des qualités secondaires de certains faits (comme la loi qui fait de toute idée la copie affaiblie d'une impression ${ }^{1}$,) ou encore de la considération des rapports d'un certain nombre de faits envisagés dans quelques-unes de leurs relations réciproques un peu superficielles, à l'exclusion des autres faits et des autres relations dont la considération serait nécessaire pour donner à la loi son vrai sens et permettre de comprendre la synthèse réelle des phénomènes (comme les lois de l'association par contiguïté et ressemblance), que de la synthèse abstraite qui réunit en une loi générale les relations réciproques et essentielles, - celles dont les autres ne paraissent que des formes particulières - d'un nombre indéfini de faits (comme la loi de l'évolution de M. Spencer), ou qui, en énonçant une loi particulière s'occupe de la mettre en harmonie avec les autres lois particulières ou avec les lois plus générales de manière à ce que le tout offre bien un système complet ou bien des éléments qui pourront, avec le progrès de la science; se coordonner en système.

1. Hume. Traité de la nature humaine. (Traduit en français par M. Pillon.) 
Les associationistes ont voulu ramener toutes les opérations de l'esprit à des associations par contiguïté et ressemblance. Nous examinerons tout à l'heure la portée réelle de ses lois, mais nous pourous roir déja qu’elles ne peurent en aucune maniere aroir la portée qu'on leur a attribuée. Si quelqu'un définissait une pendule en disant que c'est une association de verre, de marbre, d'acier et d'émail, unis suivant les lois de contiguïté de sorte que l'émail est toujours avec l'émail, le verre avec le verre, et les ressorts diver's toujours les uns avec les autres, en négligeant de nous dire que l'on se sert du tout pour mesurer le temps, j'imagine gue sa description paraitrait insuffisante et que si jamais nous n'avions vu de pendule nous aurions de la peine à nous faire une idée précise de cet objet. C'est un peu ce que l'on a fait en décomposant l'esprit en états forts et états faibles qui s'associent parce qu'ils se ressemblent ou parce qu'ils ont èté une fois ensemble selon le hasard des circonstances. Si nous démontons une pendule, nous avons des rouages et des fragments, nous n'avons plus une pendule, et si nous décomposons un esprit en idées et sensations nous n'avons plus un esprit. Si par exemple nous décomposons une croyance, et nous disons qu'une croyance est une association d'idées, nous n'arons plus une croyance et nous n'en avons pas ulle définition, mais nous avons des phénomènes dont la réunion, dans certaines conditions et selon une certaine loi, constitue une croyance: c'est cette loi qu'on détruit quand on décompose le phénomène et c'est cette loi qu'on néglige quand on ne voit dans le jeu de l'esprit que des associations par ressemblance et contiguïté. Expliquer psychologiquement un phénomène c'est le rattacher aux lois générales du fonctionnement de l'esprit, c'est-á-dire rechercher quelle est sa fonction dans la vie psychique et de quelle maniere cette fonction résulte du jeu de ses éléments - de mème 
qu'on étudie un organe à la fois en ramenant autant qule possible les propriètés de ses éléments aux propriétés générales de la matière vivante, en tàchant de compreridre comment les conditions particulières de leurs manifestations modifient ou compliquent ces propriétés, et quelle place tient l'organe en question dans la coordination générale de l'organisme. On expliquera par exemple un sentiment en montrant à quels organes, à quelles tendances générales il se rapporte, et dans quelles circonstances particulières de l'état de ces organes ou de ces tendances il se produit - et on expliquera un sentiment particulier chez une persounc détermince en le rapprochant des tendances dominantes qui constituent la persounalité au sein de laquelle il sc déreloppe, ct comment il est déterminé en ce cas par une combinaison particulière de certains éléments, combinaison qui est la caractéristique même de la personne. On ne peut rien expliquer en psychologic que par le mode de groupement des éléments qui constitue soit la finalité génèrale de l'esprit, soit le mode de finalitè particulière d'une personnalité.

Unc des causes de l'empirisme, c'est que l'on s'est hibituć à regarder les lois comme des choses purement subjectives et sans réalité. Les notions sur lc réel ont été profondément perverties par diverses influences, entre autres par l'habitude du concept de substance et l'on en est venu ì nier la réalité de ce qui ne possédait pas le caractère où l'on avait cru trouver la réalité par excellence. Il semble qu'il y ait eu ici un effet particulier de l'association par contraste. Ceux qui ont rejeté la réalité de la substance ne l'ont plus guère trouvée que dans les faits, identifiés parfois avec les perceptions, et ils ont tout ramené à ce qui leur scmblait la scule réalité possible c'est ainsi que la sensation transformée a tout expliqué et explique encore trop de choscs. Les métaphysiciens ne trouvant la réalité que dans des substances inaccessibles 
a l'expérience, les empiristes sensationnistes ou matérialistes ne l'ont trouvée que dans l'expérience sous sa forme la plus facile à constater. Les premiers n'ont pas vu que la substance est une hypothèse qui n'explique rien et ne sert a rien, les seconds n'ont pas vu qu'un fait sans loi n'est plus un fait, mais une fiction impossible à constater', et mème à conceroir ou à imaginer. Le néo-criticisme à peu prés seul a tenté de constituer un phénoménisme rationnel, mais la façon dont il comprend le phénomène et la Ioi, sa théorie de la connaissance ou sa logique générale, ne me parait pas suffisamment nette encore. Il me semble par exemple qu'elle n'est pas absolument la même chez M. Renouvier et chez M. Pillon, ni même peut-être en diver's endroits des écrits, si profonds et suggestifs d'ailleurs, de M. Renouvier. A mon sens, la réalité de la loi et celle des faits sont exactement les mêmes et j'accepte à la fois les deux formules: la loi estl'ordre abstrait des phénomènes, le phénomène est une rencontre de lois ${ }^{1}$. Il semble assez évident, d'ailleur's, que si les faits sont des composés de lois, la loi sera un abstrait des phénomènes. Elle est, a ce titre, partie des faits, et l'on doit remarquer fue, si la loi n'a pas d'existence en dchors des faits, le fait n'a pas non plus dexistence en dehor's de la loi. Nous ne connaissons pas plus de loi sans faits que de faits sans loi : ct si, par abstraction, nous retirons une loi d'un fait ou d'un groupe de faits - ce qui revient au mème, car nous ne connaissons pas de phénomène absolument simIle, - le fait n’existe plus en réalité, pas plus que la loi J'existe réellement sans lui. En réalité, les lois ne sont pas, par elles.mêmes, des abstractions isolées, il ne laut pas confondre une loi avec la formule que nous nous

1. On peut voir a ce sujet mes deux notes sur $L$ a realite des rapports et Le rapport de ressemblance dans la Critique philosophique, avril et juin 1885. 
en faisons, la formule est une représentation de la loi, elle est un composé de mots et d'éléments abstraits, mitis si l'idée d'mue loi est une pure abstraction la loi elle-míme existe dans les faits concrets. Du moment que des blements existent et forment un tout, le rapport de ces éléments est aussi réel que l'existence du fait composé qui implique essentiellement l'existence de ce rapport. Un fait en somme est esscntiellement une loi d'autres faits. L'existence d'une locomotive est un fait, et c'est la loi mème selon laquelle sont assemblées les diverses parties de la locomotive - supprimez l'arrangement, les rapports, la loi, le fait disparait, la machine n'existe plus, il n'en reste que les éléments; prenez un de ces éléments : un sifflet, un régulateur, une roue - supprimez ercore la loi, l'ordre des phénomènes, le fait disparait, vous n'avez plus que des éléments ou des morceaux - et ainsi de suite. Un fait quelconque est un résultat des rapports de ses éléments et n'existe que par leur réalité. La nature du fait et de la loi ne peù d'ailleurs ètre examinée à fond qu'avec plus de développements qu'il ne m'est possible d'en donner ici à ce sujet, et nous serions facilement amenés à aborder le problème de la connaissance. Je m'abstiens de m'engager dans cette voie et je me contente d'avoir indiqué ce.qui me paraissait se rapporter à notre sujet actuel : la critique des théories empiriques, et l'examen des lois de l'esprit.

Les psychologues associationistes ont tàché de montrer que tous les actes de l'esprit se ramènent à des associations par contiguîté et ressemblance. Je crains un peu que le verbe « se ramener " n'ait causé ou fixé quelques erreurs, - en un sens oll ne ramene jamais un fait a un autre fait, une loi a une autre loi, on peut seulement découvrir entre les deux des rapports de finalite ou de causalité, de sỹnthèse on d'analỵse - croire que l'esprit 
se ramène à des associations par contiguïté et ressemblance, ce serait admettre que l'esprit n'est que de telles associations ; c'est croire que ces formes de groupement sont les formes les plus hautes et les plus èlevẻes, comme les plus inféricures de la vie psychique. Mais tout ce que nous avons ru jusqu'ici nous autorise à croire que la théorie ainsi comprise n'est pas juste, les principes généraux de l'esprit dont l'expérience nous a montré la généralité ne sont pas ccux qu'a étudiès l'associationisme anglais. Nous avons vu que la grande loi de l'esprit c'est la finalité, l'association systématique, se présentant it nous sous diverses formes que j'ai tàché de spécifier. Nous arons ici a étudier les lois de l'école anglaise, et ce sera une nouvelle occasion de vérifier la valeur des principes généraux exposés dans le cours de ce travail. En cxaminant la thérie associationiste nous pourrons voir si les lois de contiguïté et de ressemblance sont de véritables lois, et si elles peuvent nous rendre compte réellement des fonctions de l'esprit ou bien si clles ne seraient pas entierement subordonnées à des lois synthétiques plus hautes, et si elles ne seraient pas mème des appa-rences illusoires plutôt que des lois réelles.

$\S 2$.

C'est la physiologic et la sociologie rui nous fournissent la loi de finalité, d'association systématique dont l'application à la psychologie parait indispensable. De nos jours, en efiet, la ljologie et la psychologic d'un côté, la psychologro et la sociologie de l'autre deviennent inséparables. Or', l'école associationiste a beatucoup trop négligé les phénomenes pluysiologiques et n’a pas liré des phénomenes sociaux tout re qu'elle aurait pu en tirer. On Jien les peyolologues qui, comme Bain, ont accorlé dans lents ouvrages nue flace importante a l'otude des phénomines Jhysiologirfues, ont manque de l'esprit de syuthese et se 
sont trop bornés à des descriptions ou à des analyses, it des rapprochements intéressants, utiles souvent mais qui ne se rapportaicnt pas suffisamment au probleme fondarmental de la psychologie qu'ils avaient cependant l’intention de traiter. En fait une conception d'ensemble de la psychologie et de ses rapports avec la biologie et la science sociale suffit, alor's même qu'on n'entre pas dans le détail, pour faire abandonner ou tout au moins modifier considérablement les théories acceptées trop généralement dans l'école expérimentale.

La psychologie comprend l'étude des impressions faites sur un individu vivant par son milieu, et les réactions de l'être rivant sur le milieu - toutes les combinaisons internes qui influent à quelque degré sur le mode de réception et sur le mode de réaction, sont de son domaine. Nous reviendrons plus loin sur ce point, en tàchant de synthétiser les faits et les lois qui précèdent en une conception générale et positive de l'esprit. Tous les phénomènes psychiques apparaissent ainsi comme des signes de tendances, de divers processus qui s'accomplissent dans l'organisme et qui constituent la réaction de l'individu; ce sont essentiellement ces processus, ces tendances et leurs groupements divers qui sont l'objet de la psychologie; les phénomènes subjectifs ne nous intéressent que parce qu'ils sont produits par eux et qu'ils nous les montrent à l'œurre, un fait psychique quelconque, ne prend un sens, une signification véritable, que lor'sque nous avons pu déterminer son rôle dans la réaction de l'individu.

Il paraît évident tout d'abord, que les lois de ressemblance et de contiguïté ne peuvent, à ce point de vue, servir à exprimer synthétiquement l'activité mentale. Elles ne nous donnent nullement la principale forme des actions réceptives et des réactions de l'esprit ; elles ne nous apprennent alssolument rien sur la principale fonction de 
l'esprit : la coordination des impressions et des actes. Elles font abstraction du raractère principal de notre activité, de celui sans lequel l'esprit ne serait pas l'esprit : la finalite. C'est cette coordination, rette synthese joschique, qu'il faut constater d'abord ct mettre arant tout en lumière. Alors même que l'on retrouverait toujours dans tous les actes de l'esprit, des applications de la loi de ressemblance et de la loi de contiguïté, ce qui d'ailleurs ne sc vérifie pas, il faudrait encore constater que ces deux lois elles-mêmes ne se manifestent qu'en se soumettant à une loi supérieure, la loi d'association systématique, sans laquelle l'esprit ne subsisterait plus et qui cxprime seule la forme essentielle de l'activité mentale, considéréc au point de vue synthétique de la psychologie générale.

Dira-t-on que les lois de l'école associationiste n'ont en pour but que d'expliquer la coordination de l'esprit en montrant le mode selon lequel cette coordination s'effectuc? Il y a lieu, en effet, d'examiner directement et par les faits, le rôle de la contiguïté et de la ressemblance, et c'est ce que nous allons faire, mais il n'en est jas moins vaj que, an lien de chercher at expliquer par la contiguïté et la ressemblance la finalité de l'esprit, les psychologues de l'école empirique ont eu tris souvent une tendance it remplacer par leurs nouveaux principes, toutes les lois supérieures, et il leur semblait, uon pas jue les associations par contigüté et ressemblance etairut pour ainsi dire la matiere d'une forme superienre, mais qudestestaent cette forme mème el certainement ils (1)t cru, fuand ils youl pensí: - et ils n'y ont pensé la Jlupalt du temps, gue d'une maniere assez vague, - que leur associationisue arait pour effet de dobarasser la lsylulogie de tont principe superieur - une loi plus synthetipue atyot pour enx $n 11$ aspect ruelque pen métaphy. sique - on thioologique peut-êtro. 
Aussi, le point de vue synthétique a été complètement négligé par eux. Parfois mème, on dirait qu'ils n'ont pras seulement aperçu le problème. Il leur est arrivé, quand ils voulaient se faire une idée de l'esprit, de n'en voir que les éléments, la matière, si je puis dire, les impressions, les idées, sans prendre garde à la loi générale qui unit ces idées et ces impressions et sans s'apercevoir que, si ces impressions et ces idées n'étaient pas unies dans un ensemble, dont la forme est précisément ce qui nous importe, elles ne constitueraient pas plus un esprit qu'une carrière de pierre ne constitue un monument. On n'a plus aucun doute sur ce point en examinant les définitions qu'ils ont donnée. L'esprit, a dit Hume, est un faisceau de perceptions; et Bain : "pour définir l'esprit, il faudrait enfermer dans quelques mots, par une généralisation convenable, toute la famille des faits mentaux, et en exclure tout ce qui a un caractère étranger, " et cette phrase, susceptible d'ailleurs d'une interprétation correcte, fait cependant pressentir une facon de ne pas comprendre la question qui aboutit à faire admettre que l'esprit est " l'opposé de la matiére, ou pour mieux dire de ce qu'on appelle le monde extérieur' $»$. La définition de Hume équivaut à dire que le Parthénon est un tas de picrres, celle de Bain, à expliquer la nature d'un tableau, en affirmant que c'est le contraire d'une symphonie.

M. Spencer a évité l'écueil de l'empirisme, en dẻfinissant l'esprit par l'adaptation des relations internes aux relations externes; sa conception générale, critiquable à certains égards, est, en tout cas, très supérieure à celles que nous venons de voir - de mème, les physiologistes ou les pathologistes psychologues, en Angleterre, MM. Naudsley, Ch. Bastian, Ferrier, etc., ont su se garder d'un usage exagéré des lois de l'association. L'école expérimentale française s'est égralement micux préservée que l'école anglaise des dangers de l'empirisme, au moins 
depuis Cabanis. On trouve, par exemple, dans les travaux de MM. Taine, Ribot, Espinas, un souci constant des lois générales de coordination, et l'appel aux lois de contiguïté et de ressemblance est bien moins fréquent chez eux que chez les philosophes anglais, j'en dirai autant des philosophes italiens, par exemple, Mr. Lombreso, Caporali, Sergi et Regalia.

Nous allons voir tout à l'heure l'insuffisance des lois de ressemblance et de contiguilté à rendre compte des phénomènes psychiques considérés en cux-mêmes, mais nous royons déjà que la conception générale de l'esprit que l'on a fondé sur clles est manifestement insuffisante - et l'on peut multiplier les objections. Rappelons simplement l'activité inconsciente de l'esprit et la difficulté qu'il y a à en rendre compte au moyen des lois de l'associationisme anglais. On est forcé, logiquement, par l'étude de l'habitude et de l'instinct, d'appliquer aux actes inconscients les lois de l'association. Mais pour l'associationisme, les phénomenes inconscients ne peuvent guère être que des phénomènes phỹsiologiques, et nous nous trouvons alors en présence de nouvelles difficultés analogues aux précédentes - jas plus dans l'ordre biologique que dans le domaine de la psychologie, les lois de contiguïté et de ressemlilance ne sont capables de nous donner les véritables raisons, les modes essentiels de l'enchaînement des phénomènes.

$\S 3$.

En abordant l'étude directe des lois de contiguïté et de ressemblance et de leur's manifestations dans les fhenomenes psychiques, deux questions se posent: la frenicre est de savoir si ces lois peuvent, à elles scules, rxpliqguer les phénomènes de l'esprit ou s'il faut encore adhuthé l'existence d'une loi de systématisation, de finalité qui en règle l'application; la seconde est de savoir 
si ces lois sont de véritables principes secondaires de l'association des idées, et dans quels cas elles le sont. Il est hors de doute, par exemple, que, dans un raisonnement, nous joignons des idées qui se ressemblent en quelques points : la première question à se poser est celle-ci : n'y a-t-il dans le raisonnement que des associations par contiguïté et resscmblance, ou bien ces associations sont-elles déterminées par une autre loi selon laquelle, parmi toutes les associations par ressemblance possibles, quelques-unes seulement s'effectuent à un moment donné? La seconde question est celle-ci : les associations des phénomènes semblables s'effectuent-elles à cause de cette ressemblance, ou bien la finalité expliquet-elle tout et est-ce à cause de la finalité impliquée en certains cas par la ressemblance que l'association s'effectue? - De mème pour la contiguité.

On a pu voir que j'ai ramené le raisonnement à un certain mode de systématisation des éléments psychiques, en tâchant de préciser la nature de ce mode et la nature de ce groupement, l'examen que nous avons à faire à présent servira en quelque sorte de contre-épreuve à la théorie exposée plus haut. - Prenons un exemple de raisonnement concret. Un chasseur voit un perdreau passer devant lui en volant, à quarante pas. S'il est assez peu habitué à la chasse pour réfléchir et raisonner au lieu de tirer instinctivement, il se dira, (en abrégeant beaucoup), qu'il doit jeter le coup en avant de l'oiseau et cherchera à viser en conséquence. Il sait, en effet, que pendant le temps qu'il faut pour presser la détente et faire partir le coup, l'oiseau aura fait un certain chemin et que s'il visait juste le coup porterait derrière l'oiseau - il se produit en lui, trẻs rapidement, un certain nombre de perceptions et d'idées qui sc combinent, il y a un cas très net de raisonnement. On peut y trouver une application de la loi de similarité. En effet, le vol de l'oiseau 
est assimilé a tous les mouvements que le chasseur a pu observer et les mèmes propriètés lui sont attribuées, de même le chasseur a pu déjà tirer lui-mème on voir tirer des coups de fusil dans des conditions semblables, et a pu observer des phénomènes analogues à celıi qui fait la base de son raisonnement. Mais ce que la loi de similarité ne suffit pas a expliquer c'est ceci : comment se fait-il que ce soient ces associations-lá qui se produisent et pas d'autres? Au point de vue de la loi de ressemblance, l'oiseau en volant pourrait suggérer, soit par sa couleur, soit par sa forme, soit par les caractères particuliers de son vol, bien d'autres associations qui n'auraient nullement convenu dans les circonstances supposées. Et voilà précisément pourquoi elles ne se sont pas présentées ce n'est pas la ressemblance, c'est la convenance qui est la cause directrice, le pourquoi de l'association. Parmi toutes les impressions que la ressemblance aurait pu susciter, parmi tous les états de conscience qu'elle aurait pu faire naître, ceux-la seuls se sont produits qui pouvaient s'harmoniser avec les inpressions dominantes au mème moment. Il y a ici une sélection opérée par l'appareil psycho-plysiologique, une association systématique et une inhibition que la ressemblance ne suffit pas a expliquer, et la cause du phénomène se trouve, non pas dans une association déterminée selon la loi de similarité, mais dans l'organisation acquise du système nerveux et de l'esprit.

On pourrait multiplier les exemples - j'en choisis un vulgaire et déja cmployé : je veux aller prendre le train, j'ai deux kilometres à faire à pied, je regarde ma montre ct je vois que le train partira dans cinc minutes - j'en conclus que je ne puis pas partir. Il y a encore ici des associations aIparentes par similarite; de nombreuses experiences ont pu m'apprendre le temps (ju'il faut pour parcourir a pied le chomin qu'al me faut faire et qu'il $\mathrm{y}$ a 
des chances pour que les trains de chemin de fer partent à l'heure indiquée. Mais pourquoi toutes ces idées s'éreillent-elles en moi à la vue de ma montre? Tout aussi bien la blancheur de l'émail du cadran aurait pu me faire penser à la neige ou au lis : ou la rue des aiguilles me rappeler celle des horloges que j'ai pu voir, ou des objets plus ou moins semblables, des aiguilles a coudre, des lances; ou encore ma montre me rappeler dautres montres. Aucune de ces associations ne se produit pourtant. C'est qu'il y a ici une orientation de l'esprit, un système directeur et une inhibition systématisée, c'est que je reux savoir si je puis partir et que toutes mes idées convergent vers une mème fin. Comme dans le cas précédent il n'y a pas, - ou il n'y a pas seulement - association par similarité, il y a finalité et systématisation.

De mème pour la contiguïté. Une sensation, une idée pourraient, au point de vue de la théorie associationiste réveiller par contiguïté un nombre incalculables d'idées différentes. Il est hors de doute, pourtant, qu'elles ne se réveillent pas toutes à la fois. Qu'est-ce qui détermine le choix ? Evidemment c'est l'état momentané ou durable de l'organisme et de l'esprit. Si je prends un livre dans ma bibliothèque pour le consulter, je ne pense pas pour cela à tous les volumes qui sont sur le même rayon - si j'ai égard à eux ce n'est qu'en tant que cela est nécessaire pour prendre celui dont j'ai besoin, - et si ce livre m'en rappelle un autre, ce sera sans doute un autre livre qui pourra me venir en aide en ce qu'il me fournira des renseignements utiles pour le sujet qui m'intéresse en ce moment, et non un livre qui se trouve à côté du premier ou bien qui lui ressemblera par le format et la couverture. Ici encore il y a systématisation plutôt qu'association par ressemblance ou contiguïté. Si le sourenir des livres qui sont à côté de celui-ci s'éveille en moi, c'est que j'ai d'autant plus de chances de trouver mon livre que je saurai 
un plus grand nombre de ses relations de voisinage. Et si l'on m'objecte que la ressemblance ou la contiguïté déterminent parfois la systématisation et le choix, je pense que nous aurons l'occasion d'examiner le cas tout a l'heure.

$\mathrm{Au}$ reste, les psychologues associationistes ont bien apcrcu ce fait que plusicurs séries d'associations sont généralement possibles (à un point de vuc abstrait) et que seule l'une d'elles se réalise. Bain, selon son habitude a bien décrit le phénomène, mais je pense qu'il n'en a pas tiré toutes les conséquences.

"Quand je regarde une montagne, dit-il, plusieurs séries se présentent à moi, dans lesquelles je peux m'engager à propos de l'objet qui est devant nies ycux. En vertu de la contiguité je peux penser aux autres montagnes de la chaine, aux plaines et aux villages situés audelà, à la composition minérale de la masse, a la flore, qui s'y étale, à sa structure géologique, aux évènements historiques qui s'y sont accomplis. En vertu de la similarité je peux penser aux montagnes que j'ai vues en d'autres pays, dans des tableaux ou dans les descriptions des poetes, aux formes géonétriques avec lesfuelles cette. montagne a de l'analogie, à des efrets artistiques équivalents. Toutes ces roies me sont ouvertes, mais il arrive que je m'engage dius l'une d'elles de préférence et cette préférence a un motif. Il sc pent que l'une des associations ait acquis par la répétition plus de force que les antres, pout-être suis-je labbitué à associer ensemble la montagrue ct le village voisin, et conduit comme par une transition naturelle is suivre cette association particulière. Nous trourons une autre cause dans la présence d'un second lien d'association. Si je rois la montagne attenante, je penx m'engager dans l'association qui me fait pareourir toute la chaine. Si j'ajerçois le reflet des cascades, j'aj un 
double lien de contiguïté qui porte mon esprit à la riviere qui coule du flanc de la montagne, etc.

„Je supposerai un autre cas. Une violente temucte a fait dẻborder les rivières, arraché les arbres, renversé les maisons et jeté partout la terreur. Les séries de pensées que suggère cluacun de ces incidents sont extrèmement variées et dépendent de la disposition mentale de l'observateur à d'autres points de rue, ou des idées spéciales qui se rencontrent arec le caractère qui leur est commun a toutes. La femme du marin pense à son mari qui est sur mer. Le marchand et l'assureur ont aussi leurs pensées attachées sur cet élément. Le fermier calcule la perte de ses récoltes; le meunier entreroit l'augmentation de la puissance motrice du canal qui fait tourner son moulin. Le météorologiste ètudie la direction, la durée, la force de l'ouragan et les compare arec les cas qu'il a déjà obserrẻs. Le poète contemple des effets grandioses. L'homme religieux élère ses pensées à Dieu.

" Ces exemples supposent l'existence d'une attitude habituelle de l'esprit, une émotion, une occupation, un plan toujours prêt á servir de point de départ àu mouvement de l'esprit, et se combinant arec toute stimulation qui teud à mettre en jeu les séries de l'esprit de façon à constituer un élément de l'effet composé. Les cas où la seconde association n'est présente qu'accidentellement sont régis par le mème principe " ${ }^{1}$. Si je ne me trompe, le dernier exemple donné par Bain est très propré à montrer qu'il y a dans l'esprit autre chose que les lois de ressemblance et de contiguïté ; on $\mathrm{y}$ voit que le phénomène perçu éveille en clacun de nous des séries et des complexus particuliers de phẻnomènes selon l'organisation propre de l'esprit qui le reçoit ; et c'est cette organisation que ne nous donnent ici ni la loi de contiguïté ni

1. Bain. Les sens et l'intelligence, p. 517 et 519. Trud. Cazelles. 
la loi de ressemblance, c'est cette organisation qui dirige le développement des phénomènes, c'est elle qu'il faut étudier. Il y a là un système d'habitudes qui accueille tel état de conscience, lui permet de se développer et repousse tel autre ou l'arrète dans son développement, d'après une loi bien évidente de finalité intcrne. Le fait remarquable dans le cas cité par Bain, e'est que c'est partout la tendance à l'unité qui domine, à l'unité de fin, à la systématisation des états de conscience et des tendances inconscientes ou semi-conscientes, c'est cette systématisation qui constitue ce que nous appelons une personnalité ou un caractère. Chacun a ses systèmes propres d'habitudes, ct c'est cette loi générale d'harmonie ou de finalité existant en chacun de nous sous des formes différentes qui détermine et règle le jeu de l'esprit. Le fermier calcule plutôt la perte de ses récoltes que l'augmentation de la force motrice du courant qui fait tourner le moulin parce que cette deruière considération n'a rien à faire dans sa vie, elle ne peut servir à diriger ses actes, elle serait inutile et n'a pas de raison d'ètre au point de vue de la finalité, ce qui revient à dire qu'elle ne saurait être chez lụi systématiquement associée à un certain nombre d'éléments moteurs. La psychologie du caractère serait faite le jour oì l'on aurait déterminé toutes les tendances, et toutes leurs associations possibles en vue de fins différentes.

Il n'est pas très utile sans doute de rappeler ici encore que cette finalité n'est pas absolue et complète - la vie des elements psychiques nous a permis de le constater souvent. On a dit jadis que la nature ne faisait rien en vain et c'était une absurdité, dire que l'esprit ne fait rien en vain serait moins faux, mais serait faux encore, parce gu'il n'y a pas de personnalité parfaite et que nous sommes tous incompletenent unifies mais la finalité n'en e-t jas moins comme nous l'avons vu, une toi générale de 
l'esprit et des phénomènes psychiques. Tout ce qui est esprit est harmonie, mais les harmonies particulières de deux esprits ou de deux éléments psychiques qui sont en réalité assez semblables ả deux esprits, peuvent ne pas se fondre dans une harmonie générale; un accord parfait en mi est trẻs harmonieux, un accord parfait en fa l'est aussi, mais si les deux se font entendre à la fois l'ensemble ne le sera nullement - et l'esprit est un orchestre où les musiciens ne s'accordent pas toujours, où le chef, quand il existe, n'est pas toujours suivi. La raison de l'harmonie psychique ne se troure nullement dans des formes d'association par contiguïté et ressemblance, elle se troure dans ce simple fait que l'esprit est l'expression d'un organisme et que tout organisme suppose une coordination, un fonctionnement harmonique de ses diverses parties. Si l'on roulait expliquer cette finalité, ¿̇ supposer que cela soit possible, ce n'est pas dans la ressemblance et la contiguïte que l'on pourrait trourer une explication, ce serait plutôt dans les propriétés de la matière qui auraient pernis la génération spontanée ou la formation lente d'un premier organisme.

Ce qui précède tend a faire admettre, je pense, que les lois de la contiguïté et de la ressemblance ne peurent rendre compte de l'organisation psychique; à supposer même que cette organisation ne se manifestait que par des associations de faits contigus ou semblables, elle se distinguerait encore des autres lois et devrait ètre examinèe à part, car elle seule permet d'exprimer à un point de vue synthétique l'ensemble des lois de l'esprit. Mais il ne semble pas que la loi de systèmatisation se borne à régler le fonctionnement de ces lois, elle parait aussi déterminer sans leur intervention la production de certains phénomènes, et même agrir quelquefois en un sens absolument opposé.Le mécanisme mental qui détermine un écrirain à ne pas répéter trop sourent le mème mot à des 
intervalles rapprochés pour exprimer une même chose me semble ètre un cas de ce fait général. Ici la contiguïté et la similarité paraissent agir dans le même sens pour faire répéter le mot. Je ne rois que le désir d'écrire avec une certaine élégance qui puisse s'opposer à ce qu'elles réussissent. Sans doute on peut dire que l'exemple des écrivains qui ont déjà agi de la même manière peut, par similarité, exercer une influence sur celui rui tient la plume, mais je ne vois pas pourquoi cette influence ne serait pas compensée par l'exemple de ceux qui écrivent mal si ce n'était que cette dernière influence est annuléc ou diminuée par le désir de bien écrire. L'écrivain tàcle d'arriver à faire une curre qui le satisfasse, et les phénomènes psychiques s'arrangeut dans un ordre déterminé par cette cause finale en dépit de la similarité et de la contiguïté. Et ces lois ne sauraient d'ailleurs expliquer cette cause, ou la cause qui l'a produite elle-même - nous serions ainsi amenés a remonter de cause en cause jusqu'au germe et de lá jusqu'au premier organisme dont nous ne savons rien. Nous retombons d'ailleur's dans un cas précédemment indiqué, et c'est encore l'organisation physique que nous trourons comme base et comme cause de l'organisation psychique. Il serait sans doute inutile de dire en faveur de l'associationisme que les differentes associations ont ceci de commun que ce sont des associations par finalité, et que c'est cette ressemblance qui expliquerait leur mécanisme. Une pareille interprétation, plus subtile que solide, se heurterait contre les objections générales exposées déja, ou bien elle ne pourrait signifier autre close que ceci : la finalité est la loi générale de groupement des phénomènes psychiques.

Que la loi de finalité soit d'autant plus apparente dans les phénomènes psychiques que ces phénomènes sont d'ordre plus élevé, cela n'a rien de surprenant, et c'est une vérité dont on se rend facilement compte, si l'on 
songe que la supériorité d'un phénomène ou d'un groupe de phénomènes se mesure précisément à son degré de systématisation. Aussi est-ce dans les formes les plus élevées des fonctions mentales que les adversaires de l'associationisme ont généralement puisé leurs arguments. En revanche les formes mal coordonnées, les rèves, la folie, la rêverie, les rapprochements disparates ont fourni les plus beaux cas de "l'association des idées ». Mais cu réservant ces phénomènes pour examiner tout à l'heure l'explication que les psychologues associationistes en ont donnée, nous devons dire quelques mots des formes mentales les moins complexes, mais qui fonctionnent régulièrement. Leur harmonie, si elle est plus imparfaite, comme nous avons eu plusieurs fois déjà l'occasion de le voir, en ce qu'elle résulte du consensus d'un moins grand nombre d'èléments est aussi moins imparfaite souvent en ce que ces éléments sont plus étroitement unis et nieux coordonnés au point de vue de leur fonctionnement.

Cette harmonie éclate dans les réflexes composès, dans les instincts; mais pour rester à présent sur le domaine de la vie consciente, remarquons que les phénomènes moins complexes qu'un raisonnement ou qu'une volition réfléchie, la perception, l'imagination, témoignent aussi d'une certaine harmonie qui ne parait pas réductible a l'association par contiguïté et ressemblance. C'est ce qui ressort clairement des théories d'après lesquelles toute sensation, toute perception, toute image est le résultat d'une sorte de raisonnement inconscient. L'esprit, a-t-on dit, est une chose qui raisonne et le mot n'a que le tort de trop généraliser un phénomène particulier - ce qui est vrai c'est que le raisonnement et toutes les autres opérations mentales ont ceci de commun qu'elles sont une coordination d'éléments unis de façon à pouvoir converger vers une mème fin. M. Binet, qui a très bien analysé le raisonnement dans les perceptions, a ainsi ingénieusement 
rapproché diverses formes de la vie psychique ${ }^{1}$. Mais une fois que l'on reconnait cette complication et cette coordination inféricure des phènomènes les plus simples en apparence, il semble bien que le rôle de l'association par contiguïté et ressemlılance paraisse de moins en moins important. Dans la pereeption d'un mot, par exemple, nous avons un complexus d'images nouvelles, auditives, motrices phonétiques et motrices grapliqques; comment ces diverses images se sont-elles réunies? Ce n'est probablement pas à cause de leur ressemblance. Dirons-nous que c'est par contigüté? Ce serait done parce que l'on cntend un mot quand on le prononce que l'image auditive serait associèe à l'image motrice de l'articulation. Mais pourquoi l'image visuelle s'associeraitelle à l'image motrice? Sans doute à cause de l'habitude de lire a haute roix. Il me paraît manifeste que raisonner ainsi serait prendre l'effet pour la cause. L'image visuelle ue peut s'être associée à limage motrice par la lecture que si la lecture a été possibie. Or, ce qui rend la lecture possible c'est justement cette association de l'image visuclle et de l'image motrice - il est donc inadmissible qu'elle serve à l'expliquer. Et si l'on fait intervenir comme intermediaire l'image auditive qui s'associe d'une part a l'inage visuelle, d'autre part à l'image motrice, je demanderai comment l'image motrice a pu s'associer avec l'image auditive? Quand on prononce un mot devant un enfant pour qu'il le répète et qu'il associe désormais telle image motrice et telle image auditive, on suppose évidemment que cette association est virtuellement faite; car sans les lapports anatomo-physiologiques de l'appareil moteur et de l'appareil auditif, sans la systematisation prétablie des différentscentres psychiques, aucune association entre Je son et le mot prononcé lie pourrait s'établir. Ici encore, 
ce qui dirige l'association par contiguïte, ce qui mème lui donne naissance, c'est l'organisation acquise, la systématisation étalulie qui permet aux différentes parties de l'orgauisme et du cerveau de s'associer pour nue fin commune. Il ne servirait à rien de pretendre que l'enfant apprend i parler en voyant parler et en entendant parler, et qu'il associe par contiguïté I'image visuelle d'une bouche qui s'ouvre arec le son émis, car pourquoi l'image visuelle d'une bouche qui s'ourre, la perception d'un son particulier, le pousseraient-ils à ourrir sa bouche à lui, $a$ émettre un son semblable sinon en vertu de cette propriété des éléments psychiques, la finalité, que nous arons étudiée déjà. L'image d'une bouche ourerte tend naturcllement à susciter des éléments psychiques qui puissent s'associer avec elle, la rendre plus forte et crèer autour un système d'éléments qui le soutiennent, ces éléments sont évidemment les représentations motrices qui détermineront l'ouverture de la bouche; de mème pour le son. Limitation est une forme primitire de la systématisation psychique. Je montrerais, si c'était le lieu, comment c'est une tendance naturelle, comment elle se manifeste d'une manicre fort apparente dans les formes inférieures de la vie mentale, et comment elle disparait peu à peu sous les rectifications et des inhibitions nombreuses rue le déreloppement de l'esprit lui fait subir, comment les cas anormaux et pathologiques en montrent le retour d'une manière bien curieuse. Et si nous examinions en détail la manière dont l'enfant apprend à parler, la manière dont il apprend a lire, nous rerrions comment cette tendance à l'imitation, qui ne se relie pas á l'association et à la contiguïté, est utilisée au profit de la systématisation croissante de l'esprit qui finit par l'enrayer presque complètement.

Il ne saurait ètre question ici de reprendre en détail l'examen de toutes les classes de phénomènes psychiques, 
j'indique surtont le sens général des objections qu'on peut faire, a propos de chaque fait particulier, a l'explication qu'on tenterait d'en donner all moyen des lois de conti. guïté et de ressemblance. Ce que j'ai dit dans les chapitres précédents suffit pour indiquer comment cette forme générale des objections s'appliquerait i chaque cas particulier. En somme, dans ses formes les plus hautes comme dans ses formes les moins élevées, la coordination psychique reste irréductible aux lois de l'association. Indiquons, pour terminer cette revue très rapide, un domaine très vaste de l'esprit, celui de l'activitè non pas inconsciente, mais instinctive que nous venons d'aborder déjà et où la similarité et la contiguïté ne peuvent rien expliquer. Si l'on songe que presque toute notre activité mentale se ramène à cette activité instinctive, à des réflexes psychiques, comme les a appelés M. Richet, ou à des fragments de réflexes psychiques, si l'on songe aussi que les actes qui ne s'y ramènent pas rentrent soit dans le domaine de la rolition, de la réflexion, du pouvoir personnel où la force des associations par contiguïlé et ressemblance est, sinon davantage, au moins plus visiblement dirigée par la -finalité, soit dans le domaine de l'instinct où la sýstématisation éclate avec une si grande évidence, il apparaitra, semlule-t-il, que les lois de contiguïté et de ressemblance ne sauraient en aucun cas être prises pour les véritables principes directeurs de l'esprit.

Quand une idée suscite un acte, où est l'influence de la contiguïté? Oì est celle de la similarité? L'associationisne ue peut faire à cette fuestion qu'une seule réponse: l’acte a précedé l'idéc de l'acte, l’idée de l'acte est un blément de l'acte, elle est associée par contiguïté aux autres ćléments - la sensation motrice que me donne, le monvenuent de mon loras; a éte produite par ce mouvement mème, elle s'est produite avec lui, non atvec le mouvement de ma jambe - voila pourquoi 
clle tend à le rappeler. Mais il est facile de répondre qu'ici encole ce n'est pas la contiguïté qui agit, mais la coordination; l'idée de l'acte suscite l'acte non las parce qu'elle s'est produite cn mème temps que lui, mais bien parce qu'clle est un des éléments coordomés de l'acte, et que en tant qu'élément d'un système, elle tend à susciter le système enticr.

Que cette explication soit la bonne, c'est ce qui ressort avec évidence de ce qui précẻde et aussi de la considération d'un réflexe psychique dans son ensemble, et non plus seulement de l'un de ses éléments comme dans le cas qui précède. Les lois de la contiguïté et de la ressemblance sont totalement incapables d'exprimer réellement ce fait simple et indiscutable que l'impression de la faim et que l'impression de la soif, qui sont l'expression psychologique d'un état de l'estomac ou du sang, font naitre en nous un désir de manger et de boire. Pourquoi également le sentiment de suffocation que nous éprourons dans une salle trop remplie nous fait-elle désirer l'air frais du dehors et accomplir des mouvements coordonnés nécessaires pour aller en profiter? C'est un fait qui n'est nullement impliqué par l'associationisme des psychologues anglais. La formation de toutes les associations ne peut s'exprimer que par une loi de finalité plus ou moins complexe. Comment, par exemple, expliquer les associations chez l'enfant, par exemple, des cris et du besoin de manger? J'entends bien que l'on dira ceci : l'enfant sous la pression d'un besoin quelconque qu'on ne songe pas i satisfaire s'agite, remue ses bras et ses jambes, fait mouvoir aussi les muscles de son larynx, le tout un peu au hasard, c'est la manière dont une excitation qui ne peut aboutir a son but se dépense dans l'organisme. La faim et les cris sont ainsi réunis par contigüté et comme les cris attirent l'attention des parents et peuvent déterminer la satisfaction du besoin, l'association par contiguïté tend à 
devenir indissoluble. Et cette explication me paraît bonne en un point au moins; c'est qu'clle admet précisément l'intervention de cette "idee directrice " dont l'existence n'est nullement expliquée ni par la contiguïté ni par la ressemblance. Il y a évidemment conservation des habitudes utiles, e'est-à-dire coordination et sélection. De plus il n'est peut-être pas exact de dire en général que les deux faits tendent à se suivre désormais parec qu'ils auront été une première fois produits ensemble - mais nous reviendrons plus tard sur ce point. En tout cas, les associations par contiguïté, en supposant leur réalité, sont toujours sous la direction de l'influence coordinatrice de l'esprit. L'associationisme est mème hors d'état d'exprimer par des associations de contiguïté et de ressemblance le premier cri du nouveau-né. Partout il s'agit visiblement d'une coordination d'impressions et de mouvements, d'idées, d'images, etc., dont le résultat harmonique est la conservation de l'organisme et qui ne sont que l'expression de l'unité plus ou moins avancée de cet organisme. L'habitude, dit-on, est une application de la Ioi de contiguïté. Si l'on y réfléchit un peu on verra que l'on est conduit par cette manière de voir a des conclusions absolument inacceptables. D'aprés les associationistes, il y aurait chargue fois dans un acte lhabituel, une série de faits semblables amenés chaque fois par une liaison de contiguïlé parce que plusieurs fois déjat ces faits ont été répétés ensemble. On serait conduit par un raisonnement analogue à dire par exemple que lel élat de l'organisme anenc la sensation de soif parce qu'un elat semblable llejit produit la même sensation el ì prendre pour une application des lois de contiguïté et de ressemblance ce fait fue des circonstances analogues déterminent des juccssus jeychiques analogues aussi, ce qui est dejả suffisamnent indiqué gar l'hypothèse générale de l'uniformité des lois de la nature. Au fond il vaudrait autant ramener 
a des associations par ressemblance et contiguïté le mécanisme d'une machine à vapeur, - la chaleur qui chauffe l'eau de la chaudière ressemble à celle qui l'a chaufféc déjà, et les différents effets des diverses parties de la machine ayant été produits une fois l'un après l'autre, continuent à se suivre dans le même ordre. Je crois qu'on se ferait une meilleure idée de la machine en étudiant la fin générale vers laquelle convergent les fonctions de chaque partie, la manière dont chaque partie prend part à l'harmonie génẻrale et comment ce résultat dérive de l'arrangement des éléments. Je pense mème que personne ne trouverait un intérèt quelconque dans l'autre manière de grouper les phénomènes.

Nous avons eu à parler de la difficulté qu'avaient les lois de l'associationisme à s'appliquer à la biologie, et nous avons vu, pourtant que les nécessitès de la science exigent qu'elles s'y appliquent en certains cas, si elles sont justes. Nous pouvons nous avancer sur l'autre frontière de la psychologie et nous y trouverons des raisons analogues de ne pas croire à l'importance excessive des lois de la psychologie associationiste. Dans les relations sociales - si nous considérons la société comme une sorte d'individu - comme un "organisme de consciences 》 selon le mot de M. Espinas, nous trouvons aussi en elle, des associations, au moins apparentes, par ressemblance et contiguïté, mais elles ne paraissent pas jouer un rôle plus important que dans la psychologie. Nous pouvons trouver par exemple, un phénomène qui ressemble beaucoup aux associations par ressemblance dans ce fait que les personnes qui s'occupent d'une mème chose, les marchands qui vendent une même denrée, ont parfois une tendance $\dot{a}$ se rassembler et à établir leurs magasins dans un mème quartier. Nous voyons bien deux faits sociaux semblables s'attirer en quelque sorte, se grouper. Mais il est assez 
évident que ce groupement n'est qu'une forme de la coordination sociale - c'est parce que les besoins du commerce. le demandent, pour que les acheteurs sachent où les trouver, que les marchands se rassemblent. Les halles, les bourses, toutes les constructions consacrées ainsi à recevoir des personnes qui traitent des mêmes affaires, sont des expressions, non pas de l'association par ressemblance, mais de la sytématisation nécessaire à la vie sociale. De même, il ne faudrait pas voir l'effet ou la manifestation d'une loi de contiguïté, dans ce fait, que les soldats d'un régiment, quand ils sont réunis, marchent toujours dans le même ordre, tambours et clairons en tête. On peut multiplier les exemples à l'infini. Prenons par exemple la famille, il y a ici des contiguïtés bien évidentes, mais les familles ne restent unies que par les intérêts communs, les sentiments communs ou convergents qui en relient les membres, et c'est seulement en tant que la contiguïté peut déterminer la communauté des intérêts et des sentiments, - ce qui arrive, en effet, quelquefois, que'la contiguïté nous importe, en sociologie comme en psychologie. On peut en dire de mêne à propos de tout ce qui en sociologie peut rappeler la contiguïlé de la lisycliologie, les lycées, les régiments, les cercles, etc. 11 est inutile de dérelopper plus longuement ce point de vue, j'ai roulu seulement indiquer comment les enseignements de la sociologie ainsi que ceux de la biologie venaient confimer les resultats de l'investigation psycho$\operatorname{logi}$ (pue.

De ce qui précede, il ressort au moins ceei: que les associations far contiguïté et ressemblance sont sous la deproudance d'une organisation prétablie, qui ne leur pernę re sétablir qu'en tant qu'elles sont dirigées dans un rertain sens, gu'elles sont susceptibles de prendre part it telle ou telle opération mentale, de servir telle ou trolle rendance plus ou moins importante, c'est-it-dire 
qu'elles se conforment à une loi de finalité. Il y a cependant un certain domaine de la psychologie qu'on parait leur abandonner en général assez volontiers. Je veux parler des conceptions délirantes, des imaginations de la folie, des rêves, des rèveries même. Ici le lien logique de l'esprit semble s'ètre dissous, et la forme systématisée de l'enchainement des phénomènes, parait aroir entièrement disparu. On dirait par conséquent que les lois de ressemblance et de contiguïté peuvent ici, régner à leur aise, sans qu'aucun principe supérieur les domine et en règle les manifestations. Il me semble, qu'i examiner les choses de près, on voit qu'il n'en est pas ainsi. Ce que nous trouvons ce ne sont pas des lois, mais des éléments psychiques livrés à eux-mèmes et agissant selon leurs affinitès propres, comme nous l'arons déjà vu, en dehors de toute synthèse générale, mais non de toute loi de systématisation, puisque tout élément, est par luimème une synthèse qui tend à se complèter d'une manière ou d'une autre. Dans les cas qui restent à examiner, la finalité ne disparaît pas, ou plutôt elle ne disparait que de l'ensemble - les phénomènes qui se présentent alors tout en étanı eux-mêmes des synthèses d'éléments plus ou moins nombreux, ne se coordonnent plus entre eux, de manière à produire un tout unifié. Mais nous devons ne pas aborder encore cette question et, pour rester sur le terrain de l'activité mentale coordonnée, en un mot sur le terrain de l'esprit, voir si les associations par contiguïté et ressemblance, qui n'y ont pas une importance prépondérante, et qui, en tout cas, ne peuvent exprimer la nature réelle de l'esprit, y ont une importance quelconque et sont bien l'expression d'un mode de groupement de faits psychiques, et si ce mode estréductible ou non à quelque mode différent. 


\section{$\S 4$.}

La question se pose ainsi : la loi d'association systématique nous a paru absolument irréductible aux lois de similarité et de contiguïté, il s'agit de voir à présent si le premier principe ne serait pas le fondement des deux autres, si les lois de contiguïte et de similarité ne seraient pas des eas particuliers de l'association systématisée je veux dire que peut-être les associations par ressemblance ne s'effectuent que parce que la contiguïté ct la ressemblance sont quelquefois des auxiliaires de la finalité. Il ne peut être encore question ici de passer successivement en revue toutes les catégories de phénomènes psychiques, je me bornerai à quelques exemples, et cc qui précède permettra de faire les généralisations voulues.

Quand j'ai eu l'intention d'étudier la théorie associationiste, je me suis mis a relire des fragments de divers ouvrages où cette théorie était exposcee. On peut trouver ici les éléments d'une association par ressemblance, - un livre rappelant l'idée d'un autre livre oì est traité un sujet semblable - ; nous avons vu que la ressemblance ne suffit pas pour expliquer que l'association se produise, mais sert-clle a quelque chose par elle-même, ou l'association ne se produit-elle que parce que la ressemblance est quelquefois jointe it la finalité ? Lil ressemblance de deux phénomènes psychiques estelle une raison pour que ces phénomènes s'associent ? Y a-t-il une fiacilité plus grande it unir dans l'esprit deux phónomènes semblables, ou bien au contraire, la finalité est-elle la seule cause de l'association par ressemblance? Ici, par exemple, les divers ouvrages qui reviennent à l'esprit et qui se ressemblent, en ce qu'ils traitent d'un mòme sujet, me reviennent-ils à l'esprit à cause de cette resscmblance, on bien à cause de l'utilité que je peux en tirer et du rôle qu'ils doivent jouer dáns mon activité 
mentale? On peut se poser une question semblable a propos de la contiguïté.

Il y a,deux manières de l'envisager. Si nous nous placons au point de vue d'un esprit forme, nous trouverons que la contiguïté ou la ressemblance ne paraissent pas avoir dimportance, mais nous pouvons encore nous placer au point de vue d'un esprit en formation - il est bien entendu que par esprit formé, je n'entends pas un esprit tel que l'esprit actuel de l'homme lorsqu'il s'emploie à des opérations complexes et pour lesquelles il est encore mal préparé, mais bien un esprit qui a atteint le degré d'organisation le flus élevé possible, quelque chose d'analogue à ce que nous trouvons dans l'action réflexe composée, mais avec une complexité bien supérieure.

Nous devons rechercher à ce point de vue quelle est l'importance des associations par contiguïté et ressemblance dans les opérations psychiques bien faites. Cette importance d'abord paraît grande. Dans un système complet, en effet, les rapports des éléments doivenl toujours subsister et rester les mêmes. Une machine à vapeur, par exemple, doit toujours utiliser l'eau de la mème manière, de même un organisme, en présence de la même excitation, et les circonstances internes restant les mèmes, doit toujours agir d'une manière uniforme. C'est ce qui sc rérifie dans les actes réflexes, dans l'habitude et dans l'instinct. II n'y a là qu'une application du principe de l'uniformité des lois naturelles. Remarquons qu'il est d'ailleurs très important au point de vue de la conservation de l'individu, que ces associations par contiguïté - si elles en sont - des phénomènes qui composent un des systèmes psychiques de l'homme, se r'épètent indéfiniment. Quand l'homme a besoin de respirer, il est essentiel pour lui qu'il respire. Il faut que des situations semblables, en présence d'excitations semblables, amènent des actes semblajbles. De pareils actes se composent 
d'une série de phénomènes synthétisés successifs, et on peut trouver dans leur accomplissement une application des lois de similarité et de contiguïté. Si, par exemple, chez un vautour qui a faim, la vue d'un oiseau éveille toujours une série d'états psychiques contigus, qui se terminent par les actions nécessaires a la prise de l'oiseau, il est facile de voir que cet éveil de phénomènes contigus est indispensable a la vie de l'oiseau de proic. Nous avons reconnu déjà que la contiguïté était ici complètement subordonnée à la systématisation. Tàchons de préciser son office.

Je suppose que je jette aujourd'hui une pierre dans l'eau, elle s'y enfoncera comme elle eùt fait hier, et si je recommence demain, le résultat sera le même. Il ne faudra pas voir li dedans - et je crois d'ailleurs que la pensée n'en viendrait à personne, - l'effet d'une association par contiguïté ct ressemblance. Si les mêmes actes ont eu des conséquences analogues, si l'eau en recevant la pierre l'a laisscec pénétrer en elle el se déposer au fond, et a été agritée à sa surface de manière a faire rayonner à partir du point de chute une séric de cereles concentriques, il ne faut pas en conclure qu'il y a là un effet de contiguïté et que l'eau a associé les diver's mouvements qui s'étaient produits une première fois et les a reproduits dans le mème ordr’e, mais il est bien évident, au contraire, qu'il y a simplement un effet identique amené par des conditions identiques, les unes permanentes (la pesanteur specifirue de l'eau et de la pierre et leurs diverses proprićtes qui entrent en jeu dans ce phenonéne), les autres non permanentes (le jet de pierre), une apj licalion de la loi universelle du determinisme scientifique qui se manifeste dans tous les phénomènes et ne peut en caractériser aucum. C'est dans les propriétés physiques de l'eau et de la pierre qu'il faut chercher la raison. du fait.

De même sị l'on met un morceau de savon dans la main 
d'une hypnotisée dans l'état de catalepsie étudié a lit Salpètriere 1 , elle se met à se frictionner les mains comme si elle se les lavait, et si l'experience est renouvelè la mème série de pliénomènes se dèroule, il ne fiut pas voir là dedans un effet de la contiguïté, mais la mise en jeu des propriétés du système nerveux dans certaines conditions. Les plus générales de ces propriétés c'est de réagir par une activité systématisée, c'est-à-dire de faire entrer l'impression présente dans un système d'images, de sentiments, de tendances et d'actes présentant un caractère d'unité. Dans le cas présent c'cst la mise en jeu d'une habitude aequise, qui est devenue une sorte de propriété des centres nerveux, et qui consiste dans la coordination et l'accomplissement suecessif des mouvements nécessaires pour se laver les mains.

Il est permis de se demander si toutes les manifestations des lois de similarité et de contiguïté ne se ramènent pas à des faits de cette nature et par suite à des cas de finalité. Toici un fait qui aidera à comprendre ce que je veux dire: L'autre jour je lisais je ne sais plus quel passage d'un livre ou se trouvaient ces mots "à toute heure », et ces mots réveillèrent en moi quelques phrases de cliant d'un air de baryton de Rigoletto ou ils se trouvent aussi. Il est aisé de roir en ce cas, selon la mèthode associationiste, une association par ressemblance suivie d'une association par contiguïté. Les mots éerits réveillent par similarité le souvenir des mêmes mots chantés, et les mots chantés rappellent par contiguïté les mots qui les suivent dans l'air que j'entendis chanter en moi. Mais il est facile aussi d'interpreter autrement les faits, et nous trouvons dans cette seconde interprétation cet avantage qu'elle s'accorde avec les lois générales de l'esprit. Il est très probable que ce sont les mêmes éléments neuro-psychiques rui sont mis en jeu lorsque se

1. Voir en particulier Richer. Recherche sur l'Hystèro-cipilensie. 
présente l'expression "à toute heure », - ils sont dynamiquement associés avec un systéme de sons qui composent l'air dont il est question, et c'est une association systématique qui détermine le réveil des autres mots qui les accompagnent. Nous avons donc ici un cas de cette loi de fusion que M. Binet ${ }^{1}$ a si bien mise en relief, et de la loi de systématisation - et notre interprétation a sur la première l'avantage de prendre un sens au point de vue de la psychologie générale.

Nous pouvons faire un raisonnement analogue à propos de tous les faits qui sont présentés comme des formes de l'association par contiguïté et ressemblance; toujours nous trouvons un élément commun aux deux systèmes associés qui tend par conséquent à les éveiller tous les deux et par suite a les associer - et qui le fait parfois de manière a déterminer une coordination exacte d'une série psychique conformément à une orientation donnée de l'esprit, comme cela se produit dans les opérations supérieures, parfois aussi sans que les phénomènes suscités se coordonnent dans un système supérieur, et c'est le cas de l'activité indépendante des éléments psychiques telle que nous l'avons étudiée au début de ce tra-. vail. Dans les deux cas ni la similarité ni la contiguäté ne nous apparaissent comme de véritables principes d'association. Etudions plus spécialement a présent les formes inférieures de l'activité mentale, nous rerrons si la ressemblance et la contiguïté ne sont bien en réalité que des formes particulieres de l'association systématique. Il ne nous restera plus ensuite, si c'est bien la le résultat de notre recherche, ru'a tícher de rlélerminer la part de ces formes particulières de l'association systematique dans la vie de l'esprit.

1. Binet. Psychologie du raisonnement, p. 96 et suiv. 
$\S 5$.

C'est dans les formes inférieures de l'esprit, semble-til, que l'on a vu ou cru voir le plus clairement, en général l'influence des deux lois d'association que nous examinons ici. Dans les plus remarquables de ces faits, il faut compter cette faculté que certaines personnes possèdent à un haut degré, de retenir, en la lisant une fois, une série plus ou moins longue de mots qu'aucun sens ne rattache les uns aux autres - c'est d'ailleurs une faculté qui à un degré moins éleré se trouve chez tout le monde. Je citerai ensuite comme faits de même genre, à notr'c point de vue actuel, certains phénomènes mal coordonnés comme la rêverie, les caprices de l'imagination ou des phénomènes semi-morbides ou morbides: le rève et la folie. Pour fixer les idées par des faits concrets, je citerai les suivants : de simples assonances, dit M. Luys, s'appellent les unes les autres dans des cas de folie et se groupent automatiquement ensemble; une malade citée par Parchappe, faisait souvent des associations dans le genre de celle-ci : "On dit que la Vierge est folle, on parle de la lier, ce qui ne fait pas les affaires du département de l'Allier n. M. Maury raconte des rêves où le même phénomène est très visible. "Un matin, dit-il, je me rappelai que j'avais eu un rêve qui avait commencé par un pélerinage à Jérusalem ou à la Necque; je ne sais pas au juste si j'étais chrétien ou musulman. A la suite d'une foulc d'aventures que j'ai oubliées, je me trouvai rue Jacob, chez M. Pelletier, le chimiste, et dans une conversation que j'eus avec lui, il me donna une pelle de zinc qui fut mon grand chcral de bataille dans un rêve subséquent, plus fugace que les autres et que je n'ai pu me rappeler. Voilà trois idées, trois scènes principales, qui sont visiblement liées entr'elles par les mots pélerinage, Pelletier, 
pelle, c'est-à-dire par trois mots qui commencent de même, et s'étaient évidemment associés par l'assonance".

Enfin, j'ajoutcrai un dernier fait cité par Galton et dont j'emprunterai l'exposition au rolume de M. Binet sur la psychologie du raisonnement. «Un des correspondants de M. Galton, le Rév Georges Henslow, voit toutes les fois. qu'il ferme les yeux et qu'il attend un moment, l'image claire de quelque objet. Cet objet change de forme pendant que M. Henslow le regarde avec attention. En étudiant la série de formes qui se succèdent, on remarque que le passage de l'une à l'autre est fourni tantôt par la relation de contiguïté, tantôt par la relation de ressemblance. Dans une de ces expériences, les images suivantes, se présentèrent : un arc - une flèche - une personne tirant de l'arc et n'ayant que les mains visibles - un vol de flèches occupant entièrement le champ de la vision - des étoiles tombantes - de gros flocons de neige - une terre couverte d'un linceul de neige - un presbytère dont les murs et les toits étaient couverts de neige - une matinéc de printemps avec un brillant soleil et une corbeille de tulipes - disparition de toutes les tulipes a l'execption d'une seule - cette tulipe unique de simple devient doublc - ses pétales tomljent rapidement, il ne reste que le pistil - le pistil grossit, les stigmates se changent en trois cordes ramifiées et brunes - un bouton, le bouton se plie, devient une mèche anglaise, puis une sorte d'épingle traversant un morceau de métal, et ainsi de suite ".

M. Brochard a tenté, dans un article que j'ai déjả eu l'occasion de citer, de ramener' ${ }^{1}$ la loi de similarité à la loi de contiguïté. Son analyse, qui me paraît fort juste en ce qu'elle affirme contre la loi de similarité, concorde avec celle que M. Binet a faite dans son étude de la perception. "On doit concevoir, dit M. Binet, après avoir cité le fait

1. Brochard. La loi de similarite. Revue philosophique. 
qui précède, que chacune des images se fusionne avec la précédente en vertu des points communs qu'elles offrent, et, que de plus les images successives coexistent pendant un temps très court " ${ }^{1}$. Ce sụnt des remarques analogues mais interprétées d'une manière différente qui nous ont conduit à expliquer d'une manière particulière certains phénomènes psychiques et, en particulier, la vie indépendante des éléments psychiques.

C'est a ce dernier fait que paraissent se ramener ce qu'on a appelé des associations par ressemblance - nous retrouvons bien dans ces rapprochements de phénomènes des associations systématiques, mais ces associations systématiques ne sont pas coordonnées entre elles et ne se réunissent pas en un système supérieur. Cette manière de comprendre les phénomènes, paraît à la fois mieux daccord avec les résultats de la synthèse et avec ceux de l'analyse. Je vais tàcher de l'établir brièvement - le chapitre sur la vie des éléments psychiques permettant d'écarter plusieurs développements.

Prenons un des faits en question, celui par exemple du rêve, de M. Maury, qui s'explique par les associations des mots : pélerinage, Pelletier; pelle, - nous voyons que la partie commune de ces trois mots a subsisté dans l'esprit; mais, dans chaque partie du rêve, elle a appelé à elle des éléments différents, systématiquement groupés chaque fois - ces éléments étaient d'abord d'autres lettres, d'autres sons susceptibles de s'unir à elle pour former un mot conplet, puis des images, des idées diverses, logiquement reliées au mot suscité. Seulement, tous ces systèmes au lieu de se réunir entre eux et de se combiner dans une association plus yaste, ne peuvent s'acconmoder les uns aux autres, et restent à ce point de vue incohérents. Nous trouvons done dans ce fait : 10 une

1. Binet. Ouvrage cité, p. 106. 
association systématique forméc par le groupe de phénomènes divers qui vient s'agglutiner autour de la partie persistante du mot; $2^{\circ}$ une association non systématiséc de ces différents groupes. Il est facile de roir en tout cela un cas de l'activité relativement indépendante des ćléments psychiques, et l’interprétation ne parait pas douteuse - mais examinons-la particulièrement au point de vue de la loi de similarité : la première partic rentre éridemment dans les associations systématiques, et la ressemblance n'y jouc pas un rôle sensible; étudions la seconde, c'est-à-dire l'apparence de l'ensemble du plıćnomène.

La ressemblance en parcil cas n'est pas une explication suffisante : pèlerinage ressemble sans doute a Pelletier, mais d'abord l'analyse du phénomène nous a montré que cette ressemblance tient à ce qu'une partie commune aux deux mots a persisté et s'est systématiquement associée successivement des éléments différents - d'un autre côté si pèlerinage resscmble à Pelletier et à pelle, il ressemble aussi à pèlerine, pêle-mêle, etc. Dans la plupart des cas, aucunc explication ne peut être donnéc d'une manière bien positive pour ces successions incohérentes-. mais dans le cas oì clle peut l'ètre - ce n'est pas dans la resscmllance qu'il faut la trouver c'est dans la constitution actuelle de l'esprit telle fue l'ont faite les habitudes prises, la persistance ou la force d'une impression récente, etc. - ce sont encore des considérations de finalité, de systématisation qui interviennent. - Il est probable que si une autre personne que M. Maury avait fait un rêve analogue, le chimiste Pellelịer n'aurait pas figuré dans le rêve. On conçoit ru'il soit difficile de toujours préciser les circonstances permanentes ou fugaces qui déterminent l'éveil de telle idée plutôt que de telle autre, alor's que cet éveil n'est pas amené par une orientatior bien nette de l'esprit, il n'est guére douteux 
toutefois que les préoccupations habituelles, ou la vivacité d'une impression récente ne soient des facteurs importants du phénomène, c'est-à-dire.que nous retrourons, d'une manière générale, notre loi d'association systématique, car, ètant donné qu'un certain élément persiste dans l'esprit, les états de conscience qu'il éveille, sont précisément ceux qui dans chaque individu ont le plus de facilité pour se coordonner avec lui. Les faits où le mécanisme du phénomène peut ètre assez bien connu nous permettent d'arriver à des conclusions générales un peu vagues mais qui suffisent à rendre probable notre théoric.

Toutes les associations par ressemblance son' ainsi réductibles à des formes d'activité relativement indèpendante des éléments psychiques - on pourrait multiplier les analyses, je pense que ce serait inutile après l'étude que j'ai faite plus haut de ces formes incohérentes de l'activité mentale, et des causes qui favorisent ou contrarient l'activité des éléments psychiques - la forme générale de l'analyse étant donnée. Reste l'association par contiguïté, celle-ci ne paraît pas, tout d'abord, se ramener aussi aisément à des formes de finalité. Avec un peu d'attention il me semble pourtant qu'on peut y distinguer aussi le rôle de l'association systèmatique. Afin de trouver pour les cas les plus difficiles une explication plausible, commençons par les plus simples, et les plus caractéristiques.

Si la ressemblance et la contiguïté étaient les seuls liens connus de nos phénoménes psychiques, il est un grand nombre de faits bien connus qu'on ne pourrait expliquer. Tout le monde a pu remarquer que, quand une opération suivie était brusquement interrompue pendant quelques instants, il etait souvent assez difficile de reconstituer dans l'esprit la scène entière. Si je suis occupé à lire ou à écrire, et si quelqu'un m'interrompt pendant un temps suffisamment long, pour que mon attention se porte tout 
entière sur l'objet de l'interruption, il me sera assez diffcile de reconstituer la série des états psychiques contigus qui se seront produits en moi. Je me rappellerai bien que je lisais quand on m'a interrompu, mais, à moins d'un intérêt tout particulier, je ne me rappellerai pas très nettement les phénomènes psychiques qui ont immédiatement précédé l'interruption — ou bien si je reconstitue cette série d'idées, l'interruption qui ne s'y rattache pas logiquement mais qui est un phénomène contigu aux premiers, me sortira complètement ou à demi de la mémoire. Si enfin je parviens à reconstituer la scène entière, ce sera par une série de recherches, d'actes de volonté, c'est-à-dire d'associations systématisées. Ces phénomènes qui étaient joints dans le temps, ont été complètement séparés par la différence des orientations de l'esprit auxquelles ils ont pris part, il faut pour les réunir, les rattacher tous les deux à une même attitude mentale - par exemple, à une recherche psychologique. Normalement, il se produit une sorte de trou, ou plutôt de faille dans la mémoire. C'est une faille de cette nature qui se produit entre le rêve et le réveil, et nous en avons vu des exemples. On a remarqué que nous nous rappelons souvent très bien, au moment où nous nous réveillons, le rêve que nous venons de faire - nous sommes encore orientés dans le sens du rève - mais plus tard, le souvenir a disparu, parce que les habitudes mentales de la veille sont souvent sans relation suffisante de finalité avec le rêre pour en faire revenir l'image - étant donné que la coordination mentale est beaucoup plus vigoureuse quand nous sommes éveillés que quand nous dormons - et la contiguïté, fort réelle, cependant, ne peut ricn pour le faire reparaitre. Lorsque nous venons à nous ressouvenir d'un rêve, nous oublions bien souvent les phenomènes contigus à ceux du réveil. Si au lieu de s'attacher à des analogies souvent trop superficielles entre 
les divers états de la conscience, on avait recherché les causes profondes de l'oubli, la doctrine associationiste n'aurait pas, je crois, pu s'établir - elle ne paraìt guère pouvoir rendre compte des faits que je viens de citer, et, qui n'ont cerles pas passé inaperçus des psychologues de l'école expérimentale, mais dont la plupart n'ont pas suffisamment tenu compte. Au contraire, les lois de l'association systématique et de la sélection, la théorie qui voit dans tout acte psychique le résultat d'une association d'éléments, et dans l'esprit imparfait de l'homme, la réunion d'un grand nombre de systèmes élémentaires, qui, tantôt s'harmonisent et tantôt se combattent, mais agissent toujours en un sens, chacun pour soi, explique ¿ la fois l'activité normale et l'activité anormale ou pathologique, la règle et les exceptions (ou ce qui devrait être tel.) Si nous ne nous rappelons pas exactement, dans bien des cas, ce qui s'est passé avant une brusque interruption, à laquelle nous ne nous attendions pas, c'est que, l'orientation de l'esprit a subitement changé. A un système psychique, un autre système d'èléments psychiques dynamiquement associés vient se substituer tout d'un coup et, si ces deux systèmes ne se réunissent pas en une unité supérieure, un élément du premier n'éveillera pas le second, ni un élément du second, le premier, du moins en général. On comprend cependant qu'ils puissent se rappeler l'un l'autre, s'ils ont un élément commun; on a alors une association incohérente dans le genre de celles que nous avons examinécs tout à l'heure et qui ont constitué la catégoric des associations par ressemblance; mais on comprend aussi que les deux systèmes puissent se synthétiser pour différentes raisons, par exemple, si l'on a le besoin ou le désir de se rendre compte de l'emploi de son temps. Il y a une autre raison plus générale : un système psychique en activité, qui a pour un instant la direction générale de l'esprit, ne cesse pas absolument 
son activité s'il rient à perdre brusquement cette direction et à y ètre remplacé par un autre. Son activité est plus faible, moins importante, mais clle peut persister. S'il n'y a pas une inlibition complète, si le nouveau système ne prend pas toutes les forces psychiques disponibles, les éléments psychiques qui étaient en jeu et qui ne sont pas directement inhibės, persistent tant qu'ils le peuvent en attendant le moment de reprendre le rôle prépondérant. Si je suis en train de lire un livre qui m'intéresse beaucoup et qu'on m'inter'rompe pour une chose sans grande importance, j'arrête ma lecture, et mon attention est dètournée des idées ct des émotions que cette lecture suscitait en moi ; mais ces idées et ces émotions ne disparaissent pas complètement, et elles subsistent dans une sorte d'arrière-fonds mental avec une conscience vague, étant reliées à un nombre bien moins considérable d'éléments psycho-organiques. Il cst certain que l'état total qui résulte de toutes ces conditions, peut n'être pas du tout le même que si on m'eùt interrompu au moment où je lisais un autre livre, ou au moment où j'écrivais une lettre. Or, la cocxistence de ces deux systèmes - psychiques en activité, suffit bien souvent pour qu'il s'établisse entre eux une certaine coordination plus ou moins marquée - ils sont obligés de s'adapter à un mème état de l'organisme et des haljitudes psychiques - de plus, un esprit qui est habitué à s'observer, c'est-à-dire à coordonner les impressions mentales qui lui arrivent à charque instant, les réunit au moins dans des synthèses intellectuelles, et il ne s'agit pas ici évidemment d'une association par contiguïté et ressemblance, mais d'une systématisation réclle. Nous reviendrons tout à l'heure sur ce cas; restons pour le moment à la contiguïté de succession.

L'explication que je propose me paraît absolunent d'accord avec les belles recherches de M: Delbœuf sur la 
mémoire et les conclusions qu'il tire de ses expériences d'liypnotisme. Je rappellerai ici sa conclusion : Après avoir rapporté deux expériences dans l'une desquelles le sujet avait oublié l'acte exécuté pendant le sommeil hypnotique tandis qu'il s'en souvenait dans l'autre, M. Delbcuf ajoute : "Maintenant quelle différence y a-til entre l'expérience du bonnet et celle du cigare, une seule, mais elle est capitale. Dans l'expérience du cigare, le dernier acte du rêve est le premier du réveil, en d'autres termes le sujet est réveillé au milieu d'une action et l'attitude qu'il a prise n'est explicable pour lui, comme pour les assistants, que par la suggestion sous l'empire de laquelle il agit. Dans l'expérience du bonnet, au contraire, quand on réveille le sujet, le rève est achevé. On a, si je puis ainsi dire, fermé la porte sur le rève au moment d'entrer dans la réalité. Alors le sujet ne peut renouer le fil interrompu, ou du moins, il n'est pas sollicité à le faire comme quand il se surprend lui-même dans une attitude étrange. Sans doute, la WW.... a le bonnet de M. Féré dans sa poche ; elle pourrait, à la rigueur, grâce à cet indice, reconstruire la scène qui s'est passée, et pour ma part, je ne doute même pas que dressée convenablement, elle ne pùt $\mathrm{y}$ parvenir, dans ce cas et d'autres semblables. Mais elle trouve tout aussi commode et même plus simple de supposer qu'on lui a mis cet objet dans sa poche pendant son sommeil.

"C'est ainsi que la jeune fille, à qui, sous mes yeux et sous les yeux de M. Taine, M. Charcot a fait au bras une brûlure par suggestion, a pensé qu'elle avait dù se brùler au foyer à gaz qui était allumé. Et au fond cette interprétation fausse n'est-clle pas plus plausible que la véritable? 1 .

1. Delbœuf. La memoire chez les hypnotisés. Revue philosophique, mai 1886, p. 447-448. 
J'ai cité ce dernier paragraphe parce qu'il montre clairement comment la force systematique de l'esprit change l'ordre des phénomènes et les suscite sans aroir égard à leur position dans le temps, c'est-à-dire à leur relation de contiguïté. Par le premier et par les expériences auxquelles il est fait allusion, nous royons nettement comment la systématisation possible est une condition nécessaire de l'association par contiguïté, c'est-à-dire de l'èlablissement d'un lien entre les divers termes d'une série de phénomènes psychiques. Les phénomènes passés ne peuvent ètre rappelés à l'esprit que si on les retrouve en cherchant l'explication des phénomènes actucls, c'esta-dire qu'il faut qu'une association systématique s'établisse entre les uns et les autres pour que le souvenir soit possible - on voit clairement que la contiguïté n'y suffit pas, et que l'association par contiguïté, comme l'association par ressemblance, n'est en réalité qu'une forme de l'association systématique.

Mais les expériences de M. Delbœuf et les autres faits de mème nature qui se passent ì l'état normal, à l'état scmi-morl)ide, ou il l'etat morbide, me paraissent conduire a une explication plus profonde de l'association par contiguïté et tout a fait analogue à celle qui nous a rendu compte des associations par ressemblance. Lorsque deux phénomenes qui se sont succédé dams l'esprit ont été associes a un mème systeme psychique, ou bien à une mème olientation de l'esprit, il y a des chances pour que ces deux pliénoménes se rappellent l'un l'autre - non pas paree qu'ils ont été contigus, mais parce qu'ils se rattachent a leux systèmes qui ont des éléments communs. Jos deux experiences de M. Delbouf nous le montrent hiren: dans l'une nous royons des élats contigus ne pourant se suscilop l'un l'autre, l'oriculation de l'esprit a chatuge et les deux orientations nont pas suffisamment debinents communs pour que la seconde puisse rappeler 
la premiére, dans le second cas, il n'en est plus de même, les deux orientations successives sont reliées par un même système d'actes qui commence dans la première et s'achève dans la seconde, et elles sont reliées entre elles par ce système qui est un élément commun aux deux. Si l'on joint à cette raison cette autre raison que les phénoménes successifs sont en réalité souvent unis par un lien logique, on se rendra compte, je crois, de la fréquence relative de cette forme de l'association systématique dont on a fait la classe des associations par contiguïté.

Très sourent, en effet, les phénomènes qui se suivent dans l'esprit ne se suivent pas au hasard, ils sont reliés par des liens logiques qui leur donnent de la cohésion en même temps que de la cohérence - nous avons déjà examiné le cas des idées qui se suivent dans un raisonnement, des images, des sentiments, des actẹ qui se suivent dans l'habitude, - dans les formes les plus coordonnées des actes psychiques le fait est trop évident pour qu'il y ait lieu de s'y arrêter, mais dans la rêverie même, dans les rêves, dans les imaginations de la folie il arrive non pas toujours, mais souvent, que les images sont cnchaînées les unes aux autres de manière à constituer un cnsemble offrant une certaine coordination. Prenons mème la série dans laquelle l'esprit et son activité coordo. natrice entrent pour la plus faible partic, je veux parler de la série de nos perceptions. Je ne parlerai pas des cas où nous écoutons un concert, ou bien où nous voyons défiler un régiment, mais mème dans beaucoup d'autres cas il y a un lien logique qui permet de rattacher les impressions les unes aux autres et qui fait que l'une d'entre elles pourra susciter les autres: d'abord il y a un certain classement qui s'opère dans l'esprit, fréquemment tel fait est enregistré par nous comme s'étant passé avant celui-ci, après cclui-là, de plus la séric a pour nous une certaine importance en tant que série dans une rue 
nous pouvons avoir intérèt à connaître jusqu'à un certain point la suite des maisons, pour trouver celle que nous cherchons; d’une manière générale la contiguïté est sonvent une raison d'intérèt pour nous, et c'est parce que des éléments contigus peuvent avoir, à cause de leur contiguïté, des liens logiques directs ou indirects que l'un deux peut appeler les autres.

Mais l'autre cause de l'association de contiguïté vient souvent s'allier à la première. Il est bien sûr que deux faits qui se passent l'un après l'autre ont des chances pour se trouver liés tous deus à un mème système. Je suppose que nous assistions à la représentation d'une pièce de théàtre. Il n'est pas surprenant qu'une partie de la pièce rappclle les autres - les parties réunies formant un tout unifié, bien que quelquefois pas autant quon le roudrait. Supposons un incident quelconque en deliors de la pièce qui se produise au premier acte et un autre au dernier acte par exemple; si ces incidents ont dérangé les acteurs, si d'une manière ou d'une antre ils ont pu se coordonner avec quelques-uns des éléments de la pièce, il n'est pas surprenant que plus tard l'un rappelle l'autre par le moyen des éléments communs (ou associés) auxquels chacun d'eux s'est relié. Ce cas n'est en somme qu'une complication du précédent et la distinction entre les deux ne peut être aljsolue. De mème nos perceptions successives sont souvent rattachées à une mème préoccupation, à un mème sentiment. Si nous sommes tristes, nous associons de diverses manières à notre douleur tout ce qui frappe nos sens. Ces synthèses multiples établissent un lien entre les diverses perceptions el leur permetlent plus tard de se rappeler l'une l'autre.

Tres frequemment, sans $n$ ul donte, tant que la même orientation domine dans l'esprit, tous les phénomènes p.syehiques gui se produisent en nous sont plus ou moins 
rattachés systématiquement aux tendances dominantes ou à quelques-uns de leurs éléments. L'esprit, nous l'avons vu, ne recoit que ce qui peut s'adapter à lui, rien n'entre dans l'esprit que sous la forme d'un systéme, que nous nous en rendions compte ou non. Or, les éléments de ces systèmes sont évidemment les tendances qui dominent en nous au moment d'une perception, d'une idée, d'une volition, etc., ou bien des éléments de ces tendances. Nous arons vu que lorsque l'orientation de l'esprit change, l'association par contiguïté se produit moins à cause de la modification des associations systématiques dominantes; mais quand ces associations, comme c'est l'habitude ; se maintiennent un certain temps, toutes les perceptions, toutes les idées, tous les sentiments qui se sont produits pendant ce temps-lá et ont été reliés à des éléments d'un même sysiẻme ou de systèmes convergents en gardent une tendance plus ou moins forte, et souvent très faible à se susciter l'une l'autre si le besoin s'en fait sentir. Nous retombons ici dans l'explication générale qui a pu servir à réduire l'association par ressemblance : celle qui s'appli que aux cas où des systèmes qui ont une partie commune se succèdent dans l'esprit, pour ainsi dire, autour de cette partie, qui peut d'ailleurs être insuffisamment mise en action pour donner lieu à des phénomènes de conscience.

Il faut remarquer aussi que l'orientation générale de l'esprit persiste souvent un certain temps, que les sous-personnalités, les groupes de tendances, offrent une base très stable aux évẻnements qui viennent s'y fixer, ensuite que la personnalité physiologique et morale - et la personnalité physiologique paraît jouer un grand rôle dans la mémoire, comme nous l'avons déjả reconnu - est plus persistante encore, enfin que certains groupes de tendances persistent en nous indéfiniment. On peut en conclure que la tendance des états de conscience à s'unir d'après des relations de contiguïté est une forme de 
l'association systématique qui doit se présenter assez fréquemment.

Il reste à expliquer l'association par contiguïté simultanéc. Nous nous sommes occupés jusqu'ici des états qui ayant été produits successivement se rappellent l'un l'autre; il s'agit à présent de ceux qui se produisent simultanément. Ce cas parait comporter des explications analogues á celles que nous avons précédemment exposécs. Quand deux perceptions, deux sentiments, deux phénomènes quelconques ont été présents à la fois à la conscience, si l'un d'eux par la suite vient à réveiller l'autre, c'est qu'ils ont été associés au même système d'impressions, d'idées, de sentiments, à la même cénesthésie ou à des systèmes ayant des éléments communs. Il y a donc ici encore une association systématique analogue à celles que nous arons vues déjả. Il me paraît bien, en effet, que c'est seulement dans ces conditions que peut s'effectuer l'association des idées. Cette association est d'autant plus forte en général que la systématisation est micux établic, et cquand la systématisation est très faibìe ou trés indirecte, il y a bien des chances pour que le souvenir ne se produise pas. Si l'on est fortement absorbé pạ un travilil, une lecture, on oublic facilement les petites distractions qu'on peut avoir sous l'influence de causes exterieures, si ces distractions n'ont pas de rapport avec l'occupation principale, et si elles ne sont pas assez fortes pour s'attacher par quelque élément au système psychophysiologique en activité, - si, en un mot, la systématisation est aussi failsle que la contiguïté est marquée. Qu'une mouche se pose sur notre visagre pendant que nous écrivons, nous la chasserons instinctivement, mais le souvenir de ce fait será généralement faible et clisparaîtra vite: la contiguïtés entre le fait de sentir et de chasser la mouche et le fait de live une phrase et de la comprendre ne paraît nullenent suffire pour établir une association entre les deux. 
Si, au contraire, la préoccupation intercurrente est assez forte pour entrer dans le moi, pour s'associer à un système important concurremment avec l'action principale, il est beaucoup plus probable qu'il s'établira une association durable entre les deux, association qui sera due non à la contiguïté de sinultanéité, mais bien à ce que les deux faits se rattachent à un mème système dẹ tendances, c'est-à-dire qu'il s'agit encore ici d'une association systématique. Autant que je puis interroger mon expérience personnelle, lorsqu'un passage d'un livre ou un air de musique fait vibrer en moi un sentiment autrefois éprouvé, il ramène en mème temps une notable partie de l'ancien moi que j'ètais alors. C'est un ancien système d'impressions et d'idées qui tend à se reformer et s'èbauche vaguement, une manière particulière d’ètre et de sentir et non pas une impression détachée. Encore dans quelques-uns des cas que je rappelle pourrait-on voir des associations systématiques que je n'ai pas indiquées - le rapport par exemplè d'une phrase musicale arec un sentiment déterminé. On roit, en tout cas, que les formes des phénomènes sont dans cette nouvelle forme de la contiguïté, absolument analogues à celles que nous arons déjà vues, et partout, dans les associations par contiguïtè simultanée comme dans les associations par contiguïté successive comme dans les associations par ressemblance, c'est l'association sýstématique que nous retrourons, et qui nous parait être la réelle raison de tous les phénomènes.

\section{$\S 6$.}

Nous avons envisagé jusqu'ici l'associationisme au point de vue dynamique, je veux dire au point de vue de la succession des phénomènes de conscience; on pourrait aussi l'envisager au point de vue statique, c'est-à-dire au point de vue de l'association formée d'états de conscience 
qui se présentent simultanément à l'esprit, la perception, la croyance, lc sentiment, sont des états complexes qui se composent de plusicurs éléments, ces éléments sont unis entre eux, et on ne peut guère penser que ce soit par des liens de ressemblance ou de contiguïté. Je n'ai pas besoin d'insister sur ce point après ce qui précède. Rappelons brièvement ce qui peut se rapporter à la perception. "Une perception, dit M. Binet, estle processus par lequel l'esprit complète une impression des sens par une cscorte d'images "1. Cette définition est très juste et si l'on examine la nature du rapport entre les images associées et l'impression des sens, on trouve qu'il s'agit d'une association systématique 2. La théorie de M. Binet, qui fait de la perception un raisonnement et qui y trouve une coordination d'images, s'accorde bien évidemment aveć celle que je défends ici. Les images éveillées par une impression extérieure sont celles qui peuvent s'accorder avec elles, et cela est si vrai, que l'impression extérieure ne peut déterminer qu'un fait psychique en harmonie avec ces tendances internes et que ce qui daus les causes extérieures tendrait à déterminer des phénomènes psychiques, dont la systématisation avec les images serait impossible, passe inaperȩu et reste sans effet. L'accord des images ne se manifeste pas seulement par le fait de. leur synthese, mais aussi par l'unité d'action qu'elles imposent. La perception d'un objet quelconque comprend des éléments qui nous déterminent à agir dans un même sens ou qui s'unissent au moins pour guider nos actions. Les images tactiles, motrices, etc., qui se joignent aux impressions visuelles pour consituer la perception d'un livre, s'unissent entre elles pour donner de l'unité à

1. Binet. Ouvrage cité, p. 13.

2. L'ne analyse de la perception ne peut ètre complète qu'en s'appliquant aussi à ce rapport. 
nos actes, la systématisation dans ces actes qui résultent des perceptions ou qui sont coordonnés par elles, est un signe, une preuve et un résultat de la systématisation dans les impressions. Sans doute, dans la folie, dans certains états de l'esprit mal formé, chez les enfants, chez les sauvages, dans le rève, dans l'illusion, l'harmonie des impressions et des images est moins complc̀te, mais elle ne disparait pas, et d'ailleurs ce n'est pas sur les phénomẻnes inférieurs que nous devons nous régler pour nous faire une idée de Ia nature essentielle de l'esprit. On pourrait appliquer à tous les autres phénomènes qui sont à la fois unifiés et complexes, ce qui s'est dit des perceptions, mais nous ne ferions guẻre ici que répéter ce que nous avons déjà dit. Il n'y a pas là de difficultés sérieuses et tout paraît bien converger vers les résultats principaux que nous avons déjà obtenus au sujet de la nature et du rôle des associations par contiguïté et ressemblance.

$\S 7$.

Ainsi les associations par contiguïté et ressemblance nous apparaissent simplement comme des formes particulières de la loi d'association systématique - elles résultent toutes les deux de l'activité successive d'éléments psychiques rattachés plus ou moins indirectement à un mème système au moyen d'un ou de plusieurs systèmes intermédiaires. De plus, nous avons vu qu'elles sont parfois des formes plus simples du même mode d'association; il est des cas où nous avons besoin d'associer des idées, des perceptions, etc., semblables ou qui ont étè autrefois contiguës. Mais le fait contraire se montre aussi. Le travail de l'esprit consiste souvent à détruire les associations par contiguïté et ressemblance qui ont pu se former pour les diverses raisons que nous avons énumérées, à abstraire, à briser pour ainsi dire les perceptions, les idées etc., et à composer avec les 
ahstraits ainsi formés des systèmes divers aussi bien harmonisés que possible. Toutes les impressions que l'homme reçoit sont perçues par un appareil, le système nerveux qui les analyse et les synthétise ensuite, non pas selon la ressemblance ou la contiguïté, mais, surtout dans les formes les plus élevées et les plus typiques, selon une loi de finalité, dont le résultat est la formation d'un système interne moins imparfait, et une systématisation moins incomplète des rapports de l'organisme avec son milieu animé et inanime. Nous apprenons, par exemple, la table de Pythagore et nous récitons de suite tous les produits des neuf premiers nombres, mais une telle acquisition ne nous servirait de rien, ou serait presque vaine si nous ne lırisions absolument le lien de contiguïté qui existe entre les différents produits $€ \iota$ si nous n'avions à notre service, tout prêt, le produit dont nous avons besoin à un moment déterminé, c'est-à-dire celui qui peut se systématiser avec un enscmble donné de phénomènes et former avec lui un tout innifié. De mème, dans tous les objets que nous percerons, nous isolons tous les caractères, les lois dont ils se composent et nous faisons de ces lois et de ces caractères des synthèsesque la réalité ne nous donne pas directement, mais qui nous sont indispensables. Ainsi quelquefois, c'est le côté numérique seul que nous envisageons. Si, avant de partir pour une promenade, je calcule le nombre de kilomètres que j'ai à faire, afin de bien combiner l'heure du départ, l'heure de l'arrivée, l'heure du retour, il n'est pas nécessaire, il est même nuisible que je me représente d'une manière concrète le terrain que je vais parcourir (étant donné que la route est partout également boune ou à peu près, ou que je tiens comple des differences dans mes raisonnements alsstraits). Je n'ai a prendre en considération que cette qualité abstraite, la dimension en longueur de la route, rui seule peut me servir en ce moment. Je lirise par conséfuent, un nombre 
indéfini d'associations par contiguïté et ressemblance, afin de ne garder qu'une qualité isolée qui, dans cet itat, n'a point de réalité oljective (en tant que séparée des autres) mais qui est plus apte à se systématiser arec les autres étals de conscience éreillés en moi. L'alsstraction, dont le rôle daus la vie psychique est si considerable et si essentiel, et les combinaisons des alıstractions qui forment presque toute la vie mentale de l'homme ne sont possibles que par la rupture ou l'inlibition des nombreuses associations par contiguïté et ressemblance qui existent dans les perceptions et par leur remplacement par des associations qui s'enchainent selon la loi de systématisation et de finalité.

Certains faits, en nous montrant, par exemple, la trop grande persistance des associations par contiguïlc, viennent à l'appui de ce qui précède. Le docteur Lẹden était remarquable parsa mémoire. "Je fus informé, dit Abercrombie, par une personne qui était intimement liée arec lui qu'il pouvait répéter correctement un longacte du Parlement ou tout autre document semblable, après l'aroir lu une seule fois. Un de ses amis, le félicitant, à une occasion, de sa remarquable faculté à cet égard, il répondit qu'au lieu d'ètre un avantáge. c'ètait parfois une source de grands désagréments, ce qu'il cxpliqua en disan', que lorsqu'il désirait se rappeler un point particulier dans quelque chose qu'il avait lu, il ne pouvait le faire qu'en se répélant à lui-même le document entier, à partir du commencement, jusqu'à ce qu'il arrivàt au point qu'il désirait se rappeler " l. Ici, l'association pas: contiguïté, qui, d'ailleurs est visiblement sous la dépendance d'associations systematiques, est beaucoup trop forte pour permettre aux autres associations systématiques, de s'effectuer régulièrement, elle ne peut être brisće, les fragments

1. Abercrombie. Inquiries concerning the intellectual powers, etc., p. 7 . 
ne peuvent s'isoler pour entrer dans de nouveaux systèmes, ou ne peurent le faire qu'arec une grande difficulté.

Toutefois, des faits de ce genre, comme les exemples de personnes qui peurent retenir des séries de mots, qui ne paraissent pas aroir de lien logique, sont également propres à nous montrer la force des associations par contiguïté, et cela nous amène à prendre maintenant la question au point de vue positif.

Jusqu'ici, en effet, je me suis surtout occupé de discuter la facon dont on avait interprété les relations de contitiguïlé et de ressemblance, et l'importance qu'on leur avait attribuée; j'ai tìché de montrer que ces lois n'ètaient que des formes de la loi d'association systématique, et qu'clles ne pouvaicnt servir à exprimer, au point de vue de la psychologic générale, l'activité de l'esprit. Mais nous pourons $\mathrm{y}$ roir ce que nous arons trouvé dans la loi du contraste : des formes particulières d'une loi générale, arec cetle différence, que la loi du contraste parait exprimer des pleénomènes plus essentiels au fonctionnement normal de l'esprit. Les lois de ressemblance et de contiguïté, n'en ont pas moins, si on les entend bien, un certain rôle dans le fonctionnement de l'esprit; ce rôle ayant été plutôt exagéré que méconnu, il m'a paru inutile de le développer ici; on le trouvera longuement étudié dans les ouvrages de Bain, dont j'ai critiqué la tendance générale el certaines interprétations, mais dont les analyses minutieuses et les descriptions exactes, ont rendu de grands services à la psychologic, comme j'ai cu plusicurs fois a l'indiquer dans le cours de ce travail. Je me lorne à rappeler les raisons essentielles qui expliquent les associations par ressemblance et contiguïté, et qui sont la coordination psy̌clique en général, et secondement, cette forme de coordination psychique, où nous voyous deux éléments qui ne sont pas en rapport de finalité, reliés à un même 
LOIS DE CONTIGUÏTÉ ET DE RESSEMBLANCE

système, ou bien à des systèmes rattachés à une mème orientation de l'esprit, ou bien encore, ayant une certaine partie commune autour de laquelle ils se succèdent. Les associations par contiguïté et ressemblance se ramènent aussi a une forme assez analogue a celle de l'association par contraste - mais les circonstances qui les produisent ne sont pas lés mêmes. Il ne paraît pas douteux que l'association par contraste ne soit plus essentielle, plus importante au point de vue de la vie de l'esprit. 


\section{RÉSUMÉ ET TONCLUSION}

DE LA SECONDE PARTIE.

Nous avons passé en revue, dans leurs différentes manifestations, les lois générales de l'activité psychique. La première de celles que nous arons étudiées, la loi d'association systématique, nous a paru la raison d'être générale de toutes les autres qui lui servent de complèment ou même n'en sont qu'une forme particulière. La loi d'inhibition systématisée est la contre-partie exacte de la loi d'association systematique, elle exprime le fait négatif qui correspond exactement au fait positif exprimé par la première, à elles deux ces deux lois expriment tout l'essenticl de l'esprit; la loi du contraste est une combinaison des deux premières, et les lois de ressemblance et de contiguïté ne sont que des formes particulicres de l'association systématique. Ainsi l'esprit nous apparaît comme une sorte de machine vivante extrêmement complexc, s'assimilant sans cesse de nouvelles impressions, les décomposant, rejetant ce qui ne peut lui servir, et arec les éléments décomposés, remontant des systèmes noureaux, combinant sans cesse, et détruisant sans cesse ses combinaisons pour en former d'autres, les défaire encore et revenir aux premières et à de nourelles aussi faisant avec les perceptions, les idées, les sentiments, ce qu'un imprimeur fait avec des caractères d'imprimerie, avec cette différence que l'esprit ne se distingue de ses éléments que parce qu'il est leur synthèse et que ce sont ces éléments eux-mèmes qui vivent, aiment, désirent, s'associent ct se combattent, s'arrêtent les uns les autres, se decomposent et se recomposent - parfois sans loi 
générale qui les dirige, comme des gamins qui vagabondent, quelquefois, au contraire, unis ver's une mème fin, comme les soldats d'un régiment qui manœuvrent arec ensemble, et se groupant autour d'un élément prépondérant qui les domine et dirige leur actirité. Linhibition est caractérisée par l'abandon que chacun fait, en ce cas, d'un grand nombre de ses préférences particulières, de ses désirs propres, et aussi par l'abandon semblable que chacun impose aux autres. Et, pour reprendre une comparaison qui, à mon avis, met en lumière un fait général identique réalisé dans des ordres de faits particuliers différents à beaucoup d'égards, un èlément psšchique obligé de renoncer à une partie de ses affinités pour rentrer momentanément dans un systeme complexe, me parait semblable à un homme qui sacrifie une partie de ses intérèts particuliers pour obéir à une loi sociale. Le sort d'un élćment psschique appelè à faire partie d'un système auquel il n'est pas habitué et qui doit se dégager pour cela d'une association étroite et longtemps combinée, - cela arrive fréquemment lorsque nous apprenons une nouvelle science, ou bien encore lorsque nous arous a renoncer à une croyance, à une illusion, me parait aroir des analogies sérieuses avec certains évẻnements de la vie sociale. Et l'élément psychologique, idée ou sentiment, n'est pas toujours aussi discipliné que lélément social, soldat ou fonctionnaire. Un homme doit renoncer à uné crovance qui lui était chère, il cesse, par exemple, de croire à la rie future, c'est dire que des éléments psychiques comme l'idée de sa personnalité, etc., doivent abandonner certaines associations pour en contracter d'autres, ils ne s'associent plus à des idées plus ou moins vagues d'immortalité, de bonheur éternel, de substance permanente, ils prennent place dans l'autres associations; ils s'associent étroitement avec la conception de l'organisation physiologique, de l'usure vitale, et cela ne se passe pas sans difficulté, 
les éléments qu'on reut séparer protestent et cherchent à se rejoindre. Mais l'organisation superieure de l'esprit l'emporte, il faut que la systématisation psychique s'accomplisse, la rupture des associations premières apparaît a présent comme la condition nécessaire du maintien de l'harmonie générale de l'esprit, et si l'esprit est fort la dissociation s'effectuera. Verra-t-on une fantaisie dans les comparaisons de ce fait psychologique au sort du conscrit qui est enlevé à ses travaux, à sa famille, à sa fiancée, pour prendre place dans un régiment? Ici c'est l'intérêt social qui exige le relìchement ou la rupture des premiers liens sociaux et si la société est forte, bien organisée, il n'y aura pas de résistance; l'influence seulc de l'idéc du devoir suffira à déterminer la dissociation et l'association nouvelle, Nous retrouvons dans ce fait social l'association systématique, l'inhibition, il serait facile aussi d'y roir des traces de l'association par contraste et de l'association par contiguïté et ressemblance.

Mais ce n'est pas en ce moment notre but - nous avons simplement à indiquer le sens général de ce qui précède. Nous avons vu d'abord, dans la première partie, l'activité relativement indépendante des éléments psychiques nous avons constaté dans le second leur solidarité réclle et les lois de cette solidarité. Les deux modes d'action ne s'opposent nullement. Parmi toutes les formes possibles de l'activité d'un élément psychique, comme parmi toutes les formes possibles de l'activité d'un élément physiologique ou d'un élément sociologique, il en est un certain nombre qui peuvent s'accorder avec celles des autres éléments qui s'associent avec lui, il en est d'autres au contraire qui contrarient un certain nombre de désirs des autres éléments - or, chaque forme de l'activité d'un étément tend à déterminer chez les autres les formes d'activité concordantes, celles qui peuvent s'hammoniser avec elle jour une même fin ou des tins convergentes el à 
inhiber les formes d'activité discordantes. L'harmonie de l'esprit vient de là, et elle est déterminée en grande partie par l'harmonie organique. Celle-ci, en effet, comprend des éléments relativement très stables, bien organisés et exerce une influence considérable pour l'association ou l'arrèt sur les tendances et les phénomènes psychologiques - et c'est la cause du fonctionnement de tous les phénomènes psychiques qui tendent, à des degrés divers, à la conservation générale de l'organisme - de plus chaque organe a ses représentants psychiques et nous avons ici une nouvelle cause d'association et d'inhibition. L'un de ces organes prend part non seulement à la vie individuelle, mais à la vie sociale aussi, à l'amour, à la génération, à la vie de l'espèce. Il est aussi l'élément dominant dans un nombre très important de coordinations psychiques. Le milieu social détermine en nous soit par imitation, soit par besoin de nous mettre en harmonie avec nos contemporains - et l'imitation est un moyen d'y parvenir - une autre série considérable de systémes harmoniques. Enfin certaines autres coordinations sont déterminées par des faits psychiques formés peu à peu et qui ont imprimé à l'esprit une allure particulière en rassemblant autour d'eux un groupe de tendances et d'idées - par exemple, les tendances qui dépassent le milieu social et qui ont pour but une abstraction, une forme générale, le bien, le beau que l'esprit a extraits de quelques expériences et avec lesquels il a fait une forme idéale dont il poursuit la réalisation. La vie individuelle, la vie de l'espèce, la vie sociale, la vie esthétique, scientifique et morale, voilà les quatre formes principales de la systématisation psychique, déterminées chacune par l'influence d'éléments différents, parmi lesquels les plus stables, paraissent toujours les moins élevés, mais aussi les plus forts, comme étant la condition des autres qui les impliquent. 
Ces quatre formes principales de la systematisation nsychique donneraient lieu à de nombreuses subdivisions, je suis obligé de rester ici dans les lignes les plus générales, en disant seulement ce qui est nécessaire pour donner un sens précis à la loi de systématisation qui resterait sans cela trop vague. C'est à la psychologie du caractère à étudier les différentes tendances, leur signification, leurs combinaisons possibles, la détermination des principaux types intellectuels et moraux. Mais il est un autre point sur lequel nous arons dû insister : ces différentes systématisations psychiques comportent des formẹs générales semblables de groupement des éléments : les perceptions, les images, les idées, les sentiments, les émotions, les tendances, les volitions. Quelle que soit la forme concrète de la systématisation psychique, elle implique l'existence de toutes ces formes abstraites qui restent invariablement les mèmes au point de rue abstrait quelle que soit cette forme concrète. Un raisonnement, un sentiment, comme formes générales de la mentalité, ne diffèrent pas chez un gourmand et chez un artiste, chez un savant et chez un magistrat - malgré les différenees concrètes qui peuvent exister dans le mode de raisonner ou de sentir de. chaque personne et de chaque groupe social. Nous avons done pu préciser encore la loi d'association systématique en montrant ses diverses formes générales concrètes, et les diverses combinaisons d'éléments psychiques qui servaient à la vie de l'esprit. Nous avons ainsi passé en revue et ramené à des formes particulières de l'association systématique, la perception, l'image, l'idée, le jugement, le raisonnement, la volition, la réflexion, ete., en tàchant de déterminer la nature caractéristique, pour chaque espèce 1e faits pgychologiques des éléments et de la manière dont ils sont associes. Nous avons ensuite vu la part que Inond l'inhibition a la formation de ces diverses modalites de lactivité mentale, enfin nous avons étudié, au 
même point de vue, le rôle du contraste et celui de la contiguïté et de la ressemblance. Tout a été ainsi ramené dans l'esprit à une forme essentielle: la systématisation, à une action parallèle à la première : l'inhibition, et à des formes secondaires. Nous avons vu également que les associations systématiques de plus en plus hautes étaient des parties de plus en plus considérables de l'esprit. que la personnalité enfin n'était que le cas idéal - et chacun de nous est d'autant plus une personne qu'il s'en rapproche davantage - où tous les éléments psychiques s'harmonisent en vue d'une fin, variable selon les genres de personnalités et les tendances principales, où l'inhibition est, comme l'association, parfaitement systématisée. Cette harmonie vivante, cette systématisation toujours à l'œuvre, cette direction générale qui détermine l'évolution et la dissolution, la mise en activité et l'arrèt des phénomènes psychiques plus ou moins complexes, c'est l'esprit même. Nous avons maintenant à le considérer en lui-même, c'est-à-dire à étudier l'action combinée, synthétique, concrète des lois dont nous venons d'étudier le fonctionnement abstrait. 



\section{TROISIEEME PARTIE}

\section{L'ESPRIT}

\section{INTRODUCTION}

Pour étudier maintenant dans le domaine du concret les lois que nous arons étudiées sous leur forme abstraite; pour vérifier, par une expérience plus complète, les faits généraux que l'observation et l'analyse nous ont montrés, nous avons deux moyens - d'abord prendre une portion quelconque de la vie mentale de I'homme, le langage, par exemple, ou l'amour, ou l'imagination du peintre ou du musicien, ete., et roir comment s'y combinent les différentes lois de l'esprit - ou bien suivre l'évolution d'une personnalité, depuis l'enfance jusqu'au développement complet et voir encore si ce développement est suffisamment exprimé par nos lois abstraites. Si nous pourons arriver a interpréter l'un et l'antre cas au moyen de ces lois, si elles nous permettent de ne négliger aucune partie importante du processus, nous aurons le droit d'en conclure qu'elles expriment bien la vie de l'esprit. Nous ferons done les deux tentatives, l'une après l'autre, en commençant par l'étude la moins complexe. Nous tàclierons d'en tirer ensuite une conception synthétique de l'esprit, en faisant la syuthèse abstraite des lois, déja ébauchée, au reste, dans les pages précédentes, après en avoir étudié la sýnthèse concrète. Enfin, nous aurons a rapprocher la psychologie des autres sciences, en 
recherchant s'il n'y a pas certaines lois générales de la nature qui se retrouvent dans tous les ordres de phénomènes connus, et en essayant de déterminer, en tant que cela sera possible, les ressemblances et les différences de ees lois diverses. Par là, l'esprit se trouverait rattaché à la nature, et la psychologie aux autres sciences. Notre coneeption de la partie ne peut être complétée que par la conception du tout, et si imparfaites que puissent être ces conceptions, si imparfait, par suite, que soit le résultat de la comparaison, il n'y a pas à douter que nous ne soyons obligés de la faire - on la fait toujours implicitement quand on ne la fait pas autrement. Chaque science a son couronnement dans la philosophie, et peut-être ne faut-il pas attendre qu'une science soit terminée pour en esquisser la portée générale, en supposant même que l'attente soit possible. Les généralisations les plus élevées se forment peu à peu bien souvent, comme les généralisations qui résultent des observations les plus simples; la partie philosophique d'une seience se forme parallèlement ì sa partie expérimentale. Mais restons pour le moment dans la psychologrie, et cherchons la manière dont s'incarnent les lois abstraites. 


\section{LIVRE I.}

Synthèses concrètes.

CHAPITRE PREMIER

Synthèses partielles. - L'Amour, le langage.

$\S 1$.

Prenons une tendance quelconque, l'amour, par exemple, qui est en mème temps une des plus importantes et des plus commodes à étudier. Elle est, sous sa forme la plus simple chez les êtres élevés, une action réllexe dont le point de départ est une excitation des organes de la génération, dont le point terminal est la mise en présence des deux éléments qui doivent donner naissance à un être nouveau - ou plutôt, ce n'est pas là l'amour, mais c'en est la base - le nom d'amour s'appliquant plutôt ì la masse considérable des phénomènes que le développement de l'esprit et l'état social, ont produite et rattachée ả cette tendance primordiale au rapprochement sexuel. Il serait intéressant de comparer ici les différentes formes que cette synthèse a prises dans les différentes espèces vivantes, l'amour chez les oiseaux, chez les araignées, chez les mammifères, l'amour mème considéré chez les plantes pourrait donner lieu à des analyses curieuses et instructives, mais notre but, en ce moment, n'est pas de faire la psychologie comparée de l'instinct sexuel, et en nous restreignant à l'homme nous avons encore un champ assez 
vaste pour examiner le dévcloppement des phénomènes e: l'enchevêtrement des lois.

A l'époque de la puberté le besoin sexuel naît chez l'homme, et, comme tous les besoins organiques, il tend à se satisfaire - pour se satisfaire il tend à diriger l'organisme entier, c'est-à-dire que l'impression partie des organes de la génération tend à éveiller des désirs conscients, des images plus ou moins nettes, des volitions qui tendent toutes vers une même fin : la satisfaction du besoin, l'acte de la génération. Si ce besoin pouvait être satisfait à mesure qu'il naît - comme le besoin de respirer, ou le besoin de l'irrigation sanguine, - il est hors de doute que tous les phénomènes qui constituent proprement l'amour ne se seraient pas produits, mais lc besoin sexuel, pour se satisfaire pleinement, exige le concours de deux êtres, de plus les conditions de la vie sociale ont mis divers obstacles à sa satisfaction, et voilà deux causes de complications psychologiques qui par l'inhibition imparfaite de la tendance l'ont rendue plus complexe et plus violente - et nous avons ici un effet de contraste bien marqué. D'autre part, selon la nature de l'arrêt qui lui est imposé, le sentiment prend des caracté-. res différents; les phénomènes qui viennent se joindre à la tendance primitive, qui ont compliqué l'impulsion primordiale varient avec chaque forme d'état social, "ils varient aussi selon la forme de l'organisation psychique individuelle - et nous royons la loi de systématisation et la loi d'inhibition se manifester ainsi par des phénomènes très varies. Examinons quelques eas particuliers.

On a remarqué que les premiers désirs sexuels, désirs souvent méconnus, s'accompagnent de sentiments trẻs platoniques, très dégrages en apparence et dans la pensée même de celui qui les éproure, de toute attache matérielle, quelquefois même l'éveil des sentiments amoureux se marrue par une certaine répugnance pour l'acte qui 
doit les satisfaire. Il y a là une forme très intéressante d'association psychique et qui mérite d'ètre examinée de près. L'impulsion de l'amour physique tend à rapprocher' l'homme de la femme, de plus il lui inspire, par association systématique, le désir d'accomplir tout ce qui peut le faire aimer, le mettre au-dessus de ses rivaux, le rendre digne d'admiration, il se forme ainsi tout un cortège de sentiments, d'idées, etc., dont le moteur, le point de départ, est l'impulsion sexuelle. Supposons maintenant que ces dispositions viennent à se produire dans une personnalité où dominent déjà des sentiments de timidité et un certain dédain pour la vie matérielle et les plaisirs qui passent pour grossiers. Ce jugement sur l'acte sexuel est assez naturel chez un jeune homme tel que nous le supposons, qui peut remarquer que ceux de ses camarades de son âge qui se vantent de leurs prouesses ou se complaisent à parler de certains sujets, ne sont pas les plus raffinés en général (comme il peut juger de mauvais ton, pour des raisons dnalogues, de fumer ou d'aller au café). Il faut tenir compte aussi de la gaucherie, de la crainte du ridicule, des obstacles instinctivement entrevus, de la pudeur développée par l'éducation morale, de l'espèce de honte qui s'attache aux fonctions de certaines parties du corps, de l'interdiction aux enfants de faire allusion à certains sujets, de l'éducation religieuse qui condamne les plaisirs des sens en général, de l'éducation en général qui, par toutes ses formes, tend plutôt à restreindre, de crainte d'abus, les désirs qu'à tâcher de leur préparer une satisfaction normale, - toutes ces circonstances peuvent faire que le processus qui a déterminé l'éveil de tous les sentiments, de toutes les tendances dont l'association avec lui constitue l'amour, soit enrayé dans son développement, tandis que les phénomènes qu'il a suscités continuent à persister. La tendance sexuelle, en effet, choque, dans certains cas, et pour 
certaines personnes, un certain nombre d'habitudes mentales, ces habitudes menacées réagissent (loi du contraste) et excreent une action d'arrêt sur l'jmpulsion qui ne s'harmonise pas avec elles (loi d'arrêt) - mais les idées et les sentiments éveillés par l'instinct génésique, tendresse, affection, sympathie, désir d'approbation et de louange, etc., ne sont nullement en contradiction avec les anciennes tendances de l'esprit, et peuvent très bien s'harmoniser avec elles, - elles s'accordent bien moins, au contraire, une fois qu'elles sont développécs avec l'impulsion qui cependant les a produites, et l'on comprend qu'en certains cas elles contribuent à l'enrayer. Ainsi les nouveaux sentiments et les anciennes habitudes de l'esprit s'associeront et formeront un tout très particulier, une sorte de tendresse réservèe, vive et respectueuse, ne conduisant à désirer que des échanges de paroles tendres, à peine un contact de la main, et, au moins subjectivement et plutôt dans les rêveries que dans la réalité, à des dévouements ignorés, à des sacrifices sans compensation. Remarquons, du reste, que l'impulsion sexuelle est enrayée mais non détruite, et qu'elle continue à susciter, à favoriser les sentiments et les idées qui, par les associations qu'ils contractent avee les habitudes mentales déjà existantes, tendent d'abord à l'enrayer. La crainte, le respect de la personne aimée sont pour heaucoup dans cette inhibition. C'est ainsi que par un jeu d'association systématique, d'inhibition et de contraste, l'amour platonique se forme. Et s'il arrive plus tard que les désirs physiques non satisfaits viennent à se faire sentir de plus en plus fortement et de manière à ne pouvoir plus être enrayés, ils ne pourront prendre place dans le système déjả formé, il y aura une lutte et s'ils sont suffisamment inhibés sans l'ètre trop, ils formeront un systeme a flart, un systeme noins complexe, et cette fois ils tendront à la réalisation immédiate, sans éveiller un 
cortège de sentiments semblable à celui qu'ils ont excité d'abord. Tel homme qui adorera respectueusement une reine, satisfera ses sens sans éveil de l'imagination poétique ou de sentiments chevaleresques, avec la premiere venue - l'amour qui dans une personnalité mieux systématisée, serait un complexus harmonique de sentiments, d'idées, d'images et d'impulsions, se trouve ici partagé en deux parties : amour de cœur et de tête et amour des sens qui n'auront pas grand chose de commun, se satisferont à part, et auront des objets différents.

\section{$\S 2$.}

Ce fait, comparé aux faits différents qui ne sont pas rares, est suffisant pour nous montrer comment des organisations mentales diverses peuvent, par des associations systématiques changeant logiquement avec chaque organisation et des inhibitions variant corrélativement, déterminer des modes de sentir tout-à-fait différents et même opposés en certains cas. Au reste, les conceptions individuelles de l'amour varient, même pour une même civilisation, d'une manière très marquée - et non seulement avec la constitution psychique individuelle mais aussi avec les circonstances de la vie qui sont d'ailleurs en partie cause, en partie effet de la constitution psychique. On s'en fera une idée en se rappelant la forme de l'amour que chaque écrivain a dépeinte ou bien celle qu'il a dépeinte le mieux - il n'est pas douteux que, soit qu'un auteur raconte ses mémoires, soit qu'il écrive des romans, il n'ait un penchant à dépeindre ses propres sentiments et une facilité particulière pour les exprimer. Sans doute, il faut interprêter ici aussi avec prudence, mais on peut affirmer que d'une manière générale, les différences de la description réelle ou fictive correspondent à des différences de l'organisation 
psychologique. Etudiez, au point de vue de la conception de l'amour, les mémoires, les traités, les romans des écrivains de ce siecle : Stendhal 1, Benjamin Constant ${ }^{2}$, Sainte-Benve ${ }^{3}$, Balzac ${ }^{4}$, Musset ${ }^{5}$, Hugo ${ }^{6}$, George Sand ${ }^{7}$, Stuart Mill ${ }^{8}$, Schopenhauer ${ }^{9}$, Topffer ${ }^{10}$, etc., et parmi les contemporains MM. Zola, Pierre Loti, Daudet, Barbey d'Aurevilly, Paul Bonrget, Peladan, partout rous trouvez une conception particulière de l'amour, il est sensuel et violent, sensuel, raffiné et pervers, il est passionné, despotique et sérieux, élevé audessus des lois et de la morale, il est fringant ct cavalier, souvent sensuel, vif et inégal, il est sérieux, associé à de vifs sentiments d'estime et d'amitié, scnsuel et simple, sensucl et quintessenciè, grossier et raffinè à la fois, sec et curicux, passionné et conscient, violent et triste, sentimental et timide. Analysez ces mots rous trouverez que chacun d'eux désigne une systématisation particulière ou la manière dont cette systematisation se forme. Chez Stuart Mill, par exemple, les sentiments qui forment l'amour ne sont pas les mêmes que chez Musset, et le complexus qu'ils composent n'a pas la même allure. Ici

1. De l'amour, la Chartreuse de Parme, le Rouge et le Noir.

2. Adolphe.

3. Volupte, poessies. Cf. Sainte-Beuve et ses inconnnes, par Pons.

4. Balzac. La comédie humaine, passim. Voir spécialement Ferragus, Mémoires de deux jeunes mariees. Physiologic du mariage, La duchesse de Langeais, etc.

5. Yusset, Poésies. Le sonvenir, Chansons d'Espagne et d'Italie, et surtout La confession d'un enfant du siecle.

6. Ilugo. Poésies, passim. Voir en particulier les chansons des rues et des bois.

7. Romans. Cr. Lui et Elle de Paul de Musset, Elle et Lati, de George San $\rfloor$, Laii, de $\mathbf{M}^{\mathrm{me}}$ Louise Collet.

8. Stuart Mill, Memotres.

9. Schopenhauer.

10. Topfer. Nouvelles genévoises. 
il s'agit surtout d'une profonde estime, d'une sympathie tendre, là d'une impulsion des sens, capricieuse et fantasque, mèlée aux jeux d'une imagination vive, légère, avec un arrière goùt de lassitude et de tristesse, ou lor'sque les choses deviendront plus sérieuses d'une passion sensuelle et àcre, accompagnée de révoltes de l'esprit et du cœur, de découragement, de transports violents, et d'anéantissements passagers. Analysez encore tous ces mots, et les mots par lesquels vous les remplacerez vous finirez par arriver aux faits psychiques eux-mêmes, vous verrez qu'ils ne sont les mêmes dans aucun cas, que certains éléments qui se retrouvent chez une personne ne se retrouvent pas chez l'autre, la vivacité des images, l'affection tendre, l'amertume qui résulte de la lassitude ou de la désillusion, l'estime intellectuelle, la confiance, le sens du devoir, les idées abstraites, etc., vous voyez de plus que la forme que prennent les composés est très différente, ici le but poursuivi est la jouissance, et l'exaltation qu'elle donne au sentiment et à l'imagination, là c'est plutôt la communauté de la vie, des occupations intellectuelles, des préoccupations élevées, la satisfaction de tendances sociales ou abstraites pour lesquelles chacun complète l'autre, ici les idées et les sentiments prennent une forme aiguë, ils sont vifs, fugaces, incohérents, là ils sont plus calmes, et plus solides. La fin de l'association systématique n'est pas la même, sa forme varie considérablement, ses éléments changent d'une personne à l'autre, lesinhibitions varient naturellement d'une manière corrélative et néanmoins ces deux complexus si différents reçoivent la même dénomination, uniquement parce que la tendance autour de laquelle viennent se grouper tous ces enchevêtrements de phénomènes psychiques et sous lesquels elle disparait quelquefois est bien semblable dans les deux cas et c'est l'impulsion sexuelle 'plus ou moins reconnuc. 


\section{$\$ 3$.}

Si dans une socièté les conditions d'existence et l'orientation psychique rarient d'une personne à une autre, elles varient encore bien plus d'un siècle à l'autre, d'une civilisation a une autre - certes, l'amour, chez les Français, pour l'époque actuelle par exemple, varic considérablement, cependant si nous négligeons les détails, nous parvenons à nous en fairc une idée d'ensemble qui sera incomplète, mais suffisamment exacte, prenons maintenant l'amour an $\mathrm{Xvul}^{\mathrm{e}}$ siècle, sous Louis XV, nous avons l'idéc d'un sentiment passablement différent, nous entrevoyons la prédominance de certains autres éléments: l'esprit, le raffinement des manières, l'élégance, le persiflage, l'ironie froide, le scnsualisme avoué, la sécheresse, l'effacement du sentiment proprement dit, la virtuosité, le dédain, l'égoïsme, et si nous analysons encore nous trouvons que tout se réduit à des manifestations différentes des lois d'association systématique et d'inhibition; le point de départ, la tendance essentielle est encorc la même, mais elle se satisfait d'une façon différente, ellc éveille pour les systématiser avec elles d'autres idées, d'autres images, d'autres sentiments secondaires qu'elle ne le fait de nos jours, clle inhibe au contraire, des sentiments comme la pitié, la tendresse qui dans d'autres circonstances pourraient s'harmoniser merveilleusement avec elle. Et nous avons un bon effet de contraste social et psychologique dans l'allure que prend le sentiment vers la dernière partic du siècle, sous l'influence de Rousseau. Au dix-septième siècle autre changement, autre allure gónérale de l'amour et pliénomène de contraste social très marqué entre les raffinements et les pudeurs de l'Hôtel de Rambouillet, des véritables précieuses et de leurs imitatrices et les mours grossieres de tout une partic de la jeunosse nohle. Prenez pour tenir le milieu et réaliser l'équilibre des tendances de l'époque l'amour tel que nous le 
trouvons chez les jeunes filles de Molière, Henriette surtout, et qui est une affection raisonnable, vous pouvez encore vous livrer à la même opération et trouver les éléments en jeu, la manière dont ils se combinent autour d'une impulsion centrale et les inhibitions et les contrastes qui en résultent. Chaque époque caractérisée par des traits généraux assez marqués offrirait de même une allure particulière de l'amour et toujours cette allure particulière sera produite par la nature différente des éléments, sentiments, idées, images, éveillés par l'impulsion sexuelle et qui varient naturellement avec l'individu, la race, le moment, l'exemple, les idées régnantes, la mode, toutes les autres influences, les conditions sociales dont l'équilibre momentanément établi d'une certaine manière fait la caractéristique d'une époque.

Mais on le voit, toutes ces variations que je ne puis indiquer que très sommairement, dont je n'ai pu étudier qu'un seul cas général et dont j'étudierai seulement encore un cas particulier un peu en détail, en rappelant pour les autres le sens général de l'interprétation, sont dues à un même fait essentiel : les associations systématiques et les inhibitions déterminées, soit directement, soit secondairement, et même en retour direct ou indirect par l'impulsion sexuelle, et aux phénomènes secondaires de contraste ou d'association par contiguïté et ressemblance qui en dérivent. Remarquons d'ailleurs que tout ce cortège de phénomènes est suscité par une inhibition à quelque degré de la tendance sexuelle. Celles de nos tendances qui n'ont aucune difficulté à aboutir, et c'est le cas général des tendances organiques dans l'état de santé, ne déterminent aucun complexus de phénomènes psychologiques. Celles qui se satisfont plus irrégulièrement et avec plus de peine, comme le besoin de boirc et de manger, déterminent déjà des complexus d'idées et de sentiments plus variés - nous sentons plus généralement 
l'appétit ou la faim et la soif, que le besoin de la respiration ou de la circulation du sang. Maintenant comparez la complexité des états de conscience éveillés chez celui qui a ses repas assurés et chez celui qui n'a pas de quoi satisfaire sa faim et qui cherche anxieusement comment il pourra gagner, se faire donner ou dérober quelque chose, - comparez encore l'état mental de l'homme à appétit robuste, qui mange régulièrement et se contente de mets quelconques, et celui de l'homme à santé délicate, qui est obligé de choisir soigneusement ses mets, qui se ressent longtemps du travail de la digestion, et est obligé de mettre un intervalle fixe entre ses repas. Il est incontestable que les tendances organiques se satisfont moins facilement chez l'un que chez l'autre, et que la complexité des sentiments est - toutes choses égales d'ailleurs - en raison directe de la difficulté avec laquelle la tendance est satisfaite. De même, il paraîtbien que d'une manière générale, l'amour se complique avec la difficulté de le satisfaire, il ne paraît pas que les formes les flus complexes, les plus développées de ee sentiment se rencontrent chez les peuples sauvages, où la promiscuité est l'état normal. Même chez les peuples civilisés, la différence est énorme entre le. besoin physique qui se satisfait facilement et les formes élevées de l'amour qui impliquent la sympathie, l'estime, la communauté des goùts, l'harmonie des tendances. L'état social a compliqué l'homme en faisant naitre en lui une foule de besoins, d'idées, d'impressions, de tendances, inconnus à nos premiers ancêtres - et un sentiment qui, comme l'anour, tient une place importante dans la vie, a dù s'associer à un grand nombre de ces nouveaux Ithénomènes, de lá, la complexité de l'amour moderne dans ses formes les plus riches. Mais d'un autre eôté, l'impulsion sexuelle ne peul pas toujours, pour se satisfaire, attendre d'avoir formé une association systématique avec le moi tout cutier - elle agit impèrieusement, et, 
si la civilisation a développé l'amour, si elle a enrichi le système d'éléments psychiques qui s'est formé autour de l'impulsion génésique, si elle a augmenté de plus cn plus le nombre et la complexite des phénomènes variés, des tendances avec qui cette impulsion tend a s'associer systématiquement, elle a aussi rendu plus difficile la formation de ce complexus si vaste - et elle a rendu nécessaire la survivance ou mème le déreloppement de formes infiniment plus simples de satisfaction sexuelle. Et il arrive sourent qu'une scission, analogue pour la forme à celle qui produit quelquefois l'amour platonique, mais différente dans ses résultats, rient à s'établir. L'amour disparait; la tendance sexuelle se réduit à un besoin physiologique, comme le manger et le boire, et est satisfaite de même, sans s'être associée à des partics notables de la personnalité. L'association ssstématique est ramenée ici à sa plus simple expression, et l'équilibre de l'organisme peut n'en pas souffrir, pas plus d'ailleurs que l'équilibre de l'esprit, car cela n'empêche pas ces fonctions organiques et psychiques de concourir à la vie de l'organisme, seulement elles forment comme autant de mécanismes convergents mais distincts et non directement solidaires les uns des autres; c'est en somme une forme inférieure, moins complexe et moins profondément systématisée de la mentalité. Les associations systématiques entre les diverses tendances sont ici remplacées par des actions d'arrèt, chaque tendance inhibant à son tour l'activité des autres 1 .

\section{$\S 3$.}

Dans les formes supérieures, au contraire, la vie entière s'associe à l'amour. L'arrèt est moindre, la convergence

I. Cf. Taine. Notes sur Paris. Vie et opinions de Thomas Graindorge. - Un homme de quarante ans disait : a J'ai reduit l'amour a une fonction et cette fonction à un minimum. Un jeune homme de vingt-cinq ans reprenait : * Au lieu de miuimum mettez maximum, voila mon affaire. ^ 
est supérieure. Un amour profond et sérieux ne prend pas place lans la vie mentale, comme un goùt à côté des autres, comme un nouveau rouage juxtaposé a des rouages anciens, mais il se mèle à toutes les formes de la vie mentale ou au moins aux plus importantes, et nous voyons ici d'admirables exemples de la force de systématisation des éléments psychiques et de la coordination progressive de l'esprit. L'étude de la sociologie peut être une des formes de l'amour. Voyez comment Stuart Mill parle de MI ${ }^{\text {me }}$ Taylor qui devint sa femme, après une amitié de vingt ans : "Je n'en finirais pas si je voulais dire en détail tout ce que je lui dois, ne fùt-ce qu'au point de vue de l'intelligence. Je n'en toucherai que quelques mots, qui donneront une idée générale, bien qu’imparfaile, des services qu'elle m'a rendus.... Toute ma force me venait de l'étude des sciences morales et politiques, région intermédiaire incertaine et périlleuse; j'avais accepté des solutions toutes faites, j'en avait trouvé moi-même en économie politique, en psychologie analytique, en logique, en philosophie de l'histoire, comme sur d'autres sujets, et ce n'est pas la moindre des obligations que mon intelligence doit a cette nolle femme, de m'avoir conduit à un scepticisme sage à l'ergard de ces solutions. Je ne renonçai pas pour cela à tirer des questions auxquelles j'appliquais loyalement les faculté de mon esprit, toutes les solutions qu'elles comportaient, mais j'appris à être plus réservé, à me garder de les affirmer ou de les proclaner avec une confiance que ce genre de spéculation ne saurait admettre. Bien plus, la nème influence disposa mon esprit non sculenent a accueillir mais a cmbrasser et a rechercher avec ardeur tout ee ru'ou pouvait trouver de plus clair en fatis de vues, de plus fort en fait de preuves, mème sur les guestions qui avitient fait le plus l'objet de mes médititions. On m'a souvent adressé des éloges dont, a mon avis, jo no mirite yu'une partie, pour avoir mis dans mes 
écrits, à ce qu'on dit, un esprit plus pratique que celui qu'on rencontre chez la plupart des personnes qui se sont occupées des questions les plus générales. Les œurres où l'on a observé cette qualité, n'étaient pas les ceuvres d'un esprit unique, mais de la fusion de deux esprits, dont l'un portait autant de sens pratique dans ses jugements des choses présentes, que d'élévation ct d'audace dans ses prévisions relatives à un avenir éloigné v 1 . Voilà ce que peut devenir l'amour et comment il arrire à se compliquer de psychologie analytique, de logique, d'économie politique et de philosophie de l'histoire - toutes choses dont il se passe souvent. Il est vrai qu'on pourrait dire aussi : voilà ce que peut devenir la tendance scicntifique et comment elle sait profiter des sentiments tendres, et les deux points de rue sont également bons. Les formes élevées de l'esprit sont, en effet, caractérisées par cette harmonie parfaite de toutes les tendances, et l'aide qu'elles se prètent réciproquement sans se nuire. Il ne faut pas confondre ces systématisations supérieures qui caractérisent les personnalités les plus éminentes avec cette influence réciproque et non systématisée des tendances très fréquentes d'ailleurs chez les esprits ordinaires et qui se manifestent par des produits incohérents - telle est souvent, par exemple, ce qu'on appelle l'influence du cœur sur l'intelligence, influence qui se traduit en général par l'acte d'adopter des croyances à cause de leur conformité avec tel ou tel de nos désirs, et malgré leur désaccord avec les idées justes que l'on n'abandonne pas.

Mais l'amour peut revêtir des formes diverses, même dans ses hautes manifestations. Michelet nous montre un sentiment tout à fait différent de celui qu'exprime les mémoires de Stuart Mill. La forme en est moins belle et

1. Stuart Mill. Mes mémoires, histoire de ma vie et de mes idées, p. 180-181. 
moins élevéc, et cela tient en grande partic à l'infériorité de la jeune femme que dépeint Michelet, par rapport à celle qu'a aimée Stuar't Mill. Voilà dejà une différence considérable dans les éléments de l'association systématique, cet élément de l'amour qui constitue la représentation de la nature psychique, du cœur et de l'esprit de la femme aimée varie en effet d'un cas à l'autre et d'un autre côté cette différence tient pour beaucoup à la nature même de l'amant, car ou bien il n'aime de tout son moi que la personne en qui se rencontrent les qualités qui peuvent exciter sa sympathie, ou bien il lui attribue ces qualités, si elle ne les possède pas. Mais regardons de plus près, nous verrons mieux encore la différence des idées et des sentiments qui, dans des passious d'ordre élevé, se combinent à la même tendance essentielle: "Nul doute, dit Michelet, que le grand remorqueur ne soit le désir et la force mâle. Mais, pour qu'elle soit fécondée, il faut qu'à sa sèche àpreté se mêle le fondant suave des beautés féminines. Charmant miracle de nature? Le génie, hier arrêté sur la voie de l'invention, rencontrant un de ces nœuds qu'on ne sait comment résoudre, ayant tourné tout autour, désespéré, et jeté tout, tristement s'assoit au foyer. Elle voit bien sa tristesse. "Mais qu'as-tu? Je ne veux pas, je ne peux pas nte voir ainsi.... Eh ! laisse li ton idée; oublie, je t'en - prie, sois heureux! "C'est justement ce moment d'oubli, ce bonheur qui a tout changé. Sa vue en est renouvelée, sa puissance rafraìchic : une électricité nouvelle lui reviont pour l'exécution. Il est devenu un autre homme. Comment? Aimanté de la femme, de cette grice de nature et d'aimable facilité gu'clle a et donne i toutes choses, il sourit du léger olsstacle rui l'avait arrêté la veille ${ }^{1}$. Il y a bien ici une certaine association de la tendance 
sexuelle et de préoccupations intellectuelles, mais malgré les images dont se sert Michelet, le fait n'est pas très complexe. L'influence de la femme, ici, ne ressemble pas à celle dont nous parlait Mill tout à l'heure, elle est à peu près purement physique. Il s'agit ici, en somme, d'une sorte de dỹnamogénie tout à fait comparalsle à celles que M. Féré a étudiées et lont nous avons en l'occasion de parler, la force dégagée par une association des sens et de certains sentiments parait ètre utilisée par des tendances intellectuelles dominantes, mais l'amour, et son cortège d'images, la femme aimée, ses paroles, son influence morale, n'agissent pas systématiquement pour reformer l'esprit et modifier le fonctionnement de l'intelligence. A un autre passage du mème livre, tout en retrouvant le même mode d'action, nous trouvons aussi quelque chose de plus. Michelet $\mathrm{y}$ dépeint arec chaleur à la fois et arec une précision suffisante l'harmonie de deux àmes, et nous royons encore ici ce que devient, avec les nombreuses associations systématiques qu'elle suscite, cette tendance fondamentale qui, chez Michelet du moins, n'est pas reléguée au dernier plan et ne cesse pas de montrer visiblement son influence.

"Mais enfin, écrit-il, les velléités de cette liberté résistante qui protestait par le caprice s'étant domptées elles-mèmes, le désaccord momentané de la maladie morale et physique ayant fait place à l'harmonie, .les deux àmes se sont retrouvées dans l'unité la plus tendre qu'elles eussent obtenue encore. Avec bien plus d'effusion, l'amour triompha cette fois, et se dit: "Je tiens l'infini. "

„Il y manquait une chose que la femme n'atteint vraiment que dans sa seconde jeunesse: c'est que, par un effort de coeur, elle sortit de l'état passif qui presque toujours fut le sien, prit action et mouvement, se fit lui, non 
plus par la sourde fatalité d'imprégnation, mais par la volonté, l'amour.

"Jusque là le trarail les séparait, et la femme avait ses heures; aujourd'hui toute heure est à elle, le jour, la nuit. En toute chose, il la sent utile et charmante; il ne peut plus s'en passer': c'est le jeune compagnon chéri, en qui il trouve le sérieux, le plaisir, tout ce qu'il vent, qui se transforme pour lui. C'est Viola, c'est Rosalinde, un doux ami le matin, femme au soir, ange a toute heure.

"Obéissante, elle a pourtant, au besoin, l'initiative: clle sait rouloir, agir. Et quand l'homme, soit en affaires soit en idées, faiblit, hésite, dans les nuits troubles surtout, où son âme agitée cherche, ne trouve, et semble ensorcelée, elle est lá, elle sourit. Le maurais enchantement disparait ; il en rit lui-mème. Un baiser lui rend les ailes.

"N'arons-nous pas obtenu ici ce que nous cherchions, l'écliange absolu de l'être? L'amour n'a-t-il pas l'infini ? Que la faible femme ait reçu, pris si bien l'àme de l'homme, qu'elle puisse, au besoin, la lui rendre, et que, dans la défaillance du génie viril, elle hui donne ce qu'elle n’a pas, l’étincelle génératrice, ne semble-t-il pas que ce soit le miracle de l'unité?

"Non, celle-ci peut encore se resserrer d'un degré : c'est quand tous deux se reucontrent dans une idée de bonte, sattendrissent dans la surprise d'avoir tellement le nème creur, fuand l'anour et la pitié nuclés coulent en donces larmes, c'est lo moment de fusion, oì l'amour trionphe invincible, oil l'ime renouvelle les sens, où, sonvent flus vif quan jeune ige, revient l'aiguillon du désir.

"La lonté ! oh! quelle grande chose! Tout le reste est secondaire: gradce, esprit, raison, tout cela ne vaut flue fial clle. Nome senle, elle est toute puissante. Il n'est fits rare yutoll resire nue femme paree qu'elle est bonne, 
et sans aucune autre raison. Profonde harmonie de notre être ! Il ra, par les sens aux choses deccur, il tend, par l'union phrsique, à atteindre, à posséder la suavité morale qui est lá. On y sent Dieu. C'est pourquoi on veut s'unir n 1.

Comparez cet amour dans ses formes supérieures à celui des contes de La Fontaine et des petites histoires de la Vie parisienne. Abstraitement, la tendance essenticlle - qui est elle-mème une association systématique est partout la même, voyez comme le sentiment qui en résulte varie avec les nouveaux éléments qui s'y associent, la domination de l'un ou l'autre d'entre eux (tendances intellectuelles, sensualité, amour-propre, etc.) et les inhibitions qui en résultent. Il n'est pas douteux que les lois que nous aronš ètudiées ne se manifestent ici, et, avec le jeu des éléments psychiques et des circonstances qui les farorisent ou les entravent, elles expliquent tout. J'ai dù me borner à indiquer en gros, sauf pour un ou deux cas, l'interprétation, mais elle est facile a trouver pour le reste, et ne parait pas douteuse.

Il est difficile de ne pas dire quelques mots de la pathologie de l'amour: elle nous montre des faits très intéressants, et nous fait mieux comprendre le mécanisme de l'état normal, en nous montrant ce qu'il devient quand certains éléments y manquent et qu'ils sont remplacés par d'autres au détriment de l'harmonie génćrale de l'esprit. Toutes les déviations, toutes les anomalies, toutes les perversions de l'instinct sexuel sont encore en soi des formes d'associations systématiques - nous voyons, soit dans ce que M. Binet a appelé le fétichisme dans l'amour ${ }^{2}$, une importance excessive donnée à un élément secondaire, qui devient, en quelque sorte le centre du

1. Michelet. Ouvrage cité, p. 431.

2. Binet. Le Fétichisme dans l'amour. Revue philosophique, 1887, tome II. 
système ; soit dans l'amour contre nature une substitution de certains éléments à d'autres dans la coordination psychique, avec l'inhibition des tendances normales; soit dans l'interversion des sexes, la désharmonie entre la nature des organes et l'orientation psychique ${ }^{1}$. L'association systématique, la sélection et l'activité des systèmes psychiques expriment toutes ces variétés.

\section{$\S 4$.}

Au lieu de l'amour nous aurions pu prendre un autre sentiment, l'ambition, la gourmandise, le résultat eùt été le même, et l'analyse semblable - ou bien cncore une autre forme de la vie mentale, le langage, ou la religion.

On trouve dans le langage une associalion systématique entre une idée, un sentiment, une tendance, une perception, une forme quelconque de l'activité psychique et le mot qui sert à l'exprimer - ce mot implique luimême une association d'images visuelles, d'images auditives, d'images motrices, phonétiques et motrices graphiques qui toutes convergent vers un même but : déterminer chez deux personnes différentes des états d'esprit analogues et à quelques égards identiques par l'intermédiaire des appareils de la phonation et de l'audition et des vijurations de l'air. Que ces associations varient d'une personne a l'autre par stite de la prédominance de tel ou tel élément sensible, c’est ce qui est bien établi aujourd'lui 2

1. Voyez outre les ouvrages de patho!ogie mentale déja cités: Magnan. Lerons sur les maladies mentales. - Moreau de Tours. Les aberrations du sens gendsique, etc. - On trouvera un bon résume de la question, par M. Gley, dans la Revue philosojhique. T. I. 1884.

2. Voyez en prarticulier les travaux de M. Charcot et de ses élèves: laallet, Le languge interieur et l'aphasie. Feré, Les troubles de l'usage des signes, hevue plilosophique, 1885. Bernard, De l'aphasie, et mon travail sur le Langage interieur et la pensede, Revue philosophique. Prour l'étude particulière des images auditives voir: Egger, $L a$ purole intivicure. Pour les innages motrices, Stricker, Ltudes sur le langage et la musique. 
Les différences individuelles correspondent à des différences dans les associations systématiques qui constituent le langage chez différents individus : chez I'un les idées sont plutôt associées à des images auditives, chez l'autre à des images motrices, chez dautres à des images visuelles chez d'autres encore, dans la parole intérieure, à des images abstraites. Remarquez également la complication de l'association systématique des lettres pour former les mots, des mots pour former des phrases, des phrases pour former un discours ou un livre - et pensez à ce que suppose d'associations systématiques entre des éléments moteurs graphiques, auditifs ou moteurs phonétiques ou visuels, et des idées, le fait seul d'écrire un livre où tous les éléments tendent à exprimer des idées harmoniques. L'inbibition ne se manifeste pas moins que l'association systématique - tous les éléments qui entrent dans les innombrables combinaisons qui viennent d'être indiquées ont leurs tendances qu'il faut retenir jusqu'à ce qu'elles puissent s'employer utilement. Quand nous traçons une phrase, les mots qui suivront celui que nous écrirons sont présents à l'esprit et tendent à s'associer aux images motrices qui accompagnent l'acte d'écrire - quand nous écrivons un mot les lettres qui suivent celle qui nous occupe tendent aussi à passer avant elle - et nous savons que la moindre inattention, la moindre négligence permet à ces tendances de se manifester, de là viennent bien souvent des hésitations, des lapsus, des oublis de mots ou de lettres, des confusions, qui mettent en lumière l'inhibition qui se produit normalement et qui cesse de se produire en ce cas. Les associations par contiguïté et ressemblance ne sont pas rares non plus mais elles caractérisent plutôt les déviations du langage, par exemple, quand on prononce un mot pour un autre, - les deux mots ayant quelques éléments communs - ou bien quand un mot entraine à sa suite un mot qui lui est ordinairement 
associé mais qui, cette fois, ne vient pas à propos; quant aux associations par contraste dont nous avons vu des cxemples pathologiques cliez des personnes qui disent juste le contraire de ce qu'elles veulent dire, elles se trouvent parfois a l'état normal chez celles qui emploient volontiers, pour une raison ou pour une autre, le mode ironique de parler ou d'écrire, clles constituent ainsi certaines figures de rhétorique. On aurait entièrement exprimé la nature du langage si on avail énuméré tous les éléments psychiques essentiels qui y prennent part, et indiqué les associations systématiques de ces éléments et les inhibitions excreées par clles - arec les associations par contraste, ressemblance et contiguïlé qui viennent en compliquer le jeu - on l'aurait entièrement expliqué si on pouvait donner l'origine de ces différentes combinaisons. Il serait chimérique d'cxiger actuellement de la psychologie des explications complètes et mème des analyses qui no laissent plus rien à désirer: toutefois si nous ne pourons développer ici la question, rappelons au moins que de récentes recherches ont permis de préciser beaucoup plus le mécanisme du langage, et que si son origine première, malgré de hrillantes tentatives, reste encore a bien des égards incertaine, on a pu au moins reconnaitre l'origine et les variations de certaines formes verbales, et aussi du sens des mots et ramener it des lois une grande partic des faits olservés. Dans les cas où une interprétation psychologique est possible, elle se ramene, je pense, aux lois et aux faits généraux que nous arons indiqués. On peut étudier ì ce point de vue lia transformation du sens des mots sur laquelle de hons travaux ${ }^{1}$ out appelé l'attention. Le not "ferrer" par exemple, arrive à changer considérablement de sens

1. Darmesteter. La vie des mots etudiée dans leur signification. Voyez aussi: De quelques bizarres transformations du sens dans 
dans l'expression " un cheval ferré d'argent, " - examinons cette locution a notre point de vue : le mot ferrer signifie d'abord garnir de fer, dans l'expression ferrer un cheral, sa signification se complique et se particularise ; le mot s'associe à un complexus d'images précis et bien défini, il éveille l'idée d'un morceau de métal de forme particulière que l'on fixe au sabot du clieval dans un certain but d'utilité, pour éviter qu'il ne glisse et pour éviter aussi l'usure de la corne. Dans le complexus d'images et de tendances éreillées par le mot ferrer, on voit que la nature propre du fer devient un élément secondaire, ce qui est important c'est la place du fer, c'est son rôle utile, non sa constitution chimique, un autre métal peut se substituer au fer sans que le complexus d'images soit essentiellemeṇt différent, et le mot amenè d'abord par l'élément fer, mais qui s'est étroitement associé arec le srstème d’images avec lequel il a été mis en rapport - ce qui est conforme à la loi d'association systématique, - finit par ètre associé à la forme du système, à son but, à sa fin, plutôt qu'à tel ou tel de ses éléments, mème à celui qui l'a introduit dans sa nouvelle association, il finit par ne pas ètre plus ètroitement associè à la nature propre du fer qu'il ne l'a jamais été au mot cheval il désigne une opération abstraite, c'est-à-dire une opération dont certains éléments peurent varier pourvu que la forme essentielle reste la mème et l'on dira " un cheval ferré d'argent o presque aussi bien que l'on dira "ferrer un ane. " Le mot ferrer est associé d'abord à l'image du fer, puis étant employé dans des

certains mots. Rerue philosophıque, 1876, n०1. M. Bréal, L'histoire des mots, Revue des Deur-Mondes, juillet 1887, G. Paris. La vie des mots, Journal des sarants, 187\%. Littré, Comment les mots changent de sens, publié d'abord sous le titre de Pathologie du langage dans Etudes et Glanures. Cf. Revue critique 1888 , tome II, 
conditions très spéciales où le fer jouc un rôle important; il s'associe à ces conditions si bien que l'élément peut y ètre remplacé par un aulre sans que le mot se sépare du système. L'association systémalique s'accompagne d'une inhibition corrélative, car dans l'expression ferrer un cheral, le mot ferrer n'éreille pas toutes les images auxifuelles il pourrait ètre associé, mais seulement un complexus parliculier, et dans l'expression " un cheval ferré d'argent » la tendance du not ferré à éveiller l'image ou l'idéc du fer est elle-mème inhibée en vue de la précision plus grande du sens, par l'introduction dans le systeme du mot argent, remarquons que l'ensemble du phénomène a la forme d'une association par' ressemblance. Voici un autre excmple: "Mouchoir, du sens primitif, pièce d'étoffe qui sert à se moucher, arrive au sens de pièce d'éloffe que l'on croise autour du cou." Comment s'est faite cette transformation? M. Darmesteter nous l'indique: Le hasard de nos habitudes veut que l'objet arec lequel on se mouche " soit une pièce carrée d'étoffe, soie, fil, coton, etc.. De lá, par oubli de la destination, l'idée de se moucher, et par considération mique de la nature et de la forme de loljet le mot mouchoir s'applique à des pieces d'étolle du mêne genre: se metlre un mouchoir autour du cou. Le mouchoir que les femnues mettent autour du cou retombe en pointe triangulaire sur deurs épaules. Considération d'un noureau caractire: de là le sens de mouchoir que le mot prend dans la laugue de la marine: "pièce de bois trian.

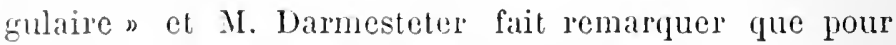
désigner la piece d'étolfe mise autour du cou on comnence à dire a mouchoir de cou » puis, par réaction, quand on veut prendre le not dans sil signification étymologique qui n'est plus sentie, on ajoute de poche»l.

1. Larmesteter. Ouv, cite, p. i6 et 130. 
Nous voyons encore ici que le fait consiste essenticllement en des associations systematiques différentes se formant autour d'un mème élément, (mot, complexus d'images audıtives, motrices ou visuelles) associé à des éléments communs à deux de ces systèmes et variant d'un système à l'autre. L'élément commun au système mouchoir, pièce de bois triangulaire, et au système mouchoir, pièce d'étoffe croisée autour du cou,est une image visuelle ou une idée de forme - l'élément commun aux systèmes mouchoir, pièce d'étoffe qui sert à se moucher et mouchoir, pièce d'étoffe que l'on croise autour du cou est' le complexus d'images visuelles, d'images tactiles ou d'idées qui constitue la représentation d'une pièce d'étoffe. On voit donc un élément qui entraîne le mot associé avec un système dont il fait partie dans une nouvelle association; c'est un exemple de l'activité des éléments psychiques, mais cette activité n'est généralement pas incohérente, le passage d'un système à l'autre s'explique souvent par des raisons de commodité, d'opportunité, de force d'une image, etc., - en tout cas les deux systèmes pris chacun à part sont manifestement coordonnés et convergent de toutes leurs parties vers une même fin. C'est ce qui s'obtient, en général du moins, et qui ne peut ètre obtenu que par une inhibition des tendances du mot et de l'élément qui l'accompagne à s'associer aux autres complexus dont il fait partie - et spécialement par l'oubli définitif ou temporaire des autres significations du mot. L'activité des éléments psychiques, les raisons qui les favorisent et les lois de l'esprit que nous avons examinées suffisent donc à exprimer le phénomène. On peut généraliser ce mode d'explication et l'appliquer non seulement à la formation, à l'érolution et à la disparition du sens des mots, mais aussi a l'érolution des formes. Appliquez par exemple ce qui vient d'être dit à la transformation qui.du latin vulgaire a fait sortir notre français, vous $y$ voyez de 
beaux exemples d'associations systématiques, d'éléments dominateurs, de concurrence et de sćlection, d'inhibition dans les lois qui expriment la persistance de l'accent latin, la cliute des royelles brèves qui précèdent immédiatement la voyclle tonique, et la disparition de la consonne médiane; rous retrouverez des opérations psychiques analogues par leur principe psychologirue essentiel, mais bien différentes par leurs formes concrètes et par les résultats philologiques dans la formation actuelle de mots norveaux ${ }^{1}$.

Ainsi les diverses parties synthétiques de la vie de l'esprit que nous examinons à notre point de vue nous paraissent toutes réductibles aux formes de mentalité que nous avons étudiées - il nous faut maintenant faire au même point de vue une étude plus complète et rechercher la manifestation de nos lois dans le développement concret d'une personnalité. C'est ainsi que nous pourrons le moins imparfaitement parvenir à comprendre dans sa forme concrète et synthétique le fonctionnement et l'érolution de l'esprit par l'effet des tendances des éléments psychiques, et des lois de l'activité mentale, que nous pourrons nous faire l'idée la moins incomplète. de l'esprit.

1. Voyez A. Darmesteter. De la creation actuelle de mots nouveaux dans la langue française et des lois qui la régissent. 
CHAPITRE II.

Synthèses générales. - La formation de la personnalité.
- Darwin.

Nous arons tous pu remarquer en nous observant rousmèmes, en observant les personnes qui sont en relation avec nous, comment les personnalités se forment - on voit une ou plusieurs tendances nées arec l'individu, croitre, s'annexer des tendances secondaires, en faire disparaitre d'autres, s'harmoniser entre elles ou se combattre, arriver enfin vers l'àge adulte à former un tout plus ou moins unifié mais mieux organisé à coup sủr, qu'il ne l'ètait d'abord (sauf dans les cas de dissolution) ou du moins, mieux organisé à certains égards, c'està-dire plus entièrement harmonisé en vue de certaines fins. Chez telle personne c'est le sens des affaires qui se développe, chez telle autre le goùt des arts, et à mesure que la personnalité se forme, les tendances dominantes dominent de plus en plus, les tendances opposées sont normalement de plus en plus affaiblies et inhibées. Chez Sainte Thérèse, par exemple, on roit le sentiment religicux se montrer de bonne heure, ètre partiellement éclipsé par des liabitudes de vie mondaine, entrer en lutte avec d'autres formes très vires de la sensibilité, puis peu a peu se fortifier par sa propre influence et, avec l'aide de certaines circonstances, finir par absorber presque en totalité les forces psychiques, et en particulier cette sensibilité affective, si forte chez Sainte Thérẻse et qui, après avoir paru enrayer quelque temps la marche de la tendance principale, semble au contraire être venue la fortifier en lui donnant un caractère de viracité, de 
force, de tendresse, de passion, qu'elle n'a pas communément ou tout au moins en augmentant ce caractére. Mais nous avons un exemple plus récent oì nous pouvons étudier arec profit cette formation d'un esprit, ce développement d'une forme particulière de l'association systématique qui finit par détourner vers elle presque toutes les lorees psychiques, presque toutes les forces vitales, fait disparaître ce qui la contrarie et arrive ainsi à l'unité plesque parfaite de la personnalité. Les publications récentes qui ont été faites sur la vie de Charles Darwin, nous permettent d'étudier en lui le développement de l'esprit scientifique en général, de son goût pour la science et de la forme concrète que prirent ses tendances : de ses idées et de ses théories principales. Il est bien entendu que nous laissons forcément de côté, dans cette étude, une partie de la personnalité de Darwin, quelques-unes de ses sous-personnalités : le père de famille, par exemple, l'homme tendre, bon et doux, que nous entreverrons à peine, mais, c'est en somme ce qu'il y a en lui de plus important, au point de vue de l'humanité que nous étudierons, et d'ailleurs la personnalité de Darwin était trés unifiée et les qualités de l'homme privé paraissent aroir été en lui celles du savant, s'exerçant sculement dans un autre ordre de faits.

Darwin naquit à Shrewshury, le 12 février 1809. Son enfance ne paraît aroir eu rien d'absolument remarquable - du moins aux yeux des personnes dont l'opinion nous est parvenue. Cependant ce qui devint plus tard sa tendance dominante se manifesta de tres bonne heure et l'on remaryua bientôt en lui un grand intérêt pour les plantes, les animaux et les collections. Au printemys de 1817, il fut envoyé comme externe à une école do Shrewshury, oil il resta un an. "J'ai entendu dire, raconte-t-il, que j'apprenais beaucoup plus lentement fur: ma plus jeune soeur Catherine, et je crois qu'a 
divers points de vue j'étais un méchant garçon. A l'époque où j'allais à cette école, mon goùt pour l'histoirc naturelle, et plus spécialement pour les collections, ctait bien déreloppé. J'essayais d'apprendre le nom des plantes et je collectionnais toutes sortes de choses: coquilles, sceaux, franchises postales, médailles, minéraux.

"L'amour de la collection qui amène un homme à être un naturaliste systématique, un virtuose ou un avare, était très ancré en moi ct était incontestablement inné, d'aucun de mes frères ou sœurs n'ayant jamais possédé ce goùt» ${ }^{1}$. Une note de M. F. Darwin confirme, d'après le témoignage d'un ancien condisciple de son père, le goùt de ce dernier pour l'histoire naturelle.

Il est curieux de voir ainsi le premier genre des tendances qui domineront plus tard la vie d'un homme; ces premiers germes sont puissants, mais mal organisés : on trouve déjà dans le caractère, dans l'intelligence, quelquesuns des éléments, dont la combinaison et l'influence formeront plus tard l'unité de la personne, mais qui sont mal diffèrenciès encore - le goût des collections, par exemple, se porte sur des sceaux comme sur des coquilles, sur des franchises postales comme sur des minéraux, de plus, ce goùt á lui seul ne décèle en rien la supériorité de l'esprit. Tout dépendait de la manière dont il s'appliquerait un jour, c'est-à-dire des autres éléments psychiques-dont il caractériserait l'association systématique.

A côté de ces germes qui se développeront, il en est d'autres qui sont destinés à s'atrophier, ou au moins, à se subordonner complètement ; les formes concrètes de l'association psychologique se transforment. Darwin enfant, était,

1. La vie et la correspondance de Charles Dawwin, avec un chapitre auto-biographique, publiés par son fils Françis Darwin. - Traduction française par Henri-C. de Varigny. 
à ce qu'il nous dit lui-même, " porté à inventer des mensonges de propos délibéré et toujours pour le plaisir de faire sensation '. "In fait nous montre ectte tendance associéc à l'intérèt qu'il portait à l'histoire naturelle. "Je racontai, dit-il, á un autre petit garcon, je crois que c'était à Leighton, qui devint par la suite un lichénologue et un botaniste bien connu, que je pouvais produire des polyanthus et des primevères de teintes diverses en les arrosant avec certains liquides colorés. C'était naturellement une fable monstrueuse, et je n'avais jamais expérimenté la chose. " Pour juger à quel point son désir de faire de l'effet s'était discipliné, combien son imagination avait appris à être dirigée par la raison et l'expérience, et conbien sa tendance au mensonge avait disparu; il suffit de se rappeler qu'il a passé vingt-deux ans de sa vie a développer, à critiquer et à rédiger l'Origine des espèces. A notre point de vue, il y a là une forme intéressante d'inhilition, son imagination n'avait pas faibli, il continuait à imaginel des théories, des faits extlaordinaires, il faisait ce qu'il appelait avee une sévérité lien excessive "des expériences d'imbécile ", mais ces idées, ces théories ne s'associaient pas étroitement à ses idées acquises sur la réalité, leur développement était enrayế, jusqu'à ce que l'expérience eùt prononcé. Il ne mentait plus ni aux autres ni à lui-même, gràce à cette combinaison d'association systématique et d'inhibition qui permet le contrôle d'une tendance par d'autres tendances, d'une croyance par d'autres croyances, et qui constitue en général le pouroir personnel - grảce, en un mot, a une systématisation supérieure de l'esprit.

De 1818 à 1825, de neuf à seize ans, Darwin alla at Shrewsloury, a la grande école du docteur Butler ; il y fut

1. Ouvrage cite., t. I, p. 32-33.

2. Idem., p. 33 . 
pensionnaire, tout en s'arrangeant pour faire à la maison paternelle de fréquentes visites, et y reçut l'instruction classique dont il ne paraît pas avoir gardé un bon sourenir. Nous avons ici une occasion de mieux connaître les qualités propres de l'intelligence de Darwin en voyant celles qu'il ne possédait pas. « L'école, en tant que moyen d'éducation pour moi, fut..... un simple zéro. J'ai été incapable toute ma vie durant, de vaincre les difficultés d'une langue quelconque. On nous appliquait spécialement à faire des vers, et je ne pus jamais réussir... Le seul plaisir que j'aie retiré de ces études m'a été fourni par les odes d'Horace, que j'admirais beaucoup »1. Il travaillait bien pourtant, et quand il quitta l'école, il n'était ni en avance ni en retard pour son àge. "Je crois, dit-il, que mes maitres et mon père me considérèrent comme un garçon fort ordinaire, plutôt au-dessous qu'au dessus du niveau intellectuel moyen ». Il faut signaler, cependant, un goùt prononcé qu'il avait pour les longues promenades solitaires. « J'ignore, dit-il, à quoi je pouvais bien songer. \Il songeait très fort en tout cas, puisqu'un jour, marchant dans un sentier sans parapets, il s'écarta, sans s'en apercevoir, de la bonne direction, et tomba d'une hauteur de sept à huit pieds. Encore ici nous voyons une autre forme mentale qui ne trouve pas encore a s'employer utilement et qui plus tard, s'appliquant à des éléments psychiques importants, leur donnẻra une allure caractéristique et contribuera à faire le savant. Nous en voyons d'autres qui s'ébauchent. « Me remémorant aussi bien que je le puis mon caractére durant ma vie d'écolier, les seules qualités pouvant être d'un bon augure pour l'avenir élaient mes goùts divers et prononcés, beaucoup de zéle pour tout ce qui m’intéressait et un vif plaisir en comprenant un sujet ou une cliose complexe.

1. Id, p. 36-37. 
Euclide me fut enseigné par un professeur particulier, et il me souvient distinctement de l'intense satisfaction que me procuraient les démonstrations géométriques» 2. D'autre part, il aimait à lire, et lisait Shakespeare, Byron et Scott. Il continuait aussi à collectionner les minéraux, “ mais sans but scientifique. Je désirais avant tout posséder des minéraux nouvellement baptisés et j'essayais à peine de les classer ». A dix ans, il s'intéressait beaucoup aux insectes et se décidait presque à former une collection de tous ceux qu'il pourrait trouver morts, " car, ditil, après avoir consulté ma sœur, j'arrivais à la conclusion qu'il n'était pas bien de tuer des insectes pour l'amour d'une collection" ".

Il avait déjà un grand nombre des élémentṣ qui devaient faire de lui un homme supérieur, mais ces éléments ne s'associaient pas, ne s'accordaient pas pour déterminer une orientation de l'esprit; il aimait l'histoire naturclle, mais en simple collectionneur; il aimait à comprendre mais ce goût se satisfaisait avec les éléments d'Euclide; il n'avait encore aucune idéc d'une recherche scientifique portant sur les insectes, les minéraux, qui n'étaient pour lui que des objets de curiosité - la coordiration mentale ne pouvait encore s'établir.

Au reste, elle ne devait pas s'établir de sitôt, en 1825 il est envoyé à l'Université d'Edimbourg pour commencer des études de médecine. On peut croire qu'il va s'y intéresser - mais non, un esprit qui n'est pas encore formé a besoin, pour s'assimiler même les éléments qui lui convienuent, que ces éléments soient présentés sous une certaine forme, faute de ruoi il les rejette ; l'anatomic le degroute et le professeur lui parait fort ennuyeux. Deus optrations anxquelles il assiste l'impressionnent iul point de le faire renoncer a suive le service de 
l'hôpital. Dans la seconde année de son séjour, il suivit des cour's de géologie et de zoologie qui lui parurent " incroyablement ennuyeux ". Le seul effet, ajoute-t-il, qu'ils produisirent sur moi fut que je pris la détermination de ne jamais lire un livre de géologie ou étudier cette science " $i$. Des faits de cette nature, qui sont d'ailleurs très fréquents, nous font très bien comprendre comment nos facultés ne sont pas et surtout ne commencent pas par être une chose abstraite, applicable à tout indifféremment, mais une forme du jeu de certains éléments psychiques. Darwin ne pouvait, évidemment, à cette époque, comprendre et apprécier la géologie que sous certaines formes; ses habitudes mentales n'étaient point assez développées, et ne s'étaient pas appliquées à un assez grand nombre de faits psychiques concrets pour trier dans l'enseignement qu'il recevait ce qui pouvait lui servir. D'autre part, le goùt de la chasse se développait en lui. Peut-être ne s'y livrait-il pas absolument sans scrupule. En tout cas, son amour des choses de l'intelligence se manifesta ici d'une singulière façon, en tàchant de s'associer à son goùt pour la chasse. "Je suppose, dit-il lui-mème, quc j'ai dù ètre à demi-honteux de cette ardeur, car j'essayai de me persuader que la chasse était presque une occupation intellectuelle : il fallait tant d'adresse pour juger où l'on trouverait le plus de gibicr et pour bien lancer les chiens ". Il paraît que cette association systématique ne fut pas facile, car elle fut remplacée par une inhibition, le goùt de la chasse finit par ètre étouffé pendant son voyage à bord du Beagle par son zèle pour les recherches scientifiques - c'est que aussi à cette époque, Darwin n'avait plus simplement un gout vague pour les occupations de l'intelligence, mais un zèle tout particulier pour un excrcice déterminé de ses

1. P. 45. 
facultés intellectuelles, pour l'observation et l'étude des animaux; des plantes; des phénomènes géologiques.

L'époque du choix d'une profession est généralement celle où une des orientations générales de l'esprit se dessine. Les éléments psychiques, les manières d'être, les habitudes mentales qui jusque lá avaient été séparées se réunissent, s'associent vers un but commun; une synthèse mentale s'organise où tout élément a son rôle spécial et où tous les éléments convergent vers une même fin, ou vers des fins harmoniques (achat et vente, plaidoiries, organisation d'une industrie, etc.). Il arrive quelquefois que chez des esprits supérieurs cette synthèse est difficile; quelques tendances fortement enracinées, mais qui n'ont pu trouver à se satisfaire sont en voie de formation, et restant encore à demi-méconnues, s'opposent à ce qu'une synthèse définitive s'établisse où elles n'auraient pas un rôle prépondérant - elles inhibent toute activité régulière imposée et sans qu'on se rende trop compte de la raison, font renoncer successivement aux diverses carrières tentées ou entrevues. C'est ce qui paraît être arrivé à Darwin, le goùt de la carrière médicale ne lui vint pas, il savait d'ailleurs que son père avait une fortune suffisante pour qu'il n'eùt pas it surmonter ses répugnances pour telle ou telle position sociale, et il ne tàchait pas de les vaincre. Son père lui proposa alors d'entrer dans l'Eglise. " $\Lambda$ juste raison, dit Darwin, il s'opposait arec véhémence à ce que je devienne un homne de sport inoceupé, ce qui semblait alors ma destinée. " C'est, en effet, ce qui arrive souvent, quand il n'arrive fas pire el cela dépend pour une bonne part des circonstances; la flànerie qui n'engage pas l'avenir et permet un exercice des facultés suffisant pour que l'ennui ne vienne pas, insuffisant pour contrarier directement les trondanees latentes dont l'organisation se prépare par une solte d'activité inconsciente, est un état assez naturel 
dans ces conditions. Darwin, du reste, sentait que son père avait raison à certains égards, il demanda seulement quelque temps pour réfléchir, au fond le parti qu'on lui offrait ne lui déplaisait pas plus qu'un autre. "D'après ce que j'avais pu penser ou entendre dire sur la question, j'avais des scrupules à l'idée d'affirmer ma foi en tous les dogmes de l'Eglise d'Angleterre. Autrement la perspective de devenir un clergyman de campagne me plaisait. Je lus avec soin On the creeds, de Pearson, et quelques autres livres de théologie, et comme je ne doutais pas alors de la stricte et littérale vérité de chaque mot de la Bible, je me persuadai vite que nos dogmes devaient être intégralement acceptés."

Pour être clergyman il fallait aller à l'Université et prendre un grade. Darwin passa donc trois années à Cambridge, et nous le voyons à la fois se développer à certains égards, et à d'autres rester invariablement stationnaire. Il y a certaines associations d'idées qui ne peuvent se former et se développer en lui - il se produit un travail d'inhibition continu. Pendant ces trois ans, dit-il " je perdis mon temps en ce qui concerne les études académiques, aussi bien à Edimbourg et à l'école. "Il étudia un peu les mathématiques, reprit les classiques et la géométrie pour se préparır à prendre son dernier grade, celui de bachelier ès-arts. a Pour passer l'examen il était aussi nécessaire de préparer les Evidences of Christianity de Paley et sa Moral philosophy. Ce travail fut fait complètement et je suis convaincu que j'aurais pu transcrire sans une faute, mais non avec la clarté de Palcy, les Evidences. La logique de ce livre et celle de sa Natural theology me procurèrent autant de plaisir qu'Euclide. L'étude consciencieuse de ces ouvrages, que je fis sans essayer d'en apprendre par cœur une partie quelconque, a été, comme je le sentis, la seule partie du travail académique dont 
mon esprit ait retiré quelque profit » 1. Ici nous voyons se former une habitude de raisonnement qui s'appliquera plus tard à de tout autres objets qu'à ceux qui l'ont développée.

Quoi qu'il en soit, son séjour à Cambridge lui fut très favorable en le mettant en rapport avec des hommes distingués qui l'associèrent à leurs travaux scientifiques. Il continuait à se livrer avec ardeur aux sciences naturelles et aussi à la chasse. Si la vocation scientifique ne se marquait pas encore très nettement, la vocation religieuse ne paraît pas s'être développée, l'orientation de l'esprit ne se formait pas. Ses occupations étaient des plus diverses, et l'on ne voit pas encore très nettement en lui le résultat, la manifestation d'une lendance dominante qui ramène tout à elle, mais bien une activité variée de tendances différentes qui se satisfont chacune de son côté, et qui donnent encore, malgré les germes de talent qu'on voit apparaître çà et là, plus d’agrément que de véritable sérieux à l'existence : « Entomolugie, équitation, chasse dans les marais, soupers, parties de cartes, musique dans la chapelle du roi, galeries du Fitz-William Museum, promenades arec le professeur Henslow, tout cela se combinait pour remplir agréablement une vie heureuse" 2 .

Malgré tout, l'esprit de Darwin se formait en attendant de se concentrer sur un sujel qui l'absorbât tout entier, et son caractère se formait aussi. Il se faisait déjà remarquer, un de ses anciens amis, M. Lerbert, parle de lui en ces termes : " Il me serait inutile de parler de ses étonnantes facultes intellectuelles, mais je ne puis terminer cette esquisse rapile el décousue sans dire - et je suis sur fue tous ses amis de college encore de ce monde, s'associerout à unoi - qu'il était le plus naturel, le plus compatissant, Je plus généreux, le plus affectionné des amis.

1. I1. p. 51 .

2. Id. p. 183. 
Ses sympathies allaient à tout ce qui est bien et vrai, il avait la haine de tout ce qui est faux, vil, cruel ou peu honorable. Il n'était pas seulement grand, mais essentiellement bon, juste et aimable" 1 . Au reste, Darwin luimême - et il n'est pas porté à se vanter - se rend compte qu'il devait avoir quelque chose d'un peu particulier, bien qu'il ne paraisse pas s'attribuer au moment où il écrit, et surtout s'ètre attribué autrefois une supériorité marquéc. " Me remémorant le passé, dit-il, j'en tire la conclusion qu'il devait $y$ avoir en moi quelque chose de supérieur à la moyenne des jeunes gens, sans quoi les hommes mentionnés ci-dessus, beaucoup plus âgés que moi et arrivés à de hautes situations académiques ne m'auraient certes pas permis de me joindre à leur société. Certes, je ne me reconnaissais aucune supériorité, et je me rappelle que lorsqu'un de mes amis de sport, Turner, qui me vit occupé à observer mes insectes, me prédit qu'un jour je serais membre de la Société Royale, l'idée me parut absurde. "

Ce moment de la vie de Darwin me paraît des plus intéressants pour nous; ses diverses aptitudes se sont développées jusqu'ici chacune pour soi, son goût pour la chasse était aussi vif que jamais ; malgré son peu de disposition pour la musique, il s'intéressait à cet art. M. Herbert nous apprend que a ce qui lui procurait le plus de jouissance étaient les symphonies de Mozart et de Becthoven avec leur ample harmonic "4. Il parlait souven "d'une sensation de froid et de frisson dans le dos produite par l'audition d'une belle musique. „ «A côté de la musique, dit M. Fr. Darwin, il avait certainement ả cette époque une grande prédileciion pour la bonne littérature, et M. Lameson me dit qu'il avait l'habitude de lire Shakespeare à haute voix chez lui et que mon

1. Id. p. 181.

2. Id. p. 185. 
père prenait plaisir à ses lectures. Il parle aussi de son intérêt, de son "amour pour les bonnes gravures, et principalcment pour celles de Raphaël Merzhen et de Müller : il restait des heures au Fitz-William Museum à regarder les épreuves de la collection."

On voit quclle grande quantité de faits psychologiques sont sysstématiquement associés en plusicurs tendances toutes importantes qui ne paraissent pas encore avoir beaucoup de rapports entre elles, ni ètre subordonnées it une tendance principale. La science à ce moment n'était pour Darwin qu'une occupation parmi beaucoup d'autres, associée peut-être plus que d'autres aux tendances fondamentales du moi, mais qui n'avait nullement accaparé les forces de l'esprit ct ne se subordonnait pas tous les. phénomènes psychiques, toutes les habitudes mentales. "A cette epoque, dit Darwin lui-même, je me serais cru fou si j'avais abandonné en faveur de la géologie ou d'une autre science l'ouverture dc la chasse aux perdrix ${ }^{1}$.

Naturellement ce goût pour la science se développe lui aussi, la lecture de Humboldt et de Herschell lui inspipèrent un zèle ardent. "Jo voulais ajouter, dit-il, si humble qu'elle frut être, ma picrre au noble édificc de. la science naturelle. $"$

En même temps son esprit se dévcloppe, son intelligence s'ouvre, sa conception des choses s'élargit, le collectionneur tend à devenir un savant. Voici un fait qui me parait intéressant comme montrant bien le travail intime qui s'accomplit dans son esprit : Il accompagnait le professeur Sedgwick dans des excursions géologiques, un jour, " pendant qu'il examinait une vieille carrière it graviers, près de Shrewsbury, un paysan me dit qu'il y avait trouvé une grande corquille usée de Volute des tropirques comme on cn voit sur les cheminées des cottages;

1. Id., p. 62 . 
et comme il ne voulut pas vendre la coquille, je fus certain qu'il l'avait réellement trouvée dans la carrière. Je racontai le fait à Sedgwick, et il me répondit (sans nul doute avec raison), que cela avait dù être jeté par quelqu'un dans la carrière, mais, ajouta-t-il, si réellement on l'a trouvée en cet endroit, ce serait une grande infortune pour la géologie, car cela renverserail tout ce que nous savons des dépôts superficiels des comtés de Midland.

"En réalité, ces lits de gravier appartiennent à la période glaciaire. Plus tard j'y trouvais des coquilles arctiques brisées.

"Mais au moment mème, je fus absolument étonné que Sedgwick ne fìt pas ravi de la surprenante décourerte d'une coquille des tropiques trouvée á la surface du sol, au cœur de l'Angleterre. Rien auparavant ne m'avait encore fait supposer, bien que j'eusse lu divers ouvrages scientifiques, que la science consiste à grouper des faits de façon à en tirer deș lois et des conclusions générales. " Nous apercevons ici bien nettement l'esprit au moment où il prend une nouvelle forme, des associations nouvelles s'établissent, la représentation d'un fait se rattache peu à peu à celle d'autres faits de manière à former un systẻme qui ne souftre pas l'introduction d'éléments hétérogẻnes, de représentations qui contrarient l'association générale établie. Les faits ne sont plus considérés en euxmêmes et pour eux-mèmes ou bien rattachés à des idées superficielles, ils's'unissent, ils se coordonnent selon une direction générale exprimée par la formule d'une loi naturelle abstraite, - une coquille, au lieu d'être admirée pour sa forme ou sa couleur, deviendra un exemple de telle ou telle faune, un signe de telle période géologique - c'est-à-dire que la représentation est rattachée à des milliers d'autres représentations réelles ou possibles et à des coordinations d'idées très complexes. Il y a là un accroissement manifeste de la systématisation de l'esprit. 
Mais la direction de la personnalité n'était pas encore tracéc, la forme de l'association systématique dominante restait encore indécise, les éléments psychiques avaient encore une vie relativement assez indépendante. Darwin avait l'àge cependant où les rocations doivent se manifester ; il fallait qu'une circonstance quelconque vint permettre it l'un de ses goùts, et ce ne pouvait guère être, semble-t-il, que son goùt pour la science, de déterminer l'orientation générale de la personnalité, ou bien que toutes ses tendances fissent entre elles une sorte d'accord tacite et qu'il se résignàt à être un bon clergyman ${ }^{1}$, soccupant à ses heures de loisir de

1. Il est assez remarquable que les deux hommes qui ont souleve peutêtre. dans notre siècle, chez le clergè et chez les croyants, l'opposition la plus vive, Darwin et M. Renan aient été destinés á l'ètat ecclésiastique. $\mathrm{Au}$ fond, il n'y a rien de bien surprenant, chacun de nous porte en lui plusieurs personnalités que les circonstances developpent, et d'ailleurs, je ne sais si un croyant de grande valeur n'est pas bien près de devenir un hérétique dangereux, il en faut si peu pour être hérétique, et quand on a commencè à l'être, qui sait si l'on s'arrêtera. Lamennais fut d'abord

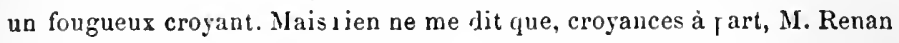
n'eút pas été un excellent évêque, et quant à Darwin, tout ce que j'ai. lu de sa vie donne cette impression, quil eût fait un admirable clergyman, de ceux dont la valeur, lintelligence, la douceur, sert a prouver la bonne influence du christianisme. Je prie le lecteur de croire que cette impression ne s'accompagne en moi l'aucune espèce de regrets. Quoi qu'jl en soit, le projet ne fut jamais formellement abandonne, il disparut pour ainsi dire lorque Darwin s'embarqua sur le Beagle, a titre de naturaliste. Sans ce voyage, pendant lequel il travailla beaucoup, observa le plus possible, recueillht les matériaux de plusieurs livres, et commença à se poser les questions qui devaient l'amener á écrire l'origine des espèces, Dar'win n'eut peut-être cultivè qu'en annateur distingué les sciences naturelles, et pour employer le temps que son ministère eút laissé libre. Comment ne pas étre disposé a le croire, quand on songe que des lihrénologues ont décidé, à la vue de la photographic de Darwin, qu'il avait "la bosse de la révérence assez développée poul dix prètres? * Le pire, est que la forme de son nez faillit empécher le voyage, le capitaine Fitz-Roy fut sur le point de le refuser comme compagnon 
science surtout, et aussi de peinture, de littérature et de musique. Heureusement une circonstance s'offrit, qui vint offrir à ses tendances favorites une admirable occasion de se fortifier et de déterminer le sens de l'orientation de l'esprit, le caractère de la personnalité définitive. Ce fut le voyage qu'il fit, du 27 déccmbre 1831 au 2 octobre 1836, à bord du Beagle "à titre de naturalistc non rémunéré. •Ici sa vocation pour les sciences naturelles passée enfin au premier plan, eut l'occasion de s'affirmer et de conquérir toute son intelligence. La force à demi latente qu'elle possédait déjà, se marque par ce fait que Darwin se montra disposé à " accepter immédiatement et avec ardeur l'offre qui lui était faite, " mais ce qui en marque les limites; c'est ceci : " Mon père, dit Darwin, s'y opposa énergiquement, ajoutant heureusement les mots suivants : "Si vous pouvez trouver un seul homme doué de scns commun qui vous conseille d'aller, je vous donnerai mon consentement. "J'écrivis le même soir et je refusai l'offre. " On sait que le voyage fut enfin décidé.

C'est l'opinion de Darwin au restc, que ce royage décida de sa vocation; il fut, dit-il, l'évènement le plus important de ma vie, et a déterminé ma carric̀re entière. "Sur le point de partir, il écrivit au capitaine Fitz-Roy : "Quclle glorieuse journéc le 4 novembre (ce devait être le jour du départ) sera pour moi. Ma seconde vie datera de cette époque, qui sera un jour de naissance pour le reste de mon existence. "

il se demandait si le propriétaire d'un semblable nez * possèderait une énergie et une détermination suffisante; pour un pareil voyage.» On voit à quoi il a tenu que les sciences naturelles ne fussent pas bouleversées, et que le nez de Darwin n'offre pas moins matière à philosopher que le nez de Cléopatre - à moins qu'on ne préfère admettre que Ja rocation de Darwin n'eút pas été enrayée pour si peu, et qu'au défaut do Darwin, quelque autre naturaliste aurait porté les mèmes coups à la croyance a l'immutabilité des espèces. Mais c'est un sujet de discussion. 
A partir de ce moment, en effet, le travail de coordination mentale s'accélère et nous apercevons aussi son développement en constatant ses effets d'inhibition. Peu à peu le zèle pour la science l'emporte sur tous ses autres goûts, dont quelques-uns étaient pourtant si vifs et si développés chez lui. "Durant les deux premiéres années, ma vieille passion pour la chasse existait presque aussi forte que par le passé, je tuais moi-même les oiseaux et animaux que je voulais collectionner, mais peu à peu, j'abandonnai mon fusil à mon domestique, car la clıasse troublcit mes travaux, surtout lorsqu'il s'agissait de reconstituer la structure géologique d'un pays. Je découvris, insensiblement et inconsciemment que le plaisir d'observer et de raisonner était beaucoup plus vif que celui des tours d'adresse et de sport " 1 , et il écrivait à Henslow en 1836 : «Depuis peu j'ai découvert que sans l'aiguillon de la science, le plaisir de visiter des pays nouveaux ne m'est rien " 2 .

Le jeu des systèmes psychiques, la lutte, la sélection, l'association systématique et l'inhibition systématisée, tels sont bien les faits généraux et les lois générales que nous apercevons dans cette genèse d'une personnalité; et nous les comprenons mieux par ce que nous dit Darwin lui-même de sa vie pendant son voyage scientifique - en même temps que nous y trouvons un effet de la coordination mentale déjà établie, nous y trouvons une cause très puissante de son développement. Darwin travaillait de diverses façons mais, dit-il, "les études variées que je viens de signaler étaient sans inportance à côté de l'habitude du travail énergique et de l'attention sontenue que j'acquis à cet exercice. Comme sujets de méditation et de lecture, je ue choisissais que ceux qui we faisaient

2. Id. ph 304 . 
directement penser à ce que j'arais vu ou à ce que je verrais probablement. Cette habitude de l'esprit dura pendant les cinq années de voyage : Je suis sùr que c'est cette discipline qui me rendit capable de faire ce que j'ai. fait dans la science " $"$.

Au retour du voyage, la personnalité était formée, elle se maintint et se fortifia; il vécut très retiré (ce à quoi contribua d'ailleurs l'état de sa santé); la science occupa sa vie entière. a Ma principale occupation et la meilleurs source de mes jouissances ici bas provient de mes travaux scientifiques, la passion excitée par ces études me faisant oublier ou chassant mes malaises. Je n'ai rien à signaler pendant le reste de ma vie (après son mariage et son installation à Down en 1842), si ce n'est la publication de mes nombreux ouvrages $n$. On sait le nombre et l'importance de ces travaux - ils ont valu à leur auteur une place parmi les plus grands philosophes naturalistes de tous les siècles. Si l'on songe que la santé de Darwin était déplorable, que " pendant quarante ans il n'eut jamais un jour de bonne santé comme les autres hommes ${ }^{2}$, " et qu'il était obligè deménager continuellement ses forces et de suspendre souvent ses occupations, on comprend combien ces obstacles à vaincre rendent admirable la grandeur de l'œuvre, et la force qu'avait aequise cette personnalité scientifique, dont nous avons vu les débuts, les tàtonnements et la formation progressive. Pour rendre compte de la grandeur de l'association systématique générale qui se manifeste dans un tel esprit, il faut remarquer aussi le nombre d'ourrages qui relèvent d'une mème habitude de l'esprit, et dont chacun suppose un nombre indéfini de recherches, d'observations, d'expériences, de réflexions, de raisonnements, qui

\section{Id. p. 66-67.}

2. Id., p. 173. 
tous convergent vers un même but et qui tous se composent d'une quantité innombralle d'éléments secondaires dont l'association systematique les rend aptes á entrer dans ces synthèses supérieures.

Naturellement l'inhibition systematisée se produit en mème temps que l'association, - chacune de ces images, chacune de ces idees, chacun de ces sentiments qui ont your fin l'observation, l'expérience, la recherche, la théorie scientifique arrète d'autres idées, d'autres sentiments qui auraient pour fin soit la chasse, soit l'émotion esthétique, soit toute autre occupation (sans parler des inhibitions qui se produisent dans le champ même de la recherche scientifique), et ces inhibitions partielles ont fini par produire parallèlement au développement des associations systématiques, des inhibitions générales systématisées - peu à peu toutes les forces de l'esprit convergent dans le mème sens, et des modes d'activité, jadis en pleine vigueur, languissent et disparaissent. Nous arons dejà vu comment le goùt de Darwin pour la chasse s'était affaibli, ses autres goûts témoignent d'une dissolution progressive analogue. L'association par contraste se manifeste de temps en temps far les regrets que lui cause la perte de telle ou telle somree de plaisir. a Il regrettait souvent que la jouissance qu'il retirait de la musique se fut affaiblic avec l'ìge », il l'écoutait encore avec quelque plaisir, mais la passion dominante avait vaincu les autres, et elle se manifestait ici par un trait bien

1. On pourrait etudier dans le mème sens la genèse des principales théories de Iarwin, pal exemple la theorie de la transiormation des espèces par le moyen de la sélection naturelle. J'ai ébauché ce travail ailleurs - et inontré que le même procédé général se trouve dans la genese de l'ceuvre d'art (en prenant, pour exemple l'histoire du roman d'Adam Bede, raconté dans George Eliot's life, by her husband G. Cross), mais la vie et la corresumdance de Dajwin n'avait pas paru a cette époque, ct il serait aujourd'hu possible de préciser bien plus que

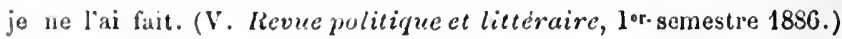


FOPMATION DE LA PERSONNALITÉ - DARWIN 501 caractéristique et que j’ai déjà signalé. " La musique, écrivait Darwin, me fait en général penser trop fortement au sujet que je viens de travailler, au lieu de me donner du plaisir » 1 . Pour ses autres goùts, en général, voici comment Darwin constate leur affaiblissement ou leur disparition :

\J'ai dit que mon esprit, à un certain point de vue, avait changé pendant les dernières vingt ou trente années. Jusqu'ả l'àge de trente ans ou environ, les poésies de tout genre, telles que les curres de Milton, Gray, Byron, Wurdsworth, Coleridge et Shelley me procurèrent un vif plaisir. Shakespeare fit mes délices, principalement par ses drames historiques, lorsque j'étais écolier. J'ai dit aussi que la peinture, la musique surtout, me donnaient d'agréables sensations. Maintenant, depuis un bon nombre d'années, je ne puis supporter la lecture d'une ligne de poésie ; j’ai essayé dernièrement de lire Shakespeare, et je l'ai trouvé si ennuyeux qu'il me dégoùtait.

- J'ai aussi presque perdu mon goùt pour la peinture et la musique..... J'ai conservé quelque goùt pour les beaux paysages, mais leur rue ne me donne plus la jouissance exquise que j'éprouvais autrefois $n$ 2.

Darwin a bien vu aussi qu'une habitude mentale avait tout envahi dans son esprit, et il arait la une belle occasion d'appliquer sa théorie de la concurrence vitale et de la sélection naturelle, mais il ne parait pas avoir considéré les choses à ce point de vue. "La curieuse et lamentable perte des goùts plus esthétiques que j'ai éprouvée, dit-il, est d'autant plus bizarre que les lirres d'histoire, les biographies et les vojages (indépendamment des faits scientifiques qu'ils peurent contenir), les essais sur toutes sortes de sujets m'intéressent autant qu'autrefois. Il me

1. Id. p. 108.

1. Ilem, page 103. 
semble que mon esprit est devenu une espèce de machine propre à extraire des lois générales d'une grande multitude de faits, mais je ne puis concevoir pourquoi cette faculté a causé l'atroplie de la partic du cerveau, de laquelle dépendent les jouissances et les goùts en question $\boldsymbol{D}$.

Son goùt pour la littérature ne disparut pas complètement, il éprouva une transformation singulière - l'émotion esthétique s'affaiblit ou s'annula, évidemment il devait aroir trop de force mentale à dépenser afin de lire ses anciens auteurs favoris, pour y prendre plaisir, mais il lui resta quelque chose de son amour pour la lecture cette tendance enrayeje sur certains points se développe à d'autres, de façon à ne pas nuire à la tendance qui avait pris la direction générale de l'esprit, et de façon à s'accorder avec d'autres tendances très fortes aussi, les sentiments de l'homme privé. Darwin se mit à lire ou à se faire lire des romans et $\mathrm{y}$ prit le plus grand plaisir il éprouvait ainsi une douce excitation de ses bons sentiments, en même temps qu'il se reposait de ses travaux et qu'il satisfaisait- les restes d'un ancien goût jour la littérature. "Les romans, qui sont des curres d'imagination, écrit-il dans son autobiographie, ceux mème qui. n'ont rien de remarquable, mont procuré pendant des annees un prodigieux soulagement, un grand plaisir, et je bénis souvent tous les romanciers. Un grand nombre de romans m'ont été lus à haute voix, je les aime tous, même s'ils ne sout bons yu'it demi et surtout s'ils finissent bien. Une loi derrait les empêcher de mal finir. ”

" Un roman suivant mon goùt, n'est une ourre de premier orlie que s'il contient queliue personuage que l'on luisse ainer, et si ce personnage est une jolie femme, tout est pour le mieux. "

Xous pourons résumer l'évolution mentale de Darwin: 
une tendance à observer, à collectionner, se manifeste dès l'enfance, elle se développe, le désir de comprendre vient se joindre au plaisir de voir et d'assembler; d'autres goùts trẻs rariés naissent aussi plus tard et se dércloppent parallelement, le goùt de la science peut d'abord ètre pris pour une aptitude moyenne comme les autres, cependant nous le royons se former de plus en plus, la conception de la science s'élargit, les habitudes mentales de coordination et de généralisation des phénoménes raturels se forment. Cette tendance à demi favorisée, à demi enrayeée par les circonstances, parait plusieurs fois sur le point d'ètre réléguée au second plan. Enfin, une occasion se présente de la faire passer au premier, l'occasion est saisie, dès lor's, pendant un temps assez long, presque toutes les occupations, toutes les recherches, toutes les méditations sont concentrées vers le même but, l'esprit s'organise, les théories générales et particulières concernant l'ensemble d'une science ou quelque point spécial, naissent et se développent, inspirent des observations, des réflexions, des expériences; l'orientation définitive de l'esprit est déterminée, les goùts secondaires s'atrophient et disparaissent ou se déforment pour ne pas nuire à la tendance dominante, qui désormais détermine à peu près seule, la direction du grand courant de la vie intellectuelle - la personnalité scientifique de Darwin est faite.

Mais il y a en Darwin autre chose que le savant, et au point de vue intellectuel, Darwin est un des esprits les mieux unifiés qu'il y ait. En réalité, pour mieux montrer la synthèse psycloologique, j'ai pris un des cas les plus simples et je l'ai volontairement simplifié encore en négligeant, de parti pris, certains côtés de la personnalité que j'ètudiais. On voit les restrictions qu'il faut apporter à la théorie de la faculté maitresse et du fait dominateur, que M. Taine a développées avec tant de force et d'éclat. Cette théorie n'est jamais complètement applicable, et 
dans les cas où elle l'est partiellement, elle s'applique au résultat d'une longuo évolution, d'une concurrence vitale et d'une sélection longtemps continuees. Il n'y a pas de personnalitẻ où tout soit harmonique, et les parties harmoniques des personnalités que nous pourons observer, se sont formées peu à peu, malgré beaucoup d'obstacles, et souvent elles gardent encore des traces de leurs luttes. Il n'en reste pas moins que la systématisation complète, l'unité de fin reste l'idéal de l'esprit - mais ce n'est ru'un idéal. D'ailleurs, le point de vue sociologique nous aile à compléter ce que le point de vue psychologique pent-être trop étroit, rendait imparfait. L'homme ne fait fras un tout à lui seul; il a besoin d'être complété par d'autres, qu'il aide à compléter à son tour. Mais il est possible, c'est ce qui arrive constamment cn fait, que le mème homme ait à remplir socialement des fonctions diverses - il est père et il est savant, par exemple, nous arons ru les inharmonies, les dédoublements qui résultent de ces exigrences sociales. Il en résulte que lharmonie et la systématisation derraient se trouver plutôt dans l'ensemble des individus que dans un individu quelconque - bien que dans l'individu mème elles puissent acquérir un très liaut degré. Disons d'ailleurs que la coordination sociale est restée aussi très imparfaite.

Ainsi, apros avoir examiné la vie des éléments psychifues, nous avons étudié les lois de l'activité mentale, c'est-it-dire la combinaison de ces éléments, et nous avons essive dexpliquer par elle toutes les formes abstraites de l'iutelligence et du caractère, la volonté, la personnalite. Passant du point de vue analytique et abstrait au point de rue coneret et syuthétique, nous arous tàche de montrer lit vie de ces elénents, et les lois de leur combinaicons analgamees dans un sentiment concret: l'amour: daus une forme importante de la vie psychique : le langatere. Enfin, nous avons étudie au nueme point de rue, la 


\section{FORMATION DE LA PERSONNALITÉ - DARWIN 505}

formation d'une personnalité donnèe. Il ne nous reste plus à présent qu’à passer du point de rue synthétique concret au point de vue synthétique abstrait, et à étudier la conception générale de l'esprit qui se dégrage de tout ce qui précède. 
LIVRE II.

La synthèse abstraite.

CHAPITRE PREMIER L'esprit comme synthèse d'éléments psychiques
et organiques.

L'étude qui précède nous permet, il me semble, de nous faire une idéc synthćtique et générale de l'esprit - et d'attacher un sens plus précis et plus complexe à cette ancienne conception : l'esprit est harmonie. C'est l'harmonie, en effet, la finalité, la systématisation, que nous avons trouvée partout dans l'esprit et reconnue pour le caractère le plus important de l'activité mentale. Mais ce concept serail resté trop vague et applicable à bien d'autres choses qu'it l'esprit si nous n'avions pas tàché d'établir quelle sorte d'harmonie était l'esprit, à quels éléments elle s'appliquait, de quelles systématisations particlles elle était la résultante et quelles formes principales clle était susceptible de prendre. Nous n'avons plus qu'ì résumer et it interpreter, au point de vue de la synthese abstraite, les faits et les lois deja cxaminés et a en tirer les conséquences qui intéressent la psychologie génèrale.

Il semble que cette activité synthétique et coordinatrice de l'esprit, reconnue implicitement par les psychologues - sans quoi ils n'auraient pu faire aucune étude psychologirque scricuse - n'ait pas été suffisamment ctudie en elle-mème, et n'ait pas pris la place qu'elle doit aroir daus la conception de l'esprit et la psychologie 
générale. J'ai déjà indiqué comment, pour cette raison, les associationistes anglais, dont les traraux ont été d'ailleurs si utiles, sont restés impuissants en psychologie synthétique : les idées et les perceptions les ont empêchés de roir l'esprit. M. Maudsley a émis des idées générales beaucoup plus justes. "L'esprit, dit-il, n'est pas une seule fonction ou faculté non composée et travaillant toujours avec la même simplicité, arec la même unité, mais une confédération de fonctions ou de facultés, qui tout en ayant leur diverses opérations subordonnées, leurs divers intèrèts, sont liées ensemble dans l'unité organique d'un tout in 1. Il n'y a à reprendre dans cette phrase que l'expression de fonction et de faculté, ce ne sont pas ou ce ne sont pas seulement des fonctions ou des facultés qui s'associent ou se combattent dans l'esprit, ce sont plutôt des touts organisés, ayant chacun leur intelligence, leur mémoire et leur volonté, des systèmes psychiques. M. Spencer a, plus que tout autre psychologue, je crois, développé une conception synthétique de l'esprit 2 . Son œuvre dans son ensemble est très belle. Mais sa loi de correspondance des relations internes et des relations externes ne paraît pas être suffisamment précisée ; elle n'exprime pas assez l'activité propre de l'esprit et la réaction de l'esprit sur le milieu; l'activité mentale consiste peut-ètre moins dans son essence à établir une relation interne correspondint à une relation externe qu'à réagir sur le milieu, de manière à déterminer une relation externe correspondant à une relation interne. Si on réfléchit un peu, on voit que c'est ce que nous faisons à propos de tout, partout ou nous le pouvons nous dérangeons l'ordre naturel des choses pour les accommoder à nos idées et à nos besoins, et les relations internes que

1. Maudsley. Natural causes and supernatural seemings, p. 156.

2. Spencer. Principes de psychologie, vol. I, partie III. Synthese générale, g. 295-415. Traduction de MM. Ribot et Espinas. 
nous établissons en conformité arec les relations externes - les idées, les croyances, l'intelligence en général - ne sont qu'un moyen d'arriver à la réaction, au réarrangement du monde extérieur ${ }^{1}$. Nous reviendrons sur ce point.

En Italie, je dois signaler la tentative hardie de M. Enrico Caporali, qui a fondé une revue, dirigée et rédigée par lui seul arec un talent distingué et un remarquable zèle, où il applique à l'ensemble des phénomènes une philosophie générale sỹnthétique, une sorte de pythagoricisme renouvelé. Ses opinions générales, malgré des divergences de détail, me paraissent pouvoir s'accorder pleinement avec la plupart des idées exposées ici 2 . Il faut rappeler aussi la doctrine de M. Sergi, qui a vu dans les phénomènes psychologiques la manifestation d'ụne loi de protection, de défense ${ }^{3}$. La théorie de M. Sergi est intéressante, mais sa conception est trop étroite - à moins que le sens des mots défense et protection ne soit trop élargi. Elle a soulevé les critiques d'un autre philosophe italien, M. E. Regalia, qui dans une intéressante brochure, a soutenu que la théorie de M. Sergi ne pouvait indiquer qu'une loi et non une cause ${ }^{4}$ des phénomènes psychiques. Le mot de Wundt: l'esprit est une chose qui raisonne, me parait sujet à la même critique que la loi de Sergi, de mème la théorie de schopenhauer, qui ne verrait au fond, dans l'esprit et dans le monde, que la

1. Il est à noter que M. Spencer, en certains passages, semble considérer les diverses classes de phénomènes psychiques comme des formes de lintelligence. Ce fait me parait venir a l'appui de ce que je dis de sa doctrine.

2. La Nuova scienza, qui paraît à Todi (Umbria), depuis l'année 1884.'

3. Sergi. L'Origine dei fenomeni psichici e loro significazione biologica. J'ai rendu compte de cet ouvrage dans la Revue philosophique. 1885 .

4. Non "Origine " ma una legge negletla dei fenomeni psichici, par l:. Regilia, Lettera al prof. Lneico Murcelli. (listratto dalla Rivista di flusofur scicntifica. Serie 2". Anno V. Vol, vi, giugno 1887). 
volonté. Dans toutes ces théories on considère surtout dans les phénomènes certains caractères généraux abstraits qui se retrouvent également dans les autres opérations de l'esprit en général et l'on applique à l'esprit entier le nom des faits particulier's que l'on a considérés. La définition ainsi obtenue a l'avantage de frapper l'esprit et de bien attirer l'attention sur ce qu'on veut dire, mais elle a l'inconvénient de donner un nom trop particulier à un mode très général d'activité et de prêter à des équiroques, car le raisonnement, comme l'entendraient les philosophes qui érigeraient en théorie la phrase de Wundt, n'est plus à proprement parler un raisonnement, et la rolonté, comme lentend Schopenhauer, n'est plus de la rolonté. Au reste, il est très légitime et souvent utile d'emplorer de pareilles métaphores en tant que métaphores.

Auguste Comte, auquel on doit tant et qu'on oublie trop, avait bien vu que la science était surtout une condition de l'action, que la systématisation générale était le but dernier de la science, de la philosophie et de la morale, mais en ce qui concerne la psychologie, ses préventions contre cette science, l'ont peut-être empêché de tirer de sa philosophie générale tout le parti possible pour la constituer. Après lui, parmi les philosophes de l'école expérimentale, M. Taine a exprimé un certain nombre de vues d'ensemble sur l'esprit, dont nous arons eu à profiter, en les modifiant quelquefois. Il a mis en relief et a mème exagérć la corrèlation des facultés dans l'esprit ${ }^{1}$.

1. On sait le parti qu'il en a tiré pour la philosophie de l'art et de la littérature. (Philosophie de l'art. Essais de critique et d'histoire, Essai sur Tite-Live, La Fontaine et ses fables, Histoire de la litterature anglaise.) II est curieux que dans la psychologie abstraite M. Taine ait eu peut-être a un moindre degré l'esprit de synthèse. Nous ne trouvons pas dans le livre de l'Intelligence, dont la valeur est d'ailleurs si considérable, toutes les lois synthétiques abstraites qu'on serait porté a y chercher. 
M. Ribot paraît aroir évité de traiter explicitement la psychologie génèrale, mais les conclusions qui se dégagent de ses divers travaux me paraissent en général tout-à-fait en harmonic avec la théorie que j'expose. Enfin tout récemment M. Richet, se plaçant surtout au point de vue de la psychologie physiologique, a insisté sur le rôle de la finalité, et M. Espinas, lans son cours à la Faculté de Bordeaux, a retrouvé la finalité dans toutes les opérations de l'esprit ${ }^{2}$. Tächons de coordonner les résultats acquis en y comprenant ceux que j'ai pu ajouter moi-mème a la science psychologique dans ce travail et dans quelques autres, et examinons les conséquences qui en résultent et la conception générale de l'esprit que l'expérience, linduction et la déduction nous conduisent à former.

\section{$\S 2$.}

L'espril est la synthèse active des éléments de l'organisme opérée par le système nerveux, de manière à determiner une coordination cosmique et une coordination sociale. Il est la synthèse systématique des conditions physiques, organiques et sociales. Telle est la conception générale qui paraît résulter de notre étude des éléments de l'esprit et des lois de l'aetivité mentale. Táchons de la rendre plus clatire et plus précise en la développant.

L'esprit est activité. La réaction qui est, avons-nous dit, sa forme principale, est préparée par la réception des excitations du monde extérieur et, de plus en plus à mesure que l'évolution se poursuit et que les formes deviennent plus élevées, par l'analyse et la synthèse plus on moins minutienses, plus on moins complexes de ces axcitations, des phénomènes qu'elles déterminent

1. J'ai eu connaissance de ces deux derniers travaux seulement après avoir à peu près arrêté les principales parties de cette étude. 
directement dans l'esprit et des éléments de ees phénomènes. Mais ces phases - réception, élaboration, réaction - qui correspondent vaguement à la division classique des facultés de l'esprit 1 , et plus exactement aux trois phases d'une action réflexe, impliquent toutes, surtout dans les formes élevées et caractéristiques la réaction qui apparait ainsi comme la partie essentielle de l'esprit.

Pour ce qui concerne la phase de réeeption, nous n'avons qu'à rappeler comment les sensations, les perceptions sont modifiées par les idées acquises, par les tendances générales de l'individu quelle différence de complexité il y a entre deux perceptions, dont l'une, ne se rapportant à rien qui nous intéresse, passe presque inaperçue - si même elle se produit, — tandis que l'autre éreille en nous des systèmes innombrables d'images et d'idées. Rappelons-nous encore une cause plus importante de l'influence de la personnalité sur la réception des excitations, c'est que nous nous plaçons toujours dans des circonstances telles que les pereeptions soient en harmonie arec la tendance qui domine, nous modifions l'ordre et la série des perceptions, de manière à recevoir principalement celles qui nous. intéressent, c'est ce que nous faisons quand nous prenons un livre, quand nous allons en royage, quand nous rentrons chez nous, etc., - il s'agit toujours d'une modification de la série des perceptions, déterminée par le besoin de satisfaire une tendance, e'est-á-dire que même la phase réceptive est déterminée par la réaction de l'organisme dans le sens

1. La division sensibilité, intelligence, volonté, a l'insonvénient de rassembler des phénomènes de nature tout-à-fait différente sous le titre sensibilité - par exemple les sensations qui sont d'ordre intellectuel au moins autant que d'ordre émotionnel - elles ne sépare pas pas suffsamment les trois phases centripète, centrale, centrifuge, de l'action psychique, et la phase centrifuge est bien incompletement désignèe par le mot volonté. 
de la șrstématisation entre les tendances dominantes et les perceptions, c'est-ì-dire entre l'organisme et le milieu.

Cette réaction est également risible dins la phase centrale - nous ne répèterons pas ce qui a été dit plus haut; rappelons que les idées ne s'enchainent pas au hasard, ni même seulement suivant leurs propres affinités, mais aussi et surtout sous l'influence d'une orientation déterminée de l'esprit, d'une tendance dominante qui rattache telle idée it telle antre, soigneusement choisie entre les nombreuses idécs que la première pourrait susciter, c'est une influence du mème genre qui détermine non sculement l'association des idées entre clles, mais aussi l'association des éléments qui composent une idée et qui sont alsstraits d'un grand nombre de perceptions et d'un grand nombre de tendances, aussi l'idée, la mème idée pour ainsi dire, l'idée d'une même chose diffère-t-elle toujours d'une personne ì l'autre, parce que les perceptions et surtout les tendances ne sont pas les mêmes. Et ce qui est rrai pour les idées et les enchaînements d'idées, est vrai pour les complexus encore moins simples. Tọjours nous retrouvons dans l'intelligence le facteur individuel. Darwin tire une sorte de conception optimiste du monde de ses théories sur la concurrence vitale et la sélection naturelle; il ne roit que les bons effets des phénomènes généraux qu'il a étudiés - d'autres en auraient tiré arec autant de raison pour le moins, une conception du monde entièrement pessimiste, c'est-i-dire que les idées éveillées par la théorie auraient été complètement différentes. Jo ne vois guère qu'un esprit infini pour apercevoir toutes les conséquences d'une theorie on d'un fait, c'est-ì-dire pour comprendre complètement un fait. Ln esprit fini, un esprit dhomme quelromrgue, voil toujours incompletement; il trie dans la robatile re qui s'aldapte a ses sentiments, à ses idées, à ses tentances, et ce qu'on peut espérer de mieux, c'est de 
voir un peu et en gros les conséquences de diverse nature qui peuvent résulter d'un fait, mais s'il est possible d'arrivcr ainsi à des idées synthétiques justes (encore ceci soulève-t-il des problèmes de métaphysique que je ne puis examiner ici) ces idées sont toujours forcément incomplètes, et, par ce qu'elles contiennent, comme par ce qui leur manque, elles portent toujours la marque de la personnalité en qui elles naissent et se développent.

En même temps les sentiments qui se mèlent aux idées et aux perceptions, les émotions, les passions, témoignent de la même vérité. Si la conception d'une chose n'est pas la même chez deux personnes, les sentiments qui l'accompagnent varient d'une manière plus visible encore, c'est que les sentiments sont, plus que les idees, trop abstraites, l'expression fidèle des tendances de chacun de nous. Un élément abstrait peut encore être le même dans deux tendances différentes, et dans bien des perceptions diverses, mais le sentiment, l'émotion, implique en général la mise en jeu d'une portion de la tendance plus concréte, plus complexe et les différences se marquent davantage. En effet, les vérités les plus alsstraites, celles des mathématiques, sont celles qui diffèrent le moins d'une personne à l'autre; encore ne sont-elles identiques qu'au point de vue logique et abstrait, car quand un écolier ou un mathématicien de génie penseiit une même formule, les deux états mentaux ne sont pas absolument équivalents, les formules abstraites n'existent pas isolément, elles éreillent toujours, d'une manière plus ou moins nette, quelques idées qui varient d'un esprit à l'autre. Si nous passons aux rérités moins abstraites la différence est plus grande, mais si nous passons aux sentiments, aux émotions qui correspondent non pas à la mise en jeu d'éléments nombreux, mais isolés, abstraits d'un grand nombre de tendances mais à la mise en jeu d'une tendance spéciale telle que la nature propre de 
l'individu, la nature de ses ascendants et les circonstances particulières où il s'est trouré l'ont faite, alors il n'y a pas de doute que la différence ne devienne plus saisissable cncore - et si ce n'est pas cncore unc vérité commune que chacun a sa manière de perceroir et de comprendre, c'en est une au moins que chacun a sa manière de sentir et d'ètre ému. Personne n'est ému cxactement par les mêmes choses que son voisin, ni n'est ému exactement de la même manière, et nous avons vu, par exemple, les différences considérables que présente un mème sentiment, l'amour, selon la personne qui l'éprouvait ou le dépeignait. Le sentiment, l'émotion, la passion, le phénomène affectif en général est donc aussi caractèristique de la personnalité, ou d'une partie plus ou moins considerable. de la personnalité, d'un système de tendances qui est seul capable de le produire exactement sous cette forme, il est lui aussi une réaction de l'organisme qui le produit.

Quant à la troisième phase, à la phasc motrice, elle est peut-être plus visiblement que toute autre sous la dépendance de la nature propre de l'individu. Nos façons d'agir, de parler, sont bien à nous. - Sans doute les actes réflexes sont moins individuels que nos actions réfléchies, il y•a en nous un certain nombre de tendances qui, comme on l'a dit, paraissent se rapporter plus à l'espèce qu'à l'individu - cependant, examinez de près, vous trouverez encore des différences individuelles, phus on moins de vivacité, de régularité, d'adresse, les fonctions orğaniques clles-mêmes sont tres variables d'une personne it l'iutre, le nombre des mourements du conr varic romme anssi la fréduence de l'inspiration et anssi la rapilité et la facilité de la digestion. Que seralce si nous nous élevons un peu plus haut sul l'échelle des reactions? les gestes les plus involontaires, les altiudes favoriles, la démarche, caractérisent un individu, et l'on a toute, non sans quelque succès, de 
reconstituer la nature intellectuelle et morale d'une personne d'après quelques lignes de son écriture: d'une personne à l'autre rien ne paraìt absolument identique. Si nous montons encore, les différences paraissent de plus en plus, chaque personne a sa manière d'ètre aimable, impertinente, grossière, polie, etc. Là même où la régularité, la banalité semble complète, l'observation fait reconnaître des différences, une originalité peu importante, insignifiante parfois, mais réelle. Erifin, si l'on regarde les manifestations supérieures de l'activité, les différences éclatent au point qu'il n'est pas nécessaire de les signaler plus longuement.

Dire que la nature de la personne se manifeste dans chacun de ses actes, c'est dire que tout se lie, s'organise en nous à quelque degré, que tout fait qui se produit en nous a son but, sa fin plus ou moins complexe et plus ou moins bien remplie, d'autant plus compliquée et d'autant mieux atteinte que les diverses parties de l'organisme, les diverses tendances de l'esprit sont mieux unifiées. Si d'ailleurs on se rappelle comment nous avons expliqué, at l'aide des propriétés des éléments pśychiques et des lois d'association systématique et d'inhibition, toutes les formes de l'activité mentale, perception, raisonnement, sentiment, volonté, on se rendra compte, à peu près, de la manière dont une immense quantité d'éléments divers viennent s'unir et converger pour constituer une personnalité, pour concourir en vue de fins déterminées par les tendances dominantes de cette personnalité, par l'orientation psychique principale, et - si l'on tient compte de ce que les éléments organiques, les, appareils de la vie de nutrition el de reproduction sont représentés dans l'esprit par des éléments psychiques, et que d'ailleurs les éléments psychiques eux-mèmes ne sont que l'activité d'une partie de l'organisme, et spécialement de quelques points de la substance grise du cerveau - on apercevra comment 
il est vrai de dire que l'esprit est un consensus, une synthèse de phénomènes organiques. Lesprit est essentiellement actif, mais ce qu'il groupe, ce sout des phènomènes physiologiques, ce qu'il ment c'est notre organisme, ou plutôt il est la loi même selon laruelle ces phénomènes organiques s'enchaînent, la forme de nolre activité.

\section{$\S 3$.}

Nous sommes ainsi conduits à examiner un peu plus longuement la question des rapports généraux de l'esprit et du corps. Le problème a été agité assez souvent - surtout il y a quelques années, et il a été parfois déclaré insoluble, parce que, jc crois, il avait été mal posé. Je n'ai pas l'intention de l'aborder ici au point de vue de la théorie de la connaissance - mais seulement au point de vue de la psychologie générale. La théorie qui a élé un. moment le plus en faveur dins l'école expérimentale, faisait du physique et du mental, deux manifestations parallèles d'une même sulystance inconnaissable ou bien deux formes différentes d'un mème fait: l'endroit et l'envers d'une même ćtofre ; le côté concave el le cớté convexe d'un verre de montre. Bain, Lewes et Leon Dumont ont défendu cette thérie; celle de M. T'aine est, a bien des égards, analogue - seulement elle donne la prépondérance au còté psychique suljectif considéré comme un texte dont le phénomène physiologique est une traduction, une apparence, la manière dont un espril se manifesterait a un autre dans certaines comlitions. Ces solutions, 'qui, mème en égard a ce qu'en atlendaient leurs auteurs, ne me paraissent pas parfailenent satisfaisantes, ne répontent nullement an jobleme tel quil est posé ici.

Il ne s'arit pas ici, en efret, de savoir quel est le rapport, ce raphort que 'T'yudall ot Dubois-Reymond onb déclaré incomatissille et incompróhensible, cutre une émotion

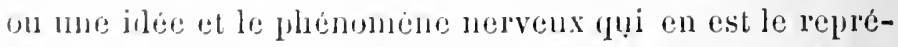
scutant physigue. Cette fuestion serait-elle résolue, la 
connaissance de l'esprit n'ên serait pas avancée de beaucoup; et d'un antre côté, nous pourrions avoir une idée très nette et suffisamment approchée de l'activité mentale au point de vue synthétique de la psychologie générale, sans qu'elle ait été définitivement résolue. Que le phénoméne nerveux soit un antécédent du fait psychique, ou un concomitant, ou une émanation de la mème substance (ce qui d'ailleurs me semble dépourvu de sens) l'idée générale que l'esprit est une sỹnthèse d'activités organiques et de conditions cosmiques et: sociales, pourrait subsister également - il n'y aurait qu'à introduire dans la formule les phénomènes psychiques, si on les. considérait comme étant essentiellement distincts des phénomènes physiologiques. La question des rapports de la conscience et du phénomiène cérébral n'est donc absolument pas la mème que celle des rapports de l'esprit et de l'organisme. Toutefois, elle se présente ici, et nous devons, en la rattachant autant que possible à notre manière de considérer l'esprit et les phénomènes psychiques, en dire quelques mots.

Je n'ai pas à développer ici les raisons qui ont conduit à donner à tout fait de conscience une base physiologique, il est admis généralement aujourd'hui que tout fait subjectif, perceptible par le sens intime, répond à un fait matériel physiologique (vibration de molécules des cellules de l'écorce cérébrales), perceptible ou qui pourrait l'être sous certaines conditions par la vue - et dans d'autres conditions, que nous n'imaginons guère, par le toucher ${ }^{1}$ : Une fois le problème posé ainsi, la question du rapport des phénomènes perçus par le sens intime avec ceux que perçoivent ou que pourraient percevoir les

1. Voyez en particulier: Spencer, Principes de psychologie, tome I. Bain, L'esprit et le corps. Taine, De l'intelligence, et Herzen. Le cerveau et l'activité cerebrale. 
autres sens, ne semule pas inpossible, ni mème peut-être très difficile à résourlre. Týndall, cependant, la déclarait insoluble, disant que nous ue possédions pas même lo rudiment de l'organe ru'il faudrait avoir pour passer du phénomene matériel au phénomène psschique.

Je remarque ici d'abord une chose singulière. On oppose couramment la matière à l'esprit comme s'il s'agissait de deux choses absolument différentes et d'ailleurs bien définies chacune de son côté. Cela se comprend dans la philosophie substantialiste où la matière et l'esprit sont des suljstances, mais il n'en est pas de même pour la psychologie positive. Ce qu'on appelle le corps est, après tout, un complexus de données de perceptions d'ordres très différents et iréductibles les unes aux autres, car je ne pense pas qu'on ait beaucoup nieux déterminé le rapport entre l'onde sonore telle que l'imagination visuelle se la figure, et l'onde sonore telle que le sens de l'oüe la perçoit, qu'on n'a déterminé le rapport de l'esprit et du corps. Dirat-ou que c'est le mème problème qui se pose? On aura pentetre raison, mats a la condition de remaryuer alors que le corrs se réduit peu it yeu it rien, cal il n'est pas doutenx yue si le son est un phenomine subjectif, une coulenr, une forme, une odeur ue le soient exactement de la mêne manière et pour une autre raison. Le problène ne lait fue clunger de forme. Evidenment les corps, quoiqu'ils yuissent ètre en cux-nuèmes et jour eux-mêmes, ne sont pour nous fue des complexus de sensations, de pereeptions, d’inages, relevant de différents sens et irréductibles l'unc it l'antre, - mitis alor's de nuême que certilins complexus de sensations visuelles el tactiles, dans contings conditions sont bgalement, ou indiquent une canse de sensation atulitive ou olfactive, pourquoi d'autres complexus de soustions visuelles el tactiles, dans d'antres conditions ne seraient-elles pas aussi ou n’indigluerationtedles pas egalenent la cause d'un phénomène 
de sens interne? En d'autres termes, si nous considérons comme faisant un tout, et constituant ou nous révélant un seul objet des perceptions qui nous sont suggéećes par la vue, l'ouie, le tact, l'odorat et le goùt, pourquoi ne considérerions-nous pas également comme faisant un seul tout des perceptions de la vue et du tact, et des perceptions du sens intime? '. Si des perceptions visuelles, olfactives, gustatives et tactiles en se synthétisant constituent pour nous une pomme, pourquoi des perceptions visuelles, tactiles et de sens intime, perçues comme celles de la pomme ou perceptibles par différents appareils et réunies eu un seul système, ne seraient-elles pas un seul tout, et ne pourraient-elles, par exemple, constituer un cerveau?

Quelle est la raison qui légitime la réunion de plusieurs perceptions en un même complexus, pourquoi puis-je considérer comme formant un porte-plume tel agrégat de perceptions visuelles et tactiles. Il y a ici évidemment des questions de finalité, de causalité, de concomitance. D'abord la sensation visuelle, ou la sensation tactile m'indique que toutes les autres perceptions qui composent l'agrégat sont renouvelables en moi sous certaines conditions déterminées, de plus ces perceptions s'unissent en un système dont tous les éléments convergent vers une même fin, - la sensation tactile, la perceptioñ visuelle, etc., sont étroitement unies et combinées en vue de l'acte d'écrire. La concomitance des perceptions, la causalité possible de quelques-unes d'entre elles par certaines autres, dans certaines conditions, l'unité finale de l'agrégat, voilà les conditions qui donnent l'unité au système de

1. J'ai développé cette idée que la perception par le sens intime est, au point de vue de la psychologie générale, entièrement analogue aux perceptions des autres sens, et suppose comme elles une synthèse, une interprétation du phénomène perçu. Voir La perception interne et la ronscience. Revue scientifique, 1888, tome $I$. 
perceptions qui constitue un oljet et nous le fait considérer comme étant bien un seul objet en réalité.

Mais quelquefois il arrive que nous ne saronis a quelles pereeptions, at quel groupe d'images rapporter telle ou telle qualité sensible qui nous arrive naturellement alsstraite it quelque degré. En fait, nos notions des choses deviennent de plus en plus concrètes à mesure que nous pouvons rattacher une nouvelle qualité à un agrégat de qualités déjí connu. Ainsi, un enfant a pu voir une flûte dans la devanture d'un marchand d'instruments sans en connaitre le son -- d'un autre côté il a pu entendre un voisin jouer de la flùte sans voir l'instrument - le son reste ainsi alsstrait, isolé, sans pouvoir être rapporté a un complexus d'images ou de perceptions visuelles, tactiles et autres; d'un autre côté, l'idée de la flùte reste aussi abstraite a quelque degré, ou, si l'on veut, moins concrète. L'expérience qui rend nos idées de plus en plus abstraites, les rend aussi, par un processus inverse et parallèle, de plus en plus concrètes, et chaque jour nous apprenons, nous découvrons de nouvelles qualités des objets rui complètent l'idée que nous nous en faisons, qui augmentent l'agrégat de perceptions, d'images et d'éléments abstraits (fue, pour nous, est cet objet.

Eht bien, nous sommes un pen, ou nous avons été longtempss par rapyort au cerveau et aux phénomènes subjectils, dans la situation de l'enfant qui aurait entendu lit flite et qui l'aurait vue, mais gui l'aurait entendue sans la voir et rui l'aurait vue sims l'entendre, qui, par suite, n'anrait pu synthétiser les diverses données des sens qui romposent l'ohijet et, si l'on trouve que le son u'est jits une pualitie essentiello de lit llùte, on peut supposer twl antre exemple (pue l'on voulla. D'un côté le cerveau a ctó coludie au moyen de l'usil et du tact, le l'autre côté los incotions, les perreptions, les idées, les sentiments ne sont ronnus comme phenomencs subjectifs que par la 
perception interne. On n'a pu encore déterminer it quel mode particulier de vibration céréhrale correspond tel ou tel phénomene subjectif, comme on a fu déterminer à quel mode de vibration d'une lame réponul le son en général, et a quel mode particulier de mourement. it quel nombre de vibrations par seconcle répond telle ou telle note. Mais aetuellement, il semble bien que nous pourons rapporter le phénomène, penséc ou émotion, à l'agrégatcerveau comme nous rapportous le phénomène son à l'agrégat lame ou vibration de corde pincée. La pensée est à ce titre une partie de l'agrégat cerveau comme le son est une partie des pereeptions dont lagrégat forme une corde de violon en vibration, comme une sensation de résistance est une partie de l'agrégät que nous appelons pierre, - avee cette différence que ce qu'on a appelé les qualités prenières de la matière diffèrent des autres parce qu'elles correspondent à des conditions plus stables, à ui mode d'activité - car toute natière est active anssi bien que tout esprit est inerte, selon le sens ru'on dome aux mots - plus durable et plus persistant ${ }^{1}$.

La pensèe est ainsi rattachée au cerveau, à lorganisme comme a son sulsstratum naturel. Le cerveau nous apparait comme domnè dans un ensenble de perceptions rariées, tactiles et risuelles smitont et, al certaines de ces perceplions visuelles et tactiles - que nous ne pouvons dailleurs avoir directement, se joignent des pereeptions différentes;

1. J'ai dî exposer brièvement toute cette conception du phénomène psychique et jai négligé plusieurs ohjections. On dira peut étre que le son est une simple apparence sulijective, que le fait objectif est le mouvement, mais le mouvement ne peut guère étre conçu que cornme un complexus de perceptions ou d'imares visuelles ou tactiles, et alors il y a quelque chose de plus permanent, de plus stable dans le mourement que dans lé son (qui ne correspond quà certaines formes de mouvements), mais, en sorme, rien de plus ni le moins objectif. La question n'avance nullement et loon ne gagne ruen à la prendre par ce cóté là. 
des perceptions de sens intime, comme à certaines perceptions visuelles et tactiles, réelles ou possibles, qui constituent un violon, vient, daus certains cas, se joindre une perception différente, celle du son. On ne peut pas préciser au juste à quelles perceptions possibles (dans des conditions impossibles d'ailleurs à réaliser) correspondent, soit la pensée en général, soit telle ou telle pensée, et pour certains autres phénomènes, du reste, (l'électricite, par exemple), la synthèse concrète n'est pas beaucoup plus avancée - mais d'une manière générale on ne peut guère nier que le phénomène psychique ne se rattache à des perceptions visuelles comme des pereeptious visuelles se rattachent elles-mêmes aux perceptions tactiles, et si nous appelons matière l'abstrait synthétique de nos perceptions visuelles et tactiles, il n'est guère douteux que la pensée soit matérielle au même titre que le son, l'odeur, le goùt, la conleur et la dureté. Elle est comme les autres données des sens, une vibration perçue d'une manière particulière qui lui donne un aspect particulier et irréductible, au moins en apparence, ì tout autre. si d'ailleurs on pense ramener toutes les pereeptions a un mème èlément, le choc nerveux, - il n'est pas douteux que les cmotions ou tout autre phènomene subjectif ne se ramènent exictement au mème élément.

Ce qui a fait aussi la difficulté de la question, c'est que les differentes perceptions ne peuvent jamais être simultanées en fait, lien qu'elles le soient virtuellement. Nous ne fonvons conmunchent ni voir, ni toucher un cerveau en activite, de plus, to phinonène cérébral ne pourrait ètre atleint patr notre esprit à cause de la grossièreté relitive ale nos sens, entin la personne qui éprouve la fereption interne, est relle rui gonrrait le moius avoir les pereptions visuclles qui hi correspondent: toutes ros lajoults ont fait fue la syulhese de la pensée et des phononenes physiologiques a eté tres longue et qu'elle 
est encore imparfaite - au lieu que le cerveau, agregat de sensations visuelles, de sensations tactiles, nous paraìt un, l'agrégat de sensations visuelles, de sensations tactiles et de perceptions internes nous parait facilement mal soudé et comprenant des choses hétérogènes. Il y a lá une vicille habitude mentale qu'il est difficile évidemment de détruire ; mais la nouvelle synthèse fait des progrès cependant ${ }^{1}$. Toutefois, mème dans l'école expérimentale, dont les doctrines cependant s'accordent parfaitement avec les idejes que j'expose ici, on retrouve des traces importantes de l'ancien dualisme. Pourquoi parler, par exemple, d'un processus à double face, l'une psychique, l'autre matérielle.? Ce n'est pas deux faces qu'il faudrait, mais bien autant de faces que nous arons d'appareils différents de perception, et le nombre en augmenterait si de nouveaux sens se formaient chez l'homme.

D'ailleurs, si nous admettons que la théorie qui prẻcède est l'expression de la réalité, nous n'en sommes pas plus avancés au point de vue de la philosophie générale qui a été celui auquel se sont généralement placés les philosophes qui ont traité la question. Nous n'arons pas cherché si la réalité dernière, la réalité rraic: dont les phénomènes seraient une apparence ou une manifestation, était une substance spirituelle, une substance matérielle, un phénomène mental, un pliénomène sonore ou lumineux - la question dernière reste intacte; je ne me suis placé ici qu'au point de rue de la science psychologique; et, à ce point de vue, il me parait légitime et nécessaire de rattacher étroitement les perceptions de sens intime aux autres perceptions qui constituent ou qui ont formé notre idée du cerveau. La comparaison de la pensẻe et de

1. Cf. Herzen, Physiologie de la volonté. - Littré, Fragments de philosophie positive et de sociologie contemporaine. - Ferrière, L'ame est la fonction du cerveare. 
l'organisme avec l'harmonie et la lyre, nous paraît après vingt siècles aussi conforme à la réalité, aussi exacte qu'une comparaison peut l'être.

\section{$\S 4$.}

Mais une fois la question des rapports des phénomènés phissiques et du phénomène psychique réglée et elle exigerait beaucoup plus de développement que je n'ai pu lui en donner ici - celle des rapports généraux de l'organisme et de l'esprit n'en serait pas comme, je l'indiquais, sensiblement plus avancée. Le phénomène psychique, en effet, n'est pas l'esprit; d'abord, les phènomènes matériels qui sont ce que nous appellerions le cerveau en activité', sont-l'esprit au même titre que lui, ensuite ces phénomènes même, l'ensemble des fonctions du cerveau ne constituent pas l'esprit 'a proprement parler. L'esprit est à la fois plus et moins que l'ensemble des phénomènes cérẹbro-psychiques qui, d'ailleurs; tous individucllement - et ruel que soit le moyen par lequel on les étudie, expérimentation, observation clinique; sens intime - peuvent être considérés comme appartenant á la physiologie.

Mais ces éléments psycho-physiologiques appartiennent a la psychologie par leurs combinaisons. "Il n'y a pas de phénomènes vitaux, disait Claude Bernard,'il n'y a que des procédés vitaux. "Tous les phénomènes de l'activité physiologique sont des phénomènes physico-chimiques. Et nous dirons de mème dans le mène sens, il n'y a pas de phénoménes psychologiques, il n'y a que des procédés psychologipres : tous les plhénomènes psychologiques sont, en somme, des phénomènes physiologiques - et de mêne, il n'y a point de phénomènes sociologiques, tous les farteur's sociologiques sont des phénoménes psychologirjues ("est-it-dire des comlinaisons de phénomènes phrsioluginges, c'est-it-dire encore des combinaisons de 
combinaisons de phénomènes phýsico-chimiques); mais il y a des procédés sociologiques, des combinaisons particulières de phénomènes psychologiques qui forment la matière d'une science nouvelle.

- La démarcation entre la psychologie et la physiologie est évidemment très peu nette, mais elle ne l'est pas beaucoup moins que la limite entre la chimie et la physiologie et elle l'est au moins autant que la séparation de la psychologie d'avec la sociologie, c'est-à-dire que les phénoménes psychologiques (l'expression est en un sens très légitime) ne sont pas beaucoup plus difficiles à différencier des faits phýsiologiques que les phẻnomènes physiologiques eux-mèmes le sont à séparer des phénomènes physico-chimiques; que les phénomènes sociaux des faits psychologiques. De même que les faits physicochimiques synthétisés d'une certaine manière sont des faits psychologiques, : de même les faits physiologiques synthétisés d'une certaine manière sont des faits psychologiques, de même les faits psychologiques synthétisés d'une certaine manière sont des faits sociaux. Une bataille entre deux grandes armées est sans nul doute un phénomène sọial - mais examinez les éléments qui la composent, ils sont tous d'ordre psychologique et physiologique, c'est un ensemble de volontés, d'instincts, de perceptions, de sentiments, de sensations douloureuses ou excitantes, etc. - de mème décomposez un phénomène physiologique, ôtez la loi; la forme qui le constitue essentiellement, vous ne trouvez que des phèromènes physico-chimiques.

Analysez aussi un phénomène psychique, vous le trouvez composé d'éléments physiologiques - prenez une sensation, vous trouvez qu'elle consiste essentiellement dans la synthèse d'impressions répétées faites sur un centre nerveux par une excitation venue du dehors par l'intermédiaire des organes des sens et des nerfs conducteurs. Les analyses de M. Taine que jai rappelées plus 
haul ue laissent aucun doute sur ce point. Il y a une multitude d'éléments physiologiques qui s'unissent et se cooldoment, prement un caractère d'unité pour constituer un phénonène nouveau, une sensation - prenez une émotion: vous y trouverezsynthétisés un grand nombre d'èléments physiologiques: mouvements du cour, monvements divers, état de divers organes, tendances diverses sont réunis et représentés dans un phénomène déterminé qui les synthélise dans un ordre spécial, cet ordre est la nature mène du phẻnomène psychologique. Une tendance comprend lorganisation de phénomènes physiologiques tres nombreux : sensations, perceptions, mouvements. Un mème mouvement (élément physiologique) peut entrer dans des milliers de tendances diverses.

Encore restons-nous ici sur un terrain mixte, oủ la limite entre la psychologie et la physiologie est impossible a tracer ; mais allons plus loin, plus haut, considérons les vais phénomènes psychologiques, ils nous apparaitront comme très distincts des phénoménes physiologiques - j'entends distincts de la même manière qu'un phénomène vital diffère d'un phẻnomène chimique, qu'un phénomène social diffère d'un phénomène psychique. Au . fond, le rrai domaine de la psychologie, e'est l'étude des combinatsons diverses de l'intelligence et du caractère. Si nous disons, par exemple, que telle persomne est bien douée pour les mathématiques ou que telle autre a le type sacerdotal, nous sortons de la physiologie et nous n'entrons pas encore dans la science sociale, mais nous constatons la maniere dont certains éléments physiologirues s'arangent, de manière à pouvoir entrer dans des combinaisons sociales; et cet arrangement particulier des phénomènes qui les rend aptes à entrer dans des combinaisons sociales, c'est l'esprit mème, de mème que le corps est une rombinaison de phénoménes plysico-chiniques groupes do firon a pouvoir entrer dans"des syntheses 
psychologiques. La façon dont les phénomènes s'ordonnent pour produire les types intellectuels et moraux, voilà vraiment le domaine de la psychologie -- les éléments de ces types sont sur une zone indécise; ils coustituent plutôt la psychologie physiologique. Ce n'est. pas à dire, au reste, que la physiologie n'ait pas son rôle dans la psychologie proprement dite; loin de là, elle apporte, elle apportera des matériaux précieux, de même que la pathologie ; mais la psychologie n'en est pas moins autre chose que la physiologie. L'esprit est une synthèse de phénomènes organiques, ou plutôt une synthèse de synthèses. Il n'est besoin que de rappeler ici comment tout phénomène psychique un peu important, exprime un état physiologique déterminé, ce que nous avons tâché d'établir en traitant de la personnalité physique, et comment aussi les états les plus èlevés de l'esprit, les plus complexes, sont des systèmes d'èléments jsychiques complexes eux aussi, et représentant, soit des tendances concrètes (sentiments, passions); soit des éléments abstraits de tendances (idées, perceptions, raisonnements). Partout le caractère qui nous a semblé le plus important, celui qui constitue essentiellement l'esprit, celui sans lequel l'esprit n'est plus; c'est la synthèse systématique, d'autant plus complexe et rigoureuse dans ses formes que l'esprit est plus élevé. Comme les éléments derniers de l'esprit sont empruntés toujours à des tendances, c'est-à-dire à des actes réflexes plus ou moins compliqués, mettant en jeu directement des appareils moteurs déterminés et, indirectement, tous ou presque tous les organes, pour jeu que l'acte soit important, c'est bien à une syuthèse d'éléments physiologiques que l'esprit, en fin de compte, doit se ramener. Et l'esprit n'est, par conséquent, ni indépendant ni séparé de l'organisme, pas plus que l'organisme n'est indépendant ni séparé des substances azotées et des corps ternaires qui le constituent 
dont il est la combinaison; pas plus que le protoplasma n'est indépendant des atomes d'azote, d'hydrogène, d'oxy. gène el de carbone qu'il contient.

"Si nous pouvions; écrit M. Fouillée, nous transporter de plus de deux mille ans en arrière chez les Grecs, au temps de Socrate et assister aux derniers entretiens du sage dans sa prison, nous entendrions ce mìme problème ile problème de la cause de nos actes ) posé et deux solutions indiquées, l'une toute mécanique, l'autie psychologique. Socrate, en effet, disait que les partisans du mécanisme universel, si on leur demandait pourquoi il était assis dans sa prison prêt à hoire la ciguë, ne manqueraient pas de répondre: c'est que les muscles de Socrate, agissant de telle manière sur ses os et sur ses membres, aboutissent a telle et telle situation de son corps. Et Socrate ajoutait: la vraic raison c'est que j'aime mieux mourir que vivre infàme et parjure o 1 . Nous pourons ici reprendre à un point de vue quelque peu différent, l'exemple cité par M. Fouillée, et qui est bien propre ànous montrerla différence entre la psychologie et la physiologie et aussi le défaut du matérialisme qui, selon la profonde vue positiviste, consiste a interprêter les phénomènes d'uns science. an moyen des phénomènes de la science inférieure. Sans séparer autant que l'a fait Comte et que l'ont fait ses disciples, les diverses sciences, on doit admettre que le défaut en question a été fréquemment une cause d'erreur. C'est celui que nous avons dù combattre en le tronvant it l'origine des théories associationistes, telles que l'école anglaise les avait formulées. Ici il se manifeste par le fait de tout ramener aux élenents sans avoir suffisamment egard à lasynthese qui les retient et leur donue un sens. ()ui, les musclos et les nerfs d'un homme étant disposes de telle et telle facon, il ne peut pas agir autrement

1. Levue des Deux-Mondes, tome 76, p. 549. 
qu'il n'agit; toutes les conditions anatomiques et pliysiologiques ètant données l'acte de courage d'un Régrulus ou d'un d'Assas s'ensuit nécessairement, mais c'est précisément cette synthèse mème qui est l'objet de la psychologie, c'est la relation qu'elle a avec les autres synthèses psychiques qui ont aussi dirige on tendı à dliriger le même organisme, e'est la série des orientations successivement ébauchécs et rejetées ou acceptées. Celui qui comparerait - le diamant au charbon, et donnerait l'un pour un morceau de même poids que l'autre, sous prétexte que les atomes qui les composent tous denx sont semblables, montrerait la mème profondeur de vue que celui qui accuserait une théorie de détruire la mocalité et l'àme, parce qu'elle ne trouve dans l'esprit et dans tel ou tel de ses actes : acte de courage on de lìcheté, de dévouement et d'égoïsme, ni substance impondérable ni indéterminisme, mais bien une synthèse de phénomènes organiques et psychiques. On n'éteint pas les couleurs de l'arc-en-ciel pour avoir prouvé que ces couleur's sont dues à l'inégale réfrangibilité de rayons qui composent la lumière blanche.

Nous avons donc indiqué en résumé comment se justifie la première partie de notre définition de l'esprit, je crois pouvoir dire qu'elle est justifiée aussi par tont ce qui précède - nous nous sommes toujours attachés à montrer le travail de systématisation qui est l'activité de l'esprit s'exerçant sur toutes les formes psychirues, lans tous les domaines de l'esprit - et toujours nous avons tàché de ramener ces éléments aux tendances, qui sont réellement l'unité psychique. L'acte réflexe, en effet, unit dans un système coordonné, rend capable de servir à une mème fin l'organe r'écepteur, le transmetleur, l'organe central, le transmetteur et l'organe moteur. La tendance est une complication de l'acte réflexe, et l'acte réflexe est le lype de l'activité mentale en mème temps que sa forme la plus simple Ramener: toute la psychologie ì l'activité synthétique des 
tendances et de leurs éléments, c'est bien montrer comment l'esprit est une synthèse de l'activité des ćléments organiques. C'est ce que nous arons essayé de faire dans tout le cours de cette étude et de résumer ici sous une forme synthétique - nous avons vu cette systématisation dans l'activité des éléments psychiques; nous l'avons vue à l'œurre par l'étude de la loi d'association systėmatique, et nous avons remarqué qu'elle se complétait par les lois de l'inhibition systématisée, de contraste, de contiguïté et de ressemblance. Voyons maintenant les autres caractères de l'esprit. 
CIIAPITRE II.

\section{Lesprit comme élément social et synthèse de produits sociaux.}

\section{$\S 1$.}

Si l'esprit est une synthèse, il est aussi un élément. Synthèse de phénomènes organiques, il est un élément du corps social. Comment l'esprit participe-t-il à la syntỉèse sociale, quel est son rôle et sa fonction, c'est ce qu'il convient d'indiquer ici, mais hrièvement, car la question relève autant de la sociologie que de la psychologie. J'ai, d'ailleurs, indiqué déjá la nature de l'activité sociale de l'esprit en plusieurs endroits de ce livre.

Les cellules et les fibres qui se forment dans l'organisme ne sont pas immédiatement aptes a remplir leurs fonetions vitales, il lenr faut un certain degré de développement pour qu'elles puissent prendre part au consensus des èléments anatomiques. De mème il faut que la jersonnalité de l'homme se soit développée, qu'elle ait manifesté ses qualités dominantes, son orientation ou ses orientations principales pour qu'elle puisse prendre part an consensus social. Pendant ruelque temps l'enfant vit d'une vie presque purement nutritive, plus tard il passe a la vie psychique, pius tard seulement il est réellement un élément actif de la société. A ce moment-là, l'homme n'est plus seulement un complexus de phénomènes physiologiques et de processus psychiques, il ne vit plus pour lui-mème, mais dans et pour un tout plus élevé et plus complexe, et ee qui caractérise sa personnalité, c'est surtout la fonction sociale qu'il a a lemplir et la 
manière dont il s'en acquitte. Et c'est ainsi que se vérifie, après la première, la seconde partie de notre définition, l'esprit est unc synthèse active de phénomènes biologiques, apte à servir d'élément dans l'activité sociale.

Que dit-on, en effet, quand on veut caractériser une cellule : on indique sa composition, ses formes et ses fonctions, et quand nous voulons caractèriser un homme? Nous indiquons son occupation habituelle et la manière dont il s'y livre, c'est-i-dire les qualités et les défauts de son caractère et de son esprit. Et chaque défaut, chaque qualité, soit de l'esprit, soit du caractère, en mème temps qu'elle indique la prédominance de certains éléments psychiques ct les formes, lentc ou vive, complexe ou simple de la tendance dominante, indique aussi l'aptitude de l'esprit à entrer dans tel ou tel complexus social, à jouẹr tel ou tel rôle dans le complexus supérieur dont il fait partie et à le jouer de telle ou telle manière. Et ces différentes choses sont indiquées, mais à un faible degré et d'une manière très vague, et parfois même complètement inexacte par la profession même de la personne dont il s'agit. De là vient qu'il faut tenir compte jusqu'à un certain point, dans beaucoup de cas am moins, mais seulement juscfu'ci un certain point, de la profession, de la josition sociale de la persomne que l'on veut connaître, et surtout du morle d'activité sociale anquel elle se livre et dont la position officielle n'indique souvent qu'une faible partie. En effel, il arrive souvent, d'autre part, que les circonstances ont empêché la vaie rocation de se déclarer et rue l'activite sociale d'un lomme ne nous indique pas pleinement et cxactement ce qu'il est, au moins au commencement de sa carrière, car, plus tard, il est possible qu'il ait fini par devenir ce qu'il parait être réellement. Il est hors de doute que la socioté laisse perdre lieaucoup de forees psychipues, beancoup d'aptitudes dont une société mieux organisee aurait pu tirer un hon parti. La 
profession est, non pas seulement le résultat de l'aptitude del'esprit à cntrer comme élément dans le toutsocial, mais en grande partie, et surtout, peut-être une des formes de la réaction de la société sur l'esprit. Ce qui earactérisc réellement l'csprit, ce qui indique l'élément social qu'il doit ètre, ce sont les formes et les aptitudes de l'intelligence et du caractère.

Chacun de nous est appelé à jouer un ròle quelconque dans la société, l'oisif même n'est pas, je ne dirai pas sans utilité, mais du moins, pour peu qu'il dépense, sans influence sur le consensus social, et cettc influence bonne ou mauvaise s'aceroit s'il se marie, s'il a des enfants, s'il a des relations ', etc. Dans une certaine mesure, ce rôle sera déterminé par la constitution mentale de chacun de nous - il n'est pas ordinaire de voir un homme exceptionnellement doué pour la poésie s'adonner a l'étude des mathénatiques, et une personnc incapable d'apprendre les mathématiques ne pourra embrasscr une carrière exigeant le passage par l'Ecole polytechnique. En cela, il y a déjả un premier fondement, un peu vague, pour une théorie des types sociaux, mais l'examen du caractère la précise un peu - ainsi la carrière militaire, la carrière sacerdotale, s'allient certainement mieux avec certaines

1. Y-a-t-il des exceptions à cette regle? En tout cas, elles sont bien rares, et on peut $y$ voir de véritables difformités psychologiques. Quelques griefs plus ou moins, légitimes et sérieus que nous ayons contre notre ètat social, nous sommes bien obligés de le prendre comme il est, sauf a tácher, dans la mesure de nos forces de laméliorer et, en attendant, d'en tirer le meilleur parti possible. Certains individus ont taché de se soustraire a peu près entièrement à l'influence du milieu social, mais on ne peut y parrenir quà la condition de se contenter d'une vie piysique, intellectuelle et morale extrèmement retrécie. Voir a ce sujet l'histoire de l'homme sauvage du Var, (V. Etude médico-psychologique sur l'homme dit le sauvage du Var, par le Dr L. Mesnet, suivie du rapport de $\mathbf{M}$. le $\mathrm{Dr}^{\mathrm{r}}$ Causse, lu à l'Académie impériale de médecine. 1865.) 
nes dispositions naturelles qu'avec d'autres. Nous voyons telle personne faire un trés bon commerçant qui eùt été un mediocre capitaine. Il est incontestable que tous les hommes n'apportent pas en naissunt, í des degrés éganx, le germe desqualités nécessaires pour ètre un bon commerçant, un bon prêtre, un bon officier, un bon ministre, malgré les opinions qui paraissent ètre actuellement en faveur. Chacun de nous, d'un autre côté, n'est pas également organisé pour être bon pẻre, bon mari, etc. De plus, chacun remplit sa fonction d'une manière assez différente el qui peut convenir à des circonstances sociales différentes aussi. Tel homme fera un bon administrateur en des temps calmes, qui en des temps de troubles restera audessous de sa tàche. Inversement, certaines personnalités qui dans une société réglée sont inutiles, sinon encombrantes ou dangereuses, peuvent, dans une crise sociale, trouver un emploi utile de leurs aptitudes et de leurs dispositions. L'orientation naturelle d'un esprit, le caractère de vivacite, de lenteur, d'olsstination, de mollesse que prend l'activité de charue personne peuvent convenil el doivent convenir a des fonctions sociales dillérentes, et c'est mème trop évident pour qüil y ait. lieu de le développer. La nature d'un esprit le rend propre âtre tel ou tel élément de la synthese sociale.

\section{$\S 2$.}

Nous avons indiqué le rôle de l'esprit dans la société, il fant etriticr un peu plus longnement peut-ètre l'influence le la sociéti surl'esprit, analogue, sans être identique it celle de l'esprit sur l'organisme, le l'organisme sur les combinaisons chimigues 1 . Il est, en eflet, certitines comfinaisons chimigues qui ne paraissent se former normalement que dans les organismes - mais alor's même que

1. I.es compraraisons entre les phénomènes qui font l'objet de plusieurs sciences et cas sciences elles-mómes paraissent indispensables. II ne s'agit 
la chimie reproduirait toutes les substances de l'ètre vivant, ce fait n'en resterait pas moins réel que c'est l'ètre vivant qui, en général, les fabrique - les plantes, par exemple, sont des appareils de synthèse qui opèrent des combinaisons inconnues en dehors des êtres vivants entre l'oxygène, l'hydrogène et le carbone. De mème l'esprit combine les phénomènes physiologiques et fait, par exemple, que le mouvement de tel muscle a une signification sociale. De même la société agit sur l'esprit, - et il ne serait pas difficile d'établir des différences très marquéés entre les phénomènes de divers ordres dont il s'agit, mais pour le moment, les ressemblances me paraissent offrir plus d'intérệt - et elle agit pour former cet esprit, pour en combiner les éléments, pour en déterminer', pour en fixer l'orientation, pour faire naître et développer certaines associations systématisées, pour provoquer certaines inhibitions, certains arrèts d'activité, êt certains arrèts de développement. Et si, dans un être vivant, les complexus de combinaisons chimiques portent la marque de l'organisme qui les a produits, si les éléments de l'organisme, la chlorophylle, la lymphe, le sang, la sève, sont des produits non seulement de telle organisation mais encore spécialement de tel organe, si de mème telle combinaison physiologique décèle l'esprit en général, et même tel esprit et telle tendance, de même les esprits portent la marque de la

pas d'ailleurs d'une confusion à ètablir entre ces phénomènes ou ces sciences. Il y a de l'use a l'autre des analogies non une identite. Quelquefois l'analogie a été forcée. Ainsi, la fameuse phrase « La société est un organisme» présente un dèfaut que j’ai dèjà relevè dans quelques autres déanitions - ou y a appliqué à deux faits différents qui présentent un caractere identique le nom particulier d'un de ces faits, au lieu d'exprimer seulement par un terme général la partie commune aux deux. Ia métaphore a d'ailleurs son utilité comme ses inconvénients. M. Espinas a évitè ce défaut en appelant la socièté, dans son beau livre sur les sociètés animales " une conscience vivante, un organisme d'idées \#, je préfèrerais encore l'expression : un organisme d'esprits. 
société dont ils sont les éléments, mais qui réagit sur eux en mille manières et - malgré les restrictions qu'on peut faire subir aux théolies qui ont exagéré l’influence sociale - il est absolument impossible de concevoir qu'un de nous, n'importe lequel, eut été exactenınt cé qu'il est s'il était né au $x_{v 1}{ }^{\circ}$ siècle au lieu de naître au $x_{1} x^{\theta}$, et en Chine, au lien de naitre en France.

Mais l'influence de la société sur l'esprit va plus loin encore - ear l'hypothèse que je viens de faire est absurde - l'hérédité, dont l'influence est certaine, est en partie caractérisée par les influences sociales qui se sont exercées sur les parents. C'est une vérité un peu vieillie peutêtre à présent que les grands hommes représentent leur temps, et pourtant on ne peut nier qu'ils le représentent en quelque chose au moins. Les trois influences étudiées par M. Taine, la race, le milieu et le moment, sont incontestables, el peut-être pourrait-on dire seulement que M. Taine a exagéré l'influence sociale en négligeant trop l'action individuelle de l'homme sur la société, action qui par l'hérédité est sans doute en partie elle-même, mais non complètement toutefois, sous la dépendance dumilieu social - et si elle ne l'est pas completement, e'est qu'une . chose n'est jamais complètement sous l'influence d'une autre et que toujours elle aura sa manière propre d'accepler celle influence et de réagir contre elle. Si la société agit sur l'individu, l'individu agit aussi sur la société, et si la maniòre mène do réagir de l'individu lui vient en lartie de l'influence sociale, cette influence sociale ellemême n'est ce qu'elle est que par les éléments psychologiques et individuels qui la composent et qui l'ont tonjours romposece.

M. Taine s'est place surtout an point de vue des diverses manifestations de l'art. Nous nous placerons, iri an point de vure de l'artivite psyeliogue en général. dors lit ronception, l'rmblryon est influencé far la 
société, par le milieu qui l'a produit; les aptitudes psychiques dont il contient le germe, ne sont certes pas identiques d'un milieu à l'autre. Mais si l'on pourait contester ce point, il n'est pas en tout cas douteux que pendant la gestation et surtout dès l'époque de la naissance, l'individu ne soit soumis a des influences sociales bonnes, médiocres ou parfois désastreuses. Sans parler des déformations que des sauvages et mème des nations civilisées font subir au cràne, en risquant de déformer l'esprit du mème coup - il est hors de doute que la manière dont les enfants sont élevés est déterminée en grande partie par les idées régnant actuellement dans la société dont ils font partie, ou plutôt dont ils feront partie un jour. De temps en temps, un esprit novateur se produit el, profitant des leçons que, sans le vouloir et sans s'en' rendre compte, lui donne la société, il tente quelques réformes et souvent tombe dans un abus opposé à ceux qu'il reut réformer et est suivi par la foule. On sait le rôle qu'a joué à cet égard Rousseau, au siècle dernier. Il se produit en petit dans des cercles plus restreints - si l'on ne suit pas un écrivain, un philosophe, on imite un ami, un parent, et l'on commence ainsi à former et ì déformer avec plus ou moins de conscience ou d'insouciance, le caractère et l'esprit qui grandissent. Bientôt l'état intervient et tàche de déterminer, sinon l'orientation du caractère et des habitudes, au moins l'orientation de l'esprit ; l'enseignement primaire, l'enseignement secondaire, l'enseignement supérieur, sont des fonctions de l'état ou tout au moins des fonctions sociales et elles ne peuvent pas être autre chose. Selon quels principes enseignerait-on, et avec quels faits et quelles consilérations meublerait-on et formerait-on l'esprit, sinon avec les principes acquis et qui dominent la société, arec les faits déjá connus et les considèrations généralement acceptées? On ne peut guẻre demander à un éducateur de refaire toutes les sciences, 
de se débarrasser de tous les jréjugés (au sens étymologique) de toutes les habitudes mentales, et de n'avoir que des idées et des connaissanees personnelles. Il est bien évident qu'une telle besogne est absolument implaticable, et qu'un seul homme ne peul pas refaire bien en quarante ans ce que l'humanité a si médiocrement fait en des siecles dont nous ignorons le nombre - sans ètre engagée par les générations précédentes. A peine s'il peut arriver, en y consacrant sa vie à se faire quelques convictions personnelles, à trouver quelques vérités, à détruire quelques erreurs - mais ces découvertes n'aulont d'influence sur l'éducation des petits enfauts et des grandes personnes, qu'en se faisant elles-mêmes, routine et préjugé 1 en devenant un mode de l'influence sociale qui, aussi bien, les a rendues possibles.

Peu à peu, le caractère se forme; ici encore, c'est en grande partie la société qui fait l'individu - par la littèrature, par les exemples proposés, par les exemples donnes sans recommandation et qui sont généralement tout différents, par la jrédication, par une foule d'autres influences, lo milieu social agit et développe ou arrête un grand nombre de ceulances, de manière a faire un composi plus on moins lammonisi en lui mème, mais capalble, at moins gue la nature frersonuelte de l'enlant ne soit deribenent trop réfractaire, de s'atapter limb bien que mal anx romlitions sociales. Des enscignements varies que lat soricte donne a l'enfant il resulte sonvent mu diveloppenent partlels de systemes pirchiques peu latrmonipues, mais tont los romblitions sociales favorisent la production simmlanéc. L'enfant pent aussi se développer surtont dans $n$ sens ou dans le sens opose, mais,

1. Cf. Ces remarquables articles de M. Tarde sur la psychologie sociale. Rie'est-ce quizne sorieté, et L'archeolngie. et l'dcononomie politipee (Revue philosophique). 
en ce cas encore, une influence sociale s'exerce, et si lè choix de l'influence dirigeante est dù en partie à l'organisme lui-même, outre que cet organisme est en partie aussi le produit des circonstances sociales passées, certaines de ses tendances sont plus ou moins arrètées ou favorisées par ce milieu, de sorte que son choix est pour ainsi dire contraint dans une certaine mesure et déterminé en partie par le milieu lui-mème. D'un autre côté, les rapports de socièté, les amitiés, exercent leur influence - et comme toutes les personnes avec qui le jeune homme entre en relation, sont formées elles aussi par le milieu social qu'elles constituent ensuite, l'influence sociale ne cesse de différentes manières selon la différence du milieu qui l'impose et de l'esprit sur lequel elle agit. Plus tard intervient un autre facteur très important, la profession qui détermine souvent pour le reste de la vie, s'il est en partie déterminé par elles, la tournure de l'esprit, les habitudes mentales et aussi, à quelques égards, le caractère de l'individu. Nous comnaissons tous des personnes à qui leurs aptitudés, soit médiocres, soit distinguées, soit supérieures, auraient laissé le choix entre plusieurs carrières et chez quila profession a déterminé l'orientation définitive de l'esprit, qui ne s'établissait pas autrement; le pli de la profession se fait peu $\dot{a}$ peu, les idses se soudent, l'esprit se forme d'une certaine manière et bien souvent, des causes purement sociales ou quelquefois fortuites, ont fait donner la préférence à telle profession plutôt qu'à telle autre et déterminé parlà, l'allure subséquente de l'esprit. Les causes sociales qui déterminent les vocations mal marquées et font pencher dans un sens ou dans l'autre, les individus que leurs aptitudes propres ne caractérisent pas suffisamment sont très nombreuses. Selon les époques, gràce à des circonstances particulières, gràce aussi à l'imitation qui est si nattkrelle à l'homme 
et qui est une forme de l'influence sociale, de la systématisation psychique et sociologigue; nous voyons telle ou telle carrière être particulièrement en faveur: la earrière militaire, politique, sacerdotale. La littérature, le théatre, nous montrent bien souvent ces tendances sociales dominantes. Le héros des pièces de théatre a été à une certaine époque, le jeune officier, puis le jeune ingénieur, plus tard, peut-être, plus volontiers le jeune savant; d'ailleurs les courants se croisent, se combinent et ne s'excluent pas absolument. On a remarqué que certains types le jeune oisif par exemple, tendent a disparaître. Au reste, sur le rôle du personnage sympathique et ses variations, on n'a qu'à consulter la Philosophie de l'Art, où M. Taine, malgré les réserves que l'on doit faire à certains égards, a magistralement développé les questions et donné une solution, sinon parfaitement juste, au moins très approchée. Les influences varient aussi selon les milieux, la famille, les relations. Il y a des savants, des ingénienrs, qui sont ingénieurs et savants parce que leur père l'a été, ou qu'ils ont un parent qui a ju leur aplanir les difficultés de la carrière. Ils sont ce qu'ils sont comme ils eussent été autre chose, si les circonstances l'eussent voulu. Enfin, il y a l'influence plus générale de la coordination sociale: bien que l'équilitıe soit assez mal établi dans les sorjetés, cependint on ne samrait nier la systématisation qai \ regne, et qui détermine un grand nomlue de phénomenes sociaux; l'activité de l'esprit des memlures dinc societé est déterminée en grande partie, non pits soulement par les desirs de ses elements, mais fratr les besoins de l'ensemble. Jone nation suffisanment organisie, troure des soldats en temps de guerre et des travalleurs en temps de paix. De temps en temps, comme nons l'avous vu cn psychologie, l'activite indefrndante des elements so manifeste et occasionne des troubles sociaux - mais tant gu'une nation dure, un 
certain consensus est la condition même de son existence en tant que nation et les activités des éléments sociaux sont inhibćes à certains égards, laissées libres à d'autres, comme cela se produit pour les éléments psychiques, et orientées dans le sens de la synthèse sociale.

Et l'influence sociale se manifeste encore de mille manières qu'il serait trop long d'énumérer - outre l'orientation générale de la grande société dont nous faisons partie, il y a l'orientation secondaire des petits groupes où presque tout le monde est engagé — religions, sociétés savantes, sociétés coopératives, sociétés pour l'amélioration de la race chevaline, etc., ctc. - sans compter les relations et la famille. Le résultat en est pour l'esprit, au point de rue psychologique, l'inhibition d'un grand nombre de tendances, le développement de certaines autres. De même que les éléments psychiques sacrifient quelques-unès de leurs associations pour entrer dans le consensus général de l'esprit, de mème font les hommes, d'une manière quelquefois réfléchie et vuulue, mais bien plus souvent automatique et inconsciente. On a pu comparer les membres de la société à des hỹpnotisés - et cette comparaison est juste à bien des égards. L'homme ne se rend généralement compte nii de l'origine sociale, ni du résultat social de ses actes. Ses actes ne sont souvent qu'une partic d'une sorte de réflexe sociologique alors qu'il croit agrir en toute indépendance et sans ètre déterminé. Et sans doutc, il agit librement, étant donné sa nature, mais c'est la socićté qui a fait en grande partie cette nature et c'est elle aussi qui bien souvent en fait jouer les ressorts, met en activité tel mécanisme, arrête le jeu de tel autre. Considèrez la même personne à vingt ans et à trente, généralement les conditions sociales de son existence ont développé certaines de ses facultés; les facultés techniques, celles qui lui servent dans sa profession, inversement un certain nombre de tendances ont 
été enrayées, telle personne d'opinions indépendantes à vingt ans a pris it trente celles de son milicu, les goùts qui ne sont pas en accord aree la profession ont souvent disparu ou se sont affaiblis - en tout cas ils se sont organisess at part et sont satisfaits it part, quelquefois en cachette, ils ne se mêlent plus comme autrefois at la personnalité tout entiere. Nous avous chez Darwin un exemple concret de ce développement et de cette atrophie de certaines parties de l'esprit et nous avons signalé l'immense quantite de faits particuliers, associations systématiques et inhibitions dont elle était l'expression définitive. Le fait se produit d'une manière plus ou moins visible pour presque tous les hommes; l'exemple concret que nous en avons étudié et qu'on peut généraliser avee les r'eserves voulues rend inutile tout développement. Remarquons le rôle que joue la société dans le cas de Darwin, ou certes les aptitudes individuelles étaient remarquablement fortes. Sams les conditions sociales qui lui ont permis d'ahord d'acquèrir une certaine somme de science, ensuite de se développer l'esprit dans la compagnie des savants qui devinèrent en lui le futur grand homme, et enfin de faire un royage dans d'excellentes conditions pour l'observation scientifique, Darwin n'cut pas été Darwin; son orientation psychique et, par suite, son rôle sucial ont été déterminés en grande partie par des conditions sociales qui, d'ailleurs, ne pouvaient produire que sur lui l'effet exact qu'elles ont produit.

Toutes les actions qui constituent l'influence sociale sont, prises a part, individuelles et psychologiques. C'est parr les pereeptions et par les systématisations qui les suivent que la société agit sur l'homme - elle détermine cului des serries coordonnées de pereptions qui déterminent naturellement, selon le mécanisme déja etudio des séries coorlonnces d'actes. 11 y at une harmonie virtuelle préćtablie par les générations qui nous ont 
précédé depuis l'origine des êtres - entre la société et l'individu - c'est par le fonctionnement de l'esprit que cette harmonie devient réelle et modific l'esprit qui est sa condition.

Ainsi nos actions, nos pensées, nos sentiments portent la marque de la société dans laquelle nous vivons. Alors même que nous pensons être en opposition complète avec notre temps et notre milieu nous nous appuyons sur lui, la réaction contre les idées qui nous ont été suggérées est encore une preuve de l'influence de ces idées - (il se produit ici une sorte d'association par contraste déterminée par une influence sociale) - on a souvent remarqué combien les hommes qui ont combattu l'un contre l'autre pour des idées cqu'ils pensaient absolument opposées, sont en somme, imbus d'un même esprit général scmblable à bien des égards et s'appuient tous les deux sur les mêmes faits, sur les mêmes idées, et mème sur des principes analogues. Dernièrement un critique rattachait Joseph de Maistre à ce xvine siécle dont il a si fortement, si âprement combattu les tendances ${ }^{1}$. Si le fond du caractère, si l'essence abstraite de l'esprit peut être la mêmc d'un siècle à l'autre, d'un milieu à l'autre, d'une civilisation à l'autre, (comme des lois plus abstraites sont les mêmes dans l'humanité entière, et des lois plus abstraites cucore dans tout le règne animal ou dans le monde entier, - et la forme générale abstraite de l'esprit correspond du reste en ce cas à une forme générale, abstraite, de la société), la forme concrètc que prend une tendance, un trait de caractère, une personnalité, les phénomènes particuliers qui en font une réalité pour ainsi dire visible et tangible, sont bien marqués par l'influence de la société dont ils font partie. On a examiné, à bien des

1. Joseph de Maistre, par E. Faguet. Revue des Deus Mondes, 15 décembre 1888 . 
points de vue, la nature du grand homme. En un sens il n'est pas contestable que le grand homme ne porte l'empreinte de l'époque où il vit - mais ce qui le fait grand ce n'est pas de résumer en lui les idées et les aspirations de son temp's, c'est qu'il fait avec les produits sociaux qui sont ses idees, ses perceptions, quelques-uns de ses sentiments, etc., des synthèses psychiques incomnues ou presque inconnues encore, c'est qu'il profite des conditions sociales pour découvrir de noureaux faits ou de nouvelles interprétations, de ṇouvelles formes esthétiques, scientifiques, industrielles, qu'il produit enfin des sýstèmes psychiques nouveaux qui, dérivant à quelque degré de l'ètat sucial, vont réagir sur cet ètat et par l'autorité, la sympathie, l'imitation, l'influence sous toutes ses formes de l'homme de génie, iront modifier dans une certaine mesure l'état psychique des autres hommes et aussi l'état de la société.

Ainsi la société existe par la synthèse des esprits et elle existe dans chaque esprit. Les éléments sociaux, unis pour la vie commune, réagissent les uns sur les autses et l'ensemble réagit sur chacun d'entre eux, comme chacun d'entre cux réagit sur l'ensemble. - Si la société vit de nous et en nous, nous vivons d'elle et en elle, c'est par elle que notre esprit existe et c'est par elle que nos actes ont un sens. Dims chacun de nos actes, ou dans chacune de nos pensées, il n'est pas malaisé de discerner non pas toutes les influences sociales qui l'ont produite, ni toutes les fins sociales vers lesquelles tend le fait psychique, et tous les résultats sociaux qu'elle doit avoir, mais au moins qucliques-unes de ces influences, quelques-unes de ces fins, quelques-uns de ces résultats.

\section{$\S 3$.}

Si an foint de vue de la sociologie, la société doit être considéré principalement comme une synthèse d'esprits, 
ainsi que nous l'avons indiqué tout a l'heure, an point de vue psyehologique, nous pouvons envisager la question par une autre face, et considérant l'esprit en lui-mème $y$ voir non pas seulement un élément social mais une ș̣nthèse de faits sociaux. Ce second point de vue n'est nullement contradictoire avec le premier, il le complète seulement, pour que l'esprit puisse ètre un élément social il est indispensable qu'il soit une synthèse d'èléments sociaux moins complexes, ou si l'on préfère cette manière de dire, une synthèse de produits sociaux.

Nous arons ru tout ì l'heure que l'esprit pouvait ètre considéré comme une syuthèse d'éléments organiques mais il n'est pas douteux que quelques-uns des éléments organiques ne soient, à quelque degré, des produits sociaux. Le cerveau par exemple, dont les rapports avec l'esprit sont particulierement intimes, et dont on pourrait dire, quand il fonctionne, qu'il est l'esprit même visible et tangible, si l'esprit ne devait pas ètre plutôt en somme rapporté à l'organisne entier quà une de ses parties, mème a celle qui coordonne les autres ${ }^{1}$, le cerveau est en grande partie un produit de la sociélé. Je n'en veux pour preuve que le développement cérébral amené par l'activite sociale systematique prolongèe, qui est la civilisation. Certaines parties, par exemple celle qui sert au langage articulé, ne sont évidemment que des produits du fonctionnement social - c'est l'influence sociale qui les a fait ce qu'ils sont. C'est elle aussi qui a déreloppé les conditions organiques de la réflexion (sous quelque forme d'ailleurs qu'on se les represente), nuais nous pouvons aller plus loin: sans rappeler ici les traces yue laissent encore sur les descendints les déformations cràniennes exercées jadis, on peut eroire que la main n'a pu devenir ce qu'elle est que par suite du mode d'activilé que les circonstances sociales ont déterminé. On peut en lire autant

1. Cf. Lewes. Spiritualisme et materialısme. (Revue philosophiqué). 
pour la forme et la capacité du cràne - et si l'on se rappelle ce qui a èté dit sur la personnalité physique, sur la corrélation particlle de toutes les parties de l'organisme et de l'esprit, on verra que tous les éléments physiologiques ressentent secondairement à quelque degré l'influcnce sociale. Mème les oróanes de la vie végétative sont certainement modifiés par la société. Il est possible aussi que Ies caractères physiques généraux de certaines races soient en grande partie sous la dépendance de certains modes d'activite d'un groupe ethnique - toutefois il y a aussi des raisons de croire que les pratiques socia. les ne déterminent pas toujours des modifications persistantes el d'aillcurs nous n'avons pas besoin de nous attacher ici à démontrer que la nature physique typique dune race est at quelque degré un produitde son mode d'activité sociologique. Il nous suffit que le cerveau soit comme je l'ai indique, incontestablement sous cette influence.

Mais si nous considérons les éléments mème de l'esprit, c'est-ä-dire le fonctionnement mème du cerveau et les produits de ce fonctionnement, la vérité devient plus évirlente encore. Tous les éléments de l'esprit sont ì quelque degré des produits socianx; pour les perceptions, il n'y a guère de doute, non seulement en ee que nos pereeptions continuelles sont - a moins que nous ne nous tronvions daus un desert - des perceptions d'oljets plus ou moins travailles par l'homme : la maison on nous demenrons, les objets dont nous nous servons, l'usine, la fabrique yuion nous fait visiter jusfu'at la campagne oì nous nous promenons, et qui n'est guère en géneral qu'une usine l'espece particuliere, une fabrique de produits spéciaux - Jes routes, les rues, le sol que nous percerous avec le pied, les mille objets divers qui nous passent far les mains, les voix, les instrmments que nous entembons, les parfuns divers jusifu'í I’odeur de lat rose, 
tout ou presque tout est un produit de l'aetivite sociale. Mais de plus, les choses qui n'ont pas éte failes par l'homme, les forèts, les fleurs, le tonnerre, nous ne les percevous qu'en complétant nos sensations par des imarges et des idées que l'activité sociale a seule rendues possibles. Notre perception du ciel n'est sùrement pas celle qu'en a la brute ou mème le sanvage ignorant. Si nous passions aux idées, aux images, aux tendances, aux souspersonnalités, à la personnalité entière, nous trouverions toujours ce même fait général, l'activité sociale entrant pour une part plus ou moins grande dans la formation de tous les éléments de notre esprit - c'est mème elle qui rend possible les instants où on l'oublie et le plaisir pénétrant qu'on éprouve à vivre quelque temps au grand air, à l'engourdissement momentané de l'esprit grisé et sainement abruti par les odeurs des prés et des bois, par la tiédeur du printemps.

Nous revenons ainsi à ce qui a èté dit tout à l'heure, il n'est pas utile que nous y insistions davantage. Si, comme je crois qu'on ne peut se refuser à l'armettre, tous les éléments de l'esprit sont des produits sociaux, l'esprit, qui est un ćlément social, est en mème temps une syuthèse de produits sociaux. Et ces produits sociaux sont en mème temps des éléments de l'esprit el des syntheses organiques partielles.

Peut-ètre aprés avoir indiqué les rapprochements de la sociologie el de la psychologie, faut-il indifuer aussi la séparation. Il est à craindre quelquefois ipue la psychologie ne soil seindée en deux, rejetée en partie d'un côté dans la physiologie, et de l'autre ramence à la sociologie. - Pour les positivistes, par exemple, les facultés psychiques étaient du ressort de la biologie, les produits : le langage, la religion, les mythes, du ressort de la sociologie. Nous tàcherons de prendre une position différente qui nous permelle, tont en sauvegardant les droits des 
deux autres sciences, 'que je pense ne pas avoir méconnus, de faire une place à la psychologie. Nous arons déjà vu en quoi cette dernière science se distinguait de la physiologie, il n'est pas très malaisé de marquer en quoi elle se distingue aussi de la sociologie. La psychologie étudie les phénomènes psychiques dans un esprit isolé, et elle étudie cet esprit mème qui en est la synthèse ; la sociologie étudie les faits et les lois qui résultent du raprochement d'esprits différents. Sans doute, ici cucore, comme entre la physiologie et la psychologie la distinction n'est pas toujour's nette : elle existe cependant. Pour prendre un exemple, la religion ou l'art, le sentiment religieux, les idées religieuses, en tant qu'ils se manifestent chez un individu, quils sont produits en cet individu par des circonstances particulières, qu'ils présentent tel ou tel caractère, sont du domaine de la psychologie. En tant yue la religion constitue une organisation sociale, en tant qu'elle réunit un certain nombre de personnes dans des croyances communes, dan's des pratiques semblables, dans un culte célébré en commun, en tant surtout qu'elle est capable de les fare marcher ensemble, de déterminer des actions d'ensemble guerrières, politiques, artistiques, ete. la religion est un phénomène sociologique. D'une maniere générale le phénomène est étudié par la psycho. logrie en tant qu'il se manifeste dans un esprit individuel, on si on le considere chez une quantité quelconque de porsomnes - mais senlement an point de vue des ressemblances et les différences d'intensité, de complexité, de composition yu'il perit présenter ehez l'une ou chez l'autre, il relève de la sociologie en tant qu'il détermine une atetivite commune de plusienrs personnes tendant vers nue fin mique on vers des lins convergentes. Eu l'esprit ainsi consiolóé est bien encore à la fois mn élément social et me syuthèce d'éléments sociamx moins complexes que lui-minn de produits socianx. 
. CIIAPITRE IV.

\section{W'esprit et le monde extérieus.}

Il nous reste à vérifier la troisième partic de notie définition de l'esprit, et, pour cela, à étudier les rapports de l'esprit et du monde inorganique, ou, en général, du milieu cosmique. L'esprit est doublement en rapport, par la perception et l'intelligence d'une part, par l'activité motrice d'autre part (et ces deux modes se réunissent généralement, la perception étant accompagnée de monvement, et le mouvement de pereeption), avec le monde qui l'entoure. Le monde extérieur agit sur l'esprit et l'esprit réagit sur lui, - nous ne saurions avoir une idée suffisamment complète de l'esprit si nous n'examinions pas brièvement les caractères généraux de cette double action.

Dans le chapitre précédent, j'ai indiqué les éléments sociaux de la perception. La perception renferme aussi d'autres éléments; par son intermédiaire, le monde cxtéricur agit sur nous, non pas seulement en tant qu'il a été modifié par l'intervention de l'homme et par les qualités qu'il doit à cette intervention, mais aussi par ses qualités propres. De plus, l'activité sociale ne peut agir sur le monde organique et inorganique que selon les propriétés de la matière organisée et de la matière non organisée, - il y a done, à ce double point de vue, une influence du monde sur l'esprit.

D'aborl, c'est le monde extérieur qui a r'endu l'organisme possible, et par suite l'esprit, c'est lui qui fournit ses matériaux à la synthèse psychique. Notre science 
n'est que la connaissance incomplète de la nature, notre art mème n'est à certains égrards que son imitation, et en tout cas son interprétation par la personnalite psychicque. Toutes les definitions de l'ceurre d'art - homo additus naturce, l'ceuvre d'art est une altération systématique de rapports réels, l'œurre d'art est un coin de la réalité vu à travers un tempérament, - le constatent. Mais, d'un autre côté, l'homme réagit sur la nature en l'interprétant, il la refait après l'avoir comprise, il la modifie après s'être modifié d'après elle, et après l'avoir reconstituée par son esprit d'après certaines données qu'il lui cmprunte il la transforme idéalement par des théories abstraites scientifirues ou philosophiques, il la transforme réellement par la mise en pratique de ces théories qui d'ailleurs précède parfois leur connaissance abstraite.

La première opération est la mieux reconnue - il n'est pas utile de démontrer longuement que l'homme observe et étudic la nature, qu'il tàche de reproduire par ses idées l'ordre abstrait de ses pereeptions réelles ou possibles ou des perceptions réelles ou possibles d'observateurs réels on implicitement supposés. Il est bien clair que par la science et la philosophie, le monde extérieur tel qu'il jeut alparaitre à un sujet sentant et pensant est plus ou noins fidelement représenté dans notre esprit. Cette adapLation de lhomme au monde, cet établissement de relations internes correspondant à des relations externes qui, d'ailleurs ofle des aspects peu connus et des points de vue curicux, a été magistralenent étudiée par M. Spencer dins ses Principes de psychologie. Le grand philosophe anglais y a vu la caractéristique principale de l'esprit en general, nous nous sommes flacés ici a un point de vue different, mais nous n'en powrons pas moins accepter comme aussi exacles ru'ingénieuses, la plupart de ses constatations et de ses généralisations secondaires, en ce gui concerne le point que jo viens d’indiquer. 
Mais l'autre côté de la question est an moins aussi important si ce n'est davantage. En effet, d'abord la phase de réception et d'élaboration a pour principal office de préparer la phase de réaction, la première ne se comprend pas plus sans la seconde que la seconde sans la première et l'ensemble des deux forme un tout indivisible. Le saroir, instinctif ou conscient, est la condition de l'action, la perception est le premier terme de l'acte. Je ne reviens pas sur ce côté de la question qui a déjá été examiné, je n'insiste pas non plus sur ce fait important longuement étudié dans un chapitre précédent: toute perception et tout acte de l'esprit impliquent déjà une réaction mentale et portent la marque de la personnalité. Mais nous devons considérer la réaction de l'esprit sur le monde extérieur et les caractères principaux de cette réaction.

L'homme est une sorte de foyer où l'univers se rencontre pour s'organiser. Les choses les plus éloignées, celles qui dans la nature n'ont entre elles aucun rapport appréciables, sont ainsi rapprochées, mises en relation et convergent en somme vers une mème fin. Les rayons lumineux que nous envoie l'étoile polaire, ou bien encore l'effet de la force magnétique terrestre sur une aiguille aimantée, viennent se combiner dans l'espril d'un homme avec les images vagues de contrees inconnues, avec les désirs de gains problématiques, avec les impressions tactiles et visuelles de différentes parties d'un vaisseau, et des excitations physiques arrivées de régions si éloignées et provenant d'objets que rien encore n'avait rattachés les uns aux autres, déterminent des séries de phénomènes psychiques systématisés et sont ainsi associées, par le fait de l'intervention humaine, pour diriger la marche d'un vaisseau. D'autres excitations, semblables ì certains égards, différentes à beaucoup d'autres, viennent se combiner encore dans un esprit d'homme et déterninent 
des séries de pensées, des séries d'actes qui conduisent aux découvertes des voyageurs, aux explorations, à la conquête de pays nouveaux, et de nouvelles richesses et les excitations venues des points les plus éloignés du monde se trouvent ainsi, grice il l'esprit de l'homme qui les recueille, les rassemble, les analyse et les synthétise, converger vers une série d'actes individuels et de faits sociaux dans la formation desquels elles sont un facteur souvent inaperçu mais parfaitement appréciable.

Tous nos actes implifuent ainsi la convergence et la réunion d'un nombre plus ou moins considérable de phénomènes physiques qui, sans l'homme, restaient, non pas peut-être absolument sans action réciproque, mais sans action appréciable. Ici les "relations exterues 》 dépendent des "relations internes » et se modelent sur celles-ci.

Si l'on considère le nombre incalculable de perceptions plus ou moins conscientes gui interviennent it chaque moment pour diriger nos actes, on comprendra l'importance de cette synthèse d'excitations venues du dehor's, qui est operée par l'esprit auquel elles arrivent. Les formes, les couleurs, les sons, la résistance des oljjets, nóus aident constamment it diriger tous nos actes, depuis la marche, jusqu'aux opérations les plus compliquées et dans charque cas nous trouvons ce fait général : des phénomènes naturels (au sens le plus général, n'excluant pas l'interrention de l'homme), sans raplorts entre eux, sont réunis par l'esprit et servent à donner de l'unité, de la régularité à nos actes. L'homme tire parti, pour son utilité, pour son agrément, c'est-it-dire en somme pour la systématisation plus ou moins importante de sa vie, d'une quantité inuombralule de faits naturellement séparée, réunis et synthétisés en lui el par lui. L'homme est une sorte de microcosme, un miroir oir le monde se reflete, mais un miroir oì il s'organise et s'unific. Ce 
n'est pas seulement au sens de l'idéalisme subjectif qu'on peut dire, arec Schopenhauer, que couper une tète c'est détruire un monde. Quoi qu'on pense de la réalité du monde extérieur, une tète est un monde condensé et coordonné, plus ou moins riche, plus ou moins bien organisé, mais qui toujour's synthétise ce qui ètait naturellement séparé, ce qui pouvait sembler destiné à l'ètre toujours et qui l'aurait toujours été sans l'homme. Chaque fois qu'un esprit se dissout, les objets qu'il réunissait en lui seul retournent à leur isolement.

On pourrait reprendre ici l'antique thème des causes finales, et les exagérations les plus ètranges deviendraient raisonnables par la différence de l'interprétation. Je renvoie mes lecteurs, pour les faits, à Fènelon et à Bernardin de Saint-Picrre, ils verront, sinon comme toutes choses ont été admirablement disposées pour l'homme, au moins combien l'homme a su tirer parti ou pourrait tirer parti d'un grand nombre de choses, - et depuis Fénelon et Bernardin de Saint-Pierre le nombre s'est augmenté de ces harmonies qui n'ont pas été faites pourl'homme, mais que l'homme a créées, instinctivement ou avec réflexion, gràce au progrès de la science et de l'industrie, aux nouvelles inventions qui ont mis à sa disposition et lui ont permis d'employer des forces auparavant inconnues ou méconnues comme la rapeur et l'électricité, et développer de mille autres manières celles qu'il connaissait déja.

La finalité n'est pas dans le monde (au moins au sens ordinaire du mot, - nous examinerons plus tard la question à un autre point de vue), ce n'est pas le monde qui a été modelé pour l'homme et adapté d'avance à lui, e'est l'homme qui s'est formé d'après le monde, qui s'est développé conformément à ses conditions d'existence, c'est-à-dire de la seule manière qui pùt rendre le développement possible. Il faut done renverser les termes de la croyance ordinaire aux causes finales - mais le fait 
mème de la finalité, entendue au sens positif comme convergence d'une certaine quantité de faits vers une fin unique, ver's des résultats harmoniques, est absolument indiscutable. Seulement c'est l'homme, ce système de partics qui toutes sont "reiproquement moyens et fins, les unes par rapport aux autres ", c'est l'homme qui produit récllement toute celte finalité que l'on a cru reconnaitre dans le monde et, parmi les hommes chacun produit dans le monde une finalité particulière, d'autant plus grande que la personnalité est plus élevée - toutes choses égales d'ailleurs. La finalité, la convergence existe pour chacun de nous en particulicr, variant d'un homme à l'autre avec le genre de vic, arec la puissance synthétique, avec les conditions d'existence de chacun, et elle existe aussi pour les êtres animés qui lous, chacun à sa manière, chacun selon ses forces, synthetiscnt aussi les impressions faites sur eux prar le monde extéricur et s'en servent pour donner de l'unité à leur conduite. L'oie, commc l'homme introduit de la finalite dans le monde, elle utilise même la resistance de l'air d'une manière que nous n'arons pu imiter encore, mais cette finalite est relativement très imparfilite pares que le systeme qui la produit est inférienr, relativement aussi bien cooldonné peut-être, mais infiniment moins complexe et noins riche en éements.

Nous avons jusquiei considéré la syuthese du monde extérienr comme s'opérant dans l'homme, el ne se manifostant all dehol's que par I'mitication de l'activite humaine, mais cette artivite modifie d'une menteve plus on noins inportante le momle exterienr lui-mène, l'aklpLition de l'homme an monde lui permet d'efrectuer

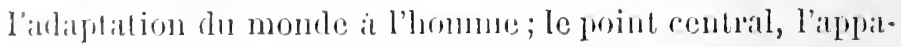
leil syuthétipue reste l'ailleurs le mòme, il est l'esprit dams les denx operations.

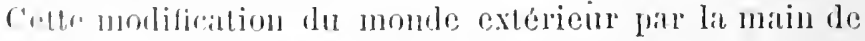

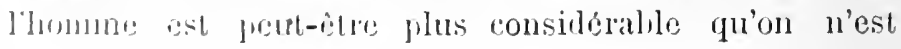


généralement porté à le penser, mais il suffit d'un peu d'attention pour la reconnaitre et en comprendre l'étendue. Depuis la modification du sol par le labourage, depuis l'acte de cueillir un fruit jusqu'au creusement des callax, jusqu'au percenient des isthmes, nous arons toute une série de modifications, dont quelques-unes sont très considérables, qui proviennent du travail de l'homme et contribuent à augmenter cette finalité que l'homme a su introduire dans le monde - et les instruments avec lesquels l'homme produit ces modifications, sont dùs eux-mêmes à des modifications préalables. Le monde est ainsi plus ou moins boulerersé; les oljets sont rapprochés, combinès, éloignés, pour rendre la finalité moins imparfaite. La table sur laquelle j'ècris, formée du bois d'arbres coupés, sciés, a été travaillée de différentes façons arec des outils formés en partie du bois dun autre arbre, en partie d'acier - et l'acier aussi a une longue histoire. L'encrier dont je me sers, l'encre arec laquelle j'écris, mon porte-plume, ma plume, tout ccla représente une quantité considérable de modifications du monde extérieur, essayez de calculer celles que représentent l'établissement d'une ligne de chemin de fer ou le percement de listhme de Suez. Chacun de nos actes occasionne généralement un remaniement plus ou moins grand des corps qui constituent le monde extérieur, et il n'est rendu possible que par les remaniements précédents qui ont mis à notre portée les objets dont nous nous servons continuellement (vêtements, outils, etc.) comme il rend lui-mème possible ceux qui viendront après lui. En fait, toutes les actions que le monde cxtérieur dirige par la concentration et la synthèse des impressions qu'il produit, conduisent à le modifier lui-mème et d'une manière non pas absolue, mais générale, à auğmenter sa finalité par rapport a l'ètre (homme ou animal quelconque) dans lequel il rient se concentrer. Je dirais presque que le 
monde extérieur se modifie lui-même, s'unifie par le moyen de l'homme, - si ce n'était pas à l'homme que revient, en somme, la part la plus importante de cette évolution, et je dirai plutôt que l'homme synthétise les impressions qui lui arrivent du monde extéricur et s'en sert pour l'adapter à lui. Les relations internes ne correspondent aux relations externes que pour que celles-ci à leur tour viennent se conformer à celles-là, et si l'on peut dire que l'homme ne commande à la nature qu'en lui obéissant, il faut ajouter qu'il ne lui obéit guère que pour lui commander.

Les synthèses internes et les réactions se compliquent réciproquement. Les premières observations faites par l'homme lui permettent de fabriquer des instruments, d'améliorer ceux qu'il a - d'un autre côté, ces instruments une fois fabriqués, lui permettent de continuer, de perfectionner ses observations. A mesure qu'il connaît mieux le monde, il peut micux le modifier; i mesure qu'il peut mieux le modifier, il peut mieux le connaitre, et comme chaque connaissance nouvelle constitue un accroissement de la finalité du monde, par rapport à l'être qui acquiert celte comnaissance, par rapport aux autres êtres qui la recoivent de lui ou qui en profitent indirectement, comme cliaque modification du monde constitue également un accroissement tout à fait analogue de la mème finalité, comme toute counaissance ef toute pratique nouvelle sont, toutes choses égales d'ailleurs ou equivaIentes, un perfectionuement de l'esprit, la supériorité de l'esprit et la systématisation du monde par rapport a cet esprit, se réveloppent simultanément - toujours en tenant compte de la même condition.

I) reste, c'est toujours dans l'esprit que la finalité, que l'organisation, en tant qu'elie nous intéresse ici, continue a lésider. En effet, les systemes d'oljets que l'homme forme liont fats leur fin en eux-mèmes; livrés à eux-mèmes; 
ils ne signifient plus rien et ne présentent qu'une harmonie factice, incapable de s'entretenir elle-mème ou tout a fait rudimentaire. Une montre a bien une sorte de vie indépendante, mais combien courte, précaire et insignifiante sans l'homme, et incapable de se prolonger ou de se reproduire. La finalité que l'homme introduit dans la nature est purement relative à l'homme lui-même ou peut-être aussi à quelques animaux - ce n'est pas à dire qu'il n'y ait pas dans la nature quelque finalité différente de celle-là, analogue à quelque degré à celle que nous trouvons en l'hommé, différant par conséquent tout à fait de la finalité du déisme; mais ce n'est pas le travail de de l'homme qui la développc ou la suscite.

Enfin, il faut remarquer que ce que j'ai attribué ici à l'esprit de l'homme, est en réalité une œuvre sociale. Presque toutes ou toutes les modifications dont j'ai parlé, sont non seulement rendues possibles par l'activité sociale en général, mais aussi directement effectuées par une association de travailleurs. Cependant, si l'on tient compte de ce que la synthèse subjective du monde est une opération facilitée et déterminée par le milieu social, mais, en somme, une opération individuelle, de ce que l'activitć sociale est une synthèse d'actions individuelles et surtout de ce que l'idéc première, l'invention qui conduit à la modification nouvelle, se produit d'abord chez un ou plusieurs individus, travaillant chacun plus ou moins isolément - on ne verra, je pense, aucune objection grave, au fait, de rapporter à l'esprit en génćral la finalité du monde, toutes réserves faites sur la part qui revient à l'action sociale en tant que telle.

Sous le bénéfice de ces réserves, nous pouvons maintenir la troisième partic de notre definition et dire que l'esprit est une sỵnthèse d'éléments fournis oul nodifies par le monde extéricur ; c'est dans l'esprit et par l'esprit que s'accomplit en tant qu'elle peut s'accomplir, la synthèse 
558

L'ESPRIT - SYNTHÉSE ABSTRAITE

du monde. Cette synthèse a deux formes principales qui se coordonnent et s'enchevètrent ; d'un côté, l'esprit fait servir des objets très différents et sans rapports objectifs appréciables entre eux, à dirigcr sa conduite et à lui donner de l'unité ; de l'autre, il modifie le monde cxtérieur pour l'adapter à l'homme et auginenter la systématisation des actions humaines. Celte harmonie entre Thomme et son milieu que produisent ces deux formes et leurs combinaisons, cette finalite, pour ainsi dire, reste toujours relative à l'homme, on plus généralement à l'esprit qui la produit, c'est dans l'esprit que réside le principe de la finalité - l'esprit est une synthèse de phénomenes cosmiques, il est le point central de la synthèse du monde. 


\section{O N C L U S I O N}

Les trois termes de notre définition de l'esprit ont été expliqués et développés, l'esprit est cssentiellement une activité synthétisant des éléments organiques et p̊ychiques en vue d'une fin sociale, déterminée par le fonctionnement de la société dont il est lui-même un élément, et synthétisant aussi des produits sociaux et des éléments cosmiques. Cette opération, qui dans tous les cas que nous connaissons reste toujours plus ou moins imparfaitc, s'effectue sclon le mode que nous avons examiné par les affinités des éléments psychiques, développécs ou retenues selon les lois de l'activité mentale, dont les deux principales sont l'association systématique et la loi de l'inhibition systématisée. Les éléments psychiques dont nous avons étudié au début de cette étude le mode d'activité, sont a la fois des synthèses organiques et des produits sociaux; pour exprimer les choses un peu en gros, l'organisme fournit la matière de ces éléments, l'influence sociale leur donne la forme ainsi que le milieu extérieur considéré à un point de vue général. L'esprit est la synthèse de ces éléments psychiques, - synthèse dont la forme est déterminée également pour une bonne part, par le milieu social surtout et aussi par le milicu en général. Les lois de l'esprit sont les modes généraux sclon lesquels les éléments psychiques se combinent pour permettre à l'activité sociale d'agir sur l'esprit, pour permettre à l'esprit d'entrer comme élément dans l'activité sociale, pour lui permettre également à la fois de mieux s'adapter a son milicu et de mieux adapter ce milieu à lui. A mesure que l'esprit se complique, a mesure que la société progresse - ce qui n'arrive pas 
toujous, il s'en faut, mais ce qui arrive quelquefois, les rapports entre ees trois groupes: le monde, l'esprit, la société deviennent de plus en plus étroits, de plus en plus nomlereux, de plus en plus harmonises. La systematisation totale s'accroìt et décroit selon les périodes d'évolution et de rlécadence, avec la croissance et la mort des hommes et des peuples. L'homme fait profite de la societé et du monde extérieur, il sert à la coordination de la société et a celle du monde extérieur, d'une manière que ne connaissait pas l'enfint et que ne connaitra plus le vieillard, de mème une civilisation à son apogée a des coordinations scientifiques, industriclles, esthétiques, qu'clle ne connaissait pas à son origine et qu'clle oubliera à son déclin, et (qui seront peut-être jerdues jour toujours après elle ${ }^{1}$.

1. La finalité de la religion ou de la philosophie du thèisme, si elle était réelle, ce qui me parait très improhable, ne serait qu'un cas particulier de celle que nous avons reconnue dans le monde, le cas où tous les élements du monde, du moins important au plus complexe seraient rétlechis et coordonnès par un esprit qui donnerait une place a chacun d'eux dans un consensus gènèral. Pour le panthéisme, la finalité du monde serait conforme à celle de l'esprit même, les éléments du monde convergerajent a'eux-mémes vers une sorte de vie de l'ensemble. Il se pourrait que le panthéisme faux aujourd'hui devint vrai un jour - mais rien ne mus antorise a croire qu'il en sera reellement ainsi. Quant au théisme, la uotion le l'esprit et de la conscience sans organisme qu'il implique, sinon logriquement dı moins en général, est un deses défauts les jlus graves. 


\section{LIVRE III.}

\section{Lois de l'esprit et lois du monde.}

$\S 1$.

Il faut maintenant nous placer it un autre point de rue. Cette finalité immanente, cette systématisation qui est la loi de l'esprit existe-t-elle à quelıue degré en dehors des ètres animés? La finalité est-elle non-seulement une loi de l'esprit, mais encore une loi du monde? Et en quel sens? Nous avous écarté déjil l'hypothèse d'après laquelle le monde serait arrangé pour l'homme, ou mème arrangé par une conscience. Mais il s'agit de saroir simplement si, en dehors de l'homme, nous trouvons une loi de finalité immanente analogue à celle que nous avons trouvée en lui.

Au-dessus de l'homme, d'abord, nous tronrons la société. Ici pas de difficulté, nous avons eu l'occasion de remarquer plusieurs fois que, si la finalité est une loi de l'esprit, elle est aussi une loi de l'organisation sociale, et que d'ailleurs elle soit aussi faible, aussi incomplète que ce qu'on roudra el bieni inférieure à ce que l'a pensé une école d'économistes, toujour's est-il qu'elle est la condition indispensable de l'existence même de la société. La société est pour le moment la forme la plus haute d'association systématique que nous puissions constater, et c'est dans les groupes sociaux les plus avancés en civilisation que celle forme d'association se manileste dims sa plus grande complexité. Que cette forme soil d'ailleurs encore - comme la forme psychique, - bien imparfaile, 
cela ne fait pas de doute et celte imperfection est due aux mèmes causes que l’imperfection psychique : l'aclivité relativement indépendante des éléments sociaux dont les désirs, les tendances ne sont pas toujours suffisamment inhibés par l'orientation sociale et qui, par conséquent, selon les mêmes lois d'association systématique et d'inhibition, tendent à occasionner des troubles sociaux (anarchie, lutle des partis, coalition des ouvriers contre les patrons, oppression des ouvriers par les patrons, grèves, action d'accaparer telle on telle substance, fraudes diverses, etc.) qui sont tellement haj)ituels, et en un sens, si nécessaires actuellement, qu'on finit presque par les considérer comme des éléments de la vie normale d'une société.

Au-dessus de la société, nous ne connaissons aucune organisation - on peut rêver une synthèse de sociétés, les Etats-Unis d'Europe, on peut aller plus loin et rêver l'association des esprits de diverses planèles, et une synthèse croissante, s'étendant à travers les mondes stellaires par l'invention de moyens de communications nouveaux, par la suggestion mentale a distance ou les pratiques du spiritisne, mais onjugera, je pense, qu'il est inutile de s'attarder pour le moment à ces considérations. Remarruons seulement que les religions offrent ì leurs fidèles des associations systématiques supura-sociales, analogues à celles-là - mais, dins tous ces cas, il y a plutól extension du type social qu'invention d'un type nouveau.

Au-dessous de l'homme, que nous arous suffisamment ctudic fonr n'avoir pas at y revenir, se trouvent les animanx - évidemment les conclusions générales de notre travail Jeur sont applicables aussi bien qu'à l'homme, la fuestion de siroir si les animax ont une ame ne se pose meme fas i notre point de rue - il va de soi que tout olganisuc a mue àne qui n'est que l'expression alustraite de ses rapports avec son milieu, de la coordination des 
impressions qu'il reçoit et des actes par lesquels il réarrit. Aprés les animaux, nous trouvons les plantes; ici encore la finalité immanente n'est pas douteuse - et bien que les relations des plantes arec leur milieu soient en général bien simples, au moins relativement à ce que nous trourous chez l'lomme, il ne paraitra pas cxcessif, je pense, d'employer le mot: esprit des plan es, ct dire que nous trouvons chez la plante l'objet d'une psychologie rudimentaire. J'ai eu, dans le cours de cette étude, l'occasion de lui faire quelques emprunts. Cependant il ne faudrait pas s'abuser sur les analogies - si les habitudes des plantes, si le mécanisme de la fécondation, de la direction centripète de la racine, de la direction centrifuge de la tige, de la disposition des feuilles, rappellent les instincts des animaux, il y a aussi entre ces phénomènes de grandes différences, et l'on est en droit, tout aussi bien, de n'appeler " esprit " que la coordination organique déterminée par l'intermédiaire d'un système nerveux - encore la question ne serait-elle pas tranchée bien nettement, car les commencements du système nerveux et mème du règne animal sont assez vaguement définis. Quoi qu'il en soit de cette question de dénomination, nos lois d'association systématique et d'inhibition se retrouvent dans la séric, dans le complexus des phénomènes qui constituent la vie des animaux inférieurs et la rie des des plantes et en se mettant en garde contre les abus, on peut tres bien considérer la vie et l'esprit comme toujours associés et réellement inséparables.

Au-dessous des plantes, quelque chose montre encore, pour ainsi dire, des traces de vic et d'organisation, e'est le cristal. On a pu parler de lil vie des mineraux, ou est mème allé jusqu'a exprimer cette proposition qui introduit dans le monde minèral l'association systématiuue, la sélection et l'inhibition : les gros cristaux mangent les petits. Mais faisons la part de lia métaphore, ne parlons 
plus ici de vie ni d'esprit, si l'on veut, il n'en reste pas moins que le cristal présente une certaine individualité, indiquée tout d'abord par la régularité de sa forme et confirmée par divers plénomènes. Il est impossible de ne pas voir une association systématique (en prenant le mot dans un sens trẻs abstrait), dans le fait du cristal qui porté dans une dissolution du mème corps ou d'un corps isomolphe, répare une perte de substance et continue a s'accroiltre. Un cristal d'alun ordinaire porté dans une dissolution saturéc d'alun de chrome continue ì s'accroitre comme rlans ume dissolution d'alun ordinaire. Mais le fait mème de la cristallisation par fusion, par sublimation, par refroidissement d'une dissolution, par évaporation indique suffisamment la tendance des molécules ou des atomes, à se rémuir en systèmes définis. Le dimorphisme est quelque peu analogue aux variations de la personnalité. Certaines substances, lorsqu'on les place dans des conditions diflérentes cristallisent dans deux systèmes distincts. Le soufre donne par évaporation à froid des octaẻdres réguliers à base rectangle, fondu à la chaleur, il donne, vers $111^{\circ}$, des prismes obliques. Il s'établit selon les circonstances des orientations différentes des tendances atomirues, comme des tendances psychiques.

Au-dessous du cristal, nous trouvons enfin deux autres systimes: la molécule et l'atome. On a reproché it certims philosophes d'animer et de douer même de conscience, la nature inorganique; de prèter une àme à un rocher, at la terre, a lis mer. Il importe de bien préciser la rulestion que je pose et la réponse qu'on croil pouvoiry faire. I'abord il me frarait sans grand interet de se demanleg si lat conscience existe dans le régne ninéral fou mène dans le rógne rogétal) : la facon dont nous avons interpretis le roble psychique de la conscience nous disJente lle nons oceluper d'elle, et si notre interpretation anos imligue mo opinion probable sur ce point, c'est que 
la conscience ne paraît exister nettement que chez les animaux supérieurs, et, en tout cas, pour les modes d'activité non complétement organisés encore pour ce que M. Caporali appelle «la nature qui se fait.» Mais nous pouvons rechercher s'il y a dans le règne minéral, dans la matière en général, quelque principe d'organisation. Or, cette organisation il ne faut évidemment pas la chercher dans des masses sans forme et sans coherence (je ne dis pas sans colhésion) comme la mer, l'air, les couches géologiques, où les éléments ont été rassemblés à cause de l'analogie de leurs propriètés ou de leurs conditions d'existence. Si l'on verse de l'huile dans l'eau, l'huile reste toute à la surface; l'eau reste toute au fond, il n'y a pas là une organisation à proprement parler. Les agglomérations de molécules chimiques sont surtout des agglomérations, non des systèmes; s'il y a de l'organisation quelque part, ce n'est pas dans les ensembles, dans les masses qu'il faut la chercher, c'est dans les éléments; ce n'est pas dans les agrégations d'atomes ou de molécules (sauf lorsque ces agrégations forment des corps vivants et des cristaux, et dans quelques autres cas particuliers c'est dans les molécules mêmes et dans les atomes.

Ici, en revanche, la systématisation est manifeste. je ne demande pas qu'on emploie les mots de vie et d'esprit, mais il est impossible de méconnaitre des ressemblances générales et de ne pas apercevoir ici des formes très différentes de celles que nous avons vues, moins complexes mais réelles de l'association systématique et de l'inhibition. Ce qui met une différence entre la psychologie et la chimie, ce ne sont pas les lois les plus abstraites, c'est la nature des éléments et de leur's combinaisons ; les éléments psychiques sont des syntheses de faits biologiques, les faits biologiques sont des syutheses de faits physico-chimiques, mais ces faits, considérés en euxmêmes et a part de l'organisme qui les combine d'une 
certaine manière. sont des șnthẻses déléments plus simples. et les grandes lois qui regissent les spntheses superieures, nons les retrourons sous dautres formes dans ces syntheses rlus simples.

La molecule chimique est une sorte de société d'atomes. Leur association srstematique est marquẻe par ce fait que des atomes, dabord sans rapport entre eux, se groupent, sumissent quand les circonstances sont farorables à la mise en jeu de leurs affinites, je dirais presque de leurs tendanes ou le Ietirs désirs, et forment désormais un ciement unique qui a son existence indépendante et qui est susceptirle dentrer comme élement dans des combinaisons sugerieures. La therie atomique a donné des symboles pour exprimer cette sorte de société des atomes rui constitue une molecule plus ou moins complexe. Il est hors de doute que les considerations des chimistes sur latomicite. sur la ralence desatomes. sur la nature monoatomique, hiatomique, triatomique des corps simples, sur la subsitution dos atomes dans les molecules, sont des illustratins fort interessantes a notre point de rue de la loi dassociation ststématique et de la loi dinhibition, car, si rous worons des affinites satisfaites, nous worons aussi de affritis enrates. Mais il faut yrendre garde de trop abrolor dans notre sens, nous sarons a peu pres ce ruestun dirgir ohez un etre organisis, nous ignorons ce

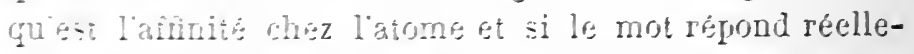

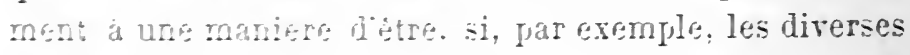
astinites dur corps correspondent a des organisations

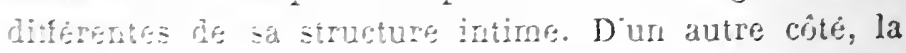

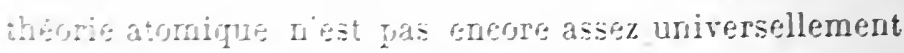

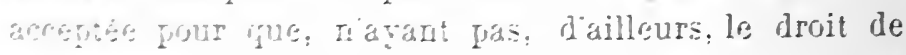

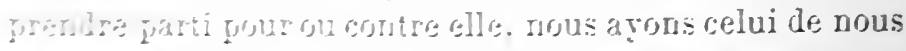

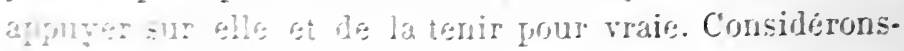

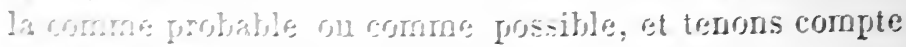

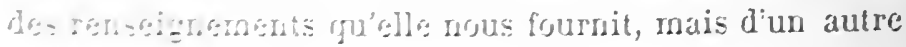


côté, reconnaissons que, si son interprétation l'un fait général, - l'association des atomes - si la manière relativement concrète dont elle tend à nous représenter les choses, peut ètre discutée, si les symboles mèmes, si le langage qu'elle a introduits dans la science chimique peuvent, au dire d'éminents adversaires, présenter quelques inconvénients - les faits gẻnẻraux qu'elle a tàché d'interpréter ne paraissent ni contestés ni contestables, et que ces faits généraux qui se rencontrent dans les combinaisons des corps, nous suffisent parfaitement non pas pour dire quelle est l'organisation de la molécule chimique, ni comment cette organisation rarie d'une molécule à l'autre, mais au moins pour affirmer qu'il y a une organisation moléculaire - et que cette organisation résulte de la synthèse, du consensus d'éléments plus simples : les atomes, dont l'existence, bien que conçue différemment, n'est niée par aucun chimiste, non pas en tant qu'il s'agirait de particules dernières absolument simples, de l'absolu en un mot, mais au moins en tant qu’ils sont les éléments des molécules.

Mais ces atomes eux-mèmes, si l'on n'en peut pas lire grand chose, au moins semble-t-il que ce qu'on en peut dire tend à établir qu'ils sont, eux aussi, des synthèses d'élèments plus simples. On connait la théorie des atomestourbillons de Sir William Thomson. L'existence de l'atome serait encore ainsi une loi de systématisation. Pour donner une idée des atomes, tels que cette conception nous les donne, M. Tait arait réalisé une expérience intéressante, les anneaux tourbillons de fumće de tabac que certains fumeurs savent fairc sortir de leur bouche, en donnent une idée approximative. "Eu lançant successivement plusieurs anneaux au mogren de la boite de Tait, on peut voir ce qui se passe quand ils se heurtent, quand l'un d'eux en traverse un autre plus grand, ctc. 
"Si l'on suppose un fluide illimité, homogène, incompressible el exempt de frottement, ou démontre par l'analyse mathematique - que des filaments tourbilons sont nécessairement fermés, mais qu'ils peuvent présenter des rebroussements et des nceuds - qu'ils ne sauraient être engendrés que par une cause créatrice extérieure - qu'à moins d'une nouvelle intervention prenant sa force hors du système, ils sont insécables et indestructibles; qu'ils possèdent tous les caractères de l'individualité et conservent éternellemrent les qualités déterminées par l'impulsion qui leur donna naissance.

- Ces propriétés font de ces petits êtres autant de sources permanentes d'énergie et les rendent éminemment aptes a expliquer les apparences que nous appelons Matière et Mouvement. Jointe à la flexibilité, leur impénétrabilité n'est plus incompatible avec l'élasticité, ce qui est la pierre d'achoppement des autres hypothèses altribuant à l'atome une étendue finie; ils exercent les uns sur les autres des actions ayant pour resultat apparent une attraction à distance, comme l'ont démontré Kirchoft et Boltzmann, leur forme annulaire s'accorde très bien arec lit forme aplatie ou allongée que l'on est conduit à supposer à certaines catégories de molécules, etc. " 1.

La molécule et l'atome paraissent donc bien présenter, à quelque degré dans leur contexture propre, cette loi de systématisation dont nous trouvons des exemples prodigieuscment compliqués 2 dans les organismes supéricurs. Ce sont là les vraies individualités du monde inorganique

1. Jouffret. Introduction de la théorie de lenergie, p. 179-180. Voir aussi, pour les questions concernant la molécule et l'atome, les traités de chimie et de physique, et Wurtz, la Théorie atomique; Berthelot, la synthese chimique, Tyndall, la Chaleur. Tait. Conferen. ces sur quelques-uns des proyres récents de la physique.

2. Les phinomenes vont exidemment en se compliquant en remontant la hierarchie des sciences, de la mécanique a la sociologie - c'est ce 
(a) part certains systèmes astronomiques ou phỹsiques). Toutefois si les concrets que forment les éléments molécule ou atome ne nous présentent en général que des rudinents negligeables de systématisation; si la finalite, dans le monde inorganique bien plus encore que dans le monde de l'esprit, est une propriété des éléments, nous retrouvons une autre forme de tendance a la finalité dans l'ordre abstrait des phénomènes, dans les lois qui expri. ment leurs relations générales de succession et qui sont probablement - lorsqu'elles ne font intervenir aucune synthèse supérieure - l'expression des propriétés èlèmentaires de diverses formes de la matière dans diverses conditions. Les lois de la physique et de la chimie, ou du moins certaines d'entre elles, paraissent bien exprimer quelque systématisation. Au reste, je n'ai pas lintention d'approfondir ici la question de la finalité dans le monde, et je me borne à signaler les faits qui me paraissent indiquer l'existence dans le moncle de lois de coordination réellement analogues à un point de vue abstrait, à celles que nous a montré l'étude des systèmes psychiques. La conclusion générale à laquelle nous aboutissons est

caractère qui fait le fondement de la classification des sciences d'Arguste Comte - toutefois cette complication vraie en un sens - en ce sens que un phénomène de l'ordre supérieur comprend toute une complication de phènornènes de l'ordre inférieur, c'est-à-dire toute la complexité des autres phénomenes plus cellequi résulte des synthèses nouvelles, qui sont précisément le phénomène d'ordre supèrieur - ne l'est peut-ètre pas autant si l'on prend la question autrement, et si, on considère seulement cette nouvelle complication. C'est, par exemple, une question de savoir si la complexité des phénomènes sociaux est aussi grande que celle des phénomènes psychologiques ou organiques, si l'on considère suulement comme complexité des phénomènes sociaux celle qui résulte des synthèses de phénomènes psychiques, abstraction faite, au point de vue sociologique, de la complexité d'éléments et de relations organiques, chimiques et mécaniques que supposent ces deruierres. C'est à ce point de rue que se plaçaıt, je pense, M. Tarde, pour déclarer la sociolcgie moins complese que la psychologie. 
celle-ci : il est v'ai qu'il y a partout de la finalité dans le monde, mais cette finalité appartient aux éléments non à l'ensemble, l'atome est un système, la molécule est un système, mais les rémnions de molécules et d'atomes sont souvent des agglomérations, fon des systemes, une pierre, un fragment de roche, sont une agglomération de molécules d'especes diverses, mais ils ne forment pas un système organisé, vivint, dont toutes les parties tendent vers une mème fin - si ceci arrive, c'est accidentellement, ou bien gràce à l'intervention d'un organisme qui par son activité propre les fait entrer dans des combinaisons dont il est le centre et l'organe essenticl.

Ainsi l'atome est le terme extrême auquel nous pouvons arriver d'un côté de l'analyse, et cet atome pur manifeste deji par sa nature propre une systématisation particulière, et par ses affinités une tendance à entrer comme élement dans des synthèses diverses (molécules), soit arec des atomes semblables, soit surtout aree des atomes differents. Remarquons que combiné arec un atome semblable il produit un corps qui ne ressemble pas absolument au prenier, ce qui impliøue bien lá systématisation, non la juxtaposilion. La molécule présente des tendiuces analogues qu'il n'est pas utile d'examiner ici en détail. Peut-ètre autrefois y a-t-il eu une tendance des molécules a se combiner en organismes tres simp, les par generations spontanées, mais si logiquement l'hypothèse est rlificile à eviter, les vérifications expérimentales n’ont janais montré sa réalisation - et si cela ne prouve assuroment pas qu'clle soit fausse, tout an moins cela maintient-il lis fuestion hors de la portéc posilive le la science

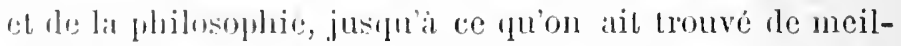
lemres raisons, directes on indirectes, de l'admetre comme vario on do la rejeler conme fausse.

Aluris lia molécule, en remontant, nous trourons une sorte de bifincation - d'un côté les éléments organiques 
nous prèsentent des synthèses de molécules, mais d'un autre côté, les molécules et les atomes s'arrangent aussi en synthèses astronomiques ${ }^{1}$, et les corps vivants rui, à d'autres points de vue, sont des syntheses, sont iri des éléments et n'agissent que par leur masse. Tous les organismes qui sont sur la terre seraient remplacés par un poids mort égal, le systeme solaire n'en scrait pas modifié - nous sommes un élément de la terre au mème titre que toute autre partie n'importe laquelle 2 . Il n'y a pas à douter que la coordination ne soit un caractère des phénomènes astronomiques - le système solaire, les systemes d'étoiles, etc., en sont des exemples aussi connus que peu discutables, si d'un autre côté le mouvement propre du soleil pouvait être rapporté à quelque autre ensemble céleste, si les étoiles de la voie lactée pouvaient être reconnues comme formant un ensemble qui tend a se conserver en rertu des forees combinées qui animent ses éléments, la systématisation serait plus complexe encore, mais ici le doute n'est pas dissipé.

Les organismes sont des combinaisons de molécules bien différentes, les différentes propriétés chimiques de l'atome y sont utilisées (et si elles se ramenaient toutes a des formes spéciales de la gravitation universelle, cela ne

1. Oa peut trouver encore des traces de coordinations clans certains phènomènes physigues, comme la circulation de l'eau par les tleures et son retour par l'air, a l'état de vapeurs qui se condensent pour alimenter de noureau les cours d'eau.

2. Si la complexité d'une science se mesurait à la complexité des faits qui en manifestent les lois, l'astronomie serait la plus complese des sciences, puisque l'animal, l'homme et la société ne sont qu'une partie d'un des éléments du système solaire. Ceci est a tapprocher de ce qui a été dit dans une note précédente - mais ici la question nollire pas de diiticulte. L'astronomie n'implique évilemment pas Ia biologie, la psychologie et la sociologie; les propr étés des corps organisés, en tant qu'organisés, ne jouent aucun rỏle dans les phénomènes astronomiques, et l'interprétation n'est ni difficile ni douteuse. 
dérangerait en rien nos conclusions), ensuite, par l'esprit, les différents éléments organiques, les différents organes sont coordonnés et liarmonisés et, par la socièté enfin, les esprits se synthétisent et forment de nouveaux systèmes ${ }^{1}$.

\section{$\S 2$.}

Par cette rapide revue des lois abstraites exprimant l'activité de l'esprit, exprimant également dans d'autres formes concrètes l'aclivité de tous les éléments de ce monde, nous pouvons remarquer une vérité que nous avons aperchne plusieurs fois. Les lois qui expriment les affinités, les désirs pour ainsi dire des éléments organiques sont fixes et immuables, le goût de l'acide sulfurique pour l'eau, de l'hydrogène et du sodium pour l'oxygène ne varie pas. On sait que l'hydrogène, à la température ordinaire, sera toujours dépossédé par le sodium qui décomposera l'eau, formera de la soude et laissera partir l'hydrogène. Quand il s'agit, an contraire, des phénomènes de la ric, la précision est moindre, à tel point qu'on a pu croire longtemps les corps vivants soustraits au réterminisme universel : quand il s'agit des phénomènes psychiques et.sociaux, la régularité est moindre eneore et l'apparence de caprice et d'indéterminisme plus frappante, et c'est ici que les adversaires de la causalité universelle sont encore les plus nombreux. On a expliqué ce fait par la complexité des phénomènes, la difficulté de tenir compte de l'entrecroisement de si nombreuses

1. Une remarque peut ètre intéressante à faire, c'est que le véritable hiatus dans la complication croissante des thénoménes, c'est le passage du monle inorganique au monde organique - les cristaus forment bien un yroupe intermédiaire aux deux groupes principaux, mais a un certain point de vue seulement, et ne fournissent pas un passage. On va facilement de l'atome a la molécule, on ra facilement aussi de l'organisme a l'enpir, le l'esprit a la socićté, mais le passage de la molécule a l'organisme ne jeat gurse étre fail actuellement que par un postulat. 
lois et notre ignorance forcée des causes, et cette raison existe en effet, et elle a eu son influence, mais il y en a une autre qui tient non pas tant à notre impuissance qu'a la nature mème des phénomènes, c'est que les lois psychologiques et les lois sociologiques ne sont pas encore toutes formées, la régularité des phẻnomènes, à cause de leur complexité, n'est pas encore obtenue - nous assistons à la formation réelle de lois psychiques et de lois sociales.

Ce n'est pas à dire pour cela qu'il y ait rien d'indéterminè actuellement dans l'esprit ou dans la société. Mais un phénomène d'ordre psychique ou social est quelquefois déterminé par des influences hétérogènes à l'ordre auquel il appartient, les tendances psychiques et sociales se manifestent sourent d'une manière irrégulière, et la suite des phénomènes peut ne pas manifester une loi psychologique qu'elle aurait manifesté si les conditions araient été plus farorables, quelle manifestera plus tard si ces conditions se présentent. Pour rendre ceci plus clair, prenons un exemple concret. C'est une loi psychologique, je suppose, que l'homme cherche son intèrèt, cette loi a été adoptée par un assez grand nombre de psychologues, elle a été adoptée par de nombreux moralistes, elle a étẻ adoptẻe par une école d'économie politique, qui, on peut bien le dire, avait singulièrement simplifié la psychologie sur ce point et sur d'autres. Eh bien, si nous examinons cette proposition nous la trouverons, en bien des cas, manifestement fausse, bien que, au fond, elle réponde à quelque chose de réel, et que, dans un monde idéal, elle dùt ètre parfaitement exacte. Sans doute l'homme cherche son intérèt quand il mange et boit modérénent, mais il ne le cherche ni ne le trouve quand il abuse des liqueurs at point de se rendre alcoolique. Ce n'est plus ici l'homme qui cherche son intérèt, c'est une tendance qui se manifeste 
aveuglément, cest un systeme psychique qui veut se compléter, un gout qui tìrlıe de se satisfaire, et je veux bien qu'en un sens ce goul, cette tendance cherche son intérèt, comme nous avons vu - mais l'homme, lui, ne lo cherche pas, il cherche, comme on dit très exactement, à satisfaire son goùt, encore ce goùt quelquefois lui répugne, lui déplait, il n'en est pas moins obligé de le satisfaire, alors même qu'il n'y trouve aucun plaisir si la tendance est devenue assez forte, si l'organisation de phénomènes qui composent ce système psychique a atleint un certain degré. Si nous supposons, au contraire, un lomme bien équilibré, ses désirs auront pour but, et la satisfaction de ses désirs aura pour résultat, la conservation de l'organisme ou son développement - l'homme recherehera réellement son intérêt. Supposons que l'état général de l'humanitè devienne semblable à celui de cet homme et la recherche de l'intérèt sera réellement une loi psychologique. C'est encore presque une loi de psychologie concrele que l'homme aime ses -enfants, mais nous connaissons des exceptions, la tendance naturelle peut ne pas exister, ou etre enrayée par d'autres; les lois psychologiques et sociales de cette nature sont des lois naturelles non encore formées. Elles correspondent à un état de l'esprit idéalisé que l'on suppose réel. Et, souvent, elles prennent la forme d'une loi morale, d'un commandement impératif. En effet, les lois morales, les lois impératives, en général, sont des ébauches de lois naturelles, des lois naturelles en roie de formation.

Si l'atome a été le résultat d'une longue érolution, cette évolıtion paraît terminée à présent; s'il est vrai, par exemple, que les différents corps simples soient en réalité complexes et ru'ils aient été formes jen a pen, par taitonnements, par des combinaisons d'une matière plus élementaire, il n'y parait plus ; les atomes de fer ou de chlore semblent aroir une individualité tout a fait 
formée et les différences d'un atome à l'autre, s'il en existe, ce qui est vraisemblable, nous sont inaccessibles. Nous n'apercerons pas de différence entre l'affinité de divers atomes d'oxygène pour l'hydrogène ou le soufre. De mème, une molécule d'eau est toujours composece dans des proportions identiques d'oxygène et d'hydrogène. Dans les ètres vivants, au contraire, les variations commencent, l'organisation n'est pas encore acherée, les systèmes qui constituent ces êtres ne sont pas réçulière-ment arrangés, ils entrent encore en conflit. Sans doute, ils poirraient être parfaits, - en tant que nous pourons concevoir leur perfection, - sans être semblables, sans former mème des groupes composés d’individualités aussi identiques entre elles que paraissent l'ètre les atomes et les molécules - car les fonctions de chaque élément dans le tout pourraient ètre différentes - mais cette action des élćments serait régulière et conforme à des lois précises qui ne se manifestent pas encore dans toute leur rigueur. Si l'homme était plus avancé en perfection, il n'hésiterait pas entre son devoir et son plaisir - et d'ailleurs, I'un ne serait pas distinct de l'autre.

L'homme est parvenu à un point de son évolution où l'organisation est encore imparfaite, mais où un état idéal de l'organisation s'impose à l'esprit et donne lieu ì une tendance réfléchic et consciente. Les lois impératives, les lois morales, les lois civiles marquent aiusi la tendance de l'homme vers un état plus ou moins élevé, elles indiquent en même temps que cette tendance n'est pas arrivée à un haut degré d'organisation. Solon, dit-on, ne voulut pas prévoir le parricide dans ses lois, ne pourant admettre cu'un tel crime fut possible - il supposait donc à tort l'existence d'une loi psỵcholorgique qui élait simplement ébauchée, et qui, si elle arait réellement existé a l'état de perfection qu'il lui attribuait, aurait, en effet rendu inutile toute loi écrite. Si la loi défend, par exemple de prendre 
le bien d'autrui, c'est que l'homme est souvent tenté de le prendre, mais c'est aussi qu'il sait résister à la tentation, au moins en certains cas; la loi morale et la loi sociale indiquent la formation d'une tendance, d'une habitude morale encore imparfaite, et qui n'existe pas en tant que loi naturelle absolue, mais qui serait une loi naturelle dans une sociêté bien organisée, arec des hommes naturellement moraux, aussi bien que les lois des proportions définies et des proportions multiples. Mais la loi morale et la loi sociale ne sont pas seulement la constatation d'un fait; par les ordres qu'elles donnent, par les peines qu'elles infligent, elles aident à la formation de la loi naturelle dont elles indiquent l'évolution.

Nous avons déjà vu que la loi de finalité ou de systématisation appliquée à l'ensemble de l'individu ou à l'ensemble de la société, ètait sommise à des restrictions nombreuses. Les luttes des éléments psychiques ou des élément sociaux font de la finalité générale de l'organisme et dı: corps social une tendance plutôt qu'une réalité. La réalité de cette finalité scrait le rẻgne de la loi morale, et la loi morale serait alors une loi naturelle, qui, au lieu de parler au mode impératif, serait simplement exprimée au mode indicatif. Mais l'existence seule de la loi morale - si imparfaitement qu'on la conçoive, est la preuve que la finalité, la systématisation générale de l'organisme et de la société est réelle bien que loin d'ètre parfaite, et c'est ce que nous avons tàché d'établir en ce qui concerne la psychologie.

Sans insister sur ces questions que je me propose d'examiner dans un autre travail ', je résume ce qui nous

1. Jo les ai abordées, mais bien imparfaitement et bien incompletement dans quelques articles publiés par la Revue philosophique. L'obligation morale au point de vue intellecinel, Liattente et le devoir. - I,es conditions du bonheur et l'érolution humains. - Le devoir et la science morale. 
importe ici au point de vue de la comparaison a faire entre les lois de l'esprit et les lois du monde: les lois synthétiques de l'esprit sont inparfaites, non pas seulement parce que la complexité des phénomenes nous empèche de les saisir, mais encore et surtout, parce qu'elles sont elles-mèmes imparfaites à cause de l'état de transition dans lequel se trouvent en général tous les esprits que nous pouvons connaitre, et particulierement l'esprit de l'homme qui est une synthèse beaucoup plus vaste mais, au point de vue de l'harmonie interne, beaucoup plus imparfaite que l'esprit des animaux en général et spécialement des animaux inférieurs. Si donc, tous les phénomènes psychiques de l'homme sont bien soumis à la loi générale du déterminisme, ce déterminisme aussi absolu sans doute, n'a pas les allures régulières qu'il a, par exemple, dans la plupart des phénoménes de la vie de nutrition et qu'il prend encore plus dans les phénomènes chimiques. Ici il n'y a pas - au moins à notre connaissance - de troubles internes; on n'a pas constaté que, tant qu'une molécule chimique se maintient, les atomes qui la constituent, puissent avoir des désirs non harmoniques qui, par leurs luttes, troubleraient l'organisation moleculaire - dans l'homme au contraire, sans que la personne disparaisse, le désordre interne peut être assez considérable, et l'anarchie des éléments psychiques, entraîner des troubles considérables de l'activité, des irrégularités et des désordres Autrement dit, le déterminisme dans l'ordre chimique est en général celui des lois de la chimie; dans les sociétés, au lieu d'ètre toujours dordre social, il peut être d'ordre psychologique, comme lorsque la volonté d'un élément, d'une personne, vient déterminer une perturbation dans les relations sociales, dans l'esprit au lieu de résulter seulement du jeu des lois de la psychologie synthétique, il peut résulter de l'activité incoordonnée de divers éléments psychiques, il peut 
même ètre purement organique et physico-chimique lorsque, par exemple, une seric de firits psychiques incohérents, est déterminéc par une comr,ression du cerveau ou une lésion de cet organc.

Il ne faudrait pas toutefois s'exagérer cette différence : pent-ètre existe-t-il dans les molécules mêmes des irrégularités que nos moyens d'obşervation ne nous permettent pas de constater. Il est à remarquer que l'instinct des animaux, faute d'avoir été assez minuticusement observé, a longtemps passé pour immuable - c'était un lien commun jadis d'opposer la perfection de l'instinct à la perfectibilité de l'intelligence, et si cette manière de voir était presque exacte, il s'ensuit forcément qu'elle ne l'était pas tout-á-fait. On a vu que l'instinct pouvait se modifier, et cette constatation est venue à l'appui des théories par lesquelles on a supposé que l'instinct s'était formé peu à peu et qu'il ne differait pas essentiellement des ébauches d'instincts que nous trouvons chez l'homme (à côté d'instincts réritables) et qui sont des formes de l'intelligence, du sentiment et de la volonté. Si l'on trouvait de même quelque variation dans les lois chiniques, on pourrait en tirer des conséquences intéressantes. Mais nous n'en avons pas besoin pour défendre les idées cxposées ici. Les lois chimiques n'ont pas toujours existé dans notre monde qui parait avoir été autrefois porté à une température à laquelle aucune combinaison ne pouvait s'effectuer. Elles existaient si l'on veut virtuellement a l'état pour ainsi dire de lois morales que les atomes se seraient formulées s'ils en avaient eu les moyens. De même aujourd'hui d'innombrables lois sociologiques et psychologiques existent virtuellenent et se manifesteront des que les circonstances le permettront, si elles le permettent jamais, des yue l'homme sera arrive, s'il y arrive, à l'etat le jerfectionnement voulu. Que maintenạnt l'état chimifue actuel se soit produit sans tàtommement, au lieu que 
les phénomènes complexes de la psychologie et de la sociologie ne peuvent se coordonner régulierement qu'en cherehant péniblement leur voie, si je puis dire; et par de longues hésitations, des raccords successifs et des tàtonnements nombreux - ou que l'évolution chimique ait été analogue à l'érolution vitale, physique et sociale, il nous importe peu en somme. Notre but était de comparer les lois synthėtiques de la psychologie avec celles du monde général, et il me semble que, des considérations qui précèdent, il ressort arec une conception particulière des lois de la synthèse psychique et sociale, une idée des lois naturelles qui diffère en quelques points de celle qui est aujourd'hui généralement acceptée !.

$\S 3$.

L'étude des lois de l'esprit et leur comparaison arec les lois de l'univers nous montrent done certaines analogies

1. Il est une deruière façon de considérer les lois de l'esprit que nous devons indiquer aussi : c'est le point de rue de la logique générale. Traiter la question en détail serait entrer dans la philosophie gẻnérale plus que je ne puis le faire ici, et d'ailleurs i'en ai déjà dit quel ques mots dans un chapitre précédent, je me bornerai à indiquer ce qui garait se rapporter le plus à notre sujet.

Pour nous, les lois sont des éléments réels des phénomènes, non pas des éléments coucrets, mais des éléments très réels, la forme des phénomènes, l'ordre selon lequel les élèments quelconques des phénomènes se groupent et se succèdent. Pour le criticisme, les lois psychologiques sont des catégories, des formes imposées à l'esprit et par l'esprit, sans les; quelles nul fait, nulle expérience n'est possible. Sans doute lorsque des phénomènes s'arrangent ou se suivent d'après une certaine :oi, cette loi est un élèment essentiel des phénomènes - le phènomène n'existerait pas suns elle, pas plus qu'elle n'existerait sans le fait. Au point de vue de la logique et de lexpérience, la conclusion est, a certains égards inattaquable, les faits ne subsistent que par les lois (non point bien entendu par lez formules que nous en donnons) et les lois ne se réalisent que par les faits. Nous nous sommes déjà espliqués sur ce point.

Ou la situation du criticisme parait moins bonne, c'est lorsqu'il se 
que nous avons pris soin de ne pas exagérer, et aussi de grandes différences parmi lesquelles les plus importantes sont d'ailleurs si évidentes que j'ai eu à peine besoin de les indiquer. D'un côté, outre la finalité que le monde reçoit de l'homme et qui est relative à l'homme, il semble bien qu'il y ait aussi une sorte de finalité immanente non pas dans le monde, considéré dans son ensemble, mais dans les éléments qui le composent et dans quelques-uns

représente ces lois comme des types immuables et sans lesqueiles aucune expérience, aucun fait n'aurait jamais étè possible, lorsqu'il affirme que vouloir expliquer par l'expérience la formation de certaines de ces lois, c'est vouloir rendre raison par l'expérience de ce qui est la condition même de l'expérience.

Il se cache souvent certaines équivoques dans les théories particulières émises à ce sujet; on a dit par exemple qüil était vain de chercher l'origine de l'idée du temps, le temps étant la forme mème de l'esprit, sans laquelle l'esprit n'existerait pas, et que l'on est obligé de postuler alors mème que l'on croit devoir en expliquer la genèse. Mais si cela tend à prouver que le temps est la forme mème de l'esprit, c'est-à-dire, je jense, que tous les états psychiques ont, pour notre expérience soigneusement appliquée, une certaine durée, cela ne prouve pas que la considération de cet élément ahstrait des phénomènes psychiques : la durée, n’ait pu varier en précision et que l'on ne puisse ètudier la formation de l'idée du temps. c'est-à-dire lidée d'un élément des phénomènes psychiques abstrait par l'esprit même, à un moment de son fonctionnement et considẻrée à part, et mème l'appréciation de cet élément dans les divers concrets où il figure. A mesure que l'homme prend pour objet d'étude son propre esprit, il est tout naturel que les formes essentielles de cet esprit deviennent pour lui un objet de considération, un sujet d'études et donnent lieu à des systèmes d'idées et d'appréciations de plus en jlus nettes, précises et cohérentes. De même pour l'espace et pour tout le restc.

En somme, il ne semble pas que la conception criticiste des lois de l'esprit doive nous faire modifier ce qui a été déjà dit précédemment, surtout en tant que nous restons dans le domaine de la psychologie et de la philosophie objectivis (le scul domaine peut-être qu'on puisse non seulement explorer mais aussi imaginer). Quant a la théorie de Ja connaissance, nous navons pas a la discuter ici. Je dirai seulement que ce nouveau point de vue ne me paraît nullement devoir modifier les résultats que nous avons obtenus d'autre part. 
des modes de groupement de ces éléments, les systèmes stellaires, les organismes, les sociétés. D'un autre côté, nous avons vu que la loi de finalité était à certains égards moins inparfaite dans les molécules que dans les éléments organiques, dans les éléments organiques que dans les éléments psychiques, dans les éléments psychiques que dans l'esprit, dans les esprits inférieurs que dans les esprits supérieurs. A mesure que les phénomènes se compliquent et que les synthèses deviennent plus vastes, elles deviennent aussi plus difficiles, et elles ne sont pas encore arrivées, en supposant qu'elles doivent jamais y arriver, à un état aussi voisin de la perfection harmonique résultant d'un consensus d'éléments parfaitement harmonisès.

Ici encore les rêves peuvent se donner carrière, on peut entrevoir, dans un avenir très vague, la venue du "règne des fins ", l'homme entièrement systématisé, la société parfaitement organisée, les relations entre êtres animés triomphant des obtacles que l'espace leur oppose et le monde intérieur lui-même harmonisé par l'esprit sorti de son sein, par l'activité systématique de ceux de ses éléments qui ont pu réussir à former les combinaisons les plus élevées. Je n'insiste pas, - ce sont là des rêves, et les plus agréables sont en général ceux que chacun fait pour lui-même. - Mais il est intéressant de constater cette sorte de long effort de la matière partant on ne sait de quel état et arrivant à l'atome d'abord, puis à la molécule, à l'organisation des systèmes stellaires, et sur un ou plusieurs des élėments de ces systèmes, aux combinaisons organiques, à l'organisation, à l'esprit, à l'état social. Si nous allons des phẻnomènes les moins complexes aux phénomènes les plus complexes, nous trouvons une systématisation croissante caractérisée par le nombre de plus en plus considérable des éléments directement unis dans le système, par le nombre de plus en plus considérable 
des éléments extérieurs aux systèmes et qui cependant sont l'éunis en lui et par lui, pour lui pernettre d'exercer et d'élendre encore son aclivité, et par la complication croissante des relations secondaires qui unissent les divers éléments du système ell des groupes syıthétisés eux-mèmes, enfin par l'élinination de plus en plus complete de tout ce qui entrave l'activité systématique, par la pureté plus grande du caractère harmonique de cette actıvité. Si nous allons des phénomènes les plus conılexes aux moins complexes, nous observons une dégradation corrélative, mais si loin que nous puissions aller dans l'étude des éléments, nous trouvons toujours cette activité systématisée dont nous avons reconnu dans l'esprit humain une forme supérieure, et il est permis de croire que c'est parce que la finalité se manifeste dans lés éléments qu'clle se manifeste - pour d'autres raisons aussi - dans les composẻs. 
Ces questions philosophiques ne doivent pas nous faire perdre de vue le but essentiel de cet ouvrage. Il'ne s'agit pas tant de comparer l'esprit au monde que de voir ce qu'il en est lui-mème, et si l'étude sommaire des lois générales de l'univers peut nous aider en cela, ce n'est cependant qu'un complément de notre étude, une interprétation générale de nos résultats qu'elle peut nous fournir.

L'esprit en lui-mème nous est apparu comme étant essentiellement une activité synthétique. Nous en avons d'abord étudié les éléments, nous avons dù reconnaitre qu'ils avaient une activité propre, et relativement indépendante, un peu comme celle des hommes, des familles, des partis qui composent une société et qui sont mieux unifiés qu'elles. Etudiant ensuite les lois de l'activité mentale, nous avons reconnu que la loi principale était une loi de finalité, la loi d'association systématique que nous avons étudiẻe successivement dans la perception, les idées, les sentiments, la volonté, la personnalité, loi complétée par la loi d'inhibition systématisée qui est, pour ainsi dire, son complément, et qui par sa combinaison avec la première, et dans certaines circonstances que j'ai tàché de préciser, donnait lieu à des fornies particulières de phénomènes exprimées par la loi du contraste et aussi par les lois moins importantes d'association par ressemblance et d'association par contiguïté. Reprenant la question à un point de vue synthétique, nous avons étudié le jeu des lois dans les phénomènes concrets, en prenant comme sujet d'étude d'abord une tendance, l'amour, puis un élément important de 
notre vie psychique, le langage, et enfin le développement d'une persomnalité l'éelle. Ensuite, résumant à la fois l'étude des éléments de l'esprit et l'étude des lois de l'activité mentale qui en expriment le groupement, nous avons trouvé dans l'esprit un une synthèse - imparfaite pour tous les esprits que nous pouvons comnaitre - d'éléments psychiques et en dernière analyse d'éléments organiques coordonn is de manière à ce que leur ensemble puisse entrer comme élément dans une synthèse sociale. Les éléments psychiques eux-mèmes nous ont semblé être fortement influencés par la société, et aussi par le monde extéricur sur lequel l'esprit réagit de son côté, et l'esprit nous a paru a la fois, en même temps qu'une synthèse organique et un élément social, une synthèse de produits sociaux et une sỵthèse d'impressions extérieures établissant entre les plénomènes du monde cxtéricur une systématisation idéale ou réclle dont il est le centre, introduisant dans ce monde une finalité qui n'est pas une illusion, mais qui est tout entière en lui, et, lui supprimé, n'existe plus. Enfin, la comparaison des lois de l'esprit et du monde nous it permis de reconnaitre au-dessus et au-dessous de l'esprit une serre d'activités synthétiques commençant à la société el finissint à l'atome, activités d'autant moins unifiées qu'elles sont jlus complexes et dont les lois, dans les domaines les plus élevés, sont encore en voie de formation. Toutefois nous avons tîché, au cours de ce travail, de déterminer ruelle était li forme particulière des lois générales (pui constituent l'esprit et les diverses catégories de phenomines psychiques. C'est ainsi que nous avons trouvis que des élements differents et des groupenents divers produisaient les divers phénomènes de l'esprit: lererptious, idees, jugements, raisonnements, sentiments, tendances, volonté, soms-persomnalites - nous avons ossily pour rlargue grompe de déterminer la nature des dements et rello de lat loi qui les missait. Quand at 
l'esprit même, à la personnalité, nous avons reconnu en elle un élément social, - la loi qui en unit les èléments, less coordonne de manière à les rendre aptes a prendre une place déterminée dans le consensus social, et le caractère nous a paru se réduire à des associations systématiques et á des formes de ces associations systématiques auxquelles la société donnait un sens et un emploi. Synthèse des phénomènes organiques et des phénomènes psychiques qui sont de véritables produits sociaux, élément du système social, principe de finalité dans le monde, voilà les trois principaux caractères de l'esprit. 



\section{TABLE DES MATIËRES}

Pages.

i.strodetion

\section{PREMIËRE PARTIE}

La vie des éléments psychiques.

chapitre premir Les elements psychiques..............

chapitro $11 . .$. L'activité indépendante des éléments psychiques.................. 17

Ch.ıpitre IL..... Eléments psỵchiqüns et facultés.........

cirnpitre $11 . .$. Les causes qui favorisent ou qui contrarient l'activité ‘les éléments psychiques...... 66 conclusioni, $\ldots \ldots \ldots \ldots \ldots \ldots \ldots \ldots \ldots \ldots \ldots \ldots \ldots$

\section{DEUXIĖME PARTIE}

\section{Les lois de l'activité mentale.}

\section{LIVRE PREMIER.}

La loi d'association systèmatique...............

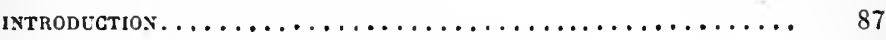

Chapitre Prenier Sensations et perceptions............ 90 chapitre Il .... L'Intelligence. - Images, idées, jugements, raisonnements ............... 106

CilaptTre III.... Phénomènes alfectifs et tendances........ 140 chapitre IF.... Le pouvoir personnel. - Conscience, attention, volonte............... 157

ChaptTre v..... La personnalité................ 180 coschusion............................. 216

\section{LITRE II.}

La loi d'inhibition systematiquc............... introdection............................ 221 chapitre premier Sensations et perceptions............. chapttre n..... L. Intelligence. - Images, idées, jugements, raisonnements ............... 239 chipltae $11 . .$. Phénomènes affectifs et tendances........ 
GHAPITaE IV.... Le pouvoir porsonnel ............ 28 t

chapitre v..... La personnalité................ 297

RÉSUMÉ ET CONCLUSION..................... 311

LIVRE III.

L'association par contraste................. 315

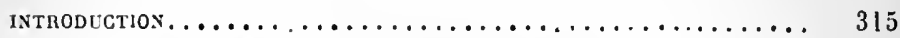

Chapitre rremier Le contraste simultané............. 317

Chapitre in .... Le contraste successif............. 365

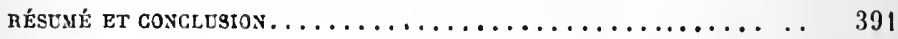

LIVRE IV.

Les associations par contiguïté et ressemblance...... 394 RÉScMé ET CONGLUSION DE LA SECONdE PAK'TIE............ 450

\section{TROISIEME PARTIE}

\section{L'Esprit.}

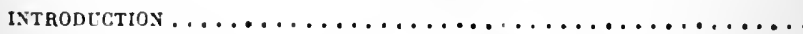

LIVRE PREMIER.

Synthèses concrètes..................... 549

ciapitre premier Synthèses partielles. - Lamour, le langage 459

chapitre il ..... Syntlıèses générales. - La formation de la personnalité. - Darwin ............

LIVRE II.

La synthèse abstraite.

Cinal'jthe premier L'esprit comme synthèse d'éléments psychiques t organiques............. 506

chapitre II ..... Lesprit comme élément social et synthèse de prorluits sociaux............. 531

Crapitne II..... L'esprit el le mondo extérieur.......... 549

conclusion............................ 559

LIVRE III.

Lois de l'esprit et lois du monde................ 561 conchtsiox............................ 583 
ix $x+8$

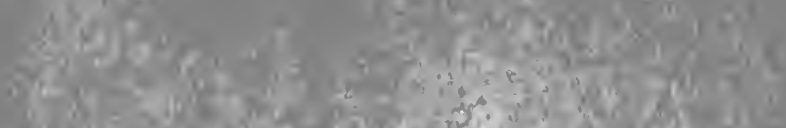

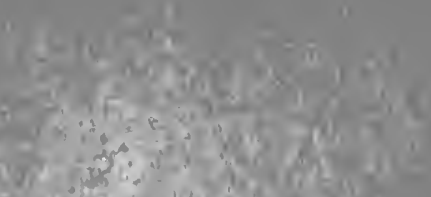

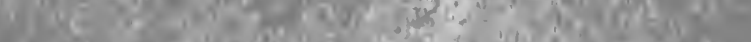

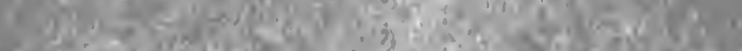

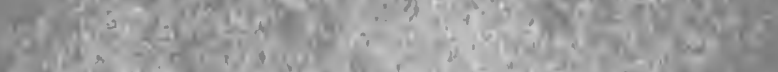

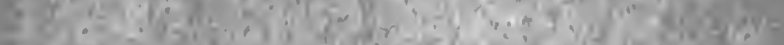

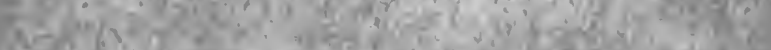

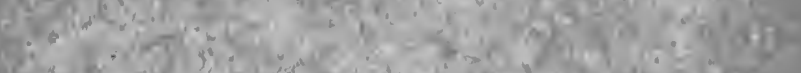

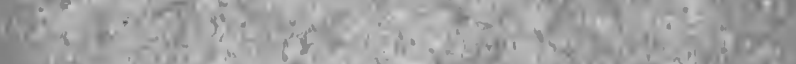

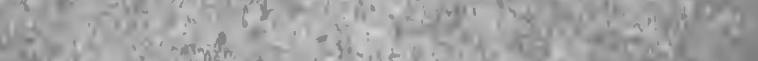

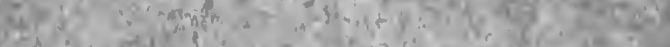

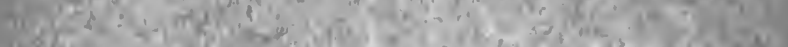

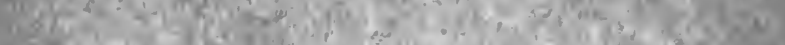

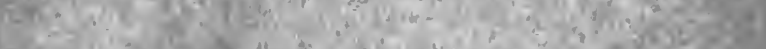

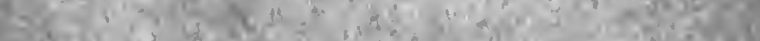

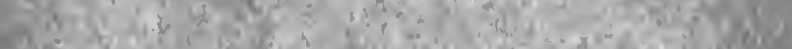

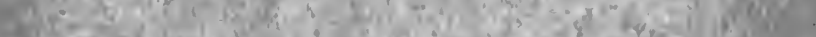

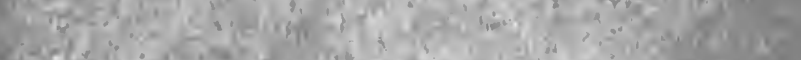

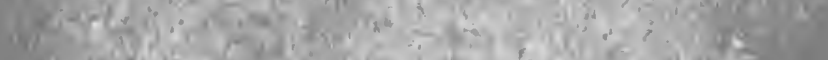

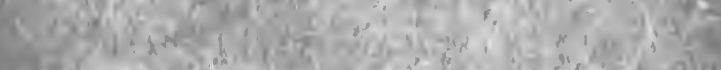

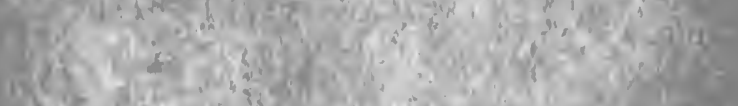

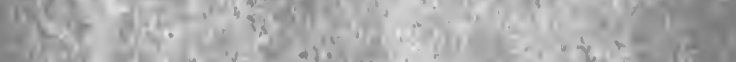

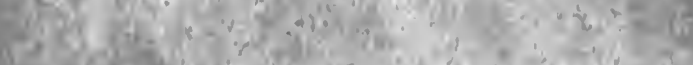

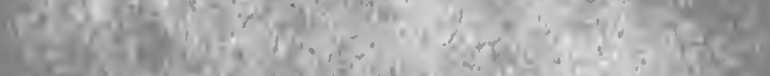

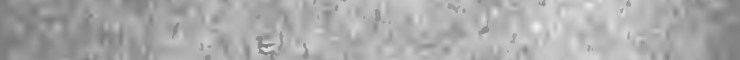

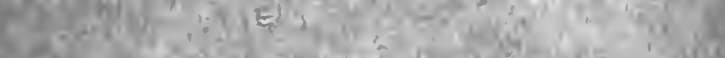

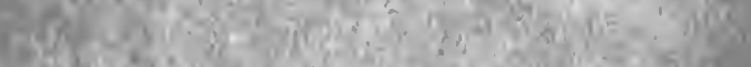

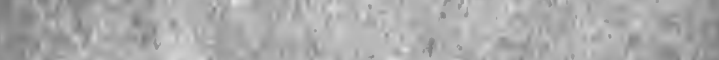

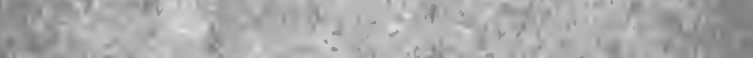

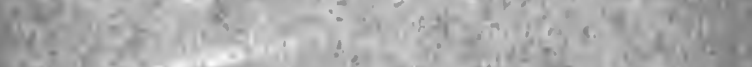

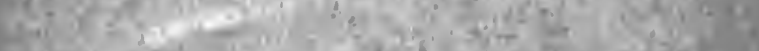

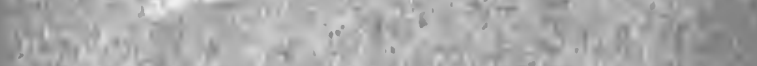

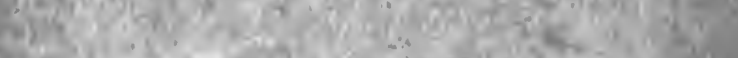

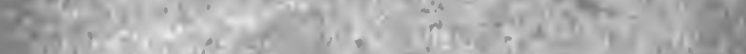

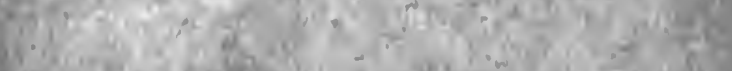
Wy

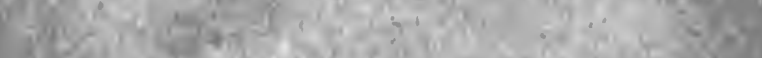

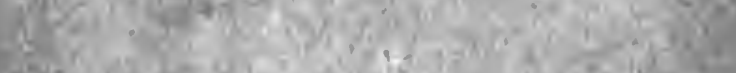

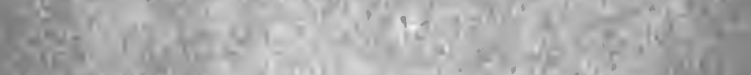

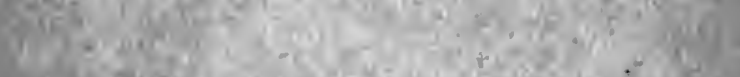

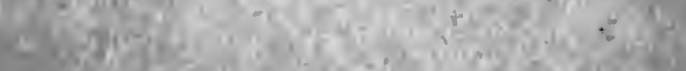

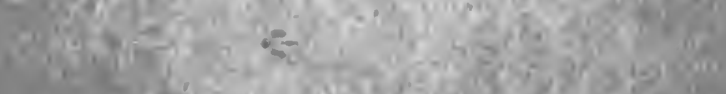

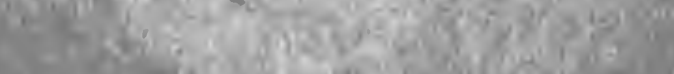

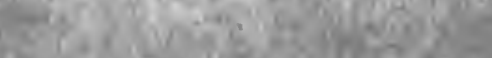

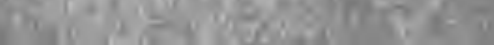




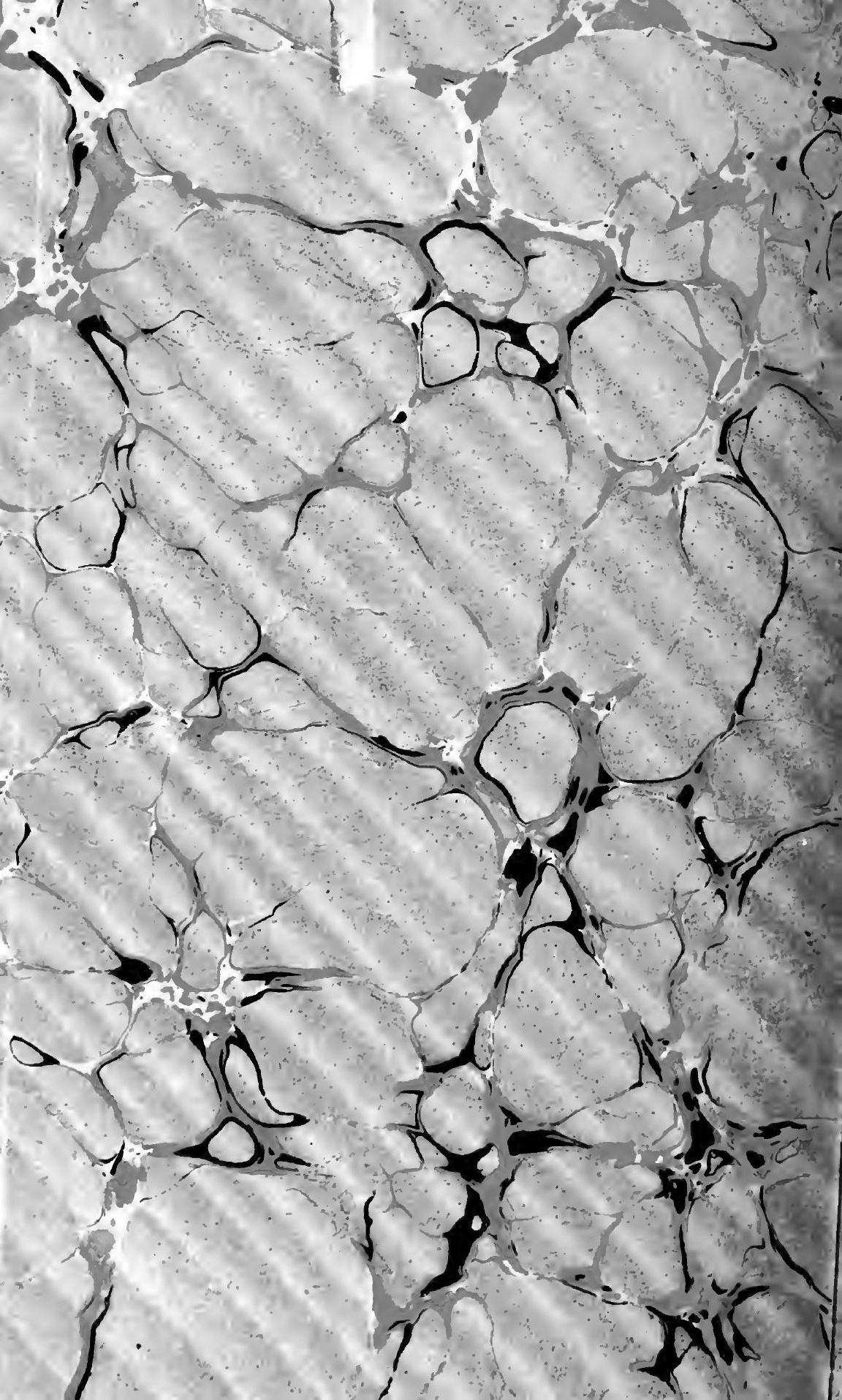




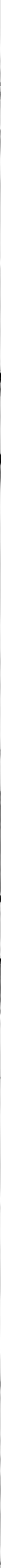


